\title{
DETLEF KOCHAN
}

\section{Die TU Dresden als eine Keimzelle der Digitalisierung im Maschinenbau}

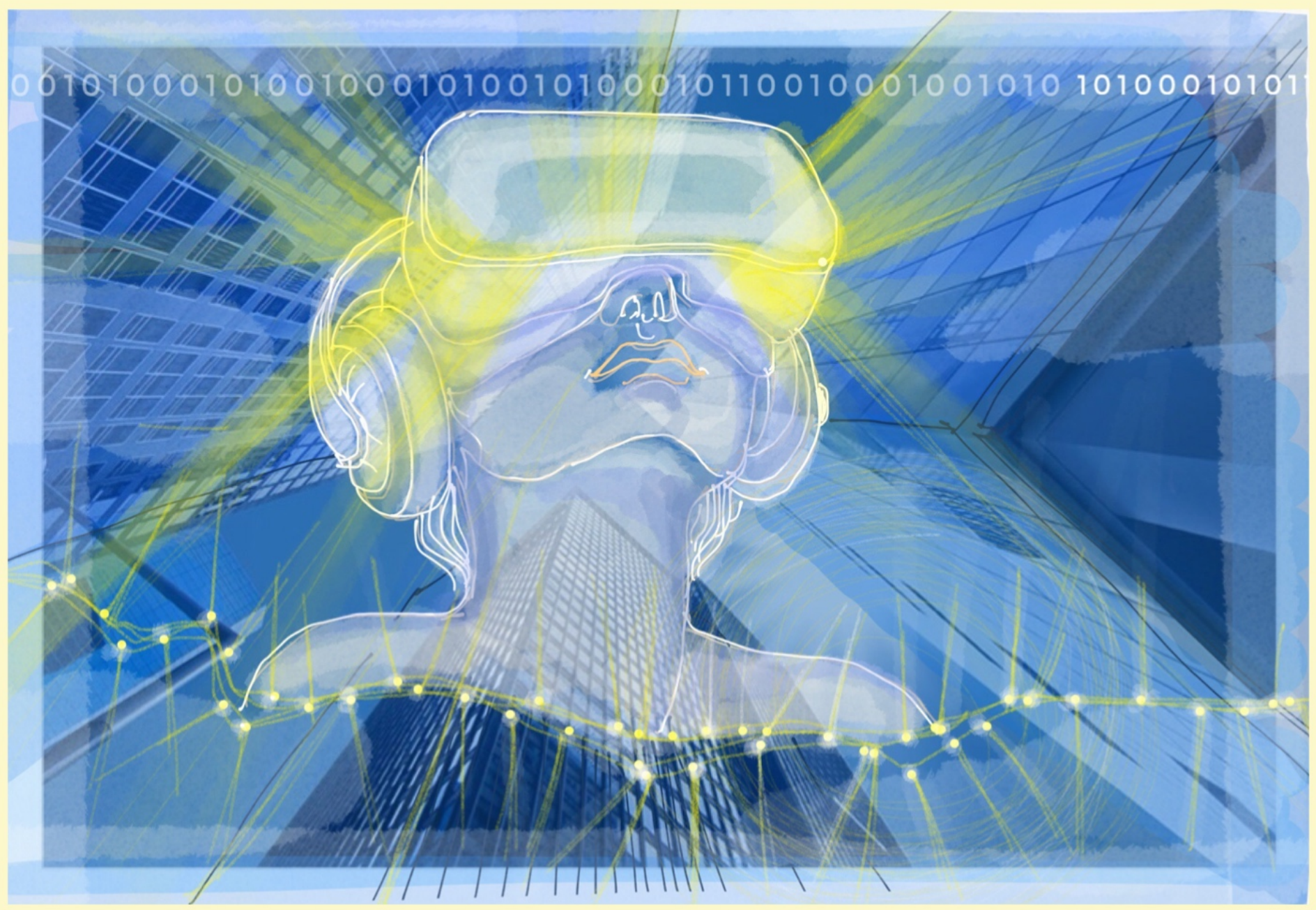

"Wer die Vergangenheit und Gegenwart nicht kennt, kann die zukunft nicht gestalten." 


\title{
AKTIVITÄTEN UND ERFAHRUNGEN IN DER DEUTSCH - DEUTSCHEN UND INTERNATIONALEN ZUSAMMENARBEIT VON 1960 BIS 2020
}

\author{
Prof. Dr. sc. techn. D. Kochan
}

MIT ERGÄNZENDEN UND VERTIEFENDEN BEITRÄGEN VON:

Prof. Dr. Dieter Weiß

Dr. Ing. Hubert Dolze

Prof. Dr. Ing. Arndt Richter

Prof. Dr. Ing. Reimund Klünder

Prof. Dr. Ing. habil. Andreas Nestler

Dr. sc. techn. Manfred Rahmig

Dr. Ing. Barbara Schennerlein

Prof. Dr. sc. techn. Peter Bonitz

Dr. Ing. Ingolf Berndt

Dr. Ing. Christine Schöne

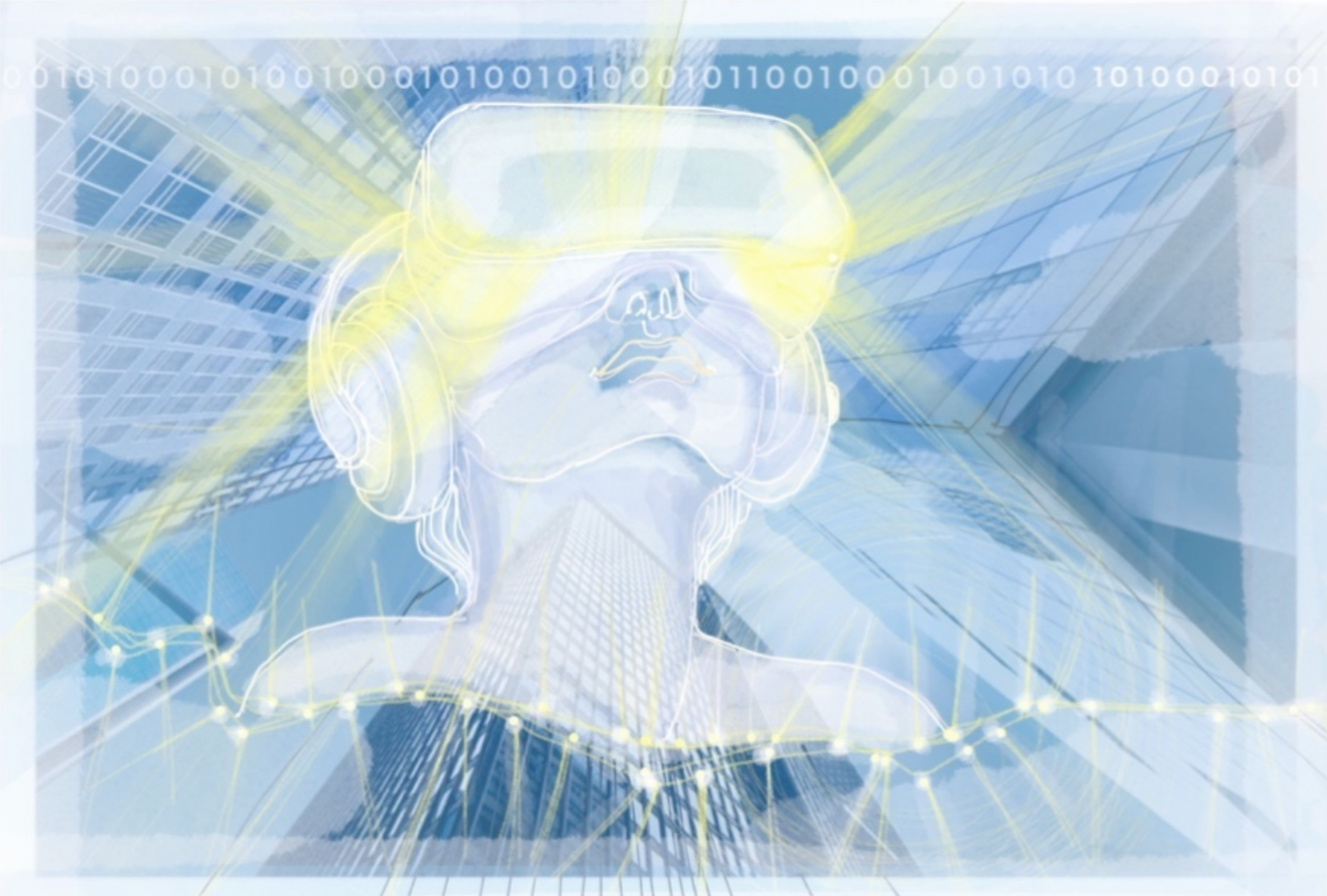




\section{Abstract}

Von Beginn der flexiblen Automatisierung mit numerisch gesteuerten Werkzeugmaschinen und der zugehörigen Programmier-Software bis zum gegenwärtigen Entwicklungsstand (Industrie 4.0) wird die historische Entwicklung von 1960 bis 2020 aus der Position eines aktiven Mitgestalters dargestellt. Interessanterweise vollzogen sich die wesentlichen Entwicklungsetappen für die ersten dreißig Jahre parallel in beiden deutschen Staaten. Aus den Lehren des Zweiten Weltkrieges wurden im Rahmen der UNESCO zum friedlichen Informationsaustausch geeignete wissenschaftliche Organisationen gegründet:

- IFIP (Internationale Föderation für Informationsprozesse, speziell Arbeitsgrupp CAM

- $\quad$ CIRP (Internationale Akademie der Fertigungstechniker)

Mit der Berufung und aktiven Mitarbeit in diesen Organisationen war eine Plattform für die deutsch-deutsche und darüber hinaus internationale Kooperation gegeben. Ein besonderer Schwerpunkt für den geordneten Informationsaustausch im Rahmen der gesamten dynamischen Entwicklung im Gebiet der Produktionsautomatisierung war dabei die im 3-Jahres-Rhythmus durchgeführte Konferenzserie PROLAMAT (Programming Languages for Machine Tools), gestartet 1969 in Rom. Im weiteren Verlauf wurde dieser Begriff viel breiter für das gesamte Gebiet der automatisierten Informationsverarbeitung und Fertigung erweitert.Ein besonderer Höhepunkt war dabei die erfolgreichste PROLAMAT-Konferenz 1988 in Dresden.

Parallel dazu erfolgten an der TU Dresden Entwicklungen in Richtung CAD/CAM -Labor und später CIM-TT (CIM-Technologietransferzentrum). Damit war an der TU Dresden 1989/90 ein Entwicklungsstand gegeben, der unmittelbar zu gemeinsamen deutschdeutschen und internationalen EU-Projekten genutzt werden konnte. Dieses hohe Entwicklungsniveau wurde zur offiziellen Eröffnung des CIM-TT-Zentrums in den Eröffnungsreferaten durch den damaligen Wissenschaftsminister Dr. Riesenhuber und Ministerpräsident Prof. Biedenkopf gewürdigt. Durch die zum gleichen Zeitpunkt verfügte veränderte Nutzung des für das CIM-TT im Aufbau befindliche Gebäude durch die neugegründete Juristische Fakultät wurde der erfolgreich vorbereitete Weg verhindert Unabhängig davon blieb meine fachliche Orientierung mit den gravierenden Weiterentwicklungen eng verbunden. Dazu trug das Sabbatical-Jahr in Norwegen und den USA 1992 maßgeblich bei. Mit dem Forschungsaufenthalt war die Entscheidungsvorbereitung für die vorgesehene Groß-Investition für das neueste generative Verfahren verbunden. Gleichzeitig mit dem fundierten Nachweis der bestgeeigneten sog. Rapid-Prototyping-Anlage vom deutschen Anbieter EOS München war die TU Dresden auf diesem neuen High-Tech-Gebiet 1992 in einer anerkannten Spitzenposition. Mit meiner Publikation eines der ersten Fachbücher im Gebiet Advanced Prototyping (jetzt Additiv Manufacturing) war darüber hinaus eine gute Basis für weitere innovative Aktivitäten gegeben Dazu gehört die Gründung einer High-Tech-Firma (SFM - Schnelle Fertigung von Modellen) mit bemerkens- 
werten beispielgebenden Ergebnissen. Hervorgehoben soll die zwanzigjährige aktive Kooperation mit der Universität Stellenbosch (RSA - Republik Südafrika), die unter anderem mit meiner Berufung zum Extraordinary Professor im Jahr 2003 verbunden ist. Mit der Eröffnung eines Technologie-Zentrums nach dem Vorbild des ursprünglichen CIM TT Zentrums der TU Dresden konnte für Südafrika ein wertvoller Beitrag geleistet werden

Das gesamte Lebenswerk ist gekennzeichnet durch die Entwicklungsschritte von der Mathematisierung über die Algorithmierung bis hin zur Programmierung vielfältiger technologischer Sachverhalte. Die Ergebnisse sind in einer Anzahl von persönlichen Fachbüchern (z.T. übersetzt in das Russische und Ungarische) wie auch Konferenzberichten und mehr als 200 Veröffentlichungen (deutsch und englisch) dokumentiert.

Dresden im April 2021

Detlef Kochan

PS: Nach Redaktionsschluß dieser Memoiren ergab sich die Gelegenheit, an einem Ideen-Wettbewerb zur Förderinitiative „Wissen schafft Perspektiven für die Region“ teilzunehmen. Mit einem jungen Team engagierter Fachkollegen im Rahmen des VDI BV Dresden wurde der Vorschlag: IAFI-,Industrierelevantes Applikations-, Weiterbildungsund Innovations-Zentrum Lausitz" erarbeitet und eingereicht. Über die Förderung der Kohleregion hinausgehend erwarte ich davon wirksame Impulse zum dringend notwendigen Erhöhen des Produktivitätsniveaus im Freistaat Sachsen. 


\section{Inhaltsverzeichnis}

\section{Abstract}

1 Ursprünge und Entwicklungen zur Digitalisierung im Maschinenbau

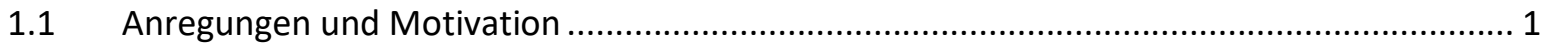

1.2 Grundsätze und Grundpositionen der wissenschaftlichen Aktivitäten im technologischen Umfeld

1.3 Internationale Organisationen und Kriterien der internationalen Anerkennung.................. 3

2 Vorreiter und Pioniere der historischen Entwicklung ............................................................. 4

2.1 Prof. Dr. Alfred Richter - Gründer des Institutes für Fertigungstechnik............................... 4

2.2 Prof. Dr. Herbert Seidel - Aufbau des Lehrgebietes „Industrielle Fertigung und

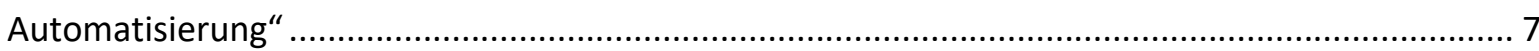

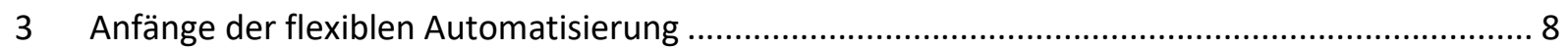

3.1 Inhaltliche Schwerpunkte erster NC-Programmiersprachen ............................................ 8

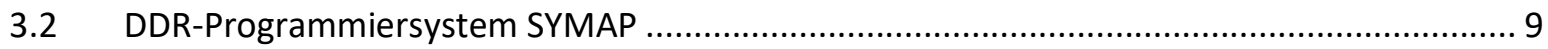

3.3 Internationale Ausstrahlung und Wirksamkeit .............................................................. 11

4 Ausbildungsinhalte und Schwerpunkte der Lehre und Forschung.............................................. 12

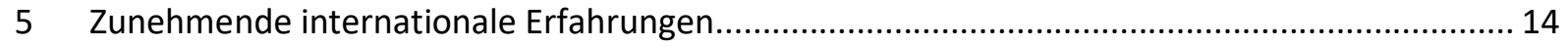

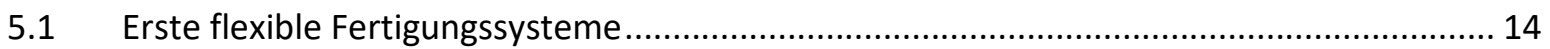

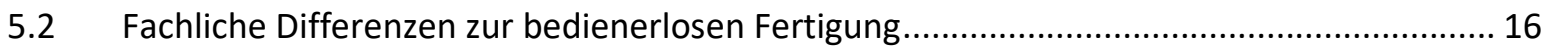

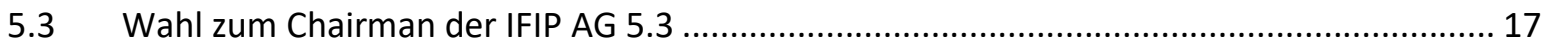

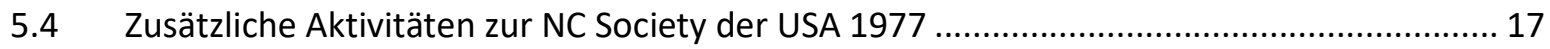

5.5 Beratung des Programmkomitees für PROLAMAT 1979 an der TU Dresden........................ 18

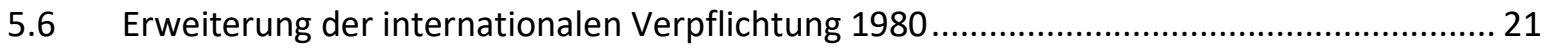

5.7 Arbeitskonferenz zur Anwendung der künstlichen Intelligenz in der Fertigungstechnik ..... 23

$5.8 \quad$ IFIP-Arbeitskonferenz zur fachlichen Vorbereitung von PROLAMAT 1988.......................... 24

5.9 Zwischenbemerkungen zu DDR-Entwicklungen der bedienerarmen Fertigung ................... 25

6 Entwicklungen zur rechnerintegrierten Fertigung ................................................................ 26

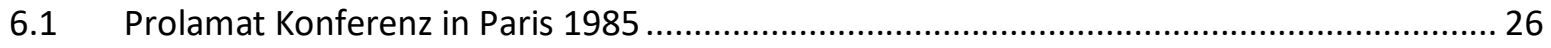

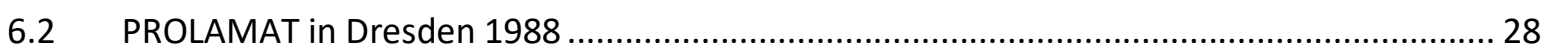

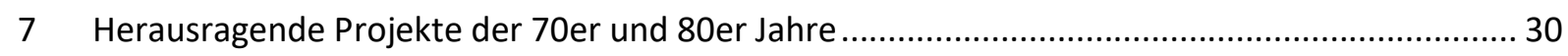

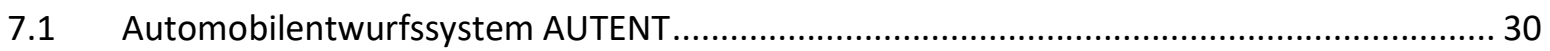

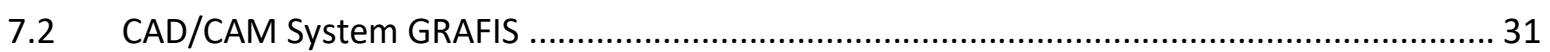

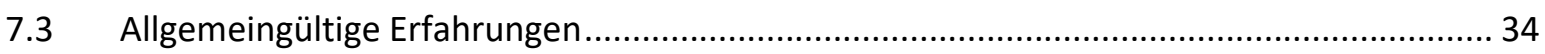

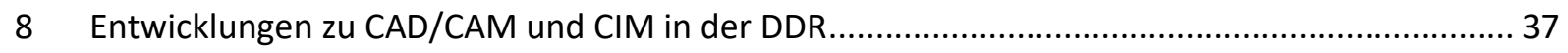

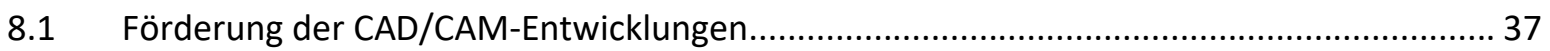

8.2 Internationale Unterstützung für Entwicklungsländer..................................................... 38

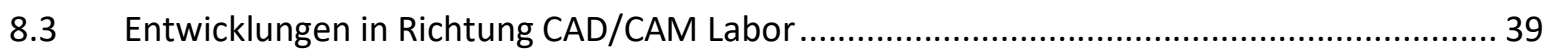


10 Internationale Aktivitäten nach der Wiederververeinigung

10.1 15. Problemseminar "Rechnerunterstützte Fertigungstechnik“.

10.2 Spezieller Höhepunkt: Sonderveranstaltung „Innovation und Motivation für den industriellen Aufschwung" 46

10.3 IFIP-Arbeitskonferenz „Prozess Planning for Complex Machinig with Al-Methods“ November 1991 in Gaußig.......

11 Entwicklungsziele vor und nach der historischen Wende 1989........................................... 53

11.1 Schlussfolgerung für künftige Kooperationen und aktuelle Situation ................................. 54

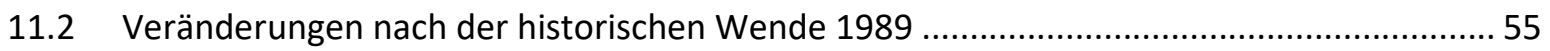

12 Forschungsjahr für revolutionierend neues generatives Fertigungsverfahren.......................... 55

12.1 Studienaufenthalte für Theorie und Praxis in Norwegen und USA.....................................5 56

12.2 Fachliche Unterstützung der neuen High-Tech-Firma EOS München.................................. 56

12.3 Nutzung hochwertiger Innovationen für verbesserte Ausbildung und Forschung ...............57

12.4 Ehrenkolloquium der CIRP für NC-Pioniere 1993 ….......................................................... 59

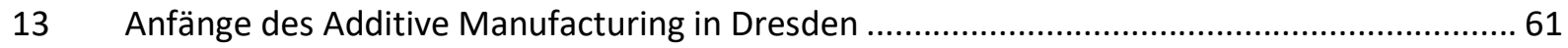

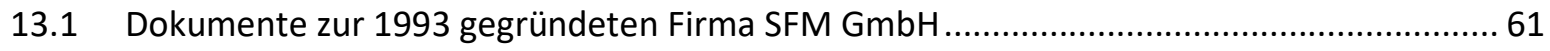

13.2 Höhepunkt der Firmenentwicklung mit der ersten Panorama-Kamera ............................... 64

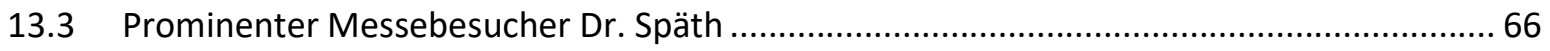

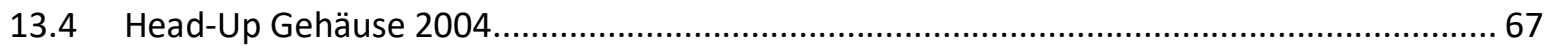

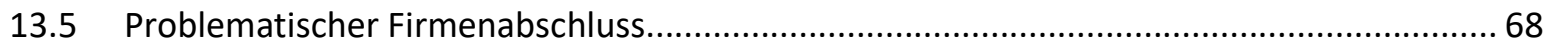

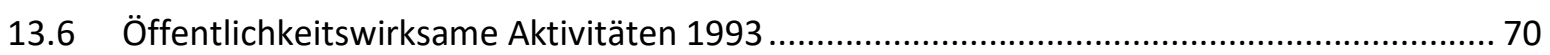

14 Beginn der aktiven Zusammenarbeit mit der NC-G für Neue Technologien $1992-1997 . . . . . . .71$

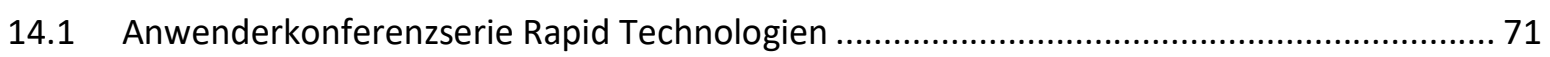

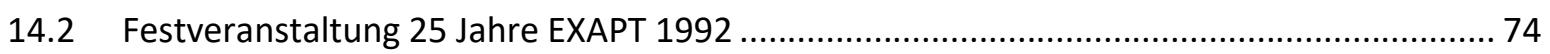

15 Öffentlichkeitswirksame Aktivitäten 1993 bis 2005 ............................................................... 74

16 Internationale Ausstrahlung in die Republik Südafrika 1994 bis 2018 ................................... 76

16.1 Aktivitäten zu einer neuen Technologie für die Kunststoffverarbeitung LOMOLD 1996 bis 201579

17 Abschließende Verpflichtung als Mitglied des IFIP TC 5 und der IFIP Arbeitsgruppe $5.3 \ldots \ldots . . .82$

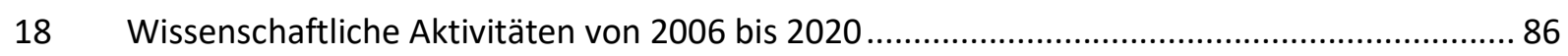

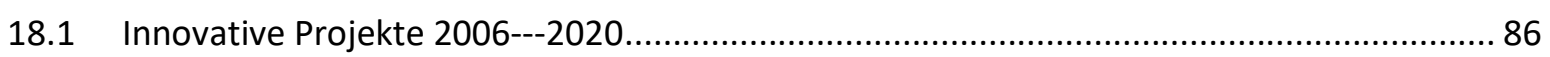

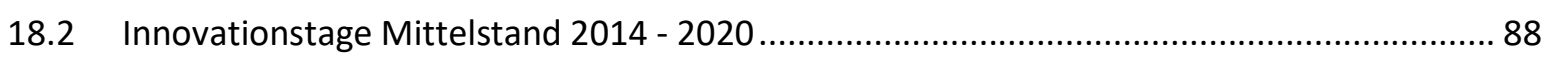

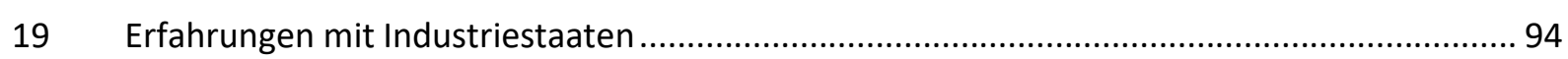

19.1 Bemerkungen zu USA-Entwicklungen in der Fertigungstechnik und dem

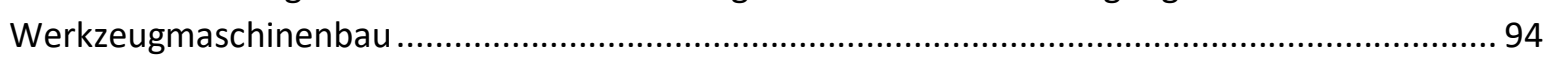

19.2 Erfahrungen mit den industriellen Entwicklungen in der Volksrepublik China ................... 96 
19.3 Überraschende Kommunikation mit Süd-Korea. 98

20 Aktivitäten in Richtung Leichtbau-Werkzeugmaschinenkomponenten 2017 bis 2020 98

21 Entwicklung der Absolventenzahlen im Gebiet Produktionsautomatisierung, Zerspanen und Abtragtechnik an der TU Dresden..... 101

22 Zusammenfassende Bemerkungen, Schlussfolgerungen und Empfehlungen 102

22.1 Generelle Entwicklungstrends in der Wissenschaft. 102

22.2 Entwicklungen der Produktionsautomatisierung 103

22.3 Digitalisierung. 104

22.4 Bemerkungen zur künstlichen Intelligenz (KI). 105

22.5 Beispiele und Schlussfolgerungen, Empfehlungen bedeutender Automatisierungsvorhaben. 106

22.5.1 Flexible Fertigungssysteme PRISMA 2 und ROTA F125 .................................. 106

22.5.2 Automobil-Entwurfssystem AUTENT ......................................................... 107

22.5 .3 CAD/CAM-System GRAFIS ..................................................................... 108

22.5.4 Hoch automatisierter Sandguss im ehemaligen Unternehmen Salzenforst/ Bautzen. 108

22.5.5 LOMOLD- Unterstützung für ein Südafrikanisches Weltpatent. 108

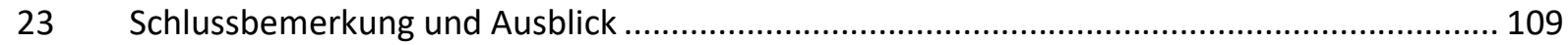

24 Besondere Würdigung herausragender deutscher Persönlichkeiten................................ 109

24.1 Professor Spur, anerkannter internationaler Pionier der Fabrik der Zukunft................... 109

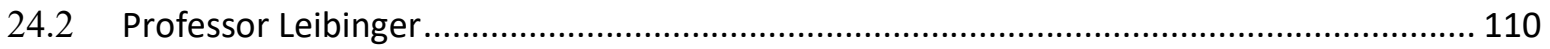

24.3 Professor H. Jäger Direktor des ILK der TU Dresden ............................................ 111

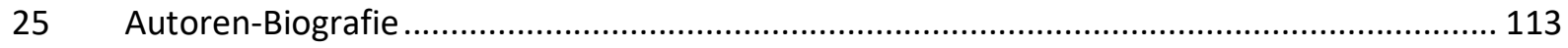

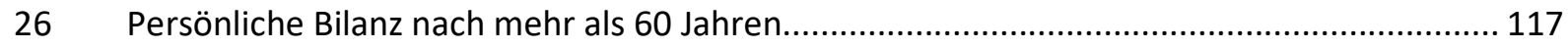

Anmerkung: Die Originaldokumente (Abbildungen) befinden sich im Privat-Archiv des Autors. 


\section{Schlüsselbegriffe}

AM

AM

AC

ACO

Al

APS

APT

CNC

Bottom Up

CAD/ CAM

CIM

CIRP

CPPS

Digitalisieren

Diaplay

EXAPT

Expertensystem

FEM

FMS

GRAFIS

IFAC

IFIP

IGES

ISO

MAP

NC-M

Programmiersystem
Additive Manufacturing (Rapid Prototyping, 3D-Drucken)

Advanced Manufacturing

Adaptive Control

Adaptive Control Optimization

Artificial Intelligence

Advanced Production Systems

Automatically Programming for Tools

Computer Numerical Control

Vorgehensweise der Einordnung eines speziellen Systems innerhalb eines komplexen Sachverhaltes (Bottom Up: vom Grund nach oben)

Rechnerunterstütztes Design/ Rechnerunterstützte Fertigung

Computer Integrated Manufacturing

Internationale Organisation für Fertigungstechnik

Cyberphysical Production System

Erfassen einer beliebigen Position, eines Bildelements oder einer 2, bzw 3 dimensionalen Darstellung und deren Umwandlung in eine rechnerinterne, digitale Koordinatendarstellung

Anzeigeeinheit oder Bildschirmgerät

Erstes einheitliches, universelles NC-Programm der BRD

Anwendung der Prinzipien der $\mathrm{KI}$ in der Technik

Methode der Finiten Elemente

Flexible Manufacturing Systems

CAD/ CAM -System der DDR-Schuhindustrie

International Federation of Automatic Control

International Federation of Information Processing

Initial Graphic Exchange Specification

International Organisation of Standardisation

Manufacturing Automatical Protocol

Numerical Control Machine

Gesamtheit der Elemente aus Eingabesystem, Verarbeitungsprogramm und Nachverarbeitungsprogramm 


\section{Ursprünge und Entwicklungen zur Digitalisierung im Maschinenbau}

\subsection{Anregungen und Motivation}

Wenn man als Ingenieur, Hochschullehrer, und auch gesellschaftlich Engagierter, insbesondere im Rahmen VDI, auch an der Bildung und Erziehung mehrerer Generationen

junger Studenten und Mitarbeiter tätig war, ist das Ergebnis der mehr als 60 jährigen Aktivitäten eine ständige Frage, die sich im fortgeschrittenen Alter besonders bemerkbar macht. Bei jeder Begegnung mit ehemaligen Kollegen und Forschungspartnern ergibt sich das natürliche Interesse wie ist die fachliche Entwicklung für jeden Einzelnen nach der erfolgreichen Promotion weiter verlaufen.

Bei den meist kurzen Begegnungen zu Konferenzen und anderen Anlässen ist meist keine Gelegenheit für einen tiefer gehenden Informations- und Gedankenaustausch.

Natürlich steht auch für mich die Frage, ob die Bilanz des fachlichen Lebens bei allen Hürden und Herausforderungen mit einer zufriedenstellenden Einschätzung verbunden werden kann.

Das wird besonders dann problematisch, wenn in der gesellschaftlichen Entwicklung Tendenzen erkennbar werden die nicht auf das Gemeinwohl ausgerichtet sind. Die gegenwärtige Entwicklung in den zukunftsorientierten Fachgebieten wie Produktionsautomatisierung und Digitalisierung im industriellen Umfeld sind nach meiner Auffassung (ganz besonders in dem ehemals leistungsfähigen Freistaat Sachsen) nicht auf dem objektiv notwendigen Niveau. Dies zeigt sich deutlich in der zu häufigen unkorrekten Verwendung gegenwärtiger Schlüsselbegriffe wie "Industrie 4.0 " und auch "Digitalisierung". Deshalb wird im Folgenden der Versuch unternommen, wesentliche Erfahrungen aus der Weiterentwicklung des

- ehemaligen Lehr- und Forschungsgebietes "Industrielle Fertigung und Automatisierung" innerhalb des Institutes für Fertigungstechnik an der TU Dresden zum

- Forschungs- und Lehrgebietes " Fertigungstechnik/Technologische Programmierung" zu dokumentieren und verallgemeinerungsfähige Schlussfolgerungen abzuleiten

- Die Motivation zum Erarbeiten wesentlicher Erkenntnisse in einem wahrhaft revolutionären Wissensgebiet der gesamten fortgeschrittenen Produktionstechnik (Advanced Manufacturing) resultiert aus der Verantwortung die jeder Ingenieur für das Wohlergehen der Gesellschaft übernimmt.

Ausgehend von dem bewährten Grundsatz "Wer die Vergangenheit und Gegenwart nicht kennt, kann die Zukunft nicht gestalten" wird aus der Sicht eines im zweiten Weltkrieg aufgewachsenen Schülers die aktive Mitgestaltung wesentlicher Entwicklungsetappen beginnend vom Übergang der analogen in die numerisch gesteuerten Fertigungseinrichtungen im Vordergrund stehen. Dabei geht es weniger um die konkreten fachlichen Fakten, die in zahlreichen Veröffentlichungen dokumentiert sind .sondern mehr um verallgemeinerungsfähige Erfahrungen.

\subsection{Grundsätze und Grundpositionen der wissenschaftlichen Aktivitäten im technolo- gischen Umfeld}

Wie in jeder technischen Disziplin geht es bei der Erarbeitung wissenschaftlicher Erkenntnisse primär darum, eine industrielle Wirksamkeit nachzuweisen. Dabei kann und muss von der bekannten Tatsache ausgegangen werden, dass mit einer innovativen Idee für ein neues Produkt bzw. einen neuen Verfahrensablauf ca. zehn Prozent des notwendigen Aufwandes erreicht sind. Von einer technischtechnologischen Innovation kann man demnach erst dann sprechen, wenn weitere ca. 90 Prozent des Gesamtaufwandes realisiert sind. 
Das bedeutet wiederum in Verbindung mit spezifischen Software- Entwicklungen für eine effiziente NC- Programmierung und weit darüber hinausgehend, dass jeder konkrete Beitrag im gesamten Umfeld der Produktionsautomatisierung experimentell erprobt sein muss.

Ausgehend von dieser Prämisse bedeutet dieser Ausgangspunkt, dass jede erfolgreiche technologische Entwicklung durch entscheidende Merkmale wie Continous Improvement und das Bottom- Up-Prinzip charakterisiert sein muss.

In Übereinstimmung mit dem deutschen Pionier der Produktionstechnik, Herrn Professor G. Spur geht es um das Verständnis des Wandels der industriellen Welt durch leistungsfähige Produktionstechnik.

Dieser Standpunkt ist in dem Hauptwerk von G. Spur Vom Wandel der industriellen Welt durch Werkzeugmaschinen, eine kulturgeschichtliche Betrachtung der Fertigungstechnik (C. HanserVerlag, München, 1991) dokumentiert:

„Zum Verständnis des Wandels der industriellen Welt gehört eine Rückbesinnung auf grundlegende Beziehungen zwischen Mensch, Arbeit und Werkzeug. Ausgehend von diesem Ursprung verdeutlichen die verschiedenen historischen Phasen fertigungstechnischer sowie arbeitsorganisatorischer Entwicklung, dass die modernen Werkzeugmaschinen einschließlich der Formen ihrer Anwendung in der industriellen Produktion Resultate konstruktiver Erfahrungen und Ideen sind, zu denen jede Epoche der Menschheitsgeschichte ihren besonderen Beitrag geleistet hat.“

In den vergangenen 60 Jahren wurde ein gravierender Wandel mit dem Übergang der analogen zur digitalen Automatisierung vollzogen. Die fortgeschrittene Produktionstechnik (Advanced Manufacturing) wird demnach durch folgende Merkmale charakterisiert.

- Befreiung von schwerer und komplizierter manueller Arbeit, einschließlich der Einbeziehung ingenieurtechnischer Problemstellungen

- Das Einbeziehen der IT- Prinzipien in Form von

-

- Maßzahlen verstehender Einrichtungen ist deshalb ein wichtiger Ausgangspunkt

- Integrationsaspekt ist dabei das Beherrschen von Computern unterschiedlicher Leistungsfähigkeit, Programmen und Steuerungen von immer komplizierteren Fertigungseinrichtungen

Diese Kerngedanken wurden in konzentrierter Form im Geleitwort des Präsidenten des VDW (Verein Deutscher Werkzeugmaschinen), Herrn Dr. B. Kapp dargestellt:

„Es ist ein faszinierender Vorgang, wie sich der Mensch, der sich in einer gezielten Anwendung von durch ihn gestalteten Werkzeugen über die Anfänge seiner Existenz hinaushob, im Laufe der Zeiten sein heutiges Umfeld schuf. Ein wesentlicher Bestandteil der Menschheitsgeschichte ist dieser Evolutionsprozess der Fertigungstechnik vom Faustkeil bis hin zur komplizierten Maschine, die ein oder mehrere Werkzeuge zur Bearbeitung von Metall präzise führt und antreibt, also zur Werkzeugmaschine. (...) Demnach ist die Werkzeugmaschine einer der Grundpfeiler des Industriezeitalters. Sie ist in vielerlei Gestalt die Mutter der Maschinen, wie die Chinesen sie nennen, und in ihrer Bedeutung für die gesamte industrielle Produktion nicht hoch genug einzuschätzen."

Bezugnehmend auf diese historischen Maßstäbe kann man davon ausgehen, dass mit der Digitalisierung im industriellen Umfeld wahrhaft revolutionäre Fortschritte erzielt wurden.

Die Einordnung in die historische Entwicklung bedingt deshalb einen angemessenen Respekt vor den wissenschaftlichen Leistungen sowohl der Vorgängergenerationen als auch der Partner in aktuellen Problemlösungsprozessen. Dabei geht es um das Fokussieren auf eigene messbare Beiträge zum technisch- technologischen Fortschritt. 
Ein wesentliches Kriterium für eine wissenschaftliche Leistung in Form von akademischen Titeln wie Diplomarbeiten oder Dissertationen war im Lehrgebiet Fertigungstechnik/ Technologische Programmierung der Nachweis industrieller Nutzbarkeit.

Ein wichtiges Kriterium in diesem Zusammenhang ist die öffentlichkeitswirksame Demonstration in Form von Veröffentlichungen bzw. Vortragstätigkeit zu Fachkonferenzen. Der angestrebte Erfahrungsaustausch zwischen Wissenschaft und Praxis war deshalb sowohl im nationalen als auch noch mehr im internationalen Rahmen ein wesentlicher Standpunkt.

\subsection{Internationale Organisationen und Kriterien der internationalen Anerkennung}

Nach dem Zweiten Weltkrieg wurden im Rahmen der UNESCO zwei wesentliche internationale Organisationen gegründet:

1960 in Paris, IFIP (Internationale Föderation für Informationsprozesse), TC5 (Computers in Industry). Innerhalb dieses technischen Komitees wurden spezifische Arbeitsgruppen gegründet, so zum Beispiel 5.3 CAM (Computer Aided Manufacturing). Die Mitarbeit in IFIP TC5- Gremien wurde so ermöglicht, dass von den interessierten Staaten ein Vertreter nominiert werden konnte. Die Mitarbeit in IFIP- Arbeitsgruppen wurde durch Berufungen auf der Basis nachgewiesener fachlicher Kompetenz ermöglicht. Eine positive Erfahrung ist mit der demokratischen Vorgehensweise in Form der Wahl der jeweils Vorsitzenden (Chairman) gegeben. Eine Wahlperiode war mit maximal 6 Jahren (min 3 Jahre)

festgelegt. (Diese strengen Regeln wurden leider nach der politischen Wende nicht mehr konsequent beibehalten-eventuell ein Einflussfaktor der reduzierten Bedeutung der IFIP) .

Die internationale Organisation für Produktionstechnik CIRP wurde ebenfalls in den 1960iger Jahren mit Sitz in der Schweiz gegründet.

Insbesondere in den 1980iger Jahren gab es zwischen beiden kompetenten Organisationen eine enge Zusammenarbeit in Form von fachspezifischen Arbeitskonferenzen, die auch in folgendem Dokument eingeordnet sind.

Für die Teilnahme am internationalen Erfahrungsaustausch in Form von Fachkonferenzen war in beiden Organisationen die Begutachtung eingereichter Vorträge durch zwei unabhängige Mitglieder des jeweiligen Programmkomitees ein wesentliches Kriterium.

Darüber hinaus war in den Statuten der IFIP-Arbeitsgruppe 5.3 festgelegt, dass insbesondere für spezifische Softwareentwicklungen der Nachweis der industriellen Wirksamkeit erbracht werden musste. Dieser Standpunkt stand demnach in voller Übereinstimmung mit den Bewertungs-Maßstäben im ehemaligen Lehrstuhl Fertigungstechnik/ Technologische Programmierung. Auf dieser Basis konnte deshalb eine langjährige aktive Mitwirkung an der Konferenzserie PROLAMAT (Programming Languages for Machine Tools) gewährleistet werden.

Schon im Zusammenhang mit der Vorbereitung der vierten PROLAMAT- Konferenz in Ann Arbor/ Michigan, USA wurde eine breitere Interpretation des Begriffes PROLAMAT „Project Research On Leading- Edge Applications and Methods for Applied Information Technology" vereinbart. Auch hierbei ist mit der Betonung der jeweils zukunftsorientierten Anwendungsausrichtung eine eindeutige Prämisse gegeben. 


\section{Vorreiter und Pioniere der historischen Entwicklung}

\subsection{Prof. Dr. Alfred Richter - Gründer des Institutes für Fertigungstechnik}

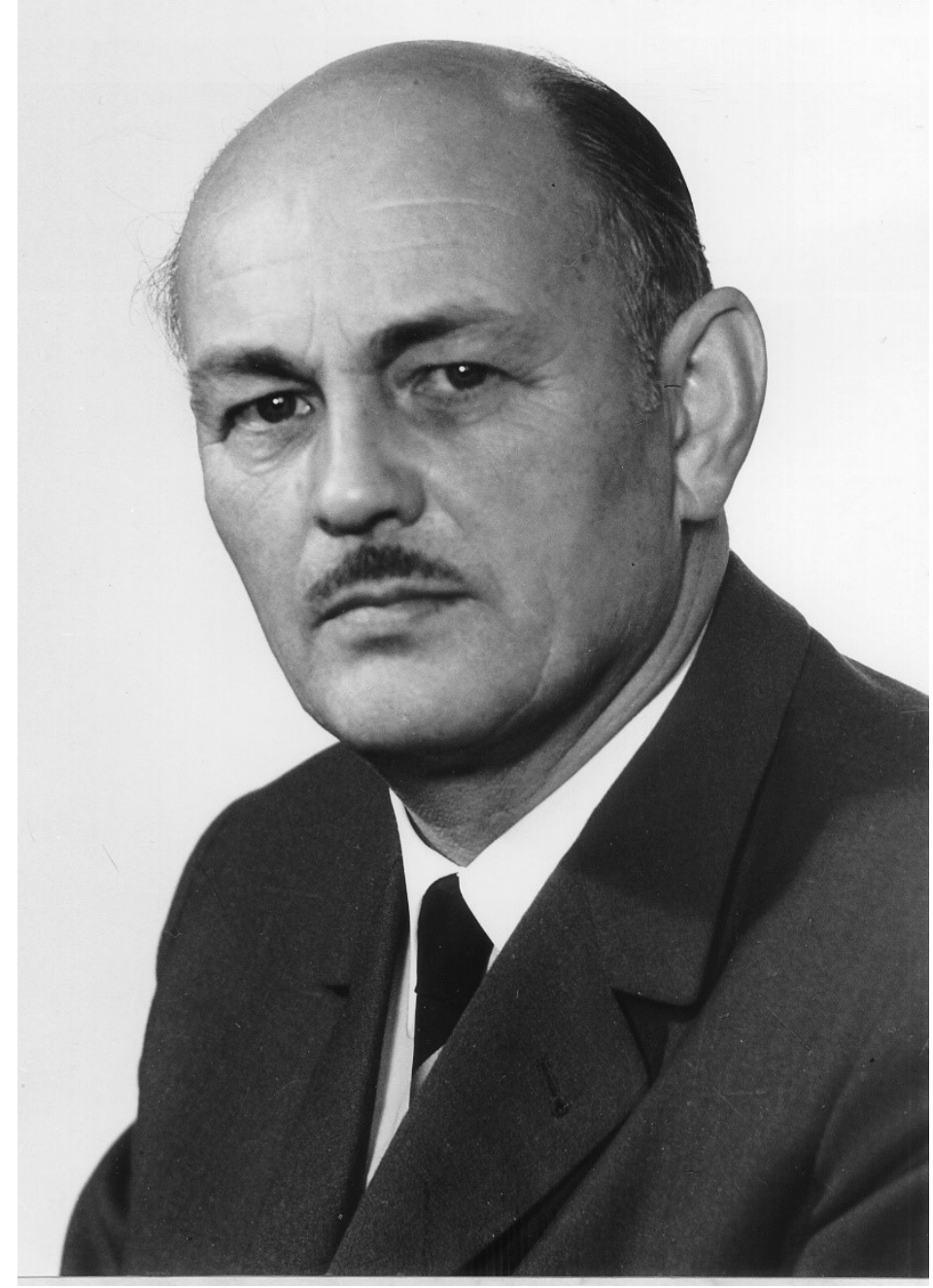

Abbildung 1: Prof. Dr. A. Richter
Das Institut für Fertigungstechnik wurde an der TU Dresden im Jahre 1953 mit der Berufung von Prof. Dr. A. Richter gegründet, wobei vier Schwerpunktgebiete in Lehre und Forschung vorgesehen waren. Umformtechnik, spanende Fertigung, industrielle Fertigung, Werkzeuge der spanenden Fertigung, Versuchsfeld. In den Folgejahren wurden weitere Lehrstühle wie Triebwerkfertigung, Fügetechnik und Montage gegründet, sodass zurecht in den 70iger und 80iger Jahren von einem Großinstitut der Fertigungstechnik gesprochen werden konnte. Gleichzeitig wurde im wachsenden Kollegenkreis der Begriff Schule der Dresdner Fertigungstechnik eingeführt. Der persönliche Schwerpunkt von Prof. Richter war das Fachgebiet spanende Fertigung, was ihm eben auch den inoffiziellen Titel „Späne Richter" einbrachte. Mit besonderem Respekte habe ich aus der Studentenzeit, d.h. wenige Jahre nach dem Zweiten Weltkrieg den Einsatz einer "Schnelldrehmaschine" in Erinnerung. In Verbindung mit dem deutschen Normenausschuss mit Untersuchungen zur Anwendung keramischer Schneidstoffe

wurden wichtige Grundlagen zu Hochgeschwindigkeitsbearbeitung (HSC High-Speed Cutting) gelegt. Darüber hinaus ist die aktive Mitarbeit im Deutschen Normausschuss herauszustellen. Schon in dieser Zeit gab es erste fachliche Kontakte zu dem deutschen Pionier der numerischen Steuerungstechnik Prof.W. Simon. 


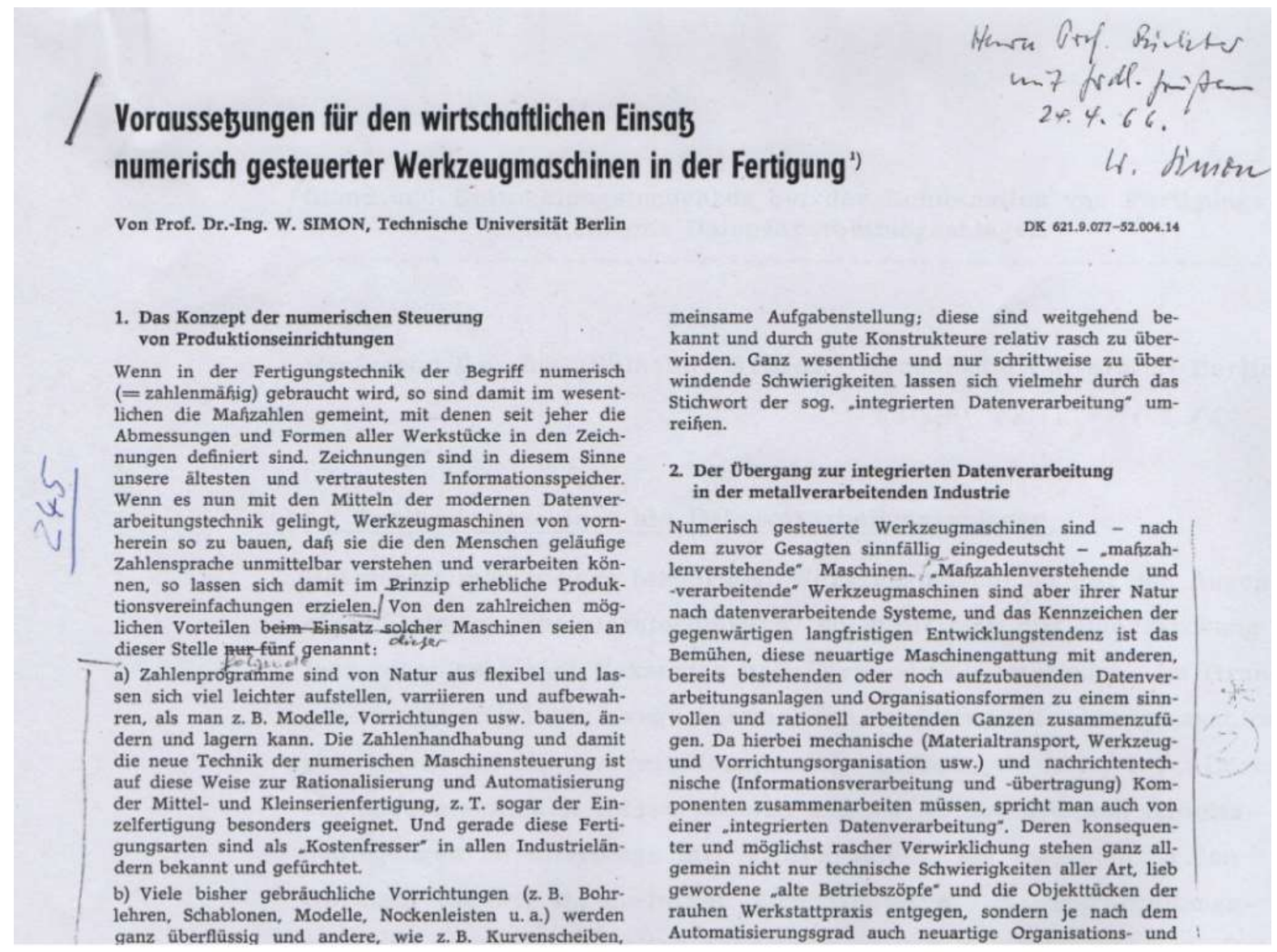

Abbildung 2: Veröffentlichung von Prof. Simon mit persönlicher Widmung für Prof. Richter

Dieser Sachverhalt wird auch mit einer für Prof. Richter persönlich gewidmeten Veröffentlichung von Prof. Simon herausgestellt. Die persönliche Widmung von Prof Simon für Prof Richter ist aus meiner Sicht ein Dokument für die deutsch/deutsche Kooperation in einer im Aufbau befindlichen High-TechDisziplin im Rahmen der "Dresdener Schule der Fertigungstechnik“. Dieser Text wurde mir von Herrn Prof. Richter zur gründlichen Auswertung übergeben. Darüber hinaus war das erste deutsche Fachbuch von Prof. Simon eine wichtige Informationsquelle für tiefergehende Aktivitäten. Das Fachbuch „Die numerische Steuerung von Werkzeugmaschinen - Einführung in die Automatisierung der Einzel- und Kleinserienfertigung mit nachrichtenverarbeiteten Mitteln"/1/ kann als ein wichtiges Ausgangsdokument für die gesamte weitere technische Entwicklung angesehen werden. „Das bemerkenswerteste und reizvollste aber auch das schwierigste bei der Durcharbeitung der neuen Automatisierungsprobleme liegt darin, dass mindestens vier bisher einander mehr oder weniger fremde technische Disziplinen eine gemeinsame Sprache finden müsse: klassischer Werkzeugmaschinenbau, allgemeine Elektrotechnik, Regelungstechnik und die neue Technik der Informationsverarbeitung. " Aus dieser komplexen Darstellung kann man herausstellen, dass die vor einigen Jahren begonnenen Anwendungen des Begriffes „Industrie 4.0" eine sehr fundierte historische Wurzel haben. Das Verständnis für diese komplexen Zusammenhänge ist auch noch heute ein wesentlicher Aspekt für deren wissenschaftliche Beherrschung.

„Das Konzept der numerischen Steuerung greift in letzter Konsequenz sehr über die einzelne Werkzeugmaschine hinaus in die gesamten Betriebsstrukturen ein; es ist vielmehr eine weitreichende Organisationsform innerbetrieblicher Nachrichtenübermittlung (in ganz Allgemeinem) und Nachrichtenverarbeitung als eine Erweiterung der zuvor genannten Maschinensteuerungsarten um eine weitere Gattung." 
„Aber man darf vor den konstruktiven Teilproblemen niemals die Gesamtkonzeption eines umfassenden Nachrichtenflusses und seine Naturgesetze vergessen." Mit der Übernahme dieser Grundpositionen von Prof. Simon war ein guter Grundstein für das angestrebte Lehr- und Forschungsgebiet industrielle Fertigung und Automatisierung gelegt.

Darüber hinaus verdient nach meiner Auffassung folgender Sachverhalt eine besondere Würdigung: Prof. Richter hat über viele Jahre die kollegiale Zusammenarbeit zu dem Dresdner Absolventen Prof. Palitzsch gepflegt. Dieser hatte an der Universität Braunschweig das Fachgebiet Holzbearbeitung aufgebaut und weiterhin aktive Kontakte zu seiner Alma Mater gepflegt. In voller Anerkennung und Würdigung dieser Aktivitäten war langfristig die Verleihung der Ehrendoktorwürde von der Fakultät Maschinenwesen der TU Dresden vorgesehen. Als der Termin im Jahr 1962 aktuell wurde, sollte wegen des Baus der Mauer im Sommer 1961 und der daraus resultierenden angeheizten politischen Atmosphäre diese Anerkennung nicht mehr stattfinden. Es verdient deshalb besondere Wertschätzung, dass sich Prof. Richter mit seiner anerkannten fachlichen Position für die Verleihung des Ehrentitels durchsetzen konnte. Diese Position des „Rückgrates“ war in den Zeiten hoher politischer Spannungen durchaus nichts Alltägliches.

In diesem Zusammenhang verdient es darüber hinaus besondere Beachtung, dass Prof. Palitzsch als Absolvent der TU (damals noch TH) Dresden, (Sachsenberg- Schule) das erste deutsche Mitglied der internationalen Organisation CIRP wurde. 

tomatisierung"

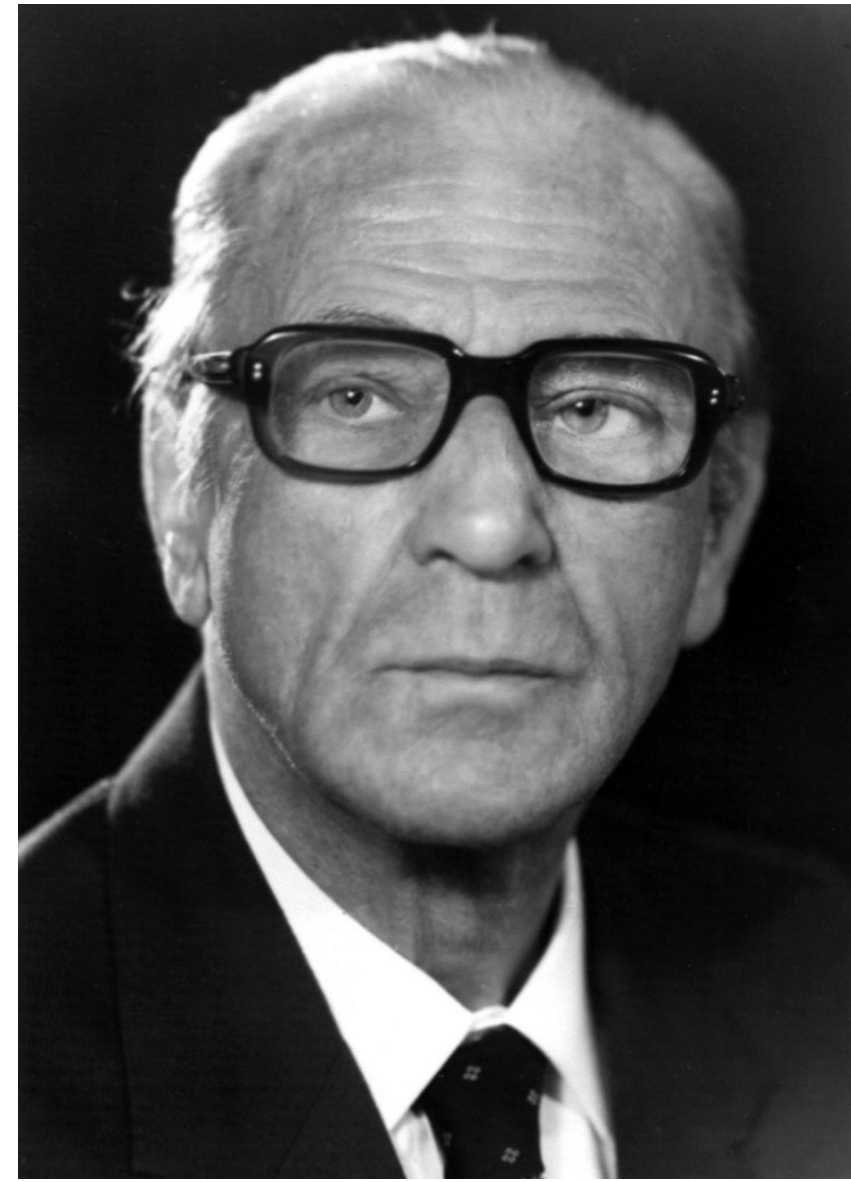

Abbildung 3: Prof. Seidel, Lehr- und Forschungsgebiet Industrielle Fertigung und Automatisierung
Prof. Seidel hatte sein Diplom an der TU Darmstadt erworben und wurde von Prof. Richter aus dem Wirtschaftsministerium an die TU Dresden berufen, um das Fachgebiet "Industrielle Fertigung und Automatisierung" aufzubauen. Von Prof. Richter war dieses Lehr- und Forschungsgebiet in den 1950iger Jahren an Prof Seidel übergeben worden, so daß ich schon als Student die Praxisbezogenheit der Lehrveranstaltungen kennenlernte. Ausgangspunkt waren die traditionellen Gebiete der industriellen Fertigung mit Gesetzmäßigkeiten der Fließfertigung, der Gestaltung von Taktstraßen und das breite Gebiet der analogen Steuerung. Als neue Fachgebiete wurden auch die Erkenntnisse zur Typisierung technologischer Prozesse (Prof. Sokolowski) und der Gruppenbearbeitung (Prof. Mitrofanow) aus der sowjetischen Wissenschaft übernommen und weiter ausgebaut. In diesem Zusammenhang wurde von Prof. Seidel der Begriff der Rahmentechnologie im Sinne bestmöglicher technologischer Prozesse entwickelt. Zu diesem gesamten Gebiet der Vereinheitlichung technologischer Prozesse wurden wertvolle methodische Grundlagen für die Verbesserungen in der Wirtschaftsstruktur der in der DDR gebildeten Kombinate geschaffen. Interessant in diesem Zusammenhang ist der Fakt, dass Jahre später an der RWTH Aachen der Gedanke der Vereinheitlichung technischer Sachverhalte und Prozesse mit dem Begriff „Teilefamilienfertigung“ geprägt wurde.

Zu Beginn meiner Assistententätigkeit war die Entwicklung der numerischen Steuerungstechnik in dem Forschungsinstitut für Werkzeugmaschinen im damaligen Karl-Marx-Stadt konzentriert. Während eines einem gemeinsamen Arbeitsbesuch mit Prof. Seidel und einem gründlichen Fachgespräch zu den Entwicklungen der numerischen Steuerungstechnik in der DDR (Prof. Russig und Dr. Wätzig) wurde ein Grundstein für eine langjährige erfolgreiche Kooperation gelegt. An der TU Dresden wurde davon ausgehend die Programmierung der Werkzeugmaschinen und das gesamte informationstechnische Umfeld als wichtiger Ausgangspunkt für die notwendigen Weiterentwicklungen gelegt. In voller Übereinstimmung mit dem deutschen NC Pionier Prof. Simon kommt es darauf an für das gesamte informationstechnische Umfeld ein systematisches Know-how über die NC Programmierung hinaus aufzubauen. Das beweisen die international führenden Werkzeugmaschinenhersteller in der Weise, dass ergänzend zu den Maschinen und Steuerungen das notwendige Know-how zum effizienten Betreiben der komplexen Anlagen ein wichtiger Teil des Verkaufspreises darstellt. 


\section{Anfänge der flexiblen Automatisierung}

\subsection{Inhaltliche Schwerpunkte erster NC-Programmiersprachen}

Im Zeitraum von 1961 bis 1965 als Assistent am Institut für Fertigungstechnik, Arbeitsgruppe "Industrielle Fertigung und Automatisierung“, tätig, wurde mit den Untersuchungen zur Programmierung numerisch gesteuerter Werkzeugmaschinen begonnen. Dabei ging es primär um die exakte Definition geometrischer Konturen, wobei der Aufwand für exakte Positionen in einem X/Y Koordinatensystem extreme Anforderungen bei z.B. Genauigkeiten von $1 / 100 \mathrm{~mm}$ erforderten. Die notwendige Erfassung der exakten Positionswerte für lineare, zirkulare parabolische und andere Interpolationsformen ist mit einem extremen manuellen Aufwand verbunden, der eine unmittelbare rechnerunterstützte Realisierung rechtfertigt.

Aus dieser Sicht wurde in Übereinstimmung mit nationalen und internationalen Trends mit der Entwicklung eines eigenständigen Programmiersystems der DDR begonnen. Gleichzeitig entstand in Zusammenarbeit mit dem Kombinat Carl-Zeiss-Jena eine kooperative Zusammenarbeit. Schon im Jahr 1963/1964 wurde von Carl-Zeiss-Jena ein Staatsplanthema initiiert, indem die Aktivitäten mehrerer wissenschaftlicher

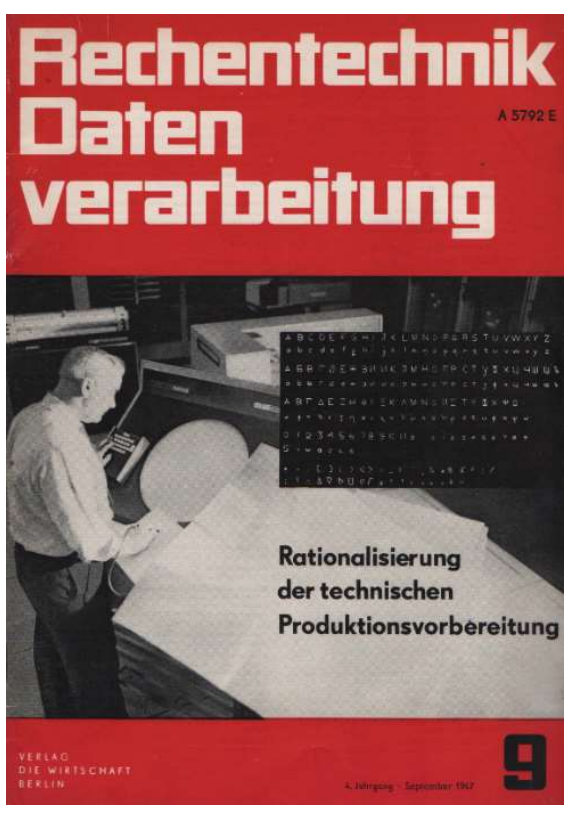

Abbildung 4: DDR-Fachzeitschrift für IT-Anwendungen Einrichtungen und Universitäten gebündelt wurden. In der Zeitschrift „Rechentechnik, Datenverarbeitung" wurde regelmäßig über zentrale Forschungsvorhaben informiert.

In einem Staatsplanthema zur "Rationalisierung der technischen Produktionsvorbereitung“ wurde ein Forschungsverbund unter Verantwortung des Kombinates Carl-Zeiss-Jena aufgebaut. Davon ausgehend erfolgte eine Berufung zum Aufbau einer Abteilung FETV4 (Forschung, Entwicklung, Technische Vorbereitung im Unternehmen Carl-Zeiss-Jena). Mit der Berufung für dieses Aufgabengebiet war gleichzeitig die Erwartung verbunden, einen Forschungsverbund für das Gesamtgebiet EDV- Anwendung für technologische Prozesse in der DDR mit aufzubauen. Mit dieser Aufgabenstellung (Begriff AUTOTECH) war die Erwartung verbunden, über konkrete Teilleistungen im Gesamtgebiet der flexiblen Automatisierung mit NC Technik eine effiziente Kooperation mit unterschiedlichen Partnern aufzubauen. Die Übernahme einer solchen komplexen Aufgabe führte für mich zu grundsätzlichen Überle-

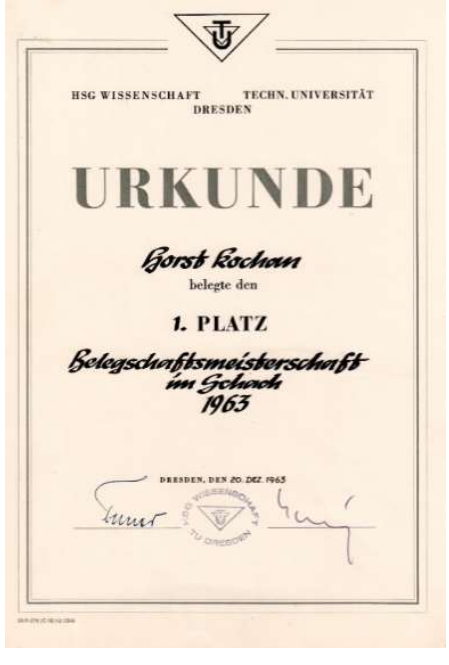

Abbildung 5: Ehrung für fortgeschrittene Schach-Kenntnisse gungen. Bezüglich der praktischen Realisierbarkeit konnte ich mir als junger Ingenieur eine solche Aufgabe zum „Wissensmanagement" mit vielen völlig neuen Fachgebieten in Zusammenarbeit mit Hochschulprofessoren in der DDR zutrauen.

Unter Beachtung meiner Erfahrungen und durchaus beachtlichen Ergebnisse als Schach-Amateur konnte ich von relativ schneller Erfassung

Komplexer Sachverhalte und strategischen Befähigungen ausgehen.

Diese Fähigkeiten und Voraussetzungen erleichterten mir die Entscheidung zur Übernahme dieser komplexen Aufgabe als Wissenschaftsorganisator.

Ergänzend zu dem fachlichen Schwerpunkt, dem Aufbau der Abteilung Forschung Entwicklung Technische Vorbereitung FETV4 im Unternehmen Carl-Zeiss-Jena von 6 auf 30 Mitarbeiter konnte ein funktionieren- 
der Forschungsverbund mit Universitäten und Forschungseinrichtungen, einschließlich der UdSSR, aufgebaut werden. Wertvolle Unterstützungen in diesem Prozess erfolgten durch den Fachdirektor FETV Dr. Klaus Gattnar und dem Direktor Forschung und Entwicklung des Kombinates Carl-Zeiss-Jena Prof. Görlich. Von beiden geschätzten Kollegen wurde eine konsequente Linie vertreten, wesentliche Schritte theoretisch gut zu fundieren und gleichzeitig konkrete messbare Ergebnisse in der industriellen Anwendung nachzuweisen. Diese von dem Physiker Prof. Görlich konsequent vertretene Grundposition war für meine gesamte fachliche Entwicklung weiterhin prägend.

\subsection{DDR-Programmiersystem SYMAP}

Ausgehend von diesen Grundpositionen wurde von einem Forschungsverbund unter Verantwortung des Kombinates Carl-ZeissJena in Zusammenarbeit mit dem Forschungsinstituten Werkzeugmaschinen und Fertigungstechnik in Karl-Marx-Stadt, sowie den Universitäten TU Dresden, TH Karl-Marx-Stadt, TH IImenau und TH Magdeburg, das Programmiersystem SYMAP entwickelt.

In diesem Zusammenhang ist es natürlich wichtig, die nationalen und internationalen Entwicklungen zu beobachten und auszuwerten.

In der BRD wurde ebenfalls in Kooperation führender Forschungsinstitute an der Entwicklung eines Programmiersystems gearbeitet. In Anlehnung an das USA System „APT - Automatically Programmed Tools" wurde der Begriff EXAPT (Extensions of APT) vereinbart. Schon im Jahre 1966 wurde das System EXAPT 1 für einfachste Fräsverfahren (Punkt- und Streckensteuerung) der Öffentlichkeit vorgestellt.
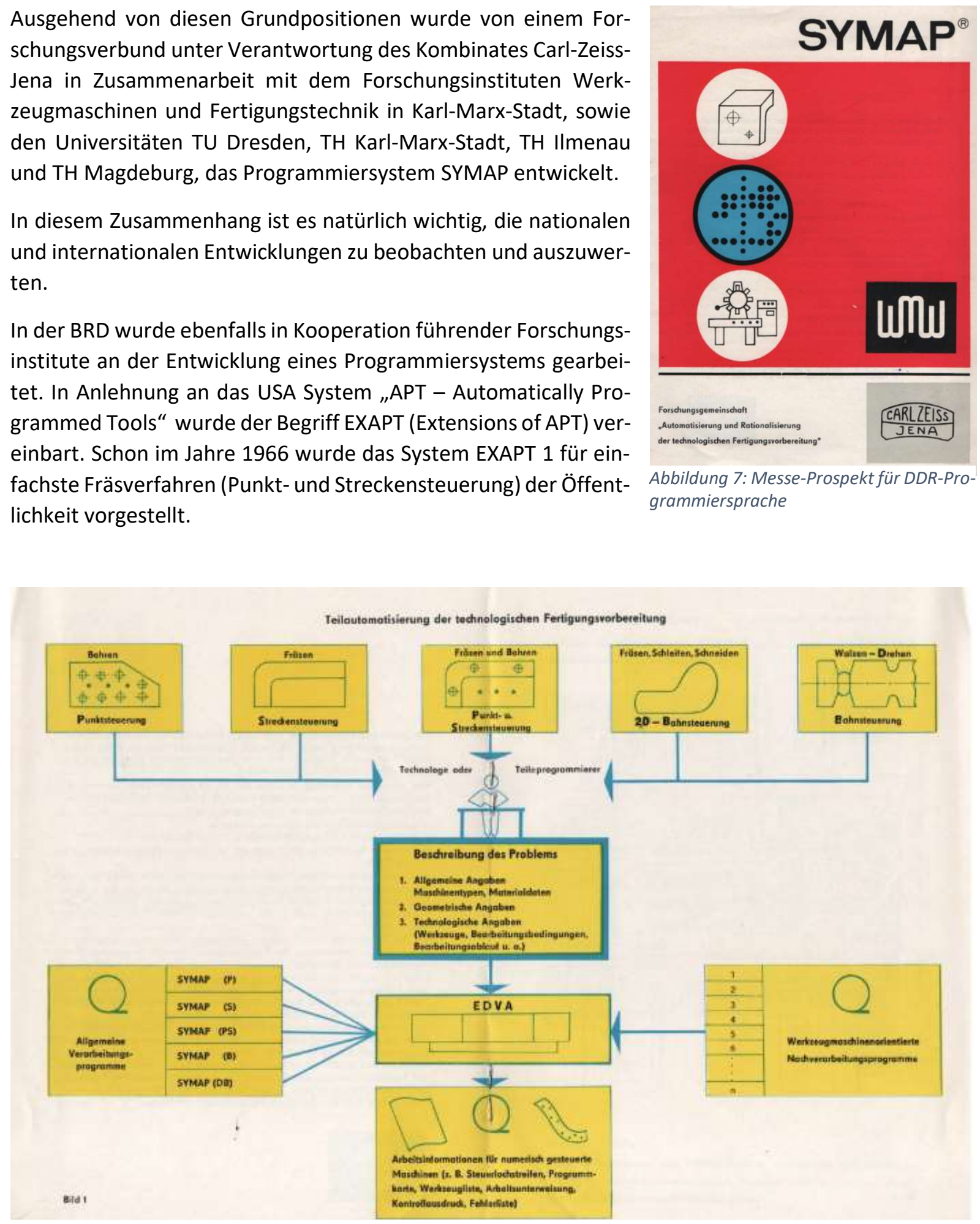
Im Jahre 1967 folgte ein Programmiersystem EXAPT 2 für Drehmaschinen mit Strecken- oder Bahnsteuerung und später EXAPT 3 für Bohr- und Fräsbearbeitung mit oder ohne Bahnsteuerungen.

Von Seiten der DDR wurde in den genannten Forschungsverbund ein System mit fünf Teilsystemen auf der Leipziger Frühjahrsmesse 1969 vorgestellt. Obwohl demnach von einem Rückstand von ca. 2 Jahren ausgegangen werden muss, verdient jedoch die erreichte Anwendungsbreite durchaus hohe Anerkennung, begann jeder Anwender die erreichte Breite der erfassten Problemgebiete durchaus anzuerkennen.

Dieses System wurde im Frühjahr 1969 zur internationalen Leipziger Frühjahrsmesse mit dem ersten Messegold für geistige Ware ausgezeichnet. Die Auszeichnung wurde vom Hauptabteilungsleiter Dr. Gattnar von Carl Zeis Jena und Abteilungsleiter $\mathrm{Dr}$ Kochan entgegengenommen. In Verbindung mit der industriellen Verbreitung des SYMAP Programmiersystems war noch wegen der Wort-Ähnlichkeit Symap mit Siemens eine Patentstreitigkeit zu bewältigen. Mit den versierten Rechtsanwälten aus dem Unternehmen Carl Zeiss

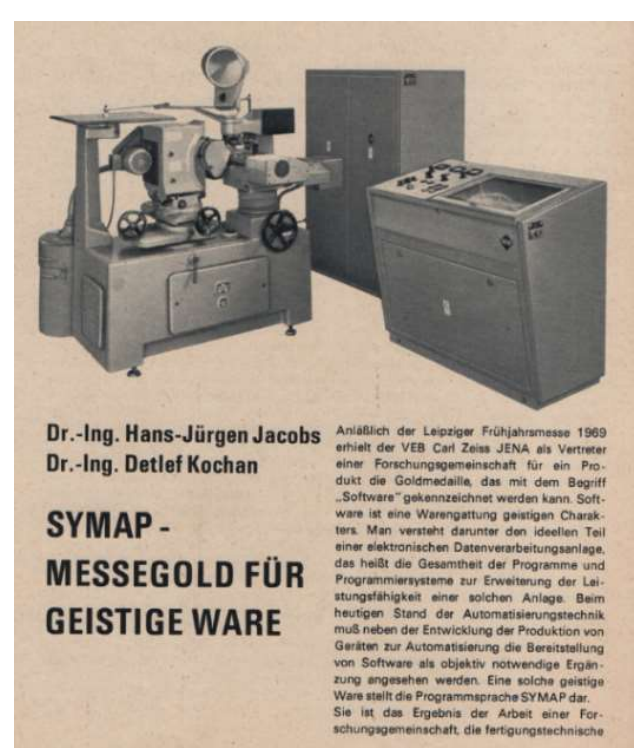

Abbildung 8: Gemeinsame Publikation TU Dresden (Jacobs) und Carl Zeiss Jena (Kochan) Jena ist das gut gelungen.

Im Rahmen der Messedemonstration gab es einen interessanten Informationsaustausch mit den EXAPT-Entwicklern aus Aachen, Berlin und Stuttgart. Diese rein fachlichen Gespräche waren von

beidseitiger Anerkennung geprägt, wobei insbesondere die technologischen Verbesserungen gegenüber der primär geometrisch orientierten USA -NC-Sprache APT ein gemeinsamer Standpunkt war.

Von Seiten der TU Dresden war in das Gesamtsystem SYMAP der Teil Bohren und Fräsen mit Streckensteuerung eingebracht worden.

Ein besonderer Aspekt war die hohe technologische Leistungsfähigkeit der verschiedenen Systemkomponenten, die in den folgenden Jahren weiter ausgebaut wurden.

Einige Jahre später wurde diese Systemkomponente vom Forschungszentrum Fertigungstechnik in Karl Marx Stadt ein eigenständiges System BoFR3 umgewandelt und wird auch unter heutigen Gegebenheiten mit noch verfügbaren Maschinen und Steuerungen in der Industrie genutzt. 


\subsection{Internationale Ausstrahlung und Wirksamkeit}

Im Sommer 1969 erreichte das Unternehmen Zeiss Jena die Information zur Durchführung einer ersten internationalen Konferenz in Rom zum Thema „Programming Language for Machine Tools (PROLAMAT)“. Die Bedeutung dieser Konferenz wurde natürlich nicht nur im Unternehmen Carl-Zeiss-Jena, sondern auch von anderen Instituten in der DDR mit Interesse aufgenommen, obwohl keine Anmeldung eines Vortrages für diese Konferenz möglich war. Bekanntlich musste sich auch die Wissenschaft der DDR noch um die internationale Anerkennung bemühen. Unabhängig davon wurden für die aktive Teilnahme vier Fachkollegen vorgesehen (Dr. Gattnar, Dr. Kochan, Dr. Wätzig, Prof. Kunert).

Im Ergebnis der Prüfung durch den sog. US-Travelport und die italienische Botschaft in Berlin reduzierten sich die Teilnehmer auf die Herren Gattnar und Kochan. Wegen einer kurzfristig entstandenen Verpflichtung von Dr. Gattnar (Dienstreise nach Frankfurt am Main) musste die Reise allein von Dr. Kochan wahrgenommen werden. Auch für die Reisedurchführung resultierte ein Jahr nach dem Prager Frühling im Jahre 1968 mit der vorgesehenen Flugreise Prag-Rom spezielle Probleme. Mit der angestrebten Reise von Prag nach Rom war auch mit einer sog. "Stand-Bye“ Lösung der ausgebuchte Flug leider nicht möglich.

Damit hing die gesamte Teilnahme an einem schwachen seidenen Faden. Mit Unterstützung des Interflug Beauftragten und mit Prager Freunden konnte die Realisierung mit einem Urlaubernachtflug von Prag nach Mailand quasi im letzten Moment noch wahrgenommen werden, nachdem zwei ursprüngliche Buchungen nicht wahrgenommen worden waren.

Für die Konferenzdurchführung im Hilton Hotel Rom musste dann noch eine weitere Hürde genommen werden. Die Teilnahme an der Konferenz machte an sich keine Probleme. Dagegen war die von der Führung des Hauses Zeiss erwartete aktive Beteiligung mit Vortrag schon schwieriger. Hinzu kam der Aspekt meiner damaligen unbefriedigenden Englisch Kenntnisse. Die Vorbereitung eines schriftlichen Vortragdokumentes konnte meinerseits mit Unterstützung von Englischlektoren der TU Dresden noch kurzfristig realisiert werden. Allerdings stellte die in jeder Konferenz übliche Diskussion in Englisch ein weiteres Hindernis dar. Durch das Entgegenkommen der Tagungsleitung, insbesondere auch mit Unterstützung des Vorsitzenden der Fachsektion Prof. Colding, (Schweden) konnte diese Hürde genommen werden.

Die Bedeutung dieser eingetretenen positiven Situation kann erst im Nachgang in Verbindung mit den weiteren Entwicklungen der gesamten Programmserie gewürdigt und eingeordnet werden. Nach dem erfolgreichen Auftakt der ersten PROLAMAT Konferenz in Rom konnte mit einem breiten industriellen und wissenschaftlichen Interesse gerechnet werden. Deshalb wurde das Mitwirken in dem kompetenten Fachgremium als eine gute Chance für die weitere persönliche und fachliche Entwicklung erkannt.

Auch in Unkenntnis dieser erfolgreichen langjährigen Konferenzserie konnte man davon ausgehen, dass die Mitwirkung in dem Organisationsteam dieser Konferenz von unmittelbarem fachlichem Interesse ist. Darüber hinaus war von Seiten der Regierung der DDR eine positive Einstellung zur aktiven Teilnahme in internationalen Gremien gegeben.

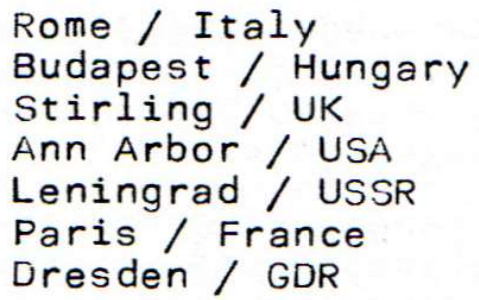

Meinerseits war bereits zu Beginn meiner Mitarbeit in der IFIP-Arbeitsgruppe CAM der Gedanke entstanden, diese nachhaltige Konferenz auch in Dresden durchzuführen, was 1988 auch gelang. 
Die im Jahre 1970/71 eingegangene Einladung zu Mitarbeit in der IFIP Arbeitsgruppe CAM (internationale Föderation für Informationsprozesse) war vom Hochschulministerium positiv aufgenommen worden, auch wenn es im unmittelbaren Arbeitsumfeld der Sektion Fertigungstechnik und Werkzeugmaschinen leider nicht immer die notwendige Unterstützung gab.

Die unmittelbare Einladung zur Mitgliedschaft in dieser IFIP Arbeitsgruppe 5.3 (CAM) war mit einer ersten Beratung in Slowenien (Ljubljana) verbunden. Erfreulicherweise nahm von Seiten der BRD der Autor des ersten NC Fachbuches Prof. Simon an dieser IFIP Beratung teil. Obwohl in der Reisedirektive explizit persönliche Kontakte mit Teilnehmern aus der BRD nicht erlaubt waren, überwiegt natürlich bei einem solchen Treffen das gemeinsame fachliche Interesse an den anstehenden Problemen. Deshalb war das erstmalige Treffen mit Prof. Simon mit ausführlichen fachlichen Diskussionen verbunden. Das führte gleichzeitig dazu, dass in der verfügbaren freien Zeit eine gemeinsame Busreise von Ljubljana in die Jama Grotten unternommen wurde.
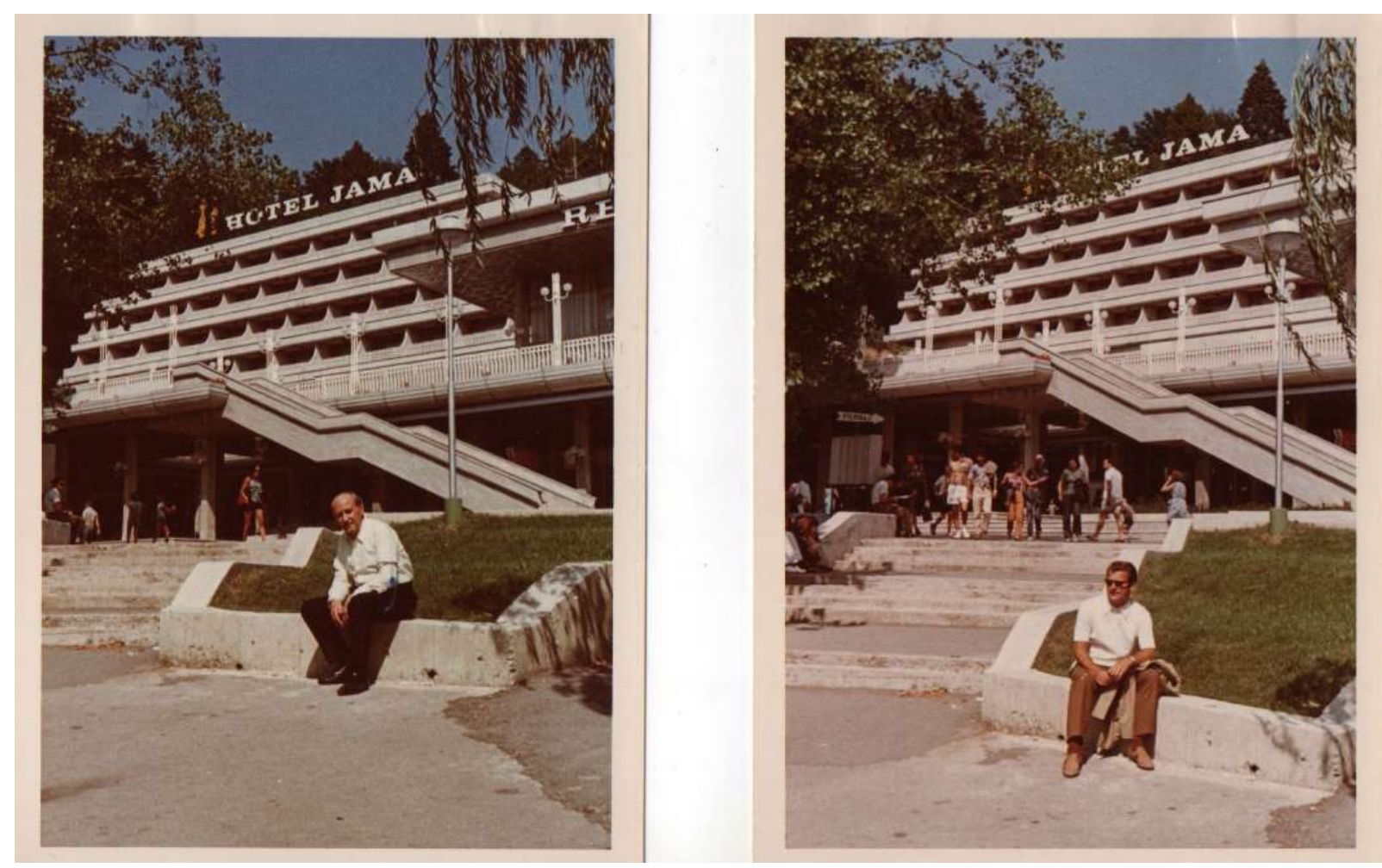

Abbildung 9: Prof. Simon als deutscher NC Pionier und D. Kochan als junger Ingenieur

Prof. Simon war zu diesem Zeitpunkt schon von der damaligen TH Darmstadt von Prof. Spur an die TU Berlin berufen wurden. Dieser Sachverhalt war gleichzeitig ein weiterer Ausgangspunkt, um auch im Rahmen der IFIP Arbeitsgruppe CAM mit Prof. Spur und auch Prof. Krause (Fachgebiet CAD) langjährige fachliche Kontakte aufzubauen.

Die Arbeitsberatung im Rahmen der IFIP Arbeitsgruppe CAM zur Vorbereitung der Konferenz 1973 in Budapest verlief ohne weitere Probleme. An der erfolgreichen Prolamat Konferenz 1973 in Budapest konnte eine beachtliche Teilnahme von Fachkollegen aus der DDR gewährleistet werden.

\section{Ausbildungsinhalte und Schwerpunkte der Lehre und Forschung}

Mit meiner Berufung an die TU Dresden im Jahre 1970 erfolgte einerseits die Übernahme der traditionellen Aufgabengebiete der Fertigungsprozessgestaltung, die auch in Übereinstimmung mit technischen Weiterentwicklungen in Übereinstimmung gebracht worden. Das gesamte Arbeitsgebiet wurde in drei Schwerpunktbereiche untergliedert und zwar Fertigungsprozessgestaltung (FPG) 1, Grundlagen 
der FPG, FPG 2 Schwerpunkt NCM-Programmierung und FPG 3 Weiterentwicklung der Automatisierungstechnik.

Das gesamte Gebiet, insbesondere der Inhalte 2 und 3, ist geprägt durch eine dynamische technologische Entwicklung. Wesentlich ist dabei der unmittelbare Praxisbezug, sodass die Installation geeigneter NC-Technik eine wichtige Voraussetzung für eine realitätsnahe Ausbildung bedeutete. Die Investition in NC Maschinen für das Drehen und Fräsen gehörte deshalb zu den wichtigen Aufgabenfeldern, die in der Sektion Fertigungstechnik und Werkzeugmaschinen zum Teil gegen den Widerstand der Spezialisten der Hydraulik durchgesetzt wurden. Die praxisrelevante Ausbildung war weiterhin geprägt durch die gemeinsame Vorbereitung der Studenten höherer Semester auf den Besuch der Leipziger Messen und die nachfolgende Auswertung der Messebesuche. Damit konnte eine gewisse permanente Aktualisierung an die technischen Entwicklungen erreicht werden. Mit der Berufung zum Professor für Fertigungstechnik/Technologische Programmierung im Jahre 1975 wurde gleichzeitig bis zum Jahre 1990 die Durchführung von sog. Problemseminaren mit den Schwerpunkten Flexible Automatisierung und CAD/CAM realisiert. Dabei wurde eine anteilige Beteiligung (50/50) von kompetenten Industrievertretern und Wissenschaftlern in den jährlichen Problemseminaren im Ferien- und Schulungsheim der TU Dresden im Schloss Gaußig gewährleistet.

Sowohl die Grundlagen der Fertigungsprozessgestaltung und die Anwendungen der Informationsverarbeitungen sind im 1977 erschienenen Fachbuch enthalten.

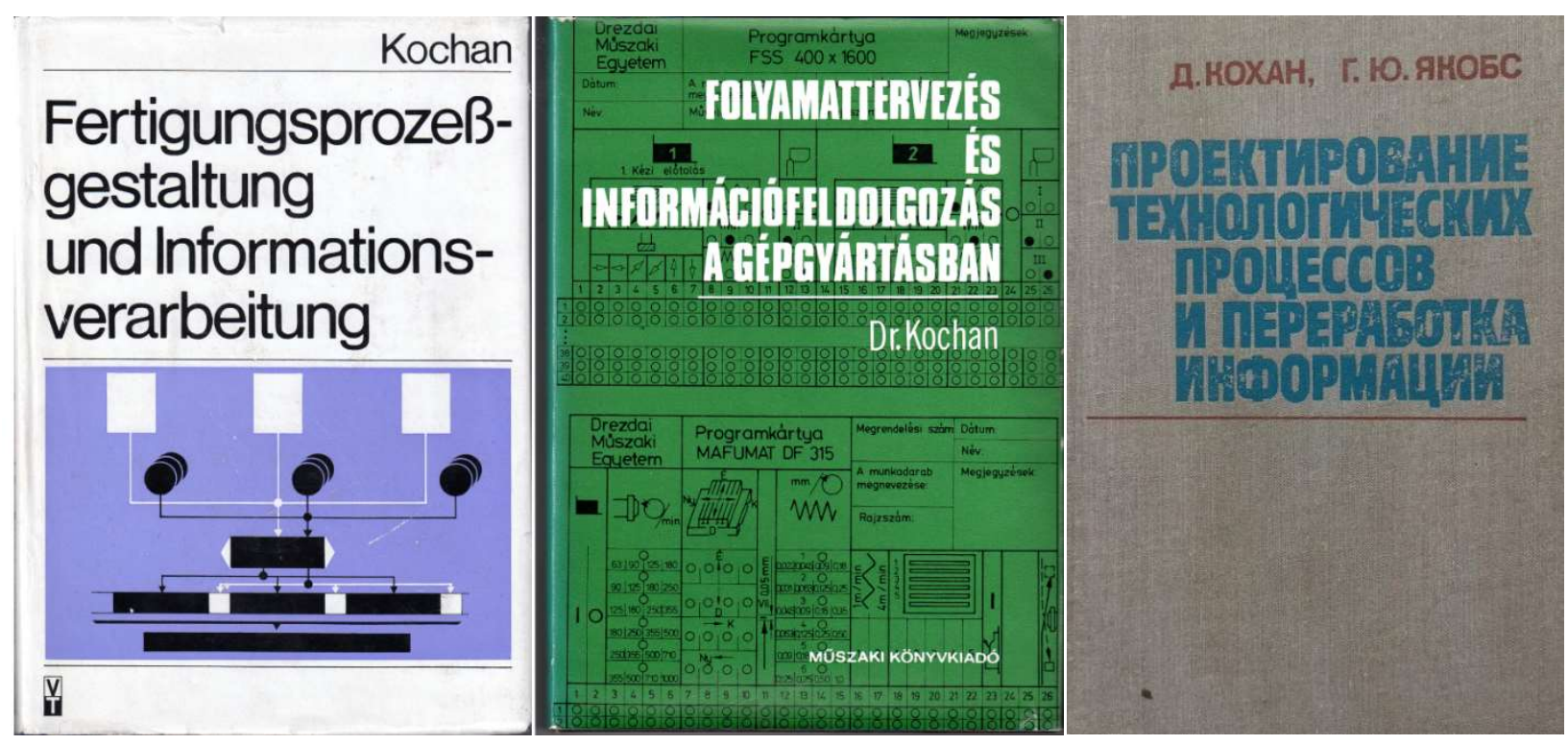

Abbildung 10: Deutsches Fachbuch der IT in der Fertigungstechnik sowie ungarische und russ. Übersetzung

Die Erarbeitung dieses Fachbuches erfolgte unter Mitarbeit von Prof. Dr. sc. techn. H.-J. Jacobs. Zum gleichen Zeitpunkt hat der Verlag Technik das Buch „Spanungsoptimierung“ von H.-J. Jacobs und D. Kochan herausgegeben. In diesem Buch werden ausgehend von den Grundlagen der Spanungsoptimierung die Aspekte der mathematischen Modellierung vertieft. Auch in diesem Zusammenhang kann auf Anregungen aus der sowjetischen Wissenschaft (Prof. Goranskie, Direktor des Institutes für technische Kybernetik in Minsk) verwiesen werden. Erstmalig wurden die Einflüsse auf den Zerspanungsprozess als ein System technischer Grenzen mit einer überlagerten Kostenzielfunktion mathematisch dargestellt und von Prof. Jacobs in Übereinstimmung mit den deutschen Normen gebracht. 
Trotz der dynamischen Entwicklungen in dem gesamten Wissensgebiet der fortgeschrittenen Produktionstechnik erfolgte im Jahre 1981 die russische Übersetzung.

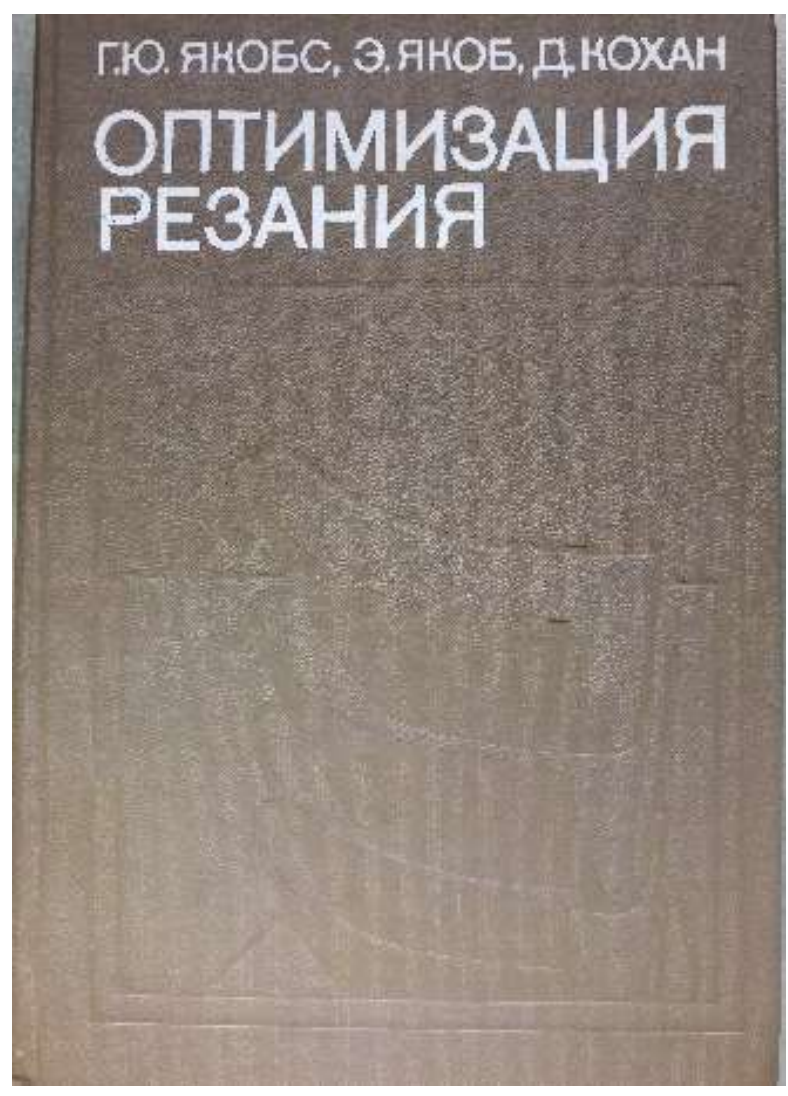

Abbildung 11: Fachbuch: Jacobs, Kochan: Zerspanungsoptimierung, russ. Ausgabe

Die Lehrinhalte der oben charakterisierten Fächer FPG 2 und 3 sind darüber hinaus in einen sog. IFIP State of the Art Report "CAM - Development Computer-Integrated Manufacturing" dokumentiert. Das Prinzip der Computer-Integrated Manufacturing (CIM) wurde in diesen internationalen Gemeinschaftswerk erstmalig umfassend dokumentiert. Dabei wurden die Entwicklungen von CAD/CAM zu CIM schon erstmalig umfassend dargestellt.

\section{Zunehmende internationale Erfahrungen}

Mit der Berufung in die IFIP Arbeitsgruppe 5.3 (CAM) war die langjährige Zusammenarbeit mit einem internationalen Expertenkreis gewährleistet.

In diesem Zusammenhang wird nochmals herausgesellt, dass eine Mitarbeit in einem technischen Fachgremium nur durch eine Berufung aus dem Ausland durch den Vorsitzenden der Facharbeitsgruppen ermöglicht werden kann. In diesem Fall war das unmittelbare fachliche Interesse in Übereinstimmung mit dem politischen Interesse zur Anerkennung der DDR in internationalen Gremien gegeben.

\subsection{Erste flexible Fertigungssysteme}

Mit Bezug einer Beratung der IFIP AG 5.3 in Cincinnati im März 1974 war gleichzeitig mit einer Teilnahme an der jährlichen Tagung der NC Society verbunden. Die Ausstattung für eine USA- Dienstreise, dem damaligen Mutterland der numerischen Steuerungstechnik, war mit nur vier Aufenthaltstagen so bemessen, dass eine Variante für einen längeren Arbeitsaufenthalt gefunden werden musste. Das 
erfolgte in der Weise, dass mit Unterstützung von Dr. Merchant, Forschungsdirektor des USA-Werkzeugmaschinenunternehmens CINCINNATI MILACRON gleichzeitig eine Vortragsreise zur Finanzierung des längeren Aufenthaltes organisiert wurde. Das Thema betraf „Flexible Fertigungssysteme und Optimierung technologischer Arbeitsgrößen“. Kerninhalt waren die ersten Erfahrungen mit dem flexiblen Fertigungssystemen PRISMA II und ROTA F250 des DDR Werkzeugmaschinenbaus. In diesem Zusammenhang kann der eindeutige Fakt betont werden, dass die DDR mit dem Entwicklungsstand und im Stammbetrieb des Kombinates FRITZ HECKERT auch realisierten technologischen Lösungen ein internationaler Spitzenstand gegeben waren. In den flexiblen Fertigungssystemen konnten acht unterschiedliche Werkzeugmaschinenbauteile mit Nutzung mehrerer Bearbeitungszentren und durch Verkettung eines automatisierten Transportsystems komplett bearbeitet werden. Die Entwicklung war schon 1969 begonnen worden und fand unter monatlicher Kontrolle eines Staatssekretärs aus dem DDR-MINISTERIUM ELEKTROTECHNIK UND ELEKTRONIK statt.

\section{Allgemeine Erkenntnis: Große komplexe Vorhaben erfordern ebenfalls große in- terdisziplinäre Teams}

Als Vertreter von Carl-Zeiss Jena war ich mit der Aufgabe der zuverlässigen Programmierung betraut. Erfreulicherweise traten dabei keine ernsthaften Probleme auf. In jedem Fall konnte die realisierte Lösung von mir im Vortrag mit Unterstützung von geeignetem Filmmaterial gut vertreten werden. Die Vortragsreise begann im damals noch führenden Unternehmen in CINCINNATI- MILACRON. Der erste Vortrag fand vor ca. 40 Ingenieuren in der besonderen Atmosphäre des sog. Adler-Konferenzraumes statt. Der hohe fachliche Anspruch war auch mit Bezug Präzisierung englischer Fachsprachbegriffe gesichert. Die Vorträge wurden u.a. an der Universität Pretoria, im Caterpillar Entwicklungszentrum und in der Firma KEARNEY UND TRECKER gehalten.

Den abschließenden Höhepunkt dieser Vortragsreise bildete das Auftreten am MIT Institut of Technology (Massachusetts). Das MIT ist bekanntlich die führende wissenschaftliche Einrichtung im Osten der USA vertreten durch Prof. N. Cook in Boston, Cambridge. Das Auftreten vor einem offenbar fachlich aufgeschlossen und interessierten Mitarbeiter- und Studentenkreis des MIT führte tatsächlich zu Standing-Ovationen für die Darstellung des damaligen beachtlichen Entwicklungsstandes der DDR.

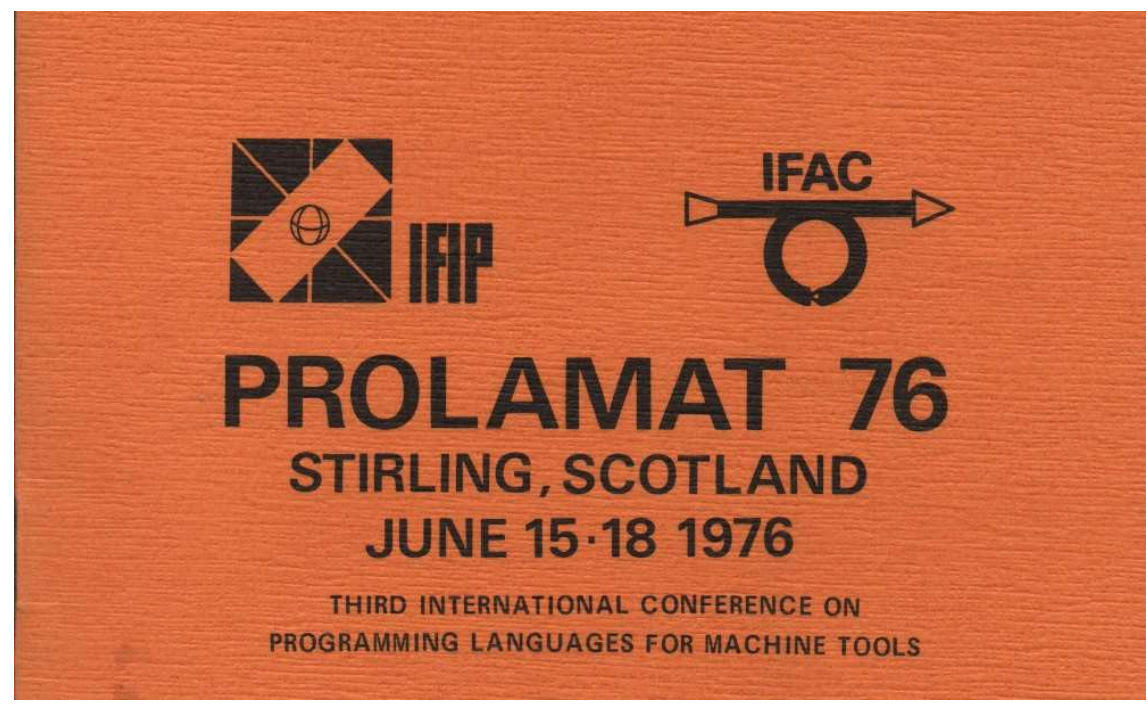

Abbildung 12: Ankündigung PROLAMAT-Konferenz 1976, Schottland 
Ergänzende Bemerkung: Eine Nachwirkung dieses Auftretens ergab sich in Verbindung mit dem Treffen der IFIP WG 5.3 im Juni 1974 in Glasgow, Schottland (Vorbereitung PROLAMAT 1976 in Stirling, Schottland). Von Prof. Iwata (Kobe, Japan) erfolgte eine Anfrage an mich mit der Bemerkung, dass vom Präsidenten der TU Dresden ein herausragender Vortrag zu flexiblen Fertigungssystemen gehalten wurde. Meine Richtigstellung zum Referenten führte zu einer Einladung zu einer Automatisierungskonferenz in Tokio 1975 mit der Bereitstellung von 1000 USD durch die japanischen Veranstalter.

Mit der Begründung vielseitiger internationaler Verpflichtungen wurde die Teilnahme an dieser Automatisierungskonferenz von der damaligen Sektionsleitung abgelehnt. Damit das gute Verhältnis zu den japanischen Fachkollegen keine negativen Auswirkungen hat wurde von mir eine Lösung angestrebt und der gemeinsame Vortrag von dem fachlich kompetenten Kollegen Jacobs in Tokyo wahrgenommen.

\subsection{Fachliche Differenzen zur bedienerlosen Fertigung}

In Verbindung mit der Beratung der IFIP Arbeitsgruppe 5.3 zur Vorbereitung der PROLAMAT Konferenz 1976 gab es mit dem Bezug der Entwicklungen zur unbemannten Fertigung von Seiten des IFIP TC 5 Vorsitzenden Mr. Vlietstra eine sehr ernsthafte Diskussion. An dem vorgesehenen Konferenzort Glasgow war zum damaligen Zeitpunkt die Arbeitslosigkeit extrem hoch. Deshalb gab es ernsthafte Bedenken, diese aktuellen internationalen Entwicklungen zur bedienarmen Fertigung ins Zentrum des Konferenzanliegens einzuordnen. Der von Prof. Hatvany vertretene Standpunkt, dass die Ingenieure die Entwicklungen zur effizienten Wertschöpfung als zentrierende Aufgabe wahrnehmen müssten, während es in der staatlichen, gesellschaftlichen Verantwortung läge, die soziale Verträglichkeit zu gewährleisten. Dieser Standpunkt wurde von mir in der lebhaften Diskussion voll unterstützt. Im Ergebnis dieser intensiven Diskussion wurden 1976 zur PPROLAMAT- Konferenz die progressiven japanischen Zielstellungen im Plenarvortrag von Prof. Yoshikava, TU Tokio wahrgenommen.

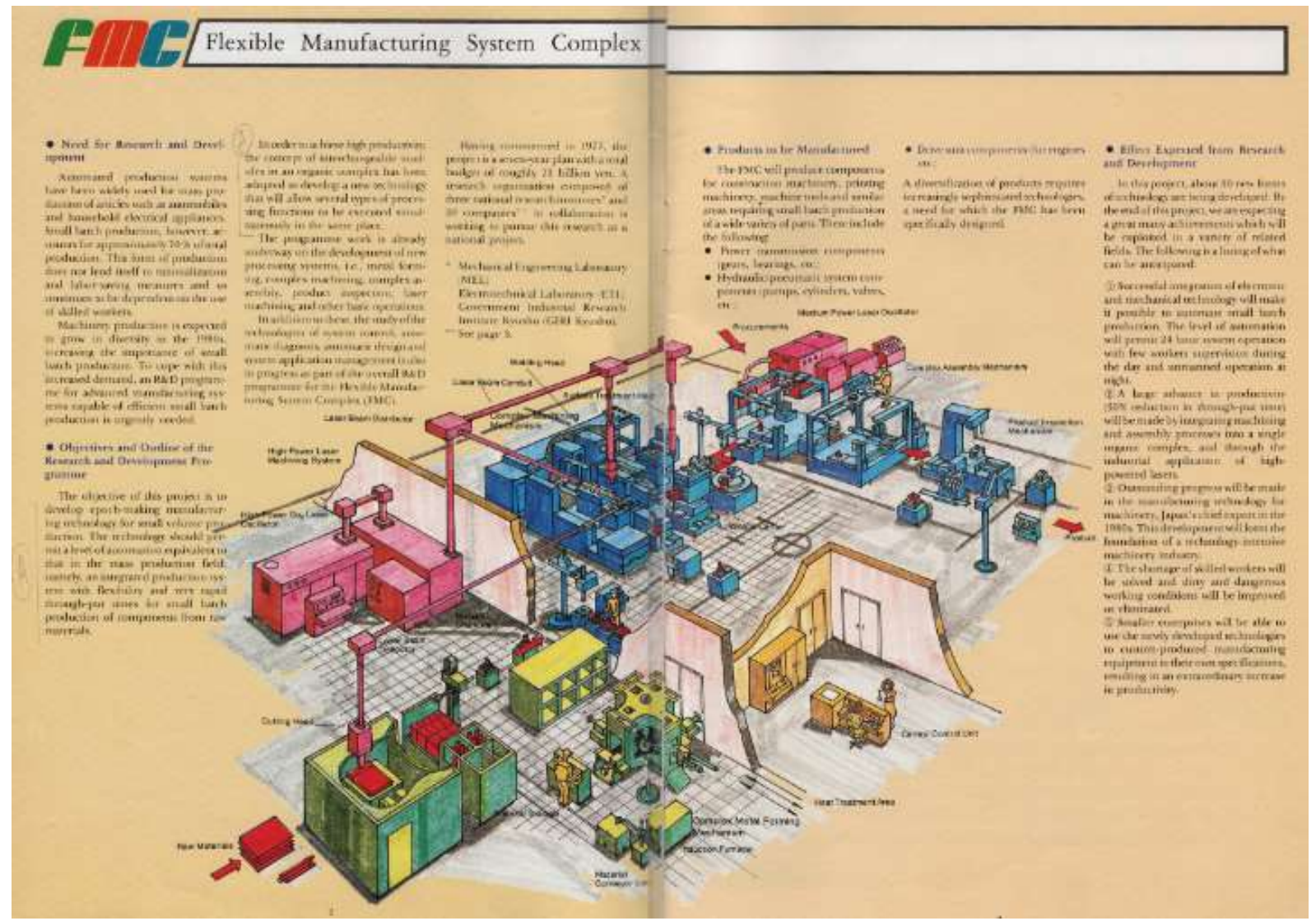

Abbildung 13: Vereinfachte Darstellung des komplexen Fertigungssystems der japanischen Industrie wie es einige Jahre später zu einer Weltausstellung in Tsukuba vorgestellt wurde 


\subsection{Wahl zum Chairman der IFIP AG 5.3}

Zur planmäßigen Beratung der IFIP Arbeitsgruppe 5.3 im Herbst 1976 stand in Dearborn, Michigan, USA die Nachfolge des Vorsitzenden auf der Tagesordnung. Der Vorsitz der Arbeitsgruppe kann nach den Regularien der IFIP normalerweise 3, maximal 6 Jahre wahrgenommen werden. Auf Vorschlag des amtierenden Vorsitzenden Prof. Hatvany wurde ich (für mich völlig überraschend ohne offizielle Reisedirektive), unterstützt vom TC 5 Vorsitzenden J. Vlietstra und weiteren internationalen Fachexperten als neuer Vorsitzender vorgeschlagen. Damit war gleichzeitig die Verantwortung für die nachfolgenden PROLAMAT Konferenzen verbunden.

- 1979 in Ann Arbor, Michigan

- 1982 in Leningrad, UdSSR festgelegt.

Im Nachhinein kann diese Wahl zum Vorsitzenden der IFIP Arbeitsgruppe CAM unter den damaligen fachlichen und politischen Gegebenheiten als ein fachlicher Ritterschlag angesehen werden.

Darüber hinaus wurde in der inhaltlichen Beratung eine neue Interpretation für der Oberbegriff PROLAMAT erarbeitet. Dieser Begriff wird neu interpretiert als „Project Research On Leading-edge Applications and Methods for Applied Information Technology" - Projektforschungen an Spitzenanwendungen und Methoden für angewandte Informationstechniken. Der Begriff Leading-edge (Spitzenanwendung) wird aus heutiger Sicht mit fortgeschrittener Produktionstechnik (Advanced Manufacturing) interpretiert und genutzt. Dabei wurde erneut die unmittelbare enge Verbindung zu industriellen Prozessketten herausgestellt.

\subsection{Zusätzliche Aktivitäten zur NC Society der USA 1977}

In Ergänzung der IFIP Verbindungen und einer USA Reise verdient die Teilnahme an einer Tagung der NC Society der USA besondere Anerkennung. In Verbindung mit der wissenschaftlichen Aufbereitung der komplexen Problemstellung des effizienten NC Maschineneinsatzes war ein Ergebnis des damaligen Doktoranden D. Dimitrov von besonderem Interesse. Obwohl zu dem damaligen Zeitpunkt die USA einen Entwicklungsvorsprung von ca. 12 Jahren in der Entwicklung und industriellen Breitenanwendung der NC Technik hatte, wurde ein Vortragsangebot zum effizienten Einsatz von Fräsbearbeitungszentren von den Organisatoren anerkannt und in das Tagungsprogramm aufgenommen. Es handelte sich dabei im Wesentlichen um Ergebnisse aus der Dissertation vom damaligen Aspiranten an der TU Dresden Dimiter Dimitrov, die von

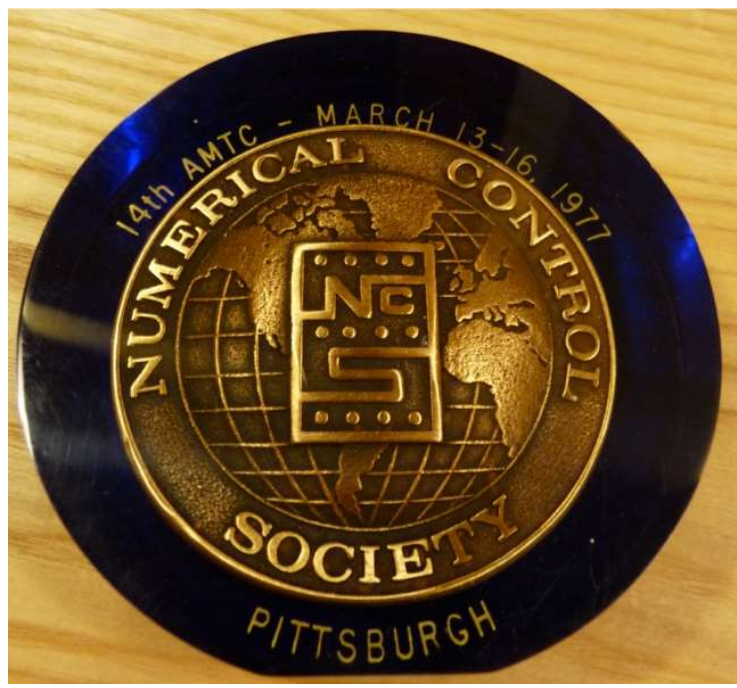

Abbildung 14: Gläsernes Souvenir für Konferenzreferenten mir zum 14. Jahresmeeting der NC Society in Pittsburgh vorgestellt werden konnten.

Mit Bezug zu der Teilnahme an dieser Jahreskonferenz der NC Society ist das Treffen mit dem damaligen Konstruktionsleiter des Unternehmens Trumpf Stuttgart von besonderem persönlichem Interesse. Dessen Chef, Dr. Leibinger. nutzte die Erkenntnisse für die fortgeschrittene numerische Steuerungstechnik für einen zügigen Ausbau der Erfahrungen im gesamten Gebiet der automatisierten Blechverarbeitung. Auf spätere erfreuliche Begegnungen mit Dr. Leibinger in Dresden bzw. Sachsen wird noch später eingegangen. 


\subsection{Beratung des Programmkomitees für PROLAMAT 1979 an der TU Dresden}

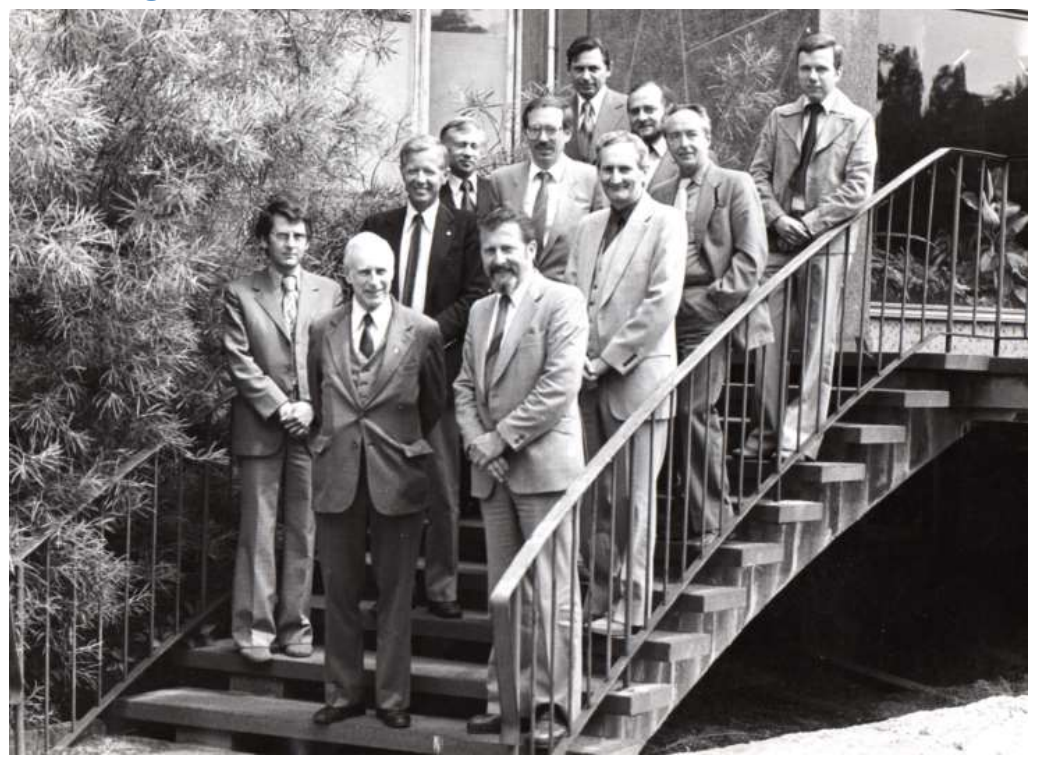

Abbildung 15: Fachkollegen des internationalen Programmkomitees für PROLAMAT 1979 an der TU Dresden (Kutzbachbau)

1978 wurde die Beratung des Programmkomitees für PROLAMAT 1979 an der TU Dresden durchgeführt. Damit war die Gelegenheit gegeben, dem internationalen Expertengremium den erreichten Entwicklungsstand des CNC- Betriebes im Versuchsfeld der Sektion Fertigungstechnik und Werkzeugmaschinen zu demonstrieren. Von Dr. Merchant wurde in diesem Zusammenhang eingeschätzt, dass an keiner USA-Universität dieser Entwicklungsstand in Lehre und Forschung erreicht war.

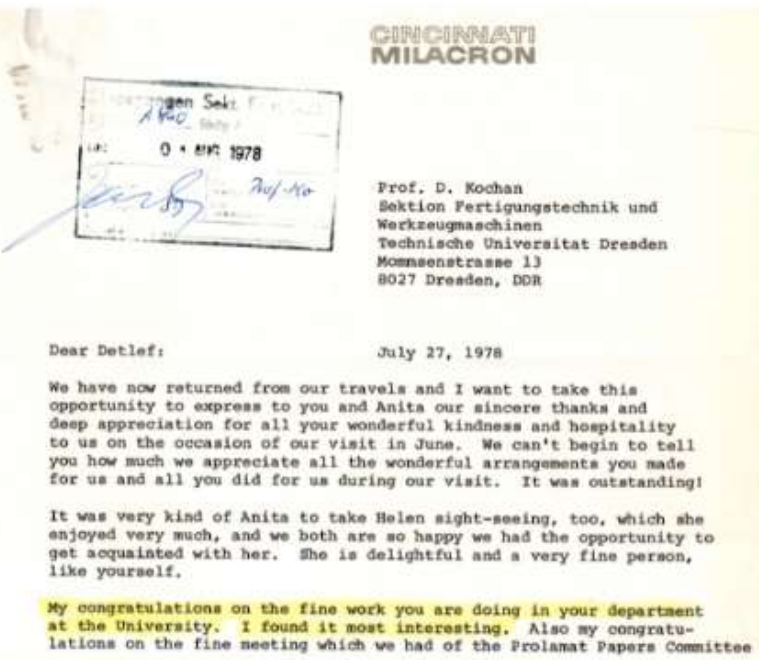

My congratulations on the fine work you are doing in your department at the University. I found it most interesting. Also my congratu-

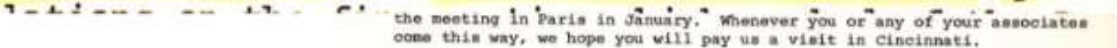

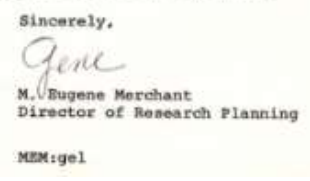


Fachkollegen des Programmkomitees für PROLAMAT 1979 in den USA, Vorn in der Mitte Dr. Merchant als Forschungsdirektor des damals noch führenden USA Werkzeugmaschinenunternehmens und hochgeschätzter Wissenschaftler für spanende Fertigung und Produktionsautomatisierung

Weitere Fachkollegen (Prof Nemes, Budapest, jetzt Australien; Prof Sohlenius, Schweden, Prof Hatvany VR Ungarn; Prof Semenkov UdSSR; Prof Krause BRD; Prof Zilienski Polen; Prof Crestin Frankreich, Prof Ponomarjov, UdSSR Prof Hartman TU Dresden)

In diesem Zusammenhang kann daraufhin verwiesen werden, dass schon ein bis zwei Jahre vorher mit der Vorbereitung des DNC-Betriebes begonnen wurde. Dazu war ein kompetenter Fachkollege von der damaligen Sektion 9 (Elektronik), Herr Dr. Becker für regelungstechnische Probleme in der zentralen Werkstatt der Sektion Fertigungstechnik und Werkzeugmaschinen eingestellt worden . Darüber hinaus fanden in diesen Jahren 1966/67 innerhalb der Sektion Werkzeugtechnik und Fertigungsmaschinen zentrale Informationsveranstaltungen statt, in denen ein größerer Kreis der Sektionsmitarbeiter davon überzeugt werden mussten, dass in der damaligen Zeit die Installation geeigneter Kleinrechentechnik zum technischen Umfeld gehört. Für diese sektionsinterne Schulungsveranstaltungen wurde ich von meinem langjährigen Mitarbeiter ,Dipl .Math. Gerhard Franz, bestens unterstützt. Bis zu diesem Zeitpunkt dominierte an der gesamten TU Dresden. auch an der Sektion 14, die Nutzung des zentralen Großrechners BESM6, die wesentliche rechentechnische Basis. Mit der objektiven Forderung, den DNC Betrieb vor Ort, d.h. in der Nähe der Werkstatt des Kutzbach-Baus, zu realisieren, konnte der Direktor des TU zentralen Rechenzentrums von dieser objektiven Notwendigkeit überzeugt werden. Die Nutzung des DNC-Betriebes (Direct Numerical Control) war dabei nur ein Teil des neugeschaffenen Rechnerlabors. Schon nach wenigen Wochen waren ca. 70 Mitarbeiter der Sektion Nutzer der nunmehr im Kutzbach-Bau verfügbaren Klein- Rechentechnik.

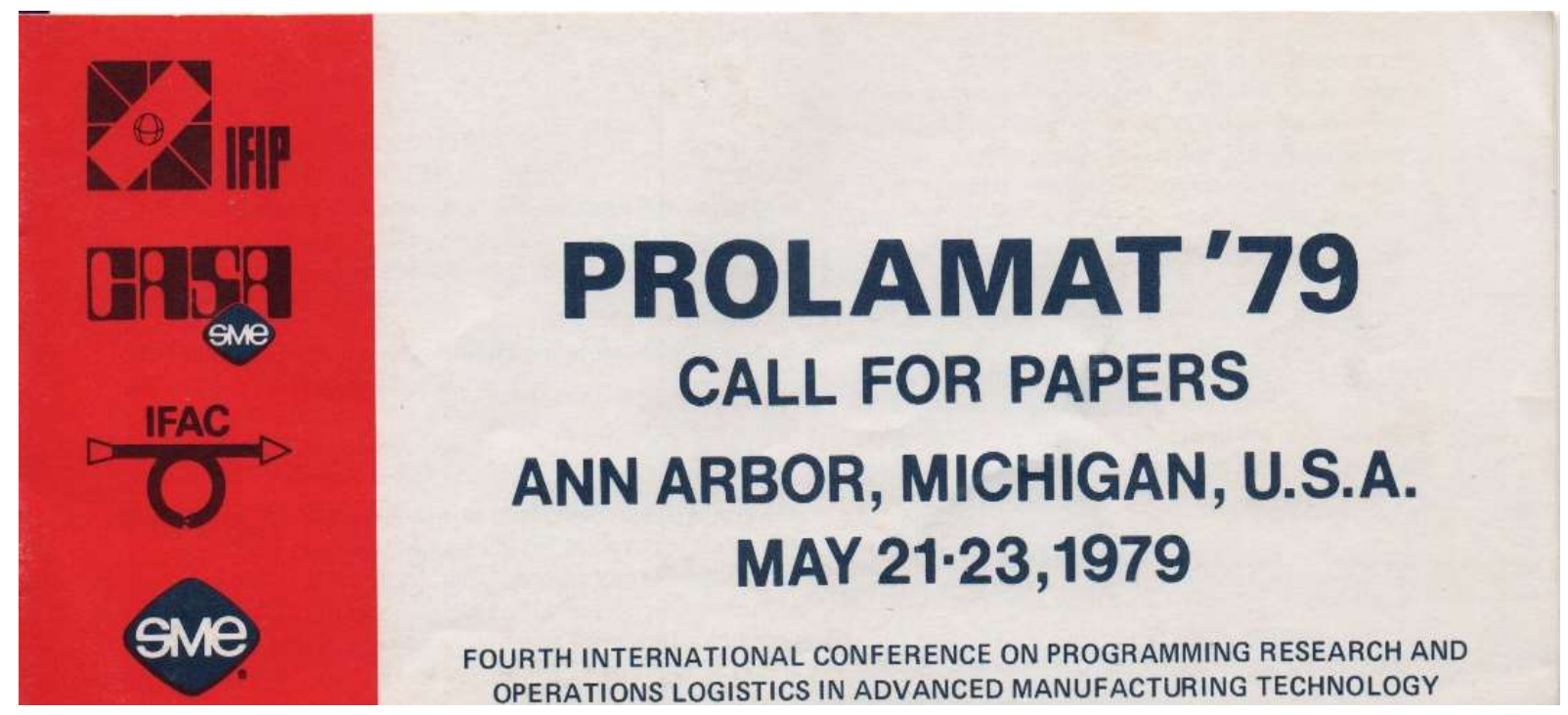

Abbildung 17: Ankündigung der vierten PROLAMAT Konferenz in den USA

1979: Zur Vorbereitung der Prolamat Konferenz war als Bankett-Speaker Mr. D. Ross vom MIT in Boston, Cambridge vorgeschlagen. Deshalb wurde meine Anreise zur Prolamat Konferenz zu einem Arbeitsbesuch des Erfinders der ersten NC- Programmiersprache APT (Automatically Programming Tools) 


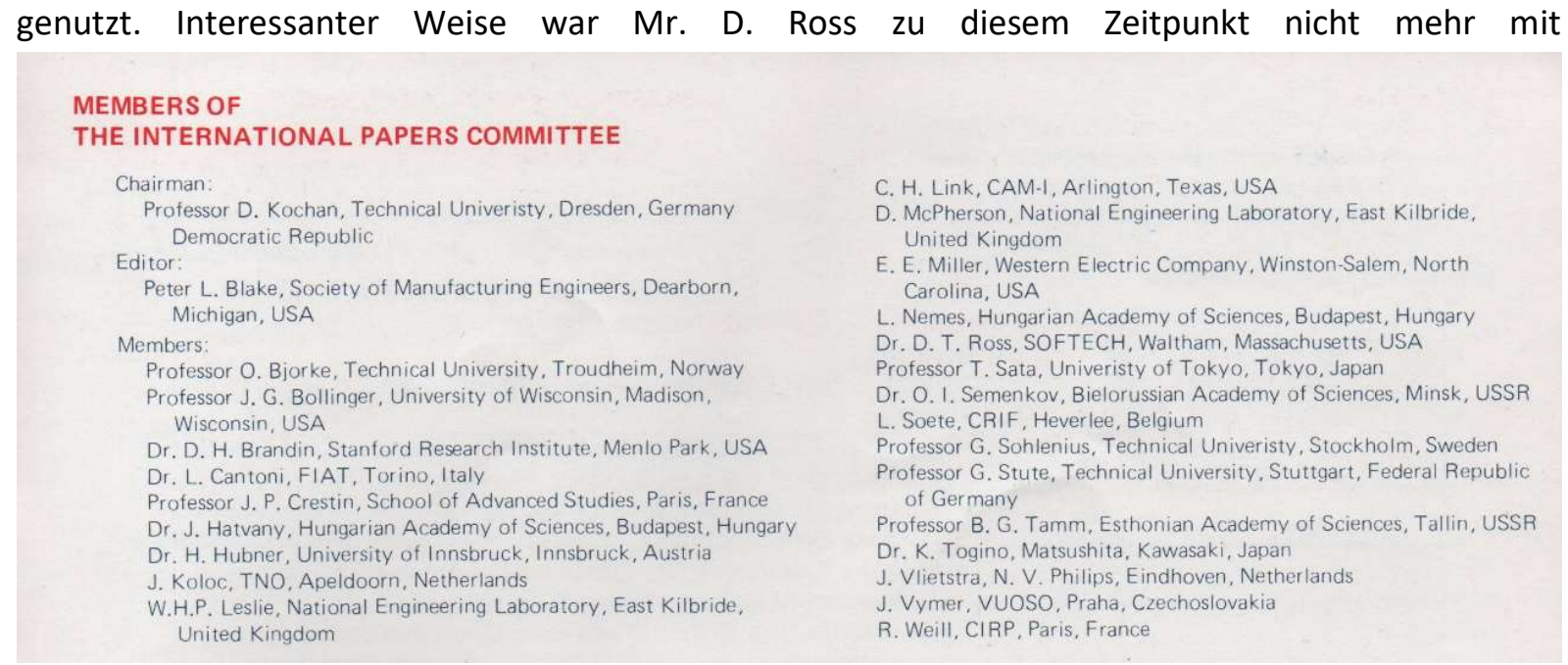

Abbildung 18: Programmkomitee der 4. PROLAMAT Konferenz

dem Problemkreis „NC Programmiersprache Weiterentwicklung“ beauftragt. Ich hatte deshalb unter anderem die Aufgabe inm einige Informationen von dem NC Programmiersprachentwicklung in Europa und in Japan zu übermitteln. Die PROLAMAT Konferenz wurde von der SME Society of Manufacturing Engineers in der Wissenschaftsstadt Ann Arbor durchgeführt. An dieser Technischen Universität ist Prof. Wu als internationaler anerkannter Fachkollege aktiv, der auch an einer IFIP Arbeitskonferenz 1983 in Gaussig (TU Ferienheim) teilgenommen hatte.

Von D. Ross wurde das private Forschungslabor , primär von der Air Force gesponsert, verständlicherweise nur zum Teil vorgestellt.

Eine besondere Herausforderung resultierte aus der Anfrage von Mr. Ross, ob ich mit ihm eine Partie Schach gegen seinen speziellen Schachcomputer spielen würde. In Erinnerung an den Sachverhalt der durchaus soliden Voraussetzungen als Amateur, habe ich diesen riskanten Vorschlag angenommen. Zum Erstaunen und ehrlicher Anerkennung wurden meine drei Erfolge (zweimal mit Weiß, einmal mit Schwarz) anerkannt und das abendliche kollegiale Meeting trotzdem freundlich beendet.

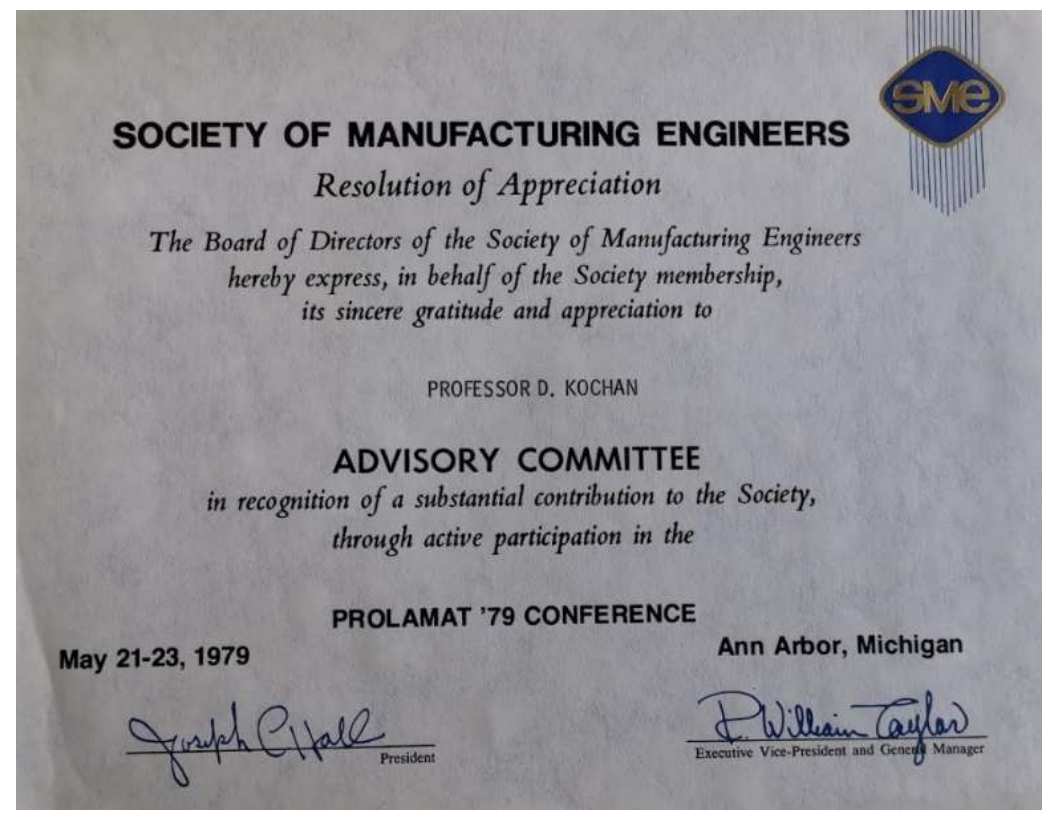


Die Konferenz wurde durch Vorträge von Experten der Freiformflächenmodellierung bereichert, wobei insbesondere Vertreter neu gegründeter Softwareunternehmen auftraten. Diese neu gegründeten Unternehmen waren aus der Luftfahrtindustrie hervorgegangn. In diesem Zusammenhang verdient die aktive Beteiligung vom Chefentwickler des DDR AUTENT (Automobilentwicklungssystem) Herr Dr. Bonitz, besondere Anerkennung. Zusammenfassend kann die PROLAMAT Konferenz 1979 in den USA als erste internationale CAD/CAM Konferenz eingeordnet werden.

\subsection{Erweiterung der internationalen Verpflichtung 1980}

Ausgehend von den anerkannten Aktivitäten der IFIP-Arbeitsgruppe CAM erfolgte die

Ernennung zum DDR- Repräsentanten im IFIP TC 5 auf Betreiben vom TC 5 Vorsitzenden J. Vlietstra. Die Mitgliedschaft in einer Arbeitsgruppe kann durch fachliche Einladungen wahrgenommen werden. Dagegen ist die TC 5 Mitgliedschaft durch die jeweilige Regierung vorzunehmen.

Obwohl mit dem Ende der DDR logischerweise diese ehrenvolle Funktion nicht mehr existiert, wurde ich weiterhin als persönliches Mitglied geführt. (s. Verantwortung für NEWPROLAMAT 2013 in Dresden)

1980 bis 1985: Im Ergebnis der TC 5 Berufung erfolgte die Bereitstellung einer spezifischen Planstelle zur Unterstützung der internationalen Aktivitäten. Diese Planstelle wurde durch die Sprachexpertin (Englisch und Russisch) Frau Dr Anett Reichel wahrgenommen.

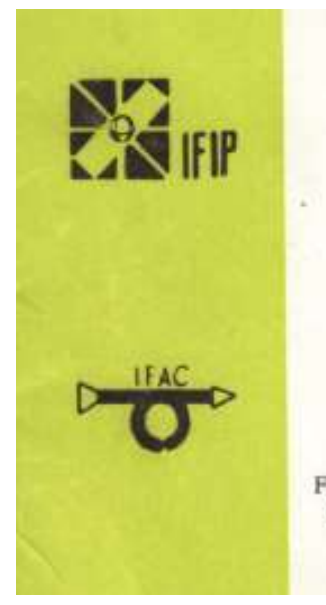

\section{IVIRCI_AMMAT'כこ}

\section{S:AILI. IFOIE FAIEEIES}

\section{IENINGIFAD, USSIZ}

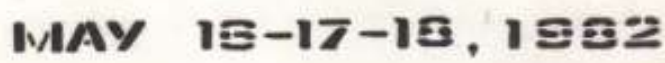

FIFTH INTERNATIONAL CONFERENCE ON PROGRAMMING RESEARCH AND OPERATIONS LOGISTICS IN ADVANCED MANUFACTURING TECHNOLOGY

Abbildung 20: Ankündigung der PROLAMAT-Konferenz 1982 in Leningrad

Für die Vor- und Nachbereitung der PROLAMAT Konferenz 1982 in Leningrad waren damit gute Voraussetzungen gegeben. Die fachliche Verantwortung für die inhaltiche Gestaltung der UdSSRBeiträge lag in den Händen der UdSSR IFIP Mitglieder:

- Prof. Ponomajov, Direktor des Universitätsrechenzentrums Leningrad

- Prof. Semenkov, Direktor des Institutes für Technische Kybernetik in Minsk

Aus der fachlichen Verantwortung dieser beiden IFIP Kollegen erklären sich die vorwiegend theoretisch orientierten Beiträge. Darüber hinaus konnte eine gute Beteiligung insbesondere auch aus dem DDR Kombinat Robotron gewährleistet werden. Ergänzend zum fachlichen Programm verdient die außerordentliche Gastfreundschaft besondere Anerkennung. 
Im Ergebnis der beiden erfolgreichen PROLAMAT Konferenzen (USA, UdSSR) erfolgte die Anerkennung mit der höchsten Auszeichnung der IFIP, dem sogenannten Silver- Core, der mir 1983 zum IFIP Kongress in Paris im festlichen Rahmen übergeben wurde. Meines Wissens hat von der TU Dresden nur noch der Mathematiker Lehmann, diese Auszeichnung erhalten.

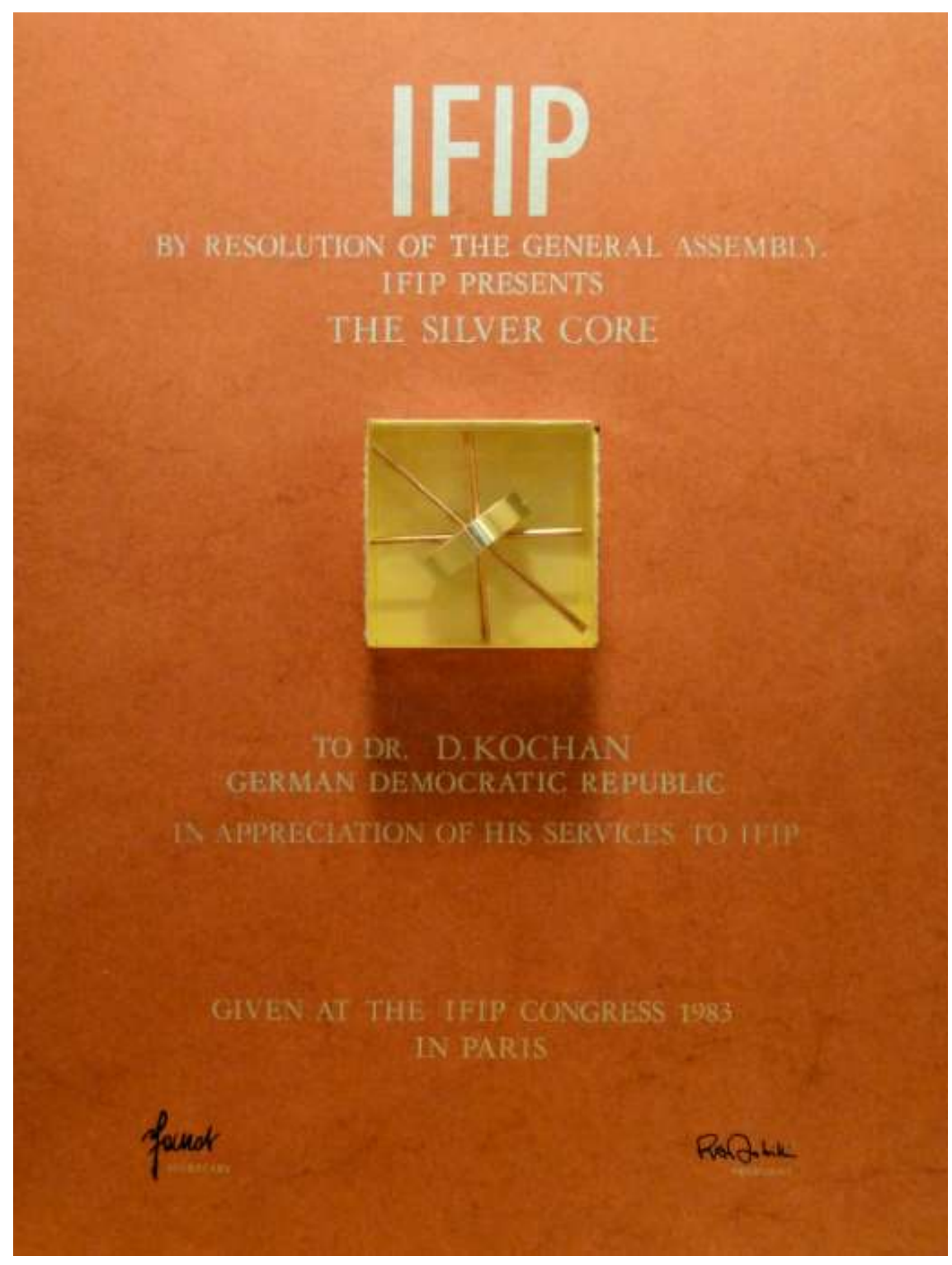

Abbildung 21: IFIP Silver Core höchste Auszeichnung der IFIP 


\subsection{Arbeitskonferenz zur Anwendung der künstlichen Intelligenz in der Fertigungs- technik}

1984: Gemeinsame internationale Konferenz (CIRP/ WG 5.3) in Tokio Künstliche Intelligenz in der Fertigungstechnik. Der gemeinsame Vortrag Kochan - Zehe zur Nutzung von CNC- Unterprogrammen für die Freiformflächenmodellierung fand besondere Anerkennung und führte zu einer Einladung, diesen Vortrag im Toyota Forschungszentrum zu wiederholen. Die hohe internationale Wertschätzung durch den internationalen führenden KFZ-Produzent ist Anlass für eine generelle Bemerkung zum Begriff der Künstlichen Intelligenz (AI - Artificial Intelligence).

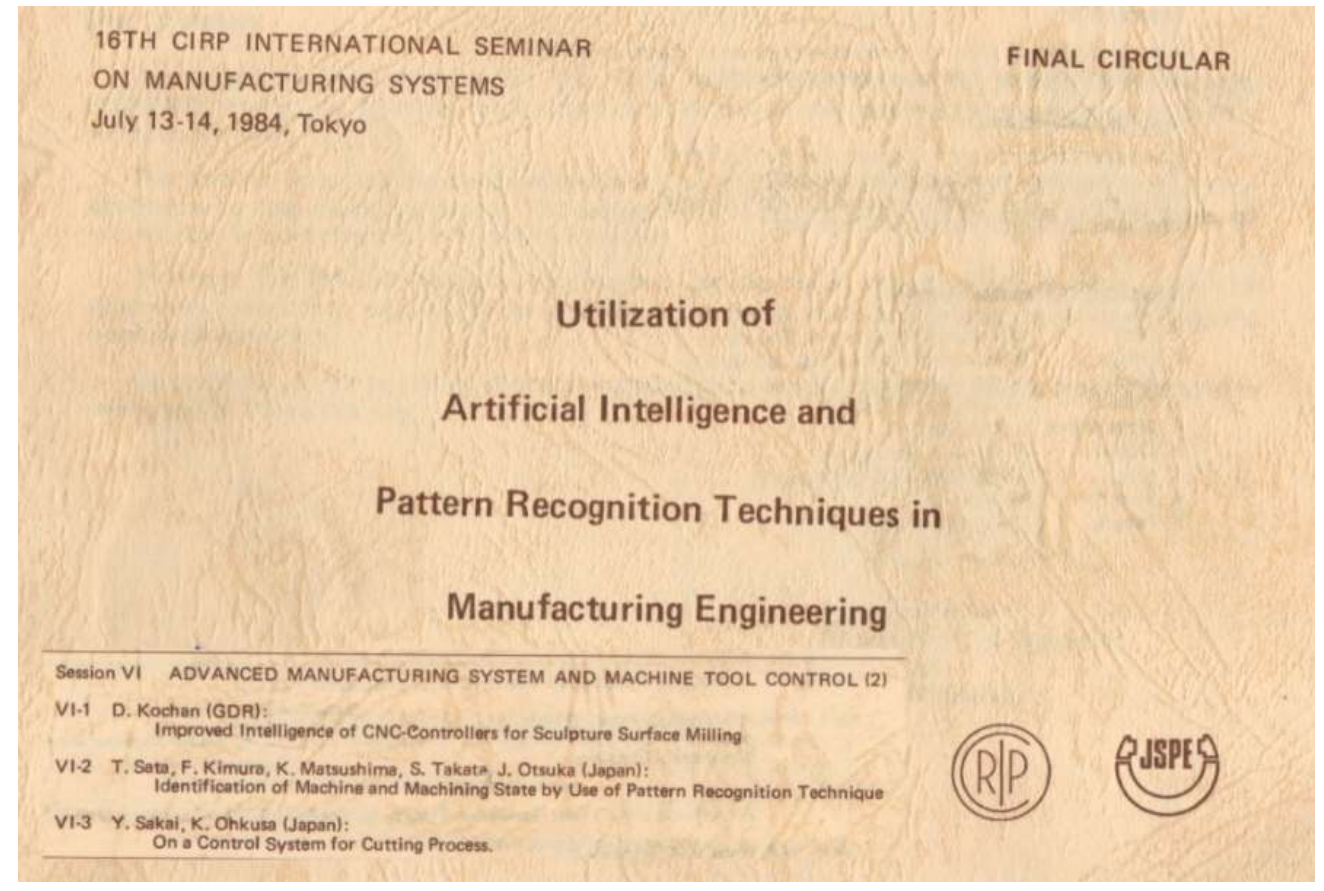

Abbildung 22:Anwendung KI und Mustererkennung in der Fertigungstechnik

Obwohl die Veranstalter CIRP und IFIP die zum damaligen Zeitpunkt international herausragende Forschungsaktivitäten zur künstlichen Intelligenz als Konferenzthema gewählt hatten, sollte man mit diesem Anspruch sehr sorgfältig umgehen. Mit künstlicher Intelligenz können mit hoher Wahrscheinlichkeit sehr komplexe Problemstellungen in der Medizin und auch in technischen Bereichen sinnvoll angewandt werden. In der Breite der industriellen Nutzung sollte man meines Erachtens primär die IT Unterstützung zur Lösung überschaubarer technologischer Problemstellungen im Sinne von Unterstützungssystemen nutzen. Da ist insbesondere mit dem von Dr. Zehe in seiner Dissertation geschaffenen spezifischer Unterprogramme. In diesem Zusammenhang kann darauf verwiesen werden, dass auch ohne ein leistungsfähiges 3D-CAD- System mit der geeignet CNC Unterprogrammtechnik durchaus Freiformflächen an technischen Objekten beherrscht werden. Dieser Entwicklungsstand führte dazu, dass mit der DDR-Steuerung CNC600 mit der Fräsmaschine des Unternehmens Auerbach schon wenige Wochen nach der Installation an der Sektion 14 Freiformflächenbearbeitungen zur Überraschung des technischen Direktors realisiert werden konnten. Dieser auch international bekannt gewordene Entwicklungsstand führte nach einem Besuch der Leipziger Frühjahrsmesse zu einem gezielten Besuch eines USA Experten. Erst nach der Wende erhielt ich von Steuerungsexperten aus dem Unternehmen Bosch die Information, dass dieser bewusste Herr im Auftrag der CIA geprüft hat ob in der DDR das für 3D-Bearbeitungen bestehende Embargo Verbot überwunden war. 


\subsection{IFIP-Arbeitskonferenz zur fachlichen Vorbereitung von PROLAMAT 1988}

1983 wurde im TU-Ferienheim Schloss Gaussig eine internationale Arbeitskonferenz (IFIP WG 5.3 und CIRP) zum Thema Integration of CAD/CAM mit gesamtdeutscher Teilnahme durchgeführt. Diese Arbeitskonferenz diente primär auch zur langfristigen Vorbereitung der für 1988 in Dresden geplanten PROLAMAT-Konferenz. Man kann davon auszugehen, dass mit der aktiven Teilnahme wissenschaftlicher Persönlichkeiten, Prof. Spur, Prof. Krause (TU Berlin), Prof. Weck (RWTH Aachen), Prof. Pritschow und Prof. Storr (beide TU Stuttgart) u.a. ein hohes Niveau der Beiträge gesichert war. Allerdings war die politische Bewertung dieser hochwertigen Arbeitskonferenz ziemlich gegenteilig. Erst viele Jahre später musste ich zur Kenntnis nehmen, das diese Konferenz als „Deutsch--Deutsche- Veranstaltung unter der IFIP- Flagge“ für mich politisch negativ eingeordnet wurde.

Als besonders wertvoll aus meiner Sicht war auch die Teilnahme von Prof. Wu Ann Arbor (USA). Prof Wu kann als der internationale Pionier der „Continious Improvement-Strategie“ anerkannt werden. Während meiner Sabbatical-Zeit im Herbst 1992 konnte ich diese fachlich wertvolle Verbindung für fachlichen Erfahrungsaustausch gut nutzen. In diesem Zusammenhang wurde zu diesem Zeitpunkt ein förderungswürdiger TU-Student (Fertigungsinformatik) für ein Zusatzstudium (PhD) von Prof. Wu aufgenommen.

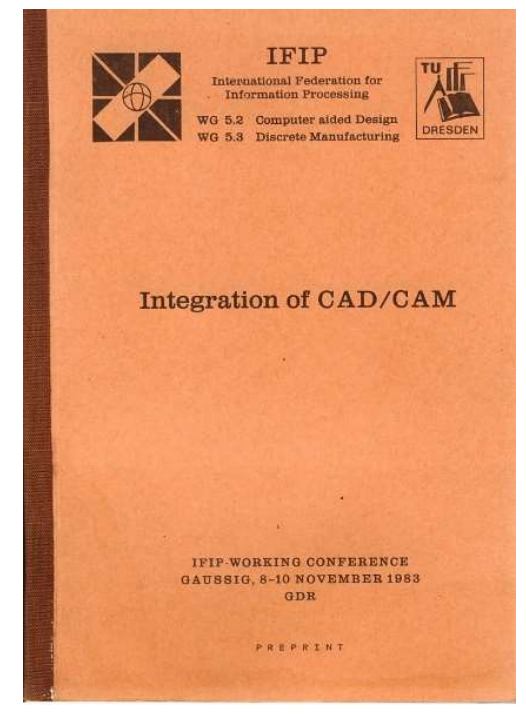

Abbildung 23: deutsch-deutsche Konferenz unter Schirmherrschaft der IFIP 


\section{Die „DDR“}

WERNER KAHL, Bonn,

Trotz strikter Geheimhaltungsversuche und semantischer Nebelkerzen, kann die "DDR" nicht länger verheirnlichen, daß es auch in Mitteldeutschland das Problem der Arbeitslosigkeit gibt. Denn seit längerem können nicht mehr alle Arbeitswilligen mit Arbeitsplätzen versorgt werden.

Ein arbeitsloser lediger Facharbeiter erhält in der "DDR" nach Angaben von Sachkennern aus Ost-Berlin einen Tagessatz von elf Mark.

Die Unterstützung ist auf sechs Monate befristet. Sobald der „Arbeitsuchende" - den Begriff "arbeitslos" kennt das "DDR“-Recht nicht wieder eine Beschäftigung gefunden hat, muß das Geld zurückgezahlt werden. Die Arbeitslosenhilfe ist zudem an die Bedingung geknüpft, daß der Empfänger bedürftig ist und nicht von Familienangehörigen unterstützt werden kann.

Nach Berichten aus Mitteldeutschland gibt es heftige Auseinanderset. zungen in SED-Betriebsgruppen, Funktionäre sehen sich wegen rüder Rationalisierungsmethoden in Belegschaftsversammlungen attackiert, und in Diskussionen gesellschaftli-

\section{und ihre Arbeitslosen}

cher Organisationen werden vor allem fehlende Perspektiven für die Jugend beklagt.

Eine erstmals vom SED-Politbüro beschlossene zentrale Arbeitsvermittlung wurde, so ist Berichten zu entnehmen, den Erwartungen in keiner Weise gerecht. Bei einer Zusammen. kunft von SED-Funktionären der „DDR"-Akademie für Gesellschaftswissenschaften mit SPD-Mitgliedern, die kürzlich in Freudenstadt (Schwarzwald) stattfand, war zu hören, da $\beta$ Jugendliche in der "DDR" sich zunehmend mit Gelegenheitsarbeiten über Wasser hielten.

Ferner werde ein Teil der Perso. nen, die nicht in geordneten Arbeitsverhältnissen stehen, von Verwand. ten, Freunden oder früheren Arbeitskollegen versorgt. Er gebe auch eine Dunkelziffer von Schwarzarbeit.

Wirklich Bedürftige, die die als ${ }_{n}$ staatliche Fürsorge " kaschierte Arbeitslosenunterstützung beziehen und nach sechs Monaten noch keine Arbeit angenommen haben, können nach den gesetzlichen Bestimmungen als "Asoziale" verhaftet werden. In einigen Fällen sollen die Behörden auf Sanktionen verzichtet haben, weil die Betroffenen von einem Partner oder anderweitig unterstützt wurden.

Die Diskussionen über den zunehmenden Verlust von Arbeitsplätzen haben sich in jüngster Zeit anscheinend verschärft. Den AnstoB gab die von der SED-Führung vorangetriebene Automatisierung und "bedienarme Fertigung ",

Was unter dem in die "DDR"-Technologie neu eingeführten Begriff "bedienarm" zu verstehen ist, erläuterte kürzlich „DDR".Professor Kochan in der "Sächsischen Zeitung “ : "Weniger Arbeitskräfte in den ersten beiden Schichten und bedienlos in der Nachtschicht."

Kochan kündigte an, „im unmittel baren Produktionsproze $\mathrm{B}^{\prime \prime}$ der $\mathrm{Fa}$ briken würden "wesentlich weniger Menschen" als heute noch in hochautomatisierten Unternehmen gebraucht. Dieser Trend habe begonnen, schrieb Kochan, ohne jedoch Zahlen zu nennen.

„Es geht darum, nicht nur einen Fertigungsbereich zu automatisieren, sondern ganze Betriebsbereiche, jawohl, bis hin zu ganzen Betrieben." Betriebe „ohne Menschen", so der Ostberliner Rationalisierungsbe auftragte, seien "für die DDR keine Zukunftsmusik mehr"

\section{Abbildung 24: Bewertung von DDR-Entwicklungen, BRD-Tageszeitung "Die Welt", 30.11.1984}

In allen bisherigen Ausführungen wurde die durchaus freundschaftliche Zusammenarbeit mit internationalen anerkannten Persönlichkeiten aus der BRD herausgestellt. Davon ausgehend ist es schon etwas verwunderlich, wenn zu den Entwicklungen der DDR von der Presse, im konkreten Fall „Die Welt", kritische Äußerungen erfolgten. Obwohl es in der DDR bekannter Weise keine Arbeitslosen gab, wird dieses Problem in Verbindung mit den Entwicklungstrends zur bedienarmen Fertigung kritisch beleuchtet. Wenn auch mit höheren Automatisierungsgraden der unmittelbare Bedienerbedarf reduziert wird, muss die Schlussfolgerung mit Bezug zu zunehmender Arbeitslosigkeit nicht voll akzeptiert werden. Wie auch in der gemeinsamen Diskussion mit den Herren Hatvany und dem TC5 Vorsitzenden Vlietstra in Schottland (siehe vorn) kann eine differenzierte Bewertung vorgenommen werden. Die Ingenieure sind in allen Ländern primär dafür zuständig, dass die industrielle Fertigung effizienter, leichter, umweltfreundlicher wird. Wie die gesamte Gesellschaft an derartigen Weiterentwicklungen und Fortschritten beteiligt wird, ist in jedem Fall eine Angelegenheit der gesamten Gesellschaft, d.h. unter staatlicher Verantwortung. Deshalb gehen diese zum Teil negativen Bemerkungen etwas an den objektiven Entwicklungszielen und der Bewertung des Entwicklungsstandes vorbei. Andererseits kannDie objektive Realität in der Breite der Industrie nicht ausgeklammert werden. So wurde vom Chefstatisker der DDR Prof. Dronda eine verdeckte Arbeitslosigkeit zu Beginn der 80iger Jahre konstatiert .Die aus den komplexen Automatisierungsvorhaben resultierenden Erkenntnisse und Erfahrungen sind demnach nicht auf die unbefriedigende Situation in der Breite der Industrie zu übertragen. 
Der schon in den 1970iger Jahren begonnene Trend zu höheren Automatisierungsgraden bis zur bedienarmen Fertigung ist logischerweise auch in der Gegenwart ein wichtiges Kennzeichen für hochproduktive Fertigungseinrichtungen und damit gleichzeitig ein wesentliches Kriterium für alle IndustrieNationen für die Position im globalen Wettbewerb.

\section{Entwicklungen zur rechnerintegrierten Fertigung}

Die enge Verbindung der Softwareentwicklungen, über den Rahmen der NC Programmierungen weit hinausgehend, führte zu dem Begriff „CIM (Computer Integrated Manufacturing)“. Innerhalb der IFIP AG 5.3 und dem übergeordneten technischen Komitee TC5 "Rechner in der Technik" entstand die Anregung den Entwicklungsstand in einem gesonderten Dokument als State of the Art Report zu erarbeiten.

Im Ergebnis der Hauptaktivitäten im TC5 und den verschiedenen Arbeitsgruppen mit jeweiligen Publikationen von den Konferenzen entstand der Gedanke, den Wissensstand im Sinne eines State of the Art Report zusammenzufassen. Unter Beachtung des industriell zentrierenden Arbeitsgebietes CAM kam die erste Anregung an die IFIP Arbeitsgruppe 5.3 (CAM). Deshalb wurde dieser Vorschlag von dem kompetenten

Fachgremium mit Leben erfüllte. Dies war der erste State of the Art Report des IFIP-TC 5.

Die enge Verbindung von Lehre und Forschung wurde in der gesamten Weiterentwicklung kontinuierlich entwickelt. Deshalb werden in den folgenden Ausführungen die Aspekte der Nutzung fortgeschrittener Erkenntnisse in der Lehre nicht explizit herausgestellt.

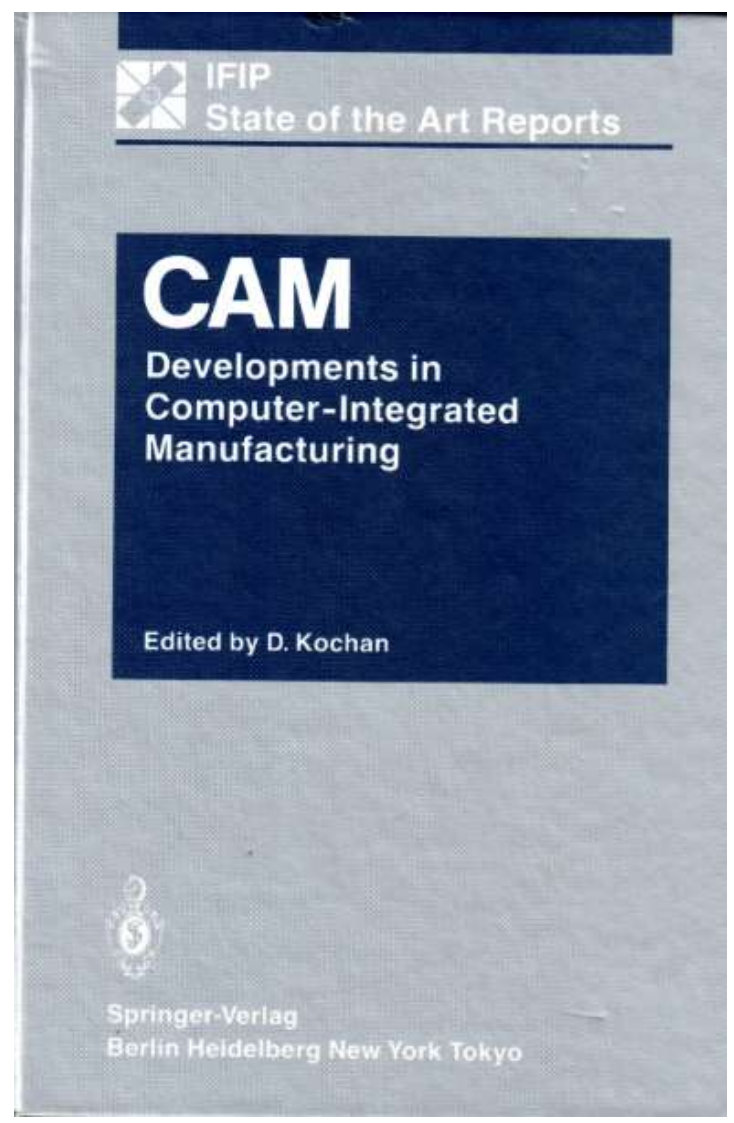

Abbildung 25: IFIP-Veröffentlichung CAM/ CIM

Als Mitautoren für dieses Vorhaben konnte eine Anzahl kompetenter Co-Autoren aus den führenden Industrienationen gewonnen werden:

Dr. Merchant, Prof. Olling, USA, Prof. Hutchinson, Dr. Kozar, Dr. Semenkov, Prof. Crestin Frankreich, Prof. Spur und Prof. Krause, BRD u.a.

Dieser erste State of the Art Report des IFIP TC5 (Rechner in der Technik) fand breite internationale Anerkennung. Bemerkenswert ist in diesem Zusammenhang die erstmalige Nutzung des Begriffes CIMComputer Integrated Manufacturing. Leider erfolgte nach der Wende an der TU Dresden konsequente

Weitere Nutzung der fachlichen Grundlagen,

\subsection{Prolamat Konferenz in Paris 1985}

Die Beratung des internationalen Programm-Komitees zur inhaltlichen Vorbereitung und Durchführung fand im Zusammenhang mit dem im Jahr 1983 in Paris durchgeführten IFIP-Welt-Kongress statt. 
Mit meiner Anerkennung durch den IFIP-Silver Core konnte ich die Pflichten als Chairman der Arbeitsgruppe CAM und gleichzeitig als Vorsitzender des Internationalen Programm-Komitees mit ruhigen Gewissen an Prof Crestin übergeben

Diese Konferenz in einem industriell hochentwickelten Industrieland kann als eine gewisse Fortsetzung der Konferenz 1979 in den USA herausgestellt werden. Mit der aktiven Teilnahme von Prof. Bézier, war einer der führenden Köpfe der 3D Freiformflächenmodellierung in Vorbereitung und Durchführung aktiv beteiligt. Damit waren auch die Weiterentwicklungen der 3D CAD Modulierungssysteme für die Auto- und Luftfahrtindustrie von besonderer Bedeutung und im Konferenzprogramm entsprechend vertreten.
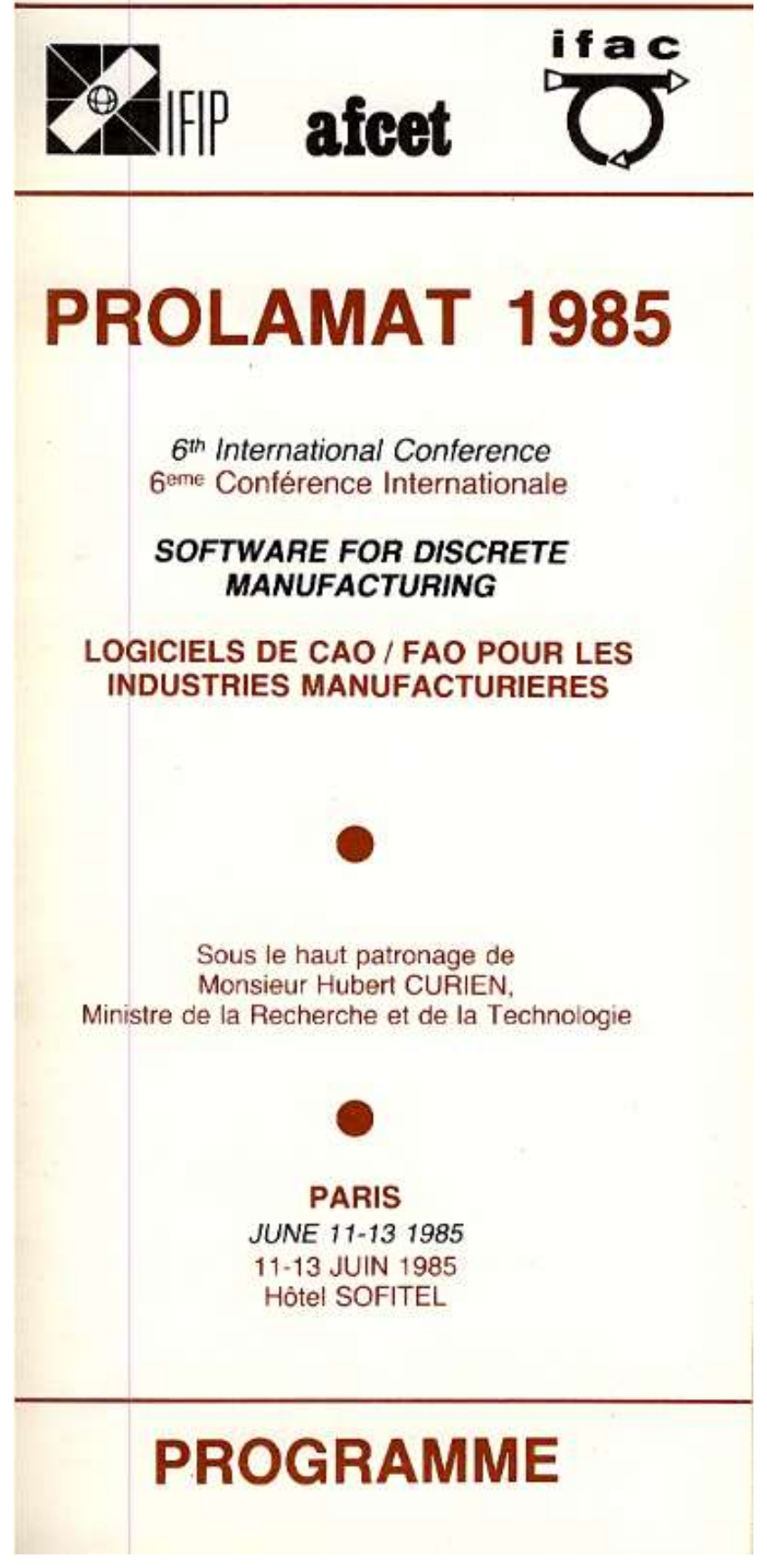

Abbildung 26: Ankündigung PROLAMAT 1985, Paris 
Die 6. PROLAMAT Konferenz unter der Schirmherrschaft eines Ministers war demnach politisch hoch eingeordnet. Das wurde auch für die nachfolgende Konferenz 1988 in Dresden entsprechend beachtet-

\subsection{PROLAMAT in Dresden 1988}

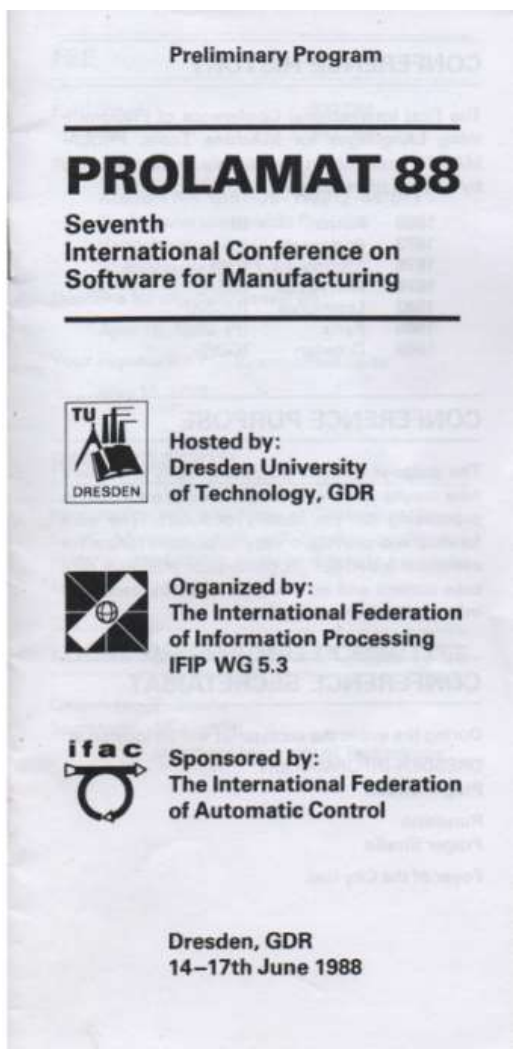

\begin{tabular}{|c|c|}
\hline NATIONAL SPONSORS & CONFERENCE PROGRAM \\
\hline H.J. Jacobs, & Tuesday, 14th June \\
\hline President of the Dresden University of Technology & \\
\hline Rat der Stadt Dresden & $9.30-10.00$ \\
\hline Ministerium & Speakers: \\
\hline für Hoch-und Fachschulwesen & $\begin{array}{l}\text { Mr. Berghofer (GDR), } \\
\text { Lord Mayor of Dresden }\end{array}$ \\
\hline $\begin{array}{l}\text { Ministerium } \\
\text { für Wissenschaft und Technik }\end{array}$ & $\begin{array}{l}\text { Prot. Roistadas (N), } \\
\text { Chairman of IFIP WG } 5.3\end{array}$ \\
\hline $\begin{array}{l}\text { Ministerium } \\
\text { für Werkzeug- und Verarbeitungsmaschinenbau }\end{array}$ & $\begin{array}{l}\text { Prof, Olling (USA), } \\
\text { Chairman IPC }\end{array}$ \\
\hline $\begin{array}{l}\text { Ministerium } \\
\text { für Elektrotechnik und Elektronik }\end{array}$ & $\begin{array}{l}\text { Prof. Jacobs (GDR), } \\
\text { President of the Dresden University of Technology }\end{array}$ \\
\hline Kammer der Technik & $10.00-12.30$ \\
\hline Akademie der Wissenschaften der DDR & $\begin{array}{l}\text { Dr. Georgi (GDR), } \\
\text { Minister für Werkzeug-und Verarbeitungs- } \\
\text { maschinenbau der DDR: }\end{array}$ \\
\hline $\begin{array}{l}\text { Forschungszentrum } \\
\text { des Werkzeugmaschinenbaues der DDR }\end{array}$ & $\begin{array}{l}\text { Entwicklungsstand und -trends zur } \\
\text { rechnerintegrierten Fertigung }\end{array}$ \\
\hline VEB Kombinat NAGEMA & $\begin{array}{l}\text { Prof. Kimura; Prof. Sata; Prof. Yoshikawa (J), } \\
\text { Tokyo University: } \\
\text { CIM-Development in Japan }\end{array}$ \\
\hline VEB Kombinat POLYGRAPH & $\begin{array}{l}\text { Dr. Merchant (USA), Metcut Research Institute } \\
\text { Cincinnati: }\end{array}$ \\
\hline VEB Kombinat ROBOTRON & CIM Tendencies in USA \\
\hline VEB Kombinat Umformtechnik & $\begin{array}{l}\text { Prof. Spur (FRG), University of Technology } \\
\text { Berlin-West: } \\
\text { Trends of CIM Development in Western Europe }\end{array}$ \\
\hline $\begin{array}{l}\text { VEB Werkzeugmaschinenkombinat .7. Oktober" } \\
\text { VEB Werkzeugmaschinenkombinat } \\
\text {..Fritz Heckert" }\end{array}$ & $\begin{array}{l}\text { Prof. Striebing (GDR), Dresden University } \\
\text { of Technology: } \\
\text { Automation of intellectual work in man-machine- } \\
\text { communication }\end{array}$ \\
\hline
\end{tabular}

Abbildung 27: Fakten zur Charakteristik des wissenschaftlichen und industriellen Wertes der PROLAMAT-Konferenz .

Zweifellos kann die PROLAMAT Konferenz 1988 in Dresden durchaus als ein wichtiger Teil meiner wissenschaftlichen Tätigkeit angesehen werden. Mit der aktiven Unterstützung meines persönlichen Freundes Prof. G. Olling und insbesondere auch der deutschen Fachkollegen konnte diese Konferenz als voller Erfolg in die gesamte Serie eingeordnet werden. Unter dem Gesamtbegriff "Software for Manufacturing" wurde die breiten industrieller Anwendungen mit dem Schwerpunkt 3D CAD/CAM zu einem doch gewissen Meilenstein in der gesamten Entwicklung. Wenn man davon ausgeht, dass der Begriff CAD/CAM bis 1985 als Anglizismus in der politisch geführten DDR, damit auch der TU Dresden, verpönt war, kann die inhaltliche Klarstellung und industrielle fortgeschrittene IT-Anwendung mit dem Schwerpunkt 3D CAD/ CAM Modellierung und Automatisierte Fertigung als wichtige industrielle Ausstrahlung eingeordnet werden. In diesem Zusammenhang muss man davon ausgehen, dass in der DDR konkret im Kombinat Umformtechnik Erfurt eine einzige 3D Work Station mit der Fähigkeit der 3D CAD Darstellung verfügbar war Dieses Gerät kann als ein Ergebnis zukunftsorientierter Forschung an der ungarischen Akademie der Wissenschaften gewertet werden.

Unkorrekter Weise wurden in den Jahren 1987 und 1988 alle Bildschirme (Displays), die in Forschungseinrichtungen und Unternehmen vorhanden waren als eine CAD Station erfasst.

In dem Konferenzprogramm ist sowohl die fachliche als auch politische Bedeutung fundiert dargestellt. In der Serie der PROLAMAT Konferenzen die üblicherweise mit Teilnehmergrenzen von über 300 Fachkollegen gekennzeichnet waren, nahmen an der Konferenz 1988 immerhin 700 Fachkollegen aus nationaler und internationaler Wissenschaft und Industrie teil. Dieses erfolgreiche Ereignis welches insbesondere von kompetenten Industriepartnern gewürdigt wurde, war verbunden mit der Aussage "Jetzt hat man erstmal begriffen, was der Begriff CAD/CAM bedeutet" und wo der internationale Stand 
nachgewiesen wurde. Allerdings wurde dieses positive Ergebnis etwas getrübt, da ich mich in Übereinstimmung mit internationalen Abstimmungen an den speziellen Status von West-Berlin gehalten habe. Danach war in den internationalen Abkommen vereinbart, dass Wissenschaftler aus Berlin-West als Vertreter der BRD (FRG) auftreten können. Die DDR -Führung sah das anders. Westberlin galt für sie staatsrechtlich als eine Art besondere politische Einheit, die von der Bundesrepublik weder regiert noch vertreten werden dürfe. Akzeptiert wurden nur gewachsene Verbindungen zwischen der BRD und Berlin-West. Die geforderte Abgrenzung und Ignorierung des besonderen Status von Berlin-West. wie es offiziell gefordert war, wurde von mir in Übereinstimmung mit den internationalen Vereinbarungen wahrgenommen. Dazu war ich im Rahmen der IFIP-Konferenz verpflichtet Der von politischer Führung in eine andere Interpretation vertreten wurde, führte das in Verbindung mit zusätzlichen privaten Kontakten zu einer ernsthaften staatlichen und politischen Disziplinarmaßnahme durch die SEDKreisleitung. In diesem Zusammenhang kann klargestellt werden, dass diese übertriebene Verfahrensweise ausschließlich der politischen Führung der TU Dresden zur Last gelegt werden kann. Da von Seiten des Berliner Hochschulministerium der Erfolg von PROLAMAT 1988 vordergründig gesehen und gewertet werden sollte. Zum Erstaunen des unmittelbar Betroffenen und von mir hoch geschätzten Prof. Spur wirkte sich diese Vorgehensweise der TU Dresden im Jahr 1989 für mich extrem negativ aus. Während ich nahezu 20 Jahre die Interessen der DDR in internationalen Gremien einschließlich der Zusammenarbeit mit westdeutschen Kollegen ordnungsgemäß vertreten habe, wurden die negativen Auswirkungen wie folgt wirksam: Im Sommer 1989 wurde auf dem Gebiet der Produktionsautomatisierung ein Gremium zwischen DDR und Westdeutschen Universitäten gegründet. Die erste offizielle Zusammenkunft fand im Institut von Prof. Spur in Berlin-West statt. Obwohl diese negative Erfahrung viele Jahre zurückliegt, bleiben Fragen, die ich leider Prof. Spur nur mit der 5-seitigen Anklageschrift aus Stasi Unterlagen beantworten konnte. Immerhin war ich in den Jahren vorher anlässlich zahlreicher Arbeitsbesuche, bei allen wesentlichen Ereignissen im Institut von Prof. Spur ( z.B. Grundstein Legung, Einweihung, Richtfest, dreijährige Konferenzen zur Automatisierungstechnik) und weiteren Anlässen im Institut Pascal Straße herzlich willkommen.

Andererseits hat sich im Jahre 1989 schon eine neue Herausforderung mit der Verfügbarkeit der völlig neuen Technologie der direkten Generierung geometrischer Objekte aus 3 D CAD Daten (damaliger Entwicklungsstand: Stereolithographie) ergeben. Es blieb keine Zeit für nutzlosen politischen Streit 


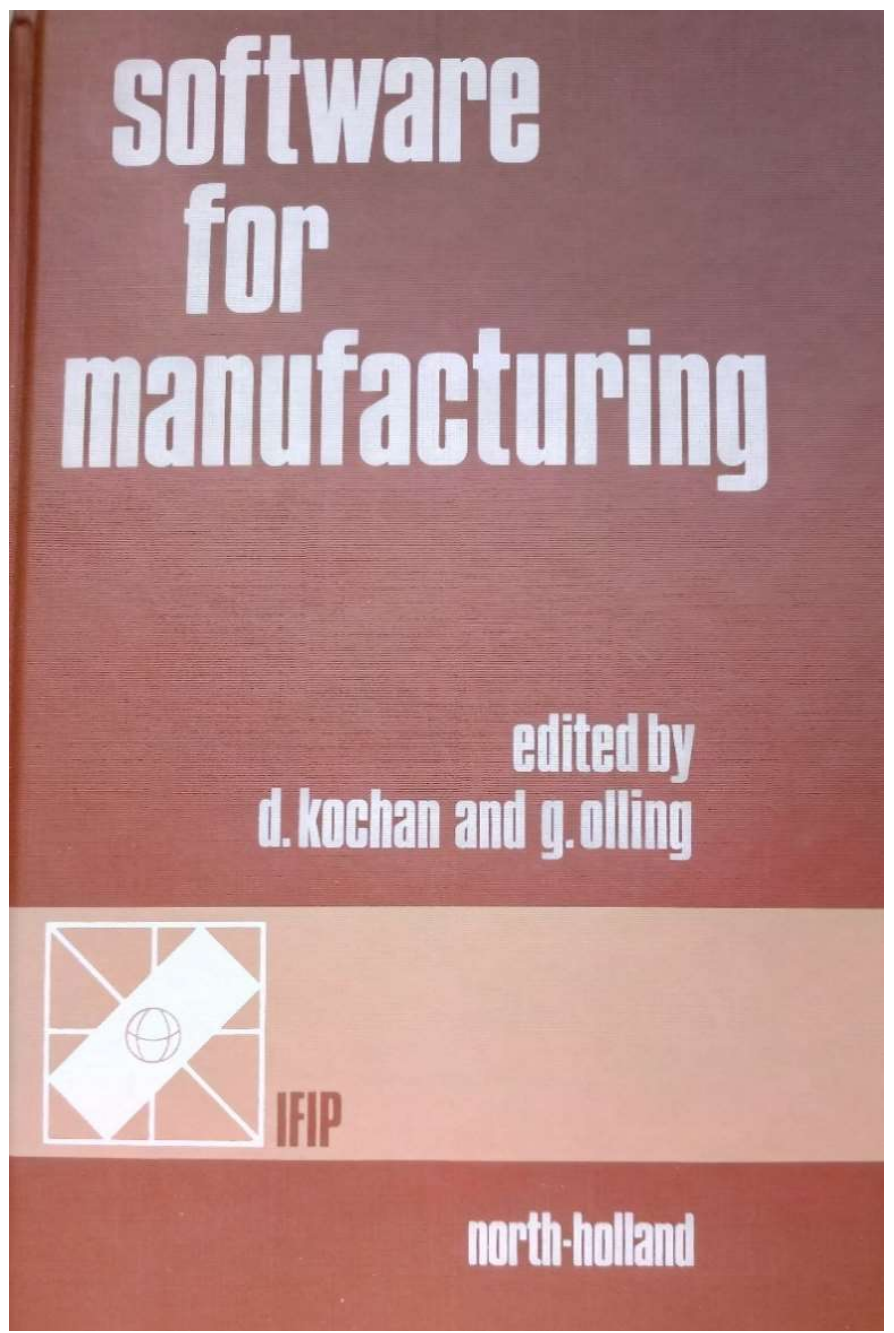

Abbildung 28: Tagungsband als Dokument internationaler Kooperation

Während alle vorangegangenen PROLAMAT- Konferenzen mit jeweils ca 300 Teilnehmern aus der wissenschaftlichen und industriellen Fachwelt besucht wurden, kann die Beteiligung zur Dresdener PROLAMAT-Konferenz mit ca 700 Teilnehmern als herausragend eingeordnet werden.

Für mich waren dabei die zahlreichen positiven Anerkennungen kompetenter Industrie- Partner das wichtigste Ergebnis, konnte doch zur inhaltlichen Klarstellung des CAD/CAM- Entwicklungsstandes maßgeblich beigetragen werden.-

\section{Herausragende Projekte der 70er und 80er Jahre}

\subsection{Automobilentwurfssystem AUTENT}

In den 70er Jahren wurden international Entwicklungen durchgeführt um auch geometrisch komplizierte Flächenformen mathematisch und rechentechnisch zu beherrschen. Bekannte Namen für diesen Entwicklungsschritt waren unter anderen Coons (Coons-Patches) und Gordon u.a. In der Fachrichtung Geodäsie an der TUD hatte zu den Problemkreis Flächenbeschreibung Herr Dr. P. Bonitz eine Dissertation erfolgreich verteidigt. Aus diesen Entwicklungsschritt entwickelte sich eine interessante Kooperation. In Verbindung mit dem Bezug zu Freiformenflächen Programmierungsproblemen waren im Lehrstuhl FT/TP insbesondere durch die Forschungsarbeiten von Dr. Zehe. Erste Grundlagen für die kom- 
plexe Beherrschung vom Entwurf bis zur Fertigung gegeben. Deshalb war die Integration in den Lehrstuhl Technologische Programmierung eine logische Konsequenz. Mit Unterstützung und Zusammenarbeit mit der VVB Automobilbau (Vereinigung Volkseigener Betriebe) wurde eine sog. Theoriegruppe aufgebaut und im Verlauf der Entwicklungen eine Anzahl Studentischer Abschlussarbeiten und daraus resultierend befähigte Mitarbeiter entwickelt wurden. Dieses gesamte Vorhaben erfuhr mit den „Raketenbeschluss" eine bedauerlicherweise erfolgte Rückstellung. Das heißt in der DDR wurde zu den Höhepunkten des „Kalten Krieges, insbesondere im Zusammenhang mit der Stationierung von Raketen in Deutschland) die LKW Produktion der PKW Entwicklung vorgezogen. Trotzdem gelang es auch, gemessen an der Größe der Aufgabe, eine beachtliche Entwicklung dieses gesamten Problembereiches. D.h. von der Freiformflächenbeschreibung bis zur in 5 Achsen gesteuerten Bearbeitung. Dieses Arbeitsgebiet gehört bekanntlich zu den für industrielle Nutzung mit Embargo Beschluss gesperrten Bereich. Das heißt es konnte auf diesem Gebiet weder Software noch entsprechende Maschinentechnik investiert werden. Mit dieser objektiv gegeben fachlichen Herausforderung wurde in konkreten Schritten das AUTENT System entwickelt und im historischen Wendejahr 1989 zu ersten praktischen Anwendungen geführt. So wurde die Motorhaube für die Anforderungen des VW 4 Takt Motors vergrößert und im Werkzeugmaschinenunternehmen Aschersleben als Werkzeug hergestellt. Darüber hinaus wurde auch für den Kofferraumdeckel ein geeignetes Werkzeug im Jahre 1989 gefertigt. Weitere Informationen zu dieser Entwicklung werden von Prof. P. Bonitz eingebracht.

\subsection{CAD/CAM System GRAFIS}

Im Jahre 1978 wurde im Forschungsinstitut für die Schuhindustrie mit dem Aufbau eines komplexen Automatisierungssystems begonnen. Dazu wurde der Begriff "GRAFIS“ genutzt. Das Wortsystem GRAFIS wurde aus den Begriffen Gradation und Forschungsinstitut für die Schuhtechnologie abgeleitet. Dieses Synonym umfasst im gesamten Bereich der entwickelten Software das Verfahrensknowhow bis zu Überleitungsstrategien zur Anwendung der Entwicklungsergebnisse in der Praxis. Diese anspruchsvolle Zielstellung wurde im Rahmen eines Schrittprogramms in einen Zeitraum von ca. 7 Jahren zur Anwendung gebracht.

Ausgangspunkt war die Vorgabe des Generaldirektors der Schuhindustrie, ein Rationalisierungsprojekt mit ausschließlicher Verwendung geeigneter IPA-Technik. Das bezog sich auf die Werkzeugmaschinen und die von ROBOtRON verfügbare Klein-Rechentechnik.

Im Jahre 1978 hatte der Direktor des Forschungsinstitutes FIS von Prof. Russig, Direktor des Werkzeugmaschineninstitutes in Karl-Marx-Stadt die Empfehlung erhalten für die Anwendung numerisch gesteuerter Maschinen, die Lehrstuhlfertigungstechnik technologische Programmierung der TU Dresden vorhandenen Erfahrungen zu nutzen . Darüber hinaus war für die Realisierung des gesamten Entwicklungsvorhabens ergänzend zu den Werkzeugmaschinen aus dem Kombinat Fritz Heckert auch die Nutzung der Kleinrechentechnik vom Kombinat Robotron von Bedeutung. 


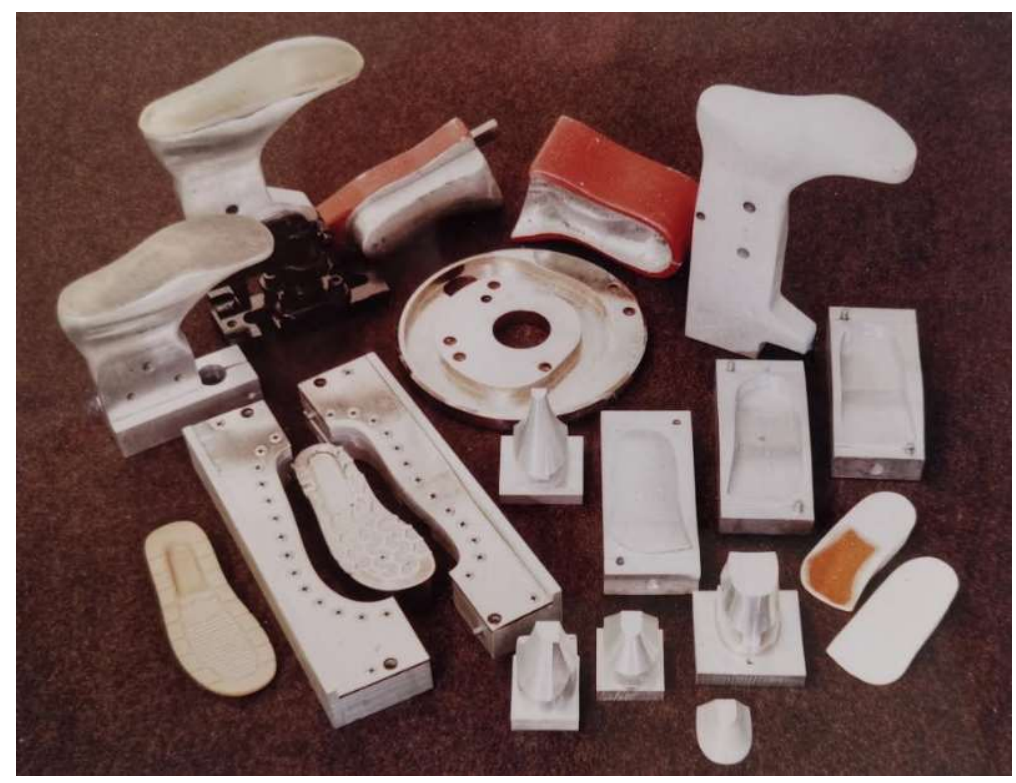

Abbildung 29: Werkzeuge, die unter Nutzung des Systems GRAFIS gefertigt wurden (auch 3D-Freiformflächen)

Auf beiden Gebieten waren im Lehrstuhl Technologische Programmierungen konkrete Erfahrungen vorhanden, die für die Zielstellungen des FIS geeignet waren. Im Ergebnis mehrerer Arbeitsbesuche in kleinen Unternehmen der Werkzeugtechnik der Schuhindustrie z.B. in Burg bei Magdeburg, Schönebeck, Weißenfels und anderen Unternehmen führte zu ersten unmittelbaren Ansatzpunkten für wirkungsvolle Rationalisierungsmaßnahmen. Dabei ging es konkret um die rationelle Fertigung von jeweils 12 unterschiedlichen Größen von Schuhsohlen, wobei die industrielle bewährte Softwarekomponente SYMAP (B) einsatzbereit zur Verfügung stand. Die damit erfolgte relativ schnelle Problemlösung war der Ausgangspunkt für eine zielstrebige Weiterentwicklung des gesamten Vorhabens. In einer dynamischen Entwicklung wurden im Werkzeugbau der Schuhindustrie folgende Teilgebiete auf ein höheres Fertigungsniveau gebracht:

- „Konstruktion und Fertigung von Schuhformteilen (Absätze, Formsohlen) als Meistermodelle für den Galvano- und Metallgussformenbau

- Konstruktion und Fertigung von Umformwerkzeugen für die Gelenkfelder- Brandsohlen- und Hinterkappen Herstellung

- Konstruktion und Fertigung komplizierter Direkt-Anspritz- Werkzeuge mit Metallleisten

- Konstruktion und Fertigung von Schablonen aus Metall, Kunststoff, Pappe

- Fertigung von Sonderwerkzeugen, wie Biegevorrichtungen, Schnittwerkzeuge, Kurvenscheiben u.v.a.m. für die Schuhindustrie und andere Industriezweige ${ }^{\prime 1}$

\footnotetext{
${ }^{1}$ Zitat aus Dissertation an der TUD 1988 von Claus Friedrich „Ausgewählte Ergebnisse der Entwicklung und Anwendung des CAD/CAM Systems GRAFIS“
} 
Die Effekte lagen vor allem in einer Beschleunigung des Gesamtprozesses der Werkzeugfertigung sowie in einer beträchtigen Qualitäts- und Präzisionserhöhung aller Konstruktionselemente. Darüber hinaus sind die durchgeführten Systementwicklungen numerisch gesteuerter Nähmaschinen besonders herauszustellen. Der gesamte Nähprozess für die unterschiedlichen Ausführungsvarianten, insbesondere für Sportschuhe wurde auf 60 numerisch gesteuerten Nähautomaten realisiert. Dabei konnten Produktivitätssteigerungen von durchschnittlich 40 Prozent in extremen Fällen bis 400 Prozent nachgewiesen werden. In diesem Zusammenhang mussten auch für spezifische Teillösungen z.B. der effizienten Ausnutzung der Ausgangsmaterialen z.B. der unregelmäßig geformten Lederausgangsrohmaterialen anspruchsvolle mathematische Optimierungsprobleme gelöst werden. Es erwies sich dabei als ein wichtiger Vorteil, dass für viele Teilaufgaben aus dem ersten Staatsplanthema von Carl-ZeissJena beschaffenen Forschungsverbund viele Kompetenzen für die unterschiedlichen Aufgabenfelder genutzt werden konnte. So sind außer der TU Dresden wissenschaftliche Graduierungsarbeiten an der TH Karl-MarxStadt und auch von der TH Ilmenau in das gesamte Ergebnis eingeflossen.

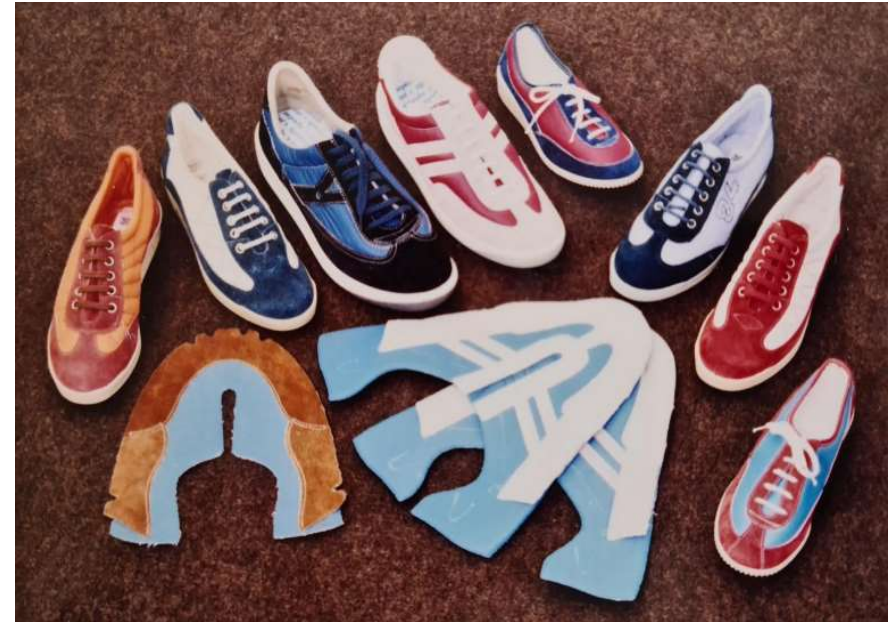

Abbildung 31: Schuhschäfte und Schuhe, die auf numerisch gesteuerten Näöhautomaten unter Nutzung von GRAFIS hergestellt wurden

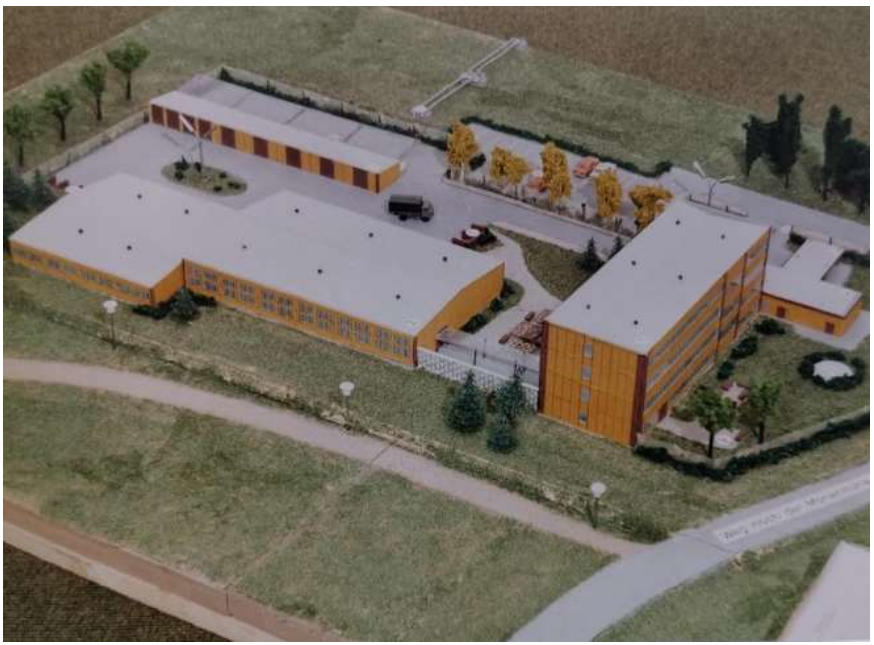

Abbildung 30: Betrieb für rechnergestützte Formteilentwicklung und Werkzeugfertigung 
PROF. DR.

\section{DETLEF KOCHAN}

\section{FÜR HERVORRAGENDE WISSENSCHAFTLICH-TECHNISCHE LEISTUNGEN \\ BEI DER ENTWICKLUNG UND REALISIERUNG \\ DES CAD/CAM-SYSTEMS „GRAFIS“ ALS BEISPIEL \\ ZUR RATIONALISIERUNG DER FERTIGUNGSVORBEREITUNG \\ UND AUTOMATISIERUNG DER FERTIGUNGSPROZESSE

\author{
DEN NATIONALPREIS \\ DER \\ DEUTSCHEN DEMOKRATISCHEN REPUBLIK
}

I. KLASSE

FUR WISSENSCHAFT UND TECHNIK

IM KOLLEKTIV

BERLIN, DEN 7. OKTOBER 1985

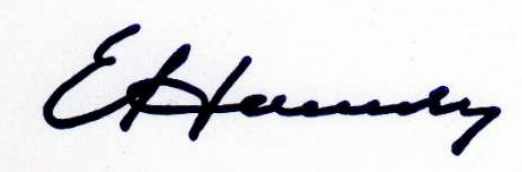

Abbildung 32: Nationalpreis I. Klasse

\subsection{Allgemeingültige Erfahrungen}

Ein wesentliches Merkmal dieser gesamten zukunftsorientierten und industriell bewährten Lösung waren mit dem Aufbau eines Technikums verbunden. In diesem Sinn konnten die Entwicklungen der notwendigen Software und die Einsatzerprobung der numerisch gesteuerten Maschinen und Nähautomaten erprobt werden. Gleichzeitig war dieses Technikum Ausbildungsstätte für die Qualifizierung des Personals. Für das effiziente Nutzen aller Komponenten des CAD/CAM System GRAFIS gegeben. Diese wichtige Erfahrung steht im Zusammenhang mit jeder Form der komplexen Automatisierung in der Fertigungstechnik, wie es sich in der industriellen Entwicklung von der einzelnen NC Maschine über die Bearbeitungszentren und flexiblen Fertigungssysteme bewährt hat. Am Beispiel GRAFIS wird die in der 
Einleitung dieses historischen Dokumentes herausgestellte strategische Vorgehensweise "Continiuous Improvement" bekräftigt .

Für diese herausragenden Forschungsergebnisse wurde 1985 der Nationalpreis 1. Klasse für das Entwicklungskollektiv vergeben. Nach Aussage vom Direktor des Universitäts-Archives der TU Dresden,

Herr Dr. Lienert, gab es in den 1970iger und 1980iger Jahren keinen weiteren Nationalpreis 1. Klasse an der TU Dresden

Darüber hinaus wurden diese beispielgebenden Lösungen als Muster für andere Aufgabengebiete genutzt.

Obwohl die genutzte DDR - Technik (Werkzeugmaschinen und Rechentechnik aus dem Kombinat Robotron) nicht den internationalen Entwicklungsstand entsprach, war z.B. das führende Schuhunternehmen Salamander daran interessiert, die gesamte Systemlösung zu kaufen und im eigenen Unternehmen zur Wirkung zu bringen. Der dazu notwendige Qualifizierungsaufwand und die Nutzungseffekte innerhalb der DDR waren der Ausgangspunkt für die Ablehnung der Interessen von Salamander.

Zusammenfassend verdienen die charakterisierten Ergebnisse des Entwicklungsvorhaben CAD/CAM Lösung GRAFIS aus folgenden Gründen besonderer Anerkennung. In Verbindung mit dem Besuch von Präsident Gorbatschow am historischen 7.10.1989 in Berlin wurde ihm als eine der wenigen Spitzenleistungen der DDR das CAD/CAM System GRAFIS vorgestellt. Dieses Ereignis ist im Schuhmuseum Weißenfels dokumentiert.

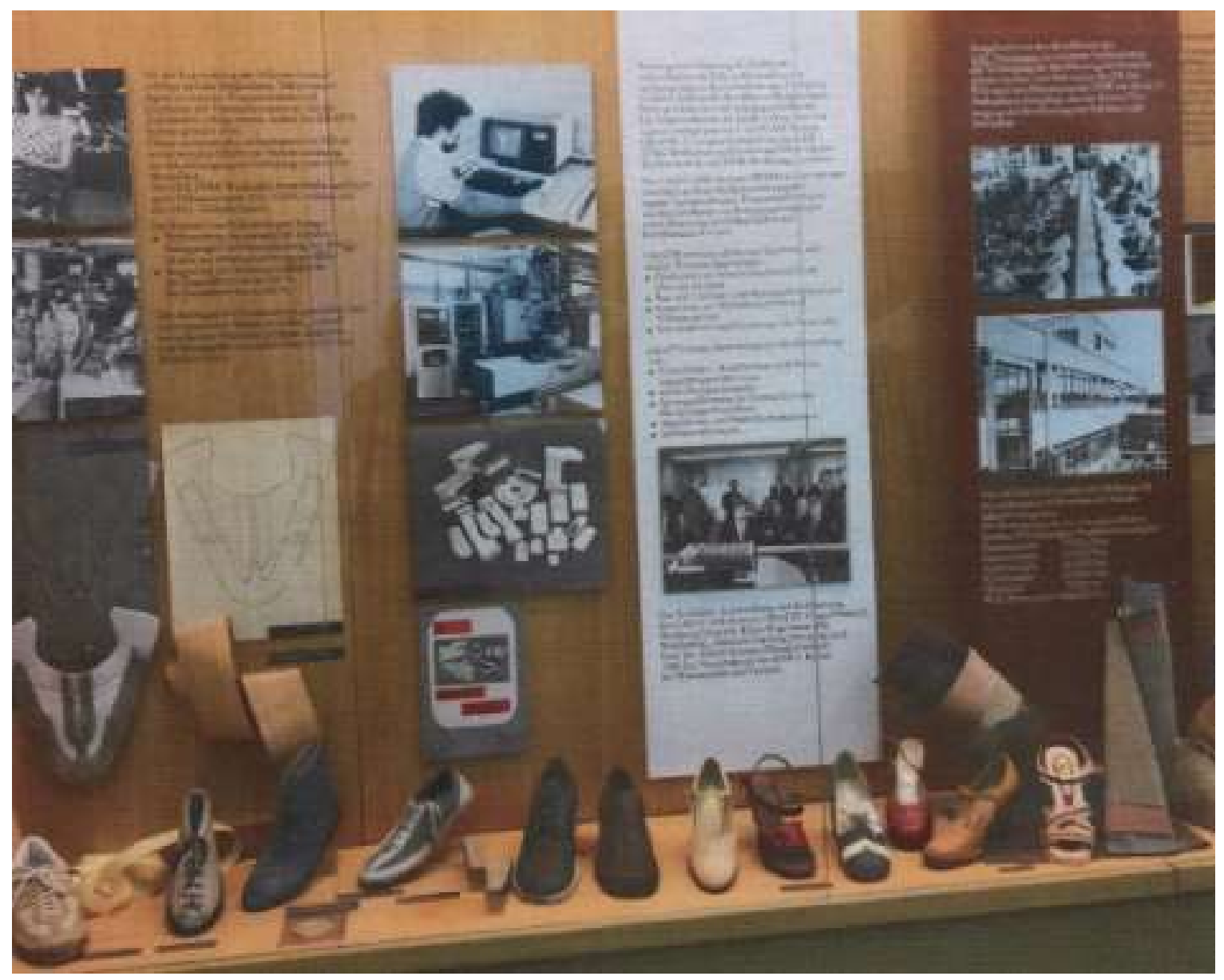

Abbildung 33: Ausstellung im Schuh-Museum Weißenfels 


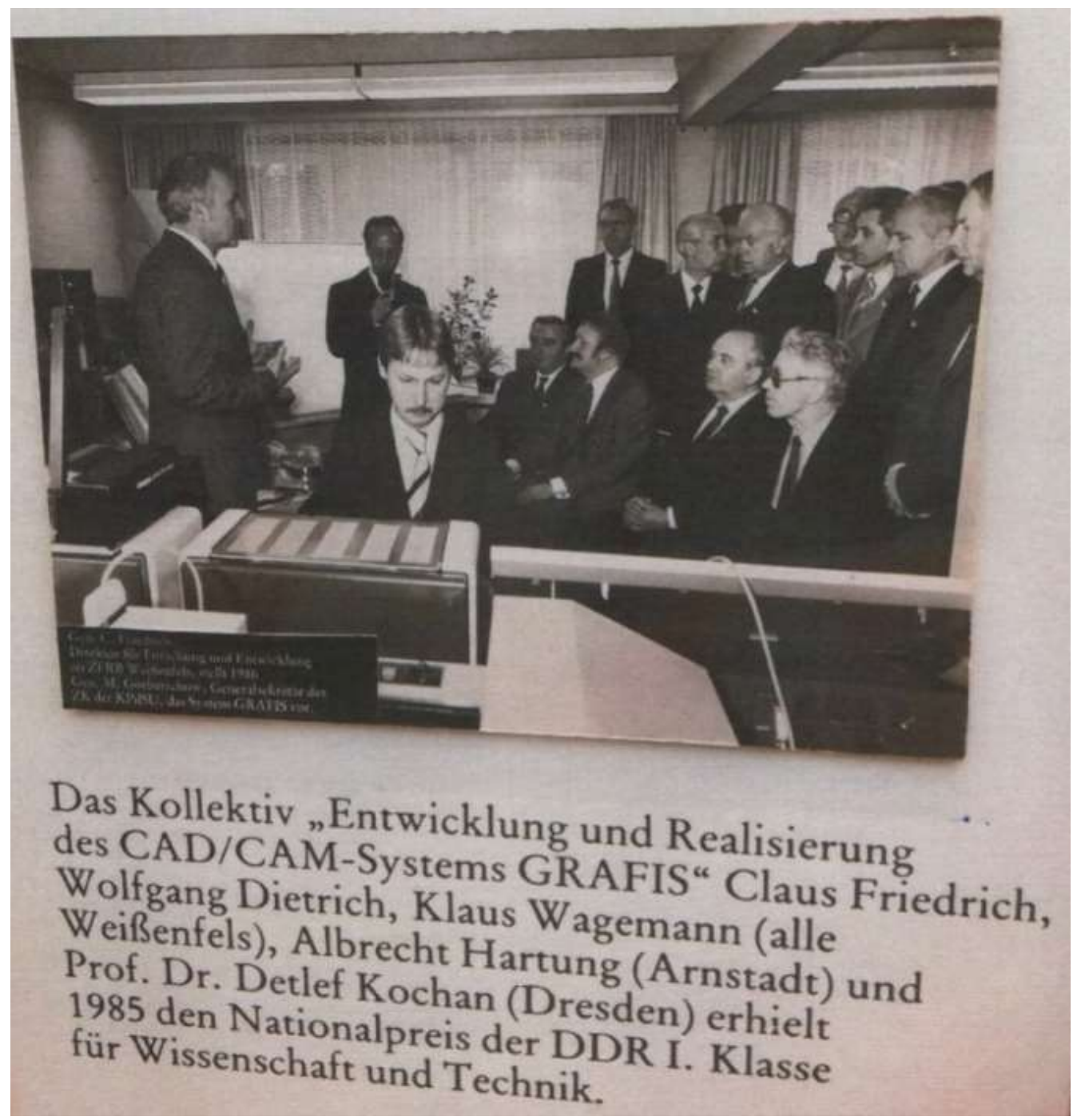

Abbildung 34: Präsentation der DDR-Spitzenleistung durch Prof Friedrich vor Gorbatschow im historischen Oktober 1989 


\section{Entwicklungen zu CAD/CAM und CIM in der DDR}

\subsection{Förderung der CAD/CAM-Entwicklungen}

Nachdem in der DDR bis ca. 1984 der Anglizismus CAD/CAM von der politischen Führung verpönt war, erfolgte ein krasser Wechsel im Jahr 1985. Mit meiner Berufung als CAD/CAM Beauftragter des Hochschulministers war eine neue Plattform gegeben, die gleichzeitig mit hohen Erwartungen verbunden waren. Zum breiten Verständnis in den gesamten Entwicklungen war zweifellos die erfolgreiche Realisierung der internationalen PROLAMAT- Konferenz 1988 in Dresden ein wichtiger Beitrag (siehe oben). Darüber hinaus sollte in möglichst kurzer Zeit ein Buch mit dem Begriff „CAD/CAM Schlüsseltechnologie als Intensivierungsfaktor" geschaffen werden.
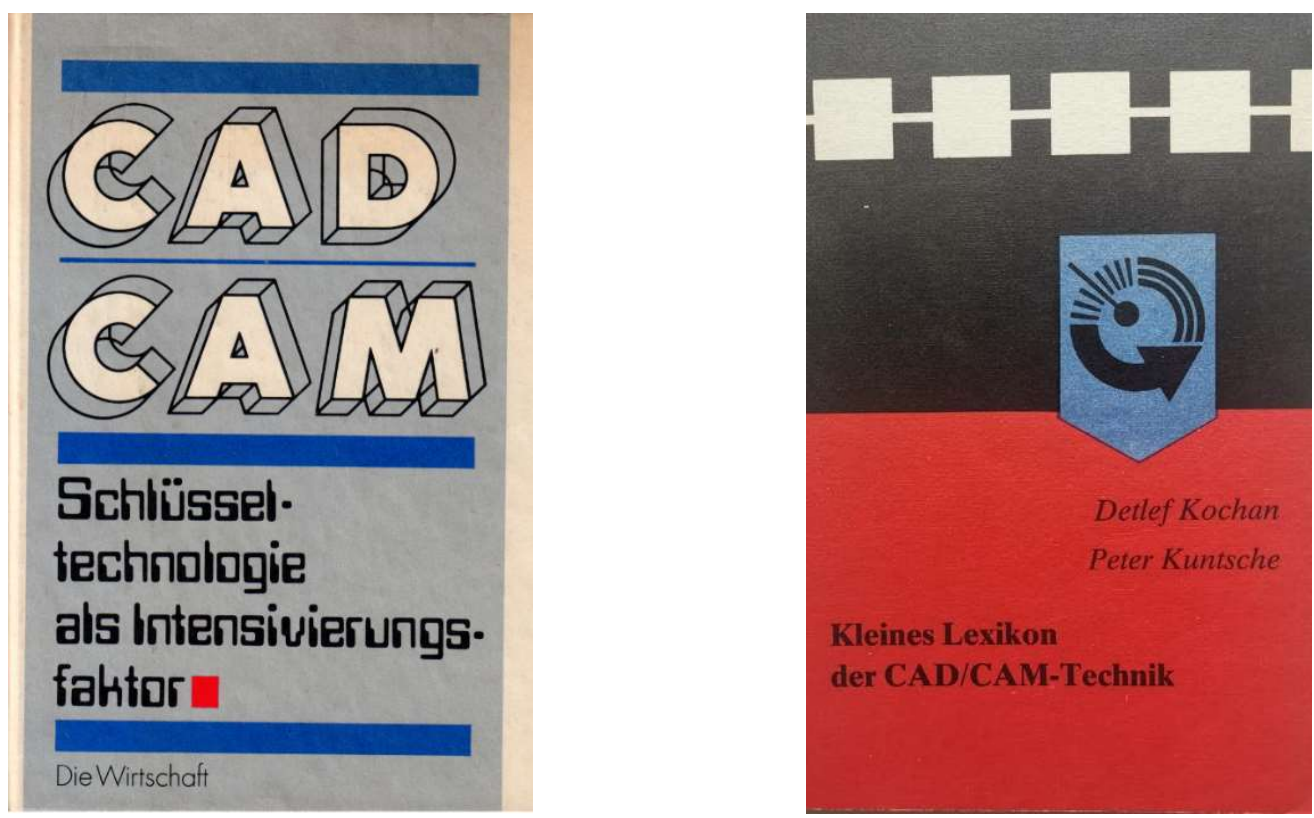

Abbildung 35: Erste CAD/ CAM -Veröffentlichung in der DDR (I.) sowie Fachbegriffe der neuen Wissensdisziplin (r.)

In Verbindung mit meiner Mitgliedschaft im Wissenschaftlichen Beirat des Hochschulministeriums und in einer Arbeitsgruppe (Forschungsrat im Ministerium für Wissenschaft und Technik) war mir ein groBer Kreis anerkannter Fachkollegen bekannt. Davon ausgehend konnte kurzfristig ein Arbeitskollektiv zu dieser anspruchsvollen Aufgabenstellung gewonnen werden. Es dürfte zu den besonderen Leistungen im Hochschulwesen gehören, dass ein praktikables Fachbuch in weniger als einem Jahr realisiert werden konnte. In einer Begegnung mit Herrn Dr. Heinze, der nach der Wende die IBM Vertretung in Sachsen aufbaute, wurde auch unter Beachtung der totalen Veränderungen der Strukturen eine positive Einschätzung zu diesem Buch gegeben. In dem ausgewiesenen Entwicklungsstand wurden viele Gemeinsamkeiten zwischen Altbundesländern und Neubundesländern festgestellt. 


\subsection{Internationale Unterstützung für Entwicklungsländer}

In Verbindung mit den Statuten von UNESCO Organisationen, wie auch die IFIP (Internationale Föderation für Informationsprozesse) ist die Verpflichtung zur Unterstützung von Entwicklungsländern fester Bestandteil wesentlicher Aktivitäten. Aus dieser Gegebenheit resultierte der Vorschlag und die Einladung, die erste CAD/CAM Konferenz 1985 in Indien (Bangalore) mit geeigneten Vorträgen zu unterstützen. Gemeinsam mit Prof. Encarnacao (zuständig für CAD), Dr. Ketel Bo (für CAE), Prof. Kochan (CAM, TU Dresden) wurde die Zusage mit entsprechenden Vorträgen gegeben. Ursprünglich war eine zweitätige Konferenz in einem Hörsaal vorgesehen. Die entsprechenden Vorträge mit der Stundenaufteilung erfolgten auf dieser Grundlage. Auf Grund der überraschenden hohen Anzahl von Teilnehmern erfolgte kurzfristig unmittelbar

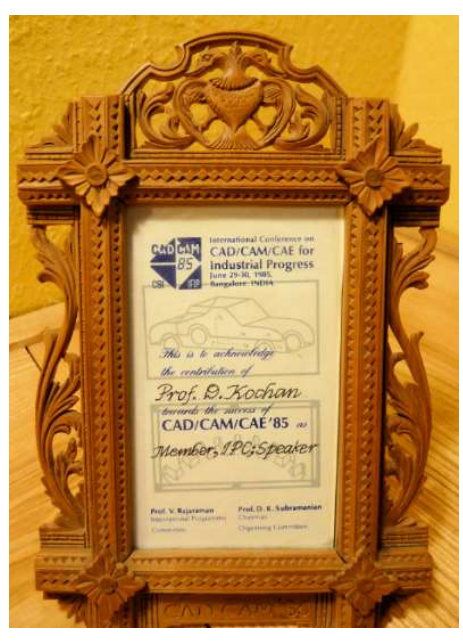
vor dem Tagungsbeginn die Aufteilung der Teilnehmer in zwei Hörsäle des Hotelgebäudes. Damit verbunden war das Anliegen die vorgesehene Zeit zu verdoppeln. Das war eine besondere Herausforderung auch unter dem Aspekt, dass die Veranstalter gleichzeitig darum gebeten haben, dass die Vorträge gespeichert werden sollten und deshalb eine permanente Beleuchtung mit Tiefstrahlern erfolgte, wobei in der Diskussion die Anfragenden kaum erkannt werden konnten. Auch diese spezielle Herausforderung wurde gut gemeistert, aber der Elan für nachfolgende Veranstaltungen dieser Art war etwas gedämpft, bei allem Interesse für das Entwicklungsland Indien. Trotzdem erfolgte im Jahre 1988 eine Wiederholung mit entsprechenden Veranstaltungen in Mexico .und später (schon in der "Neuen Zeit" )in Kuba. 


\subsection{Entwicklungen in Richtung CAD/CAM Labor}

In Verbindung mit der Charakteristik des CAD/CAM Buchvorhabens muss herausgestellt werden, dass die erfolgreichen Aktivitäten des Lehrstuhls Fertigungstechnik/Technologische Programmierung insbesondere auf den jeweiligen Praxisbezug aufbauen. Deshalb stand von Beginn der Förderung des CAD/CAM Anliegens die übergeordnete Zielstellung darin, ein geeignetes CAD/CAM Labor für Forschung, Ausbildung, und Technologietransfer, aufzubauen.

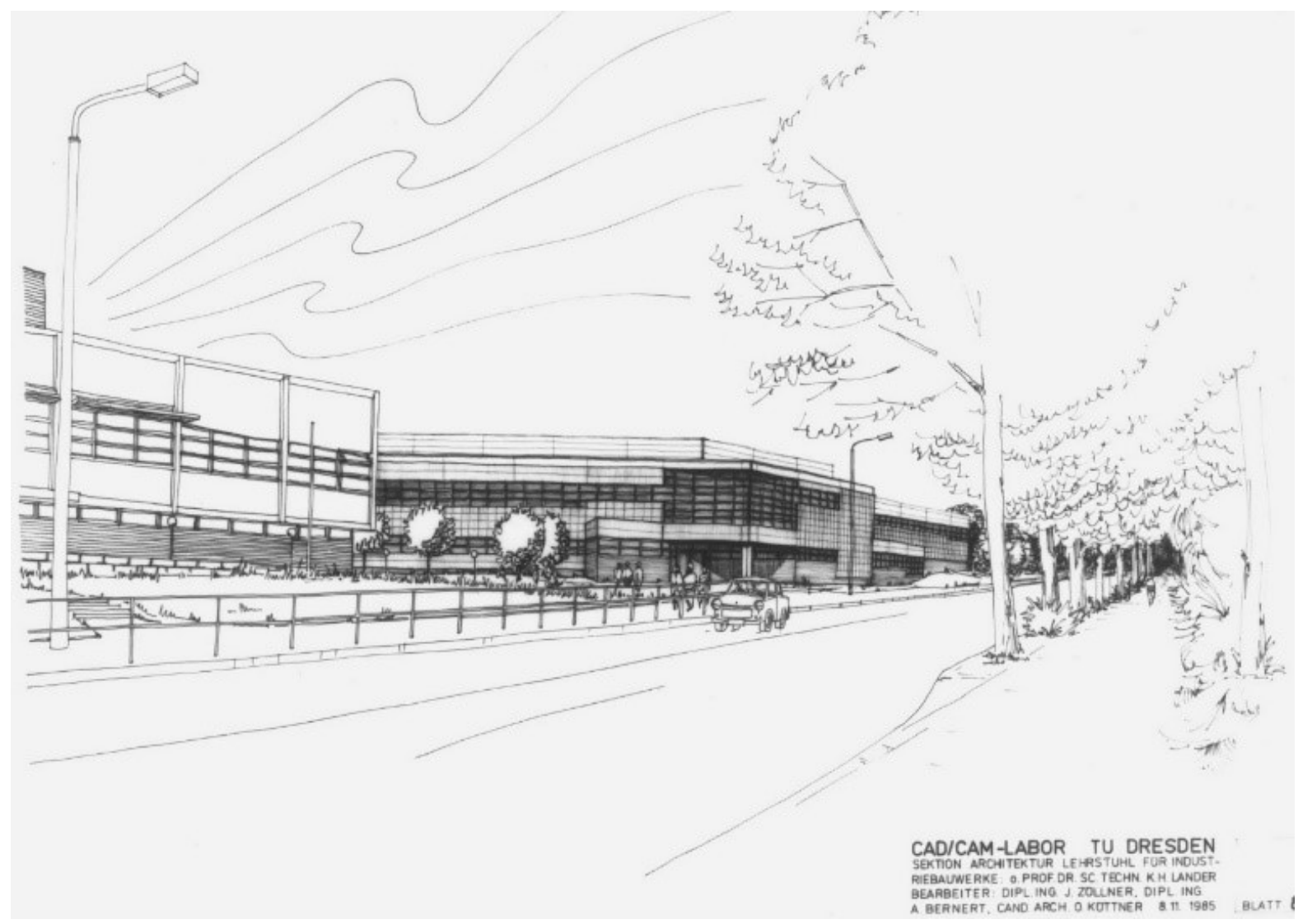

Abbildung 36: Entwurf CAD/CAM -Labor

Dazu wurden schon im Jahre 1985 erste konkrete Inhaltliche und auch bauliche Konzepte entwickelt. Die inhaltlichen Aspekte standen dabei im engen Bezug mit der gesamten Einordnung der Entwicklungen in ein umfangreicheres Automatisierungsvorhaben der Sektion Fertigungstechnik und Werkzeugmaschinen (Sektion 14), genannt AUTFERT (Automatisierte Fertigung in der Sektion 14). Für die baulichen Entwicklungen wurde als kompetenter Architekt Herr Prof. Lander von der TU Dresden gewonnen. Aus den zahlreichen baulichen KonzeptEntwicklungen wird das beigefügte Bild gezeigt.

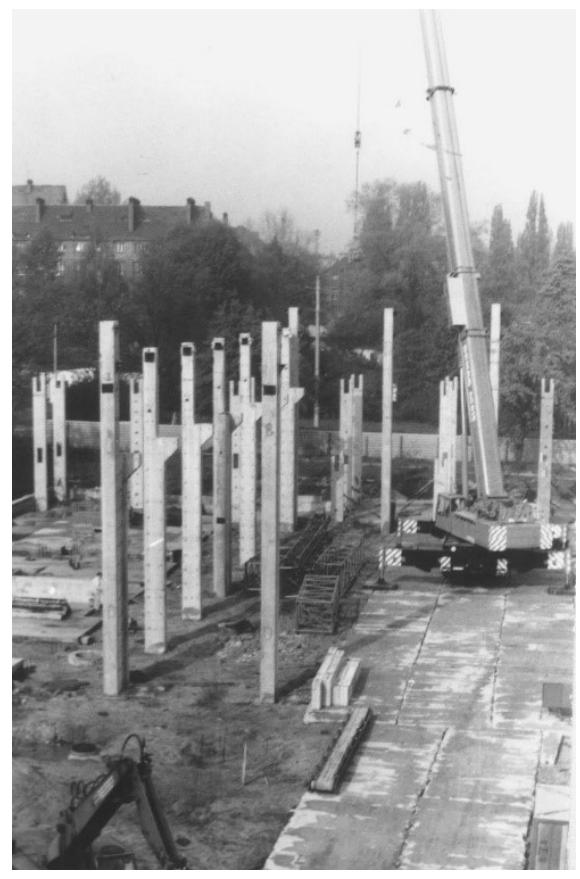

Abbildung 37: Fundament CAD/CAM Labor 
Ergänzend zu den baulichen Vorbereitungen war auch die inhaltliche Verbreiterung des CAD/CAMAnliegens ein wichtiger Aspekt. Mit der im Jahr 1986 erfolgten Um- Berufung von Professor W. Krug von der TH Köthen an die TU Dresden wurde gleichzeitig eine neue Struktureinheit CAD/CAM-Labor (später CIM-Technologie- Transferzentrum) geschaffen. Damit war für die vielfältigen IT-Anwendungen eine breitere Basis für Forschung und Ausbildung gegeben. Mit den wissenschaftlichen Arbeitsgebieten der Modellierung, Simulation und Optimierung wurden durch Prof. Dr. W. Krug mit konkreten Leistungen für die Produktionstechnik, Organisation und Logistik vor und nach der Wende national und international anerkannte Ergebnisse geschaffen.

Ausgehend von diesem baulichen Konzept wurde schon vor der Wende mit dem Bau des CAD/CAM Labors in der Bergstraße neben der Neuen Mensa begonnen. Ergänzend zu den theoretischen Fundierungen des neuen Wissensgebietes CAD/CAM war die Notwendigkeit gegeben eine praxisrelevante technologische Basis für problembezogene Softwareanpassungen, spezifischen Technologietransfer und fortgeschrittene Ausbildung mit einer geeigneten Ausrüstung zu schaffen. Mit Bezug zu Beschlüssen, die von der politischen Führung in Abstimmung mit unterstützenden Industriepartnern vereinbart waren, wurde am 6.Oktober 1988, d.h. ein Jahr vor der historischen Wende, mit dem Aufbau des CAD/CAM Labors begonnen.

Der Rohbau des Fundamentes und bauliche Fortschritte in den Jahren 1990 wird in den Bildern dargestellt.

Entgegen den fachlichen und politischen Zielstellungen zur Spitzenforschung und gleichzeitigen Technologie Transfer, wurde der Bau des CAD/CAM Labors leider nicht fortgesetzt und das gesamte Bauvorhaben für die neu gegründete Juristische Fakultät der TU Dresden zur Verfügung gestellt.

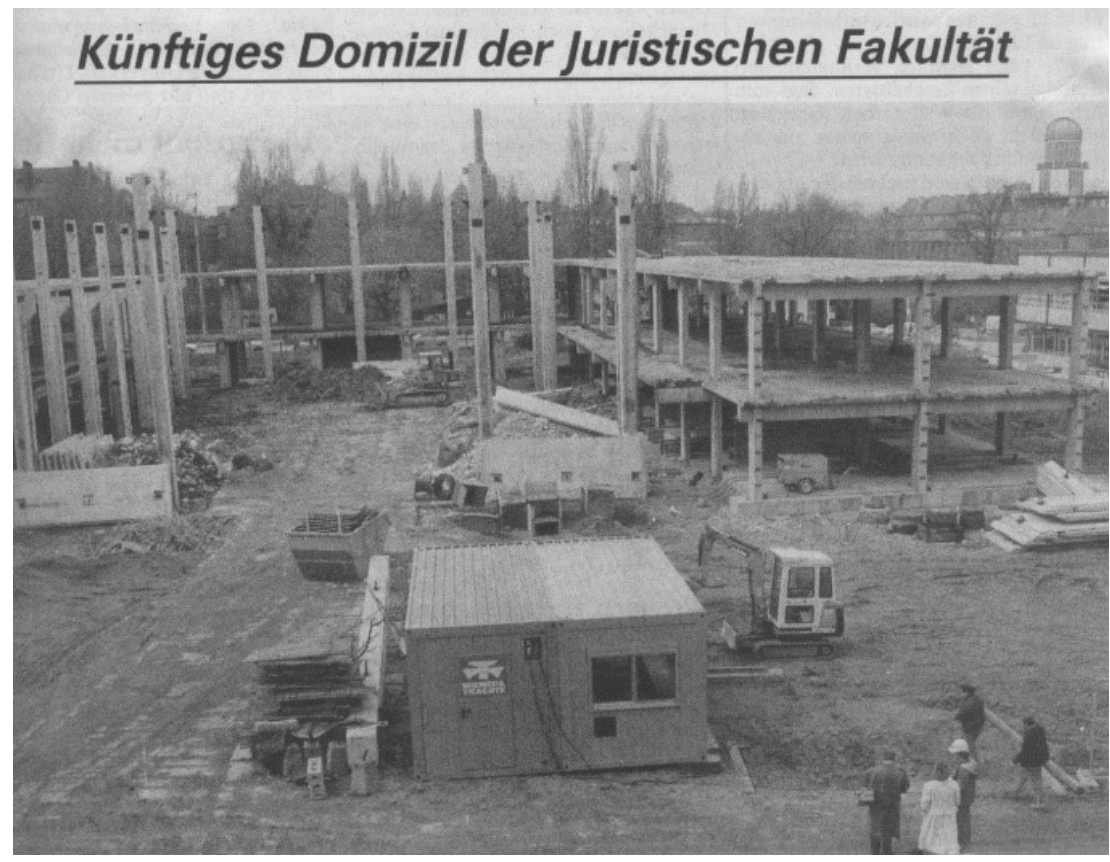

Abbildung 38: Baufortschritte im Jahre 1990

\section{Eröffnung CIM-TT-Zentrum}

Trotz fehlender Kompatibilität der älteren Rechnergeneration sind die inhaltlichen Schwerpunkte und die fachlich und politisch kompetenten Festredner erkennbar. Hier ergibt sich die Frage: Warum fand die wenige Tage vorher in Gaußig durchgeführte internationale Konferenz keine Beachtung? 
Veranstaltungsprogramm

Termin. 5.Dezember 1991

Vortragsveranstaltung

Von 10.00 bis zur Er'ffnung der Veranstaltung sind $\mathrm{f}$ r die Laborr, ume ge"ffnet.

$10.00 \mathrm{Uhr}$

Magnifizenz Prof.Dr.rer.nat.G. Landgraf, Technische Universit,t, Dresden

Begr áung

Ministerpr ${ }_{n}$ sident Prof. K.Biedenkopf, Freistaat Sachsen

Bundesminister Dr.?? Riesenhuber, Bundesministerium $f \quad r$ Forschung und

Technologie

Ziele und $\mathrm{M}$ "glichkeiten der Bundesregierung zur Finanzierung von Forschung und

Technologie insbesondere in den neuen Bundesl ${ }_{n}$ ndern

Prof. Dr.h.c.mult. Dr.-Ing. G. Spur, Technische Universit,t Berlin

Dr.-Ing. J. Buámann, NC-Gesellschaft Ulm

Neue Forschungskonzepte auch $f \quad r$ Klein- und Mittelbetriebe

Prof.Dr.-Ing. W. Voelkner, Technische Universit „t Dresden

Das Profil des Dresdner CIM-Technologie-Transferzentrums

Prof.Dr.rer.nat. W. Hacker, Technische Universit,t Dresden

Prof.Dr.-Ing. M. Rentzsch, Technische Universit, t Dresden

Menschbezogene Arbeitsorganisationsgestaltung - einige Grundlagen und Probleme

Herr ?? Schnelle, Industrie- und Handelskammer Dresden

Technologietransfer und Wirtschaftsf"rderung im Regierungsbezirk Dresden

\section{Demonstrationen}

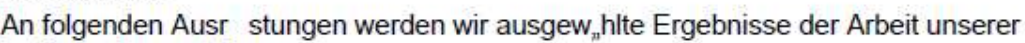
Institute demonstrieren:

Maschinenhalle Kutzbachbau

* CNC-Drehmaschine Gildemeister CTX 400

* CNC Drehmaschine NILES DFS 2/2 SINUMERIK 3TT

${ }^{\star}$ CNC-Bearbeitungszentrum AUERBACH CW 400 CNC 600

${ }^{*}$ CNC 5 Achs-Bearbeitungszentrum MAHO

* CNC-Fr, smaschine DECKEL

* 3D-Meámaschine Leitz

* 2-St, nderpresse ERFURT

* Koordinatenbohrmaschine MIKROMAT BKOE 630

\section{Abbildung 39: Programm der festlichen Eräffnung des CIM-TT-Zentrums}

Der beachtliche Anteil von CNC-Maschinen und Bearbeitungszentren wurde über das Lehr- und Forschungsgebiet Fertigungstechnik/ Technologische Programmierung hinausgehend auch von anderen Partnern innerhalb der Sektion Fertigungstechnik und Werkzeugmaschinen mit genutzt. In Übereinstimmung mit dem Entwicklungsstand der Software-Lösung AUTENT wird auf die herausragende, schon im Jahr 1991 installierte Fünfachs-Bearbeitungstechnik (MAHO) hingewiesen. Mit diesem Dokument zur Eröffnung des CIM-TT-Zentrums werden folgende Fakten charakterisiert:

- Mit dem fachlichen Plenar-Vortrag von Prof. Spur wurden die langjährigen nationalen und internationalen Kontakte dokumentiert

- Die mit der NC-Gesellschaft unmittelbar nach der Wende aufgebauten neuen Kontakte zeigten sich im zweiten Fachvortrag des damaligen Sprechers der NC-Gesellschaft, Herr Dr.

Bussmann 
- Die Inbetriebnahme des Fünfachs-Bearbeitungszentrums war gleichzeitig eine wichtige Voraussetzung zur aktiven Beteiligung am ersten EU-Projekt der TU Dresden (FAME- Five Axis Manufacturing Environment)

Bedauerlicher Weise wurden die geschaffenen Startpotentiale nach meiner Auffassung nur unzureichend-gemessen an den Zielstellungen (Technologietransfer) genutzt und weiter ausgebaut.

\section{Minister Riesenhuber eröffnete das neue CIM-Technologietransferzentrum Dresden}

BMFT fördert die Einführung und den Ausbau der rechnerintegrierten Fertigung "ClM in den neuen Bundesländem

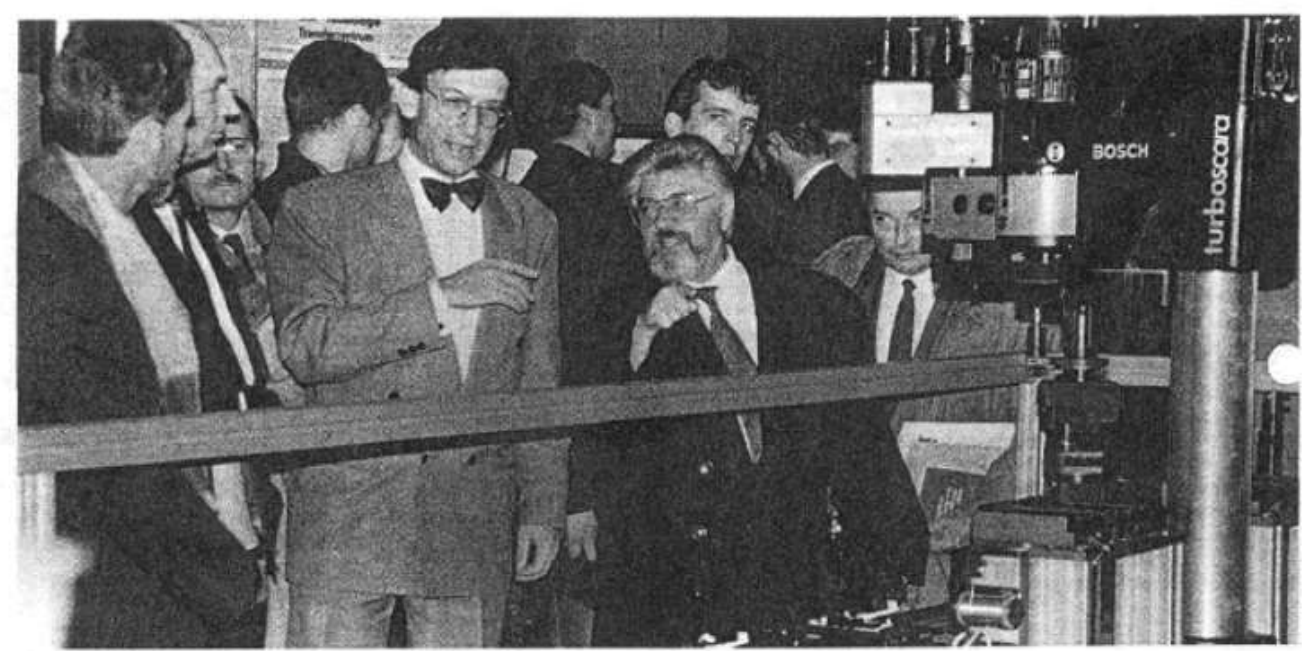
Amt 5, Dezent minister fut Forschung und Technologie (BMFT). D. Heinz Aresenthubet, im Bessein des Mirtiterpasidenton des Freistastes Sach. sen, Prot. Dr, Kurt Biedenkopt, das CIM. Technologidfansterzentrum (CIM-TT) an der Tochnischon Univorsitlit Dresden. Die neve Einrichtung ist ein wichtigor Buitrag zut wift. schattichen Entwickiung des Raumes Sach. sen und darüber hinaus aller neven Bundos. lander. Minister Piesontuber stelis tût den Be. trieb des CIM-TT.Zentrums Dreaden 44 Milso. nen DM zur Vertügung.

Koordinien und betreut werden die Aktivitaten dieses and waiterer CIM.TT.Zentren im Aut. trag des BMFT durch den Projakttrâger Fert: gungetechnik und Qualiatseichenung (PFT) des Karntorschungszentrums Karlaruhe (KIK)

100 Mio. DM für ClM-Einführung in den neuen Bundesländern

Bei der Eröfnungsveranstaitung, an der über 340 Vertreter der Wirtschatt, der Wissenschast und der Politik aus der gesamten Bundesrepu. bilk und dem Ausland tesinahmen, gab der BMFT dik Wiederautnatme der indirekt-spez. fischen Fördedera von industriollon Entwick.
In fachllichem Disput bei der Enoffaung: Bundesminister Dr. Heinz Pliesenhuber (links) und ber Leitor der newen Eintichtung. Prot. Dr.-ing. abil. W. Voelíner

Fotos: K. Eckold, Dresdher Univlouinal (2)

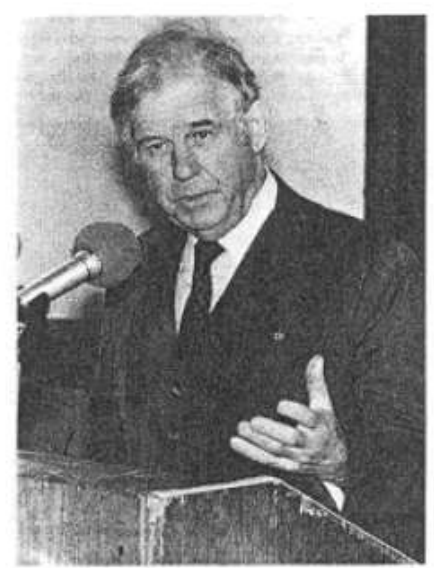

Ais eine investition in die Zukunt' bowertote
Das CIM-T-Zentrum Dresden ist Bestandtei ines bundesweiten Netzes von insgesamt 21 CiM-TT-Zentren. Zusàtzlich zu den bestehen. cen CIM-TT-Zentren in der alten Bundeslancern wurden 1991 neben Dresden vier weitere Zentren in den neven Bundestandern eingefichtet und zwar in Chemnitz Sachsen. Magdeburg-Sachsen-Anhalt. Suhi/Thüringen und WismariMecklenburg-Vorpommern.

Aile CIM-Tr.Zentren sind an Hochschuilnst. en angesiedelt. Ihre Aulgabe besteht darn das Sactwiseen und die Ertahrung der Hoch. schulinstitute beschleunigt in die industrielle Anwendung zu bringen und insbesondere die kiein- und mittelatandische Industrie bei der Losung von CIM betreffenden Problemen wettbewerbsneutral $\mathrm{zu}$ informioren und zu unter. stützen. Die Einrichtungen verbindet aine enge Zusammenarbeit und ein gegenseitiger Ergebhis:- und Ertahrurgsaustausch. Jedes Zentrum hat antevechend seinen Moplightreiten und der jeweiligen Tracition der Universibat die Fe. certone tor ausgewathlo Arbeitogebiete in CIM-Beseich Übernommen.

Fur die Wiederautnahme des indirekt-spezitischen Förderprogramms CIM, das in den alten fundesilandern seit 1988 tauth und 1992 endet. tele der BMFI fur die neuen Bundeslande too Mio. DU zur Vetiogurg. CMu, das bedeo

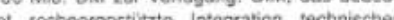


Magnifizenz!, lieber Herr - darf ich sagen Kollege - Riesenhuber, Streiter seit vielen Jahren, gemeinsame, meine sehr verehrten Damen und Herren!

Angesichts der fortgeschrittenen Zeit möchte ich mich kurzfassen. Die Gründe für die Verzögerung sind für die ganze Lage in den neuen Bundesländern charakteristisch. Hier wird etwas Neues geschaffen und vor der Tür stehen die Menschen, die in dem Alten gearbeitet haben, das wir nicht weiterführen können. Die einen sind schon wieder beim Aufbau und die anderen müssen noch die Konsequenzen des

Zusammenbruchs bewältigen. Deshalb schien es gerechtfertigt, erst mit denen zu reden, die nach dem Zusammenbruch auch ihre eigene persönliche Lebensplanung bewältigen müssen, ehe wir dann zu denen kommen, die mit uns allen sich darüber freuen, daß etwas Neues begonnen wird. Das CIM-Technologie-Transferzentrum ist für uns in mehrfacher Hinsicht von Bedeutung.

Einmal ist es ein wichtiger Schritt auf dem Weg, Brücken zu bauen zwischen der Wissenschaft und der Praxis, zwischen der Wissenschaft und der Wirtschaft, zum zweiten wird es ergänzen und verstärken, was wir insbesondere im Dresdner Raum, aber ganz besonders im Freistaat Sachsen an Beiträgen zur Entwicklung neuer Schlüsseltechnologien leisten wollen. Wir wissen, dieser Teil Deutschlands, der nach dem ersten Weltkrieg die Industrieregion war mit dem höchsten Bruttosozialprodukt pro Kopf der Bevölkerung im ganzen damaligen Deutschen Reich wird den Anschluß an die Zukunftsentwicklung nur erreichen können, wenn neben die hervorragenden Qualifikation der Facharbeiter und der Handwerker, der Ingenieure, auch eine wissenschaftliche Entwicklung tritt, die wettbewerbsfähig ist mit den modernsten Wissenschaftszentren in der Welt. Deshalb ist die Staatsregierung glücklich darüber, daß in der Fülle der Programme, über die wir diskutieren und von denen, wir hoffen, daß wir sie im angemessenen Umfang in die Hochschulerneuerung und in die Wissenschaftserneuerung einbeziehen und damit auch finanzieren können, daß neben all diesen Programmen auch hier der Bundesminister für Forschung und Technologie und damit der Bund seine helfende Hand reicht und mithilft, Neues aufzubauen. Wir erhoffen uns von der Arbeit des CIM-Technologie-Transfers, wie es hier auf der Einladung heißt, eine Investition in die Zukunft. Wir erhoffen uns von dieser neuen Einrichtung, daß sie - wie Magnifizenz das schon gesagt hat - die kleinen und mittleren

Unternehmen mitbefruchten möge und auch die Struktur der praxisnahen Forschung weiter voranbringt. Die Technologieparks, die Technologie-Transferzentren sind Entwicklungen in der jüngeren Vergangenheit in der ganzen westlichen Welt. Wir können aus diesen Entwicklungen lernen. Wir können insbesondere daraus lernen, daß, wie das in manchen alten Bundesländern zunächst versucht wurde, sich Technologie-Transfer nicht bürokratischer Methoden bedienen. Das entscheidende für den Technologie-Transfer ist, daß die Menschen zusammenkommen, die über das Wissen verfügen, daß sie gemeinsam an Projekten arbeiten und daß auf diese Weise das Wissen derer, die sich der Wissenschaft gewidmet haben, übertragen wird auf die, die wissenschaftlichen Erkenntnisse in die Praxis umsetzen sollen. Alles was wir tun können, auch was der Freistaat Sachsen tun kann, Magnifizenz, und meine sehr verehrten Damen und Herren, um den Erfolg dieses Projektes zu unterstützen, werden wir tun; und zu dem gehört es jetzt auch, sich kurzufassen, damit Herr Riesenhuber zu

Wort kommt.

Abbildung 41: Vortragstext des Ministerpräsidenten Prof. Biedenkopf

Aus diesen zukunftsorientierten Aussagen ist nicht zu erkennen dass das Bauvorhaben CAD-CAM-Labor mit den komplexen inhaltlichen Zielstellungen des CIM-Technologiezentrums verbunden werden sollte. Die ersatzlose Umorientierung trotz des schon erfolgten Baufortschrittes des CAD-CAM-Labors als Räumlichkeiten für eine im Aufbau befindliche juristische Fakultät ist schwer zu verstehen.

Frage: Übereinstimmung von Theorie und Praxis? 


\title{
Richtfest für den "von-Gerber-Bau» vor 25 Jahren
}

\author{
Einst als CAD-CAM-Labor geplant, wurde das Gebãude Sitz der furistischen Fakultät
}

Fioland it Millor

Das Jubriam vom a, Oktober 1902 ist Anlass, an dive Interessinto Fntetio hunpiposehschite des ersten Nevbuss far elne Untwersitat tn ơinen der neuen Piundeslander nach dor Wondo ru erfnnern. Das Hischtfost wunde auf ofner

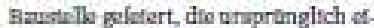
nam anderen cotoude gawidmat war. Crundateinlegung unter anderem mit Hans Modrow, damals 1. Sekratar der SED Bextrkslestung Dresden grgebon. In a CAD-CAM Lobor und Cate Away Entwurfinentrume sollto der Tochnologkoforschung nouen schwung vorlat. ben, um den durch die sozlatistieche Thenvirtschaft und die Embergopolitik der RaD oingatretanen Reckstand der DOS zufruholen, hrw. wioder An. schluss an ifo wastlichen Intustrie. stauten tu gusitanen: Dusa war berofts am 10. Oktober 10sis of Vertrog mit dom wEH Kombiriat Sperfaltechnike peschlossen worden Dansch sollto dto TUD das Haugrundetock rur Warforong stelkn, flit dis wissanch hattletwen bew. personollon kapaztiman sorgen und das K.ombenat die Finanziorung usw. das Vochabens efnachtiolisch der Beschaf. fung der speriellen Aasristung (wie Import von Ilochiontochnik aus dem nicht sortalintischen Wirtsenaftsgebses: tobornohmen. Am z Oktober wass hatte

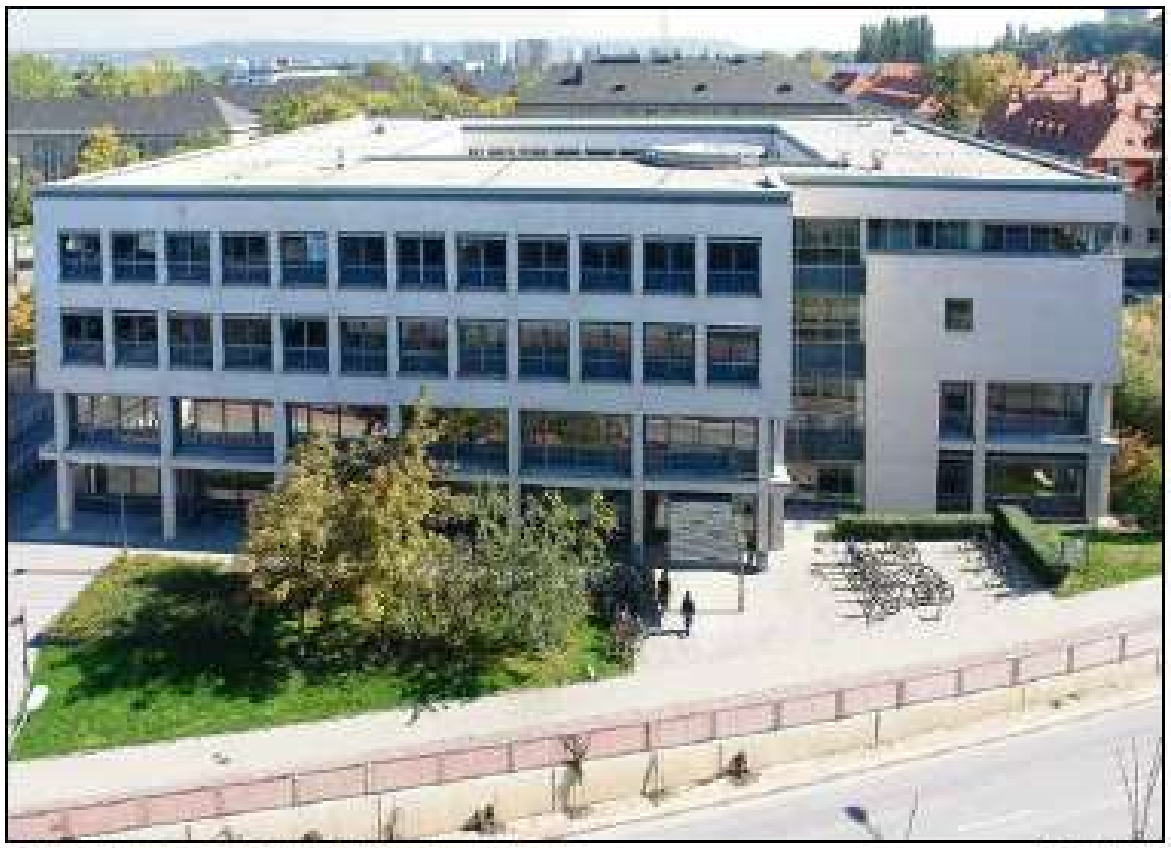

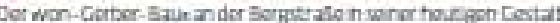

Foto Lyfrosid center Mfttaz, der sullowwaltigo for dro Wirtschaitspolatk im Fuhnugrazro. mitum der SED und der DOR, affentllch des TUD EIn solkes lisborgiblade trutz der Priforitat von Wohnangy- und MFlinarbaton zogeagt. Do Alarungsarbetten warden darainitin solort durct den aKombtnatsbetricb Forschung und Profoktbrunger des oWEB RMKe auigeDominson. Vorarbetten des Iehnstuhis Industrisobu der TUD, der Drojektierungevinntchtungen dos Mintstertums an der TUD sowieder als Nutzer vorpe. sobenen TUD-Skittionen und Wissen. schaftsgebtete flossun darin ofn. Die

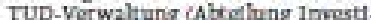
tfonony fanglerte als Baruaftraggeber

and hammerte stch arn die konkrete Aninshme des Vortabena fn dre Volks wirtschaftaplamang der DDEl. Dabel war man stch aber bewuset, dese das ivterliche Virsprochen von ctenter M: tag und fas Vertragswork ntcht aurnt. chen, um bald mit dem naw an begln. aen, donn der Engpass war vor allorn die verfagburs Rukbpazltat des BMX. Var tber unenilich vile Bernahungen

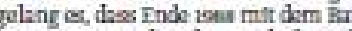
begonnen wurde. Aber aech danach ging es mur schlappend vxan, donn es Hautachnth.

Erst soo berann trofols des Wogfill er Miritzrtoution im wahrotan sinn des Wortes der Hohben schlagartig mu

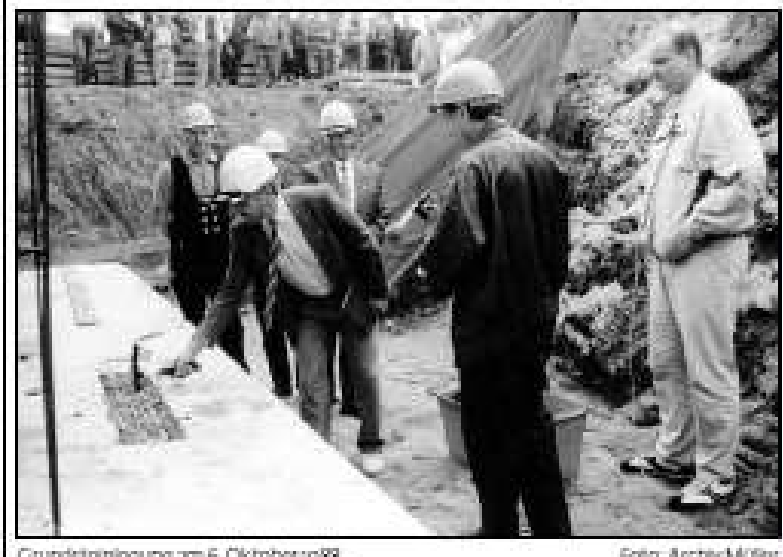

wacheon. Berets vor der ersten ireten Volkekammenwal war jodbch zbeeh bar, dass woder das CAD-CAM-1sbo in der geplanton Walse afne Zukunft hatte, noch dass der Baru an sfch ben Anforderungen etnes moblerner Hochsctalleuses und den Stanfiarde der Hill entsproction wordie. Vor alkm aber wat dfo Herstsstelhing der Finenumitte nixbt mohr pewshirbtotot. Aar begrel fichon orunden drangta fodoch der to zwischen privatisterte Bububtriob gof Einhaltung der Vortrige und worlang sogar efine nistitzliche Dramfo, um das Fertfgstellungralel sL. Dexember 200 . etrumalon. De Dramatkund don Op

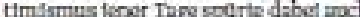
die das Bawortuben fodorfinhrende $A b$ teflang der TUD. Vorwaltung, In thre Baracke gaben iflch llatgober und inte. roseterto Investuren aus den aken Par. deslindern die Kilinke fo the Hand. Is war fodoch himiner berett, die Fertfgetel lung dos Wortubens zo finanxieren. $\mathrm{Da}$

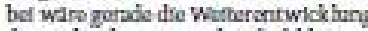
der Technologfo efn Znkunitssold gewe. der Tochinalogie ein Znkunitssila gew. TUD thre Wotthewarbsithi gott hatren bewsisen bionen. Dm drohendon wedbewelisen bibnnen. Do drohendon wed. teron Zahlungeforderungen for den Besu as entyohen, wurde nach der Wilh. rungureform, nachdern dis Nutrungel terrnatsve Tachnologite-und Gitndernestrum masschfod, etn llaustopp werfugt.

Der zu erwartende Ansathes der DD in dio HilD und thr Binchteystem broch te visele Frazen auch zur Zukanft der TUD mit sich. Antworten wurden be der entstehendin Landererferung posucht Drew hatte anfangs thron sils in worlgen Berurtumen anf alnem Cang
Im Gebatude des Wraberigen tiotes des

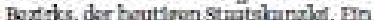
wotdsutscher Ministorla lboumter liell dart Anfang Soptomber woo nach diner sttzang mit dorn desgribanim Mintster-

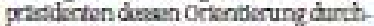
bicken dass is Tochnische Untvenstist. ils kenftire volluntverstat auch cine Auristis Gefor thatz brw. Harum geschaffen wordon mass Von doom Momers an two sich alles daranf, das I aborgotbiode

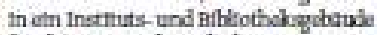
fur dio Jartatische Fakulat umizumandeln Al Tartner dafitr hatte sich ein Doternehmen aus Stuttgart gofundon. Doni war amp soptember iboo dine Vosefnhorung zwischen dem Rokter Drut. cinther tanigral und den Handhungbevollmkchiligten der stattgarter ribrungstirma abgeschicreen woader. Desor warvn sohr intensfve Beratungen, Vorhandlangen und Ortsbedictutgun. grn worangogangun. Dobel gehätson dor.

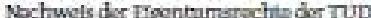
In dom Pangrundstoct ond dio Vertif: an don

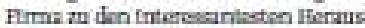
fordinungon fener Zdt.

Das Statlsche Hochbsumit des Fre. intates Sachen ubornahm bald daraut do Vorabetten der Inxwischen in das: ssch zebist Unfwersttatstret- und Entwicklungreteng ungostaloten Ab trofung Investttonen. Danach kam es

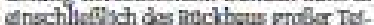
le der Rohbrusubteni des CAD-CIM-

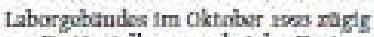
zur Fertigetellung, wobel der Kosten-

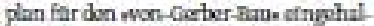
tan wande. 


\section{Die Juristische Fakultät an der TUD existiert nicht mehr, ein echtes CIMTT Zentrum wird dringend benönigt.}

In diesem Beitrag aus der Universitätszeitung der TU Dresden ist die gesamte Entwicklung des CAD-/ CAM- Labors bzw. des CIM-TT (Technologietransfer)-Zentrums konzentriert und korrekt dokumentiert.

Für die weiteren mit guten Vorsätzen gestarteten Aktivitäten zum gezielten CIM-Technologietransfer insbesondere für die KMU im sächsischen Umfeld gab es in der Weiterführung leider nicht die angestrebte Breitenwirkung in der Fertigungsindustrie.

Wenn man als Maßstab und Orientierung die Steigerung des produktionstechnischen Niveaus der sächsischen Industrieunternehmen zugrunde legt, resultiert zwangsläufig die Fragestellung: Sind die formulierten Zielstellungen annähernd erreicht?

Mit dem dreißig Jahre nach dem Mauerfall erreichten produktionstechnischen Niveau von ca. $80 \%$ kann diese Frage leider nicht positiv beantwortet werden.

\section{Internationale Aktivitäten nach der Wiederververeinigung}

Unabhängig von den gravierenden politischen Veränderungen wurde unter Nutzung der gegebenen neuen Möglichkeiten eine zielstrebige wissenschaftliche Kooperation fortgesetzt.

\subsection{Problemseminar „Rechnerunterstützte Fertigungstechnik“}

In dem historischen Wendejahr 1990 fand das 15. Problemseminar zum Thema rechnerunterstützte Fertigungstechnik im November im Schulungsheim der TU Dresden als deutsch-deutsche Veranstaltung, zum Teil mit internationaler Beteiligung (Norsk Data, Norwegen) statt. In den 5 Tagen wurden insgesamt 27 Vorträge in der schöpferischen Atmosphäre des Schlosses Gaußig gehalten, so dass von einer inhaltsreichen Arbeitskonferenz gesprochen werden kann.

Eine besondere Bereicherung war in dem Vortrag von Dr. Tucholke von der Universität Bremen zum Thema "Stereolithographie in der Prototypen-Fertigung" gegeben.

Für die an der TU Dresden weiterhin vorgesehene Installation einer Stereolitographie-Anlage war und ist bekanntlich die Verfügbarkeit in sich geschlossener 3D-CAD-Datenmodelle eine entscheidende Voraussetzung. Mit besonderer Begeisterung konnte unser langjähriger und verdienstvoller Dipl.-Math. G. Franz über seine „Erfahrungen mit dem CAD-System TECHNIVISION (T 2000) von Norsk Data" berichten. Die Entscheidung war deshalb für die Installation an der TU Dresden gefallen, weil es die leistungsfähigste europäische Software ohne Embargo-Restriktionen darstellte.

Wenn man davon ausgeht, dass sich im gesamten Gebiet der rechnerunterstützten Fertigungstechnik in den vergangenen dreißig Jahren dynamische Weiterentwicklungen vollzogen haben, verdienen die in Thesen vorliegenden Kernaussagen des einführenden Vortrages besondere Beachtung. Die grundsätzlichen technologischen Zusammenhänge im betrieblichen Umfeld kann man über mehrere Jahre

als nahezu gleichbleibend betrachten. Nur das informationstechnische Umfeld ist durch eine dynamische Entwicklung gekennzeichnet. Mit der zunehmenden Leistungsfähigkeit der Computer und

Steuerungen wird zur effizienten Lösung vergleichbarer Problemstellungen immer mehr technische Unterstützung bereitgestellt, die wiederum von den fachlich kompetenten Personal bestmöglich ausgenutzt werden kann. 
Deshalb wurden zum Bekräftigen dieser grundsätzlichen Aussage zumindest die ersten zwei Seiten und die zugehörigen Bilder in die vorliegende Dokumentation übernommen:

Im Rahmen des 15. Problemseminars fanden gleichzeitig inhaltliche Abstimmungen für ein gemeinsames EU-Forschungsprojekt zum Thema: „Five Axis Milling Environment" statt.

Gleichzeitig erfolgten Vorbereitungen zur intensiveren Zusammenarbeit mit dem EXAPT-Verein Aachen.

\subsection{Spezieller Höhepunkt: Sonderveranstaltung „Innovation und Motivation für den industriellen Aufschwung"}

In Anbetracht der tiefgreifenden industriellen Veränderungen in den Neuen Bundesländern wurde der zweite Teil des 15. Problemseminares am 30. November und 1. Dezember 1990 mit der Teilnahme von kompetenten Führungskräften aus der Industrie durchgeführt.

Für diese als Seminar durchgeführte Sonderveranstaltung war das exakte Programm leider nicht mehr auffindbar. Aus der noch vorliegenden Teilnehmerliste können folgende wesentliche Fakten entnommen werden:

-an der Veranstaltung nahmen 26 Führungskräfte aus der Industrie teil.

-das waren ehemalige Fachdirektoren (z. B. Prof. Dr. Klaus Mütze Carl Zeiss Jena, Prof. Dr.A. Russig Forschungszentrum Werkzeugmaschinen Karl Marx Stadt) aus der Wissenschaft.

-Persönlichkeiten aus der Industrie (z.B. ehemaliger Generaldirektor Prof Dr. H.J. Hahn, Kombinat Elektromaschinen. Dr. A. Schwarz, IFA Personenkraftwagen AG)

-weitere Vorstandsmitglieder, Betriebsleiter, Geschäftsführer u.ä.

-9 Teilnehmer aus der Wissenschaft, insbesondere TU Dresden.

-aus der BRD Prof W. Meinig Universität Bamberg; Automobilwirtschaft.

Von dem weiteren Teilnehmer vom Unternehmen Carl Zeiss Jena, Herr H. Dietrich, ist mir bekannt, das er später bei der Umgestaltung durch den ehemaligen Ministerpräsident Dr. L. Späth dessen „Rechte Hand“ darstellte.

Das Thema war dem Anliegen „Innovation und Motivation für den industriellen Aufschwung“ gewidmet. Zweifellos war der Eröffnungsvortrag durch den damaligen VDMA-Präsidenten, Dr. Leibinger ein besonderer Höhepunkt (s. auch gesonderte Würdigung S. 97) mit wichtigen Auswirkungen auf die industrieelle Entwicklung im Freistaat Sachsen. 


\subsection{IFIP-Arbeitskonferenz „Prozess Planning for Complex Machinig with Al-Methods" November 1991 in Gaußig}

Diese IFIP-Arbeitskonferenz fand praktisch nur wenige Tage vor der offiziellen Eröffnung des CIM-Technologie-Transfer-Zentrum statt.

Nach der ersten IFIP-Arbeitskonferenz 1984 in Tokio (gemeinsam mit CIRP) war es die 2. Konferenz, in der Methoden der künstlichen Intelligenz thematisiert wurden. Dieses Arbeitsgebiet wird gerade zur gegenwärtigen Zeit sehr oft im Zusammenhang mit Digitalisierung angesprochen ohne, dass dabei auf konkrete inhaltliche Bezüge orientiert wird. Deshalb soll mit der Schwerpunktsetzung zu dem gesamten Gebiet effizienter Wertschöpfungs-Prozessketten hingewiesen werden. Die schon 1990 erreichte Komplexität flexibler Fertigungssysteme erforderten einen zunehmenden Softwareaufwand und insbesondere Rückkopplungen aus den in unmittelbaren vielfältigen Bearbeitungsprozessen. Das gesamte Gebiet des Advanced Manufacturing kommt an die Grenzen der Mathematisierung und Algorithmierung hochkomplexer Fertigungsprozesse. Daraus resultiert die Anforderung einer möglichst realitätsgerechten Erfassung des spezifischen Erfahrungswissens.

Der einführende Hauptvortrag wurde vom Direktor des Unternehmens „Fritz Werner Werkzeugmaschinen AG Berlin“, Her Dipl. Ing. H. Hammer gehalten. Von diesem Unternehmen war das Erste funktionsfähige flexible Fertigungssystem (FMS) der BRD 1983 zur Europäischen WerkzeugmaschinenMesse in Paris vorgestellt worden. Im Ergebnis der vielfältigen Erfahrungen weltweiter FMS- Installationen unterschiedlicher Baugrößen und Funktionalitäten konnte in Übereinstimmung mit dem Anliegen der Arbeitskonferenz die herausragende Bedeutung intelligenter Software herausgestellt werden. Der anteilige Wert der benötigten Software an den Gesamtkosten eines FMS kann dabei bis zu 50\% betragen.

Dabei hat die komplexe Bearbeitung komplexer Geometrien viele Aspekte. In der IFIP-Arbeitskonferenz wurden deshalb wesentliche Merkmale, welche den fortgeschrittenen Entwicklungsstand des Computer-Integrated- Manufacturing aus technologischer Sicht charakterisieren, herausgestellt.

Derartige wesentliche Aspekte sind:

- $\quad$ Flexible Automatisierung der gesamten Fertigungsprozesse für definierte Teilefamilien von Werkstücken.

- $\quad$ Entwicklung und Anwendung von Fertigungszellen für Multioperationen. 
- Weiterentwicklung von Fertigungsverfahren durch verbesserte Werkzeugmaterialien, Spindeln und Steuerungen, mit denen Hochgeschwindigkeitsbearbeitung (HSC) ermöglicht wird.

- Neue Prinzipien von Fertigungsverfahren zum damaligen Zeitpunkt als „Rapid Prototyping“ bzw. "Solid Freeform Fabrication“ bezeichnet.

Der generelle Entwicklungstrend in Richtung komplexe Bearbeitung in Bearbeitungszentren, Fertigungszellen und flexiblen Fertigungssystemen als typische Ausstattungen für die flexible Automatisierung erfordern einen hohen Grad von Softwareunterstützung. Insbesondere der Trend zu CIM erfordert immer mehr intelligente und auf Erfahrungswissen basierende Unterstützung.

Alle diese typischen und spezifischen Richtungen der komplexen Bearbeitung sind die Grundlage für eine verbesserte methodische Unterstützung durch differenzierte Lösungsansätze von erfahrungsbasierten Methoden.

Diese Entwicklungstrends erfordern die Automatisierung vieler Funktionen für den gesamten Fertigungsprozess, wie z.B.:

- $\quad$ Bearbeitung mit unterschiedlichen Fertigungsverfahren.

- $\quad$ Werkzeugwechsel, Werkzeugmanagement und Überwachung

- Werkstückhandling, Speicherung und Transport

- $\quad$ Supervising, Qualitätskontrolle und andere Hilfsfunktionen

In die Arbeitskonferenz wurde eine Vielzahl von praktischen Erfahrungen, einschließlich der TUD, eingebracht.

Interessanterweise wurde von Seiten der TU Bremen ein Vortrag zu ersten Erfahrungen der Anwendung von Rapid Prototyping in Form der Stereolithographie-Technik von 3D-Systems -USA gehalten. Damit wurde das in Bearbeitung befindliche Konzept der Investition einer Stereolithografie Anlage an der TUD inhaltlich gut ergänzt.

Der zunehmende Automatisierungsgrad insbesondere im Gebiet der spanenden Fertigung mit der Kombination vielfältiger spanender Verfahren führt zu neuen Ansätzen für die IT unterstützte Prozessbeherrschung.
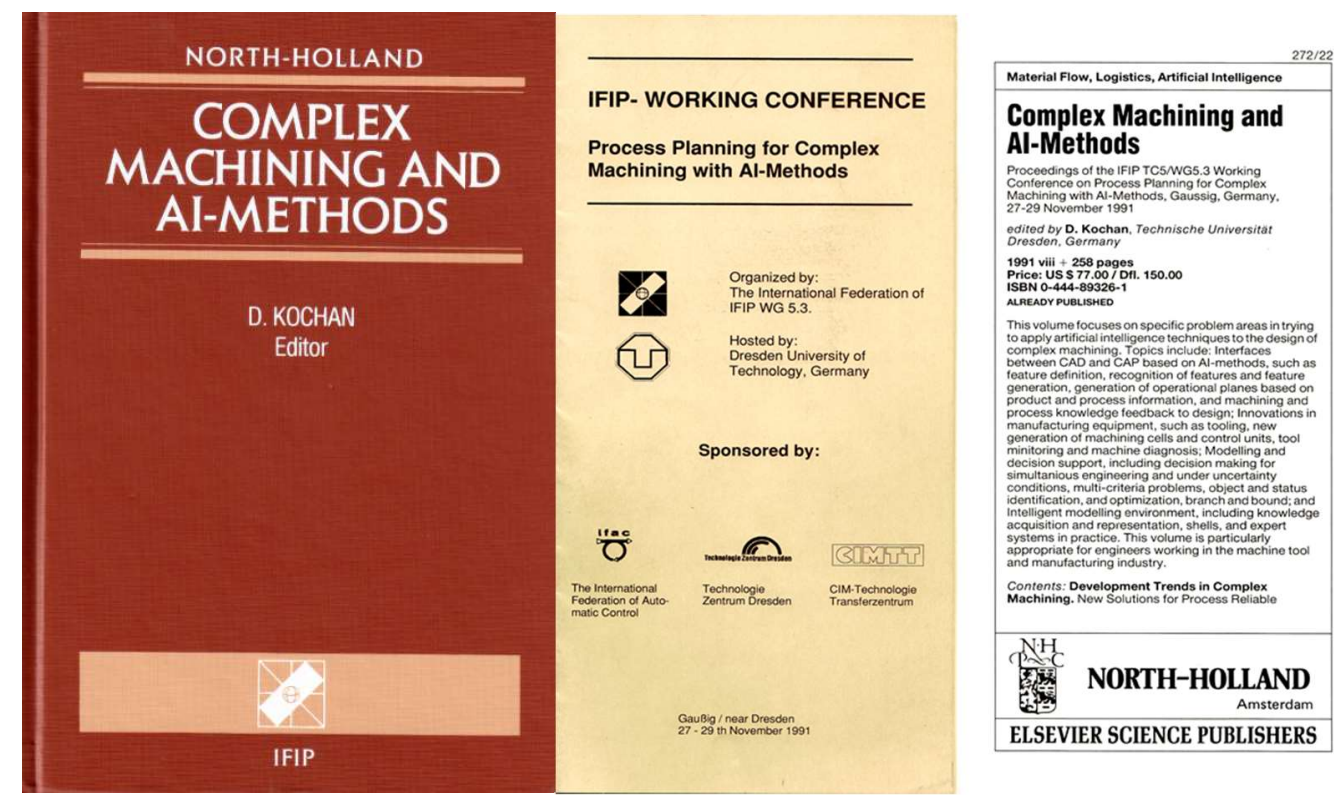

Abbildung 44: Zweite IFIP-Arbeitskonferenz zu Anwendungen der künstlichen Intelligens 
Um diesen erhöhten Anspruch an die notwendige Unterstützungssoftware zum Ausdruck zu bringen war zum damaligen Zeitpunkt der anspruchsvolle Begriff Al Artificial Intelligence geläufig. Zu diesem Anspruch wurden einige Vorträge auch aus dem Lehrgebiet FT/TP gehalten.

Man kann demnach davon ausgehen, dass sich das sich das Team des Lehrgebietes Fertigungstechnik/ Technologische Programmierung zu diesem Zeitpunkt (1991) noch auf Augenhöhe mit dem internationalen Spitzenstand befand.

Diese Aussage wird mit den nachfolgenden originalen Dokumenten bekräftigt. 
Tu Drewden

Prof. Dr. sce,tenchr. Moctian
7.11 .90

kowich thengat

The sen far den Vortrag in Gausig 1990

\section{Aktuelie Maglichkeiten, neue MaBstabe und Anforderungen im CAD/CAM-} Beresch

\section{Eintoitang}

Din Entwicklung und Anwendung von Methoden der rechnerunterstitztein

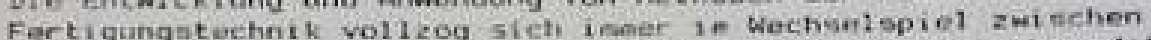
Leistingsvermagen verfigbarer Hardware und algorithmischen-deterininierbaren Fntscheidungskomplexen. Trotz der progressiv steigenden toistungsmertiche nische Aufgaben rechneruntor butzt zu lowen, vortivft sich datiei zu-

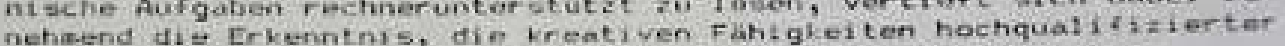
Ingenteure in Frar von Nersech-Maschine Systemen ei nzubezintert.

2.

Dife aktielien Moglichteriten ncehleistungsfahiger workstationer und Steworungstechnik setzen dabei gleichseitig neue Maßstabn und Anforderungen. Auch mit der zunehesenden Verfugbarkeit von geetgnetar Vomminikationstechnil sind neus Vorausestzungen goschaffen, die uafas send ausgenutet werden iönnen und müvser.

Dabei kann es nicht nur darum gohem, Notriebsiat tol- und Prozobiaten, Noreative M.a. bereitzusteller und zu uberwachen, sondern genereil affehtive nuse Strukturen und Arbeitsteilung uber alle Aufgabengebit te hirweg anzustreber.

3. Prablewsteliung; gesamtbetriebilche Prozesse als Optimierungeaufgabe

Der var 70 Jahrien von Taylor gepragte Ausspruch, die Dividende eines Unternehmens hangt von der Schneide ab, wurde jber Jahrzehnte hinweg von Ingeni eurgener ationen von Fertigungstechnikern wit. Recht und ubarzeugung vertreten.

Aus heutiger Sicht nub gan fedoch der von Warnecke vertrotenen Auffassung, das alle betrioblichen Beroicho anteilizg zum hohen Gesantergebnis erines Unternehmens beizutragen haben, voll zustimaen.

n.h. Dptimierungsobjekte sind die wesenti ichen Besantprozesse, wobet für die Fertigungstechniker der technische Intormationsfluß (CAD/CAM) in Vordergrund stehen aus.

Unter der Pramisese, daf stabile und zuverlassige Prozesse al a erste Voracssetzung gowahr leistet bind, gewinnen unter dea Aspekt des harten internationalen Wettbewerbs Optimiorungoziele, wie 
- Cualitat

- Reaktionsfahigkest bei hoher flexibilitat

- Durchl aifzeil tverkarzung

zunelsaind an Bedeutung.

4.

In veresnfachenden Prinzipdarsteliungen wird zum Erreichen dieser Zielstethungen der durchgängige Dateriflup und zahlreiche Ruckkopplungen uber unterschiedilche ProzeBstufen hinweg entsprechend Bild 1 a verteutlicht. Ein reater betrieblicher Ablauf ist in ebenfaliu noch stark vereinfachter Darstellung tatsachlich wesentilch vielfaltiger und trosiptencer (Bitid $\mathrm{i}$ b).

5.

Den Ansitzen durch Parallelsierung von Abl bufen mit den englischen Begriffen "Simultanions-Engineering" bzw. "Concurrent-Engineering" sind dabei objeictiv bestimmte Grenzen gasetzt. Gravierende Veranderungen und Verbesserungen sind demach nur durch prinzipiell neue Creganisationsformen, die elne Grupperverantwortung und Ubernatime komplexer Aufgabenstellungen wichert, zu errachen.

6.

Eine entscheidente Voraussetzung dafür ist eine unfasserve Wissensbereitstollung für wesentliche Aufgabenkomplexe in der technischen Produstionsvorbureitung.

Unter Beachtung der unterechiedlichen Kostenverantwortung muB dabei oin wi-ssensbasierte Rïckkopplung zum uberwinden gegerwartig gegelsener abjektiver Hemanibse geschaffen werden (sh. Bild 2).

7.

Gravierende Problese der bisherigen Arbeitsweise - auch mitteis verfugbarer CAD/CAM-Systeme - resultiaren aus der Trennung zwischen Konstruktion und Arbeitsvarboreitung:

- konstruktive tosungen gaher unzuroichend von den fertigungstechnische Moglictikeiten aus;

- das gegebene fertigungstechnische Wissen der Konstrukteure kann objuktiv nicht die zunehmende Palette neuer Verfahren, insbesondere aus der Sicht kostennabiger Auswirkungen, erfassen;

- spezifisches fertiqungstechnisches Wissen wistiert nur in den Kopfen bestianter Mitarbeiter. Daraus resultiert die große Gefahr des Wistans- und Erfahrungsverlustes.

B.

Zur Problemiösung kann nur mittels einer umfassenden Bereitstellung
von Daten. Fakten, Methoden und Wisgen zur jeweiligen fortigng von Daten, Fakten, Methoden und wissten zur jeweiligen fortigungstech-
ischen Lósung beigetragen werden. Damit kann und muß ein weitgehendes Parallelisieren von Produkt- und
Prozeßgestaltung erreicht werden. 


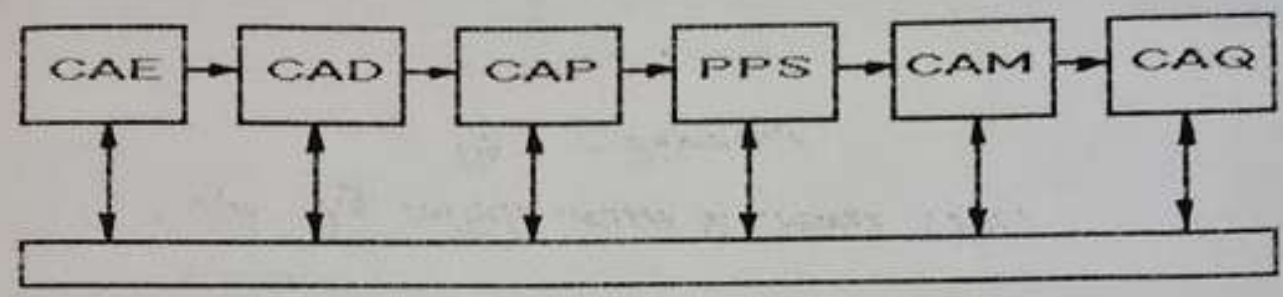

Bild 1a: Informationsfluss und Rückkopplungen in CAD/CAM-Bereich

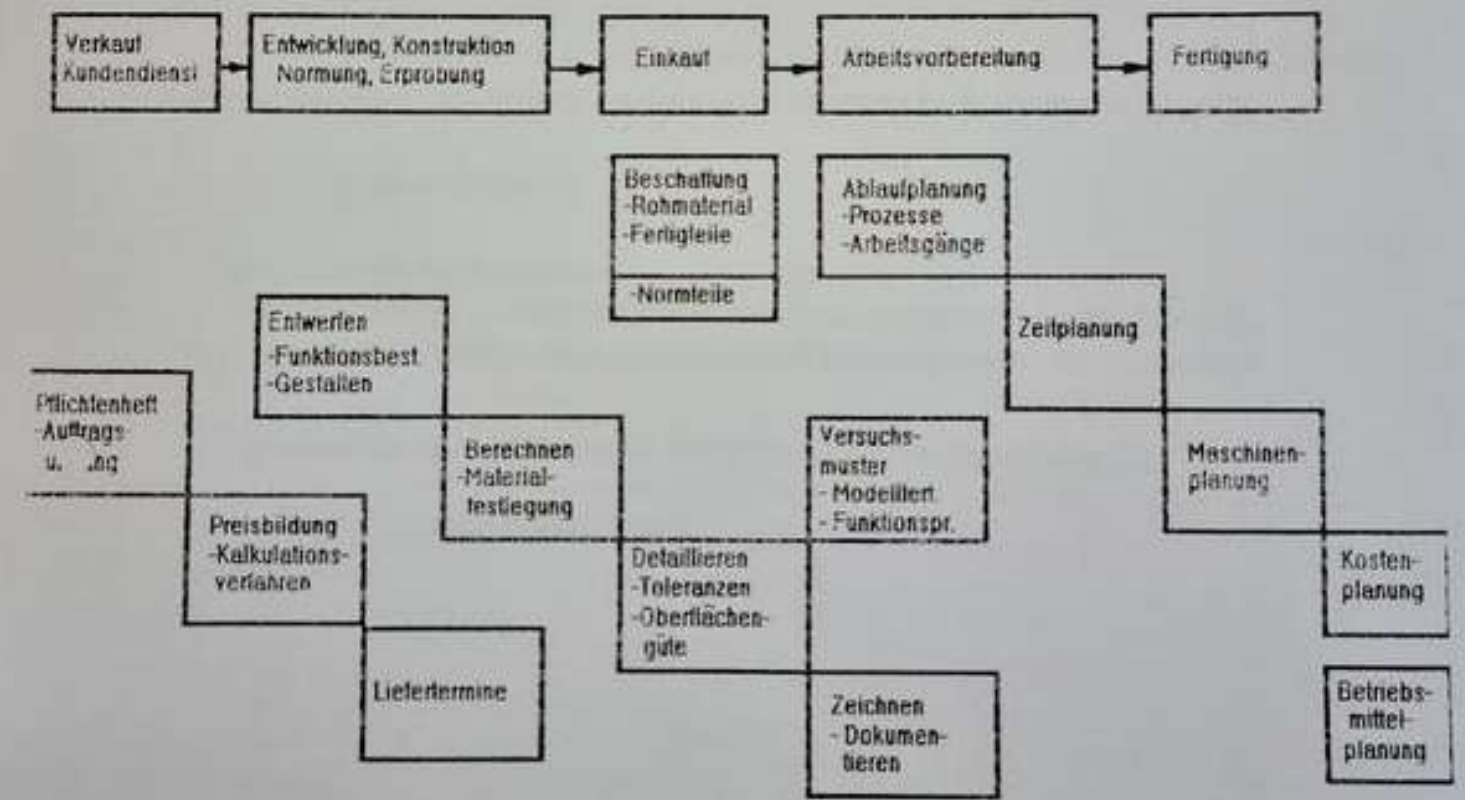

Biid Ib: Aulgaben und informationsbeziehungen in der lechnischen Produktionsvorbereilung (vereinfacht) 


\section{Entwicklungsziele vor und nach der historischen Wende 1989}

Im Mai 1989 gab es in einer Laserzeitschrift eine Information zu einem völlig neuen technologischen Prozess mit der Bezeichnung Stereolithografie. Mit Hilfe von Laserstrahlen kann eine polymere Flüssigkeit gezielt gehärtet werden, wenn aus 3D Daten abgeleitete Schnittdarstellungen informationstechnisch aufbereitet werden können. Diese in der Tat revolutionierende neuartige Technik war in Verbindung mit der Produktionsautomatisierung eine neue Herausforderung. Diese in den USA entwickelte neue Technik wurde in Darmstadt von dem damaligen Unternehmen Spectra- Physics (später 3D-Systems) vertreten. In dieser schon politisch komplizierten Zeit konnte nur mit Unterstützung des Unternehmens Carl-Zeiss-Jena ein Lösungsweg gesucht werden. Deshalb konnte kurzfristig ein Arbeitsbesuch bei meinem ehemaligen Fachdirektor Dr. Claus Gattnar zum damaligen Zeitpunkt 1989 Wissenschaftlicher Direktor des Kombinates Carl-Zeiss, beitragen. In dem Gespräch mit Dr. Gattnar ergabsich als Schlussfolgerung, dass eine Lösung nur mit Unterstützung eines mir bis dahin Unbekannten in der Person von Schalk Golokowski möglich war. Dazu musste ein fundiertes Konzept erarbeitet werden, um notwendige Devisen beantragen zu können.

Wichtiger Ausgangspunkt war deshalb ein kurzfristiger Arbeitsbesuch in Darmstadt, der über den fachlichen Kontakt mit den IFIP TC5 Partner Herrn Prof. Encarnacao (TU Darmstadt) organisiert wurde.

Das freundlich geführte Informationsgespräch im Unternehmen Spektra-Physics im Juni 1989 begann mit der überraschenden Information, dass diese neue Technik entgegen der üblichen Verfahrensweise nicht unter den Embargo (COCOM) Bestimmungen stand. So wurden z.B. auch Verkaufsverhandlungen mit einem Institut im damaligen Leningrad geführt. Zum vollen Verständnis für das neue Funktionsprinzip, inzwischen als 3D Druck allgemein bekannt, wurden anschauliche Informationsmaterialen einschließlich einer Videodemonstration zur Verfügung gestellt. Ausgehend von diesen Informationen wurde im kleinen Kreis der TU Leitung nach der Präsentation und in Abstimmung mit Carl-Zeiss-Jena ein gemeinsames Realisierungskonzept entwickelt. Danach war vorgesehen, dass eine Stereolithografie-Anlage an der TU Dresden installiert wird und in Jena, im Unternehmen Pentacon Dresden und in den optischen Werken ROW (Optische Werke Rathenow) geeignete 3D CAD Arbeitsplätze installiert werden. Insbesondere zu dem damaligen unter fachlicher Verantwortung von Carl-Zeiss stehenden Großbetrieb Pentacon (6000 Beschäftigte in Dresden) war eine gute Voraussetzung für die schnelle Nutzung der geplanten Investitionen gegeben. Diese Einschätzung kann von mir mit Nachdruck im Ergebnis eines IFIP Arbeitsbesuches im Oktober 1989 in Tokio bestätigt werden. In diesem Rahmen erfolgte im international führenden japanischen Unternehmen Nikon ein Informationsbesuch, wonach keine gravierenden Unterschiede im produktionstechnischen Niveau festgestellt werden konnten. In diesem Zusammenhang wurde auf 3D Technik von Norsk-Data (Norwegen) orientiert, weil dies bezüglich keine Embargo Anforderungen anstanden. Dieses gemeinsame Konzept wurde im September 1989 an das Wirtschaftsministerium übergeben und der notwendige Betrag von 2 Millionen D-Mark (ValutaMark) im September 1989 vom Staatssekretär bestätigt. Interessanter Weise fand an dem historischen 09.11.1989 im Wissenschaftsministerium der DDR ein Gespräch mit fachkompetenten Partnern zu der entscheidenden Frage statt, ob die DDR in der Lage wäre, Stereolithografietechnik selbst zu entwickeln. Dabei wurde folgendes Ergebnis erzielt: Eine technische Lösung und auch der Aufbau einer geeigneten 3D CAD Software wurde in Verbindung mit der Akademie der Wissenschaften (Arbeitsgruppe Prof. Iwainski) und weiteren Industriepartnern durchaus als realisierbar eingeschätzt. Allerdings wäre ein derartiges Projekt an der Leistungsfähigkeit der chemischen Industrie gescheitert. Das zur damaligen Zeit führende Schweizer Unternehmen Cibay Geigy, welches diese spezielle mit Laserstrahl aushärtbare chemische Substanz auch in kleinen Mengen produziert hat, konnte die für große Mengen „Tonnagen“ ausgerüsteten chemischen Industrie der DDR nicht realisiert werden. 
Trotz dieses unbefriedigenden fachlichen Ergebnisses war der kurze Arbeitsbesuch am 09.11.1989 in Berlin zur Eröffnung der Mauer ein emotionales Ereignis, was die weitere Entwicklung geprägt hat.

\subsection{Schlussfolgerung für künftige Kooperationen und aktuelle Situation}

Interessanter Weise hatte ich Ende November, 14 Tage nach der Maueröffnung, (9.11.1989) einen Vortrag zum Kongress der Wirtschaftsingenieure der BRD im Hörsaal des Institutes für Konstruktionen und Produktionstechnik (Prof. Spur) zu halten. Mein Vortrag beinhaltete die Entwicklungen von "CAD/CAM und CIM in der DDR". In meinem Abschlusskommentar dieses Vortrages wurde meine Überzeugung zum Ausdruck gebracht, dass wir in Deutschland eine gute Basis durch eine Zusammenlegung der gegebenen Potentiale für gemeinsame Weiterentwicklungen haben. Konkret wäre durch eine Zusammenführung der vorhandenen intelligenten Potentiale (Neubundesländer) im Gebiet der Produktionsautomatisierung mit den Voraussetzungen leistungsfähiger Technik (Altbundesländer) eine gute Basis vorhanden. In der noch immer euphorischen Stimmung der erfolgten Öffnung der Berliner Mauer wurde mein fachlicher Vortrag mit der Schlussfolgerung mit großem Beifall aufgenommen. Allerdings verlief auch im Gebiet der Wissenschaftskooperation die Entwicklung nicht in Übereinstimmung mit den angestrebten idealen Möglichkeiten. Die auf der unmittelbaren fachlichen Arbeitsebene gegebenen Kontakte wurden leider durch übergeordnete politische Einflüsse nicht optimal zur Wirkung gebracht.

Die unmittelbare Übersiedlung in die Industrieregionen der BRD eines beachtlichen Teiles meiner Absolventen und insbesondere der vertieft ausgebildeten Beststudenten der Fertigungsinformatik sind einerseits der Beweis für das anforderungsgerechte Ausbildungsniveau an der TU Dresden. Andererseits sind die damaligen und leider bis in die heutige Zeit anhaltenden Übersiedelung gut qualifizierter Absolventen von beträchtlichen Nachteil für die NBL.

Obwohl dieses Problem allgemein bekannt und als Solches auch anerkannt wird, geschieht m.E. zu wenig, um nach 30 Jahren "Deutsche Einheit“ , um diesen unbefriedigenden Zustand zu verändern.

In den vergangenen ca 10 Jahren wurden an das Sächsische Wissenschafts- und das Wirtschaftsministerium, sowie an Abgeordnete Vorschläge zur Verbesserung eingebracht, ohne das entsprechende Reaktionen erfolgten. 


\subsection{Veränderungen nach der historischen Wende 1989}

Ausgehend von den dargestellten produktionstechnischen Entwicklungsstand bestand für mich nach der historischen Wende kein Grund von dem inhaltlichen Zielstellungen abzuweichen. Im Gegenteil. Durch konsequente Orientierungen auf Produktivitätsverbesserungen, konnte weiterhin auf den grundsätzlichen Zielstellungen aufgebaut werden. Das bezieht sich im Bereich der spanenden Fertigung insbesondere auf die Verfügbarkeit von 5-Achsbearbeitungstechnik für das Fräsen geometrisch komplizierter Geometrien. Deshalb war die aktive Mitwirkung in einem ersten EU Projekt an der TU Dresden ein wichtiger Beitrag. Die gegebenen guten fachlichen Kontakte zum Institut von Prof. Spur / Prof. Krause (Berlin) und auch Norsk-Data in Norwegen wurde in dem Projekt FAME - Five Axis Manufacturing Environment zu Grunde gelegt und weiter ausgebaut. Um auch dafür eine experimentelle Basis zu schaffen gehörte die Investition einer geeigneten 5-Achsbearbeitungstechnik zu einer wesentlichen Aufgabe. Für eine hochwertige und zuverlässige Lösung wurden umfangreiche Recherchen in Deutschland bis nach Österreich (Hallein) und Arbeitsbesuche durchgeführt. Die vorrangig für Lehrzwecke in Österreich produzierten Fräsmaschinen für Ausbildungsaufgaben erwie-

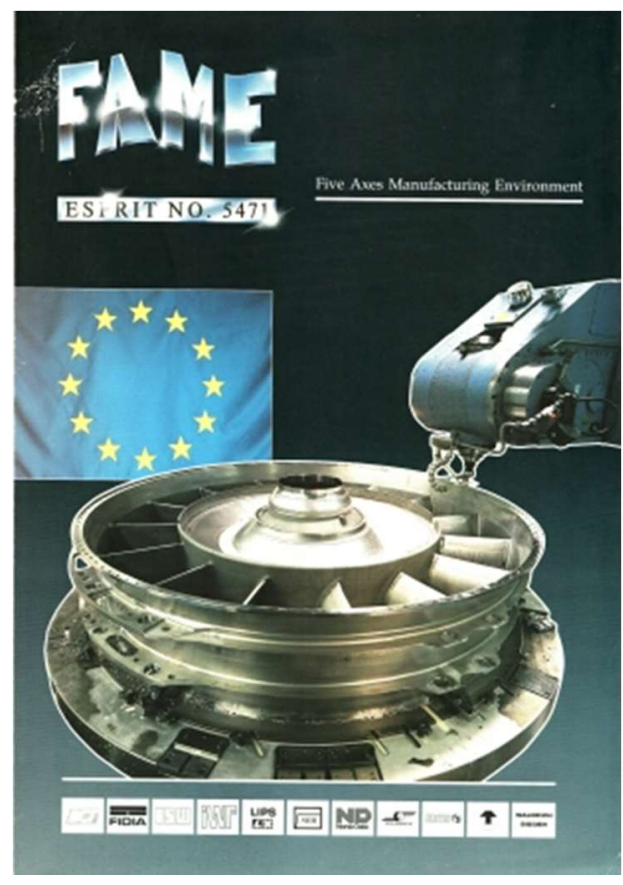

Abbildung 48: Charakteristik EU-Projekt FAME sen sich für anspruchsvolle Forschungszielstellungen als weniger geeignet. Zum Abschluss der durchgeführten Recherchen fiel die Entscheidung für ein Bearbeitungszentrum vom Unternehmen Maho (s. auch CIM TT, Abschnitt 11 ). Diese an der TUD 1991 installierte Technik war mehr als 20 Jahre die einzige Anlage für anspruchsvolle Ausbildungs- und Forschungsaktivitäten im Gebiet komplizierter Geometrien an der TU Dresden.

\section{Forschungsjahr für revolutionierend neues generatives Fertigungs- verfahren}

Nachdem mit dem Bekanntwerden des völlig neuen Fertigungsverfahrens mit dem Begriff „Stereolithographi“ im Frühjahr und Sommer 1989 nur ein theoretisches Vertraut machen mit dem neuen Wirkprinzip und den zughörigen Anforderungen möglich war, konnten nach dem Fall der Mauer tiefergehende Untersuchungen erfolgen. Dazu konnte ein Sabbatical Jahr genutzt und vorbereitet werden. Dabei ging es primär um die Frage, welche Anlagentechnik außer dem USA -System vom Unternehmen „3D-Systems“ kommt für eine Investition an der TU Dresden noch in Betracht. Bekannt waren darüber hinaus Anlagen aus Japan. Israel und erfreulicherweise auch aus Deutschland vom Unternehmen EOS (Elektro-optische Systeme). Diese neuartige Anlagentechnik wurde von EOS gemeinsam mit BMW entwickelt so das von einer hohen Praxis-Relevanz ausgegangen werden konnte. Unabhängig davon mussten natürlich tiefergehende Untersuchungen durchgeführt werden, da von Seiten des USASystem-Anbieters ein beachtlicher Marktvorsprung gegeben war.

Schwerpunkt der Untersuchungen war die Vorbereitung und Durchführung eines spezifischen Benchmarks. In dessen Ergebnisses erfreulicherweise die EOS Anlagentechnik als funktionell bestgeeignete nachgewiesen werden konnte. 


\subsection{Studienaufenthalte für Theorie und Praxis in Norwegen und USA}

Logischerweise war auch nach der Wende im Jahre 1990 die unter dem Sammelbegriff Rapid Prototyping stehende Anlage noch immer eine wichtige Herausforderung. Deshalb wurde das ursprüngliche Konzept mit weiteren Recherchen ausgebaut und vertieft. In diesem Zusammenhang und unter Beachtung von Entwicklungen aus Japan, Israel und auch Deutschland war ein relativ breites Spektrum von technischen Möglichkeiten gegeben. Deshalb war es sinnvoll und gerechtfertigt tiefergehende Untersuchungen im Rahmen eines Sabbatical Jahres 1992 anzustreben. Wenn auch kein volles Jahr realisiert werden konnte, wurden mehrere Monate an dem damaligen in Europa führenden Institut an der Universität Trondheim (Norwegen) wahrgenommen. An diesem von Prof. Björke geleiteten Institut war eine USA Stereolithografieanlage und eine Anlagetechnik Cubital von Israel im Einsatz für Lehre und Forschung. Die zweite Hälfte der Sabbatical Zeit wurde mit Unterstützung von Dr. Merchant am Institut IAMS (Institute for Advanced Manufacturing Sciences) durchgeführt. Dieses Institut in Cincinnati hatte selbst keine Anlagentechnik, aber die Zugänge zu den führenden Erstanwendern

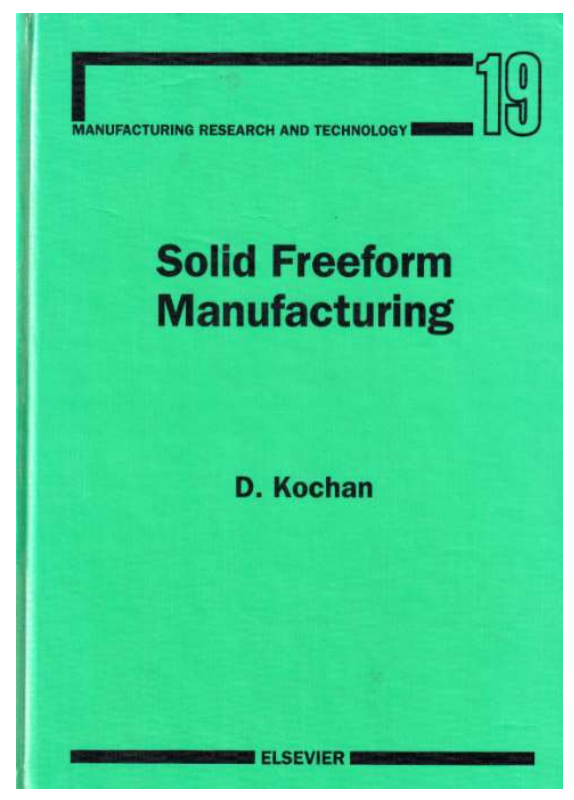

Abbildung 49: Ergebnis des Sabbatical-Jahres der Stereolithographietechnik in den Automobil - Unternehmen (Chrysler, General Motors, Ford) war eine gute Voraussetzung zum Studium des realen industriellen Entwicklungsstandes. Die Sabbatical Zeit wurde deshalb primär zur Bewertung der damals am Markt verfügbaren Systeme für die TUD Investitionsentscheidung und zur Bearbeitung eines Fachbuches genutzt. Der in der Fachwelt damals genutzte Oberbegriff „Rapid Prototyping“ befand sich allerdings in einer Überlappung mit den auch in der Softwareentwicklung genutzten Begriff Rapid Prototyping. Deshalb wurde in Übereinstimmung mit führenden USA-Fachexperten der Oberbegriff „Solid Freeform Manufacturing - Advanced Rapid Prototyping", genutzt. Obwohl von Seiten der USA schon eine beachtliche Anzahl unterschiedlicher Systeme über die Stereolithografietechnik hinaus, industriell genutzt wurden, erwies sich die relativ schnell zur Marktreife geführte Stereolithografietechnik des Unternehmens EOS München als besonders nutzerfreundliche Anlagentechnik. Deshalb erfolgte im Ergebnis der gründlichen Bewertungen die Auswahl auf die Anlagentechnik von EOS 400 vom Unternehmen EOS München.

Die im Jahre 1993 an der TUD installierte Anlage EOS Electrical Optical Systems 400 war gleichzeitig der Ausgangspunkt für die Gründung eines Unternehmens mit dem Begriff „SFM GmbH - Schnelle Fertigung von Modellen".

\subsection{Fachliche Unterstützung der neuen High-Tech-Firma EOS München}

Als in den Jahren mit anderen zur industrieellen Reife entwickelten Stereolithografieanlagen ( $z . B$ Japan, BRD u.a.) folgten schwierige Patentstreitigkeiten mit dem führenden Unternehmen 3D-Systems (USA) .Deshalb musste auch von EOS München eine geeignete zukunftsorientierte Lösung gefunden werden. Für die anstehenden Patentstreitigkeiten wurde von mir auf Anfragen des bayrischen Wirtschaftsministeriums eine aktive fachliche Unterstützung für EOS München gegeben. Allerdings wurde von Seiten der USA mein sachlich fachliches Urteil der verbesserten Funktionsmerkmale mit der Begründung abgelehnt, dass man als Käufer der Anlagentechnik für den Sachverhalt befangen ist. Unabhängig davon wurde meine Unterstützung für EOS München von dem bayrischen Wirtschaftsministerium mit einem spezifischen Gutachten meinerseits unterstützt. Nach dem verlorenen Patentstreit, 
wonach von EOS kein weltweiter Vertrieb aufgebaut werden konnte, war nochmals eine kritische Situation für das Unternehmen EOS München gegeben. Für die Weiterentwicklung der Nutzung von 3D CAD Datenmodellen zum direkten Aufbau geometrischer Objekte, musste deshalb ein neuartiger Ansatz entwickelt werden. Das geschah in der Weise, dass geeignete pulverförmige Materialen ebenfalls in einem Schichtenbauprozess mit dem Begriff "Selective Laser Sintering - SLS" entwickelt und patentrechtlich abgesichert wurden. Diese konsequente Entscheidung wurde auch vom bayrischen Wirtschaftsministerium im Ergebnis eines meinerseits unterstützenden Gutachtens gefördert. Damit war eine wichtige Voraussetzung geschaffen um mit einer Vielzahl geeigneter Materialen zunächst hochwertiger Kunststoffe eine breite Basis geschaffen . Darüber hinaus wurde mit zunehmend leistungsfähiger Lasertechnik der

Zugang zu metallischen Werkstoffen insbesondere auch Stahl ermöglicht. Diese geschaffenen Voraussetzungen sind die Voraussetzungen für die vom Unternehmen EOS München erreichte Weltmarktführerschaft im Gebiet hochwertiger Kunststoffe und metallischer Werkstoffe.

\subsection{Nutzung hochwertiger Innovationen für verbesserte Ausbildung und Forschung}

\section{Installation der Stereolithographie-Anlage EOS $\mathbf{4 0 0}$ für Lehre und Forschung an der TU Dresden}

Mit der erfolgreichen Inbetriebnahme des leistungsfähigen Stereolithographie-Systems von EOS München im Jahr 1993 waren hervorragende Voraussetzungen für Aus- und Weiterbildung in Übereinstimmung mit den Zielstellungen des CIM-TT-Zentrums geschaffen. Im Ergebnis des Sabbatical-Jahres in Norwegen und den USA und in diesem Rahmen durchgeführter Benchmarks aller am Markt verfügbaren sog. Rapid-Prototyping-Anlagen waren die anwenderfreundlichen Merkmale der EOS-Anlage eindeutig nachgewiesen worden. Damit wurden die funktionellen Merkmale gegenüber der Stereolithographie-Anlagen vom damaligen Weltmarktführer 3D-Systems, USA für die installierte EOS-Anlage als gravierender Vorteil genutzt. Die fachlich objektive Entscheidung für die funktionell anwenderfreundliche Anlage von EOS München mit einer Benchmark-Bewertung von damals 4 in Betracht kommenden

Anlagen konnte von mir in einem Vortrag zu einer CIRP-Ehren-Konferenz für die USA-NC-Pioniere 


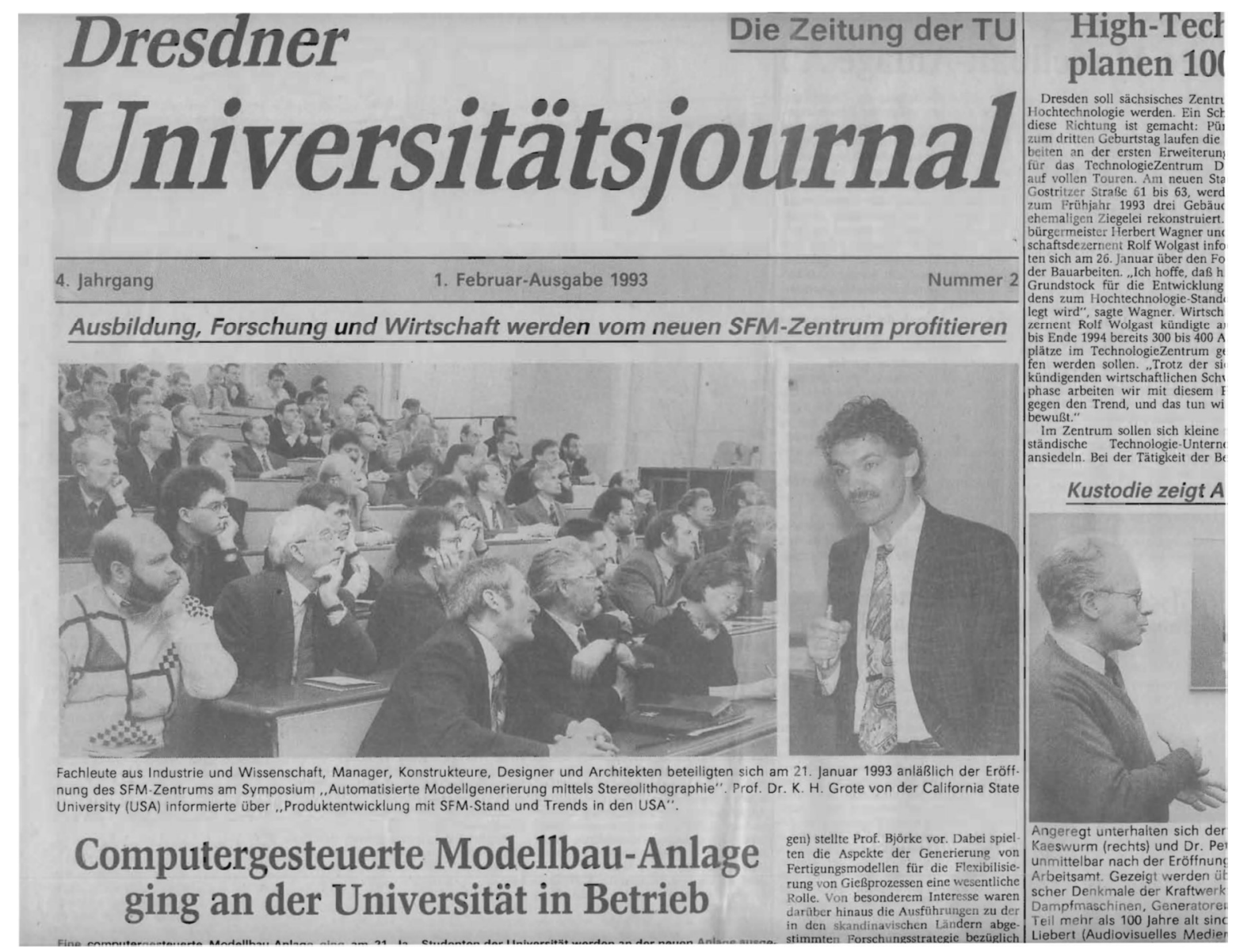

Die Veranstaltung zur Inbetriebnahme der EOS-Stereolithografie Anlage fand an der TU Dresden

großes Interesse, wie es an dem gut gefüllten Hörsaal erkennen kann. In dem Vortrag von Professor Karl H. Grote wurde der aktuelle Entwicklungsstand der Produktentwicklung in den USA dargestellt. Prof Grote war zu diesem Zeitpunkt Inhaber einer "Greencard“ und an der California State University tätig. Wir hatten in dieser Zeit aktive fachliche Zusammenarbeit über viele Jahre , die auch nach seiner Rückkehr nach Deutschland als Dekan an der Fakultät Maschinenbau der TU Magdeburg fortgesetzt wurde. 
Dresdner Universitätsjournal 3/93

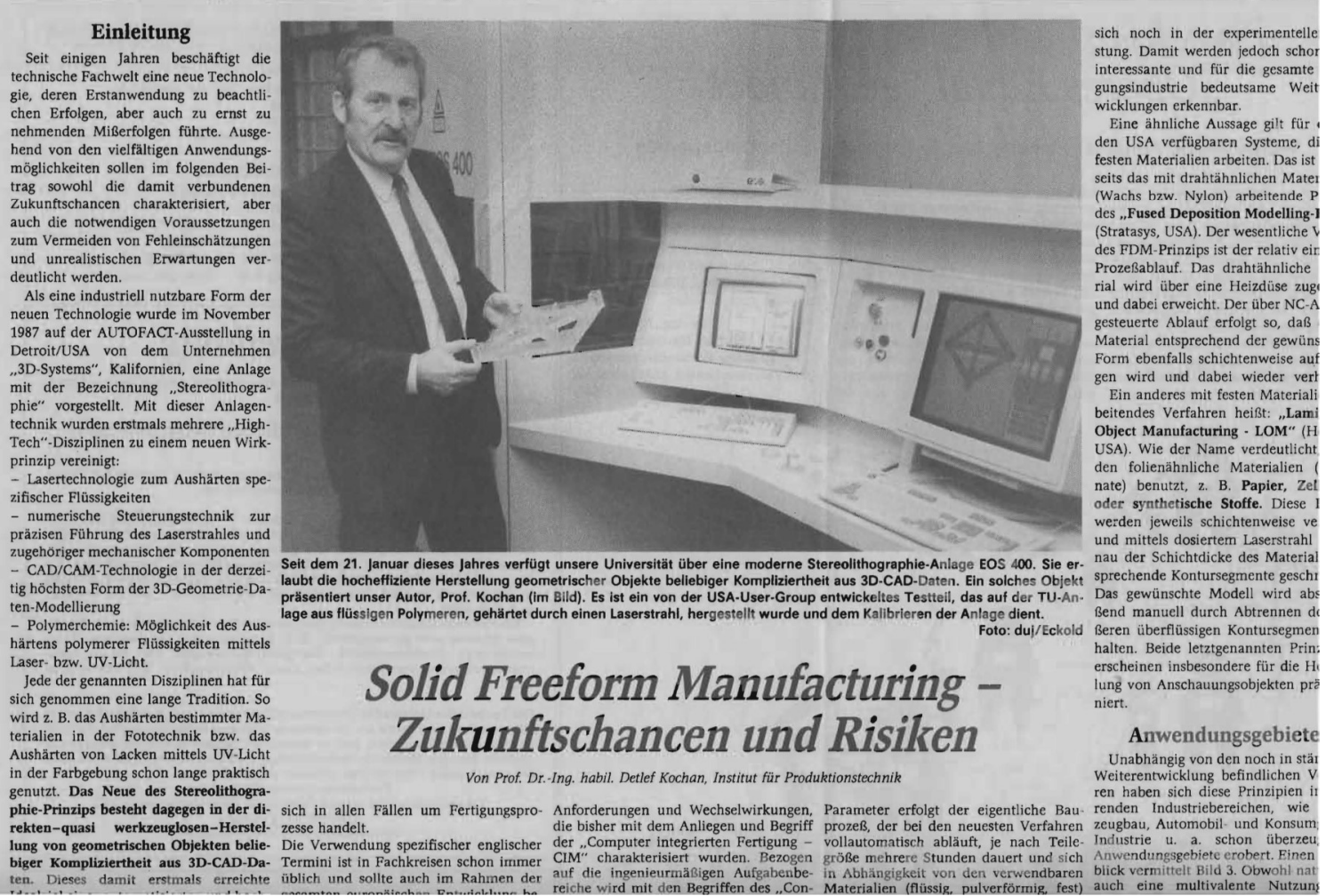

Abbildung 51: Inbetriebnahme der ersten Rapid Technology -Anlage in den NBL

\subsection{Ehrenkolloquium der CIRP für NC-Pioniere 1993}

Im Jahr 1993 fand in Slowenien eine Fachkonferenz für 2 Pioniere der numerischen Steuerungstechnik statt. Unmittelbarer Anlass war die Würdigung des 25th Jubileums der CIRP-Seminare zum Thema

„Flexible Fertigungssysteme“. Veranstalter waren die international führenden Wissenschaftler der CIRP, d.h. der Weltorganisation der Fertigungstechniker. Unmittelbare Organisatoren von diesem Ereignis waren die Professoren Spur (D), Sata (Japan), Colding (Schweden/ USA) und Peklenik (Slowenien).

Anlass war die Feier zur Würdigung der historischen Entwicklung der Flexiblen Automatisierung mit der numerischen Steuerung von Werkzeugmaschinen. Das Thema der CIRP- Konferenz war "Flexible Manufacturing Systems -Past- Present- Future" (ISBN 86-7217-100-4) J.Peklenik)

Mit 15 Fachvoträgen wurden geehrt:

- Dr J. Merchant (USA)für die Entwicklung der Grundprinzipien Flexibler Fertigungssysteme und Integration von Fertigungsverfahren

- Dr j. t. Parsons (USA) als Erfinder der Numerischen Steuerungstechnik ( s. Abbildung 52)

Meine aktive Teilnahme mit dem aktuellen Vortrag "Solid Freeform Manufacturing- the New Generation of Flexible Manufacturing Systems" als einziger ostdeutscher Professor vor einem kompetenten Teilnehmerkreis kann auch als eine besondere Wertschätzung der von mir vertretenen TU Dresden angesehen werden. Es muss schon als ein bedenklicher Widerspruch eingeordnet werden, wenn im gleichen Jahr 1993 meine weitere Mitarbeit an meiner "Alma Mater" nicht mehr gefragt war. 


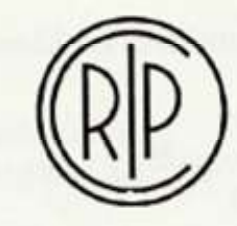

College International pour l'Etude Scientifique des Techniques de Production Mécanique International Institution for Production Engineering Research

Internationale Forschungsgemeimshaft für Mechanische Produktionstechnik

dedicated the 25 th jubilee CIRP.

International Seminar on Manufacturing

Systems to honour no great

manufacturing engineers and scientists

\section{Dr. M.E. Merchant, USA}

who, in the early sixties, established some basic principles of the FMS and integration of manufacturing technologies, triggering a significant impact in the development of the factory automation

and

Dr. J.T. Parsons, USA

who, in the late fourties, invented and introduced the principles of NC-technology, creating a revolution in manufacturing methods and making the FMS realizable.
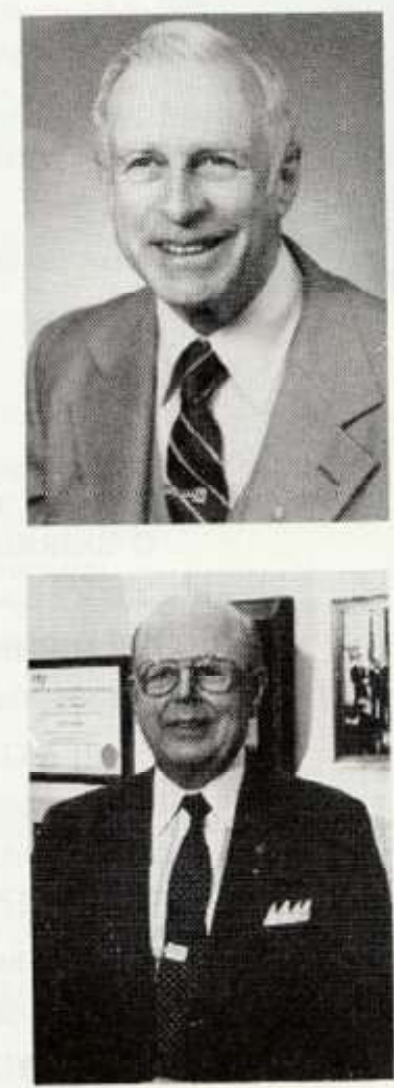


\section{Anfänge des Additive Manufacturing in Dresden}

\subsection{Dokumente zur 1993 gegründeten Firma SFM GmbH}

Für die industrielle Anwendung der zum damaligen Zeitpunkt führenden „Rapid Prototyping -Technologie“, jetzt Additive Manufacturing bzw. 3D-Drucken war in Übereinstimmung mit den Zielstellungen des CIM-TT-Zentrums eine anteilige Nutzung für die erfolgte Firmenausgründung vereinbart worden.
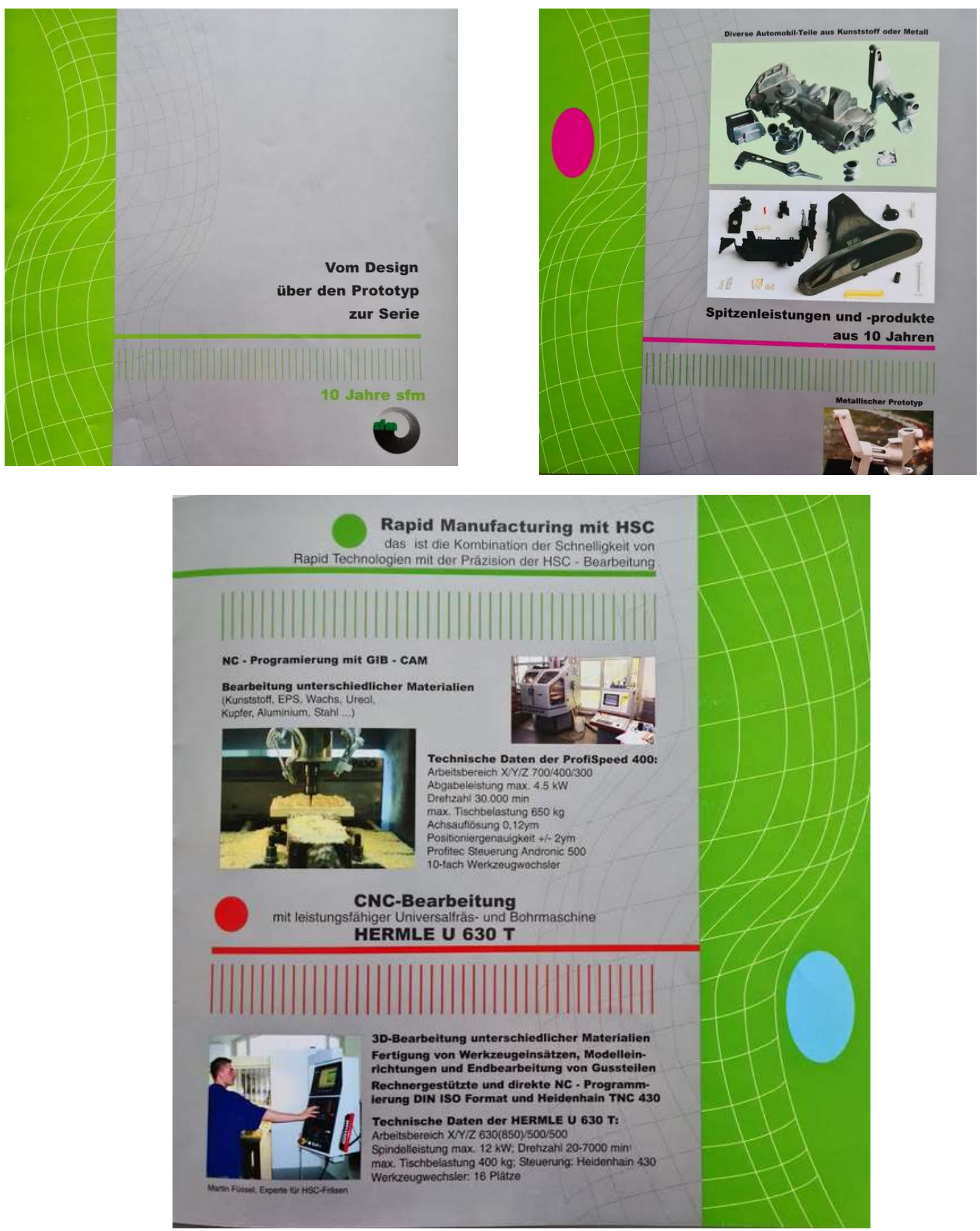

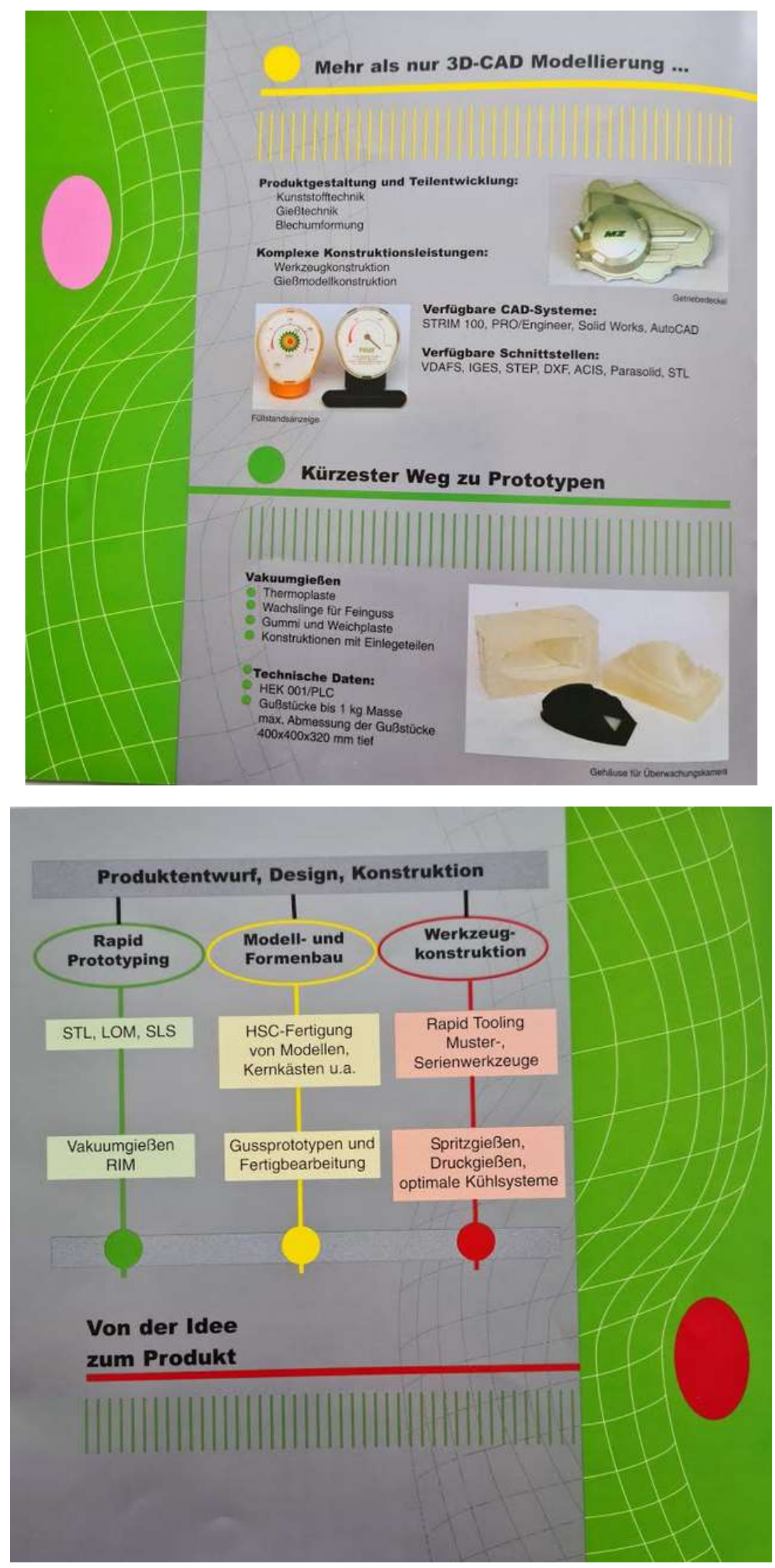


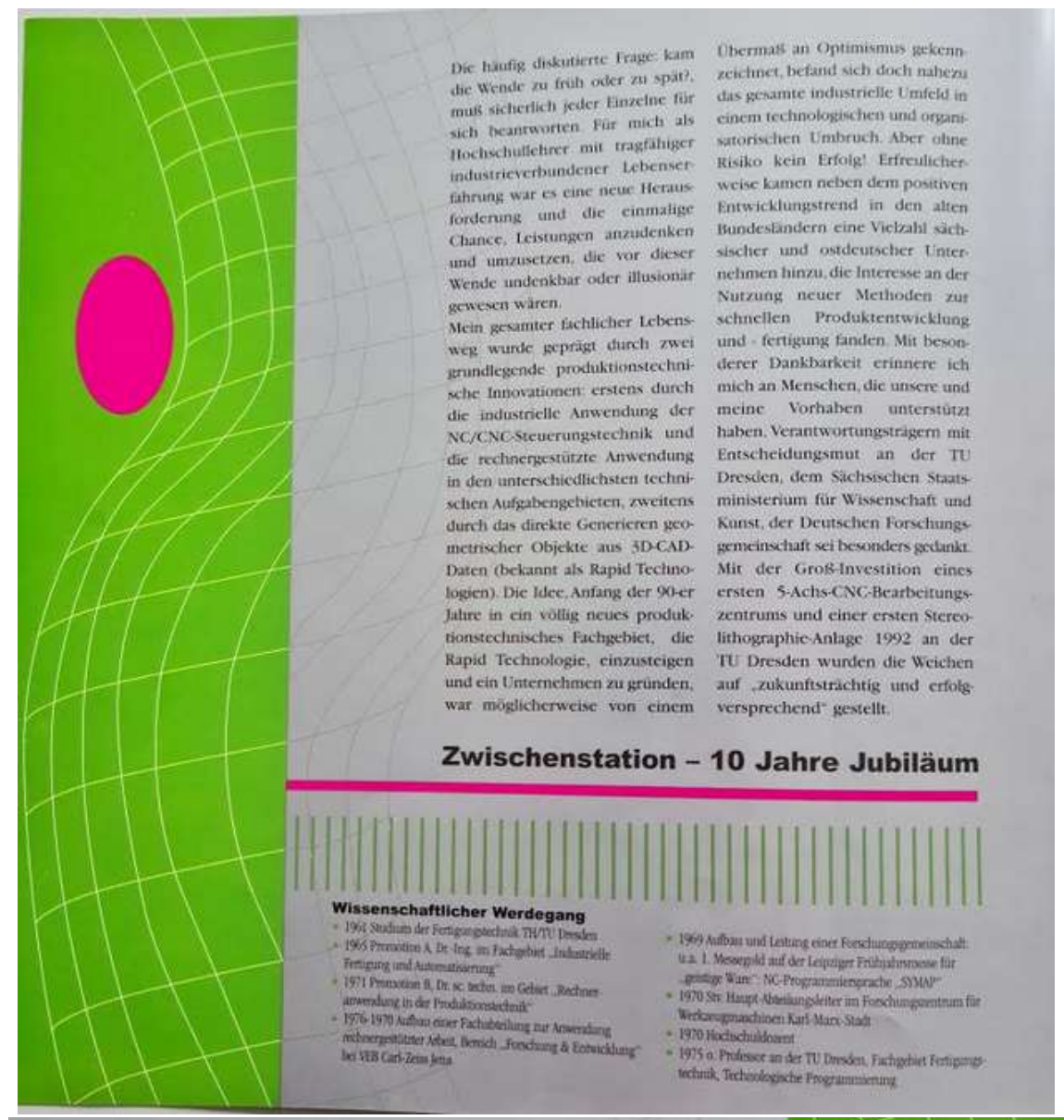

Finen besonderen Beitrag zu erfolg. gus Heidenau" za einem in reich realisierten Problemlosungen Deutschland fohrenden Unterneh haben die Stereolithographic und men der Druckgusstechnik geführ das Vakuumgiefen in unserer hat, der mit Familie in den Osten Branche und in unserem zog. rahireciche Arbeitsplizze schu Unternclumen peletset Obne sic und der auch unerem Lntemeh

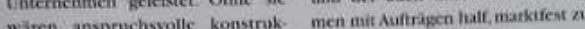
waren anpore

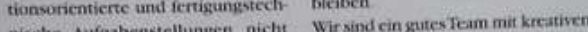

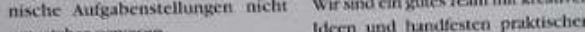
umsetzbar gewesen.

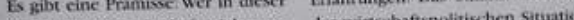

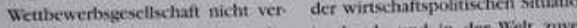
sagen will, muss innovativ, markt. im Lande und in der Wh und teamfahig sein. Olne einen sichtich fur die Zukuntt.

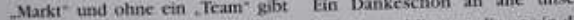
es keine innovation. Das forkea wir Ko rechtzeitig erkannt und danach 10 jahren unterstutzen. gehandelt. Den Markt haben wir thr Prof. Dr. Detlef Kocha uns erechloesen und dabei Partiner Geschaiftsfuther gefunten for permeinesme Problembetown Als besonderes Gluck

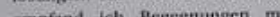

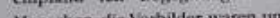

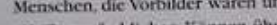
mit ihrem fachilchen kongen uber zeugten. Mit Hochachitung gedenke ich anlisstich des 10-jattrigen Jubiàums, des leider zu fruih ver storbenen Lnternehmers Peter Schuchandt, der als liebenswerter Wess: das Lnternehmen .Druck-

\section{Zwischenstation - 10 Jahre Jubiläum}

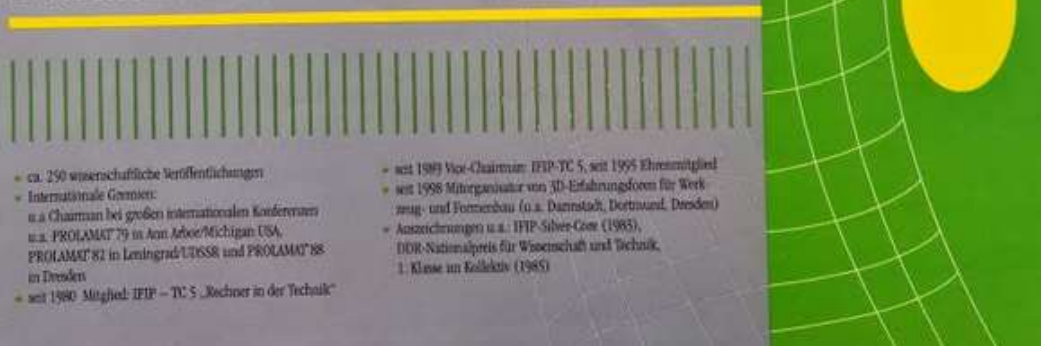




\subsection{Höhepunkt der Firmenentwicklung mit der ersten Panorama-Kamera}

\section{Erfahrungen mit dem neugegründeten Unternehmen SFM GmbH}

Die Unternehmensgründung aus der TU Dresden heraus war in der insgesamt problemreichen Entwicklung des industriellen Umfeldes durchaus vonvornhinein mit Risiken verbunden. Trotzdem konnte eine durchaus erfolgreiche Entwicklung mit einer Anzahl unterschiedlicher Projekte und Aufträge realisiert werden. Als ein repräsentatives Beispiel wird auf das aktive Mitwirken an der Entwicklung einer neuen Panoramakamera verwiesen. Aus dem großen Unternehmen Pentagon war eine Firma mit dem USA Besitzer Sir Noble herausgelöst worden und mit einem kleinen Entwicklungsteam, die Konstruktion einer neuartigen Panoramakamera vorangetrieben worden. Die damit verbundenen Aufgaben zu 3D CAD Modellierung und Herstellung erster Prototypen war deshalb eine interessante und anspruchsvolle Aufgabenstellung. Mit dem im Jahre 1993 begonnenen ersten Aufgaben der 3D CAD Modellierung und der funktionellen Bauteile für die Panoramakamera war deshalb eine hohe Herausforderung, weil möglichst zu einer Photokina Messe im Jahr 1994 in Köln eine Demonstration erfolgen sollte. In enger Zusammenarbeit mit dem fachlichen Kopf und dem erfahrenen Designer konnte eine konstruktive Entwicklung der tragenden Konstruktionsbauteile erfolgen. Wobei speziell die für das angenehme Handling viele Detailabstimmungen zwischen 3D CAD Modellierer und Designer notwendig waren.

Im oberen Bild ist der mit Stereolithografie-Technik hergestellte tragende Funktionsteil der Panoramakamera gezeigt. Darüber hinaus ist unteren Bild ein mittels der sog. LOM (Laminated Object Manufacturing) Anlagentechnik hergestellter Vollkörper gezeigt. Zu dem tragenden Teil gehörten dann noch eine Anzahl weiterer kleiner Funktionsteile. Der als Vollkörper mittels LOM hergestellte Prototyp wurde als Vorlage für die notwendige Verpackung hergestellt.

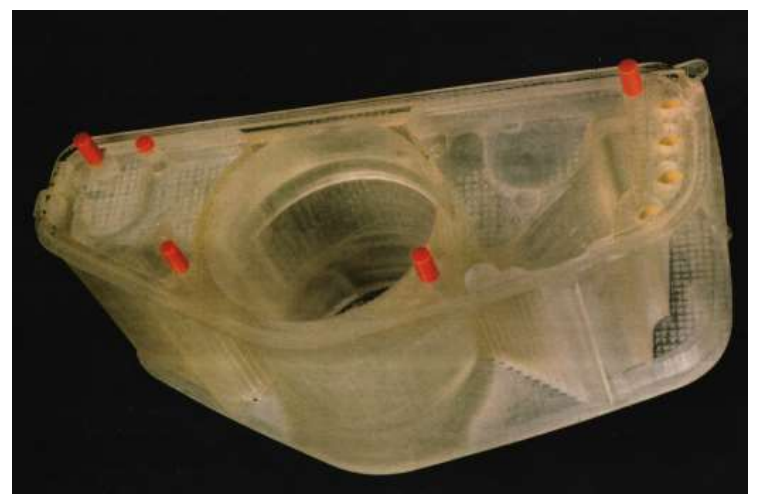

Abbildung 53: Stereolithographie-Aufnahme Grundkörper Panoramakamera

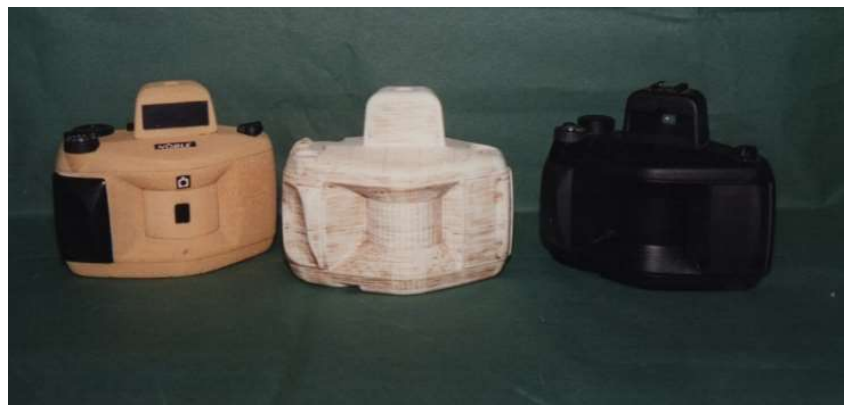

Abbildung 54: Ur-Modell Panoramakamera 
Ohne weitere Details für die gesamte Präsentation zu erläutern, wurde das „sportliche“ Ziel ,Teilnahme an der Photokina Messe in Köln, erfolgreich gelöst.

Im Bild wird das Prospekt gezeigt, welches auf der Basis von 20 funktionsfähigen Prototypen hergestellt wurde. In der weiteren Entwicklung konnte diese Panoramakamera als industriereifes Produkt für Interessenten einen beachtlichen Absatz erreichen. Während einer Reise nach Singapur 1996 (Einladung von Prof Chu Chee Kai) habe ich persönlich viel Freude an der Nutzung der Panoramakamera für besondere Objekte gehabt.

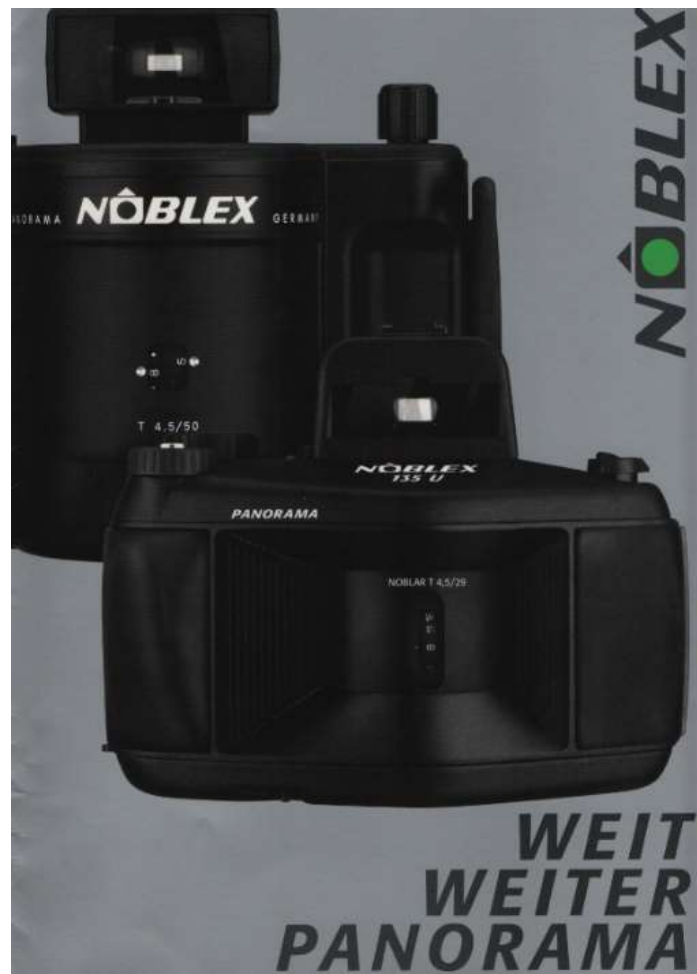

Abbildung 55: Messeprospekt Fotokina 1994

Im Ergebnis dieser insgesamt erfolgreichen Entwicklung und meiner fachlichen Kontakte zu den USA, insbesondere den jährlichen Fachkonferenzen zum Thema „Rapid Prototyping and Manufacturing" hatte ich ein Vortragsangebot bei den Konferenzorganisatoren eingereicht. Dieses Angebot wurde erfreulicherweise angenommen, sodass eine gute Chance für eine mit dem Fachvortrag verbundenen Marketing gegeben war. Aus dieser Sicht erfolgte mit dem Unternehmensbesitzer und seinem Sohn die Vorbereitung eines spezifischen Vortrages. Dabei war abgestimmt, dass von meiner Vortragszeit 5 Minuten für den als Verkäufer in der USA tätigen Sohn von Sir Noble vereinbart war. Entgegen aller Absprachen war leider der Herr Noble Jr. nicht am Konferenzort und hat sich für diesen Fauxpas auch nicht entschuldigt.

\section{World Leadership for Panorama-Cameras Based on new Technologies}

Prof. Dr. D. Kochan, SFM-GmbH Dresden, GFaI LV Sachsen Sir John H. Noble, Kamerawerke Noble GmbH Dresden

\section{$\underline{\text { Abstract }}$}

The increased utilisation of the potentials of the new technologies for the direct generation of geometrical objects of any complexity based on 3D-CAD-Data has to consider more then the substitution of traditional technological procedures.

For an exact assessment and best possible exploitation the impact on the entire value added process chain is of importance.

This will be demonstrated by the accelerated development of a new Panorama Camera

\section{Introduction}

The international competitiveness reguired more and more the development of new products in shorter times, lower costs and better quality. This general requirements can be fulfilled by

Abbildung 56: Veräffentlichung zur USA-Konferenz Rapid Prototyping and Manufacturing 西 


\subsection{Prominenter Messebesucher Dr. Späth}

Über diese Erfolgsgeschichte hinausgehend wurden eine Anzahl weiterer technologischer Projekte realisiert, wobei ergänzend zu der Herstellung von Kunststoff-Prototypen insbesondere auch Modelle, bzw. Komponenten für Formwerkzeugen für das Druckgießen hergestellt wurden.

Die positive Entwicklung des Unternehmens SFM GmbH in den 90er Jahren konnte auch auf den jährlichen Euromold Messen in Frankfurt am Main demonstriert wer-

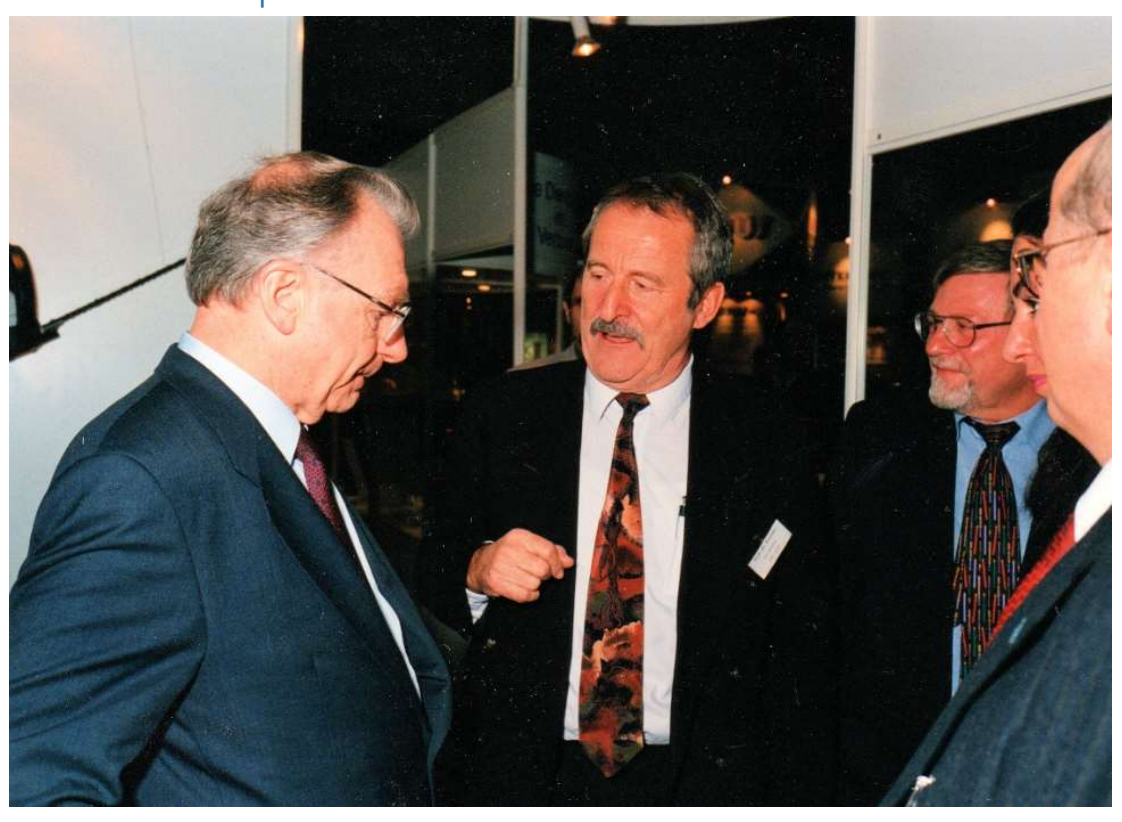

Messe Euromold 1995, Dr. Späth als Messebesucher

den. Zu den besonders exponierten Besuchern gehörte auch der ehemalige Ministerpräsident von Baden-Württemberg Herr Dr. Späth (links im Bild). Bekanntlich hat sich Dr. Späth nach der Wende für die Sanierung der Unternehmen Carl-Zeiss-Jena und Jenoptik besondere Verdienste erworben. 


\subsection{Head-Up Gehäuse 2004}

Aus der Zusammenarbeit mit dem Werkzeugbau des Unternehmens Siemens Automotiv Babenhausen erreichte mich eine anspruchsvolle Anfrage zur Mitarbeit an der Weiterentwicklung eines Head-Up Gehäuses für BMW.

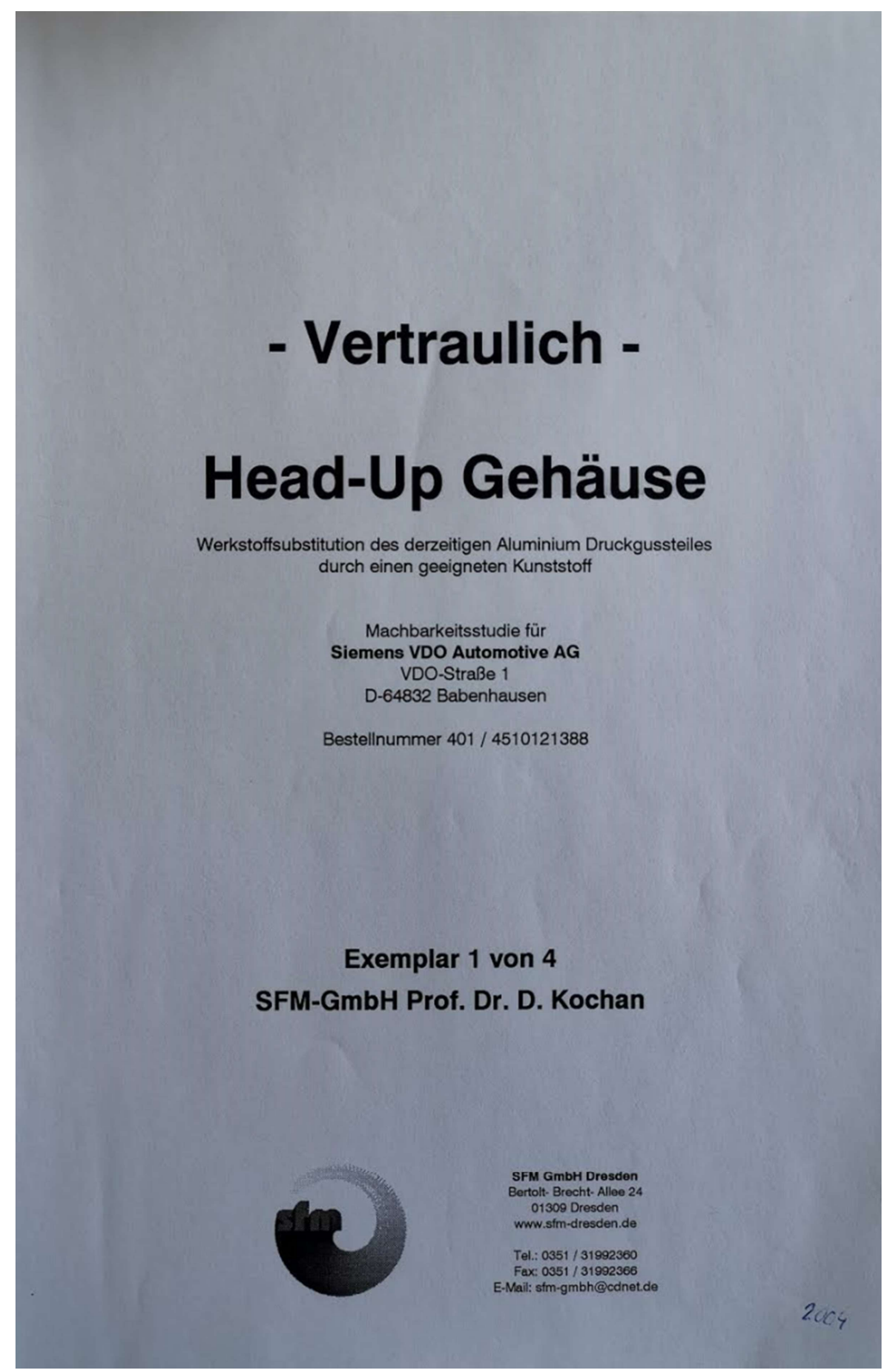

Ein komplett funktionsfähiges Head-Up Gehäuse lag als eine Aluminium Druckguss Komponente vor. Dabei war das besondere Merkmal, dass für den Strahlengang mit mehreren Spiegeln extrem hohe Genauigkeitsanforderungen erfüllt werden mussten.

Aufgrund thermischer Einflussfaktoren zeigten sich in der praktischen Anwendung spezifische Genauigkeitsprobleme. Davon ausgehend stand die generelle Frage, ob es einen besser geeigneten stabilen Kunststoff gibt, der keine negativen thermischen Eigenschaften ausweist. Nach Recherchen in der Kunststoffdatenbank mit 6000 unterschiedlichen

Materialien konnte ein geeigneter Werkstoff gefunden werden.

Der nächste Schritt war das Gewinnen eines Kunststoffproduzenten und eines erfahrenen Werkzeugbauers. Beide Unternehmen konnten im Mittleren Erzgebirge gefunden werden.

Abbildung 57: Beteiligung an einer innovativen Entwicklung Head-Up-Display 


\subsection{Problematischer Firmenabschluss}

Leider fanden die hoch interessanten Jahre als Unternehmer keinen guten Abschluss. Im Jahre 2006 war von einem bayrischen Unternehmer in Salzenforst bei Bautzen eine hochautomatisierte Fabrik für das sandgebundene Aluminiumgießen in Betrieb genommen wurden. Das wäre durchaus eine gute Chance für eine Kooperation und gegebenenfalls für eine spätere Übernahme vom Unternehmen Automatisierter Sandguss gewesen. Allerdings erwies sich dieser Gedanke als nicht realisierbar. Obwohl sich das Unternehmen SFM GmbH in einer nicht ganz unproblematischen wirtschaftlichen Situation befand, wäre gerade mit der rationellen Herstellung von Gießmodell-komponenten eine gute Voraussetzung gegeben zur stabilen Weiterentwicklung gegeben. Allerdings kann man im Nachhinein konstatieren, dass es für den „Unternehmer" Weigl offenbar zum Geschäftsmodell gehörte in Betracht kommende Partnerunternehmen in die Insolvenz zu treiben. Das ist offenbar auch mit anderen Unternehmen (Gießerei Finsterwalde, Spanende Fertigung Zittau und Glauchau u.a.) praktiziert worden. Wie in dem Zeitungsbeitrag klar dargestellt, wurden im Gesamtumfang 50 Millionen Fördermittel und Gläubigerschulden im wahrsten Sinne des Wortes in den Sand gesetzt. Leider wurden diese Fakten erst zu einem späteren Zeitpunkt bekannt, sodass die gegebene Situation einer privaten Insolvenz neben weiteren Einflussfaktoren gemeistert werden musste.

Als eine allgemeingültige Schlussfolgerung zu diesem negativen Sachverhalt, muss man von folgender realen Einschätzung ausgehen: Wie in der gesamten Automatisierungstechnik kann auch für eine hochautomatisierte Gießerei nicht davon ausgegangen werden, dass eine schlüsselfertige Lösung auf der grünen Wiese aufgebaut werden kann. Das ist bedauerlicherweise mit der kompletten Anlagentechnik genannt DISAMATIK vom dänischen Unternehmen DISA eingetreten. Wie am Beispiel GRAFIS System auBerordentlich erfolgreich nachgewiesen, erfordern komplexe Automatisierungslösungen einen parallelen Know-How Aufbau von qualifizierten Mitarbeitern bis zu promovierten Hochschulabsolventen. Die geometrisch und technologisch anspruchsvollen Problemstellungen können nur mit entsprechend qualifizierten Personal realisiert werden.

\section{Neue Festnahme im Krimi um Sachsen-Investor Weigl}
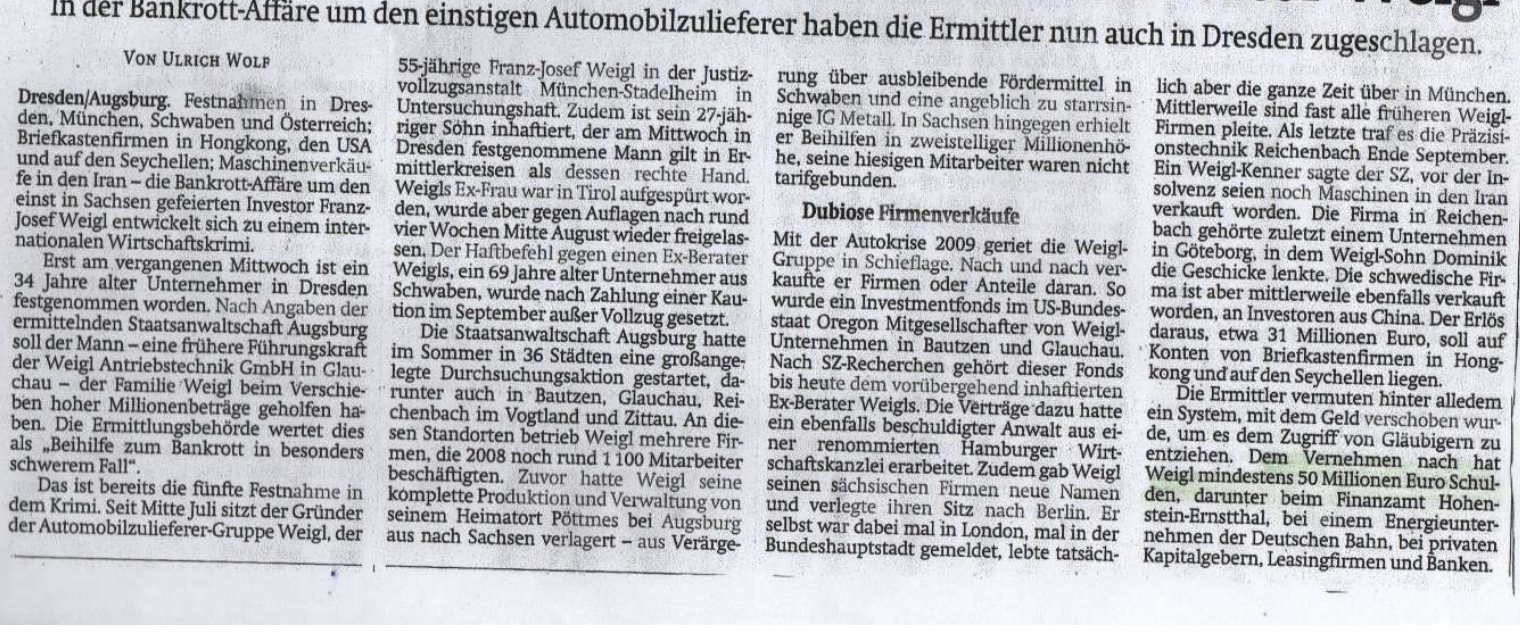

Abbildung 58: Dokument eines Investors mit unkorrektem Geschäftsmodell

Das heißt in diesen konkreten Fall der DISAMATIK Anlagentechnik das für jedes gießtechnisch herzustellende Teil, Simulationen für die unterschiedlichen Prozessschritte dringend notwendig sind. In dem mir 
vorliegenden umfangreichen Rechtsstreit zwischen dem Unternehmen Weigl und der dänischen Firma DISA sind diese Fehler die mit einem erheblichen Kostenaufwand für die sächsische Staatsregierung und einige Gläubiger verbunden waren, leider nicht beachtet wurden. Darüber hinaus erscheint es nach meiner Auffassung weiterhin problematisch, wenn mit den Entwicklungs-trends zu höheren Automatisierungsgraden (Industrie 4.0) derartige elementare Qualifikationsanforderungen nicht beachtet werden. Andererseits sollten auch derartige negative Beispiele von großen Automatisierungs-vorhaben in geeigneter Form ausgewertet werden. Schlussfolgerung: Für fortgeschrittene Produktionstechnik qualifizierte Ingenieure sollte automatisierter Sandguss Herstellung zum Grundwissen gehören. 


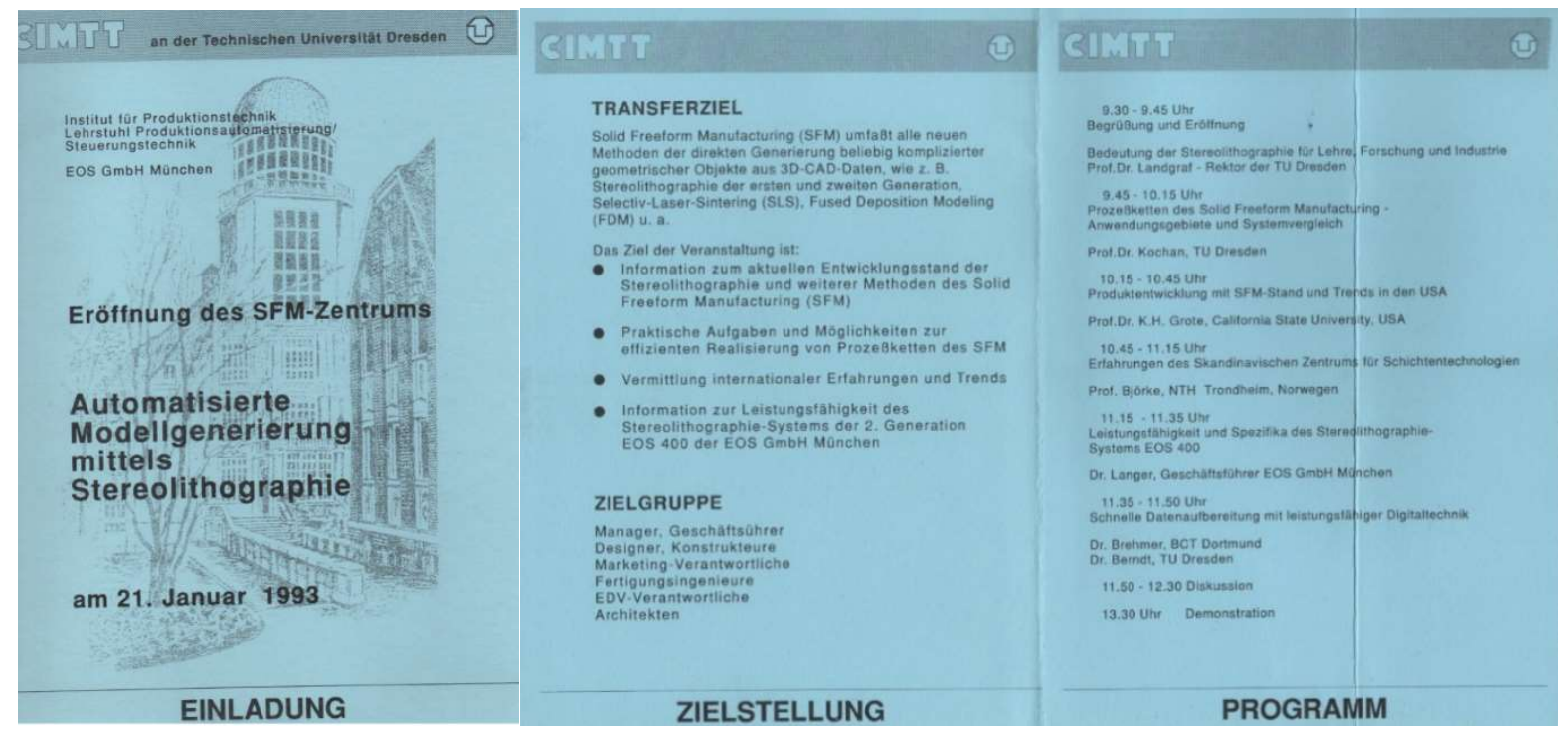

Abbildung 60: Inbetriebnahme Stereolithographie-Anlage

Die Investition einer Stereolithographie-Anlage an der TUD kann als ein wichtiger Baustein für die Erhöhung des produktionstechnischen Niveaus in Lehre und Forschung an der TUD eingeordnet werden. Das wurde unmittelbar öffentlichkeitswirksam mit einer Informationsveranstaltung zur Vorstellung der neuartigen „Rapid Prototyping Anlagetechnik". In dem fachlichen Programm wurden die neuen Möglichkeiten mit wertvollen internationalen Erfahrungen aus den USA durch Prof. Grote ergänzend eingebracht. Die damit verbundenen Zielstellungen sind von dem für Wissenschaft zuständigen Journalisten Herr Stephan Schön in seinem Beitrag in der Sächsischen Zeitung klar dargestellt.

Mit der Investition der ersten leistungsfähigen Stereolithografieanlage in den neuen Bundesländern waren beste Voraussetzungen geschaffen um die begonnenen Aktivitäten im neu gegründeten CAD/CAM Labor zu komplettieren. Das CAD/CAM Labor war gleichzeitig ein Bestandteil des in den 80iger Jahren aufgebauten Automati-

\section{HOCHSCHULE}

\section{TU Dresden erhält weltweit neueste Modellbau-Anlage}

Neue Nahtstelle zur Wirtschaft in der Region geschaffen

Seit heute ist die Technische Universi- - und Institute damit auszurüsten, so Prof tat Dresden um eine Attraktion reicher. Kochan. Die Amerikaner allen voran. In Nach Aachen, Stuttgart und Bremen er- Deutschland fand dieses Systern ab 1991 halt sie als vierte Universität in Deutsch- Verwendung. "Doch in Dresden steh land eine computergesteuerte "Modell- nun das derzeit weltweit modernste Ge bau-Anlage". Heute nahm sie ihren răt." Dies würden ausgiebige Analysen Betrieb für Lehre Forschung und als rand Dies wurden ausgiebige Analysen Dienstleister für die Region auf. bestätigen, meint Prof. Detlef Kochan den Begriff „Stereolithographie sich ein Systereolithographie verbirgt Preis dieser Anschaffung. Die eine Hälfte kompliziertesten, Modelle mit Hohlräu- davon zahlte Sachsen und die andere die kompliziertesten Modelle mit Hohlräu- Deutsche Forschungsgemeinschaft.

men und verschlungenen Kanälen răum- Deutsche Forschungsgemeinschaft.
lich exakt hergestellt werden kōnnen.

Auf einem CAD-Rechner erarbeitete TU ist diese neue Großgerät ganz siche: Konstruktionsdaten werden per Diskette ein nicht zu unterschätzendes Plus. Abe in die Anlage eingespeist. Der systemin auch die Wirtschaftsregion werde davon in die Anlage eingespeist. Der systemin- profitieren, meint Prof. Kochan. Die serstrahl, der auf ein mit Harz gefülltes schnelle Uberleitung von neuen Ideen Becken gerichtet ist. Dort, wo der Strahl und Design in die Produktion werde imauf die Flüssigkeit trifft, verwandelt er mer stärker zu einem entscheidenden diese in einen festen Körper. Schicht für Wettbewerbsfaktor. Deshalb stehe diese Schicht, jeweils 0,1 Millimeter, setzt der Anlage auch den Firmen der Region zur Laser das gewūnschte Modell zusammen. Verfügung.

Es könnte dann beispielsweise als Guß- Die ersten kleinen und mittleren Unvorlage für Metallteile dienen. Was mit ternehmen Sachsens signalisierten beherkommlicher Technik Wochen dauerte reits ihr Interesse, und rund 50 Firmen oder gar nicht zu modellieren war, ist hatten sich für das heutige Eröffnungs: nun in wenigen Stunden möglich", symposium angesagt.

schwarmt der TU-Professor Detlef Ko. Prof. Kochan jedenfalls rechnet mit chan von seiner neuen Anlage. In erster etwa 25 bis 30 festen Partnern aus der InLinse werden daran die Studenten der dustrie. Für sie wäre die Uni dann ein Universität ausgebildet, um mit dem "Dienstleistungsunternehmen“ im Highneuesten Stand der Technik auch wirk. Tech-Bereich. Denn immerhin ein Dritlich praktisch umgehen zu können. Aber tel der Kapazitāt dieser Anlage wolle auch für grundlegende Forschungen sei man für die Industrie reservieren. Und diese Anlage gedacht. Denn das auch auswärtige Institute und HochschuVerfahren ist noch relativ jung. Erst seit len kommen nicht zu kurz weg, sie erhalAbbildung 59: Inbetriebnahme der ersten Stereolitografiel-Anlage in den $N B L$ 
sierungslabors an der Sektion Fertigungstechnik und Werkzeugmaschinen, kurz AUTFERT genannt. Der Begriff AUFFERT stand für automatisierte Fertigung an der ehemaligen Sektion 14 der TU Dresden und ist außer der verfügbaren NC Technik durch eine Roboterzelle und einem automatisierten Komplex für die Blechbearbeitung gekennzeichnet. Mit der Stereolithographie-Anlage waren dann die Voraussetzungen gegeben geschlossene Prozessketten vom konstruktiven Entwurf (Prototypen) zu unterschiedlichen technologischen Prozessen herzustellen.

\section{Beginn der aktiven Zusammenarbeit mit der NC-G für Neue Technologien 1992 - 1997}

\subsection{Anwenderkonferenzserie Rapid Technologien}

Schon vor meiner Sabbatical Zeit in den USA hatten sich Kontakte zu dem rührigen Geschäftsführer der deutschen NCG entwickelt. In Übereinstimmung mit den gesamten Titel für "Neue Technologien" war der agile Herr Pflug natürlich an den neuen Rapid Technologien (mein Buchtitel SFM) interessiert. Im Ergebnis des aktuellen Informationsaustausches von Norwegen und den USA war der Vorschlag einer ersten Informationsveranstaltung zu diesem Thema in Deutschland (Ulm) entstanden.

Von Herr Pflug wurde dafür der Begriff "Anhörung" (Hearing) vorgeschlagen.

Die erste Veranstaltung im Herbst 1992 in Ulm fand trotz kurzer Vorbereitung schon ein beachtliches Interesse. Deshalb war eine fachlich begründete Fortsetzung im Rahmen einer jährlichen Wiederholung zum gleichen inhaltlichen Anliegen eine logische Konsequenz mit der Fortsetzung im Jahr 1993 in Form eines internationalen Anwender-Kongresses. 

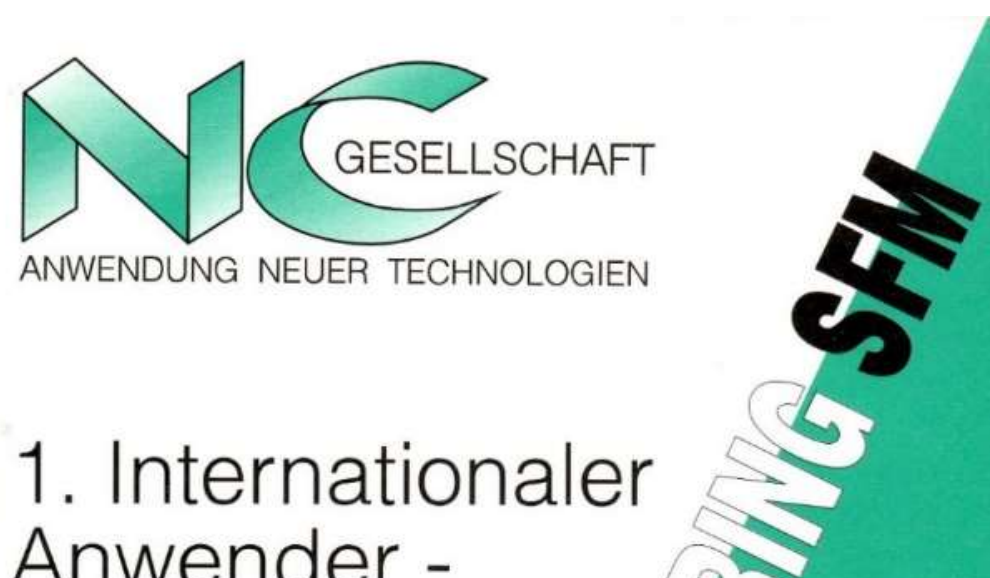

1. Internationaler Anwender Kongreß

\section{TU Dresden 28. - 30.10 .93}

Schirmherr:

Staatsminister

Prof. Dr. H. J. Meyer
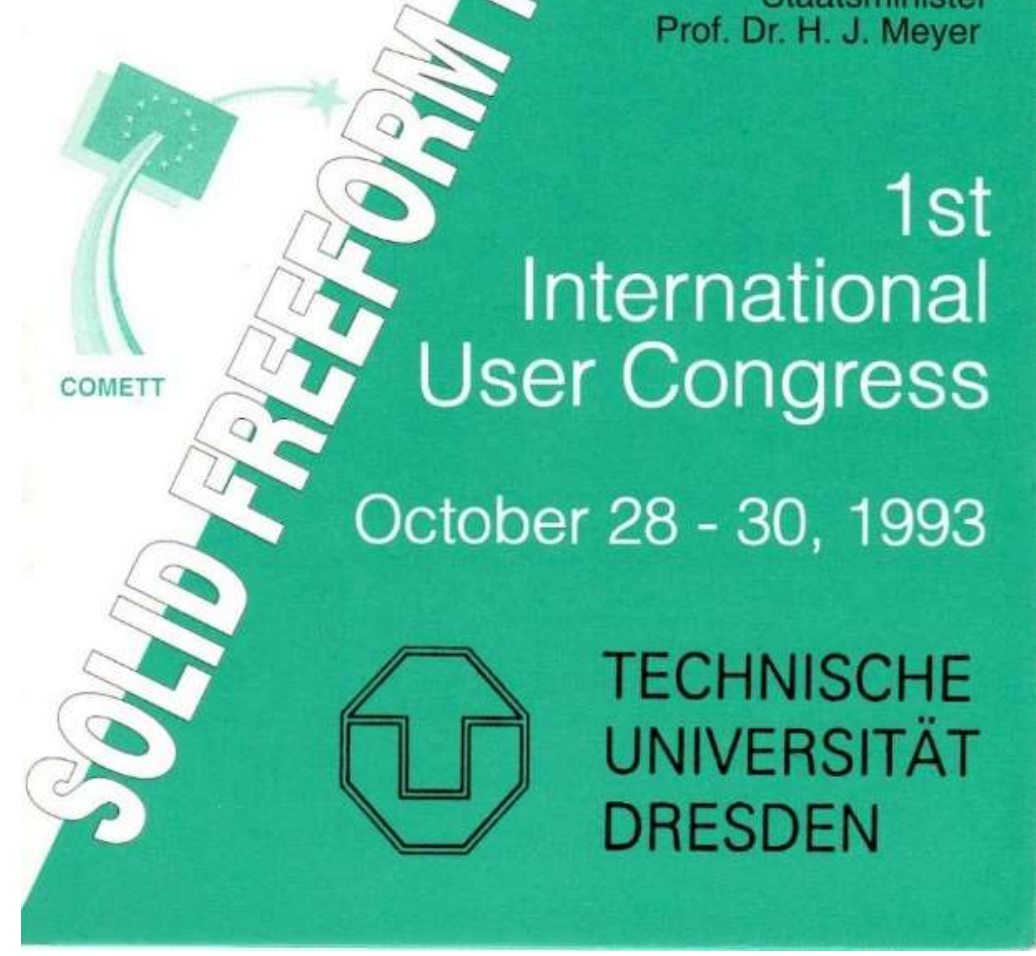

Abbildung 61: Einladung zum ersten internationalen Anwenderkongress Rapid Technologien 


\section{Fertigungs-Elite in Dresden}

Dresden (ADN). Die Fertigungstechniker der Welt wollen mit ihrem ersten Anwender-Kongreß des Solid Freeform $\mathrm{Ma}$ nufactoring (SFM) vom 28. bis 30. Oktober 1993 in Dresden einen Meilenstein des Industrie-Zeitalters setzen. Wie die Technische Universität Dresden und die NC-Gesellschaft für Anwendung neuer Technologien als Kongreßveranstalter informierten, haben bislang Referenten aus den USA, Singapur, Japan, Norwegen, Belgien, Österreich und Deutschland ihre Teilnahme zugesagt. Die sich hinter SFM verbergenden neuen Verfahren sind Expertenaussagen zufolge in der Lage, CAD/CAM-Daten direkt in "handfeste" Modelle umzusetzen. dem Entwickler oder In. Damit werde licht, sofort zu erkennen, rungen in Form, Funktion werbessegungstechnik nötig sind.

Abbildung 62: Würdigung durch den Wissenschafts-Journalisten der SZ

Nach dem erfolgreichen Auftakt der ersten Anwenderkonferenz zum Thema Rapid Technologien (jetzt Additive Manufacturing bzw. „3D-Drucken“) folgten jährliche Wiederholungen.

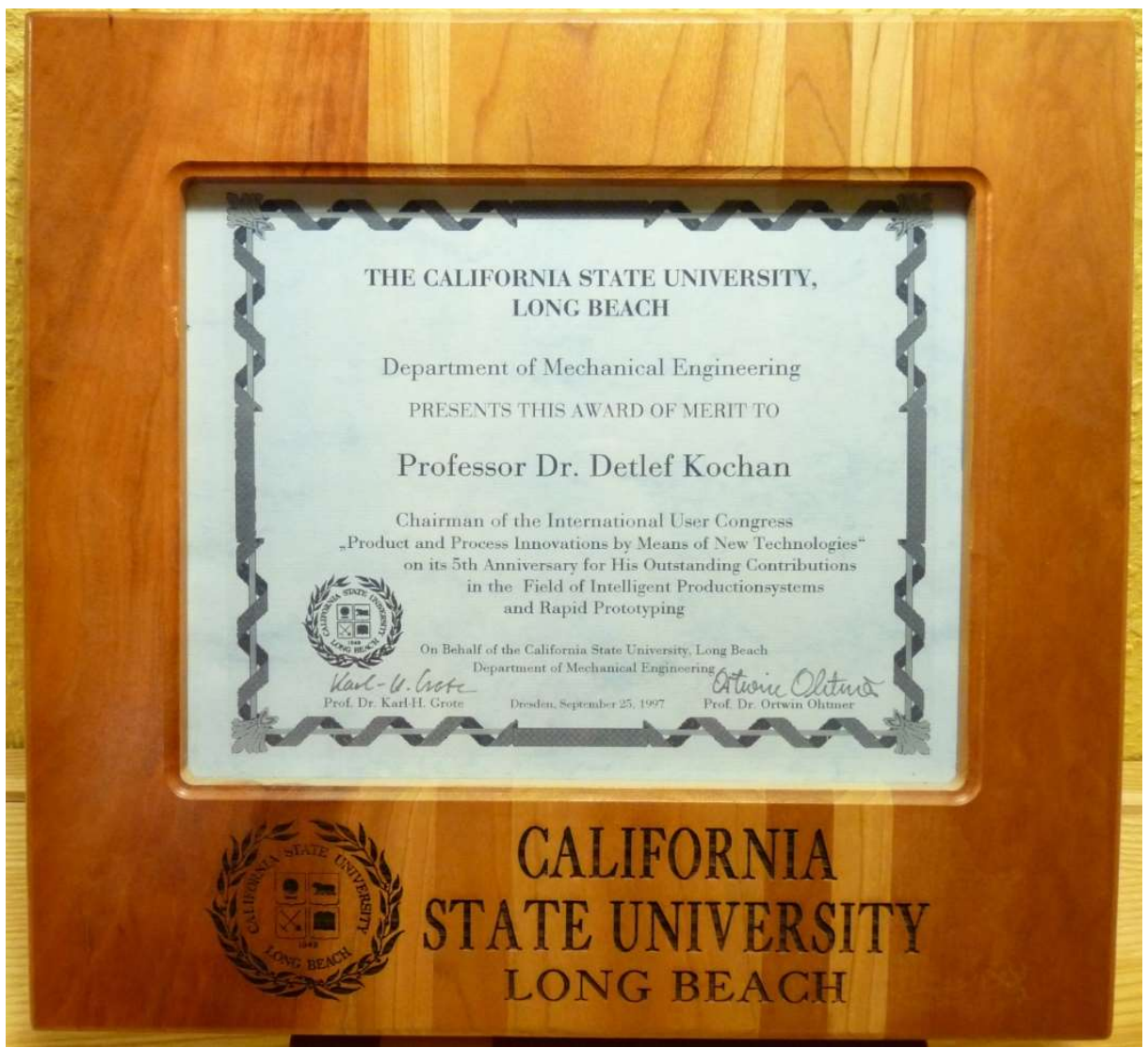

Abbildung 63:Wertschätzung der Anwenderkonferenzserie durch USA-Kollegen 
Die 5. Fachkonferenz im Jahr 1997 - wieder in Dresden - führte zu einem speziellen persönlichen Auszeichnung durch die in den USA lebenden deutschen CAD/CAM Pioniere Prof Otmer und Prof Grote (s. Abbildung 62).

Auf Anregung vom deutschen Pionier der Hochgeschwindigkeits-Bearbeitung (HSC/HPC Herr Prof H.Schulz von der TU Darmstadt fanden ab 1998 gemeinsame 3 D Erfahrungsforen zu Formen- und Werkzeugbau (Darmstadt, Dortmund, Dresden) statt.

\subsection{Festveranstaltung 25 Jahre EXAPT 1992}

Die traditionell kollegiale Zusammenarbeit mit den Pionieren der NC Programmierung und die beidseitige fachliche Anerkennung führte zur ehrenvollen Einladung zum 25-jährigen Bestehen des EXAPT Vereins. Obwohl ich in dieser Zeit mein Sabbatical Jahr in Trondheim verbrachte, war die ehrenvolle Teilnahme mit einem Vortrag eine angenehme Verpflichtung. Gleichzeitig kann in diesem Zusammenhang herausgestellt werden, dass mit dem Aufbau einer EXAPT AuBenstelle in Dresden ein wichtiger Schritt zur weiteren Verbreitung des EXAPT Systems und Ausbau der Kooperation wahrgenommen wurde (siehe Teilbericht Prof. A. Richter).

\section{5 Öffentlichkeitswirksame}

\section{Jahre EXAPT}

\section{Einladung}

Feierstunde

am 10. Juni 1992, EUROGRESS, 5100 Aachen uhr Forschungsprojekt "Technologiedatenbank" unterstūtzt EXAPT
Beginn: 11.00 uhr Einführung in den neuen Bundeslāndern Herr Prof. Dr. Kochan, TU Dresden

Dr. Jürgen Linden
Oberbürgerneister der Stadt Aachent Prot. Dr. Dr. Th.c. mult. Günter Spur

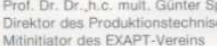
Mitinitiator des EXAPT-Ver Dr. Mantred D. Roh Vorstand des EXAPT-Vereins

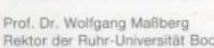
Poiktor der Auntr-Universitat Bochum der Forschungsvereinioun

Abbildung 64:Dokument der langjährigen fachlichen Zusammenarbeit

\section{Aktivitäten 1993 bis 2005}

Mit den realisierten Investitionen .insbesondere der ersten Stereolithografieanlage in den neuen Bundesländern waren gute Voraussetzungen gegeben, mit völlig neuen Prozessketten die traditionelle Fertigungstechnik gravierend zu bereichern. Die dynamische Entwicklung in den vergangenen 30 Jahren auf diesem Gebiet führte dazu, dass der Oberbegriff „Additive Manufacturing“ im korrekten Sprachgebrauch bzw. 3D Drucken im landläufigen Sprachgebrauch genutzt wird. Zur angestrebten Wissensverbreitung wurden schon im Jahre 1993 mit der damaligen Gesellschaft für "NC Technik und neue Technologien" eine gemeinsame Konferenzserie zwischen Ulm und Dresden im Wechsel begonnen. In Verbindung mit den fortgeschrittenen Fertigungsprozessen wurde der Vorschlag von Prof. $\mathrm{H}$. Schulze, TU Darmstadt, dem deutschen und internationalen Pionier der Hochgeschwindigkeitsbearbeitung, eingebracht, eine gemeinsame Konferenzserie zu realisieren. Diese Konferenzserie mit der Bezeichnung „Erfahrungsforum Werkzeug- und Formenbau“ wurde nach entsprechenden Vorbereitungen 1998 begonnen. Der ergänzende Titel „3D Erfahrungsforum“ beinhaltete gleichzeitig die Gründerstädte Darmstadt, Dortmund und Dresden. Zu einem späteren Zeitpunkt schloss sich die TU Berlin diesem gemeinsamen Forum an. Das 3D Erfahrungsforum „Werkzeug- und Formenbau Rapid Technologien“ wurde mit Unterstützung des Dresdner VDI Bezirksverein im Jahr 2004 durchgeführt. Davon ausgehend kann man

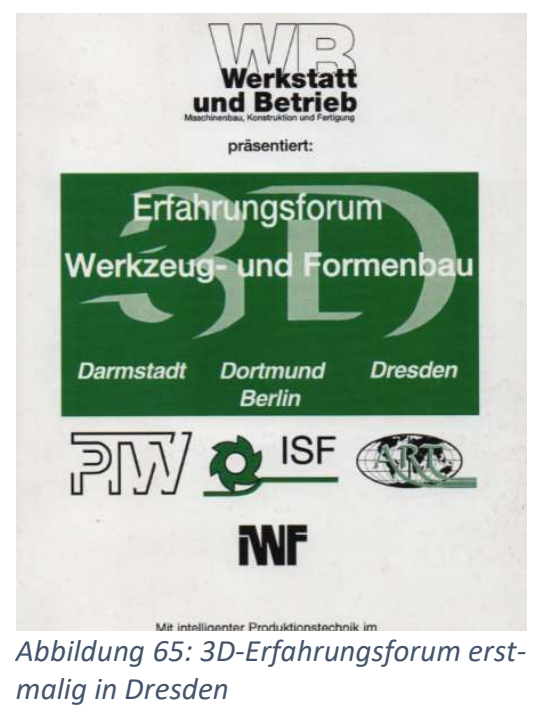


feststellen, dass mit der gesamtdeutschen Beteiligung wertvolle Erkenntnisse zur Realisierung der Hochgeschwindigkeitsbearbeitung und der Nutzung der Rapid Prototyping Technologien, insbesondere auch mit den Anwendungen in Richtung Rapid Tooling wesentliche Erkenntnisse vermittelt wurden.

Mit besonderer Freude erinnere ich mich daran, dass wir den leider inzwischen verstorbenen herausragenden Wissenschaftler der Hochgeschwindigkeitsbearbeitung, Herr Prof $\mathrm{H}$. Schulze als Ehrengast des 3D Erfahrungsforum im Jahre 2004 in Dresden die verdiente Anerkennung für sein Lebenswerk zum Ausdruck bringen konnten. Im Jahre 2005 fand mit der letztmaligen Beteiligung von Dresden das 3D Erfahrungsforum planmäßig in Darmstadt statt.

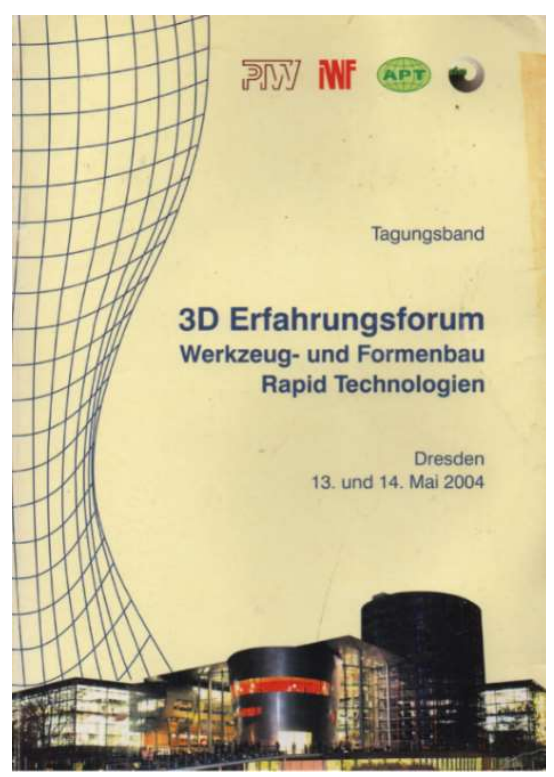

Abbildung 66: Zweites 3D-Erfahrungsforum in Dresden
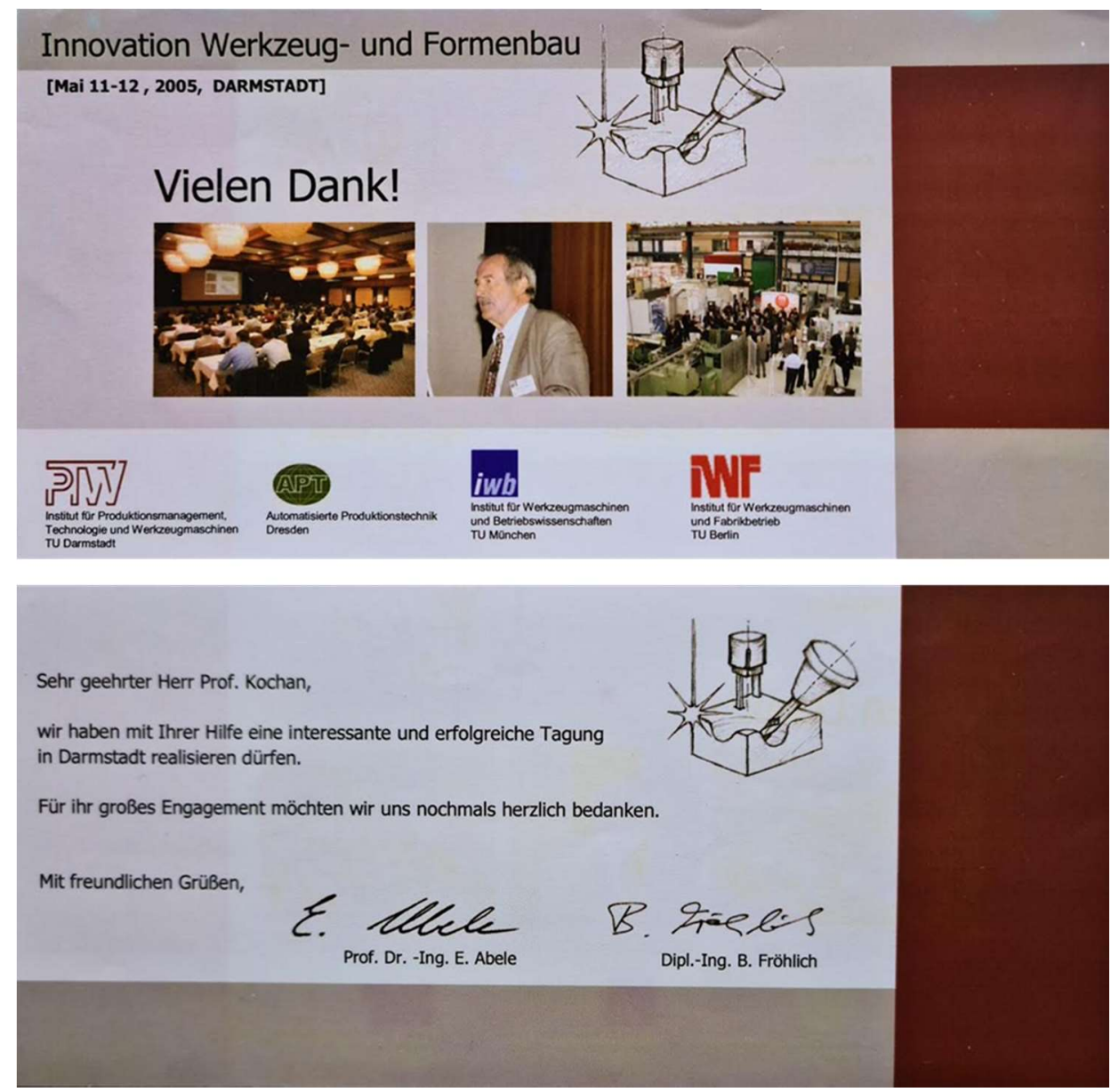

Abbildung 67: Anerkennung durch die Fachkollegen des PTW Darmstadt

Die 1996 auf Initiative vom deutschen Zerspanungsexperten, Prof. H. Schulze gemeinsam mit der NC-Gesellschaft NCG gestartete Konferenzserie 3D-Erfahrungsforum Werkzeug- und Formenbau fand aus verschiedenen Gründen nach 2005 leider keine Fortsetzung. Aus verschiedenen Gründen hatte ich vergeblich versucht, für mein begründetes Ausscheiden aus dem Team der Organisatoren einen Nachfolger aus dem Dresdner Raum zu finden. 


\section{Internationale Ausstrahlung in die Republik Südafrika 1994 bis 2018}

Auf der Grundlage der gesammelten Erfahrungen im Gesamtgebiet Advanced Manufacturing einschließlich Additive Manufacturing (Rapid Prototyping) war der ehemalige bulgarische Doktorand Dr. D. Dimitrov 1994 als interessierter Fachkollege zu einem Arbeitsbesuch in Dresden. Er lebte zu dieser Zeit schon einige Jahre in Kapstadt in Südafrika und hatte sich vergeblich für eine Professur an der Hochschule Schmalkalden beworben. Von den in Dresden vorhandenen neuen Möglichkeiten insbesondere den ersten Erfahrungen

zu Stereolithografie war er spontan begeistert und bat um eventuelle Unterstützung für gemeinsame Aktivitäten mit der Republik Südafrika. Die Empfehlung zur offiziellen Unterstützung dieses Anliegens konnte nur mit staatlicher Hilfe erreicht werden. Das ist Dr. Dimitrov relativ schnell gelungen, sodass schon 1995 erste Vereinbarungen zur wissenschaftlich technischen Zusammenarbeit (RSA/BRD) auf dem Gebiet Rapid Prototyping fixiert waren. Diese Zusammenarbeit begann schon im Jahr 1996 wobei die Aspekte der schnellen Produktentwicklung (Rapid Product Development) mit Rapid Prototyping Anwendungen zunächst im Vordergrund stand. Die jeweils für 2 bis 3 Jahre vereinbarten Forschungsprojekte waren die Voraussetzung, dass bestimmte technologische Problemstellungen mit studentischer Unterstützung (Praktika, Abschlussarbeiten) realisiert werden konnten. Mit Beginn der Projekte im Jahr 1996 wurde von mir mit Industriepartnern (Kunststoffverband und Gießereiverbund der RSA) und in mehreren Fakultäten der Universität Stellenbosch zum Aufbereiten des fachlichen Interesses durch detaillierte Erfahrungsberichte beigetragen wurde. Auch in diesem Zusammenhang standen industrielle Problemstellungen im Vordergrund, wobei die aus Dresden HTW/TUD) einbezogenen Studenten von Dr. Dimitrov an der Universität Stellenbosch in der ersten Woche des Aufenthaltes mit dem 3D CAD System Pro-Engineer vertraut gemacht wurden. Die durchaus ergebnisreiche Vorgehensweise führte wiederum zu solchen Ergebnissen, die im Rahmen der südafrikanischen Organisation Rapid Product Development in jährlichen Fachkonferenzen vorgestellt werden konnten. Diese Aktivitäten wurden im Jahre 2003 mit dem Titel „Extraordinary Professor“ der Universität Stellenbosch gewürdigt.

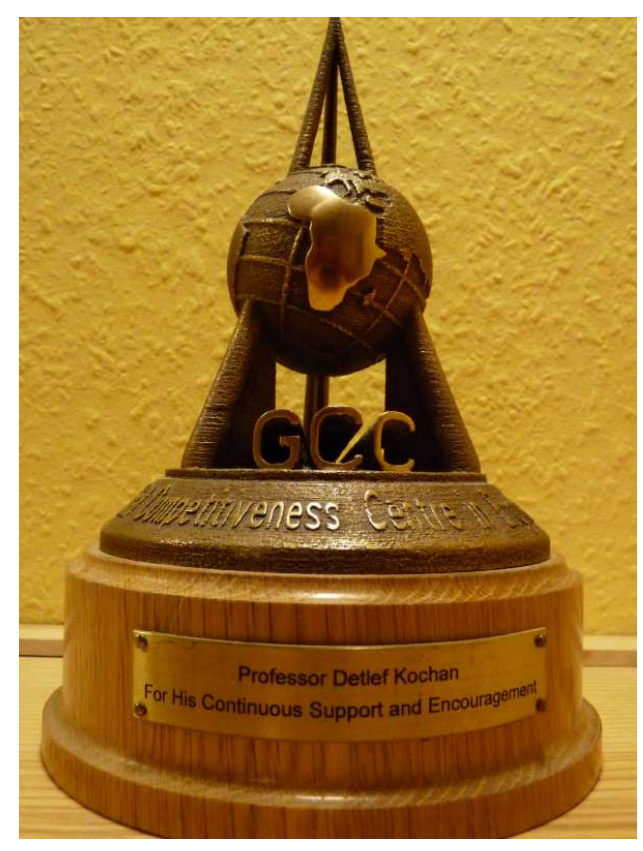

Abbildung 68: Pokal des Center of Cpmpetitiveness 
Die gemeinsamen bzw. koordinierten Forschungsaktivitäten unter Verantwortung Prof. Dimitrov wurden an der Universität Stellenbosch mit zwei Schwerpunkten vorangetrieben.

- RPD - Schnelle Produkt Entwicklung

- Advanced Tooling - Fortgeschrittene Werkzeugtechnik

Für diese Aufgabenfelder wurde mit Unterstützung der Industriepartner und des Staates ein kontinuierlicher Aufbau von Forschungs- und Experimentiertechnik an der Universität Stellenbosch realisiert. Dazu gehörte außer Rapid Prototyping Anlagen ein in 5Achsen gesteuertes Fräsbearbeitungszentrum des Unternehmens Hermle (Deutschland), später ein Bearbeitungszentrum von Maho (Deutschland). Hinzu kommt eine ausreichende Anzahl von leistungsfähigen CAD Arbeitsplätzen, die von jungen Mitarbeitern und Studenten genutzt werden konnten. Diese für Lehre und Forschung wertvollen Ausstattungen waren gleichzeitig eine wichtige Voraussetzung, um im Jahre 2001 von der Universität Stellenbosch ausgehend eine Konferenzserie mit der Bezeichnung „COMA - Competitiv Advanced Manufacturing" zu starten.

Diese Konferenzserie wurde von Beginn an von der Internationalen

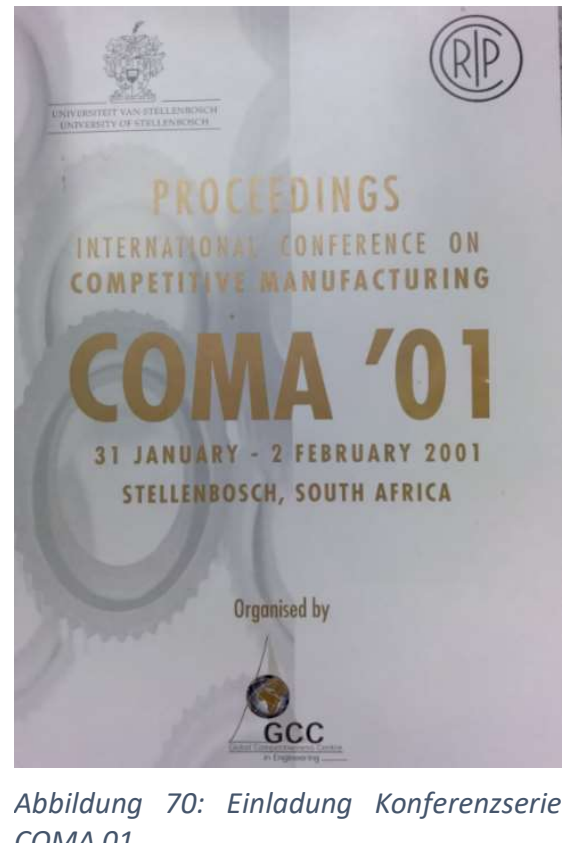
Forschungsgemeinschaft CIRP unterstützt, wobei für die Vortragsauswahl durch die Bewertung von zwei unabhängigen Gutachtern ein solides inhaltliches Niveau gewährleistet werden konnte. Diese im 3-JahresRhythmus durchgeführte internationale Konferenzserie war gleichzeitig durch den reizvollen Konferenzort Stellenbosch in der deutschen Fachwelt sehr beliebt, was u.a. zu jeweils mehr als zehn Vorträgen vom Fraunhofer IWU Chemnitz-Dresden und der RWTH Aachen führte. Auch andere deutsche Universitäten waren mit Vorträgen beteiligt. Die von Prof. Dimitrov persönlich verantwortete Konferenzserie endete 2016 mit dem gleichzeitig fertiggestellten Technologiezentrum an der Universität Stellenbosch.

Mit dem plötzlichen Tod von Prof. Dimitrov im Jahre 2018 waren gleichzeitig die unmittelbaren fachlichen Kontakte beendet.

Zwar überschattet vom Ableben von Prof Dimitrov war das Gesamtergebnis der langjährigen Zusammenarbeit mit dem südafrikanischen wissenschaftlichen Institut sehr überzeugend und beidseitig ertragreich.

Die ursprünglich an der TU Dresden vorgesehene Realisierung eines CIM Technologietransferzentrums war nun

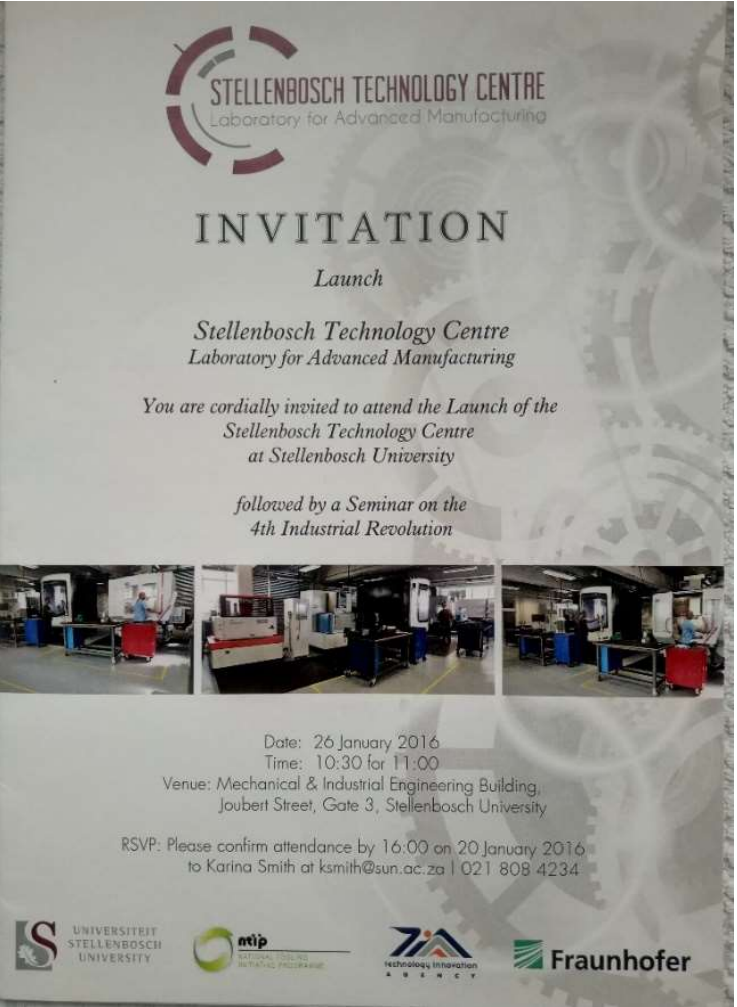

Abbildung 71: Einladung zur Eröffnung des Labors für fortgeschrittene Fertigungstechnik auch in Südafrika verwirklicht.

Mit besonderer Freude erinnere ich mich an die fachlich 
gehaltvollen Gespräche mit den Aachener Kollegen, insbesondere Prof Klocke und damals noch Dr (jetzt Prof) Berg.---Die COMA-Konferenz-Serie hatte darüber hinaus einige Mal einen besonders interessanten Plenar-Referent, Prof Ni aus Ann Arbor Michigan. Der Nachfolger des von mir hoch geschätzten Prof Wu ist gleichzeitig Institutsdirektor in den USA und monatlich für eine Woche Dekan an einer chinesischen Spitzenuniversität in Schanghai-Basis für hochwertige Fachgespräche!

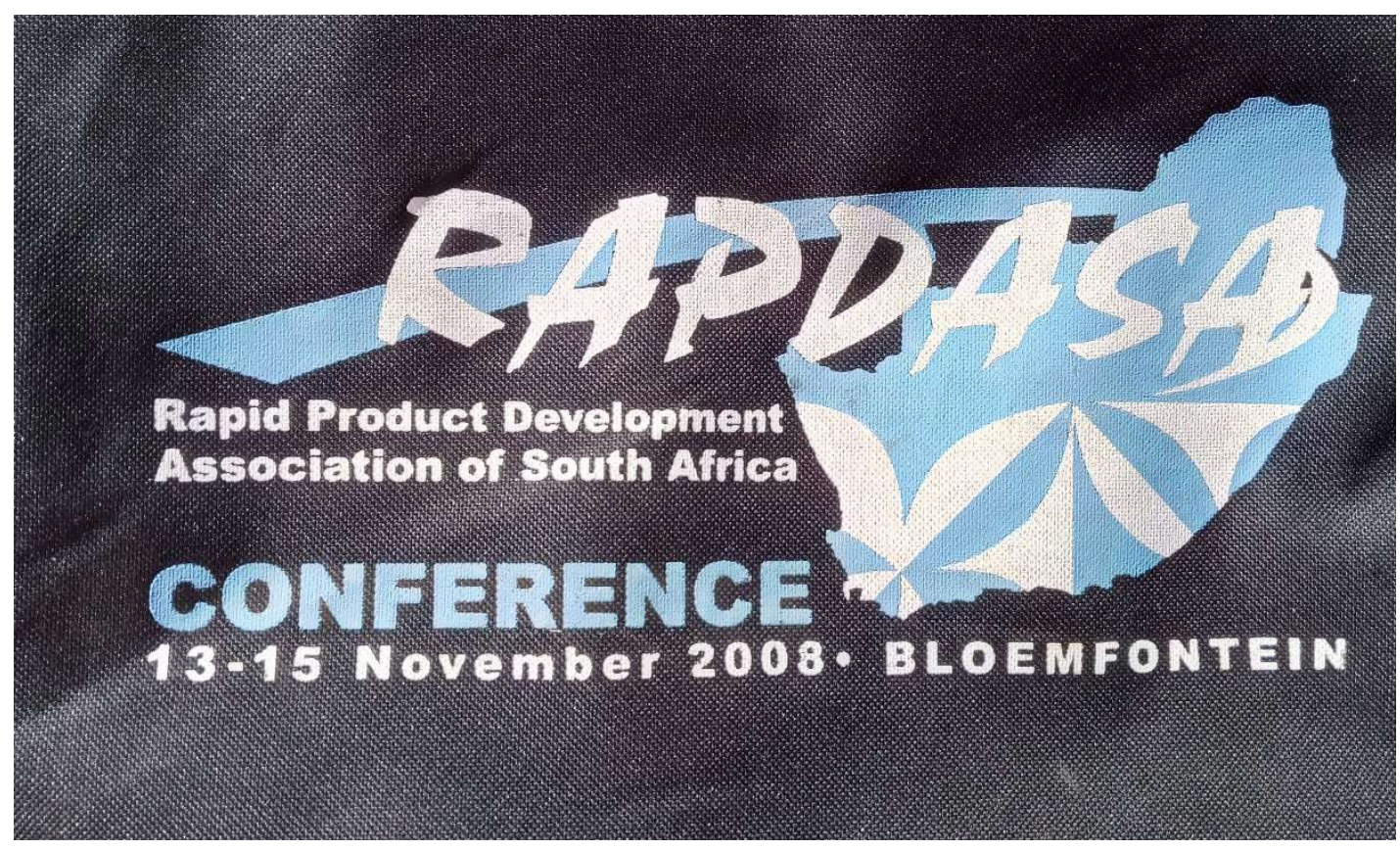

Abbildung 72: Jährliche Veranstaltung der RPD Association of South Africa 
Die von Prof. Dimitrov initiierte und in Stellenbosch gestartete Konferenzserie RAPDASA wurde als jährliche Veranstaltung in den technisch ausgerichteten Universitätsstädten durchgeführt. Insbesondere in Bloemfontein war eine hohe Konzentration unterschiedlicher Rapid-Prototyping-Anlagentechniken konzentriert. Dabei spielten schon zu diesem Zeitpunkt die Anwendungen im medizinischen Bereich eine wichtige Rolle. Für mich bedeutete die mehrfache aktive Teilnahme mit Vorträgen die RAPDASA-Konferenz in Bloemfontein eine besondere Rolle. Nach dem auch in der Republik Südafrika sehr bekannten USA-Pionier Terry Wohlers und ersten Ehrenvorsitzenden der RAPDASA-Organisation wurde ich als zweiter Ehrenvorsitzender dieser Konferenzserie gewählt.

\subsection{Aktivitäten zu einer neuen Technolo- gie für die Kunststoffverarbeitung LOMOLD 1996 bis 2015}

Schon bei meinem zweiten Ar-

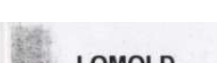

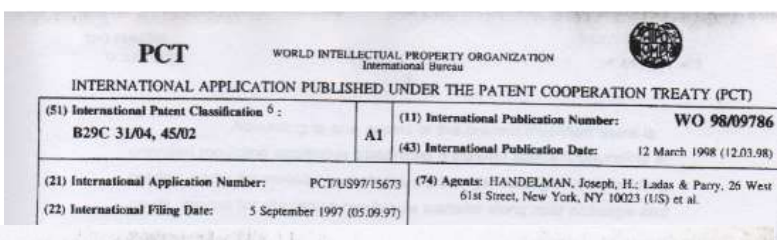

LOMOLD

Vorentwicklungsprojekt beitsaufenthalt im Jahre 1996 in Südafrika kam es zu einer Begegnung mit dem Erfinder eines neuartigen technologischen Verfahrens mit der Bezeichnung "LOMOLD“. Die Bezeichnung wurde abgeleitet aus einer Anzahl von positiven Merkmalen, die mit der Charakteristik Low (niedrig) verbunden waren. Der südafrikanische Erfinder Herr P. du Toit war von Haus aus Informatiker und für das Unternehmen IBM Südafrika tätig gewesen und zum Zeit-

Zu überprüfende Vorteile der Lomold-Anlage gegenüber Spritzgießanlagen:

$\Rightarrow$ geringe mechanische Beanspruchung des Materials

geringerer Druckaufbau im Werkzeug

geringerer Werkzeugverschleiß

geringere Schließkräfte

geringere Aufschmelztemperatur

geringere Kühlzeiten

$\Rightarrow$ geringere Zykluszeiten

$\Rightarrow$ geringerer Energiebedarf

geringeres Bauteilgewicht durch geringere Wandstärken

$\Rightarrow$ geringerer Materialbedarf

$\Rightarrow$ geringerer Preis des Werkzeugwerkstoffes

$\Rightarrow$ geringere Flächenbedarf der Anlage

geringerer Instandhaltungsaufwand

Modularer Aufbau der Anlage

$\Rightarrow$ geringere Kosten der Anlage

$\Rightarrow$.

Das Werneugird

Das Werkzeug wird zur Anlage

C.

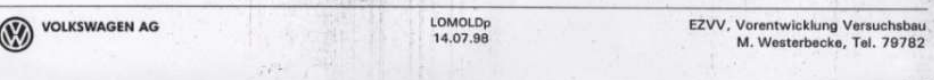

I.

Abbildung 73: Weltpatent LOMOLD

punkt des Treffens Farmer-und weiterhin techno-

logisch aktiv. Herr du Toit hatte von einem südaf-

rikanischen Erfinder das LOMOLD Patent erwor-

ben und war davon überzeugt, dass es beachtliche Fortschritte als Ergänzung für das weitverbreitete Kunststoffspritzgießen gewährleisten kann. Von dieser Überzeugung ausgehend, hat er als Farmer eine relativ große Kuhherde verkauft um ein entsprechendes Weltpatent einzureichen. Davon ausgehend bat mich der Erfinder um Unterstützung für das Gewinnen deutscher Automobilfirmen zur Nutzung des Lomold-Patentes. 
In Realisierung dieses Anliegens wurden im folgenden Jahr Arbeitsbesuche in den deutschen Firmen VW Wolfsburg, BMW Landshut und Daimler Stuttgart durchgeführt. Im Ergebnis dieser Informationsund Werbebesuche gab es eine relativ schnelle und positive Reaktion vom Unternehmen VW Wolfsburg. (s. Bild)

Die im Patent angeführten Merkmale und Vorteile sind in dem Arbeitsdokument vom Unternehmen Volkswagen ausgewiesen. Ein we- Abbildung 74: Von VW anerkannte Vorteile des LOMOLD-Prinzips sentlicher handschriftlich ergänzter Vorteil besteht darin, dass mit dem neuen Verfahren auch langfaserige CFK oder GFK Materialen verarbeitet werden können.

Das Grundprinzip des LOMOLD Patentes besteht darin, dass für das Spritzgießen nicht mit einer üblichen Extruder-Schnecke gearbeitet werden muss, in der üblicherweise Fasermaterial in kleine Teile getrennt wird. Es kommt demnach primär darauf an eine exakt für einen Teil dosierte Menge in einen Druckzylinder auf die notwendige Verarbeitungstemperatur zu erwärmen.

Im nebenstehenden Bild wird die Anwendung des LOMOLD Prinzips in einem 3-fach Werkzeug für unterschiedliche Bauteile veranschaulicht, wie es meines Wissens aber nicht zur Anwendung gelangt ist.

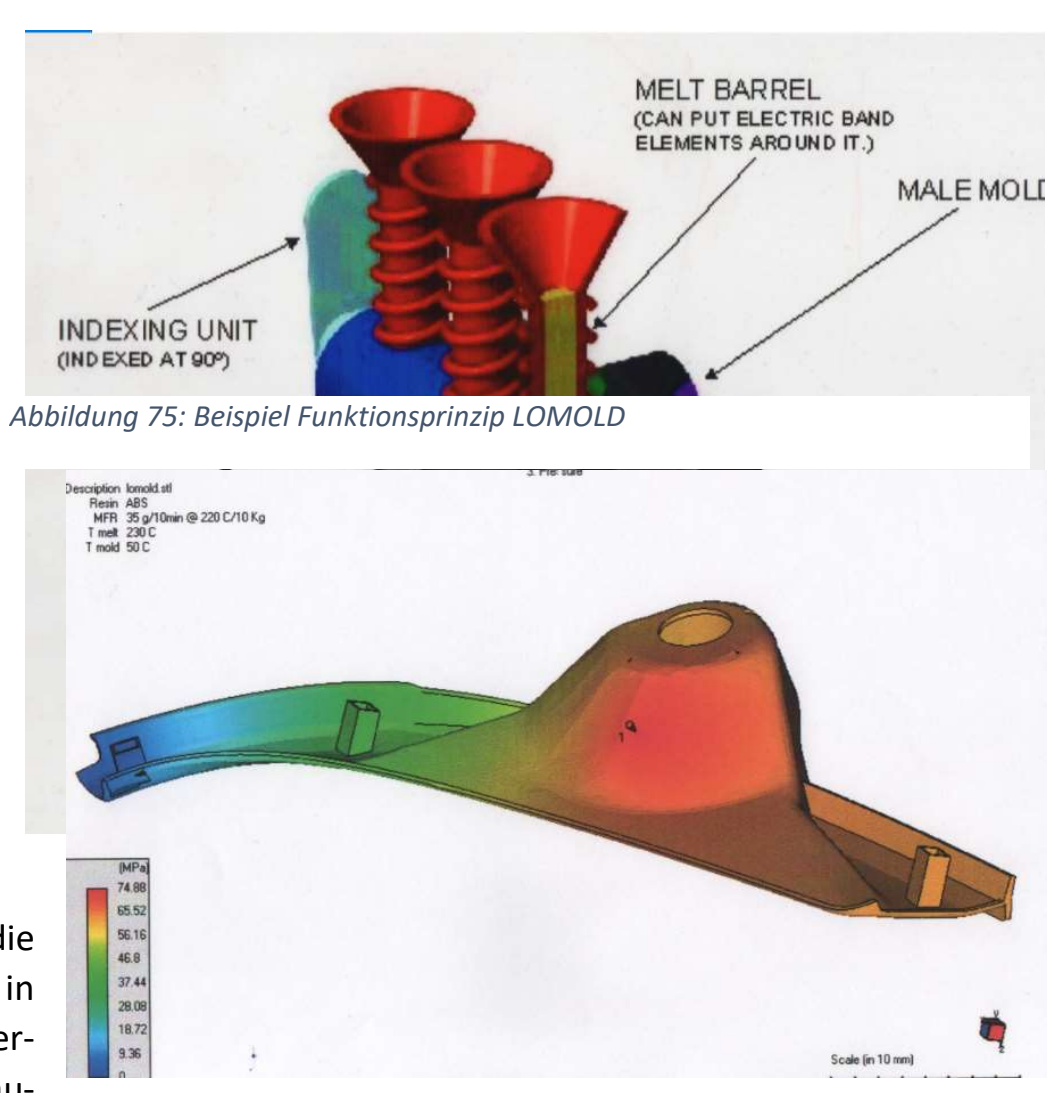

Abbildung 76: Versuchsteil für LOMOLD-Erprobung bei VW

Im Allgemeinen hat man sich auf jeweils eine Teilegeometrie konzentriert. Das ist auch der Fall für ein Versuchsteil wie es im Entwicklungsbereich von Volkswagen als Testobjekt ausgewählt wurde, wofür eine spezielle Konstruktion entwickelt und erprobt werden musste.

Im Bild 76 wird die in 3D CAD vorliegende Geometrie des Versuchsteiles veranschaulicht. Mit den damit vorliegenden Volumendaten konnte von mir ohne Probleme die Auslegung des notwendigen Druckzylinders ermittelt werden. Die über mehrere Wochen notwendige Verarbeitung des Versuchsaufbaus und der technologischen Realisierung wurde von mir betreut und mit den fachlichen Partnern insbesondere Dr. Westerbecke und dem Technikerteam aus dem VW Versuchsbereich realisiert. Das erfreuliche Erfolgsergebnis wird im folgenden Bild 77 gezeigt. 


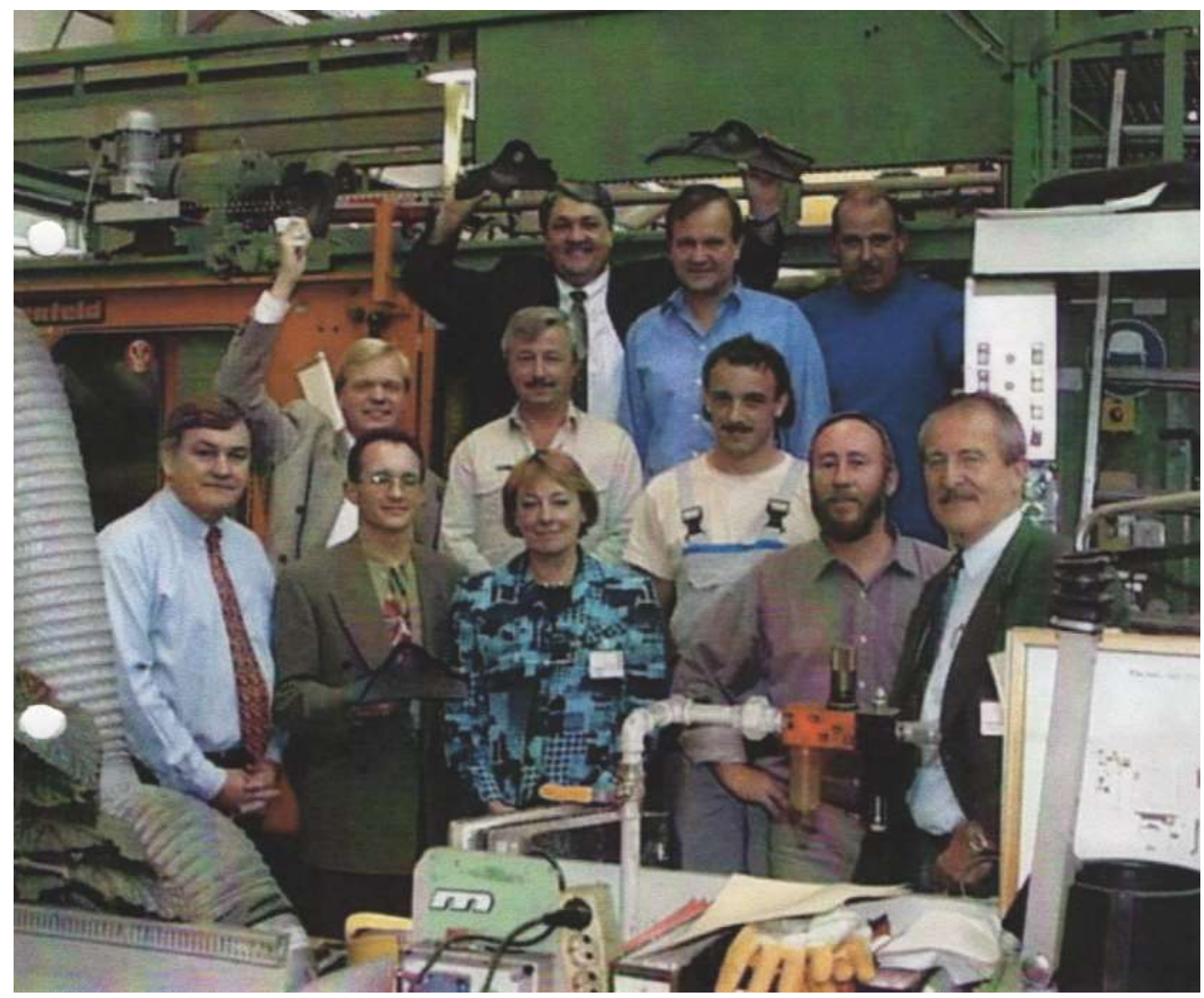

Abbildung 77: Abschlussdemonstration zum ersten LOMOLD Versuchsteil im Unternehmen VW

Für weitere kommerzielle Verhandlungen waren aus Südafrika die im linken Teil vorn gezeigten Personen von Pricewaterhouse mit anwesend (der Herr vorn links und die Frau in der Mitte sowie dazwischen der Kunststoffexperte aus dem südafrikanischen Forschungsinstitut).Dahinter Herr Westerbecke von VW und oben links der zufriedene Erfinder Pieter du Toit.

Dieses wichtige Ergebnis hatte interessante Folgen . Seitens VW erfolgte eine Zusage, dass man die LOMOLD Teile sowohl in einer ersten kompletten Versuchsanlage und auch weitere Produktionsanlagen von einem industriellen Hersteller kaufen würde. Diese Zusage war wiederum der Ausgangspunkt zu einer Beratung mit der sächsischen Wirtschaftsförderung .Die Zielstellung bestand dabei in der Konstruktion und Erprobung einer entsprechenden Anlagentechnik, wobei von den personellen Voraussetzungen davon ausgegangen werden konnte, dass mit dem in die Insolvenz gegangen Unternehmen Kuasi in Freital geeignetes Fachpersonal relativ schnell gewonnen werden konnte. Diese Beratung konnte leider nicht zum Erfolg geführt werden, da die Forderung für die Übernahme der alleinigen Nutzungsrechte mit einem Preis von 0.5 Mio D-Mark als zu hoch angesehen wurde. Wenn man andererseits bedenkt, dass für weniger innovative Maschinenanlagen zum Teil mehrere Millionen ausgegeben werden, erscheint im Nachhinein diese Preishürde für durchaus gerechtfertigt. Der weitere Ablauf kann kurz dargestellt werden. An der Universität Stellenbosch erfolgten weitere Versuche mit einer umgebauten Spritzgieß-Anlage. Von mir wurden dazu 2 studentische Abschlussarbeiten (Master)

an der Universität Stellenbosch mit betreut. Davon ausgehend wurde später die kommerzielle Verwertung durch ein Unternehmen in Taiwan wahrgenommen. Die erfolgreiche technologische Umsetzung wurde vor einigen Jahren auf der jährlichen Kunststoffmesse „JEC“ in Paris mit einem Award anerkannt. 


\section{Abschließende Verpflichtung als Mitglied des IFIP TC 5 und der IFIP Arbeitsgruppe 5.3}
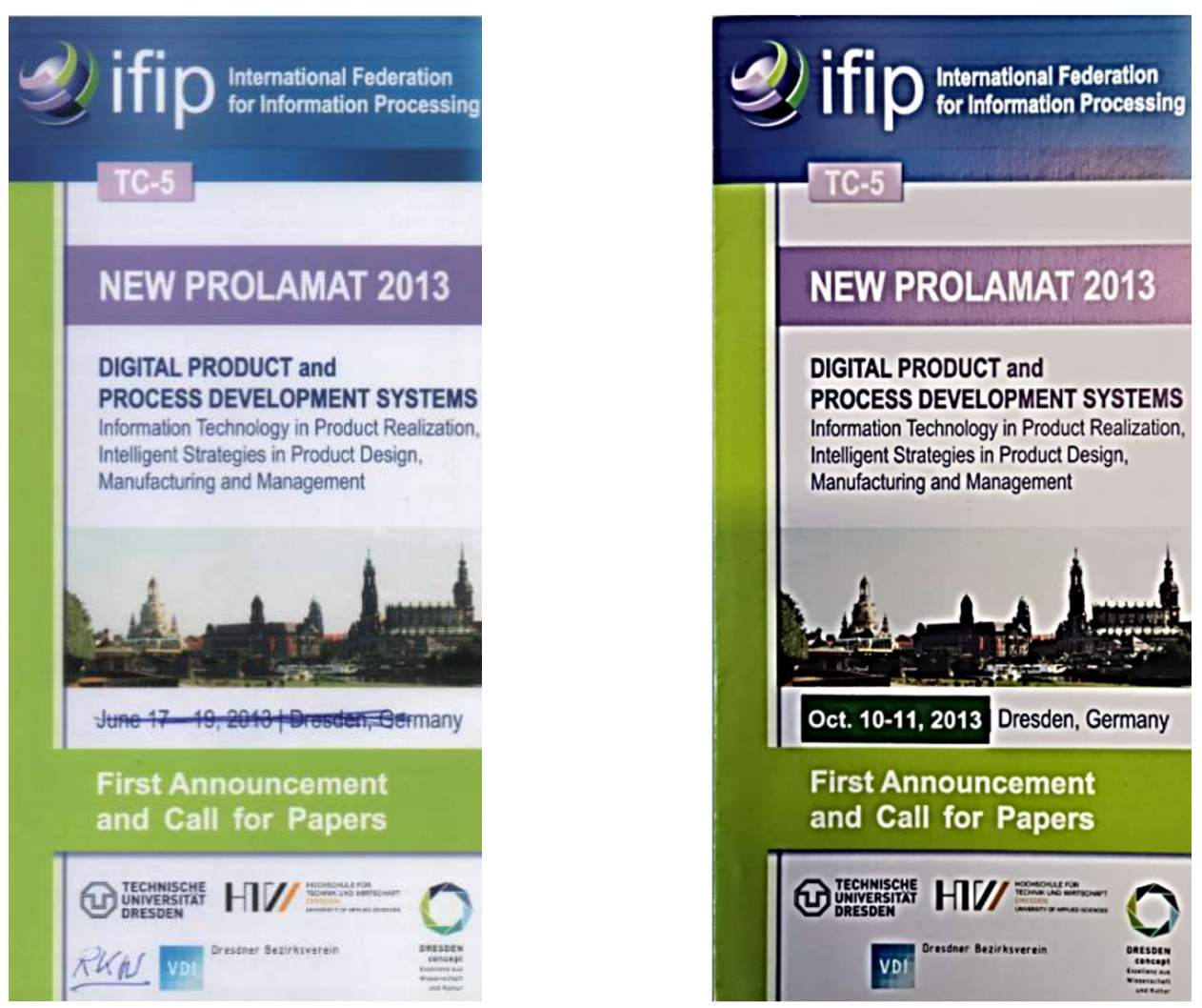

Abbildung 78: Einladung für New Prolamat-Konferenz 2013

Obwohl ich als DDR - Repräsentant der IFIP im Jahre 2012 natürlich nicht mehr in dieser Eigenschaft tätig sein konnte, erreichte mich die überraschende Einladung zu einem Meeting des TC 5 im Sommer 2012 in Wien. Dieses Treffen erschien mir als eine gute Gelegenheit mit den Fachkollegen aus den führenden Industrienationen noch einmal zu einem fachlichen Informationsaustausch zusammen zu kommen. Deshalb nahm ich die Einladung an, ohne an unmittelbare Verpflichtungen gebunden zu sein. Darüber hinaus interessierte mich natürlich der Fortgang der bewährten Konferenzserie PROLAMAT. An den PROLAMAT Konferenzen 1993 in Berlin (Prof. Krause) und 1996 in Trento, Italien hatte ich noch mit eigenen Vorträgen teilgenommen. An einer letzten internationalen PROLAMAT Konferenz 2004 in Shanghai konnte ich leider nicht teilnehmen.Aber ich war natürlich weiterhin neugierig wie es weitergehen sollte, da in der Einladung für das Wiener Treffen ein Tagungsordnungspunkt "New PROLAMAT“ vorgesehen war.

Die im Jahr 2004 in Shanghai durchgeführte letzte PROLAMAT-Konferenz wurde vom Teilnehmer Prof. Kovac kritisch bewertet. In den zuständigen IFIP-Gremien wurde mehrfach ohne Ergebnis über eine Fortsetzung beraten. Mit Bezug zu der herausragenden PROLAMAT-Konferenz 1988 in Dresden ergab sich an mich die Anfrage zur eventuellen Übernahme der Verantwortung für die New PROLAMAT Konferenz.

Diese Anfrage bedeutete für mich eine gewisse moralische Verpflichtung. Hatte ich doch über viele Jahre mit der internationalen Fachwelt eine erfolgreiche Zusammenarbeit gepflegt. Darüber hinaus betrachtete ich diese Anfrage als eine Möglichkeit, in den inzwischen nicht mehr auf fortgeschrittenen 
produktionstechnischen Niveau befindlichen neuen Bundesländern, einen Beitrag zum Aufholen zu leisten.

In der gesamten Beratung entwickelte sich die Diskussion in eine Richtung, wonach die erfolgreichste PROLAMAT Konferenz (1988 in Dresden) mit aktuellen Inhalten möglichst noch einmal wiederholt werden sollte. Damit stand für mich noch einmal eine schwierige Entscheidung an. Einerseits fühlte ich mich der internationalen Organisation weiterhin verpflichtet, war sie doch in Zeiten des Eisernen Vorhanges für mich die Basis mit den Fachkollegen aus den Industrienationen von Japan bis in die USA aktiven fachlichen Erfahrungsaustausch zu pflegen. Andererseits war ich mir natürlich bewusst, ohne eine geeignete fachliche Unterstützung und ein entsprechendes Hinterland, konnte ich in dem Rahmen der Beratung des TC 5 keine Zusage abgeben. Deshalb habe ich den TC 5 Vorsitzenden Herr E. Neuhold (Österreich) um eine Bedenkzeit zur Prüfung des Vorschlages.

Mit dieser Motivation führte ich einige Gespräche zur Klärung der notwendigen fachlichen und organisatorischen Unterstützung durch die TU Dresden, das Wirtschaftsministerium und kompetenten Organisationen. Die Unterstützung für das Anliegen war sehr differenziert. Während einige Organisationen, z:B. NC-Gesellschaft Ulm und das RKW Sachsen die volle Unterstützung anboten, hielt sich die TUD und das Wirtschaftsministerium sehr zurück. Das vermittelte mir den Eindruck des nicht Verstehens der aktuellen Lage auf dem wichtigen Gebiet der Produktionstechnik. Im Gegensatz dazu konnten für die Durchführung der Konferenz mit der fachlichen Expertise und Empfehlungen von Prof. Spur ausgewiesene Fachkollegen gewonnen werden.

In einem diesbezüglichen kurzen Gespräch mit dem damaligen Dekan der Fakultät Maschinenwesen der TU Dresden und gleichzeitig Direktor des Fraunhofer Institutes IWS (Prof. Bayer) in Dresden, wurde eine Zusage zur Unterstützung gegeben. Wie im Jahre 1988 von einem fachlichen Rückstand im gesamten Fachgebiet der fortgeschrittenen Produktionstechnik (Advanced Manufacturing) ausgegangen werden musste, hat sich an dieser Situation Produktivitätsvergleich Alte und Neue Bundesländer" leider nicht Gravierendes geändert. Im Jahr 2012 wurde dieser gegebene Produktivitätsrückstand in den statistischen Unterlagen mit ca. 25 bis $30 \%$ ausgewiesen. Dieser Fakt war demzufolge eine weitere Triebkraft zu Verbesserungen in der industriellen Breite beizutragen. Ausgehend von diesen genannten kritischen Fakten war ich dann gleichzeitig davon ausgegangen, dass von Seiten des Wirtschaftsministeriums mit einer gewissen Unterstützung des gesamten Anliegens gerechnet werden konnte. Auf dieser Basis konnte mit den inhaltlichen Vorbereitungen und der Bildung eines nationalen Programmkomitees begonnen werden. 
Unter Beachtung der für die IFIP Veranstaltung generell gegebenen Verpflichtungen die Vorträge in englischer Sprache zu halten, wurde unter Beachtung der für kleine und mittlere Betriebe gegeben sprachlichen Voraussetzungen mit dem IFIP TC 5 vereinbart, dass die Vorträge auch in deutscher Sprache gehalten werden können. In guter Zusammenarbeit mit den aktiven Fachkollegen aus dem Programmkommitee wurde zunächst eine erste Ankündigung „First Announcement and Call for Papers" erarbeitet.

Der gewählte Titel der digitalen Produkt- und Prozessentwicklungssysteme kann auch aus heutiger Sicht nach wie vor als hochaktuell eingeschätzt werden. Nach den auf der Grundlage des dargestellten Call for Paper die intensive inhaltliche Vorbereitung vorangebracht wurde, zeigte sich, dass die erhoffte aktive Unterstützung von Seiten der TU Dresden nicht ganz den Erwartungen entsprach. Unabhängig davon wurde auch mit dem Vertreter der deutschen NC Gesellschaft, Herr Dipl.-Ing. Pflug, eine Zusammenarbeit angeregt. Von dieser industriell breiten Fachorganisation war eine Unterstützung zugesagt, wenn auch vom sächsischen Wirtschaftsministerium eine finan-

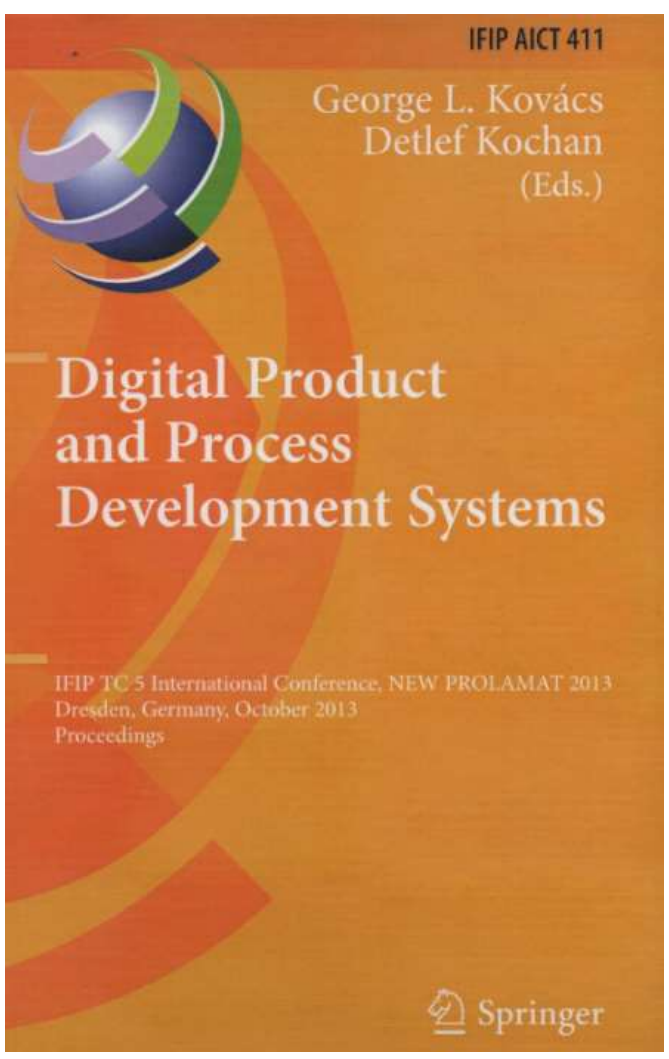

Abbildung 79: Titelseite IFIP-Konferenzschrift New PROLAMAT zielle Unterstützung erfolgen könnte. Auch dieser Lösungsansatz konnte leider nicht mit Leben erfüllt werden. Erfreulicherweise wurde für die organisatorische Unterstützung das RKW Sachsen (Herr Vogel) gewonnen und damit konnten die weiteren Vorbereitungen gesichert werden. Im Zusammenhang mit einem Arbeitsbesuch bei Herr Prof. Spur in Berlin konnte ich wertvolle Hinweise zu aktuellen und fachlichen Themen bezogenen geeigneten Forschungsaktivitäten erhalten. Damit waren wesentliche Eckpunkte auch gesamtdeutscher inhaltlicher Ausrichtung gegeben und für die weitere terminliche Vorbereitung zu Grunde gelegt. Die leider unzureichende Unterstützung von Seiten der TU Dresden zeigt sich insbesondere darin, dass der vorgesehene Termin Juli 2013 kurzfristig auf einen späteren Zeitpunkt geändert werden musste (siehe Korrektur im Flyer ( Call for Paper). Trotz aller aufgetretenen Probleme konnte im Oktober 2013 eine durchaus gehaltvolle Konferenz gesichert werden. Insbesondere die Vorträge von

- Dr. Filos (EU Kommission) - Manufacturing Innovation and Horizon 2020

- Prof. Kimura (Universität Tokio) - Manufacturing Innovation and Horizon 2020

- Dr. Trippner (BMW) - Challenges for Digital Product and Process Design Systems at BMW

- Dr. Katzenbach (Daimler Stuttgart) - JT Format (ISO 14306) and AP 242 (ISO 10303): The Step to the Next Generation Collaborative Product Creation

- K. Müller-Lohmeier (Festo Stuttgart) - Additive Manufacturing as Integral Part of the Digital Solution Process - An industrial Short Note

waren herausragende Beiträge und es wurden darüber hinaus weitere sehr gute Fachvorträge gehalten. Gemeinsam mit der Unterstützer-Organisation RKW konnten wertvolle Schlussfolgerungen für das Wirtschaftsministerium des Freistaates Sachsen abgeleitet werden, die leider nicht wie erwartet in entsprechender Form umgesetzt wurden. 
Eine Nutzung dieser Empfehlungen ist leider in der Innovationsstrategie des Freistaates Sachsen nicht erkennbar. Trotz ergänzender Hinweise durch Standpunkte des VDI Arbeitskreises Dresden sind keine wirkungsvollen Maßnahmen erkennbar.

Unabhängig von diesen realen Fakten habe ich die gegenüber der IFIP Organisation übernommene Selbstverpflichtung ordnungsgemäß erfüllt.

Obwohl die Bemühungen zur Realisierung von Produkt- und Prozess- Innovationen leider in den sächsischen Umfeld nicht die erwartete und notwendige Unterstützung fanden, ist die vom Springer-Verlag im August 2020 übermittelte Information des mit den Verkaufszahlen nachgewiesenen weltweiten Interesses ernsthaft zu beachten. Im Rahmen des VDI-Bezirksverbandes Dresden wurde mehrfach auf die Bedeutung der verstärkten Orientierung auf das Gesamtgebiet der fortgeschrittenen Produktionstechnik (Advanced Manufacturing) hingewiesen.

Deshalb sollte die weltweite Verbreitung der fortgeschrittenen Erkenntnisse als konkrete Beiträge zur Erhöhung des Produktivitätsniveaus in den neuen Bundesländern als ein aktuelles Signal verstanden werden (s. offizielle Nachricht vom Springer-Verlag).

\section{Springer}

$28 / 08 / 2020$

\section{Dear Detlef Kochan,}

Today we are pleased to share your Annual Book Performance Report with you, which summarizes the number of chapter downloads* in the first half of this year, the calendar year 2019 and previous years as applicable.

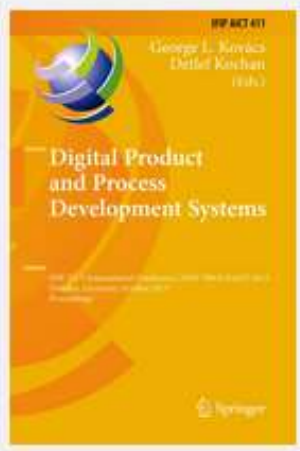

\section{Digital Product and Process Development Systems}

IFIP TC 5 International Conference, NEW PROLAMAT 2013, Dresden, Germany, October 10-11, 2013, Proceedings

$\begin{array}{ll}\text { Year } & \text { Usage } \\ 01 / 2020-06 / 2020 & 8108 \\ 2019 & 17584 \\ 2018 & 23562 \\ 2017 & 13631 \\ 2016 & 16879 \\ 2015 & 16215 \\ 2014 & 20904 \\ 2013 & 6066\end{array}$


Die international weiterhin beachtliche Anerkennung mit dem Nachweis einer Spitzenposition im Ranking der aktuellen Fachliteratur im Gebiet Advanced Manufacturing ist einerseits für das Autorenteam erfreulich. Andererseits bleibt die Frage weiterhin offen, wann im Freistaat Sachsen dieses auch für KMU bedeutende Fachgebiet einen gebührenden Stellenwert erhält.

\begin{abstract}
Schlussfolgerung: Die Erkenntnis dieser nüchternen Fakten und realen Gegebenheiten führte zu der Schlussfolgerung, mit breitenwirksamen Aktivitäten im Arbeitskreis Rapid Innovation die grundsätzlichen Zielstellungen Verbesserung des produktionstechnischen Niveaus in geeigneter Form fortzusetzen.
\end{abstract}

Um die weiterhin gegebene Situation des Produktivitätsrückstandes wurden deshalb seit 2014 Aktivitäten im Namen des VDI Bezirksverbandes begonnen, mit der Bezeichnung Innovationstage Mittelstand.

\title{
18 Wissenschaftliche Aktivitäten von 2006 bis 2020
}

\subsection{Innovative Projekte 2006---2020}

Ausgehend von den vielfältigen Möglichkeiten der Nutzung generativer Verfahren wurden im Rahmen der aktiven Zusammenarbeit mit der Universität Stellenbosch (RSA) mit ausgewählten Studenten konkrete Industrieprojekte realisiert (s. Abschnitt 16)

Dafür wurden insbesondere Studenten der HTW, z.T. auch der TU Dresden gewonnen.

Das war wiederum ein Ausgangspunkt, um mit den fachlich interessierten Professoren der HTW gemeinsame Innovative Industrie-Projekte durchzuführen. Inhaltliche Schwerpunkte waren dabei die sich in Entwicklung befindlichen neuen Möglichkeiten der konturbezogenen Temperierung beim Spritzgießen. Mit den Fortschritten im Gebiet der generativen Verfahren (SLS-Selektiv Laser Sintern u.a.) konnte später auch das extrem anspruchsvolle Druckgießen in die Untersuchungen einbezogen werden.

Wesentliche Forschungsprojekte waren:

Neue Werkzeugkomponenten für intelligente Werkzeugtechnik 1.12. 2006-30.11.2007 mit Prof Otto Homogen beheizbare Formwerkzeuge für Faserverbundwerkstoffe. 1.5.2009--31.10.2011 Prof Dietrich Energieeffizienz, Produktivitäts- und Qualitätssteigerung durch homogenisierte Temperierung beim Druckgießen (EPQ) 01/2011-12/2012 Prof Otto

Prozessaktiv temperierte Druckgießformen zur Verbesserung der Verfahrenseffizienz (PDE) 01/201303/2016 Prof Otto

Konturbezogene Heizung von Formeinsätzen mit Heizkeramikketten und .Netzen (BFH) 09/2014$08 / 2016$

Optimiertes Druckgießen mittels neuartiger Werkzeugtechnik 05/2015-04//2017 (DWT)n aktuelles Projekt ist das erst 2020 begonnene Projekt „Echtzeittemperaturmanagement für Druckgießwerkzeuge durch Additiv Manufacturing -EDAM“ welches wegen Corona bedingter Insolvenz des Industriepartners gegenwärtig ruht. 
Nach der inhaltlich gehaltvollen internationalen Konferenz „New PROLAMAT“ wurden mit der Zielstellung für KMU Unterstützungen zu geben eine neue Konferenzserie im Rahmen des VDI-Bezirksvereins gestartet.

\section{Echtzeittemperaturmanagement für Druckgießwerkzeuge durch Additive Manufacturing „EDAM“}

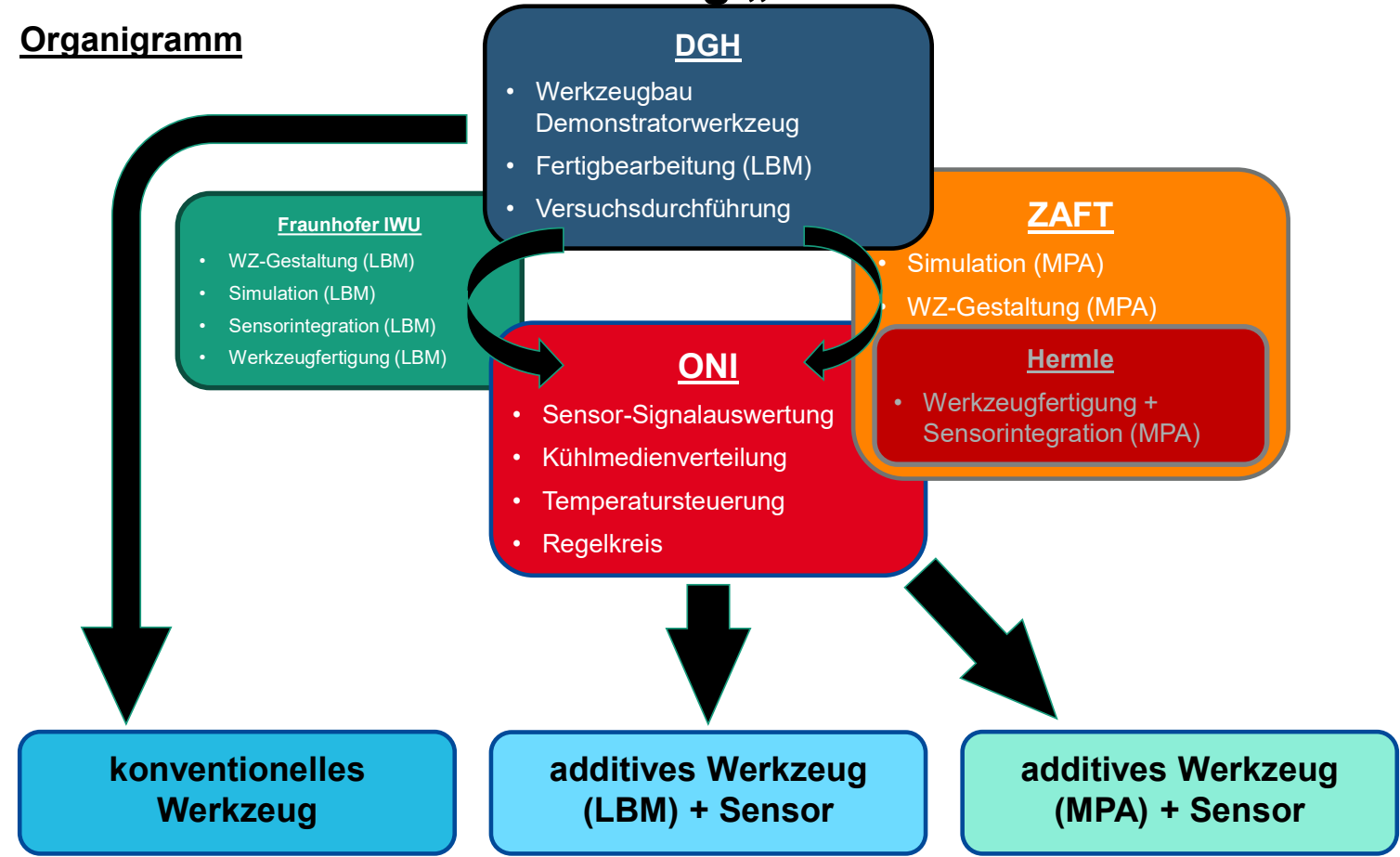

Abbildung 81: Übersicht Kompetenzlage der EDAM-Partner

Der Zielpartner für dieses von der SAB (Sächsische Aufbau Bank) geförderten Projekt ist einer der führenden Automobil-Zulieferer Druckguss Heidenau. Für ein ausgewähltes Referenzwerkstück geht es darum, mit Hilfe eines neu gestalteten Werkzeuges zu einer Produktivitätssteigerung und gleichzeitig eine Qualitätsverbesserung zu erreichen. Wissenschaftliche Partner sind dafür das Fraunhofer IWU Dresden welches Erfahrungsträger und Anwender des generativen Fertigungsverfahrens LBM ist und das ZAFT (Zentrum für Angewandte Forschung und Technologie-an der HTW Dresden) mit dem Industriepartner Hermle München -Inhaber und Anwender des patentierten MPA (Metall-Pulver Auftragsverfahrens) Für die Temperierung, d.h. die konturbezogene Kühlung wird für den Aluminium-Druckguss erstmalig reines Kupfer als Wärmeträger erprobt. Darüber hinaus wird durch den Forschungspartner ONI-Rhytemper erstmals anstelle des üblichen Thermo-Öls auch Drucküberlagertes Wasser eingesetzt. Für die gesamte Echtzeit-Temperatur Überwachung sind spezifische Sensoren vorgesehen

Für meine Aufgabe als Projektleiter am ZAFT ist ein aktives Mitwirken nur mit Unterstützung junger

Absolventen der Produktionstechnik möglich, die eine fundierte Ausbildung in den Anwendungsfeldern der thermischen, mechanischen und Strömungs-Simulation erhalten haben. Aus diesen Erkenntnissen werden gleichzeitig Schlussfolgerungen für eine Zukunftsorientierte Ausbildung abgeleitet. 


\subsection{Innovationstage Mittelstand 2014 - 2020}

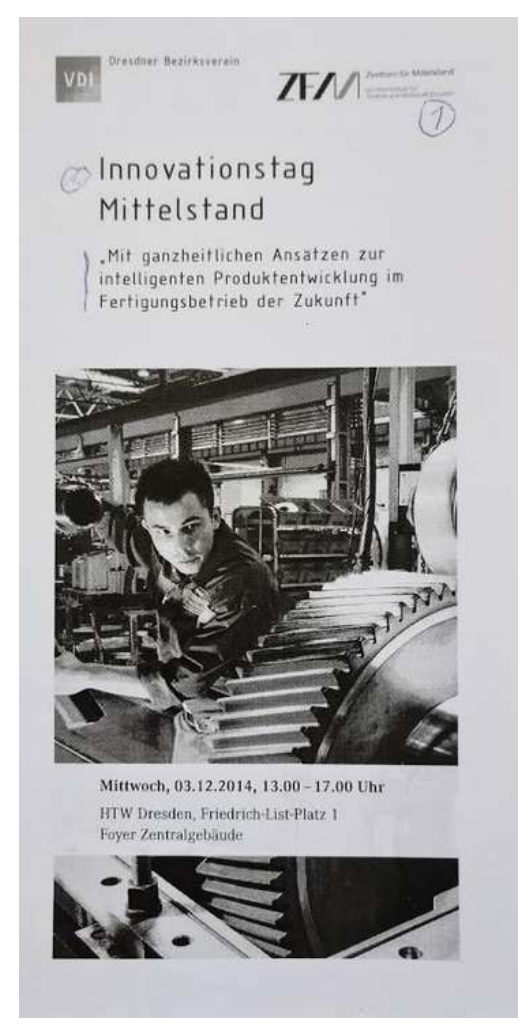

Abbildung 82: Einladung erster VDI-Innovationstag

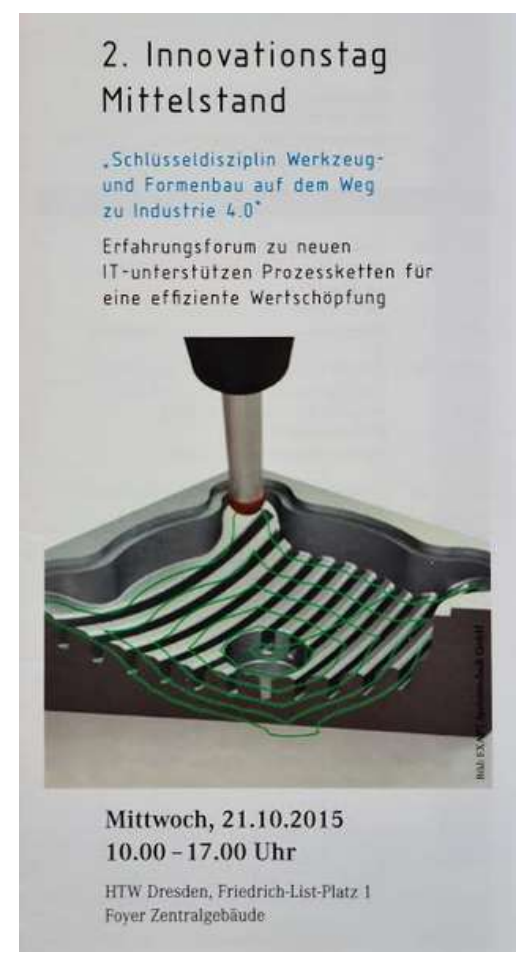

Abbildung 83:Einladung zweiter VDI-Innovationstag
Der erste Innovationstag Mittelstand wurde am 03.12.2014 in der HTW mit folgender Zielstellung durchgeführt: „Mit ganzheitlichen Ansätzen zur intelligenten Produktenwicklung im Fertigungsbetrieb der Zukunft." Mit kompetenten Industrievertretern wie z. B. Herr Warnatsch, Geschäftsführer MIKROMAT Dresden und Dr. Hartmut Freitag, Geschäftsführer der XENON Automatisierungstechnik $\mathrm{GmbH}$, Dresden wurden fortgeschrittene Erfahrungen sächsischer $\mathrm{KMU}$ vermittelt. Zur praxisrelevanten Vermittlung in intelligenter Software für effiziente Wertschöpfungsprozesse trugen die Beiträge vom Geschäftsführer der Schwindt CAD/CAM-Technologie Dr. Ralf Seidler und Herr Dietz, Beauftragter Lehre und Forschung der Siemens Industry Software maßgeblich bei. Mit der anschließenden intensiven Diskussion auch fortgesetzt im kleinen Interessentenkreis wurde der Zeitplan der ersten Veranstaltung weit überschritten. Daraus resultiert die Schlussfolgerung, diese Konferenzserie fortzusetzen.

Zum zweiten Innovationstag Mittelstand (21.10.2015, HTW) wurde folgendes Motto zugrunde gelegt: „Schlüsseldisziplin Werkzeug-und Formenbau auf dem Weg zu Industrie 4.0 - Erfahrungsforum zu neuen IT-unterstützen Prozessketten für eine effiziente Wertschöpfung."

Mit Bezug zu zwei aktuellen Forschungsprojekten wurde der Erfahrungsaustausch mit kompetenten Industriepartnern durchgeführt. Dabei verdient die erstmalige Teilnahme der Entwickler des neuen MPA (Metall-Pulver-Auftrag) Prinzipes besondere Beachtung: „Die MPA-Technologie als Unterstützer intelligenter Werkzeugtechnik für thermische Abformverfahren" von Herrn Derntl und Herrn Müller.(Hermle Ag München Ottobrunn)

Für die Anwendung im Spritzgießbereich konnte vom Geschäftsführer des Unternehmens BACH Resistor Ceramics GmbH mit dem Vortrag „Heizkeramikanwendung im Freiformflächenbereich“ ein wertvoller Beitrag eingebracht werden. 
Der dritte Innovationstag Mittelstand (09.11.2016, HTW) wurde unter folgendem Motto durchge-

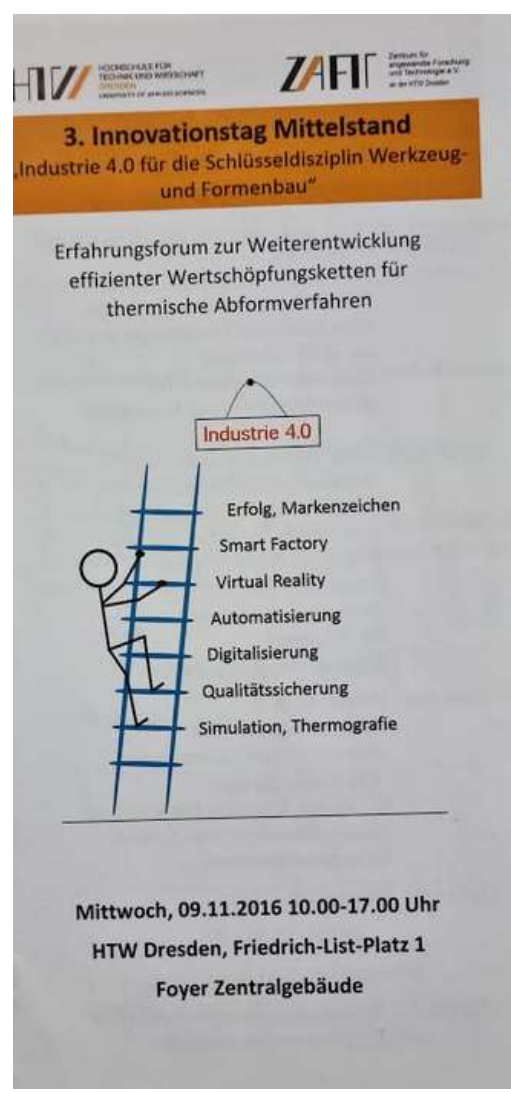

Abbildung 84: Einladung dritter VDIInnovationstag

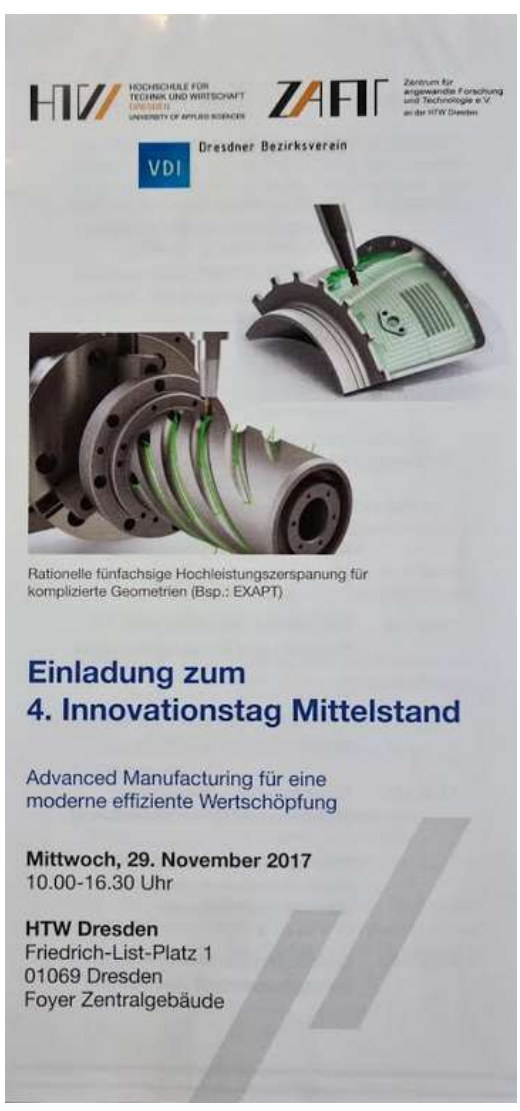

führt: „Industrie 4.0 für die Schlüsseldisziplin Werkzeug- und Formenbau - Erfahrungsforum zur Weiterentwicklung effizienter Wertschöpfungsketten für thermische Abformverfahren." Mit kompetenter Beteiligung der Aachener Werkzeugbau Akademie zum Thema "Neue Technologieansätze in Werkzeug und Formenbau - Schlüssel für Leistungsfähigkeit zur Fertigungsgestaltung“ wurden wertvollen Orientierungen von dem fortgeschrittenen Aachener Entwicklungstand übermittelt.

Von Prof. Jäger, Sprecher des Vorstandes des Institutes für Leichtbau und Kunststofftechnik der TU Dresden wurde das Thema „Hybrider Leichtbau - Die Leichtbau-Allianz Sachsen“ vorgestellt.

Die ersten industriellen Erfahrungen zur Verwendung von CarbonFasern wurden vom Dr. Werner, Geschäftsführer Thyssenkrupp TechCenter Carbon Components Dresden mit dem vielversprechenden Titel "Geht nicht, gibt's nicht - wie die Carbon-Faser doch noch in die Autofelge kam."

Der gesamte Konferenzrahmen wurde darüber hinaus zum Erfahrungsaustausch der aktuellen Spritzguss- und Druckgussprojekte des ZAFT Dresden genutzt.

Der vierte Innovationstag Mittelstand (29.11.2017, HTW) zum Thema: „Advanced Manufacturing für eine moderne effiziente Wertschöpfung" vermittelte starke Impulse aus der Forschung und gesicherte Erfahrungen aus der industriellen Praxis.

Dazu trug der herausragende Plenarvortrag von Prof. E. Uhlmann (IPK Berlin) zum Thema „Perspektiven digital integrierter Produktion für den Mittelstand" maßgeblich bei.

Das hochaktuelle Thema Leichtbau wurde nochmals von Prof. Jäger (ILK der TU Dresden) mit dem Thema „Megatrend Leichtbau mit Produkt- und Prozessinnovation" eingebracht. In einem kollektiven Vortrag der Partner des aktuellen Forschungsprojektes für den Magnesium-Druckguss wurde von Herrn Wilhelm (ONI Rhytemper) mit dem Titel „Effiziente Wertschöpfungskette für das Druckgießen" vorgestellt. Dabei konnte die effektive Nutzung von Simulationsmethoden verdeutlicht werden und die darauf aufbauende Schaffung von Arbeitsplätzen in dem KMU ONI Rhytemper nachgewiesen werden. Herausragende Vorträge waren darüber hinaus vom geschäftsführenden Gesellschafter Direkt-Form GmbH Freiberg und von Herrn C. Hüsken, Konstruktionsleiter der RENISHAW GmbH Lüdenscheid mit dem Thema „Additive Manufacturing im Formen- und Werkzeugbau - mit konturnaher Kühlung zu optimierten Prozessen“. 
Abbildung 85: Einladung vierter VDIInnovationstag

Der fünfte Innovationstag Mittelstand (27.11.2019) fand im Fraunhofer-Institut für Forschungstechnik und Angewandte Materialforschung IFAM statt. Durch die aktive organisatorische und inhaltliche Unterstützung durch den Informationsverbund Maschinenbau Sachsen (VEMAS) war der Untertitel „Von der Praxis für die Praxis“ voll gerechtfertigt. Dabei stand das Anliegen „mit Hochleistungsbearbeitung zu höherer Produktivität und Qualität" im Vordergrund.

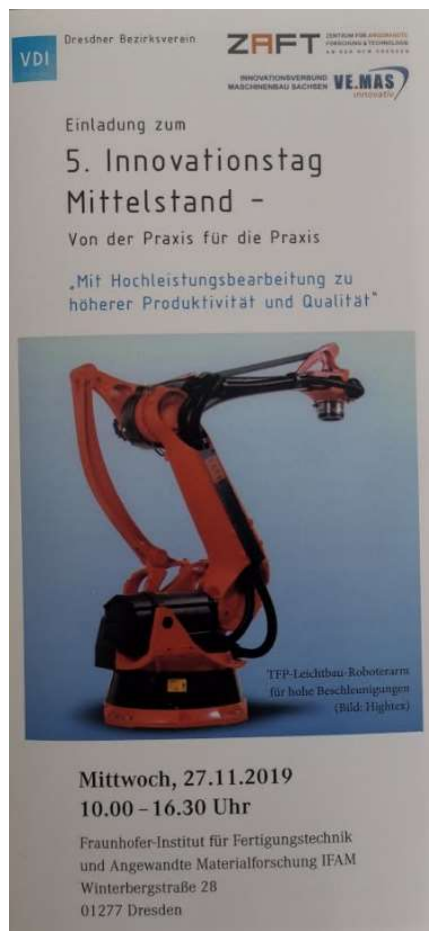

Abbildung 86: Einladung fünfter VDI-Innovationstag 


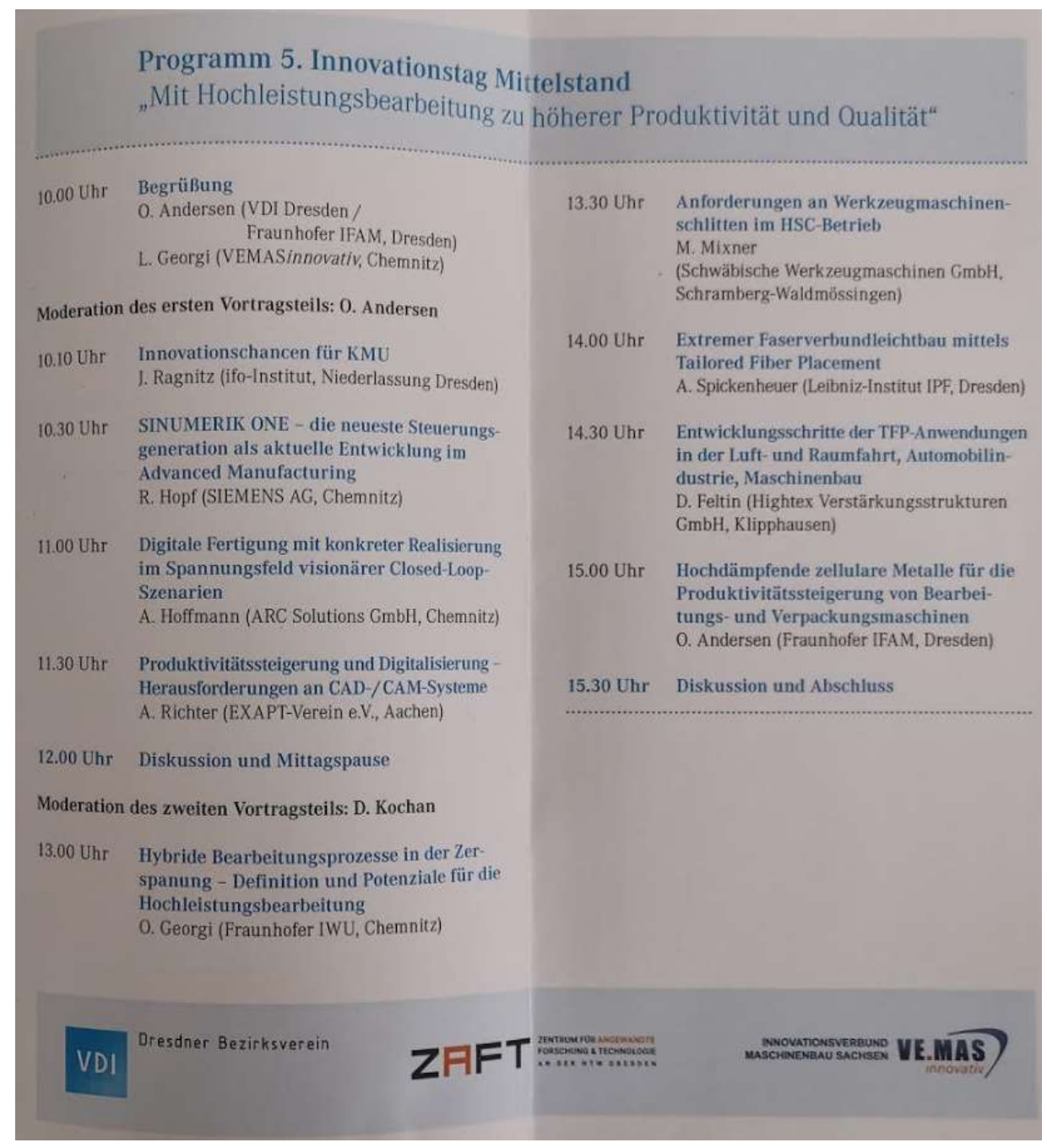

Abbildung 87: Programm fünfter Innovationstag

Mit den eingebrachten Vorträgen wurde gleichzeitig eine hohe Praxisrelevanz und Zukunftsorientierung erreicht. Gleichzeitig kann das gesamte Vortragsprogramm als ein Dokument für die gute Kooperation auf der fachlichen Arbeitsebene im Maschinenbau zwischen Dresden und Chemnitz angesehen werden. In diesem Zusammenhang wurde in der Moderation auch das Motto „Innovationen aus Sachsen" herausgestellt. Aus dieser Sicht wäre aus meiner Sicht eine höhere Beteiligung (Ist-Stand ca. 60 Besucher) durchaus gerechtfertigt gewesen.

Während in den vorangegangenen Innovationstagen über abgeschlossenen bzw. in Bearbeitung befindliche Forschungsprojekte des ZAFT und jeweiligen Industriepartnern im Mittelpunkt standen, konnten zum 5. Innovationstag zunächst die guten Voraussetzungen von Schlüsselpartnern für ein neues hochinnovatives Forschungsvorhaben ProLaMaS (Prozesskette optimierter Leichtbauwerkzeugmaschinenschlitten). Das gegebene Ausgangsniveau ist insbes. In den Vorträgen

- Mixner: Anforderungen an Werkzeugmaschinen im HSC-Betrieb

- Dr. Spickenheuer: Extremer Faserverbundleichtbau mittels TFP

- Dr. Feltin: Entwicklungsschritte der TFP-Anwendungen in Luft-, Raumfahrt, Automobilindustrie

- und Maschinenbau

- Wenn man davon ausgeht, dass das neue Verfahren des Tailord Fiber Placement schon seit ca 25 Jahren am Leibniz IPF (Institut für Polymer Forschung) entwickelt wurde, dann müsste noch viel mehr zur Verbreitung dieses bedeutenden Leichtbau-Prinzipes getan werden. 
- Leider hat sich nach der altersbedingten Beendigung meiner VDI - Aufgabe im Arbeitskreis

- „Rapid Innovation“ noch kein Nachfolger gefunden.

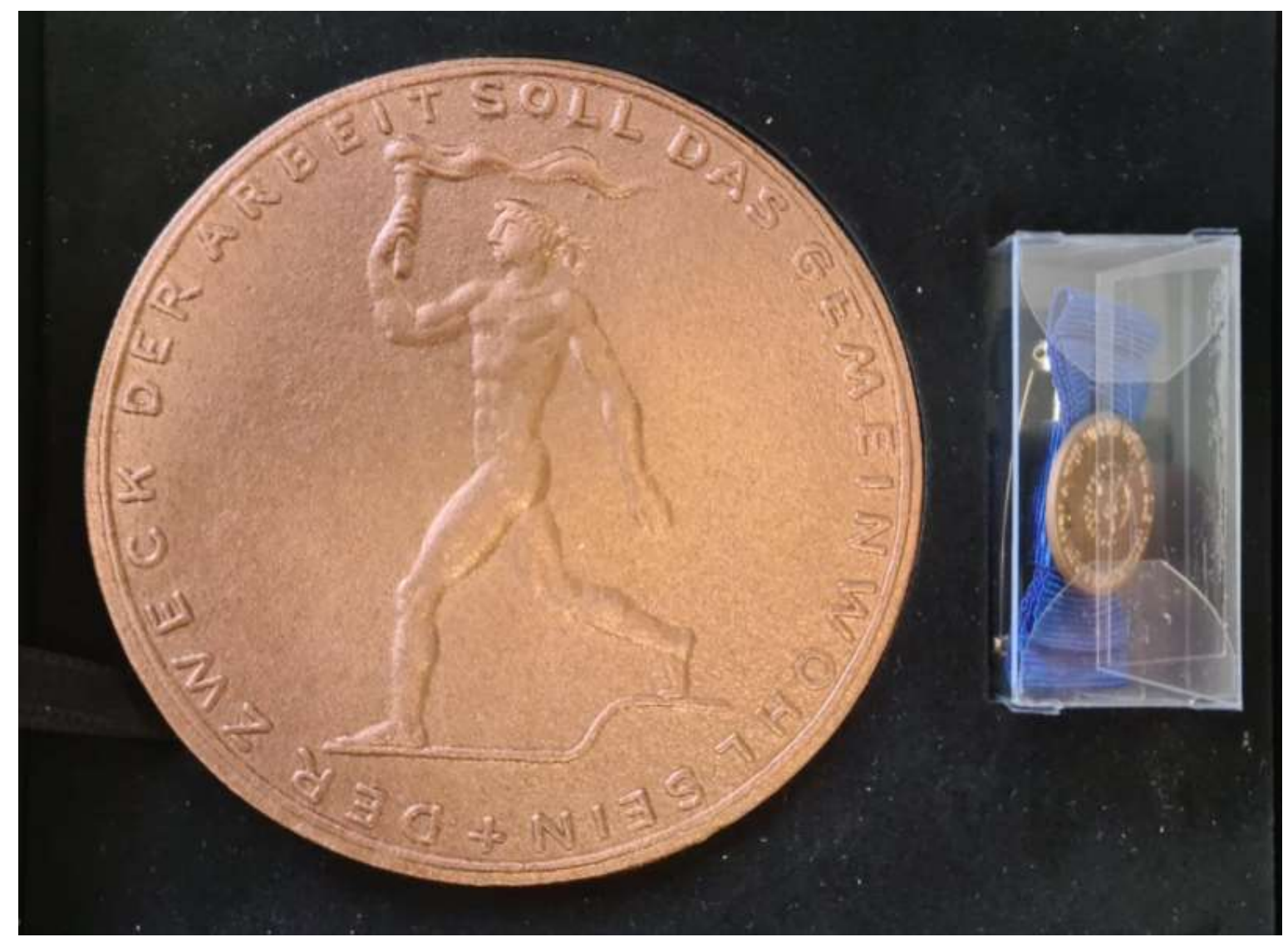

Abbildung 88: Ehrenplakette des VDI

Mit besonderer Freude habe ich den gewichtigen Text „Zweck der Arbeit soll das Gemeinwohl sein" aufgenommen, charakterisiert er doch mein langjähriges Engagement im Gesamtgebiet der fortgeschrittenen Produktionstechnik. 


\section{Ehrenurkunde}

Verein Deutscher Ingenieure - Dresdner Bezirksverein e. V.

Herr Professor Dr. sc. techn. Detlef Kochan

erhält die

\section{Ehrenplakette des VDI}

in Anerkennung seines engagierten Wirkens als Leiter des Arbeitskreises Rapid Innovation des Dresdner Bezirksvereins. Detlef Knchans besonderes Anliegen war und ist es, den VDI als neutrale Plattform fur aktuelle Themen der Fortschrittlichen Produktionstechnologien zu etablieren, wo offener fachlicher Austausch, Wissenstransfer, aber auch Förderung des ingenieurtechnischen Nachwuchses im Mittelpunkt stehen. In zahireichen Gremien der Wirtschaft und Wissenschaft bringt er seit vielen Jahren sein Fachwissen aktiv ein und vertritt dort die Zlele des VDI.

Der Vorstand spricht Detlef Kochan seinen aufrichtigen Dank aus.

Dresden, den 6. Mărz 2020

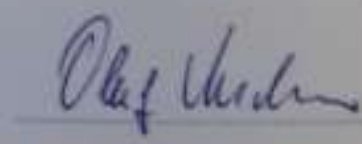

Dr. ing Olat Andersen

versitzenider

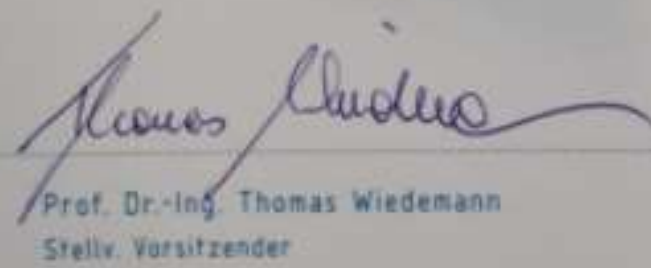

Stelly. Varsitzender

Abbildung 89: Urkunde über Verleihung der VDI-Ehrenplakette 


\section{Erfahrungen mit Industriestaaten}

\subsection{Bemerkungen zu USA-Entwicklungen in der Fertigungstechnik und dem Werk- zeugmaschinenbau}

Etwas überraschend erreichte mich 1995 die Würdigung als Senior-Mitglied der Society of Manufacturing Engineers (SME). Diese Anerkennung resultiert offenbar aus einer Anzahl von Aktivitäten in unterschiedlichen Wissensgebieten innerhalb der SME. Das insbesondere:

- Teilnahme an mehreren Konferenzen der N.C. Society der USA

- fachliche Verantwortung für die bedeutende vierte PROLAMAT-Konferenz (1979)

- relativ häufige Teilnahmen an den SME Veranstaltungen „Rapid Prototyping and Manufacturing Engineering" in den 90er Jahren (einmal mit Vortrag, mehrere Male als Berichterstatter für deutsche Fachzeitschriften)

Dieser erfreuliche Fakt der fachli-

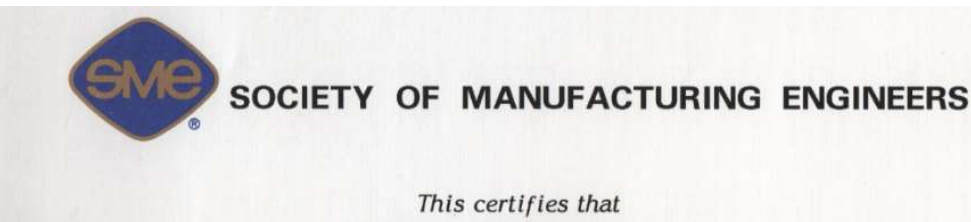

KOCHAN H DEHEF

was elected to the grade of SENIOR MEMBER

in this Society, an institution for the advancement of scientific knowledge in the field of Manufacturing Engineering
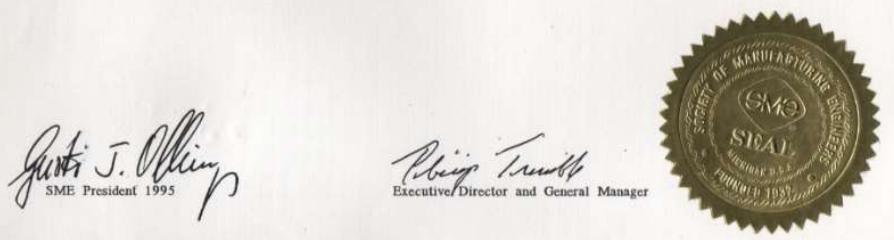

chen Anerkennung durch die

Society of Manufacturing Engineers ist für mich ein Anlass einige generelle Bemerkungen zu den industriellen Entwicklungen in den U.S.A. zu machen:

- die Werkzeugmaschinenindustrie hatte sich nach dem Ersten Weltkrieg in Verbindung mit der dynamischen Entwicklung der Autoindustrie zu einer Weltmarktführerschaft entwickelt

- nach dem zweiten Weltkrieg wurde insbesondere mit Unterstützung der U.S. Airforce die numerische Steuerungstechnik relativ schnell entwickelt

- über die N.C. Society hatten die industriell führenden Länder (auch Deutschland) Zugang zu diesen Entwicklungen

- aus dieser Position heraus war mein erster Vortrag 1974 im Mutterland der N.C. Technologie eine spezifische Herausforderung

- die Vorstellung des anerkannten DDR-Spitzenstandes im Gebiet der flexiblen Fertigungssysteme war dabei eine gute Voraussetzung

- ausgehend von der führenden Position in der gesamten flexiblen Automatisierung mit numerischer Steuerungstechnik vollzog sich relativ schnell ein Rückgang des Werkzeugmaschinenbaus in den 80iger Jahren

- diese Veränderungen resultierten aus dem gleichzeitigen Aufstieg und der Verbreitung der japanischen Werkzeugmaschinenindustrie in den USA

- sehr konkret konnte ich dies während meines Sabbatical Aufenthaltes 1992 in Cincinnati feststellen 
- während von dem japanischen Unternehmen Yamazaki (MAZAK) ein Unternehmen auf der anderen Seite des Grenzflusses Ohio-River in Kentucky eine große Werkzeugmaschinenfirma aufgebaut wurde erfolgte gleichzeitig ein Abziehen von Fachkräften aus dem Unternehmen Cincinnati-Milacron

- dieser Sachverhalt des Werkzeugmaschinenrückganges betrifft eine Anzahl weiterer ehemals bekannte US-Unternehmen

- die gegenwärtige Situation kann dadurch charakterisiert werden, dass sich die führenden Industrieländer Japan und Deutschland in einem harten Wettbewerb befinden in dem zunehmend auch China eine beachtliche Rolle spielt

- dieser gegebene Sachverhalt sollte für wissenschaftsstrategische Entscheidungen eine wichtige Rolle spielen, wobei insbesondere in dem ehemals führenden mitteldeutschen Raum spezifische Förderungen eine stärkere Rolle spielen sollten (siehe Eröffnungsrede des ehemaligen Ministerpräsidenten Prof. Biedenkopf zur Eröffnung des CIM-Technologiezentrum am 5.12. 1991 )

Erfahrungen mit dem Industriestadtstaat Singapur

Im Jahr 1993 hatte ich eine erste Begegnung mit dem jungen Dozenten Chua Chee Kai aus Singapur, anlässlich einer Tagung „Prototyping and Manufacturing“ in den USA. Seine Frage für eine eventuell gemeinsame Veröffentlichung in der IFEP-Fachzeitschrift "Computers and Industrie“ konnte ich deshalb positiv beantworten, weil für mich trotz ausreichender Englischkenntnisse natürlich eine sprachliche Unterstützung möglich war. Mit Bezug zu meinem Fachbuch und der fundierten Erfahrungen aus dem dem Unternehmen SFM zur Gestaltung effektiver Rapid-Prototyping-and-Manufacturing-Prozessketten war eine geeignete Kompetenz gegeben.

Davon ausgehend erfolgte einige Jahre später (1916) wahrscheinlich auch aus Dankbarkeit durch Mr. Chua Chee Kai eine Einladung zu einem Besuch in Singapur an der NTU. Der einwöchige Aufenthalt war mit mehreren Vorträgen an Universitäten und Forschungsinstituten voll ausgefüllt. Die jeweils folgenden Fachdiskussion in den besuchten Einrichtungen waren mit hohen fachlichen Herausforderungen als Berater verbunden. Trotz der extremen Hitze und Schwüle und der begrenzten Freizeit habe ich diesen Aufenthalt in bester Erinnerung. Die weiteren Kontakte mit Prof. Chua Chee Kai beschränkten sich auf wenige unmittelbare Begegnungen zu internationalen Konferenzen.

Im Jahr 2016 kam Prof. Chua Chee Kai für einen Kurzbesuch (ein Tag) nach Dresden und übergab mir eine Kurzdokumentation zu aktuellen Vorhaben im Gebiet Rapid Prototyping und Advanced Manufacturing. (Gemeint ist damit das gesamte weitere Gebiet der Produktionsautomatisierung)
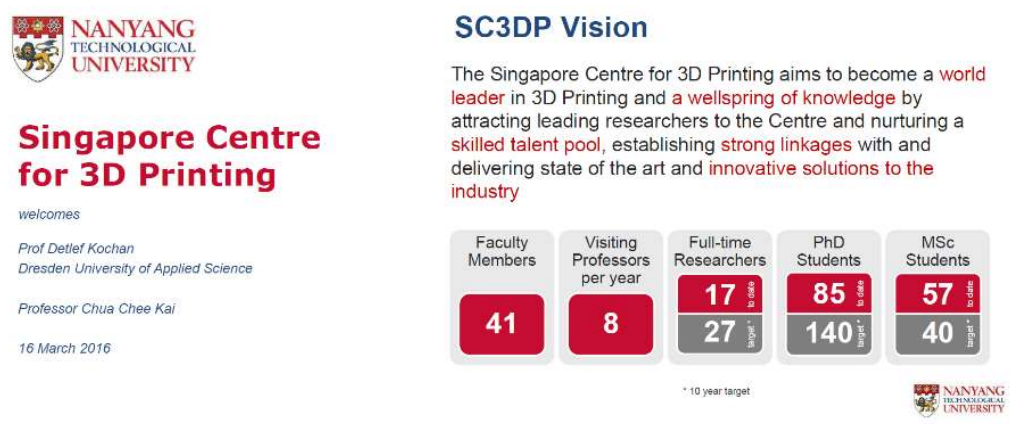

SC3DP Funding

A $\$ \$ 150 M$ Centre supported by:

- National Research Foundation (NRF)

- Economic Development Board (EDB)

- SPRING Singapore

- Agency for Science Technology and Research (A*STAR)

- Ministry of Education (MOE)

- Nanyang Technological University (NTU)

- Industry Partners 
Die beachtliche Förderung im Umfang von circa 150 Mio. Singapur-Dollar (100 Mio. US-Dollar) resultierte aus Informationen aus den USA. Vom damaligen US-Präsidenten Obama wurde eine großzügige Förderung zur Anwendung der 3-D-Printing (Additive Manufacturing) Möglichkeiten für innovative Weiterentwicklung in der gesamten Industrie veranlasst.

Erfreulicherweise wurde in den realisierten Investitionen in Singapur auch noch deutsche Technik gebührend eingeordnet (Additive Manufacturing und hochautomatisierte Werkzeugmaschinen). Interessant ist in diesem Zusammenhang, dass hier die Gestaltung der vierten industriellen Revolution (Industrie 4.0) in Singapur mit dem Begriff Advanced Manufacturing gleichgesetzt wird. Dieser Sachverhalt wurde auch in mehreren Sonderbeilagen der VDI-Nachrichten zu Entwicklungen in Singapur herausgestellt.

Aus meiner Sicht ist der wesentliche Entwicklungstrend der fortgeschrittenen Produktionstechnik (Advanced Manufacturing) die fachlich fundierte Begründung für die Gestaltung effizienter Wertschöpfungsprozesse. Davon kann wiederum eine Erklärung für das in Singapur realisierte hohe produktionstechnische Niveau gegeben werden. Mit Unterstützung von Prof. Ragnitz (IFO-Institut) wurde ein Vergleich des produktionstechnischen Niveaus mit Bayern und Baden-Württemberg vorgenommen. Danach übertrifft Singapur beide führende deutsche Bundesländer.

\section{Die sächsischen Ingenieure und Studenten des Maschinenbaus würden sich mit Sicherheit begeistern lassen, wenn mit hoher Konzentration auf die Entscheidung der vierten industriellen Revolution (Industrie 4.0) in Übereinstimmung mit den zukunftsorientierten Strategien Singapurs stärker orientiert würde.}

\subsection{Erfahrungen mit den industriellen Entwicklungen in der Volksrepublik China}

Die Bezüge zu den wissenschaftlichen und industriellen Entwicklungen zur fortgeschrittenen Produktionstechnik resultieren aus zwei Teilnahmen an IFIP Konferenzen in Peking (einmal DDR-Zeit und 1993) anlässlich einer IFIP-CAE Konferenz.

Der Entwicklungsstand zu dieser Zeit lässt sich mit dem Begriff „Fahrradnation“ charakterisieren. Die beachtlichen Weiterentwicklungen konnten beginnend mit Jahr 2015 kennen gelernt werden.

Im Mai 2015 erreichte mich eine Anfrage von einem chinesischen Professur Yao zur Teilnahme einer Konferenz zum Hauptthema Industrie 4.0. in China. Diese Anfrage entstand durch die Empfehlung von Altmagnifizienz Prof. Neumann (HTW). Zwischen Dresden und der 20 Millionenstadt Huang Zhou für das Jahr 2016 war eine große internationale Konferenz zum Thema „Industrie 4.0“ geplant. Nach meiner erfolgten Zusage stellen sich in Verbindung mit der notwendigen beschleunigten Visa-beschaffung ein schwerwiegender Irrtum zu dem angegebenen Termin heraus. Es handelte sich tatsächlich um eine Konferenz Ende Juni 2015. Das bedeutete wiederum eine extrem kurze Zeit für die qualifizierte Vorbereitung eines Fachvortrages zu den aktuellen Entwicklungen in Richtung Industrie 4.0. Nach der beschleunigten Klärung der gegebenen Randbedingungen konnte die Reise ordnungsgemäß durchgeführt werden. Dabei zeigte sich schon auf der Fahrt vom Flugplatz in das Hotel und beim schnellen Autoverkehr auf den Stadtautobahnen, dass ich in einem neuen China angekommen war.

Trotz der kurzen Vorbereitungszeit und Einordnung als Plenarvortrag wurden die von mir vertretenen Auffassungen zum Entwicklungsstand Industrie 4.0 gut aufgenommen. Die gesamte Veranstaltung war offenbar durch prominente Parteivertreter und auch von Seiten der Industrie z.B. chinesischer Repräsentant von Siemens und des deutschen Vertreters der Organisation Trade ß Invest besucht. 
Diese positiven Eindrücke wurden im Rahmen der Exkursionen in modernen Rechenzentren vertieft. Auch die Zentrale des Unternehmens Alibaba konnte besucht werden .Dieses Unternehmen ist angetreten und offenbar auf einem guten Weg .den Weltmarktführer Amazon zu übertreffen.

Zwei Monate später erreichte mich eine Einladung zum Besuch des Unternehmens DETAO. Natürlich war ich ordnungsgemäß vorbereitet meinen Vortrag auch in dieser Forschungseinrichtung in Shanghai in der angekündigten Konferenz zu präsentieren - ebenfalls zum Thema Industrie 4.0. Obwohl diese Reise durch das einladende Institut wie üblich bezahlt wurde, passierte für mich etwas Erstmaliges

Überraschenderweise war mein offenbar im China bekannter Vortrag in dieser nationalen Konferenz nicht gefragt. Dagegen war der Gastgeber an drei Schwerpunkten interessiert:

- Teilnahme an einer Strategieberatung zur Weiterentwicklung in Ausbildung und Forschung - die Forschungs- und Entwicklungseinrichtung DETAO ist primär mit den Aufgabenfeld Design mit verschiedenen Anwendungsfeldern Bauwesen, Texiltechnik, Verkehrswesen u.a.aktiv .Mit meinen Empfehlungen sollte das Gebiet CAM (Computer Aided Manufacturing) stärker aufgebaut werden

- im Nachgang zu der nationalen Konferenz zum Thema „Internet plus“-Industrie 4.0 fand ein spezielles Fachgespräch zur weiteren Entwicklung in Richtung Advanced Manufacturing statt.

Moderator für diese Veranstaltung war ein chinesischer Professor aus einer südkalifornischen Universität mit dem Schwerpunkt Luftfahrt.

- abschließend nach allen Beratungen wurde mir ein mehrseitiger Fragebogen ausgehändigt um Vorschläge für ein Ausbildungszentrum im Gebiet der fortgeschrittenen Produktionstechnik zu erarbeiten

- diese Anfrage wäre meinerseits als einmalige Aufgabe ausgehend vom derzeitigen Entwicklungsstand möglich. allerdings auch mit beachtlichem Aufwand verbunden gewesen.

Die Anfrage wurde meinerseits deshalb nicht angenommen, weil damit eine permanente Unterstützung über das Internet zu unvertretbaren Verpflichtungen geführt hätte

2017 : Auf Anfrage von Professor Yao wurde eine weitere Chinareise geplant wobei von meiner Seite noch zwei Fachkollegen teilnehmen konnten. Die Auswahl geeigneter Fach Kollegen war mir allein überlassen.Dafür wurden der Geschäftsführer von dem Werkzeugmaschinenunternehmen Mikromat Dresden Herr Warnatsch und Herr Prof. Dr. Arndt Richter Geschäftsführer von EXAPT einbezogen und vom Einladenden bestätigt. Die gemeinsame Reise nach Hung und darüber hinaus in den chinesischen Süden, d.h. insbesondere auch die Automobilregion war für uns von besonderem Interesse, da unter anderem das Unternehmen Gily besucht werden sollte. Die mehrtätige Reise in Unternehmen Forschungsinstitute und Universitäte waren bis zur letzten Stunde voll ausgefüllt. Im Ergebnis der Betriebsbesuche wurde an uns mehrfach die Anfrage gerichtet ob es Möglichkeiten zu aktiven Unterstützung durch geeignete Entwicklungskapazitäten für Konstruktion und Fertigung geben könnte. Leider wurde konnten diese Anfragen auch nach Prüfung der Möglichkeit in sächsischen Umfeld nicht erfüllt werden.

Im Jahre 2018 erfolgte meinerseits eine Reise in die Industrieregion Yinan organisiert durch Herrn Mattes. 


\section{3 Überraschende Kommunikation mit Süd-Korea.}

ausgehend von einer Veröffentlichung in einer internationalen Fachzeitschrift erreichte mich im Jahr 2003 eine Anfrage von einen mir unbekannten Prof Kim aus Südkorea. Er meldete sich für einen Informations-Besuch mit dem Bezug Erfahrungen mit dem LOM (Laminated Objekt Manufacturing) Bauprozess an.Im Unternehmen SFM-GmbH hatten wir erste Erfahrungen mit LOM-Formwerkzeugen für keramische Bauteile- auch in Zusammenarbeit mit dem Dresdener Fraunhofer IKTS.

Nach der erfolgten Terminabstimmung

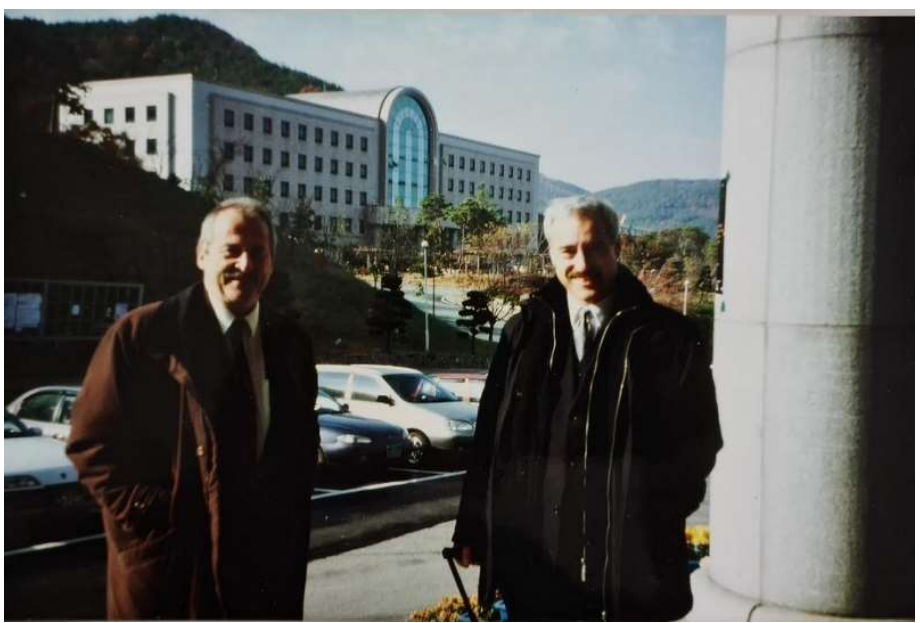

Abbildung 91: Gemeinsamer Aufenthalt mit Prof. Exner (TH Mittweida) und seiner verspäteten Anreise stellte es sich heraus, das im noch verfügbaren Zeitrahmen von ca 1/2 Tag nach dem SFM-Firmenbesuch keine ausreichende Zeit für fachliche Gespräche mehr zur Verfügung stand. Deshalb erfolgte kurz danach eine Einladung nach Südkorea zu Vorträgen an Universitäten und Forschungsinstituten. Nach der Ankunft in Soul kam es zum Treffen mit Prof Exner aus Mittweida, der ebenfalls zu einer Vortragsreis eingeladen war. Das hatte gleichzeitig den Vorteil, dass wir den gemeinsamen Aufenthalt zum fachlichen Austausch nutzen konnten, der dann in der Heimat zu gemeinsamen Forschungsprojekten (Laseranwendungen) genutzt werden konnte. Die Kommunikation mit Prof Kim führte zu keinen weiteren Aktivitäten obwohl die Informationsbesuche durchaus interessant verliefen.

\section{Aktivitäten in Richtung Leichtbau-Werkzeugmaschinenkomponen-} ten 2017 bis 2020

In Abweichung von den bisher dominierenden Arbeitsgebieten Intelligente Werkzeugtechnik wurden aus einer Anfrage von MIKROMAT Dresden 2017 gezielte Aktivitäten gestartet. Das Unternehmen MIKROMAT gehörte neben dem Druckmaschinenhersteller PLANETA Radebeul (jetzt KBA) mit 3500 Beschäftigten zu den größten Maschinenbauunternehmen der DDR. Die Mitarbeiteranzahl im Unternehmen MIKROMAT war in den Jahren bis 2003 auf 40 reduziert, bevor es von den Herren Warnatsch und Dr. Hermsdorf übernommen werden konnte.

Es gab in den Jahren bis 2017 von Seiten HTW nur einige wenige Aktivitäten in Form betreuter studentischer Arbeiten. Anfang des Jahres 2017 erfolgte eine überraschende und interessante Anfrage bzgl. Weiterentwicklungen in Richtung Leichtbau-Werkzeugmaschinenkomponenten. Der Ausgangspunkt dazu war die langjährige Zusammenarbeit mit SW (SCHWÄBISCHE WERKZEUGMASCHINEN) zur Fertigung von Strukturbauteilen, insbesondere auch den dynamisch hochbeanspruchten Werkzeugmaschinenschlitten.

Aus meiner Grundüberzeugung, hohem Respekt und Erfahrungen der beispielgebenden familiengeführten Unternehmen in Baden-Württemberg war diese Anfrage eine besondere Herausforderung. Davon ausgehend folgten einige konkrete Schritte zur Informationsgewinnung:

- Hannover Messe Industrie, 2017 und

- Europäische Werkzeugmaschinenausstellung EMO, Sept. 2017 z.T. gemeinsam mit MIKROMATGeschäftsführer Herr Warnatsch 
- Kontaktaufnahme mit dem ILK - Institut für Leichtbau und Kunststofftechnik der TUD

- Durchführung erster Simulationen zum Nachweis der Stabilitätsbereiche

Darüber hinaus wurden gemeinsam mit Herrn Mixner (SW) in Betracht kommende Institutionen und Unternehmen besucht, um Realisierungsvarianten zu erarbeiten:

- FHG IWU zur Information des Entwicklungsstandes in Richtung Leichtbau und Simulationsvoraussetzungen

- Institut für Leichtbau und Kunststofftechnik der TU Dresden und Leichtbauallianz Sachsen

- TU BeRgaKademie Freiberg zum Ermitteln der Grenzen bzgl. dünnwandigem Guss

- Unternehmen Q-PoINT, erfolgreicher CFK-Anwender für Leichtbau-Sportflugzeuge und Sportwagen: Dieses kleine Unternehmen stand länger im besonderen Fokus wegen der Anwendung der sogenannten RTM-Light-Prozesskette

- Unternehmen KÄPPLER UND PAUSCH Neukirch: Erfolgreicher Blech-Verarbeiter mit Nutzung von mehr als 40 unterschiedlichen Anlagen vom Weltmarktführer TRUMPF Ditzingen/Neukirch für Nutzung dünnwandiger Blech-Konstruktionen für den SW-Schlitten

- LSZ (LASERZENTRUM SACHSEN) mit Durchführung einer Machbarkeitsstudie bzgl. geeigneter CFKVarianten

Alle diese Recherchen und tiefergehenden Untersuchungen verschiedener Realisierungsvarianten mit Topologie optimierten Wandstärken erwiesen sich in Verbindung mit den dargestellten massen- und Kostenabschätzungen als nicht tragfähig. Trotz der anerkennenden Bewertung auch von Seiten SW reichten die positiven Ansätze noch nicht zu einer vertiefenden experimentellen Ersterprobung.

Nach den vielfältigen Aktivitäten ohne einem messbaren Fortschritt einer konkreten industriellen Umsetzung kam aus der Tagespresse ein entscheidender Impuls zum Entwicklungsstand des Unternehmens High-Tex (Dresden-Klipphausen). Die erfolgreichen Anwendungen des TFP-Prinzips (Tailored Fiber Placement) in der Luft- und Raumfahrt, Automobilindustrie und anderen Anwendungsbereichen wurde als ein geeigneter Lösungsansatz für dynamisch hoch beanspruchte Werkzeugmaschinen- Komponenten erkannt. In einem fachlichen Informationsaustausch mit dem HighTex-Geschäftsführer Dr. Feltin im Frühjahr 2018, wurde der Grundstein für tiefergehende Aktivitäten gelegt, die in einem ZIMProjektvorhaben ProLaMaS zusammengefasst sind (s. Abbildung 92).

Die Kurzbezeichnung für das gemeinsame Projektvorhaben entstand in den intensiven Abstimmungsberatungen, insbesondere mit dem Partner aus der Wissenschaft, dem Leibniz Institut für PolymerForschung (IPF) in Person von Dr Spickenheuer : „ProLaMas-Prozesskette für einen Leichtbau MaschinenSchlitten“. Damit wurde gleichzeitig die Übereinstimmung erzielt, das es um die Entwicklung und Erprobung einer neuen Prozesskette geht, die auch für andere dynamisch hoch beanspruchte Maschinen-komponenten genutzt werden kann. Der sogenannte Z-Schlitten des Industrie Partners SWSchwäbische Werkzeugmaschinen ist dabei das ausgewählte Referenzobjekt. Wesentlich ist dabei, das die schon jetzt vom Spitzen-Unternehmen der Hoch -Geschwindigkeits- Bearbeitung SW erreichbaren Parameter noch weiter gesteigert werden können. Die erstmalige Anwendung des TFP-Prinzipes kanndurch aus als eine revolutionäre Zielstellung angesehen werden, die von allen Partnern ergänzend zu den vorhandenen Erfahrungswissen die Nutzung fortgeschrittener Erkenntnisse und Methoden bedingt.Für mich persönlich ist die Ähnlichkeit zum Begriff PROLAMAT- meine langjährige wissenschaftliche Herausforderung eine besondere Verpflichtung für meine voraussichtlich letztmalige aktive Teilnahme an einem bedeutenden Projektvorhaben. 


\title{
SW sснмuн, Hightex, ZFFT [ipf]
}

Anhang zu Anlage 4

Projektbeschreibung

OPTIMIERTER LEICHTBAU-

WERKZEUGMASCHINENSCHLITTEN

\section{ProlaMaS}

\author{
Projektzeitraum
}

$01.09 .2020-31.08 .2022$

Die Weitergabe dieses Anhangs zur Arlage 4 des ZiM-Antrags volistëndig oder in Auszūgen bedarf der schriftlichen Genehmigung aller Projektparner, da wetbewerbsrelevente Technologien im Rahmen dieser Vorhabensbeschreibung detailliert vorgestellt werden.

Abbildung 92: Titelblatt Vorhabensbeschreibung ProLaMaS 


\section{Entwicklung der Absolventenzahlen im Gebiet Produktionsautoma- tisierung, Zerspanen und Abtragtechnik an der TU Dresden}

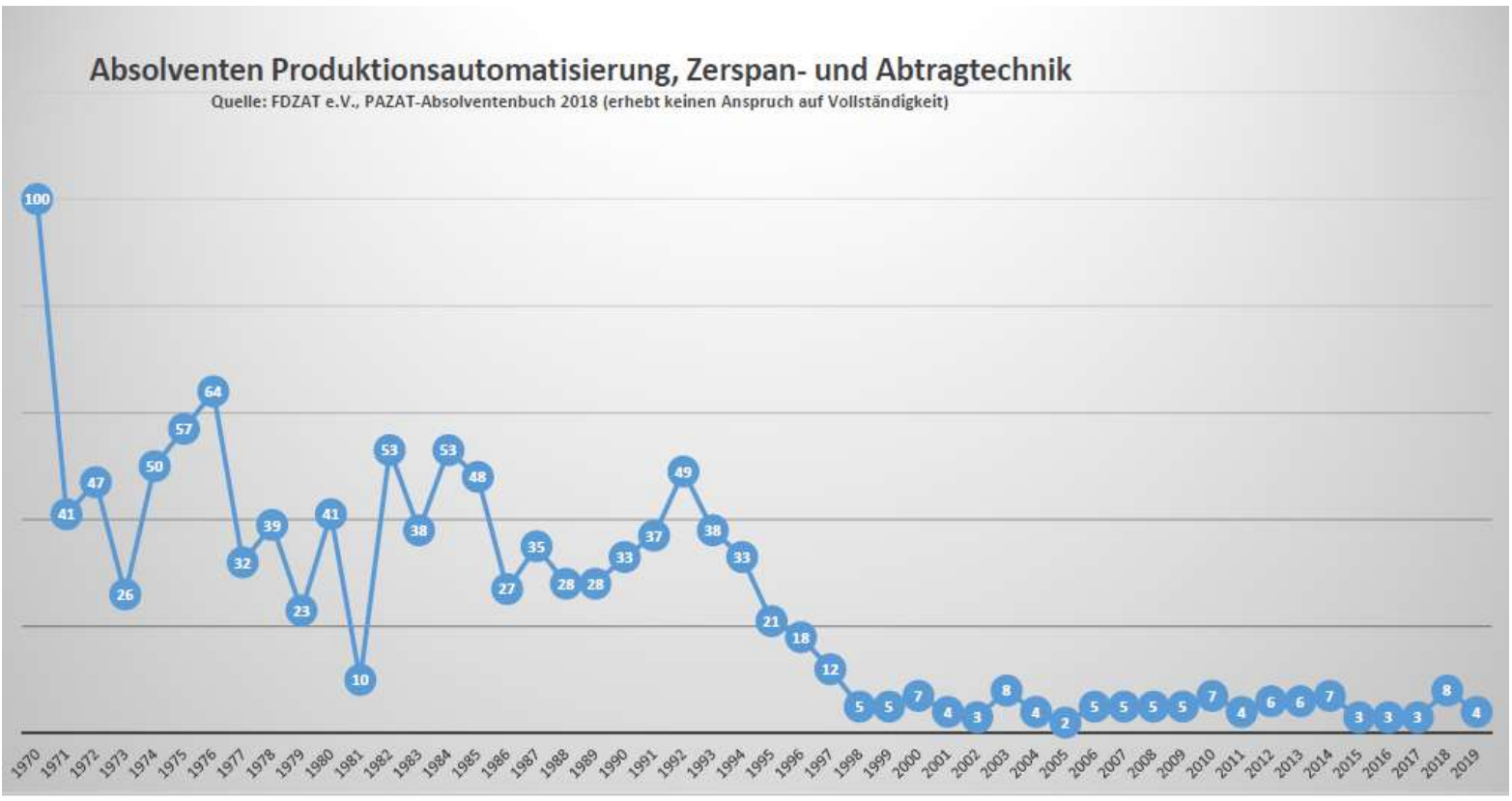

Abbildung 93: Entwicklung Absolventen Produktionstechnik und spanende Fertigung an der TU Dresden

Die gesamte technologische Weiterentwicklung wird im bedeutenden Umfang von befähigten und engagierten Studenten in den abschließenden schöpferischen Ausbildungsabschnitten (Praktika, Diplomarbeiten, Dissertationen u.a.) erbracht.

Besonders gute Erfahrungen wurden an der TU Dresden mit einer spezifischen Form der Besten-Förderung erreicht. Im Rahmen der neu geschaffenen Struktureinheit CAD-CAM-Labor erfolgte die vertiefte Ausbildung als Forschungsstudium mit dem Schwerpunkt Fertigungsinformatik. Auch für alle anderen Studenten der Produktionsautomatisierung war damit eine Grundlage für ein attraktives Gesamtstudium in Übereinstimmung mit den industriellen Anforderungen der zunehmenden IT-Anwendung in vielen Arbeitsbereichen. Diese Ausstrahlung auf viele Studienjahrgänge von 1970 bis 1990 wird in Abb. 93 dargestellt. Das zeigt sich auch in den hohen Studentenzahlen Der steile Abfall von 1990 bis 1996 ist eindeutig zu erkennen. In den Folgejahren sind die Absolventenzahlen auf 5 bis 6 Studenten pro Jahr insgesamt gesunken! Dieser Fakt ist deshalb besonders unter dem Bedarf der gesamten Fertigungsindustrie als extrem kritisch zu bewerten. In der jährlichen offiziellen Statistik des VDMA (Verein Deutscher Maschinenbauer) wird der Anteil der industriellen Wertschöpfung mit hochautomatisierten Fertigungsprozessen der spanenden Fertigung mit mehr als 50\% ausgewiesen. Unter diesem Aspekt verwundert es nicht, dass sich ein Produktivitätsrückstand in den Neuen Bundesländern (NBL) von 20\% bis $25 \%$ beinahe verfestigt hat.

Im 1991 gegründeten CIM-Technologie-Transferzentrum waren wesentliche Voraussetzungen, z.B. 5achsiges Bearbeitungszentrum und erste Stereolithographie-Anlage, geschaffen worden, um beispielgebend Hightech-Verfahren in den KMU anzuwenden und weiter zu entwickeln.

Die effiziente Ausnutzung hochproduktiver Fertigungsanlagen für die jeweilige Prozessvorbereitung und -Durchführung befähigte Ingenieure und Facharbeiter, die im Rahmen des CIM-TT fachspezifisch vertieft ausgebildet sein müssen. Mit dem Abstand von nunmehr dreißig Jahren ergibt sich für Technik-interessierte Historiker die Frage, ob das CIM-TT die Erwartungen aus dieser Sicht annähernd erfüllt hat. 


\section{Zusammenfassende Bemerkungen, Schlussfolgerungen und Empfehlungen}

\subsection{Generelle Entwicklungstrends in der Wissenschaft}

In allen Wissensgebieten vollzieht sich ein dynamischer Erkenntnisfortschritt. Damit verbunden ist eine starke Differenzierung und eine Entwicklung in viele spezifizierte Fachdisziplinen.

Die gesamten Trends der wissenschaftlichen Entwicklungen können demnach in zwei Hauptrichtungen eingeordnet werden:

- Differenzierungsprinzip, d. h. Spezialisierung in viele Fachgebiete

- für eine stabile, harmonische technische Entwicklung muss deshalb ein

- Integrationsprinzip als Ergänzung bzw. Ausgleich wirksam werden.

Dieser Sachverhalt kann am Beispiel der industriellen Entwicklung in Sachsen mit den besonderen Leistungen von dem Gründer der technischen Bildungsanstalt Andreas Schubert charakterisiert werden. Bekanntlich war Prof. Schubert zugleich als Maschinenbauer (Entwicklung erster sächsischer Eisenbahn -Saxonia) und als Bau-Ingenieur (Göltzschtal-Brücke) erfolgreich tätig. Wesentliche Voraussetzungen für das Wirksamwerden in unterschiedlichen Fachgebieten war das notwendige fortgeschrittene Wissen. Für die technologische Realisierung musste vielfältiges Wissen aus unterschiedlichen Gebieten integrativ zusammengefasst werden. Die historischen Pionierleistungen konnten nur durch konsequente Anwendung beider Grundprinzipien vollbracht werden. Für diese, in der damaligen Zeit herausragenden Leistungen ging A. Schubert von seinem bewährten Grundsatz aus:

\section{Die konkreten Beispiele sind in der Ausbildung für die Vermittlung des technischen \\ Fortschrittes der wesentliche Ausgangspunkt!}

Aus heutiger Sicht muss man davon ausgehen, dass sich nach dem Differenzierungsprinzip eine Vielzahl unterschiedlicher Wissensgebiete entwickelt haben Deshalb muss man für ein konkretes Wirksamwerden im Sinne des technischen Fortschrittes das Integrationsprinzip bestmöglich beachten. Auch in der Technik, insbesondere im Maschinenbau, müssen alle integrativen Verbindungen zu anderen fortgeschrittenen Fachgebieten für die Gestaltung und Realisierung effizienter Wertschöpfungsketten beachtet werden.

Diese elementaren Grundsätze wurden leider in der Innovationstrategie des Freistaates Sachsen vernachlässigt. Konkret bedeutet dieser Sachverhalt, dass die sogenannten Key Enabling--Technologies (KET) überbetont sind. Das bedeutet konkret, dass z.B. diese Sonderverfahren nur einen Anteil von ca. $5 \%$ an der industriellen Wertschöpfung haben. Im Gegensatz dazu wird dafür ungleich höhere Förderbeträge eingesetzt werden. Dagegen wird dem dominierenden Arbeitsgebiet der fortgeschrittenen Produktionstechnik (Advanced Manufacturing) entsprechend seiner Bedeutung zu wenig Aufmerksamkeit gewidmet.

Auf diese Fakten habe ich mit Unterstützung des VDI Dresden mehrfach das Wissenschafts- und Wirtschaftsministerium hingewiesen, leider ohne sichtbares Ergebnis. 


\subsection{Entwicklungen der Produktionsautomatisierung}

In dem beigefügten Bild 93 zur Entwicklung der Automatisierungstechnik bis zur 4. Industriellen Revolution (Industrie 4.0) ist der Ausgangspunkt der sogenannten flexiblen Automatisierung mit numerisch gesteuerten Maschinen in den Jahren 1950 bis 2020 ausgewiesen. Der Anteil an der industriellen Wertschöpfung für spanende Fertigungsprozesse von 50 \% ist von Beginn an in gleichen Größenordnungen Die gesamte Entwicklung der flexiblen Automatisierung vollzog sich im Wesentlichen in zwei Hauptrichtungen:

Erhöhung des Automatisierungsniveaus, z.B. NC-Maschine für bestimmte Verfahren.

- CNC (Computer Numerical Control) mit frei programmierbarer Steuerung

- DNC (Direct Numerical Control)

- Bearbeitungszentren mit automatisiertem Werkzeugwechsel

- Flexible Fertigungssysteme, verkettete Bearbeitungszentren mit automatisiertem Werkstückwechsel

- Breite Anwendungen der flexiblen Automatisierung in weiteren Verfahrensbereichen, z.B. Ur- und Umformtechnik und Fügetechnik

Parallel zu den Entwicklungen des Automatisierungsumfanges vollzog sich über die unmittelbare NCProgrammierung hinausgehend das gesamte Gebiet der rechnerunterstützten Fertigungstechnik (CAM: Computer Aided Manufacturing). Ein wichtiger Bestandteil war dabei die Modellierung von geschlossenen 3D-Datenmodellen. Das war gleichzeitig ein wesentlicher Ausgangspunkt für das gesamte Anwendungsgebiet des CAD (Computer Aided Design), d.h. der rechnergestützten Konstruktion. Im Ergebnis dieser evolutionären Entwicklungsschritte waren die Voraussetzungen für einen, in der Tat, revolutionären Fortschritt gegeben.

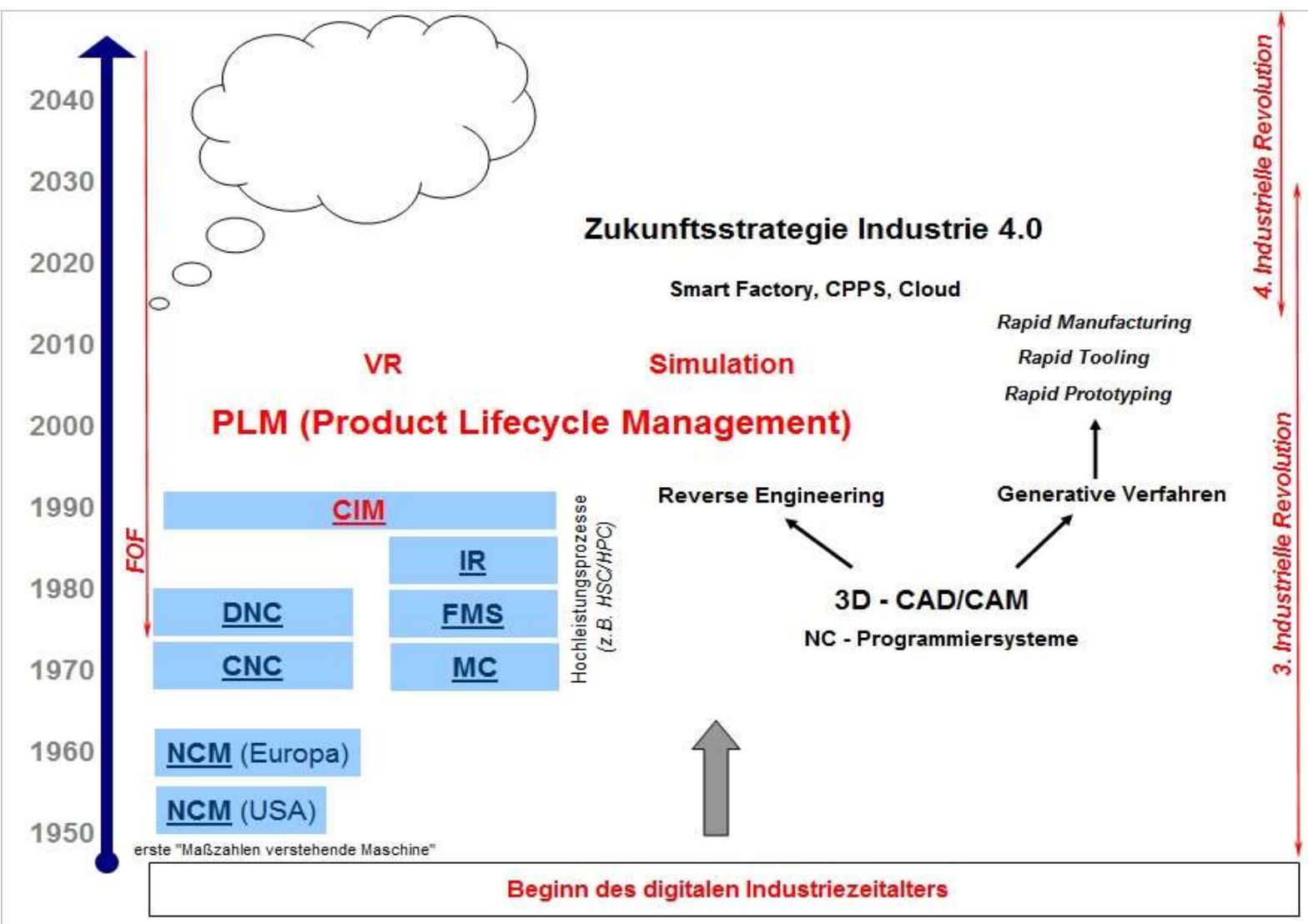

Abbildung 94: Zukunftsstrategie Industrie 4.0 
Für alle charakterisierten Entwicklungsschritte wurde konsequenterweise von dem Prinzip der Kontinuierlichen Verbesserung (Continous Improvement) ausgegangen. Davon ausgehend, dass mit den vielfältigen Entwicklungen zur Erhöhung des Automatisierungsgrades und den zahlreichen Anwendungen der flexiblen Automatisierung in der gesamten Fertigungstechnik wird es damit klar, dass wirkungsvoll Weiterentwicklungen primär mit dem Bottom-Up-Prinzip verwirklicht werden.

\section{Main Strategies in direction of Industrie 4.0}

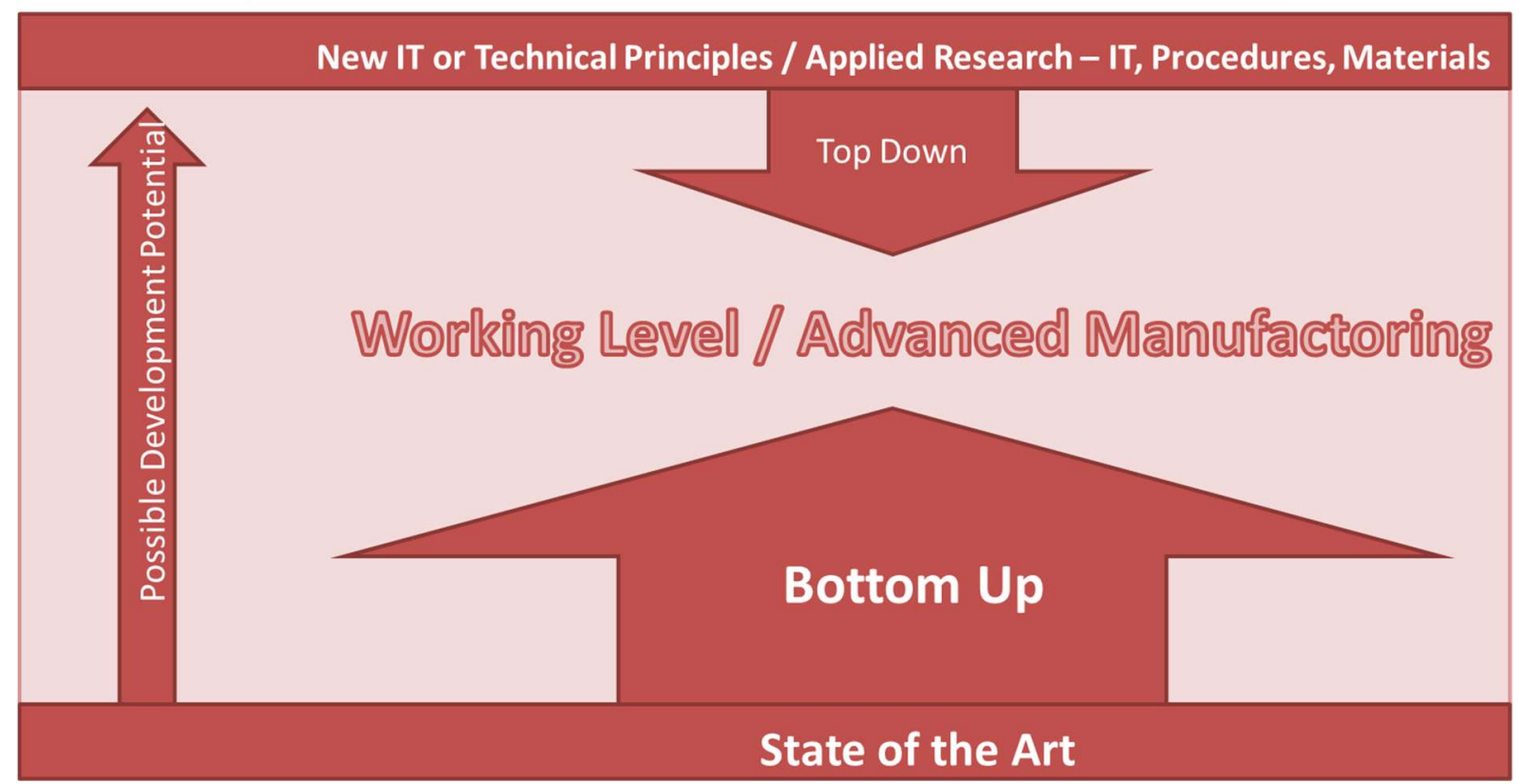

Abbildung 95: Forschungsstrategien im Gebiet der Produktionstechnik (Advanced Manufacturing)

Im Jahr 1988 wurde in den USA mit der Entwicklung der ersten sogenannten Stereolithografie-Anlage die Fertigung beliebig komplizierter geometrischer Objekte ohne traditionelle Werkzeuge ermöglicht. Voraussetzungen dafür waren geschlossene 3D-Datenmodelle der jeweiligen Objekte. Nach der inzwischen dreißigjährigen Entwicklung, begonnen mit dem Begriff Rapid Technologien und auch SFM (Solid Freeform Manufacturing), dominieren gegenwärtig die Begriffe 3D-Drucken im allgemeinen Sprachgebrauch bzw. Additiv Manufacturing nach aktueller ISO-Norm. Die effiziente Ausnutzung aller dieser Möglichkeiten ist ein wichtiger Maßstab für das produktionstechnische Niveau in den Unternehmen der Fertigungsindustrie.

\subsection{Digitalisierung}

In den vergangenen Jahren wurde auch für die breite Öffentlichkeit die Bedeutung der Digitalisierung bekannt und häufig als wichtige strategische Orientierung herausgestellt, ohne auf konkrete Anwendungsbereiche Bezug zu nehmen. Dieser grundsätzliche Standpunkt ist durchaus zu akzeptieren, sollte jedoch zwingend auf konkrete Anwendungsbereiche (bspw. Medizin, Pädagogik, Logistik u.A.) fokussiert werden.

Bezugnehmend auf die Gesamtentwicklungen der Digitalisierung ist nach dem in den vergangenen sechzig Jahren vollzogenen Trend der Erhöhung des Automatisierungsgrades die Verbesserung der industriellen Wertschöpfung weiterhin der Kernaspekt für das Wohlergehen der Gesellschaft. In Verbindung mit der allgemeinen Polemik zur Digitalisierung wird leider dieser wesentliche Bezugspunkt zur Automatisierung nicht gebührend beachtet. 
In einem von der Bevölkerungsanzahl mit dem Freistaat Sachsen vergleichbaren Stadtstaat Singapur wird konsequenterweise von einer derartigen Position ausgegangen. Mit dem Aufkommen des Begriffes Industrie 4.0 gleichgesetzt mit dem Kernanliegen des Advanced Manufacturing wird diese Position als entscheidend für die Wettbewerbsfähigkeit betrachtet (vgI. VDI-Nachrichten, 17.11.2017).

Bekanntlich hat Singapur eine höhere Produktivität im Vergleich zu unseren führenden Bundesländern Bayern und Baden-Württemberg. Das sächsische Wirtschaftsministerium wurde mit konkreten Fakten auf diesen Sachverhalt meinerseits mehrfach hingewiesen.

\subsection{Bemerkungen zur künstlichen Intelligenz (KI)}

Der Begriff der "Künstlichen Intelligenz" wurde erstmals von Wissenschaftlern der Informatik geprägt und als spezifische Disziplin dieses Wissensgebietes ausgewiesen. Primäre Anwendungen sind im Zusammenhang mit der systematischen geordneten Aufbereitung großer Datenbestände (z.B. in der Medizin) gegeben. Darüber hinaus gibt es im Gebiet der Fertigungstechnik noch weitere Anwendungsgebiete die über die Mustererkennung (Pattern Recognition) weit hinausgehen. Dabei handelt es sich um neue wissenschaftliche Erkenntnisse, die in der Anwendung bestimmter Automatisierungslösungen als ergänzendes Erfahrungswissen (Know how) erarbeitet werden. Dieses umfangreiche verfahrenzbezogene Erfahrungswissen kann man als eine wichtige Ergänzung algorithmischer und damit programmierbarer Erkenntnisse betrachten. Das jeweilige verfahrensbezogene Know how ist demnach für jedes innovationsorientierte Unternehmen ein wichtiger Bestandteil geistiger Ware, ohne das dabei

in vielen Fällen der Begriff der künstlichen Intelligenz genutzt wird. Für das außerordentlich wertvolle Zusatzwissen industrieller Wertschöpfungsprozesse gibt es leider keinen prägnanten Kurzbegriff. Von diesem Sachverhalt sollte man

im gesamten Gebiet des Advanced Manufacturing ausgehen, damit dieses wertvolle Zusatzkapital produzierender Unternehmen entsprechend beachtet und nach Bedarf auch geschützt wird wird. In den folgenden Ausführungen wird der Begriff KI mit beiden inhaltlichen Bezügen (Informatik:methodische Anwendungen; Fertigungstechnik: systematisch aufbereitetes Erfahrungswissen.)

Die Möglichkeiten der Nutzung der künstlichen Intelligenz in der Fertigungstechnik wurden in zwei internationalen IFIP-Arbeitskonferenzen behandelt, an denen ich als Referent (Tokyo) bzw als Veranstalter aktiv beteiligt war:

- Utilization of Artificial Intelligence and Pattern Recognition Techniques in Manufacturing Engineering (1984, Tokio, s.S. ...)

- Complex Machining and Al-Methods (1991, Gaußig/ Dresden s.S ...)

Mit diesem Bezug zum Gebiet der rechnerunterstützten Fertigungstechnik (CIM) spielten die unmittelbaren methodischen Vorgehensweisen aus informationstechnischer Sicht nicht die wesentliche Rolle. Zweifellos haben derartige Methoden der geordneten Datenaufbereitung für extrem hohe Datenbestände (Big Data), insbes. in der Medizin eine wichtige Rolle, wenn es darum geht umfangreiche Datenmengen geordnet zu strukturieren. Auch in der Fertigungstechnik lassen sich bestimmte Sachverhalte mit derartigen Prinzipien einer Lösung zu führen ,(z. B. Mustererkennung) ohne dass sich dafür wesentliche industrielle Anwendungen ergeben haben. In der Fertigungstechnik spielt über die informationstechnische Aufbereitung und Anwendung algorithmierbarer Sachverhalte eine wesentliche Rolle. Konkret bedeutete das, dass der Wert von hochautomatisierten Fertigungsanlagen mit der zugrundeliegenden Software gegeben ist. Der Begriff KI als methodische Disziplin der Informatik spielt demnach in diesem Zusammenhang komplexer Automatisierungsanlagen keine Rolle. 
Mit Bezug zu den Fachvorträgen zur Gaußig- Arbeitskonferenz wird dieser Sachverhalt an folgenden typischen Beispiel charakterisiert:

Ein leistungsfähiges flexibles Fertigungssystem besteht z.B. aus 3 Bearbeitungszentren mit 4 und 5achsigen Steuerungen integrativ durch ein Roboterhandling-System verbunden.

Die Bearbeitungszentren sind mit ca 60-80 Werkzeugen ausgestattet mit denen jeweils zeitlich parallel $60-80$ unterschiedliche Bearbeitungsabläufe mit verfahrensbezogen ebenso vielen unterschiedliche Verfahrens-Parameter genutzt werden. Dabei sind auch unter Beachtung der HSC-Hochgeschwindigkeitsbearbeitung die jeweiligen Werkzeugstandzeiten für die Verfahrensrealisierung zu berücksichtigen. Man kann davon ausgehen, das für die programmtechnische Entwicklung und technologische Umsetzung jahrelange Vorleistungen eingebracht werden müssen, damit effiziente und qualitätsgerechte Fertigungsprozesse realisiert werden können.

Darüber hinaus ist für jede Automatisierungslösung das jeweilige Erfahrungswissen aus den unmittelbaren Bearbeitungsprozessen eine wichtige Komponente, die ggf. gesondert erfasst werden muss. Konkret bedeutet dieser Sachverhalt, dass der Wert automatisierter Fertigungseinrichtungen ergänzend zu der unmittelbaren Hardware und Steuerungstechnik mit der zugehörigen Software gegeben ist. Diese gesamte Software besteht aus dem verfahrensspezifisch algorithmierbaren Anteil, ergänzt um das verfahrensspezifische Erfahrungswissen (Know-How). Der wertmäßige Anteil kann in Größenordnungen von 20 bis $50 \%$ angenommen werden.

An dieser Stelle wird deutlich, dass die von Vertretern der Informatik- Intelligenz vertretene Auffassung nach dem Top-Down Prinzip in der Fertigungsindustrie wirksam zu werden, an objektive Grenzen stößt.

In leistungsfähigen Automatisierungs-Anlagen ist soviel intelligentes- programmtechnisch digitalisiertes Potential enthalten, das mit dem notwendigen fachlichen Respekt zu behandeln ist.

Die Nutzung der künstlichen Intelligenz sollte nach meiner Auffassung deshalb immer mit dem konkreten Anwendungsbereichen in Übereinstimmung gebracht werden.

Damit die stets aktive Rolle der kreativen Ingenieure und verallgemeinert der schöpferischen Menschen in allen Entwicklungsetappen der Realität entsprechend eingeordnet wird, sollte auch zukünftig für hochautomatisierte Fertigungssysteme der Begriff „Mensch-Maschine- Systeme “ wieder stärkere Beachtung finden.

\subsection{Beispiele und Schlussfolgerungen, Empfehlungen bedeutender Automatisierungs- vorhaben.}

Schlussfolgerungen und Empfehlungen mit Bezug zu historischen Projekten. Im Verlauf meiner fachlichen Entwicklung war ich im unterschiedlichen Umfang an verschiedenen Projekten beteiligt. Dabei war stets ein Bezug zu fertigungstechnischen Problemstellungen und deren Lösung mit informationstechnischer Unterstützung gegeben. Obwohl die meisten Aktivitäten schon einige Jahre zurückliegen können durchaus verallgemeinerungsfähige Erfahrungen aus heutiger Sicht abgeleitet werden.

\subsubsection{Flexible Fertigungssysteme PRISMA 2 und ROTA F125}

Flexible Fertigungssysteme sind aus heutiger Sicht wesentliche Merkmale zur Charakteristik des produktionstechnischen Niveaus. Deshalb sollten einige historische Fakten sachlich zur Kenntnisgenommen werden. Eine objektive Bewertung erscheint immer sinnvoll. In diesem Sinn wird im Folgenden versucht, wesentliche Schlussfolgerungen aus den historischen Entwicklungen abzuleiten. 
Die Idee zur Entwicklung Flexibler Fertigungssysteme begann Ende der 1960iger Jahre, wobei von der DDR eine führende Position erreicht wurde. Nachdem 1968 im Forschungsinstitut Werkzeugmaschinen Karl Marx Stadt ein erstes Konzept erstellt war, konnte durch eine koordinierte intensive Zusammenarbeit über die bestehenden Kombinats- und Betriebsgrenzen hinaus im Jahr 1971 mit der Bezeichnung Prisma2 im Stammbetrieb des sogenannten "Fritz-Heckert- Kombinates" die komplexe Inbetriebnahme erfolgte. (ausführliche Beschreibung siehe "Werkzeugmaschinenbau in Sachsen" Naumann/Neugebauer- Verlag, Heimatland Sachsen, Chemnitz 2003)

Zum Vergleich: Von der BRD wurde ein erstes Flexibles Fertigungssystem" zur EMO (Europäische Werkzeugmaschinen Messe) in Paris (Firma Fritz Werner Werkzeugmaschinen AG Berlin) vorgestellt, d.h. ca 12 Jahre Rückstand. Anlässlich meiner ersten USA- Reise (beginnend im WZM-Unternehmen Cincinnati-Milacron) mir nur erste Konzepte für FMS vorgelegt werden.

\section{Strategische Schlussfolgerung und Empfehlung:}

Unter Beachtung der besonderen Stellung des WZM-Baus ("Mutter aller Maschinen") und der internationalen Wettbewerbssituation

- Rückgang der ehemaligen Weltmarktführerschaft der USA

- hohe Leistungsfähigkeit des japanischen WZM-Bau

- dynamische Entwicklung der chinesischen Industrie, einschließlich WZM Bau

sollte die deutsche WZM-Industrie in der Förderstrategie der BRD eine höhere Beachtung finden. Ausgehend von den in die deutsche Einheit eingebrachten wertvollen Erfahrungen im gesamten Gebiet des Advanced Manufacturing könnten m.E. für die ostdeutsche Industrie beachtliche Potentiale durch zielgerichtete Maßnahmen geschaffen werden.

\subsubsection{Automobil-Entwurfssystem AUTENT}

Dieses Beispiel kann dadurch charakterisiert werden, dass bedingt durch die Embargo-Bestimmungen objektiv ein Rückstand gegeben war. Trotzdem wurden für die Industrie nutzbare Ergebnisse geschaffen. Als wesentliches Resultat der von meinem Lehr-Forschungsgebiet vertretenen Position der Orientierung am internationalen Entwicklungsstand kann die Situation zur Wendezeit wie folgt charakterisiert werden:

- alle ehemaligen High-tech-qualifizierten Mitarbeiter wurden von VW, bzw in diesem fachlichen Umfeld leistungsfähiger Software-Unternehmen aufgenommen

- - ein Teil der hochqualifizierten Mitarbeiter gründeten Unternehmen.

Schlussfolgerung: Die dynamischen Entwicklungen im gesamten Gebiet der Produktionsautomatisierung und IT-Anwendungen bedingen für eine zukunftsorientierte Ausbildung eine permanente Orientierung am fortgeschrittenen Entwicklungsstand. Dabei geht es um den Erwerb von Wissen und Befähigen zum Mitwirken der Gestaltung komplexer Problemlösungsprozesse. Zum Gewährleisten der Anwendbarkeit neuer Prinzipien ist die Orientierung an industriereifen Methoden und Lösungen ein wesentlicher Ausgangspunkt. Für das Zusammenwirken von Forschungsaktivitäten und Lernfabriken werden in den NBL 


\subsubsection{CAD/CAM-System GRAFIS}

Dieses beispielgebende Rationalisierungsvorhaben der Leichtindustrie der DDR kann als einen gelungenen Beweis angesehen werden, das man mit geschultem Personal und verfügbarer Technik im effektiven Zusammenwirken durchaus herausragende Ergebnisse schaffen kann. Es ist natürlich auch klar, dass mit leistungsfähiger Technik durchaus eine Effizienzerhöhung möglich gewesen wäre. Warum das nicht geschehen ist, bleibt unklar. Unabhängig davon erscheint mir eine Schlussfolgerung wesentlich. Ausgehend von der vom deutschen Handel vertretenen Vorgehensweise (von Strategie sollte bewusst nicht gesprochen werden) der Produktionsverlagerungen in Billiglohnländer ist eine konsequente Orientierung auf effiziente Hightech Anwendungen für wesentliche Fertigungsbereiche der bessere Weg nicht zuletzt im Interesse deutscher Arbeitnehmer.

\subsubsection{Hoch automatisierter Sandguss im ehemaligen Unternehmen Salzenforst/ Bautzen.}

Bei diesem Beispiel ist der hohe Respekt für die technologisch exzellente Lösung durch das dänische Unternehmen Disa verbunden mit leider zu häufig auftretenden gravierenden Fehler mit komplexen Automatisierungs-Lösungen. Eine solche Fehlleistung sollte in einer technisch gebildeten Nation nicht noch einmal passieren! Eine hoch automatisierte Fabrik kann nicht ohne personelle Voraussetzungen in Betrieb genommen werden. Leider wird von den Journalisten zu häufig das nicht vorhandene Problem des möglichen Verlustes von Arbeitsplätzen in das öffentliche Bewusstsein gebracht.

\footnotetext{
Schlussfolgerung: Es ist mir zwar nicht genau bekannt, welcher konkrete Betrag von den vom Investor insgesamt veruntreuten 50 Mio Eur in den "Sand" gesetzt wurden. Der Anteil von Fördermitteln des Freistaates dürfte beträchtlich sein. Wiekann eine kompetente Begutachtung gewährleistet werden, wenn das gesamte Gebiet des Advanced Manufacturing im Freistaat Sachsen nicht den objektiv notwendigen Stellenwert hat? Ein weiterer Aspekt ist m.E. auch die sachlich objektive Auswertung in geeigneten High-Tech Demonstrationszentren
}

\subsubsection{LOMOLD- Unterstützung für ein Südafrikanisches Weltpatent.}

Wie schon in der vorliegenden historischen Dokumentation dargestellt, ergab sich in Verbindung mit der Zusammenarbeit mit einen südafrikanischen Erfinder und Weltpatent-Inhaber eine Chance zur angestrebten "Wiederbelebung" eines Dresdener Unternehmens (Spritzguss-Maschinen- Hersteller (KUASI Freital) beizutragen. Ausgangspunkt war das Interesse des Erfinders (Mr Pieter du Toit) die deutsche Autoindustrie und ein produzierendes Unter- nehmen in Deutschland zu finden.

Trotz erfolgversprechendem Start konnten die Zielstellungen nicht realisiert werden. Das neue effektive Spritzguss- Verfahren auch für langfaserige Materialen ist für den Leichtbau weiterhin von Interesse.

Schlussfolgerung: Prüfung der ehemaligen Kooperation zur Aktualisierung, Da die Gültigkeit des Patentes aus dem Jahr 1998 inzwischen ausgelaufen ist, sollte die aktuelle Auswertung des Lomold-Prinzips im Rahmen der Leichtbau-Allianz Sachsen geprüft werden. 


\section{Schlussbemerkung und Ausblick}

Meine persönliche fachlische Biografie kann mit dem Jahr 1920 erfreulicherweise noch nicht abgeschlossen werden. Das aktuelle Forschungsprojekt EDAM (s. Abb. 81) erfordert weiterhin einige Anstrengungen,damit die innovativen Lösungsansätze zur konturbezogenen Temperierung in der Werkzeugtechnik für das Druckgiessen im Partnerkreis zur industriellen Wirksamkeit geführt werden.Die damit verbundene Vertraulichkeit verbietet in diesem Rahmen weitere Erläuterungen.

Darüber hinaus kann aus gleichen Gründen auch kein konkreter Ausblick zu notwendigen Entwicklungen im Gebiet der Fortgeschrittenen Produktionstechnik in diese Dokumentation aufgenommen werden.Konkrete Aktivitäten sin im Rahmen des VDI im Gange.

-Ausgangspunkt für notwendige Überlegungen sind folgende Fakten:

- der traditionsreiche Industriestandort Sachsen ist nach 30 jahren Deutsche Einheit mit einem Produktivitäts-Rückstand von ca $20 \%$ im Vergleich zu den ABL noch immer in einer unbefriedigenten Position.

---die unmittelbar nach der Wende vollzogene Eröffnung des CIM-TT Zentrums (Festredner Prof.Biedenkopf,Prof.Riesenhuber, Prof. Spur u.a.) war mit wertvollen Zielstellungen und Erwartungen verbunden, die im Laufe der Entwicklungen leider nicht annähernd erfüllt wurden.

--die von Prof Spur vorgeschlagene stärkere Kooperation TU Berlin mit der TU Dresden und die angestrebte Zusammenarbeit mit den Westdeutschen Spitzen-Forschungseinrichtungen (Aachen u. Stuttgart) auf "Augenhöhe“ im Gebiet der fortgeschrittenen Produktionstechnikzusammen zu arbeiten blieb ein nicht realisierter Vorschlag.

--der gravierende Rückgang der Studenten zahlen in den Schlüsseldisziplinen Produktionsautomatisierung und Spanende Formgebung (s. Bild...) entspricht nicht den Anforderungen der sächsischen mittelständigen Industrie.

Für einen realistischen zukunftsorientierten Ausblick bleibt deshalb nur eine Vision:In Übereinstimmung mit dem VDI-Leitmotiv „Der Zweck der Arbeit ist das Gemeinwohl“sollte die Angleichung des produktionstechnischen Niveaus von Sachsen (und auch weiterer Regionen) auf dasvon Baden-Württemberg und Bayern erreichte bundesdeutsche Niveau sein

Ein Lösungsansatz kann derAufbau von anwendungsbezogenen Forschungseinrichtungen in enger Verbindung mit Aus- und Weiterbildung zum Befähigen der effizienten Ausnutzung intelligenter Software und Automatisierungstechnik.

\section{Besondere Würdigung herausragender deutscher Persönlichkeiten.}

\subsection{Professor Spur, anerkannter internationaler Pionier der Fabrik der Zukunft}

Im Verlauf meiner gesamten fachlichen Entwicklung habe ich vielfältige Formen der Zusammenarbeit auf "Augenhöhe" und sachdienlicher Kooperation mit unterschiedlichen Bezugsebenen kennen gelernt

Außer meinen ersten Hochschullehrern und weiteren profilierten Fachkollegen aus Deutschland und der Welt verdienen zwei herausragende wissenschaftliche und menschliche Persönlichkeiten eine besondere Würdigung: Prof. Dr. Dr. h.c. mult G.Spur wird mit voller Berechtigung als Pionier der Produktionstechnik anerkannt.

Mit besonderer Freude kann ich darauf verweisen, das über seinen "Doktorvater" Prof Palitzsch (wiederum Absolvent des legendären Prof Sachsenberg der TU Dresden) die historischen Wurzeln nach 
Sachsen reichen. Mit seinem fachlichen Weitblick war es ihm gelungen den deutschen Pionier der numerischen Steuerungstechniik (Prof. Simon) von Darmstadt nach Berlin zu berufen und gegen den Widerstand konservativer Maschinenbauer erste NC-Maschinen für Lehre und Forschung an der TU Berlin einzusetzen. Vergleichbare Erfahrungen habe ich ebenfalls gemacht, musste ich mich für die erste Investition einer NC-Maschine gegen eine konservative fachliche Umgebung an der TUD, insbesondere aus dem Wissensgebiet Hydraulik durchsetzen.

Davon ausgehend .konnten auch an der TUD alle wesentlichen Entwicklungsetappen nach dem Vorbild der TU Berlin (und natürlich auch der RWTH Aachen, TU Stuttgart u.a.) mit Unterstützung leistungsfähiger Industriepartner der DDR wirkungsvoll mitgestaltet werden. Das bezieht sich auf die erste deutsche NC-Programmiersprache EXAPT, die Weiterentwicklungen der gesamten flexiblen Automatisierung (BAZ, FMS), die DNC-Betriebsweisen u.a.m. Mit der Berufung von Prof Krause für das Arbeitsgebiet Rechnerunterstützte Konstruktion und Arbeitsplanung (CAD) an der TU Berlin waren die CAD/CAM-Wertschöpfungsketten bestens ausgestattet und insbesondere auch die Verbindungen zum IFIP-TC 5 mit beiden Arbeitsgruppen WG 5.2 CAD und WG 5.3 CAM stabil verbunden.

Mit ehrlicher Freude habe ich die zahlreichen hohen Auszeichnungen von Prof Spur zur Kenntnis genommen und die persönlichen fachlichen Gespräche als besonders wertvoll eingeordnet.

Die aktive Teilnahme von Prof. Spur an den von mir verantworteten Fachkonferenzen (Gaussig 1983, PROLAMAT 1988) trug zum hohen Niveau und zur vollen Anerkennung bei.

Auch sein orientierender Vortrag zur Eröffnung des CIM-TT Zentrums an der TU Dresden verdient besondere Anerkennung, auch wenn die weiteren Entwicklungen leider nicht in seinem Sinn verliefen.

Die verantwortungsbewusste Position zur bestmöglichen Ausgestaltung der weiteren gemeinsamen Entwicklungen im wieder vereinten Deutschland zeigte sich unmittelbar nach der Wende in folgenden Fakten: Unter Beachtung der ungeklärten Situation in der Akademie der Wissenschaften wurde eine Arbeitsgruppe (Automatisierungstechnik) unbürokratisch in sein Institut übernommen.

Damit der Lehr- und Forschungsbetrieb an der TH Berlin-Wartenberg ohne Unterbrechungen weiter geführt werden konnte, wurden die von der TU Dresden gekommenen ehemaligen Kollegen aus der damaligen Sektion 14 durch den Einfluss von Prof Spur ohne lästige Eignungsbewertungen in ihren Ämtern bestätigt.(z.B. mein langjähriger Oberassistent Prof Dr.J. Hartmann, und Prof Bahmann u.a.)

\subsection{Professor Leibinger}

Als junger Ingenieur war mir als ebenso junger Kollege Herr Leibinger zu einer Tagung der NC-Society erstmals in den USA begegnet (1974 in Cincinnati) Herr Leibinger war zu dieser Zeit schon Konstruktionsleiter im Unternehmen Trumpf und interessierte sich natürlich für die Möglichkeiten der NC-Bearbeitung im Gebiet der Blechbearbeitung .Die Nutzung der automatisierten Fertigung in diesemAnwendungsgebiet führte zu einer außerordentlichen positiven Entwicklung des Unternehmens Trumpf. Die Spätere Übernahme und der weitere Ausbau war die logische Konsequenz.

Diesen erfolgreichen Aufstieg konnte ich natürlich nur von der größeren Entfernung beobachten und bei fachlichen (IFIP)-Treffen von Stuttgarter Kollegen (z.B. Prof. Storr, Prof. Pritschov) erläutern lassen. Auf diese positiven Merkmale konnte ich mich besinnen, als es im Herbst 1990 darum ging einen Beitrag zur industriellen Umgestaltung der ostdeutschen Wirtschaft zu leisten .Ausgehend von der langfristigen Planung stand im November 1990 das Schloss Gaußig für das traditionelle CAD/CAM Problemseminar zur Verfügung. Deshalb konnte eine Aufteilung in der Weise vorgenommen werden, in dem für 2 Tage primär Führungskräfte aus der Industrie eingeladen wurden.(z.B. Prof Mütze Carl Zeiss Jena; 
Prof Russig WZM-Institut Karl-Marx-Stadt). Als Plenarreferent konnte der damalige Präsident Dr Leibinger des VDMA gewonnen werden. Das war deshalb möglich, weil Dr.Leibinger diese Reise mit einem Informationsbesuch in Neukirch (Entwicklungsbereich des Kombinates Landmaschinen) verbinden konnte. Der orientierende Vortrag von Dr Leibinger endete mit einer denkwürdigen Empfehlung an die Industrie-Partner: "Bemüht Euch um erste Kontakte mit leistungsfähigen und zuverlässigen BRD-Unternehmen als verlängerte Werkbänke-realisiert aber baldmöglich zukunftsorientierte eigene Entwicklungen". Dieser Erfolgs-Grundsatz wurde in dem neu gegründeten Unternehmen Trumpf-Sachsen in Neukirch vorbildlich verwirklicht.

Vom langjährigen Trumpf- Neukirch Geschäftsführer Herrn Strehle (TUD-Absolvent Fertigungsgestaltung) erfuhr ich später, das sich Dr.Leibinger ernsthaft um den Kauf des Schlosses Gaußig bemüht hat. Es sollte als Schulungsheim weiter genutzt und ausgebaut werden. Leider konnte dieser gute Vorsatz nicht verwirklicht werden. Von Herrn Strehle erhielt ich 2008 die Information und Einladung an einem Vortrag von Prof Leibinger im Industrieklub Dresden (Schloss Eckberg) teilzunehmen.Prof. Leibinger hielt einen Vortrag zu seinen Bindungen nach Sachsen, wobei auch seine persönliche Wertschätzung zu Erich Kästner eine besondere Rolle spielte. Nach der angeregten Diskussion im relativ großen Plenum ergab sich die Gelegenheit zu einem 4-Augen-Gespräch mit dem hoch geschätzten Fachkollegen Leibinger inzwischen auch Mitglied im Senat der TU Stuttgart.

Auf meine Frage nach Verbesserungen der noch immer mit ca 25\% Produktivitäts-Rückstand gegebenen kritischen Gesamtsituation in Dresden und Sachsen (Hauptursache unbefriedigende Position zum Gebiet Advanced Manufacturing) wurde mit einer Empfehlung beantwortet: "Halten sie sich an den Spruch von Erich Kästner:-,,Tue Gutes und rede darüber" Dieser gewichtige Ratschlag war für mich ein Anlass, insbesondere auch im Rahmen des VDI in den folgenden Jahren auf dringend notwendige Veränderungen der sächsischen Forschungsstrategie und den zu verbessernten Technologie-Transfer hinzuweisen. Die bisherige fachliche Bilanz bietet Raum für weitere notwendige Aktivitäten. Der Respekt vor einer herausragenden deutschen Wissenschaftler- und Unternehmer-Persönlichkeit ist eine lebenslange Verpflichtung!

Das bedeutet konkret:

- Realisieren geeigneter Forschungsprojekte mit Industriepartnern und öffentliches Wirksamwerden

- Fachliche Einflussnahme mit VDI-Partnern auf politische Entscheidungsträger

\subsection{Professor H. Jäger Direktor des ILK der TU Dresden}

In Verbindung mit der Orientierung auf das Gesamtgebiet Leichtbau, angeregt durch die Anforderungen des Unternehmens SW als wichtiger Auftraggeber für das Unternehmen MIKROMAT Dresden entwickelte sich eine wertvolle Zusammenarbeit in Form eines regelmäßigen Informationsaustausches. Ausgangspunkt war die beidseitige Grundposition, den Freistaat Sachsen mit geeigneten Beiträgen zu einem höheren Produktivitätsniveau zu unterstützen. Das ILK (Institut für Leichtbau und Kunststofftechnik) mit seinem erfahrenen Direktor verdient meines Erachtens desbesondere Anerkennung und Würdigung, weil mit fachlich hohem Niveau und hoher Industrieverbundenheit maßgeblich zur Anerkennung der TU Dresden als Exzellents Universität wirkungsvoll beigetragen wird.

Ich bin Kollegen Jäger auch deshalb zu besonderem Dank verpflichtet, weil er mich bei der Realisierung meiner Grundposition zum „lebenslangen Lernen“ so unbürokratisch unterstützt hat.

Besonders wertvoll waren auch die z.T. gemeinsam mit Prof A. Richter geführten "Stategiegespräche“ zur verbesserten Förderung des gesamten Fachgebietes „Advanced Manufacturing“ in der sächsischen Wissenschaft und Industrie. 

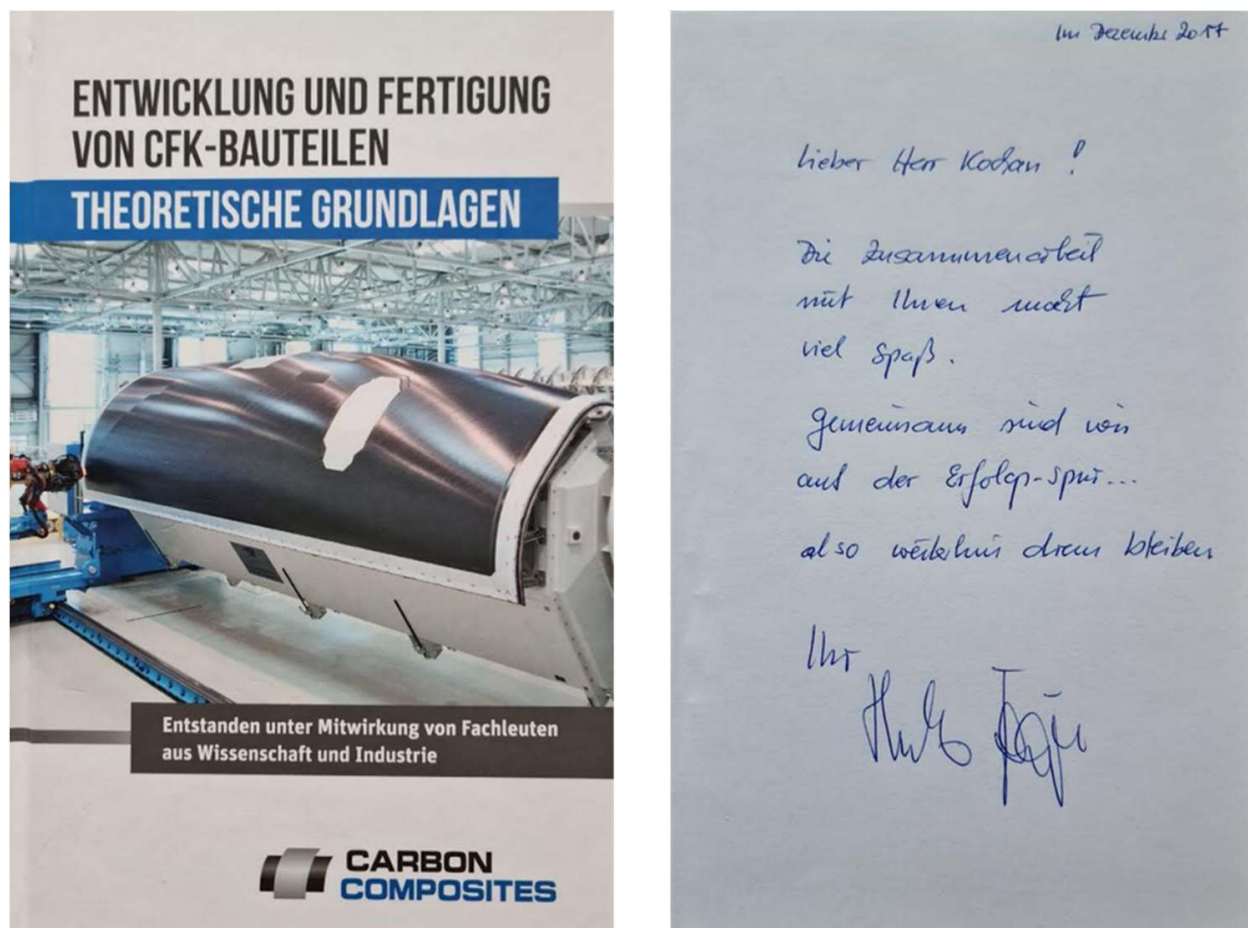

Abbildung 96: Aktuelle Fachliteratur mit persönlicher Widmung von Prof. Jäger

Von Seiten Prof. Jäger als Nachfolger von Prof. Hufenbach sind auch mit der Gründung der Leichtbauallianz Sachsen hervorragende Voraussetzungen gegeben. Meinerseits konnte im regelmäßigen Informationsaustausch der aktuelle Entwicklungsstand im Maschinenbau eingebracht werden. Mit zwei gehaltvollen Vorträgen zu den Innovationstagen Mittelstand wurde durch Prof Jäger Dresden beigetragen. Darüber hinaus erfolgten tiefergehende Untersuchungen zur Nutzung der CFK-Potenziale für Anwendungen dynamisch hochbeanspruchter Werkzeugmaschinen-Komponenten .

Als besonders wertvoll halte ich den zukunftsorientierten Gedankenaustausch zum Wirksamwerden in Richtung eines zielgerichteten Ausbaus des Kenntnisstandes für Ingenieure im gesamten Gebiet des Advanced Manufacturing. Mit besonderen Dank würdige ich die moralische Unterstützung meiner Bemühungen zur Verbesserung des sächsischen Produktivitätsniveaus. Meinen Respekt verdient Prof Jäger für sein persönliches Engagement für die Entwicklung der Wissenschaft und Industrie im Freistaat Sachsen. 


\section{Autoren-Biografie}

Horst Detlef Kochan (geb. 28.06.1935 in Gera)

Auf Empfehlung eines versierten Luftfahrtingenieurs (HeinkelDessau) wurde nach dem Abitur eine Ausbildung als Maschinenschlosser realisiert, was sich meines Erachtens absolut positiv auswirkte.

Ich habe ein Studium am Institut für Fertigungstechnik an der TU Dresden von 1955 bis 1961 mit dem Abschluss als Diplomingenieur absolviert. Den Titel „Doktor Ingenieur" erhielt ich am 01.03.1966 Dissertationsthema: „Fertigung rotationssymmetrischer Werkstücke auf programmgesteuerten Spitzendrehmaschinen. Ein Beitrag zur Ermittlung technisch optimaler Einsatzmöglichkeiten programmgesteuerter Werkzeugmaschinen und technologischer Anforderungen an die Konstruktion"

Doktor der Wissenschaften (Dr. sc. techn.) wurde ich am 05.11.1971

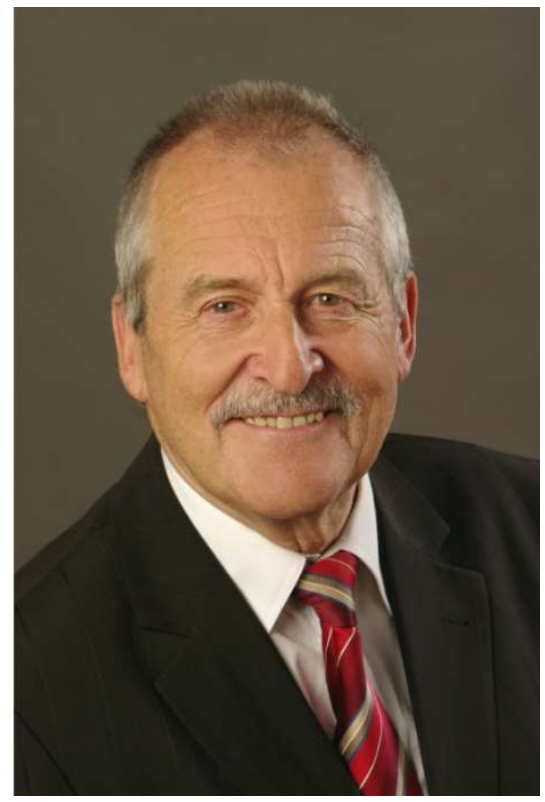

Im Gegensatz zu einigen Fachkollegen wurde nach der Wende auf die Umwandlung dieses Titels in „habil“ verzichtet. Mit dem Titel „habil“ wird primär die Lehrbefähigung dokumentiert. Die Lehrbefähigung (Facultas Docenti) war meinerseits schon am 01.06.1970 nachgewiesen.

Die mit dem Titel „Dr. sc. techn.“ verbundene Anerkennung:

„Aufgrund hervorragender wissenschaftlicher Befähigung auf dem Gebiet Fertigungsprozessgestaltung und erfolgreicher Tätigkeit als Leiter wissenschaftlicher Kollektive“

wird mit dem Begriff „habil“ nicht ausreichend dokumentiert.

Weitere fachliche Entwicklungsschritte:

Beruflicher Werdegang

1961 Diplomabschluß am Institut für Fertigungstechnik der TU Dresden

Ab 1961 wissenschaftlicher Assistent am Institut für Fertigungstechnik, Bereich Industrielle Fertigung und Automatisierung

Ab 1965 Wissenschaftlicher Oberassistent am gleichen Institut

Seit 1967 Abteilungsleiter im Direktionsbereich F/E im VEB Carl Zeiss Jena; Aufbau der Abteilung F/E Technische Vorbereitung (mittels Rechneranwendung) von 7 auf 30 Mitarbeiter

2/1970-9/1970 Tätigkeit als stv Hauptabteilungsleiter im Forschungszentrum Fertigungstechnik KarlMarx-Stadt zur Überführung der Forschungsergebnisse an den Werkzeugmaschinenbau (u.a. für Steuersoftware für das 1. Flexible Fertigungssystem PRISMA 2 der DDR)

01.10.1970 Berufung als Dozent für das Fachgebiet Fertigungsgestaltung an der TU Dresden

01.09.1975 Berufung zum ordentlichen Professor für Fertigungstechnik/ Technologische Programmierung (im Folgenden FT/TP) an der TU Dresden

01.10.1975 (bis 1983) Berufung zum stellvertretenden Direktor für Forschung an der Sektion Fertigungstechnik und Werkzeugmaschine der TU Dresden 
Tätigkeit in wissenschaftlichen Gremien/Inland

- 1968/1969 Mitwirkung bei der Ausarbeitung der Nationalen Konzeption zur „Automatisierung der technischen Produktionsvorbereitung"

- (ca 1970) Prognose zur Entwicklung der technologischen Prozesse der Stoffbe- und -verarbeitung beim Ministerrat der DDR

- Ca 1970/71) Mitarbeit bei der Vorbereitung und Realisierung des Regierungsabkommens zur "Automatisierung der technischen Vorbereitung im Maschinenbau „ mit der UdSSR

- 1972-1990 Aufbau und Leitung einer Nationalen Arbeitsgruppe „Rechner in der Technik“ bei der Ingenieurorganisation „Kammer der Technik“

- 1978-1980 Federführende Ausarbeitung einer Wissenschaftskonzeption „Technologie der metallverarbeitenden Industrie mit einer Arbeitsgruppe von 20 Professoren der DDR

- 1978-1984 Mitglied des wissenschaftlichen Beirates für Maschineningenieurwesen und Vorsitzender der Arbeitsgruppe „, beim Forschung, Wissenschaftsentwicklung und wissenschaftlicher Nachwuchs „, beim Ministerium für Hoch- und Fachschulwesen.

- 1982-1985 Mitglied des wissenschaftlichen Rates der komplexen Forschungsaufgabe „Automatisierte Produktion"

- 1985-1989 Mitglied des Zentralen Arbeitskreises „Rationalisierung der technischen Produktionsvorbereitung" beim Forschungsrat der DDR; Gleichzeitig Arbeitsgruppe „Anwendung der Mathematik und Datenverarbeitung in der Volkswirtschaft"

- 1986-1089 Beauftragter des Ministers für Hoch- und Fachschulwesen und des Rektors der TUD

- Für die Entwicklung und Anwendung der Schlüsseltechnologie CAD/CAM. In dieser Eigenschaft federführende Ausarbeitung einer langfristigen Strategie der Grundlagenforschung im Gebiet CAD/CAM bis zum Jahr 2000

\section{Mitarbeit und Funktionen in internationalen Gremien}

- seit 1972 Mitglied in der IFIP (Internationale Föderation für Informationsprozesse) ,Arbeitsgruppe CAM, gleichzeitig Mitglied des Internationalen Programm- Komites (IPC) der 3-jährigen PROLAMAT_Konferenzen

- 1977-1983 Chairman der IFIP AG CAM;

- Vorsitzender des IPC für die PROLAMAT-Konferenzen 1079 in ANN Arbor USA und 1982 in Leningrad, UdSSR

- Seit 1980 DDR Mitglied im IFIP-TC 5 (Rechner in der Technik)

- 1979-1990 Mitglied im Redaktionsbeirat der Zeitschrift "Computers in Industrie“

- Seit 1992 Initiator und Mitwirkender für mehrerer IFIP-Arbeits- Konferenzen.

- 1988 Fachlicher Höhepunkt. Erfolgreichste PROLAMAT-Konferenz in Dresden

\section{Öffentlichkeits-wirksame Aktivitäten nach 1990}

- 1991 IFIP- Arbeitskonferenz in Gaussig/Dresden „Fortgeschrittene Produktionstechnik undKünstliche Intelligenz"

- 1992 -1997 Anwender-Kongresse SFM mit NC-Gesellschaft (UIm; Dresden)

- 1998- 2005 3D Erfahrungsforen Werkzeug -und Formenbau (Darmstadt-Dortmund-Dresden; Berlin)

- 2001-2016 Internationale CIRP -Konferenzserie (Aller 3 Jahre) COMA-(COMPETITIV MANUFACTURING) an der Universität Stellenbosch- RSA (2001 als Co-Chairman, alle weiteren Konferenzen als Mitglied des Programm-Komitees)

- 2013 nochmalige IFIP-Konferenz NEW PROLAMAT in Dresden

- 2015-2020 VDI-Konferenz-Serie Innovationstage Mittelstand in Dresden 


\section{Zusammenstellung wissenschaftlicher Publikationen}

1. Bücher, Broschoren, Lehrbriefe

1.1. Bucher, Broscharen

1. Kochan, D.; Strenpel Progranngesteuerte Werkzeugsaschinen und Ihr Binsatz; Reihe

Automatisierungstechnik, VEB Verlag Technik Berlin 1969

2. Blume, F.; Mitautor und Autoren-

Einfürung in die Pertigungstechnik; kollektiv

3. Jacobs, H. -J. Spanungsoptimierung Jacob; Kochan, D. VEB Verlag TEchnik Berlin 1977

Lizenz in Ungarn und UdSsR

4. Kochan, D. Jacobs, H. -J.

FertigungsprozeBgestaltung und Informationsverarbeitung; VEB Verlag Technik, Berlin 1977; Lizenz in Ungarn u. UdSSR

5, Kochan, D. Enderlein, H. Fichtner, D.

Mehrmaschinenbedienung im Maschinenbau VBB Verlag Technik Berlin 1979, Lizenz in Bulgarien

6. Kochan, D. u.a.

Integration of CAD/CAM; North Holland

Publishing House 1983

7. Sohiffer, F. und Autorenkollektiv Mitautor

FertigungsprozeBgestaltung VEB Verlag Technik Berlin, 1982. 1988

8. Mälier, G.

Lexikon Technologie Autorenkollektiv

Verlag Technik Berlin, 1985

9. Kochan, D. u,a.

Developments in Computer Integrated Manufacturing state of the art-CAM: Springer Verlag, Heidelberg, Berlin, New York, Tokyo, 1986

10. Kochan, D. U,a.

CAD/CAM Schlusseltechnologie als Intensivierungsfaktor; Verlag Die wirtschaft, Berlin; 1988

11. Koohan, D. olising, $G$.

12. Kochan, D. Kuntsche, $\mathrm{P}$.

13. Kochan, D.

\section{Software for Manufacturing} Proceedings

PROLAMAT 'BB; Dresden; florth-Holland Publishing-House Aasterdan; 1989

Kleines Lexikon der CAD/CAM Technik

Verlag Technik, Beriln, 1989

Lizenz Hauser Verlag, München, 1989

Conplex Machining and AI Methods North-Holland, Ansterdam, 1991 
14. Kochan, D.

15. J. Feldhusen

K. H Grote

Autorenkollektiv

Mitautor

16. George L. Kovacs Detlef Kochan
Solid Freeform Manufacturing - Advanced Rapid Prototyping (Elsvier, Amsterdam, 1993)

Pahl/ Beitz

Konstruktionslehre, Springer 2013

Digital Product and Prozess Development System,New PROLAMAT, Springer, 2013

\subsection{Lehrbriefe}

1. Lehrbrief II (Projektierung teohnologischer Prozesse) 1967

Tu Dresden - Industrielle Fertigung (1) Verlag Technik

2. Lehrbrief III (Projektierung technologischer Prozesse) 1968 TU Dresden - Industrielle Fertigung (1) Verlag Technik

3. Lehrbrief IV (Projektiorung technologischer Prozesse) 1969 TU Dresden - Industriel1e Fertigung (1) Verlag Technik

4. 1. FertigungsprozeBgestaltung II (Technologische Pro- 1972 gramilerung) Lehrbrief I; Verlag Technik

2. Fertigungsprozeßgestaltung II (Technologische Pro- 1972 grannierung) Lehrbrief II; Verlag Technik

3. FertigungsprozeBgestaltung III (Technologische 1973 Programmierung) Lehrbrief V; Verlag Technik

4. PertigungsprozeBgestaltung I Lehrbrief IV (1) 1975 Verlag Technik

5. FertigungsprozeBgestaltung I Lehrbrief $V(1)$

5. Lehrbriefroihe CAD/CAM $(1-7) \quad 1985$ Verlag Technik

Publikationen in Fachzeitschriften ca 200

Gutachten für Dissertationen: 126 


\section{Persönliche Bilanz nach mehr als 60 Jahren}

Als ich im Frühjahr 2020 zum Abschluss meiner Verpflichtung als Vorsitzender des VDI-Arbeitskreises "Rapid Innovation" mit einer Auszeichnung verabschiedet wurde, war in der Ehrenplakette das VDI Motto: "Der Zweck der Arbeit soll das Gemeinwohl sein" eingeprägt .Eine persönliche Bilanz sollte deshalb von diesem generellen Kriterium ausgehen. Dabei wurde mir klar, warum ich einen beachtlichen Fachkollegenkreis mit besonderer Hochachtung begegnet bin und auch deshalb meine persönliche Bilanz nach diesem übergeordneten Kriterium einordnen kann. Zu dem wichtigen Aspekt der positiven Einstellung der Bereitschaft zum lebenslangen Lernen, gehört dazu die Orientierung an fachlichen Vorbildern. Die fachliche Kooperation mit anerkannten Erfahrungsträgern aus der Wissenschaftlich und Industrie gehört deshalb zur "Sonnenseite" meiner Bilanz, die ich aus heutiger Sicht in ziemlich exakt in 2x30 aktive Lebensjahre einteilen kann, wobei sich die "Höhen und Tiefen" in beide Zeitabschnitte verteilen.

Die Zeit 1960--1990 war gekennzeichnet durch mehrere Ausbildungs- und Qualifizierungsetappen in Verbindung mit der Teilnahme an zukunftsorientierten Forschungsvorhaben. Ein ausgeprägter Schwerpunkt der eigenen wissenschaftlichen Aktivitäten war dabei von Beginn an die effiziente Anwendung informationstechnischer Fortschritte im gesamten fertigungstechnischen Umfeld. Das internationale Entwicklungstempo in diesem Hightech -Gebiet war deshalb eine permanente Herausforderung, die durch die politischen Grenzen des "Eisernen Vorhanges" mit dem damit verbundenen Embargo- Schranken noch gesteigert wurde. Das ständige Bemühen, um zu technologischen Fortschritten beizutragen, kann man durchaus als eine auch "sportliche " Herausforderung einordnen, der sich nicht alle Kollegen im unmittelbaren fachlichen Umfeld stellen wollten oder konnten? Es war zum Teil bequemer, sich einen eigenen Weltstand zu definieren und auf international anerkannte Veröffentlichungen zu verzichten. Allerdings bleibt es für mich unklar, mit welcher Befugnis der so genannte "Mittelbau" nach der Wende die Arbeitsleistungen in den eingesetzten Kommissionen zu bewerten hatte.

Es hat mir natürlich einige Mühen, aber auch viel Freude gemacht mich für mein Heimatland, welches mir meine fachliche Entwicklung ermöglicht hat, bestmöglich einzusetzen. Für mich bleibt eine positive Bilanz, auch wenn nach der historischen Wende, auf mein Mitwirken zur weiteren Ausgestaltung der erarbeiteten nationalen und internationalen Position an meiner ehemaligen " Alma Mater" verzichtet werden musste. Mit besonderen Bedauern bleibt zum Nachteil meines jetzigen Heimatlandes der Fakt bestehen, das das bedeutende Wissensgebiet "Advanced Manufacturing" nicht mehr den objektiven Anforderungen entsprechend angemessen vertreten ist.

Der Zeitraum von 1990--2020 kann nochmals etwas differenziert in die gesamte Bilanz eingeordnet werden. In den Jahren bis 1993 konnte auf den fortgeschrittenen Erkenntnisstand unmittelbar mit leistungsfähigen Ergänzungsinvestitionen zur verbesserten Ausstattung des CIM-TT beigetragen werden. Damit waren gleichzeitig gute Voraussetzungen geschaffen, um mit gezielten wissenschaftlichen Veranstaltungen neueste Erkenntnisse insbesondere im Gebiet des "Additiv Manufacturing" ( damalige Begriffe SFM bzw Rapid Prototyping) zu vermitteln. Noch im Jahr 1991 fand unter meiner Verantwortung eine IFIP Arbeitskonferenz zum Thema "Künstliche Intelligenz in der Fertigungstechnik" statt .Noch heute sollten die grundlegenden Erfahrungen Beachtung finden

Obwohl mit Schreiben vom Wissenschaftsminister Prof Meyer (6.10.1993) einer Weiterbeschäftigung an der TU Dresden nichts im Wege stand, erfolgte bekanntlich

die damals übliche Bedarfskündigung. Das bleibt aus der jetzigen historischen Sicht etwas widersinnig, da mir im gleichen Jahr eine besondere Ehrung durch die CIRP-die Elite der internationalen Fertigungstechnik zuteil wurde .Ich war als einziger "Ost-Prof" mit Vortrag zu einem Ehrenkolloquium der USA Pioniere der Produktionsautomatisierung nach Slowenien eingeladen. 
Mit einer solchen moralischen Stärkung, konnte ich mir -im Gegensatz zu einigen ebenfalls Betroffenen- juristische Zeit Verschwendungen einsparen. Mit gesunden Optimismus und den erarbeiteten Erfahrungen im damals völlig neuen Wissensgebiet der direkten Generierung geometrisch beliebiger geometrischer Objekte aus 3D-CAD-Daten konnte ich als nicht mehr ganz junger Unternehmer meine eigene Ausgründung aus der TUD realisieren. An dieser Stelle verdient es besondere Anerkennung und Wertschätzung, das noch einige Zeit die als Großinvestition eingeordnete Stereografie-Anlage noch einige Jahre mit genutzt werden konnte. Die hoch interessanten, heraus forderten , abwechslungsreichen 13 Jahre als "Jungunternehme" ,ergänzt durch vielseitige internationale Aktivitäten, insbesondere der Republik Südafrika sind mit wunderbaren Erinnerungen verbunden. Als einzigen dunklen Schatten muss ich die Jahre 2005-2006 einordnen, u.a. auch gekennzeichnet durch eine "kleine Autokrise". Aus verschiedenen Gründen war es Zeit das Unternehmen an fachlich geeignete Interessenten zu übergeben. Mit dem fachlich am besten geeigneten "Investor" gab es leider keinen guten Abschluss .Entgegen getroffenen Vorabstimmungen kam es zu betrügerischen Handlungen bis zur Insolvenz .Nur wenige Jahre später kam es mit dem hochinteressanten Automatisierungs -.Vorhaben wegen falscher Personalpolitik zu einen ebensolchen Ende. Die in dem Gerichtsverfahren nachgewiesenen veruntreuten ca 50 Mio EUR hätten wahrlich eine bessere Verwendung verdient.

Die folgenden Jahre als Technologie- Berater und insbesondere als Projektleiter Produktionstechnik am ZAFT (Zentrum für Angewandte Forschung) an der HTW Dresden brachten ständig neue Herausforderungen. Mit Unterstützung der Professoren der Fertigungstechnik und weiterer Disziplinen wurden inzwischen mehrere Generationen von Studenten in Form von Praktika und Diplomarbeiten in anspruchsvolle Projekte einbezogen. Mit dem dabei jeweils engen Bezug zum Wissensgebiet der Fortgeschrittenen Produktionstechnik (Advanced Manufacturing) wird unmittelbar zur Stärkung der mittelständigen Industrie beigetragen. Die damit charakterisierte durchaus erfreulicherweise positive Gesamtbilanz bringt mich noch nicht auf den Gedanken das aktive Wissenschaftler- Leben abzuschließen. Insbesondere für das "Sächsische Gemeinwohl" gibt es noch Einiges zu tun!

Nach 60 herausfordernden, hoch interessanten und auch erfolgreichen Jahren kann ich eine durchaus zufriedene Bilanz ziehen Dafür möchte ich mich ganz besonders bei allen ehemaligen Mitarbeitern, Kollegen, Doktoranden und natürlich auch der großen Anzahl von Studenten sehr herzlich bedanken .Ohne diese stets vertrauensvolle Zusammenarbeit wäre mein fachliches Leben nicht so erfolgreich verlaufen

.Die längste Zeit der wissenschaftlichen Aktivitäten wurde auf Augenhöhe mit der deutschen und internationalen Elite der Fertigungstechnik und weiterer MINT-Partner gestaltet. Stellvertretend für die häufig aus der fachlichen Zusammenarbeit resultierenden persönlichen Freundschaften möchte ich Prof. Dr.-Ing habil. Hans Göldner hiermit einen Gedenkstein setzen. Prof. Hans Göldner führte mich als Hochschullehrer über die Hürden der Technischen Mechanik- und später konnten wir gemeinsam im Beirat Maschinen Ingenieurwesen des Ministeriums für Hoch-und Fachschulwesens der DDR viele nützliche Beiträge zur Verbesserung der Lehre und Forschung einbringen. Eine besondere Würdigung verdient über einen Zeitraum von mehr als 40 sportlichen Jahren seine anerkannte Position als „Präsident" der Professoren Sportgruppe Volleyball der TU Dresden bis zu seinem Tod 1920 im Alter von 92 Jahren.

Dresden im April 2021

Detlef Kochan 
Ergänzende und vertiefende Beiträge 
Ergänzende und vertiefende Beiträge zur kollektiven Entwicklung des Lehr-und Forschungsgebietes Fertigungstechnik/ Technologische Programmierung

Mit einem Teil der von mir betreuten Doktoranden gab es nach der historischen Wende noch fachliche und auch persönliche Kontakte, insbesondere auch im Rahmen des Freundeskreises der Absolventen der Produktionstechnik und spanenden Fertigung (PAZAT).

Dabei gab es meist nur die Gelegenheit für kurze persönliche Gespräche und keine Zeit für vertiefenden Gedankenaustausch zu den jeweiligen fachlichen Weiterentwicklungen.

Mit meiner Einladung zu meinem 85. Geburtstag Mitte Juni 2020 ergab sich die Möglichkeit zum Nachholen dieses Anliegens.

Die ursprüngliche Orientierung der elektronischen Speicherung entsprechender Redebeiträge, erwies sich unter den Corona-Bedingungen als eine unsichere Variante.

Deshalb wurde von den 15 Teilnehmern an dem "gemütlichen" Beisammensein der Vorschlag einer schriftlichen CV-Dokumentation von 10 Teilnehmern wahrgenommen.

Damit ist eine wertvolle Ergänzung und Vertiefung-wie erhofft und erwünscht-für meine Memoiren entstanden.

Die beachtliche Breite und Vielfalt der fachlichen Weiterentwicklungen ist m.E. ein wertvolles und interessantes Zeit-Dokument, wie mit praxisorientierten wissenschaftlichen Ergebnissen auch nach der Promotion zu messbaren Fortschritten beigetragen wurde.

Dafür gilt mein herzlicher Dank und besondere Anerkennung meinen geschätzten Mit-Autoren.

Detlef Kochan 


\begin{tabular}{|l|l|l|}
\hline Zeitabschnitt & Inhaltliche Schwerpunkte & $\begin{array}{l}\text { Absolvent/ Mitarbeiter/ } \\
\text { Doktoranden }\end{array}$ \\
\hline $\begin{array}{l}\text { Ursprünge } \\
1950-1960\end{array}$ & Institut für Fertigungstechnik & \\
\hline $\begin{array}{l}\text { Anfänge } \\
1961-1970\end{array}$ & $\begin{array}{l}\text { Lehr- und Forschungsgebiet industrielle } \\
\text { Fertigung und Automatisierung }\end{array}$ & [Dipl.-Math. G. Franz] \\
\hline $\begin{array}{l}\text { Weiterentwicklungen } \\
1970-1975\end{array}$ & $\begin{array}{l}\text { NC-Programmierung, } \\
\text { Produktionsautomatisierung }\end{array}$ & $\begin{array}{l}\text { [Prof. Dr. J. Hartmann] (1972) } \\
\text { Prof. Dr. Weiß (1973) }\end{array}$ \\
\hline $\begin{array}{l}\text { Weiterentwicklungen } \\
1975-1980\end{array}$ & $\begin{array}{l}\text { Algorithmisierung, Mathematisierung, } \\
\text { Optimierung }\end{array}$ & $\begin{array}{l}\text { Dr. H. Dolze (1976) } \\
\text { Prof. Dr. A. Richter (1978) } \\
\text { [Prof. Dr. D. Dimitrov] (1978) }\end{array}$ \\
\hline $\begin{array}{l}\text { Weiterentwicklungen } \\
1980-1985\end{array}$ & CAD/CAM, PLM u.a. & $\begin{array}{l}\text { Prof. Dr. Klünder (1981) } \\
\text { Prof. Dr. A. Nestler (1984) } \\
\text { Dr. Dr. Rahmig (1985) }\end{array}$ \\
\hline $\begin{array}{l}\text { Weiterentwicklungen } \\
1985-1990\end{array}$ & CAD/ CAM- Labor, CIM-Entwicklung & $\begin{array}{l}\text { Dr. B. Schennerlein (1985) } \\
\text { Prof. Dr. P. Bonitz (1986) } \\
\text { Dr. I. Berndt (1987) } \\
\text { Dr. Chr. Schöne (1987) } \\
\text { [Prof. Dr. C. Friedrich] (1987) }\end{array}$ \\
\hline $\begin{array}{l}\text { Weiterentwicklungen } \\
\text { und Veränderung } \\
1990-2020\end{array}$ & $\begin{array}{l}\text { CIM-TT-Zentrum; } \\
\text { Additive Manufacturing }\end{array}$ & \\
\hline
\end{tabular}

Mit dieser Dokumentation möchte ich gleichzeitig die leider schon verstorbenen ehemaligen Kollegen Dipl.-Math. G. Franz , die Prof.Dr. D. Dimitrov, Dr. C. Friedrich und Dr. J. Hartmann würdigen. 
Zum 85. Geburtstag von Prof. Dr. sc. techn. Detlef Kochan

\author{
Persönlicher Beitrag \\ über fortgeschrittene Fertigungstechnik und Digitalisierung
}

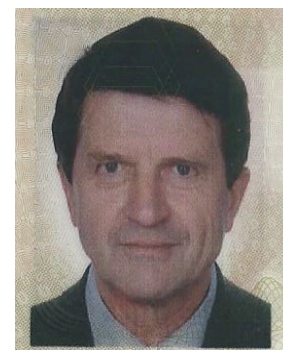

\title{
A. Etappen der fachlichen Entwicklung nach der Promotion (ab Juni 1973)
}

\section{Zeitraum 1973 bis 1976: Kombinat Umformtechnik Erfurt}

Mitarbeiter Technologische Planung /Abteilungsleiter Technologische Rationalisierung

Schwerpunkte der Tätigkeit waren die Auswahl und Einführung geeigneter technologischer Verbesserungen. Die Einführung neuer Verfahren wie zum Beispiel: Gewindewirbeln bei Druckspindeln, Ejektorbohren, Zahnflankenschleifen für große Module, Wendeplattentechnik beim Bohren und Fräsen (Kooperation WKS), Glattwalzen, sowie Einsatz neuer Vorrichtungen - waren eine permanente Aufgabe. Bei der Bearbeitung von großen Schweißbaugruppen (Kopfstücke, Seitenständer, Tische, Stößel) für Pressen wurde der schrittweise Übergang zur Komplettbearbeitung zur wichtigen Herausforderung. Die Produktion auf konventionellen Horizontal-Bohrwerken, Radialbohrmaschinen mit einem beachtlichen Anteil von Handarbeit (z.B. Anreißen) erforderte viele Transporte mit Hallenkränen, führte zu langen Durchlaufzeiten, körperlichen Anstrengungen und Unfällen. Als fertigungstechnische Lösungen wurden in betrieblichen Projektteams das Flexible Fertigungssystem "Skoda -PC3“ und die Portalfrästechnik von Waldrich Coburg nach ausgiebigen Sortimentsanalysen ausgewählt, Investitionsvorbereitungsmaßnahmen umgesetzt und schließlich realisiert. Neben der Mitarbeit am Gesamtkonzept oblag mir die Entwicklung der Spanntechnik in Kooperation mit dem FZW Karl-Marx-Stadt. Infolge der materialsparenden Konstruktionsweise der Baugruppen galt es, besonders

Schwingungsanfälligkeiten zu überwinden. Merkmale des PC 3: arbeitsteiliges System aus sich ergänzenden und ersetzenden Maschinen und Spannplatz darunter ein CNC- Bohrwerk, rechnergeführter Werkstücktransport.

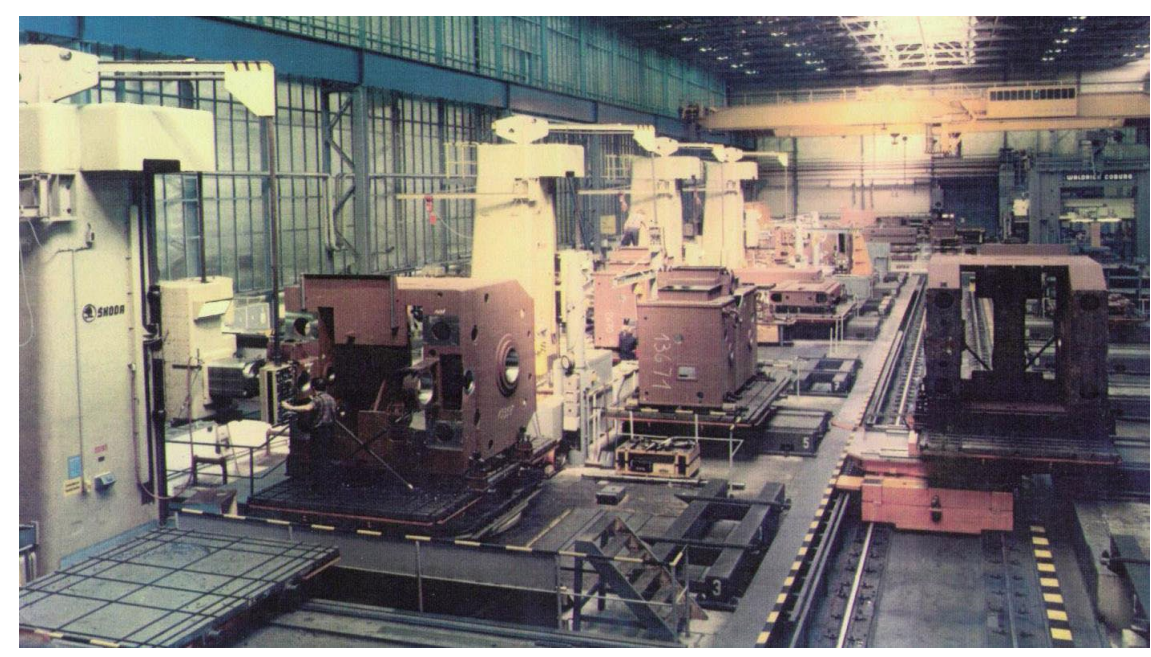

Komplettbearbeitung mit flexiblen Fertigungseinrichtungen im Schwermaschinenbau - ab ca. 1976

Der Einsatz des genannten Fräsportals (3,20 m Durchlass, Tischlänge als Doppeltisch 12m) erfolgte für flache Baugruppen (z.B. Aufspannplatten, Seitenständer) mit dem Ziel der 5Seiten-Bearbeitung in einer Aufspannung. Winkelfräsköpfe mit hohen Antriebsleistungen 
waren dazu Voraussetzung. Das Tiefbohrverfahren Ejektorbohren für Ziehstiftbohrungen ergänzte die Ausstattung. Da beide Systeme mit unterschiedlichen Konzepten der Komplettbearbeitung mit moderner Werkzeugtechnik ausgestattet waren, konnten auch die Prozesszeiten deutlich gesenkt werden. Beide Lösungen wirkten nachhaltig und befanden sich noch nach 2012 im Einsatz. Dem ersten Fräsportal FP 320 folgten im zeitlichen Abstand noch weitere, eines sogar mit Bestückungsroboter für Werkzeugwechsel. Für beide Technologiekonzepte mussten umfangreiche Wirtschaftlichkeitsnachweise erarbeitet werden. Die Beteiligung daran war für mich auch ein Lernprozess.

Nebenberuflich übte ich in dieser Phase eine Lehrtätigkeit an der Außenstelle der Ingenieurschule Schmalkalden in der Betriebsakademie in Erfurt aus.

\section{Zeitraum 1977 bis 1981: Fahrzeug- und Jagdwaffenwerk Suhl}

Mitarbeiter des Haupttechnologen / Abteilungsleiter Produktionsvorbereitung Fahrzeuge

Statt Schwermaschinenbau erlebte ich nun die Massenfertigung von Mopeds. Die Abteilung war zuständig für die technologische Prozessplanung (Verfahrensauswahl, Maschinen- und Werkzeugauswahl, Erprobungen, Nullserien, Rationalisierung und neue Produktionskonzepte) und gliederte sich in mehrere Gruppen. Ein besonderer Schwerpunkt lag in dieser Zeit in der Entwicklung und Serieneinführung der neuen Motorreihe M531/541. Der Gesamtumfang an Maschinen - Investitionen umfasste eine Vielzahl von Standard- und Sondermaschinen inclusive einer automatischen Taktstraße für Motorengehäuse. Dazu kamen unzählige Werkzeugausstattungen, Sondereinrichtungen, Mess- und Prüfmittel. Die Nullserie war kompliziert, weil zu dem geplanten Abschlusszeitpunkt noch nicht alle SerienAusrüstungen vorhanden und erprobt waren. Der hohe Anteil an Einzweck - Sondermaschinen für viele einzelne Arbeitsgänge bedingt durch hohe Stückzahlen wurde dem Unternehmen nach der politischen zur Wende zum Verhängnis, als mit dem Markt die Stückzahlen wegbrachen und die nötige Flexibilität der Ausrüstungen fehlte - was auch auf den damals geringen Anteil an CNC-Maschinen zurück geführt werden kann.
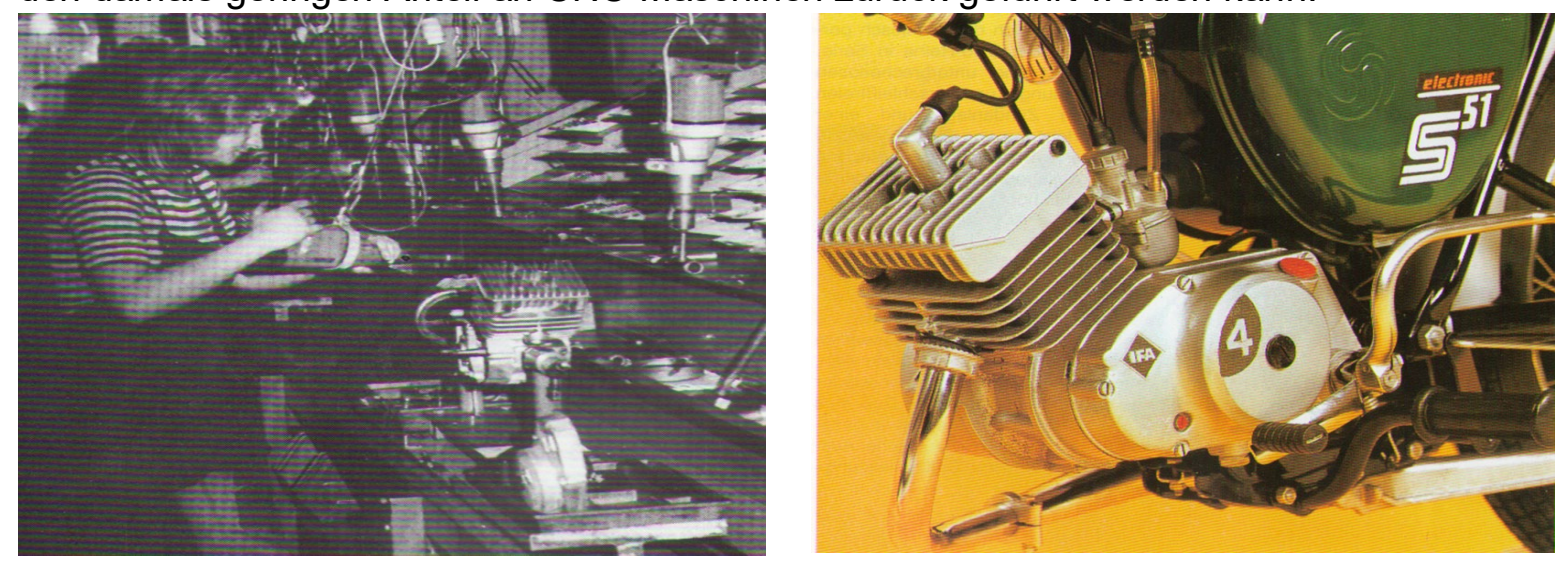

Zusammenbau des Motors M531/541 als Gruppenmontage /Nestmontage;

Bild aus E. Dähn: Motorräder aus Suhl

Die Montage der neuen Motorenreihe wurde von Fließbandmontage (36 Arbeitsstationen) auf Gruppenmontage in 12 Montagenestern umgestellt. Durch Selbstbestimmung der Arbeitsabläufe konnten die Austaktungsverluste und die Monotonie der Arbeit erheblich reduziert werden. An der technologischen Vorbereitung war neben den Kollegen der Montagetechnologie auch die Hochschule Zwickau (Prof. Enderlein) und Prof. E. Müller (TU Chemnitz) - damals noch als Diplomand - beteiligt.

Auch in dieser Zeit war ich nebenberuflich tätig - an der TH IImenau als Dozent für NCProgrammierung und Fertigungsprozessgestaltung. Darüber hinaus war ich zeitweilig Dozent an der Simson -Betriebsakademie. 
Zeitraum 1982 bis 1992: Geräte- und Pumpenbau Merbelsrod (GPM)

Gruppenleiter Betriebsmitteltechnologie und Werkzeugbau / Leiter der Arbeitsvorbereitung

Das Produktspektrum des Unternehmens - Kühlmittelpumpen für alle Typen von Verbrennungsmotoren, Ölpumpen sowie Gleitringdichtungen für Pumpen aller Art erforderte eine komplette persönliche Neueinarbeitung in die produktbezogenen Prozessspezifika. Die Prozesse umfassten alle Fertigungsformen sowie alle Fertigungsarten von der Einzel- bis zur Großserienfertigung. Neben Leitungsaufgaben wurden Eigenkonstruktionen von Vorrichtungen, Prüfständen und Prüfmitteln erstellt. Operative Arbeiten - wie zum Beispiel räumliche Umstrukturierungen des Werkzeugbaus waren häufig an der Tagesordnung. Hinzu kam der Aufbau einer eigenen Druckgießerei. Dazu erfolgte ergänzend die Einführung des CNC - Fräsens und CNC-Senkerodierens im Werkzeugbau. Nach der Wende kam als neuer betrieblicher Schwerpunkt die Aufbereitung von Wasserpumpen für in hohen Stückzahlen hinzu. Es erfolgte nun auch die verstärkte CNC Maschinen - Einführung für die Teilefertigung. Hohe Positioniergenauigkeiten und Rekord verdächtig geringe Nebenzeiten, die noch zum Teil zeitlich überlagert mit der Bearbeitung waren, erhöhten das technologische Niveau beträchtlich. Die Hauptzeit wurde durch diese Parameter wieder interessant - somit auch die Optimierung der Schnittwerte.

Es wurden betrieblich intensive Aktivitäten zum Ausbau bestehender Kundenbeziehungen und zur Gewinnung neuer Kunden ausgeführt. Trotz aller Bemühungen trat ein massiver Stellenabbau in Kraft, um die Existenz des Unternehmens zu erhalten.

\section{Zwischenfazit zum Praxisteil:}

Das Studium und Forschungsstudium (Fertigungstechnik und Fertigungsprozessgestaltung) an der TU Dresden haben mir ermöglicht, betriebspraktische Aufgaben erfolgreich zu lösen, und sich somit für mich ausgezahlt. Digitale Planungsmethoden wurden in der Praxis erst allmählich eingeführt und in Teilbereichen (z.B. NC-Fertigung) durchgesetzt. In der Praxis standen immer das Produkt und eine optimale Wertschöpfung im Vordergrund. Präferiert wurden schnelle technologische Maßnahmen zur Produktabsicherung. Neben der Zeit spielte das Qualitätsmanagement eine wichtige Rolle. Auch Kosten- und Wirtschaftlichkeitsrechnungen müssen heute vom Fertigungsingenieur auf dem jeweils aktuellen Methodenstand beherrscht werden.

\section{Zeitraum 1993: Bildungswerk der Thüringer Wirtschaft / Aktivitäten}

- Anpassungsausbildung mit REFA- Qualifizierung zum Industrial Engineer, Auffrischung Informatik/Steuerungstechnik/Hydraulik/Pneumatik/Wärmetechnik u.a.

Die gestufte REFA -Ausbildung (Grundlehrgang, Fachlehrgang, Seminar Industrial Engineering) erwies sich für die spätere Tätigkeit an der FH Schmalkalden als nützlich.

\section{- Berufungsverfahren an der FH Schmalkalden:}

Nach der Bewerbung auf die Professorenstelle Arbeitsvorbereitung /Industriebetriebslehre erfolgte die Berufung mit Wirkung ab Beginn Sommersemester 1994. Mein Berufungsverfahren erfuhr in dankenswerter Weise durch Prof. Kochan und Prof. Hartmann Unterstützung in Form von Beratung und Gutachten.

- Mitwirkung an Unternehmensberatungsaufgaben (insbesondere REFA -Zeitstudien).

Zeitraum 1994 bis 2013: Fachhochschule Schmalkalden / Hochschule für angewandte Wissenschaften, Fakultät Maschinenbau, Professur für Arbeitsvorbereitung und Industriebetriebslehre.

Weitere Lehrfächer im Direktstudium, Fernstudium und Anpassungsstudium für Ingenieure des Maschinenbaus und Wirtschaftsingenieurwesens waren Fertigungsprozessgestaltung, Arbeitswissenschaft, Ergonomie und Prozessgestaltung (WIW), Computergestützte Prozessplanung (MB Masterstudium), Kostenrechnung, Wirtschaftlichkeitsrechnung, Technische Investitionsvorbereitung, Vertretung Mathematik. Mir oblag neben den 
Vorlesungen und Übungen die Betreuung von über 300 Abschlussarbeiten für die Grade Dipl.-Ing (FH), Bachelor of Engineering, Master of Engineering. Dazu kamen noch viele zu betreuende Betriebspraktika. Als Ergänzungsqualifizierung im Maschinenbaustudium organisierte und realisierte ich die REFA Grundausbildung über fast 20 Jahre. Die interne Gremienarbeit in Fachbereich / Fakultät Maschinenbau und Hochschule gab mir vielfältige Einblicke und umfangreiche Erfahrungen. Die Gremien waren: Konzil, Studienkommission WIW -Vorsitz, Prüfungsausschuss Maschinenbau, mehrere Berufungskommissionen - davon zweimal als Vorsitzender.

Nebenamtliche, zeitweilige Tätigkeiten außerhalb der Hochschule erforderten zusätzlichen Zeit- und Kraftaufwand und lagen aber häufig auch im Interesse der Hochschule. Dazu gehörte auch die externe Mitarbeit in der „Schmalkalder Forschungs- \& Bildungs-Fördergesellschaft e.V." (FBF).

Die Vielzahl der wahr genommenen Aufgaben ermöglichten laufend eigene Lernprozesse, ließen aber auch keine Freiräume für komplexe Forschungsarbeiten. Die Mitarbeit in der FBF führte im Laufe der Jahre trotzdem zu mehreren öffentlichen Tagungsbeiträgen - soweit diese inhaltliche Nähe zum Berufungsgebiet besaßen.

\section{Zeitraum seit 2013: Ruhestand mit nachberuflichen Aktivitäten}

- Nebenamtliche Lehrtätigkeiten für die Hochschule Schmalkalden in mehreren Fächern, für den REFA - Landesverband Thüringen als Dozent in unterschiedlichen Lehrgängen und als QM-Beauftragter sowie für die Duale Hochschule Gera -Eisenach als nebenamtlicher Dozent im Lehrmodul Ergonomie

- Tagungsbesuche: FDZAT/PAZAT - Tagungen an der TU Dresden, FBF Fachtagungen in Schmalkalden, Vortragsreihen des VDI- Arbeitskreises EKV an der TU IImenau.

\section{B. Beiträge zur Digitalisierung im Maschinenbau}

\section{B1. Strukturelle Konzepte der Arbeitsplanung / Fertigungsprozessgestaltung}

„Zwischen Tradition und Business Reengineering - die neue Arbeitsvorbereitung im Kontext mit der flexiblen Unternehmensorganisation" war ein zeittypischer Beitrag zur 15. Schmalkalder Fachtagung 1999. Um diese Zeit stand im Zusammenhang mit flexiblen Unternehmenskonzepten die Perspektive der Arbeitsvorbereitung auf der Tagesordnung. Zu diesem Thema arbeitete ich auch in der AG „Prozessorientierte Arbeitsvorbereitung“ des damaligen Ausschusses für wirtschaftliche Fertigung (AwF) mit. Die Fortschritte durch die Digitalisierung sowohl in der Fertigung (enorme Zunahme des CNC-Einsatzes) als auch in der Arbeitsvorbereitung selbst, wo digitale Planungs- und Steuerungswerkzeuge verstärkte Verwendung fanden, führten sehr schnell zu einem Bedeutungszuwachs der AV. Es wuchs die Einsicht, dass AV-Wissen und hochwertige Planungs- und Steuerungsdaten sowie schnelle Planungsprozesse auf Basis moderner digitaler Informationstechnik mit Gruppenarbeit nicht ersetzt werden können.

Außerdem bot die Integration von Produkt- und Prozessplanung neue Chancen und neue Planungsmodelle.

Im Fachbereich Maschinenbau der FH Schmalkalden wurde von mir in dieser Zeit 2001 ein AV-Labor eingerichtet, das neben lokal vernetzter Hardware auch einen variablen Montagearbeitsplatz umfasste. Hier wurden Übungsprojekte mit ständig neuen digitalen Planungswerkzeugen (z.B. HSiplan, IImoplan, CAD-CE, digitale Planzeitkataloge, enginObject, Machine Tool Selection sowie REFA Planspiel und eigenen Programmen) bearbeitet. 


\section{B2. Integration von Produkt- und Prozessplanung}

Der Prozessorientierung als Prinzip für die Gestaltung von Unternehmensprozessen kommt seit Ausbreitung der Produktionskonzepte der schlanken Produktion die Rolle des tragenden Grundgedankens zu.

Unter dem Aspekt Prozessorientierung eröffnete der Einsatz digitaler Werkzeuge sowohl in der Produkt- als auch in der Prozessentwicklung neue Integrationsmöglichkeiten beider Ingenieurbereiche. Ihre Erscheinungsformen entwickelten sich rasant bis zur Digitalen Fabrik. In einem kurzen Forschungsbericht zum Tag der Forschung 1999 mit dem Kurztitel "Logische und informationstechnische Integration von Produkt - und Prozessentwicklung“ versuchte ich, die Grundlagen aus Sicht der Arbeitsvorbereitung für Studierende zu verdeutlichen. Diesem Anliegen entsprachen auch verschiedene Lehrbriefe zur Arbeitsvorbereitung für das grundständige Studium Wirtschaftsingenieurwesen in den neuen Bundesländern

Die 25. Schmalkalder Fachtagung 2009 schließlich thematisierte das Thema „Digitale Fabrik“. Bei dieser Gelegenheit hielt ich den Vortrag „Integration der Prozessplanung in die Digitale Fabrik“. Berücksichtigt wurden dabei sowohl Einzellösungen als auch die Netzwerkseinbindungen und Integrationsmöglichkeiten der digitalen Prozessplanung. Im folgenden Bild wird eine mögliche Grundidee zur logischen Verknüpfung von Konstruktion und Arbeitsplanung gezeigt, die auf der Verwendung der Feature- Technologie beruht. Basis ist die Konstruktion eines Festkörpermodells mit Hilfe von Features aus einer Bibliothek in Verbindung mit der automatschen Generierung des Fertigungsablaufes und von Ausführungsdateien. Das Beispiel wurde ausgewählt, weil es bereits mit Erfolg in der betrieblichen Praxis eingeführt war. Die gezeigte Art der integrierten Prozessmodellierung gehört in die Modellierungsebene der Detailplanung. Dieser gehen in der Regel die Ebenen der strukturorientierte (Fertigungs- oder Montagestrukturen, Digitale Fabrik) und die der gestaltorientierten Modellierung (Produktentwurf, Verfahrens-, Prozessauswahl) voraus (Beispiele folgen unten). Die Ebenendefinitionen ermöglichen durchaus eine sinnvolle Systematisierung.

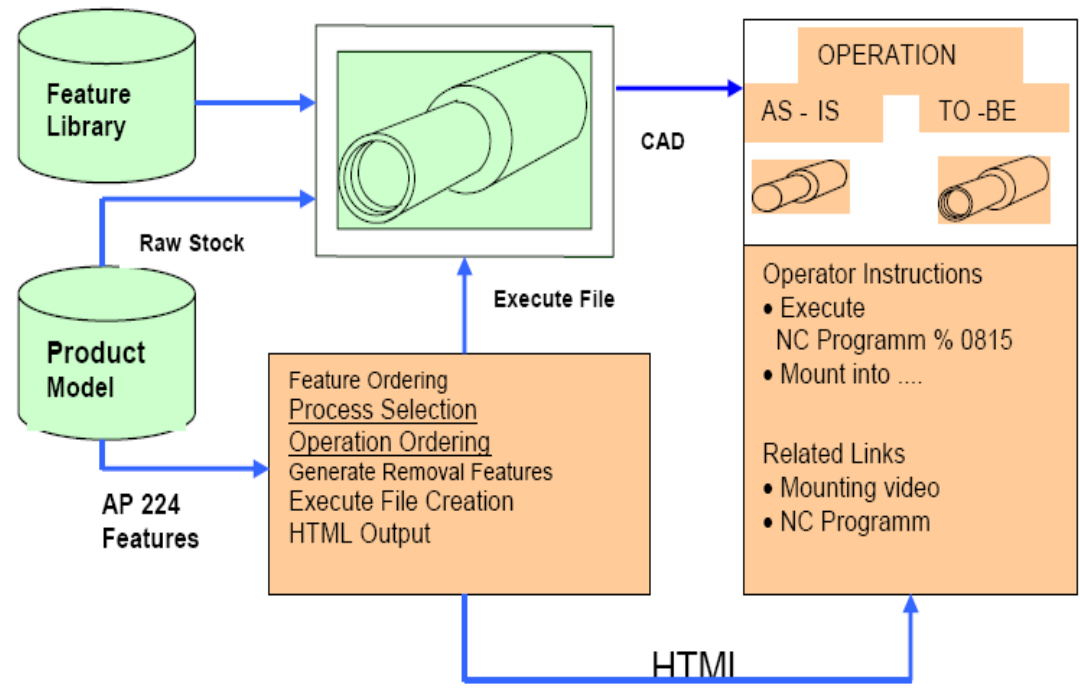
Automatisierte Prozessplanung in der Produktentwicklung in Anlehnung an: Laliberty, Gilman - „Noch schneller zum Produkt"“, it.AV 6/98

Als Zeichen aktueller Bedürfnisse der Industrie standen und stehen vielfach betriebliche Konzepte für die Integration von Produkt - und Prozessentwicklung im Rahmen von Abschlussarbeiten auf der Tagesordnung. Folgende Beispiele verdeutlichen je nach Modellierungsebene verschiedenartige Problemlagen. 
(1) EDAG Fulda/ 2005: „Entwicklung eines ganzheitlichen Planungsprozesses unter wirtschaftlichen Gesichtspunkten für den Karosseriebau in der Automobilindustrie bei besonderer Berücksichtigung digitaler Werkzeuge" als Beispiel für eine strukturorientierte Modellierung.

(2) Eine gestaltorientierte Modellierung wurde 2004 bei der Hella KG aA \& Co Lippstadt untersucht: „Überarbeitung und Optimierung des Produktentstehungsprozesses durch Integration der Ergebnisse von punktuellen Optimierungsprojekten“.

(3) Ein Beispiel für die Lösung von detailorientierten Digitalisierungsproblemen wurde 2007 bei Sandvik Tooling Supply Germany / Schmalkalden bearbeitet: Erstellung einer optimierten digitalen Prozesskette mit dem Inhalt , Rechnergestützte Prüfplanerstellung mit der Software CALYPSO der Fa. ZEISS, auf der Grundlage unserer mit dem

Konstruktionssystem UNIGRAPHICS NX 3 erstellten 3D-Volumenmodelle und der messvorbereitenden Software BCT-INSPECTOR V 9 der Fa .BCT-Technology AG.Rvon“". Hier ergaben sich optimierte Datenformate, eine verbesserte CAD -Modellierung und kürzere Prozesszeiten. Es wurde eine Entscheidungsgrundlage für den Kauf und die Installation der Softwaremodule geschaffen.

\section{B3. Optimierungskonzepte}

Einen wichtigen Aspekt der Digitalisierung bildet das Zusammenspiel von Simulation - einem typischen Merkmal der Digitalen Fabrik - und Optimierungsmodellen. Auch bei der Kombination von Simulation und Optimierung sollten die unterschiedlichen Zielbereiche der eingesetzten Optimierungsmodelle beachtet werden. In einem kurzen Überblick sollen drei unterschiedliche Konzepte auf drei Modellierungsebenen charakterisiert werden.

Als methodische Gemeinsamkeit wird jeweils ein regelungs-technisches Zusammenwirken von Optimierungsmodulen und Simulationsmodellen nach dem in der folgenden Graphik dargestellten Prinzip gestaltet:

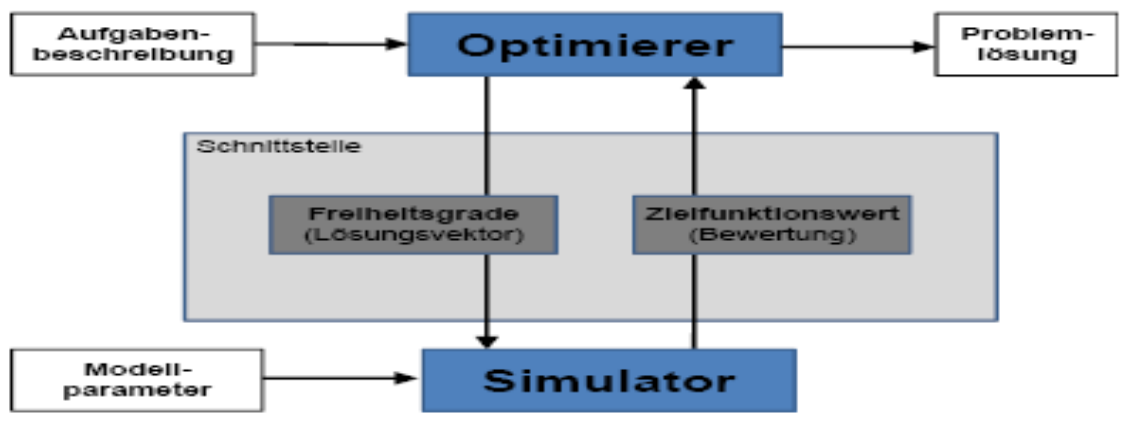

Prinzip der simulationsbasiert en Optimierung (i.A. an DA Weisheit)

Beispiele für kombinierte Simulations-und Optimierungskonzepte -Überblick

\begin{tabular}{|l|l|l|l|l|}
\hline System & Anwendung & Simulation & Optimierung & Fallbeispiel \\
\hline $\begin{array}{l}\text { Dualis } \\
\text { GmbH (1) }\end{array}$ & Strukturoptimierung & 3D-Create & $\begin{array}{l}\text { ISSOP 2.0 } \\
\text { (Methodenpaket) }\end{array}$ & $\begin{array}{l}\text { Günstigster } \\
\text { Montageablauf durch } \\
\text { Variantenoptimierung }\end{array}$ \\
\hline $\begin{array}{l}\text { Engin Soft } \\
\text { GmbH (2) }\end{array}$ & $\begin{array}{l}\text { „Prozessintegrierte } \\
\text { Produktentwicklung“ } \\
\text { (PIDO), } \\
\text { Gestaltoptimierung }\end{array}$ & $\begin{array}{l}\text { CAE- } \\
\text { Methoden, } \\
\text { Machining } \\
\text { Simulation }\end{array}$ & $\begin{array}{l}\text { modeFrontier } \\
\text { (Methodenpaket) }\end{array}$ & $\begin{array}{l}\text { Optimale Spritzgiess- } \\
\text { parametern für } \\
\text { optimales Spritzguss- } \\
\text { teil }\end{array}$ \\
\hline $\begin{array}{l}\text { FHS/Sandvik } \\
\text { (3) }\end{array}$ & $\begin{array}{l}\text { Schnittwertoptimierung } \\
\text { Detailoptimierung }\end{array}$ & COROGUIDE & SWO (EXCEL) & $\begin{array}{l}\text { Werkzeugauswahl mit } \\
\text { Kostenbewertung }\end{array}$ \\
\hline
\end{tabular}

(1) Als strukturorientiertes Beispiel wurden in einer Diplomarbeit Varianten eines realen Montageprozesses mit Hilfe verschiedener möglicher Optimierungstrategien bewertet.

(2) In einem sehr lomplexen Beziehungsgeflecht werden im zweiten Beispiel Produkt- und damit also gestaltorientierte Optimallösungen entwickelt. Im Vergleich zum klassischen 
Dresdener Optimierungsmodell werden bei EnginSoft alle technischen Grenzen sowohl des Produktes als auch des Fertigungsverfahrens durch Simulationsmethoden (FEM und andere) und nicht deterministisch bestimmt. Durch Integration fertigungstechnischer Kompetenzen wird die Produktentwicklung qualitativ verbessert.

Das Optimierungsteil „modeFrontier" kann viele unterschiedliche Algorithmen für anspruchsvolle multikriterielle Optimierungen zum Einsatz bringen (Simplexvarianten, MOGA, Spieltheorie). Die Produkteigenschaften werden zum Beispiel mit FEM berechnet und unter fertigungstechnischer Simulation (Schmieden, Gießen, Zerspanen,...) optimal gestaltet. Die Optimieralgoirthmen steuern die Simulation.Endziel ist das unter optimalen Fertigungsbedingungen zu erzielende optimale Produkt
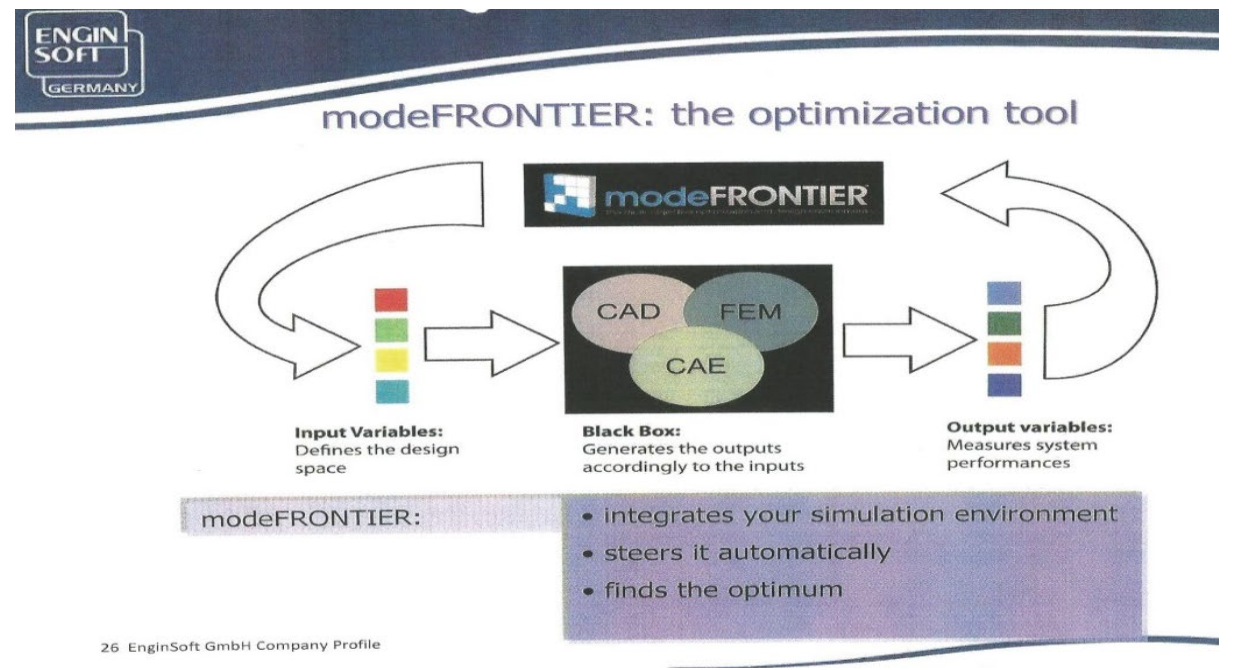

\begin{abstract}
Gestalt orientierte Anwendung, ,PIDO= prozessintegrierte Designoptimierung“"
\end{abstract}

(nach ENGINSOFT)

(3) Mit dem Werkzeugauswahlprogramm „Coroguide“ von SANDVIK Coromant erhält der Planer zunächst für eine Operation aufgrund seiner Planungvorgaben aus der Grobplanung (Durchmesser, Länge, Werkstoff,..) mehrere Werkzeugempfehlungen. Dazu gehören Typ und Größe der Wendeschneidplatte, Schneidstoff, technische Grenzen des Werkzeuges, das graphische Lösungsfeld des Werkzeuges (!) sowie die in der aktuellen Simulation entstehenden Ergebnisse: Schnittzeit,Standzeit, Leistungsbedarf Mit dem Optimierungsprogamm „Schnittwertotpimerung“ (SWO) können für beliebig viele Lösungsvarianten von jedem Werkzeugtyp die optimalen Parameter schnell ermittelt und bewertet werden. Das Modell ist für die Anwendung in der Lehre gedacht.

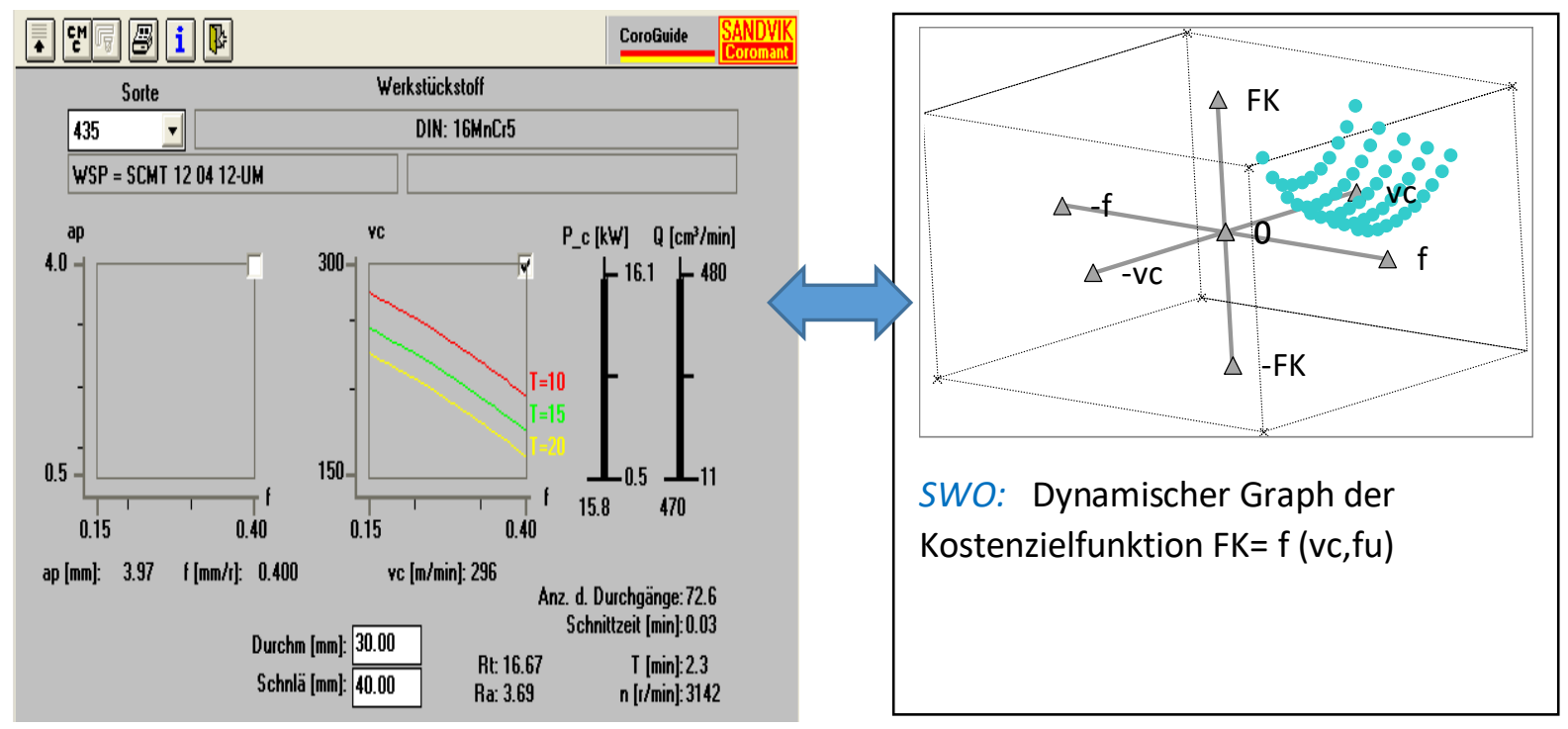

Simulationsprogramm Coroguide (SANDVIK Coromant) und Optimierungsmodul SWO im Zusammenspiel 
Das Optimierungsmodell der Dresdener Schule der Fertigungstechnik wurde für Lehrzwecke in Form von EXCEL-Programmen aufbereitet und weiter entwickelt- zum Beispiel in Richtung der graphischen Interpretation von technischen Grenzen und Kostenzielfunktion, der Erkennung und Interpretation von technologischen Zusammenhängen im Modell, der Erkennung von Schwachstellen und Verbesserungspotenzialen.

Vorteile der Nutzung sind: gutes Lehrmittel zum Kennenlernen des technologischen Arbeitssystems, Berechnungsformeln sind leicht änderbar und nachvollziehbar, sehr schnelle Ergebnisse, leichte Erstellung von Richtwerttabellen, vielfältige, aber einfache simulative Untersuchungen.

Anwendungen in Form ausgewählter Abschlussarbeiten

(1) Renault Automation Castres, 1997: Untersuchung des Bearbeitungsverfahrens Achparalleles Drehfräsen von Kurbelwellenhublagern.

Ergebnis als Kalkulationsprogramm zur Verfahrensbeschreibung und zur orientierenden Schnittwertoptimierung und Standzeitvorhersage zwecks Gestaltung neuer Maschinen.

(2) Stahlwerk Thüringen $\mathrm{GmbH} 2000$ : Vergleich der Leistungsfähigkeit von fünf Walzendrehereien in der ARBED-Gruppe, objektivierte Vergleichsbasis waren optimierte Schnittwerte, um individuelle Einflüsse auszuschließen.

(3)X-FAB Dresden GmbH \& Co. KG 2011: Erstellung und Optimierung univariater Modelle zur Bewertung von PVD - Metallisierungsprozessen im Halbleiter - Waferprozess.

\section{B3: Sonstige interessante Konzepte der Fertigungsprozessgestaltung}

(1) Der eigene Beitrag zu „Methodische Betrachtungen zur Integration ökologischer Ziele in die Fertigungsprozessplanung" - in Krah: Aspekte ökologiegerechter Fabrikprozess- und Fabrikplanung, 2009, baute auf den Grundlagen der Arbeitsplanung auf.

(2) Das Konzept „CAD/CE - Teile - Bewertung im Konstruktionsprozess" (CE = Cost Estimation) wurde vom Montage Design Studio /Prof. Holle initiiert und über gemeinsame Tagungsbeiträge Holle/Weiß veröffentlicht.

(3) Zeitdatenermittlung: Hierzu gehörten die Zeitaufnahme mit PC inclusive

Rechnerauswertung mit Software - Modulen für statistische Auswertung, Planzeitermittlung und für Systeme vorbestimmter Zeiten im AV-Labor.

4) Instandhaltungsmanagement: Die Befassung mit Problemen der Instandhaltung ist ein Beispiel dafür, wie sich Aufgaben verteilen, wenn Umstrukturierungen - hier die Auflösung der Vertiefungsrichtung Instandhaltung am Fachbereich Maschinenbau - stattfinden.

Benchmarking von Instandhaltungsprozessen: Ein externes Projekt der FBF endete 2002 mit dem Abschlussbericht: "Benchmarking des Instandhaltungs- Controllings im Werk Rastatt der DaimlerChrysler AG“. Davon abgeleitet hielt ich auf der 19.Schmalkalder Fachtagung einen Vortrag zum Thema Benchmarking. Zum Themenkreis Instandhaltung wurden mehrere Abschlussarbeiten betreut.

Die Vielzahl der betreuten Abschlussarbeiten ist dadurch begründet, dass die Themenstellungen vorrangig aus der Praxis und nicht aus eigener Forschung kamen. Betriebliche Gutachten waren neben der Bewertung der studentischen Leistung auch Gradmesser für die Anerkennung der präsentierten Hochschulausbildung. Viele Absolventen sicherten sich mit der Abschlussarbeit ihre erste Arbeitsstelle.

\section{B. Zusammenfassung}

Möge dieser Beitrag den Erwartungen gerecht werden. Aus der Erfahrung als Lehrender weiß man, wie schwer das sein kann. Und: Digitalisierung ist längst nicht alles.

Sehr geehrter Prof. Kochan, ich hoffe Sie können alle diese Entschuldigungen annehmen! 


\section{Sehr geehrter Jubilar, sehr geehrte Fachkolleginnen und Fachkollegen,}

herzlichen Dank, Herr Prof. Kochan, für die heutige Einladung. Die Zusammenkunft anlässlich Ihres 85. Geburtstages hat verursacht, dass ich mich nochmals mit der Dissertation Doltze / Otto beschäftigt habe.

Gestatten Sie mir daher einen kurzen Rückblick in das Jahr 1976 also vor 44 Jahren.

Die Dissertation Doltze / Otto befasste sich damals mit der wirtschaftlichen Erzeugung technologischer Verarbeitungsprogramme und hier speziell mit der Generierung von Postprozessoren für das Verfahren Drehen. Ziel war die automatisierte technologische Fertigungsvorbereitung unter Einsatz der elektronischen Datenverarbeitung, d.h. aus einer Menge von Drehteilen waren für die Bearbeitung die wirtschaftlichsten NC-Maschinen auszuwählen und deren Steuerinformationen zu erstellen. Auf Grund der Komplexität dieses Vorhabens lies sich das nur mittels Modultechnik oder wie damals genannt, dem Bausteinprinzip realisieren. Das waren inhaltlich geschlossenen Programmabschnitte, die einerseits technische Inhalte (Verarbeitungsbausteine) und andererseits programmtechnische Aspekte (Eingabe- und Ausgabebausteine) aufwiesen. Nun war noch ein Generierungsprogramm erforderlich, welches die Bausteine in geeigneter Form zusammenfügte und über entsprechende Schnittstellen interagieren lies. In Kurzform hieß das, man gibt die Rohteil- und Fertigteilform des Werkstückes ein und erhält die wirtschaftlichste Maschine und deren Steuerinformationen für die Bearbeitung.

Die Vorteile der modularen Programmierung sind: Übersichtlichkeit, Flexibilität bei der Programmerstellung, Erweiterungsfähigkeit und Modifizierbarkeit, leichte Änderungs- und Testfähigkeit.

Soviel zur Geschichte und damit zurück zur Gegenwart.

Die damals erkannten Grundlagen gelten heute immer noch, modulare Programme und Apps sind Stand der Technik geworden. Wenn man so will, hat die Fertigungstechnik damals eine Vorreiterrolle gespielt. Die heutige Entwicklung hat zu sehr hohen Speicherplatzvolumen und sehr schnellen Prozessoren geführt. Die Miniaturisierung der Hardware führte zu Anwendungen u.a. in der Kommunikation (Smartphon) bis hin zu Haushaltsgeräten.

Es gibt kaum noch einen Wirtschaftsbereich, der von der Digitalisierung ausgeschlossen ist. Nicht alle Entwicklungen sind dabei positiv einzuordnen, die Möglichkeit über Datensammlung Verhaltensmuster zu erfahren und Dateien über Nutzer anzulegen, bürgen das Problem der missbräuchlichen Nutzung in sich.

An diese Herausforderung haben wir vor 44 Jahren nicht im Entferntesten gedacht.

Ich bleibe daher auf der Seite der positiven Entwicklung und will einige Beispiele von CAD / CAM Anwendungen im Bauwesen nennen. In diesen Bereich habe ich nach der Wende gewechselt. Um Missverständnissen vorzubeugen, ich war nicht direkt im CAD/CAM Bereich tätig sondern ich war als Hausbauer der Nutzer der Ergebnisse dieses Bereiches.

Mit den heute zur Verfügung stehenden CAD Systemen kann man im Hausbau sowohl die zweidimensionale als auch dreidimensionale Darstellung wählen und diese aufgliedern in Fundament, Etagen und Dachstuhl. Weiterhin ist es möglich die Einzelelemente, wie Türen, Fenster, Balkone oder Wintergärten herauszulösen und deren Gestaltung vorzunehmen. 
Im Ergebnis werden die Zeichnungen für die Ausführungsplanung mit allen notwendigen Details erstellt.

Im Massivhausbau hat die z.B. Firma Heinritz und Lechner eine Montagevariante entwickelt, die es erlaubt, ein individuelles Haus per CAD System zu planen. Die geschosshohen Wände werden dann in Einzelsegmente aufgeteilt und zwar so, dass sie in der Fabrik gefertigt und später transportiert werden können. Sämtliche Öffnungen für Fenster und Türen sowie Kanäle der Elektroinstallation sind vorhanden. Das System weist je Segment die notwendigen Vorrichtungen und deren Anordnung für die Fertigung in der Fabrik aus. Auf der Baustelle werden die Segmente per Kran montiert und mittels Zuganker fest verbunden. Der Baustoff besteht aus zementgebundenen Tonkugeln (Handelsnamen Liapor oder Klimpor) und dieser erfüllt die gültige EnEV in den Wärmedämmwerten. Die Montagezeiten für ein EFH mit ca. $150 \mathrm{~m}^{2}$ Fläche liegen ab OK Bodenplatte bzw. Kellerdecke bei 5 Tagen.

Eine andere Variante im konventionellen Hausbau, bei denen das Haus z.B. aus YtongSteinen gebaut wird, ist die Aufteilung sämtlicher Wände per CAD System in ganze, dreiviertel, halbe und viertel Steine, so dass auf der Baustelle keinerlei Steintrennung erfolgen muss. Der Maurer erhält einen Setzplan, nach dem er mit den diesen Steinen immer ganzzahlig setzen kann sowohl bei Öffnungen als auch am Mauerende. Die Steine werden auf Paletten in der richtigen Zusammensetzung pro Mauer auf der Baustelle angeliefert.

Bei der Dachstuhlherstellung werden aus den CAD-Unterlagen in den Abbundzentren in Kombination mit CAM Systemen sämtliche Zuschnitte für die Pfetten, Sparren und Stützen passgenau auf CNC-Maschinen hergestellt, so dass der Zimmermann nach Plan auf der Baustelle nur noch montieren muss.

Im Fenster- und Türenbereich kann man über CAD Systeme in freier Konstruktion Pfosten und Kämpfer, Sprossen jeder Art, Gläser und Füllungen sowie Beschläge positionieren. Auf den Fertigungslisten sorgen Schnitte, Skizzen und Ansichten mit Bemaßung für exaktes Arbeiten. Für jede Konstruktion erzeugt das System die ganze Arbeitsvorbereitung mit allen Informationen direkt aus dem Fenster- bzw. Türdesign.

Egal ob PVC, Holz, Alu als Fenster oder Türen in Standard oder Sonderausführung, über entsprechende CAM Systeme stehen die entsprechenden Maschinendaten für Zuschnittsäge, Bohr-, Schweiß- und Beschlagautomat oder Bearbeitungszentrum zur Verfügung.

Für die Wiedererrichtung historischer Gebäude mit neuem Nutzungskonzept gibt es noch einen besonderen Anwendungsfall für CAD Systeme. In meinem Wohnort, der Ortschaft Altfranken, laufen gegenwärtig die Planungen für den Wiederaufbau des Schlosses von Graf Felix von Luckner, welches 1939 abgerissen wurde. Als Nutzungskonzept für den Neubau ist ein Hotel vorgesehen. Um die geringeren Deckenhöhen eines Hotelbaues in der Außenfassade zu kaschieren, war es erforderlich, die etagenhohen Fenster der historischen Fassade so abzuwandeln, dass mit minimalen Eingriffen der ursprüngliche Charakter erhalten blieb. Das gelingt am besten, wenn die alte Fassade über die neue gelegt wird und dann die Retuschen vorgenommen werden. Das erzielte Ergebnis lässt den unverwechselbaren Charakter des im Tudor-Stil (Neogotik) erbauten Schlosses wieder erkennen.

Mit diesen Beispielen will ich es belassen und wende mich wieder unserem Jubilar zu. 
Sehr geehrter Herr Prof. Kochan, vor fast einem halben Jahrhundert begann an Ihrem Lehrstuhl eine Entwicklung, die bis heute immer noch anhält und an Breite gewinnt. Mit Fug und Recht kann man sagen, da war bei Ihnen wissenschaftliche und organisatorische Weitsicht vorhanden, aber auch eine politische Loyalität im eigenen Hause wie auf internationaler Ebene, die für damalige Verhältnisse nicht selbstverständlich war.

Ihr permanenter Wille, bis ins hohe Alter wissenschaftlich tätig zu sein, zeigt auch Ihr Engagement im Zentrum für Angewandte Forschung und Technologie und nicht zu letzt in der heutigen Veranstaltung mit der Sie auch uns, dank Ihrer Tagesordnung, wieder in den Wissenschaftsbereich mitgenommen haben.

Alles Gute und ganz herzlichen Dank.

Hubertus Doltze 


\title{
„Advanced Manufacturing und Digitalisierung der Produktion“"
}

\begin{abstract}
Beiträge zur Dresdner Schule der Fertigungstechnik mit Bezug zum Lehr- und Forschungsgebiet Fertigungstechnik/ technologische Programmierung
\end{abstract}

\section{anlässlich des 85. Geburtstages von Professor Detlef Kochan}

\section{Wissenschaftlicher Werdegang von Arndt Richter}

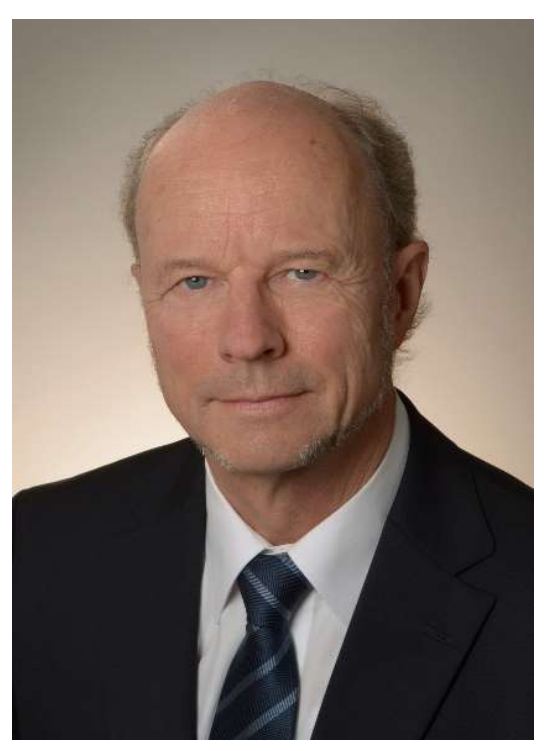

Arndt Richter studierte an der TU Dresden, Fakultät Maschinenwesen, Fachrichtung Fertigungstechnik/ Fertigungsprozessgestaltung. Nach dem Studium war er wissenschaftlicher Assistent am Lehrstuhl Technologische Programmierung und promovierte 1978 zum Dr.-Ing. auf dem Gebiet der Optimierung spanender Bearbeitung. Anschließend arbeitete er über 10 Jahre im Elektromaschinenbau. Danach wechselte er zur EXAPT Systemtechnik $\mathrm{GmbH}$ und leitete über mehrere Jahre die Geschäftsstelle Dresden dieser Gesellschaft. 2004 wurde er zum Geschäftsführer der EXAPT-Gruppe in Aachen bestellt und übte diese Tätigkeit bis zum Ruhestand 2016 aus. Jetzt ist er Vorsitzender des Aufsichtsrats der EXAPT Systemtechnik $\mathrm{GmbH}$, Aachen und Vorstand im EXAPT-Verein. Seit 2018 vertritt er als Honorarprofessor das Lehrgebiet Computerintegrierte Produktion an der HTW Dresden.

\section{Wissenschaftliche Arbeit am Lehrstuhl „Technologische Programmierung“}

Das Studium von 1969 - 1973 begann mit der Immatrikulation an der Sektion Fertigungstechnik und Werkzeugmaschinen der TU Dresden. Zu der damaligen Zeit fand bei der Immatrikulation noch keine weitere fachliche Vertiefung statt. Alle Studenten wurden einheitlich für die gesamte Sektion ausgebildet. Erst nach dem 4. Semester erfolgte die Orientierung auf die verschiedenen Vertiefungsrichtungen.

Die Diplomarbeit 1973 zum Thema „Untersuchungen zur rationellen Fertigungsvorbereitung von skulpturartigen Werkstücken für numerisch gesteuerte Werkzeugmaschinen" wurde gemeinsam mit Karl-Heinz Bäse unter Betreuung von Herrn Prof. Dr.sc.techn. D.Kochan nach 8 Semestern geschrieben. Für die experimentelle Untersetzung der Ergebnisse stand im Versuchsfeld Kutzbachbau eine FKrW 900, einem umgerüsteten Bohrwerk BFT 80, mit PARAMAT 3D (IfW-30) Steuerung zur Verfügung. Die PARAMAT-Steuerung war eine numerische Bahnsteuerung auf Relaisbasis mit parabolischen Inneninterpolator und 
Lochstreifeneingabe. Der Platzbedarf für die Steuerschränke war beachtlich und füllte einen großen Teil der Hallenwand.

Das Forschungsgebiet der mathematischen Beschreibung von Freiformflächen und deren Nutzung in der maschinellen Programmierung von NC-Maschinen stand zu dieser Zeit am Beginn einer langen Entwicklungsphase. Das betrifft sowohl die mathematische Flächenbeschreibung als auch die NC-technische Umsetzung. Grenzen waren dabei insbesondere durch die Leistungsfähigkeit und Verfügbarkeit der Rechentechnik als auch durch den Entwicklungsstand der NC-Steuerungen und NC-Maschinen gesetzt. Der Start dieses Forschungsgebietes erwies sich jedoch, trotz aller Schwierigkeiten, langfristig als eine wichtige Säule des Lehrstuhls.

Während seiner an das Studium anschließenden Assistenzzeit am Lehrstuhl technologische Programmierung (TP) lagen die Tätigkeitsschwerpunkte sowohl im Bereich der Ausbildung als auch auf dem Gebiet der Rationalisierung der Arbeitsvorbereitung durch den Einsatz von Rechentechnik.

In der Lehre waren insbesondere die Seminare „Fertigungsprozessgestaltung I“ (FPG I) zum technologischen Variantenvergleich für die mechanische Bearbeitung von Werkstücken und „Fertigungsprozessgestaltung II“ (FPG II) zur Arbeitsvorbereitung und Programmierung von numerisch gesteuerten Werkzeugmaschinen zu betreuen. Für die Übungen zur maschinellen Programmierung von NC-Maschinen kamen zunächst das sprachbasierte Programmiersystem SYMAP (Symbolische Maschinelle Programmierung), eine Entwicklung von Carl-Zeiss Jena, und später AUTOTECH DR4 und AUTOTECH BOFR1 vom Forschungszentrum für Werkzeugmaschinen (FZW) Karl-Marx-Stadt zum Einsatz (Bild 1).

In den Lehrveranstaltungen wurden Studenten im Direkt- und auch im Fernstudium ausgebildet. In den damaligen Jahren wurden ca. 280 Studenten im Direktstudium und ca. 120 Studenten im Fernstudium pro Jahrgang an der Sektion Fertigungstechnik und Werkzeugmaschinen immatrikuliert. Von den Direktstudenten hatten sich ca. 70 - 90 Studenten in die Vertiefungsrichtung „Fertigungsprozessgestaltung“ eingeschrieben und davon ca 30 Studenten in die Spezialisierung "Technologische Programmierung“.
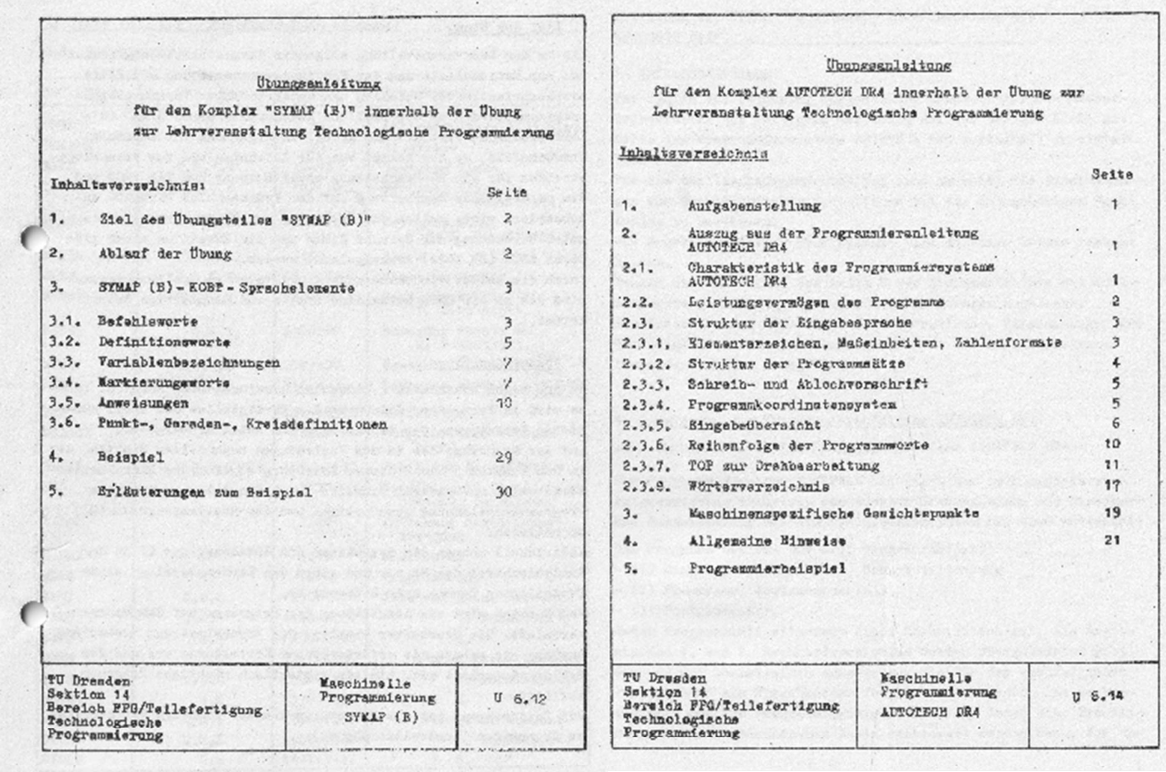

Bild 1:

Übungsanleitungen für SYMAP und AUTOTECH DR4 am Lehrstuhl Technologische Programmierung 
Für diese Studenten waren die notwendigen Praktikums- und Diplomarbeiten vorzubereiten, die Aufgabenstellungen abzustimmen sowie die Betreuung und Bewertung zu übernehmen.

Zusätzlich zu den Aufgaben in der Lehre, zu der auch die operative Übernahme von Vorlesungen und die Mitarbeit bei der Erstellung von Ausbildungsmaterialen (Bild 2) gehörte, war die Vorbereitung und Durchführung von wissenschaftlichen Veranstaltungen ein wichtiger Teil des Aufgabengebietes.

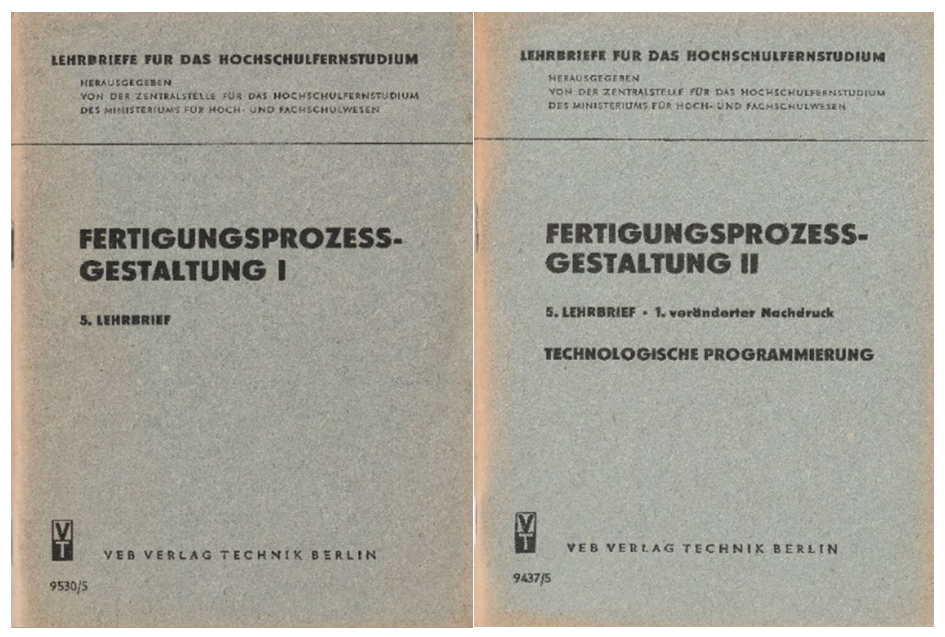

Bild 2: Lehrbriefe Fertigungsprozessgestaltung I und II

Besondere Höhepunkte waren dabei die Fachtagungen INFERT an der TU Dresden, Sektion Fertigungstechnik und Werkzeugmaschinen oder die Problemseminare „Rechnergestützte Fertigungstechnik“ in Gaußig, die vom Lehrstuhl Technologische Programmierung getragen wurden. So wurde anlässlich der internationalen Fachtagung INFERT 74 im Versuchsfeld Kutzbachbau ein erster DNC-Betrieb auf

einer NC-Fräsmaschine FKrS 250 mit BNC3 Steuerung (Bild 3) und als DNC-Server einem KSR4000 (Bild 4) realisiert.

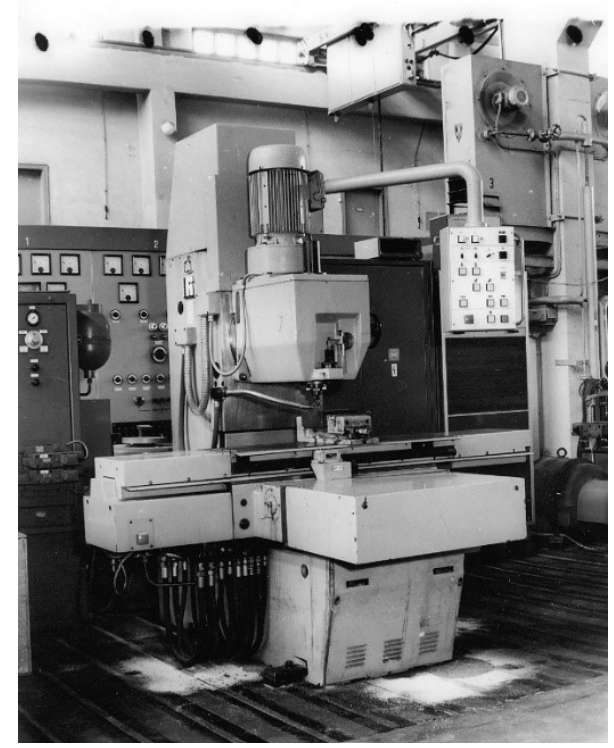

Bild 3: NC-Fräsmaschine FKRS 250 mit BNC3 Steuerung

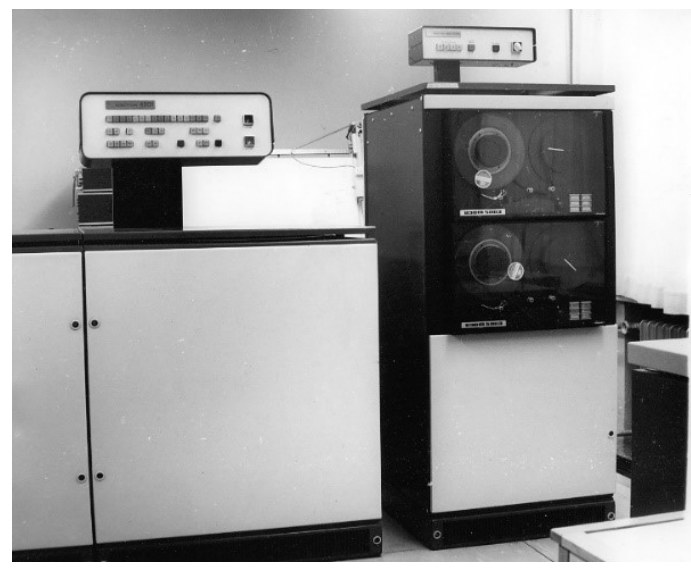

Bild 4: Kleinrechnersystem Robotron KRS4200 
Der KRS 4000 (Kleinrechnerśsystem) von Robotron war die erste dezentrale Rechentechnik am Lehrstuhl und eine wichtige Basis für die weitere Forschung und Ausbildung. In der ersten Ausbaustufe war das im Bild $4 \mathrm{zu}$ sehende Spulenmagnetbandgerät noch nicht verfügbar. Der Hauptspeicher war mit zunächst $16 \mathrm{~K}$ später $32 \mathrm{~K}$ sehr begrenzt. Anwendungsprogramme wurden segmentiert und führten in mehreren Durchläufen zum Ergebnis. Die Datenübergabe zwischen den einzelnen Programmsegmenten erfolgte in der Startphase über Lochstreifen. So waren zur Erstellung eines NC-Programms mit AUTOTECH DR4 zwei Durchläufe des Prozessors und dann ein weiterer Durchlauf für den Postprozessor erforderlich.

Auf dem wissenschaftlichen Gebiet wurden zunächst die Erkenntnisse aus der Diplomarbeit weiterverfolgt. Der Schwerpunkt lag dabei auf den fertigungstechnischen Aspekten bei der Freiformflächenbearbeitung. Die mathematische Durchdringung dieses Themengebietes sowie die technischen Möglichkeiten waren zu der damaligen Zeit jedoch noch nicht so weit fortgeschritten, dass eine fertigungstechnische Umsetzung zielführend möglich gewesen wäre.

Im Gegensatz dazu lagen auf dem Gebiet der technologischen Optimierung der spanenden Bearbeitungen entsprechende Ergebnisse vor, die auch unter dem Gesichtspunkt des Einsatzes von numerisch gesteuerten Werkzeugmaschinen weiterentwickelt werden sollten.

Mit der Strategie zur technologischen Optimierung KOFA (Kostenoptimierung fertigungstechnischer Arbeitsgrößen) und den daraus entstandenen Lösungen für Drehen KOFA 1 (D.Kochan, H.-J.Jacobs) und KOFA 2 (E.Jacob) waren die Grundlagen gelegt, die spanende Bearbeitung unter Berücksichtigung von Spanungsprozess und Wirtschaftlichkeit zu betrachten. Die darauf aufbauenden Arbeiten von G.Raschke und W.Wunderlich, die sich insbesondere auf den Einfluss der Komponenten der Werkzeugmaschine auf den Spanungsprozess beim Drehen bezogen, führten zu der Ausbaustufe KOFA 3.

Diese Arbeiten wurden fortgeführt und durch weitere wissenschaftliche Ergebnisse angereichert. So wurden z.B. die Ergebnisse zur Berücksichtigung der Spanform (A.Knörrich) integriert, die insbesondere für das Drehen von außerordentlicher Bedeutung sind. Das Restriktionssystem konnte deutlich erweitert und besser an die praktischen Bedingungen angepasst werden (Bild 5).

Zum Nachweis der breiten Nutzungsmöglichkeiten der mathematischen Modellierung des Spanungsvorganges wurde zunächst das mathematische Modell in ein EDVProgramm umgesetzt. Dafür stand der Großrechner BESM-6 mit der Programmiersprache FORTRAN IV im zentralen Rechenzentrum der TU Dresden zur Verfügung. Das Programm wurde so konzipiert, dass zusätzlich zur Evaluierung des mathematischen Modells ebenfalls Beispielrechnungen für Industrieanwendungen realisiert werden konnten. Dazu wurden umfangreiche Primärdaten für Werk- und Schneid-stoffe, Werkzeuge, Spannmittel sowie Werkzeugmaschinen erfasst und eine Datenbasis aufgebaut. 
Der experimentelle Nachweis der Funktionsfähigkeit des mathematischen Modells erfolgte auf einer Drehmaschine DFS400 mit Steuerung NC450 (Bild 6) im Versuchsfeld Kutzbachbau. Die anhand des mechanischen Kraftmodells berechneten Restriktionen, wie z.B. der Futterspannkraft bei fliegender Futterspannung oder der Formabweichungen vom Zylinder und vom Kreis durch Deformation des Werkstückes durch Schnittkraft und Futterspannkraft konnten auf diesem Wege ebenso wie die Wirkung der neuen Restriktionen für die Spanformung positiv evaluiert werden. In späteren Einsatzuntersuchungen in der Industrie wurde das System weiter spezifiziert und vervollständig.

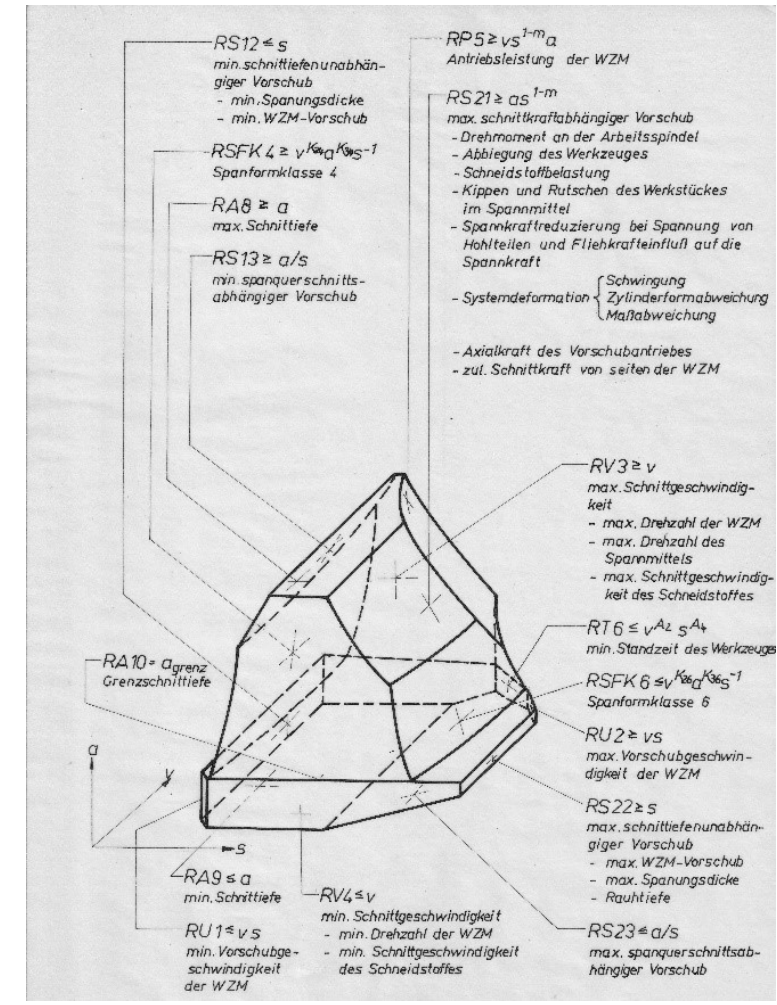

Bild 5: AUTOTECH / KOFA3 Restriktionssystem

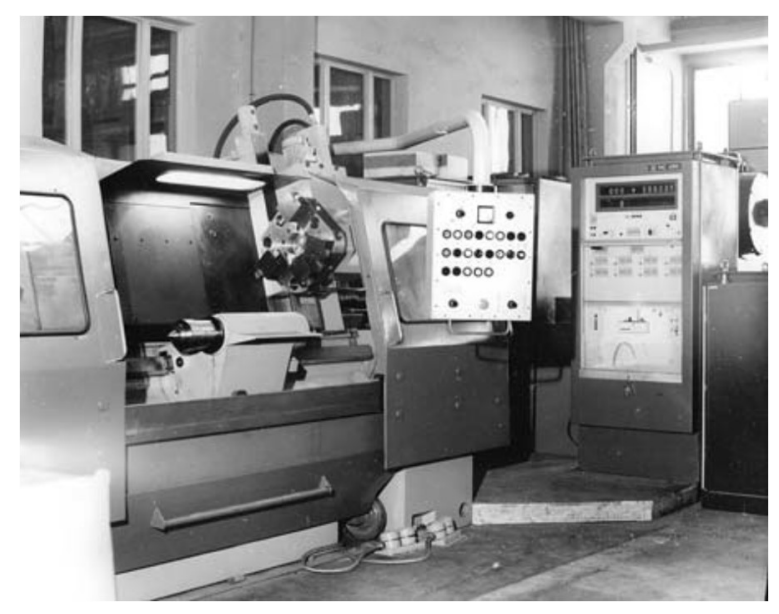

Bild 6: NC-Drehmaschine DFS 400 mit Steuerung NC450

Zur einfacheren und schnelleren Bewertung der Optimierungsergebnisse, die nur in zahlenmäßiger Darstellung über Druck möglich waren, wurden Wege zur grafischen Visualisierung eingeschlagen. Zur Verfügung stand dafür der grafische Zeichentisch Digigraph 1008 im zentralen Rechenzentrum der TU Dresden. Damit konnte das jeweils aktuelle Restriktionssystemen geplottet und somit eine schnellere visuelle Bewertung vorgenommen werden. 


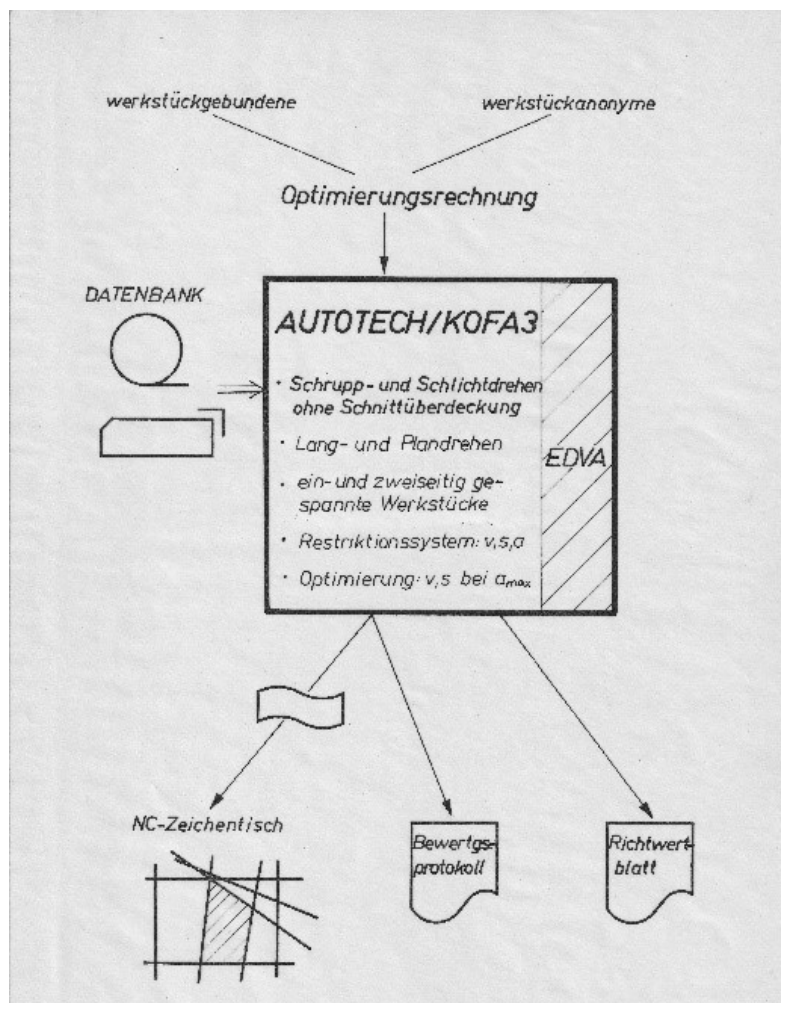

Unter dem Gesichtspunkt des damaligen begrenzten Zugriffs zur Großrechentechnik wurden die Arbeiten in Richtung der Darstellung von werkstückanonymen Spanungsrichtwerten unter Nutzung komplexer technologischer Optimierungsmodelle für die Drehbearbeitung vorangetrieben. Ausschlaggebend war dabei unter anderen auch die Entwicklung des zentralen Schnittwertspeichers in Magdeburg, mit dem sich eine enge Zusammenarbeit ergab.

Für die AUTOTECH/KOFA3-Lösung wurde ein breit nutzbares Systemkonzept entwickelt (Bild 7).

Bild 7: AUTOTECH / KOFA3 Systemkonzept

In der weiteren Folge wurden die Arbeiten zur Spanungsoptimierung für Drehen in Richtung der fertigungstechnischen Bewertung von Fertigungsmitteln ausgebaut. Insbesondere die Betrachtung von Werkzeugen war auf Grund der hohen Verbrauchskosten von Bedeutung. Die Zielstellung bestand in der Auswahl optimal geeigneter Werkzeuge für die jeweilige Fertigungsaufgabe.

Die verdichteten Ergebnisse der Arbeiten zur Modellierung des Restriktionssystems für die externe Spanungsoptimierung beim Drehen sowie zum Aufbau und zur Evaluation des System AUTOTECH/KOFA3 wurden in der Dissertationsschrift „Untersuchungen zur Weiterentwicklung der Modelle und Programme für die Spanungsoptimierung - dargestellt am Beispiel des Fertigungsverfahren Drehen“ zusammengefasst. Die Promotion erfolgte 1978 mit dem Referenten D.Kochan, H.J.Jacobs und F.Lierath.

\section{Rationalisierung der Arbeitsvorbereitung mit Kleinrechner im Elektromaschinenbau}

Nach Abschluss der wissenschaftlichen Assistenzzeit an der TU Dresden erfolgte ein Wechsel in den Bereich Prozessorganisation und CAD/CAM-Anwendung des Forschungs- und Entwicklungszentrums für Elektromaschinen in Dresden. Dieser Bereich hatte die Aufgabe, Rationalisierungs- und Organisationslösungen als Unternehmensstrategie für alle Elektromaschinenbaustandorte zu entwickeln und in den verschiedenen Standorten zum Einsatz zu bringen. Als Mitarbeiter Forschung und 
Entwicklung lag der Tätigkeitsschwerpunkt zunächst in der Rationalisierung der Arbeitsvorbereitung insbesondere durch den Einsatz der dezentralen Kleinrechentechnik KRS 4200 zur Normzeitermittlung im Bereich spanender Bearbeitungsverfahren.

Als Projektleiter Prozessorganisation und CAD/CAM-Anwendung wurde ab Anfang der 80-er Jahre, zunächst gemeinsam mit H.-J. Bock, das Thema des Rechentechnikeinsatzes zur Prozessausarbeitung im Elektromaschinenbau vorangetrieben. Dabei stand sowohl die Unterstützung der detaillierten Ausarbeitung einzelner Arbeitsgänge einschließlich der NC-Programmierung als auch die Rationalisierung der Erstellung von Fertigungsunterlagen im Vordergrund. Der Schwerpunkt lag dabei zunächst auf dem Einsatz der dezentralen Kleinrechentechnik KRS 4200. Für diese Aufgaben wurde die Lösung AUTOTECH/PRO vom Forschungszentrum für Werkzeugmaschinen (FZW) Karl-Marx-Stadt übernommen, für den Elektromaschinenbau adaptiert und zu dem System PROKEM (Prozessausarbeitung im Kombinat Elektromaschinenbau) ausgebaut. Entsprechend den sehr unterschiedlichen Produktionsprofilen der potenziellen Anwender war eine sehr hohe Flexibilität bzgl. der Anpassung an unterschiedliche Bedingungen gefordert. Einfache Generierungsvorschiften für Ablauf, Input, Layout wurden geschaffen und die Lösung in mehreren Betrieben zur Anwendung gebracht.

Mit der Verfügbarkeit von Minicomputern, der Bürorechentechnik BC A5120/5130 später PC 1715 und A 7100/A7150 von Robotron, rückte die Nutzung dieser Technik für die Rationalisierung der Arbeitsvorbereitung in den Mittelpunkt des Aufgabengebietes.

Von der TU Chemnitz (D.Tischendorf) wurde für diese Technik das System PRO15 zur Prozessausarbeitung übernommen. Das System PRO 15 war auf Basis von Redabas (Relationales Datenbanksystem) von Robotron (ein logisches Äquivalent von dBASE II) entwickelt worden und hatte einen Maskengenerator für das Bildschirmund Drucklayout. Gemeinsam mit der TU Chemnitz wurde das System weiterentwickelt und in die PROKEM-Strategie integriert. Das System war dialogorientiert und weitgehend modular aufgebaut. Den logischen Rahmen bildete die Folge der Arbeitsgänge des Fertigungsprozesses in Form der Arbeitsplanstammkarte. In das Rahmensystem konnten verschiedene Module zur Detaillierung der einzelnen Arbeitsgänge integriert werden.

Dafür wurde z.B. mit dem Programm DR 15 eine Lösung zur optimalen Schnittwertund Normzeitberechnung für das konventionelle Drehen entwickelt. Ergebnisse der Arbeiten an der TU Dresden wurden dabei weiterverarbeitet und von Großrechentechnik auf Bürotechnik umgesetzt.

Parallel dazu erfolgte die Ausweitung des Bürocomputereinsatzes von dem Hauptprozess auf die Hilfsprozesse. Die Ursache lag dabei auch auf dem zu betrachtenden Datenumfang und den damals zur Verfügung stehenden Speichermöglichkeiten. Die Datenmenge in den Hilfsprozessen ist im Vergleich zum Hauptprozess geringer. Für den Werkzeug-, Muster- und Versuchsbau als sehr wichtige aber damals in sich abgeschlossene Bereiche des Elektromaschinenbaus, wurde in der Folge die Lösung PROKEM weiterentwickelt und die gesamte Materiallogistik bzgl. Einkauf und Lagerung abgebildet. 
In Fortführung der Strategie zum Softwareeinsatz in der technischen Vorbereitung und Organisation erfolgt nach der Wende die Berufung zum Leiter des CAE-Centers der VEM Antriebstechnik AG, Dresden, mit der Verantwortung für den Rechentechnikeinsatzes in den technischen Bereichen des Gesamtunternehmens. Entsprechend der Konzernstrategie wurde auf die Softwareprodukte SIGRAPH auf Workstation WS70 der Siemens AG orientiert.

Parallel zu den Aufgaben im Forschungs- und Entwicklungszentrum des Elektromaschinenbaus erfolgte im Rahmen des KdT Arbeitsausschusses „Rationalisierung der technologischen Fertigungsvorbereitung“ (Kammer der Technik) zunächst als Mitglied und zuletzt als Leiter des Arbeitsausschusses eine intensive Arbeit und fachlicher Informationsaustausch mit Fachkollegen aus unterschiedlichen Industriebereichen. Neben themengebunden Informationsveranstaltungen wurden Seminare zur Weiterbildung sowie Fachtagungen ausgerichtet.

\section{Vertriebsleitung und Projektmanagement für das EXAPT-CAM-System und -Werkzeugorganisation}

Im Rahmen der internationalen Zusammenarbeit auf dem Gebiet der CAD/CAMEntwicklung hielt D.Kochan gute Verbindungen auch zu den Kollegen, insbesondere zu W.Budde, von EXAPT aus Aachen. Nach 1989 vermittelte er auf dem Problemseminar „Rechnergestützte Fertigungstechnik“ in Gaußig Kontakte zu EXAPT, in dessen Folge Anfang 1992 der Wechsel zur EXAPT Systemtechnik GmbH erfolgte. Als Vertriebsleiter für die neuen Bundesländer bestand die Aufgabe in der Erschließung des Marktes für EXAPT in den neuen Bundesländern. In diesem Zusammenhang wurde eine EXAPT Geschäftsstelle in Dresden aufgebaut, die bis 2003 für die Betreuung der Kunden und Interessenten in dem Vertriebsgebiet verantwortlich war.

In Folge der starken Verbindungen und Abhängigkeiten der produzierenden Betriebe in den neuen Bundesländern zu den Muttergesellschaften im Westen war eine ausschließliche Orientierung auf das Vertriebsgebiet neue Bundesländer langfristig nicht ausreichend. Es erfolgte deshalb zunehmend die Übernahme von Vertriebsaufgaben und dann ebenfalls des Projektmanagements für konzernweite Großprojekte in verschiedenen Sparten.

In der Auswertung der Vertriebsaktivitäten und der Kundenanforderungen wurden gemeinsam mit der EXAPT-Entwicklungsleitung Anforderungen an die strategische Weiterentwicklung des EXAPT Systems formuliert und entsprechende Kundenprojekte gestartet sowie das Projektmanagement geführt. In diesem Zusammenhang bestand Ende der 90er-Jahre die Aufgabe in der Mitarbeit zur Konzeption einer neuen EXAPTSoftware-Generation, die gemeinsam mit einem großen OEM definiert und umgesetzt wurde 


\title{
Gesamtverantwortung für das EXAPT-CAD/CAM-System mit integrierter Produktionsdatenorganisation
}

Im Jahr 2003 erfolgte eine Verlegung des Dienstsitz in die Unternehmenszentrale der EXAPT Systemtechnik GmbH nach Aachen und 2004 die Bestellung zum Geschäftsführer des Unternehmens sowie des Vereins zur Förderung des EXAPTSystems e.V. (EXAPT-Verein) und der Forschungsvereinigung Programmiersprachen für Fertigungseinrichtungen e.V. (FVP).

Die Jahre als Geschäftsführer bis zum Ruhestand 2016 waren geprägt durch die Weiterführung der Entwicklung und Markteinführung der EXAPTsolid-

Systemgeneration. Die Aufgabe bestand dabei insbesondere darin, EXAPT von der 2D- in die 3D-Welt zu transformieren und gleichzeitig die steigenden Anforderungen hinsichtlich der organisatorischen Einordnung in den gesamten Prozess zu berücksichtigen. Die umfangreiche und sehr leistungsfähige EXAPT-Technologie für das Drehen, Bohren und 21/2D-Fräsen war dabei zu übernehmen und ständig fertigungstechnisch weiterzuentwickeln sowie mit neuen Bearbeitungsstrategien im simultanen 3- bis 5-Achsbereich zu verbinden (Bild 8).

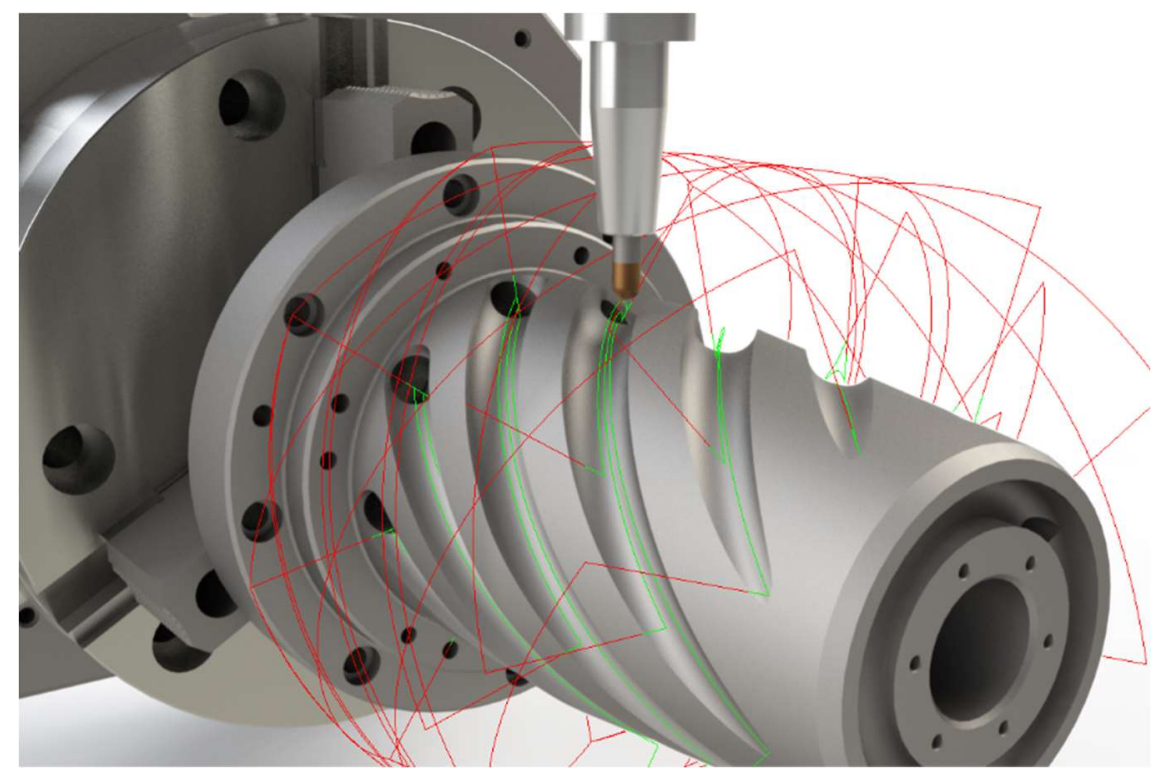

\author{
Bild 8: \\ Komplettbearbeitung \\ eines \\ Wendelverteilers \\ auf einem Dreh- \\ Fräszentrum \\ (Bild: EXAPT \\ Systemtechnik \\ $\mathrm{GmbH}$, Aachen)
}

EXAPTsolid ist als featurebasierendes CAM-System konzipiert mit automatischer Featureerkennung und Verarbeitung. Die systemeigenen Bearbeitungszyklen können mit anwendereigenen Arbeitsabläufen ergänzt werden. Kundeneigenes FertigungsKnowhow wird damit geschützt. Eine große Herausforderung bestand darin, den Anforderungen aus der Praxis gerecht zu werden, die eine Ergänzung der automatischen Verarbeitung mit interaktiven Arbeiten forderten, bei gleichzeitiger Assoziativität zum CAD-Modell als Voraussetzung für eine effektive Änderungsprogrammierung.

Zusätzlich waren die Innovationen auf dem Werkzeug- und Werkzeugmaschinenmarkt sowie die sich verändernden Produktionsstrategien der Kunden, insbesondere 
hinsichtlich kleiner Stückzahlen und Komplettbearbeitung zu berücksichtigen, die sich besonders im Großanlagenbau widerspiegelt haben. Hinzu kamen die sich ständig weiterentwickelnden Techniken und Möglichkeiten bezüglich der Visualisierung und Virtualisierung des Planungsprozess (Bild 9).

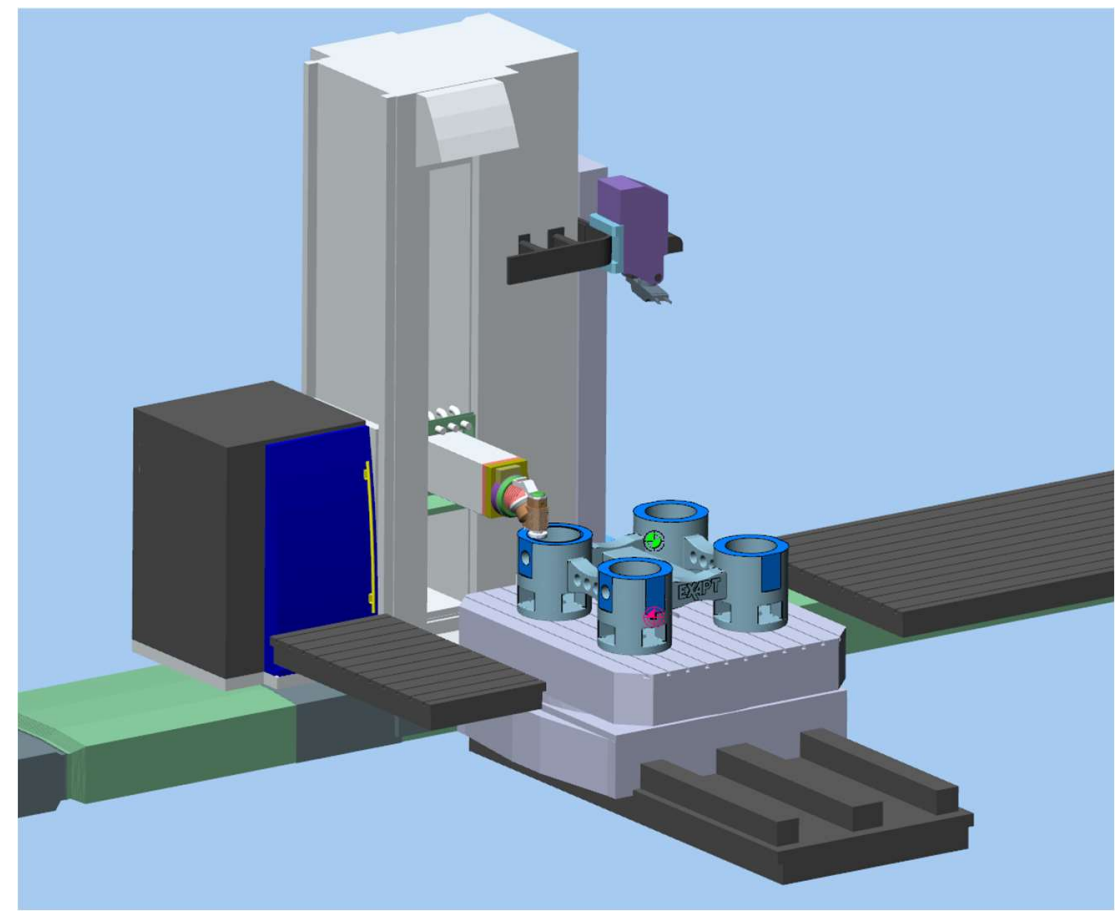

Bild 9: Virtuelle Planung und Simulation einer Großteilebarbeitung mit dem EXAPT CDA/CAM-System (Bild: EXAPT Systemtechnik $\mathrm{GmbH}$, Aachen)

Ein leistungsfähiges CAD/CAM-System sowie die virtuelle Abbildung des Fertigungsprozesses in der Planungsphase erfordern umfangreiche Datenbestände über Werk- und Schneidstoffe, Werkzeuge, Spannmittel, Werkzeugmaschinen sowie Planungsparameter. Die vorhandenen singulären Datenverwaltungslösungen waren zu einer durchgängigen Produktionsdatenorganisation und Technologieplattform weiterzuentwickeln (Bild 10) sowie die 3D-Modelle der Fertigungsmittel, die für die Simulation benötigt werden, zu integrieren.

Eine entsprechende Kommunikation zur realen Fertigung und Rückführung von Prozessparametern zur kontinuierlichen Verbesserung der Datenbasis ist zwingend erforderlich, um langfristig die Qualität der Planungsergebnisse zu gewährleisten. Dafür wurden internetbasierende Lösungen am Markt eingeführt.

Im EXAPT-CAD/CAM-System ist deshalb die hoch ausgebaute NC-Planung untrennbar verbunden mit der Produktionsdatenorganisationen, die alle benötigten Ressourcen abdeckt. Die integrierte Kommunikation mit der Fertigung und den anderen Ebenen der betrieblichen Planung wird unterstützt und damit ein durchgängiger Workflow der betrieblichen Daten gewährleistet. 


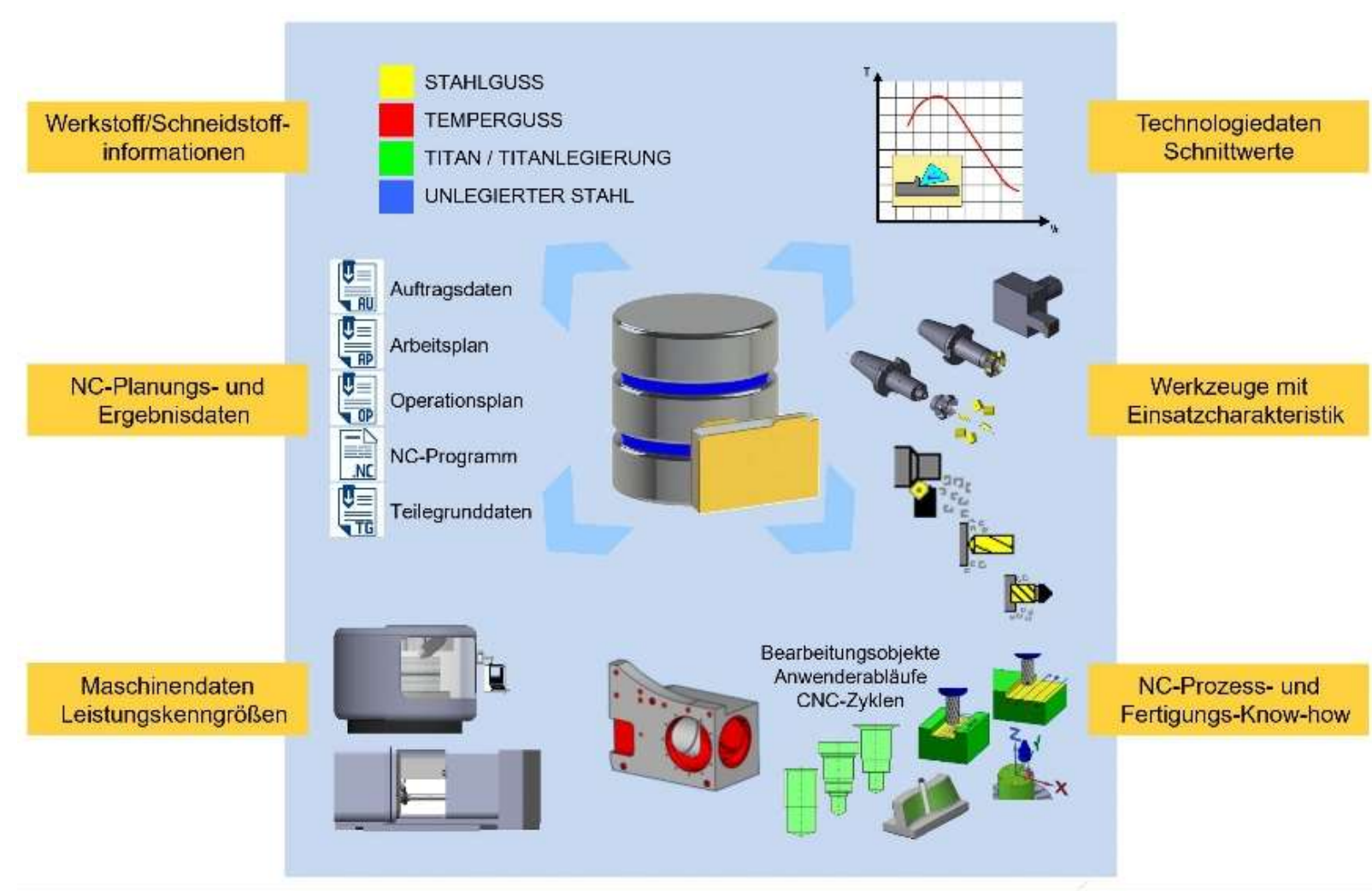

Bild 10: Fertigungs- und Technologieerfahrung in der Produktionsdatenbank als Technologieplattform (Bild: EXAPT Systemtechnik GmbH, Aachen)

\section{Industrielle Forschung zur Absicherung des wissenschaftlichen Vorlaufes}

In der Forschungsvereinigung Programmiersprachen für Fertigungseinrichtungen e.V. (FVP) wurden Forschungsvorhaben, die u.a. von Seiten der Europäischen Union, der Bundesministerien bzw. des Landes Nordrhein-Westfalen öffentlich gefördert wurden, unter dem Gesichtspunkt der Vorlaufforschung bearbeitet. Themengebiete waren die CAD/CAM-Strategie und die Organisation in der Fertigung vor allem unter Nutzung neuer Techniken. Schwerpunkte waren dabei die weitere Qualifizierung der fertigungstechnischen Abläufe u.a. auch für nichtmetallische Werkstoffe, die Verfahrenskombination mit der Laserbearbeitung, der Einsatz von CAD/CAMTechniken im Bereich additiver Fertigung und beim Robotereinsatz sowie das breite Gebiet der Nutzung des Internets für die Produktionsorganisation.

Hervorzuheben sind die Projekte:

- "Technologiedatenbank" welches 1991 gemeinsam mit der TU Dresden, Institut für Fertigungsinformatik, Lehrstuhl Technologische Programmierung gestartet wurde und wesentliche Impulse zur Ausgestaltung der Werkzeugdatenorganisation von EXAPT brachte und für den Vorlauf bei der Zielstellung des Aufbaues einer Technologieplattform wesentlichen Anteil hatte. 
- „KOMBIMASCH - Verkürzung der Prozesskette zur Fertigung rotationssymmetrischer Bauteile durch Verfahrenskombination in modular aufgebauten Werkzeugmaschinen"

- „Untersuchung der Potenziale und Einsatzmöglichkeiten von STEP-NC für Kleinund Mittelbetriebe“"

- „Automatische Featureerkennung als Beitrag zur Effizienzverbesserung in der CAD/CAM-Integration“

- "Integration der NC-Planung in das Product-Lifecycle-Management“

- „Frühzeitige Prozessabsicherung in der CAD/CAM-Planung unter Einbindung einer realitätsnahen Fertigungssimulation“

- „Laserunterstütztes Drehen thermisch gespritzter Metall-MatrixVerbundwerkstoffe"

- "CAMCloud - CAM System in der Cloud"

- „Innovative Fertigungstechnologie für komplexe Rotationsbauteile zum Ersatz von Sonderwerkzeugen durch Standardwerkzeuge in der CAx-Prozesskette“"

Wesentliche Forschungspartner waren das WZL der RWTH Aachen, das FraunhoferInstitut für Produktionstechnologie (IPT), Aachen, das Institut für Werkzeugmaschinen und Fertigungstechnik der TU Berlin, das Fraunhofer-Institut für Produktionsanlagen und Konstruktionstechnik (IPK), Berlin, das Institut für Steuerungstechnik der Werkzeugmaschinen und Fertigungseinrichtungen der Universität Stuttgart (ISW) sowie die TU Dresden, Lehrstuhl technologische Programmierung später das Institut für Produktionstechnik bzw. Institut für Fertigungstechnik.

Die Forschungs- und Entwicklungsergebnisse und deren Umsetzung bei Kunden sowie Anwendererfahrungen beim Einsatz des EXAPT-Systems wurden auf den jährlichen Technischen Tagungen des EXAPT-Vereins und der FVP mit Fachvorträgen aus Industrie und Forschung untersetzt.

Nach Eintritt in den Ruhestand erfolgte die Wahl in den Aufsichtsrat der EXAPT Systemtechnik GmbH und zu dessen Vorsitzenden sowie in den Vorstand des EXAPTVereins und der FVP jeweils zum 2. Vorsitzenden.

\section{Vermittlung der Erfahrungen im Fachgebiet an Fachkollegen und Studenten}

Die Aufrechterhaltung der Verbindung zur TU Dresden sowie zu anderen wissenschaftlichen Einrichtungen war ein wichtiges Anliegen zur ständigen Aktualisierung des eigenen Wissens sowie Anregung für die weitere Arbeit.

Aus der engen Verbindung zur TU Dresden und zu anderen Hochschulen resultiert auch die Begutachtung mehrere Dissertationen als Referent aus der Industrie. 
Resultierend aus der Zusammenarbeit mit Hochschulen und wissenschaftlichen Einrichtungen erfolgte auch die Weitergabe der Erfahrungen und Ergebnisse aus der eigenen Arbeit an Fachkollegen und Studenten.

Auf unterschiedlichen nationalen und internationalen Tagungen, Veranstaltungen Printmedien wurden ca. 75 Publikationen und Fachvorträge vorgestellt. Höhepunkte waren Vorträge auf der PROLAMT 88 gemeinsam mit A.Nestler und J.Sämisch, dem Aachener Werkzeugmaschinen-Kolloquium, 2008, gemeinsam mit C.Brecher, K.Großmann, W.Herfs, F.Hoffmann, A.Meidlingern, R.Rieger und R.Siegler sowie auf der NEW PROLAMT 2013 und eine Vortragsreise in China zum Thematik Industrie 4.0 im Jahr 2016.

In Ergänzung zu der Öffentlichkeitsarbeit erfolgte eine Tätigkeit in der studentischen Ausbildung.

So wurden von 1985 bis 90 als nebenamtlicher Fachschuldozent für Fertigungstechnik das Fachgebiet Abtrenntechnik an der Ingenieurschule für Schwermaschinenbau Roßwein, Außenstelle Dresden betreut und von 1998 bis 2000 als nebenamtlicher Dozent für Rechnerintegrierte Fertigung am Steinbeis Transferzentrum Produktionstechnik und Entsorgungslogistik, Dresden Vorlesungen gehalten.

Seit 2007 erfolgten regelmäßige Gastvorlesungen auf dem Gebiet CAD/CAM und Produktionsdatenorganisation an der Hochschule für Technik und Wirtschaft Dresden im Studiengang Maschinenbau / Produktionstechnik. 2018 erfolgte die Bestellung zum Honorarprofessor für Computerintegrierte Produktion an der HTW Dresden.

Für das von J.Dietrich in Nachfolge von H.Tschätsch langjährig herausgegebene Fachbuch „Praxis der Zerspantechnik" wurden die Kapitel "CAD/CAM“ und „Produktionsdatenorganisation" erarbeitet und als Co-Autor in die 13.Auflage 2020 eingefügt. 


\section{Chronikbeitrag anläßlich des 85. Geburtstags von \\ Prof. Dr. sc. techn. Detlef Kochan}

Verfasser: Prof. Dr.-Ing. Reimund Klünder

Für den Verfasser (Jahrgang 1951) ergab sich 1974 die Attraktivität einer Tätigkeit bei dem Dozenten Dr. Kochan sowohl aus dessen Persönlichkeit als auch aus dem Spektrum der Aufgaben. Dass einige Gralshüter des überkommenen Wissens die rechnerunterstützte Planung von Fertigungsprozessen mit Argwohn beäugten, stachelte den Jungassistenten eher an, denn bei „Ko“ und der Mehrzahl der Doktoranden stand das Suchen im Vordergrund, nicht das Verwalten des Wissens oder das Beschreiten vorgegebener Wege. Bereits während des Ingenieurpraktikums und der Diplomarbeit bei dem verdienstvollen Dr. Wilfried Rothe im Dieselmotorenwerk Rostock war die Überzeugung gewachsen, dass es Ko ohne perfektionistische Attitüden um Beiträge zur Entwicklung innovativer Planungsprozesse für die automatisierte Fertigung ging. Offensichtlich kam dieses angemessene Herangehen auch in internationalen Fachkreisen sehr gut an.

Über den Auftrag, an der Entwicklung eines NC-Programmiersystems der RGW-Länder mitzuwirken, war der Verfasser begeistert und ist dem Jubilar dafür bleibend dankbar. Das gemeinsame Arbeiten und die Freizeitaktivitäten mit den osteuropäischen Kollegen trugen enorm zur Horizonterweiterung bei. Gewiss waren die unterschiedlichen Modellvorstellungen zum Ablauf spanender Prozesse nur schwer vereinbar. Aber sie zwangen immerhin zur selbstkritischen Auseinandersetzung mit den Lehrinhalten im eigenen Hause. Letztlich trugen diverse Versuche an der DFS 400 NC 450, deren Ergebnis ausgeglühte Wendeschneidplatten waren, zur Akzeptanz osteuropäischer Fachkompetenz bei.

Natürlich blieben auch Irritationen nicht aus. Eine Zeitlang schien es beispielsweise, als wäre am Lehrstuhl TP das Maß der Wertschätzung proportional zur Zahl der NC-Achsen, in denen der Betreffende unterwegs war. Im Hinblick auf eindimensionale Dünnbrettbohrer war das angebracht, doch fataler Weise hatte sich der Verfasser gerade mit einer überlangen Eilgangbewegung in negativer z-Richtung massiv blamiert. Gefühlte zwei Arbeitstage musste er dann Herrn Franz zur Hand gehen, der mit Engelsgeduld alle Abnahmebedingungen einer Werkzeugmaschinenspindel messtechnisch durchexerzierte. Diese Erinnerung erleichtert 
bis heute die Demontage- und Montagearbeiten an CNC-Maschinen, wenn mal wieder eine NC-Achse oder der Werkzeugwechsler festgefahren wurde.

Von 1981 bis 1986 war der Verfasser in der Berliner Werkzeugmaschinenfabrik tätig. Das Vordringen numerisch gesteuerter Maschinen erforderte eine flächendeckende Rechnerunterstützung beim Erstellen und Verwalten der kompletten NC-Dokumentation. Die Vielfalt der praktizierten NC-Programmiermethoden und der Steuerungen sowie die volle Breite der spanenden Fertigungsverfahren stellten hohe Anforderungen an den Aufbau und Betrieb einer ganzheitlichen Systemlösung.

Aber die Fähigkeit und Bereitschaft, Herausforderungen zu erkennen und anzunehmen, hat uns Assistenten geprägt, denn sie war uns von unserem Chef vorgelebt worden. Unter Insidern wird bis heute die Adaption des Kempa-Tricks, der aus dem Handball stammt, als seine vielleicht verdienstvollste Leistung angesehen: Detlef Kochan antizipiert die Entwicklung, fliegt in das Zuspiel und zieht den Wurf durch, während andere noch staunend am Boden kleben.

Eine derartige Herausforderung an den Verfasser stellte Mitte der 80er Jahre das Mitwirken beim Aufbau einer (voll-)automatisierten Produktionsstätte dar. Auf der Grundlage unausgereifter Hardware war für eines dieser sog. "Automatisierungsvorhaben" die komplexe Transport- und Lagersteuerung zu entwickeln und schrittweise in Betrieb zu nehmen.

Ab 1987 konnte der Verfasser seine Expertise der NC-Fertigung in CAD-CAM-Kopplungen einbringen, die das ZKI der AdW in Kooperation mit mehreren Betrieben des Werkzeugmaschinenbaues entwickelte. Im Hinblick auf die fertigungstechnische Machbarkeit wurden Methoden erarbeitet und implementiert, mit denen die fehlerbehaftete interne Geometriebeschreibung validiert, teilweise korrigiert und in das CAM-Zielsystem transformiert werden konnte. Mittels dieser Schnittstellenmodule erhielt die Industrie durchgängige Systemlösungen für Blechteile, später auch für prismatische Teile.

Bei der ab 1991 folgenden Tätigkeit am Fraunhofer-IPK ließ sich auf diesen Vorkenntnissen aufbauen. Als es um CAD-CAM-CAQ-Prozessketten für den Schienenfahrzeugbau ging, entwickelte der Verfasser in einem Dreierteam eine Methodik zur Integration von Bearbeitungsfolgen in CAD-Modelle. Konstruktion und Arbeitsplanung konnten so ihre Ergebnisse in einem gemeinsamen, stets aktuellen Teile- bzw. Baugruppenmodell ablegen. Über die Instanziierung in der Familientabelle wurden die Änderungen der parametrischen Modelldaten von Arbeitsgang zu Arbeitsgang nachgeführt. Darüber hinaus war so gewährleistet, dass bei konstruktiven Weiterentwicklungen die Konsistenz zur Arbeitsplanung und zur Zeichnungserstellung gewahrt blieb. 
Parallel zu diesen CAD/CAM-Aktivitäten waren im EU-Projekt TOLOMEO einschlägige Kenntnisse zur Struktur und zum internen Ablauf bei automatisierten Fertigungssystemen gefragt, insbesondere zur Werkzeuglogistik.

1996 nahm der Verfasser einen Ruf als Professor für Maschinenbau an die damalige Berufsakademie Berlin, die heute ein Fachbereich der HWR Berlin ist, an. Schwerpunkte der Lehrtätigkeit waren fertigungsnahe Sachgebiete, CAD/CAM und Erneuerbare Energien. Auf Grund der Breite des Fächerspektrums und ohne Laborausstattung war es lange Zeit schwierig, sich aktiv in die Wissenschaftslandschaft einzubringen. Nach jahrelangem Ringen gelang es schließlich doch, Forschungsaktivitäten als freiwillige Teilaufgabe eines Professors zu etablieren. Als Vorsitzender der Forschungskommission konnte der Verfasser hier zu einem allmählichen Wandel beitragen.

Um so wichtiger waren in den wissenschaftlichen Dürrejahren Angebote von außen, teilweise auch von ehemaligen Mitstreitern des Lehrstuhls TP. Zu nennen ist hier vor allem die Möglichkeit, in den Beiräten des EXAPT-Vereins und der Forschungsvereinigung Programmiersprachen mitzuwirken, woraus immer wieder Impulse für die eigene Tätigkeit resultierten. Darüber hinaus bot ein früherer Kollege aus IPK-Zeiten in seinem Unternehmen die langjährige Möglichkeit, bei anspruchsvollen Aufträgen auf dem Gebiet des Produktdatenmanagements im In- und Ausland mitzuwirken.

Mit Genugtuung war zu beobachten, dass in dieser Zeit einigen ehemaligen Assistentenkollegen späte, aber wohlverdiente akademische Ehren zuteil wurden. In den Berufungen von Dimiter Dimitrov, Christian Otto, Arndt Richter und Andreas Nestler kann man auch ein Stück nachholende Gerechtigkeit erkennen. All dies wird jedoch zweifelsfrei von dem unverwüstlichen Engagement unseres gemeinsamen Doktorvaters getoppt.

Dessen eingedenk hat der Verfasser beim altersbedingten Ausscheiden aus der regulären Lehrtätigkeit einige Forschungsaufträge eingeworben. Bei den Machbarkeitsuntersuchungen zum Einsatz bio-basierter Werkstoffe in Mikro-Windenergieanlagen wurden mittlerweile alle Hauptgruppen der Fertigungsverfahren einbezogen, zuzüglich einiger Anleihen bei der Verfahrenstechnik. Ernüchternd ist allerdings, dass z.B. bei der täglichen Arbeit an einem Bohr-/Fräs-Bearbeitungszentrum manche vermeintliche Gewissheit aus der Lehre hier nicht weiterhilft, z.B. im Hinblick auf Genauigkeitsaspekte. Erst der Rückgriff auf das angesammelte Methodenwissen schafft dann (manchmal) die Grundlage für Teilerfolge.

Das Methodenwissen und die Erfahrungen mit Mehrmaschinensystemen bilden darüber hinaus eine geeignete Basis bei der Ausprägung dieser Windkraftanlagen als Multiturbinensysteme. Strukturelle Fragen der Funktionsverteilung, der Konfiguration aus gleichen bzw. verschiedenen Komponenten sowie der technischen Betriebsführung 
einschließlich An- und Auslauf weisen Ähnlichkeiten mit entsprechenden Problemstellungen bei Flexiblen Fertigungssystemen und Flexiblen Transferlinien auf. Hier schließt sich ein Kreis, der ohne den Lehrstuhl Fertigungstechnik/Technologische Programmierung niemals zustande gekommen wäre.

Prof. Dr.-Ing. Reimund Klünder

Juni 2020 


\section{Dokumentarischer wissenschaftlicher Lebenslauf}

\section{unter dem Aspekt „Beiträge zur Digitalisierung und zum Advanced Manufacturing“" anlässlich des 85. Geburtstages von Prof. Detlef Kochan}

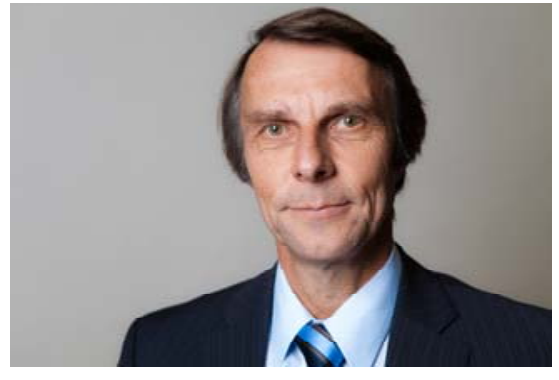

Andreas Nestler (geb. 1954) studiert an der TU Dresden im "Maschineningenieurwesen“ die Fachrichtung „Fertigungsprozessgestaltung“. Die Diplomarbeit am Institut für Fertigungstechnik und Werkzeugmaschinen, Lehrstuhl Fertigungstechnik/ Technologische Programmierung zum Thema "Weiterführung der rechentechnischen Testung des Programms AUTOTECH/KOFA3 mit dem Ziel der praktischen Erprobung der optimalen Arbeitswerte auf der DFS 400 NC" betreuen Prof. Dr.-Ing. sc. techn. Detlef Kochan und Dipl.-Ing. Arndt Richter. Nach dem Studium arbeitet er als Entwicklungsingenieur im Forschungs- und Entwicklungszentrum für Elektromaschinen Dresden, bevor er an die TU Dresden wechselt. Ausgewählte Etappen, Tätigkeiten und Themen in seiner Entwicklung an der Universität sind in der Folge chronologisch dokumentiert.

\section{Entwicklungsweg als Mitarbeiter der Technologischen Program- mierung und Produktionsautomatisierung}

An der TU Dresden beginnt im Jahr 1977 bei $D$. Kochan die Tätigkeit als Wissenschaftlicher Assistent am Lehrstuhl Fertigungstechnik/Technologische Programmierung (FT/TP) mit Aufgaben in Lehre und Forschung.

Für seine Lehrtätigkeit bezieht inn $D$. Kochan sehr schnell in die Lehrveranstaltung „Fertigungsprozessgestaltung II (FPG II)“ ein. Schwerpunkt des Einsatzes ist das Komplexpraktikum FPG II, speziell zur Fertigungsvorbereitung von numerisch gesteuerten Drehmaschinen. Später folgen erste Vorlesungsvertretungen zur rechnerunterstützten NC-Programmierung. Mit der Zielstellung zur Erreichung einer Promotion erhält er für die wissenschaftliche Arbeit die Aufgabe zur Weiterentwicklung von KOFA3 unter den beiden Aspekten der Ausnutzung des Informationsgehaltes zur Bewertung von Fertigungsmitteln und der Einbeziehung des Optimierungsmodells in technologische Programmiersysteme.

\section{Grafisch-interaktive Werkzeug- und Schnittwertermittlung}

Die Forschungstätigkeit beginnt in dem von Dipl.-Ing. A. Richter bearbeiteten Arbeitsfeld zur "Kostenoptimierung Fertigungstechnischer Arbeitsgrößen“ (KOFA) Drehen. Auf der Grundlage der Verfahrensoptimierung ${ }^{1}$ und unter Einsatz des speziellen digitalgeometrischen Arbeitsplatzes (DGA) entsteht an der TU Dresden eine erste, auf der grafischen Programmierung gestützte Realisierung zur Werkzeugauswahl, Schnittwertoptimierung und Fertigungsmittelbewertung ${ }^{2}$, speziell ein

\footnotetext{
${ }^{1}$ ein Arbeitgebiet der Fertigungstechnik, welches die beiden Professoren Kochan (FT/TP) und Jacobs Wissenschaftsbereich Fertigungstechnik/Fertigungsgestaltung (FT/FG) bedienen.

2 in Zusammenarbeit mit Institut für Schiffbau Rostock, Wilhelm-Pieck-Universität Rostock und dem Schiffsdieselmotorenwerk Rostock (Dipl.-Ing. W. Rothe) entstanden bereits ähnliche Lösungen für die Verfahrensoptimierung Fräsen.
} 
grafisch-interaktiver Preprozessor zur Werkzeug- und Schnittwertermittlung für die Drehbearbeitung. Die Dialogprogramme zur Prozessausarbeitung und -bewertung stellen eine Alternative zur bisher üblichen Dateneingabe und -ausgabe mit Hilfe von Eingabeformularen und Bewertungsprotokollen dar. Andererseits ist der Informationsgehalt der Verfahrensoptimierung für komplexe Entscheidungen, z. B. zur Auswahl eines geeigneten Werkzeuges, interaktiv nutzbar. Die technologischen Entscheidungsschritte sind aus Funktionalitäten zur Auswahl, Ermittlung, Simulation, Manipulation und Kontrolle aufgebaut. Als Prozessor fungiert KOFA3 ${ }^{3}$. Im Ergebnis der Interaktion liegen fertigungstechnisch begründete Prozessvarianten vor. Die damit erreichte Problemlösung setzt jedoch noch in den Entscheidungsschritten Kenntnisse bzw. Erfahrungen des menschlichen Experten zu den fertigungstechnischen Zusammenhängen des Gesamtsystems Maschine-SpannmittelWerkstück-Werkzeug (WSWW) voraus bzw. ist durch „trial and error" interaktiv zu ergründen (Bild 1 und Bild 2).

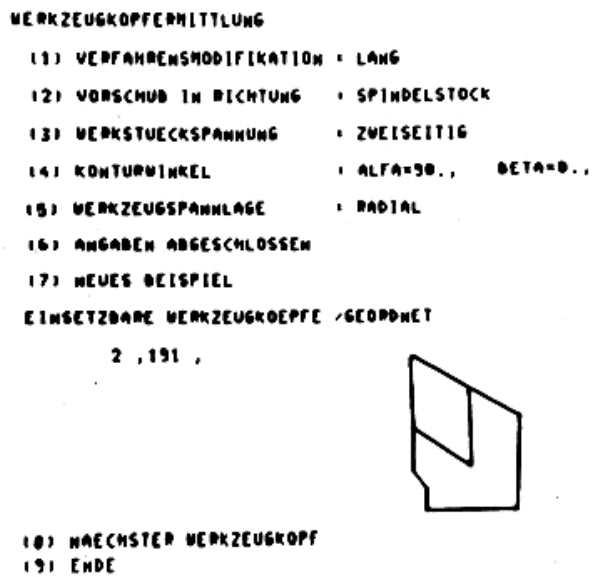

Bild 1: $\quad$ Prozessausarbeitung - Dialog zur Werkzeugermittlung

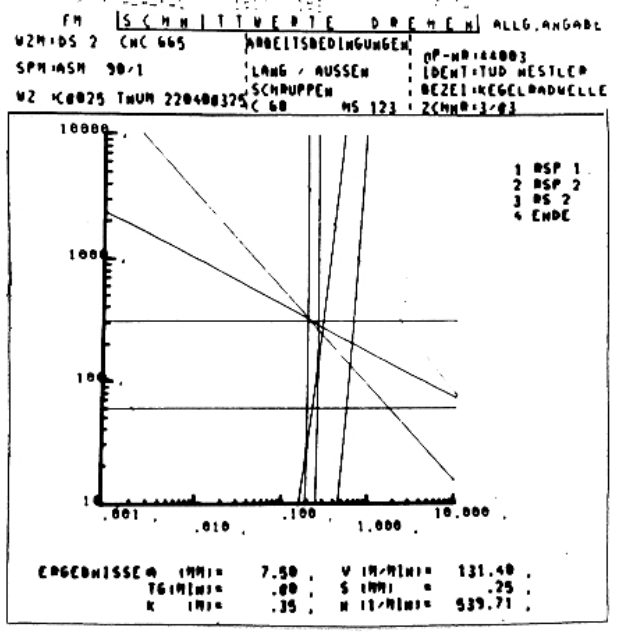

Bild 2: Prozessbewertung - Dialog zur Schnittwertermittlung

Die Arbeiten von $A$. Nestler sind mit den technischen Voraussetzungen einerseits der Drehmaschine DFS 400 mit Bahnsteuerung NC 450 (Bild 3), später DS 2/2 CNC 665 zur praktischen Testung im Versuchsfeld Kutzbach-Bau und andererseits mit dem Großrechner BESM 6, später ESER 1022 sowie mit dem grafisch-interaktiven Display GD'71 (Gerätetechnik mit Ein- und Ausgabemedium Bildschirm und den peripheren Einrichtungen Lichtstift, Positionierkugel, Funktions- sowie alphanumerische Tastatur) aus Ungarn ${ }^{4}$, koppelbar mit KRS- und ESER-Rechnern, im Willers-Bau (Bild 4) verknüpft.

Für die entwickelten FORTRAN-Anwendungsprogramme zum grafisch interaktiven Dialog dient ein KRS 4201 mit dem Betriebssystem GIST als Steuerrechner für das grafische Display GD'71. Zum Zugriff auf das Anwendungsprogramm KOFA3 dient eine Kopplungsvariante, über die das grafische Terminal mit dem Großrechner EC 1022 angeschlossen ist.

\footnotetext{
${ }^{3}$ das Optimierungsprogramm KOFA3 ist auch als 3. Niveaustufe ein Bestandteil des DDR-zentralen Schnittwertspeichers Magdeburg

${ }^{4}$ In Zusammenarbeit mit Sektion Mathematik, Wissenschaftsbereich Mathematische Kybernetik und Rechentechnik (Dr.-Ing.rer.nat. R. Ortleb)
} 
1984 promoviert $A$. Nestler bei D. Kochan zum Dr.-Ing. mit der Arbeit „Bildschirmunterstützte Werkzeug- und Schnittwertermittlung beim Drehen“.

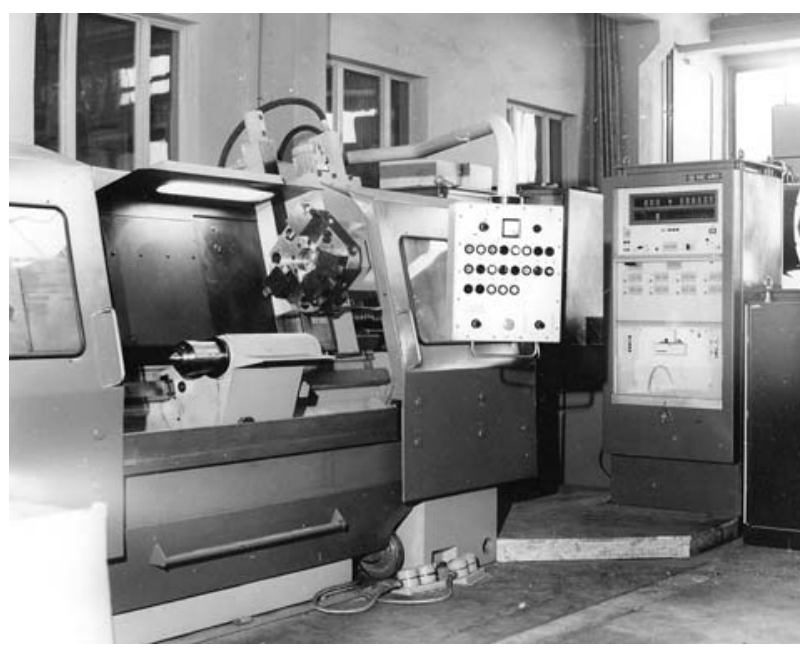

Bild 3: Drehmaschine DFS 400 NC 450

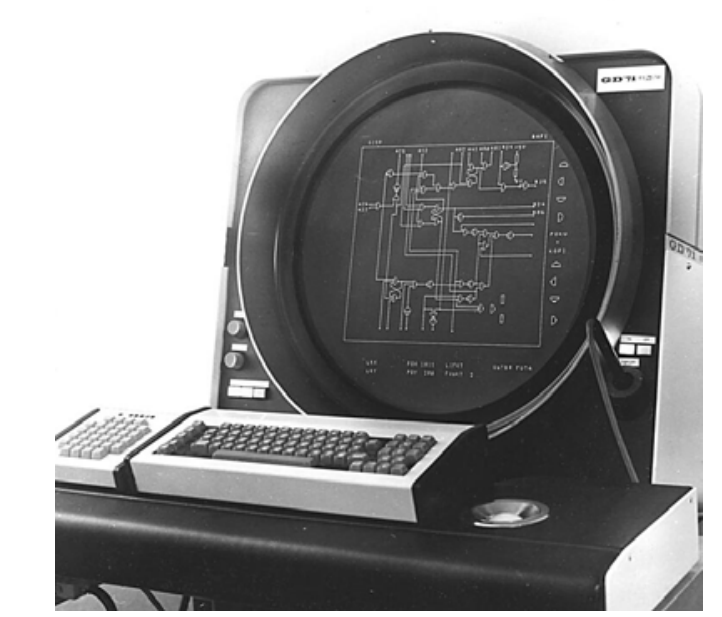

Bild 4: Grafisch-interaktives Display GD'71

\section{Technologisch leistungsfähige NC-Programmiersysteme}

Diese Forschung steht im Zusammenhang mit der Anforderung zur Entwicklung der technologischen Leistungsfähigfähigkeit von NC-Programmiersystemen für das Drehen. Es existieren bereits Konzepte und Prototypen zur Einbeziehung der vorliegenden Bausteinkomplexe KOFA 3 und der Bausteine zum "Einheitlichen System zur Maschinellen Programmierung" (ESMP) in das kommerziell verfügbare NCProgrammiersystem AUTOTECH-NCM/ROTA vom Leitzentrum für Anwendungsforschung (LfA) Schwerin (erste Phase etwa1979-1982, Konferenz INFERT'82).

In der weiteren Forschungstätigkeit baut $A$. Nestler aus den vorliegender Arbeiten zum Schnittwertoptimierungssystem KOFA (Dr.-Ing. A. Richter) und Kinematiksystem ESMP (Dr.-Ing. R. Klünder, Dipl.-Ing. K. Beyer) einen integrierten Modul ESKO (ESMP in Verbindung mit KOFA) Drehen. Damit entsteht eine technologisch leistungsfähige Schnittwert- und Kinematikermittlung für technologische Operationen und geschlossen beschriebene Bearbeitungssegmente, die über den Stand der Technik zur NC-Programmierung hinausgeht. Umfangreiche Zerspantests auf der Drehmaschine DS 2/2 CNC 665 (Dipl.-Ing. Bernholz) im Versuchsfeld Kutzbach-Bau belegen die praktische Anwendung der Berechnungsergebnisse.

Eine etwa 500 Seiten umfassende Dokumentation nach der Rahmenmethodik der Datenverarbeitungsprojektierung mit den Bestandteilen Anwenderdokumentation, Programmdokumentation, Problemdokumentation und Dokumentation Rechenzentrum ist über mehrere Jahre in Arbeit. AUTOTECH/ESKO bildet den Ausgangspunkt für weitere Aktivitäten.

Mit der Möglichkeit zur Einbeziehung von ESKO in das NC-Programmiersystem AUTOTECH-NCM/ROTA über eine Erweiterung der AUTOTECH-Eingabesprache entsteht eine neuen Qualität zur Ausarbeitung der Arbeitsstufen zum Arbeitsgang Drehen (Bild 5). Damit ist AUTOTECH/ESKO sowohl autonom als auch über weitere Fachsprachen (APT-Dialekte wie EXAPT, EUROAPT) flexibel anpassbar und im Verbund mit NC-Programmiersystemen nutzbar (zweite Phase etwa 1981-1984). 


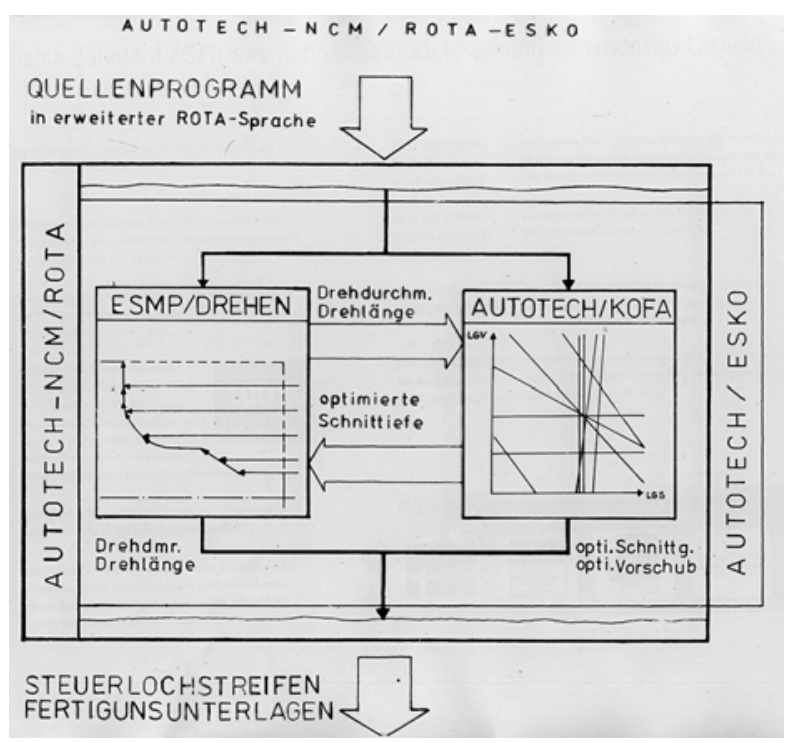

Bild 5: Struktur zu AUTOTECH-NCM/ROTA mit Einbeziehung von AUTOTECH/ESKO

Diese Entwicklung ist Anfang der 80er Jahre wiederum integraler Bestandteil des Konzeptes für Durchgängig Automatisierte Informationssysteme (DAUS), speziell zum Arbeitsgangprogramm AUDRES für das Drehen (Dr.-Ing. J. Hartmann). In Konzeptionen (6. Seminar „Rechnerunterstützte Fertigungstechnik“, DAUS-Seminar 1981 in Hartenstein) ist der fortgeschrittene Stand zum Drehen ein grundlegender Bestandteil. Realisiert für ESER-Rechner mit dem Betriebssystem OS/ES liegt das Ergebnis im Jahr 1984 als Komponente der Pilotlösung des CAD/CAM-Systems ${ }^{5}$ TU-ROTA-P für rotationssymmetrische Einzelteile (Dr. J. Sämisch) vor ${ }^{6,7}$. Im Rahmen einer Preprint-Reihe der TU-Dresden sind diese Sachverhalte u. a. zu „CAD/CAM für rotationssymmetrische Einzelteile“ zusammengefasst.

In dieser Etappe sind AUTOTECH-Produkte zu modular aufgebauten CAM-Teilsystemen weiterentwickelt. Mit dieser Gesamtlösung lassen sich nun die Grundlagen zur rechnerintegrierten Fertigung an die Industrie herantragen. Erste Voruntersuchungen zur Anwendung finden u.a. in der Berliner Werkzeugmaschinen Fabrik in Marzahn und im Druckmaschinenwerk Planeta in Radebeul statt.

Von 1983 bis 1989 dominiert bei $A$. Nestler die Industrietätigkeit zur Weiterentwicklung und Einführung technologisch leistungsfähiger Systemkomponenten für die Arbeitsplanung und NC-Programmierung innerhalb von CAD/CAM-Lösungen als Mitarbeiter Forschung/Entwicklung im Polygraph Druckmaschinenwerk Planeta Radebeul. Die Arbeiten ordnen sich in die betriebliche Anwenderlösung „CAD/CAMScheiben/Trommeln“ (Dr.-Ing. B. Brönner) ein. Die Weiterentwicklung von ESKO richtet sich auf die Umstellung für DOS3-Bedingungen, die softwaretechnische Entkopplung von NCM/ROTA unter Beibehaltung der Fachsprachenverbindung zu übergeordneten Systemen sowie auf Inhalte zu ESKO-Teilsystemen. Außerdem konzentriert sich die Arbeit auf die Erweiterung von Inhalten zum zugehörigen FZWFertigungsmittelspeicher. Darüber hinaus ist die Kopplung von Arbeitsgangbausteinen mit dem Schnittwertspeicher Magdeburg (DDR-Informationszentrum für

\footnotetext{
${ }^{5}$ Mitte der 80er Jahre wurde für DAUS endgültig das weltweit übliche CAD/CAM übernommen

${ }^{6}$ in Zusammenarbeit mit VEB Berliner Werkzeugmaschinen Fabrik (BWF) Berlin-Marzahn (Dr.-Ing. R. Klünder, Dr.-Ing. G. Mittelstädt), VEB Forschungs- und Entwicklungszentrum für Elektromaschinen Dresden (Dr.-Ing. A. Richter) und VEB Fortschritt Erntemaschinen Neustadt (Dipl.-Ing. K. Beyer)

${ }^{7}$ Nachnutzung über VEB Großdrehmaschinenbau Karl-Marx-Stadt, ein Betrieb des VEB Werkzeugmaschinenkombinat „7. Oktober“ Berlin.
} 
Schnittwerte der spanenden Teilefertigung ${ }^{8}$ ) gefordert. AUTOTECH/ESKO V2.0 als eine Großrechnervariante DOS3/ES zur Stapelverarbeitung des Systems einschließlich der Projektdokumentation wird im Jahr 1986 dem Betrieb übergeben; ein Jahr später folgt im Rahmen des Vertrages über den gemeinschaftlichen Vertrieb und die Weiterentwicklung die Übergabe als Variante OS/ES an das LfA Schwerin.

Zum Industrieeinsatz der Software kommt es nicht. Gründe liegen u.a. darin, dass die Komplexität der Gesamtlösung, die erforderlichen Datenbasen sowie die über Großrechner-Terminals eingeschränkte Mensch-Maschine-Kommunikation dem industriellen Einsatz noch nicht gerecht wird. „Wir sind mit der technologisch anspruchsvollen Lösung zur Arbeitsgangausarbeitung nach dem Optimierungsprinzip der Zeit noch zu weit voraus" (Aussage H.-J. Jacobs zum Abschluss der Arbeiten). Außerdem steht bereits die nächste Rechnergeneration zur Ablösung bereit.

\section{CAP/NC-Module zur Arbeitsgangausarbeitung Drehen}

Neue rechentechnische Herausforderungen und Möglichkeiten eröffnen ab Mitte der 80er Jahre die vollständige Übernahme der Software auf sog. Personalcomputer (PC). Den ersten IBM-PC im Wissenschaftsbereich erhielt $A$. Nestler völlig überraschend von $D$. Kochan, den er als IFIP-Vertreter anlässlich einer aktiven Teilnahme in Berlin-West mit Unterstützung eines Kollegen von Prof. Spur einkaufte. Ein PC, noch ohne Festplatte, mit 3,5-Zoll Diskettenlaufwerk und MS-DOS sowie WINDOWS $1.0^{9}$, wozu damals allerdings keine Anwendungen verfügbar sind.

Mit der zunehmend in der Breite verfügbaren PC-Technik ${ }^{10}$ treten Forschung und Entwicklung für Modell-, Methoden- und Datenbasen sowie zur Kommunikation in den Vordergrund. Unter maßgeblicher Mitgestaltung entsteht nach einer neuen Grundkonzeption (Dr. J. Sämisch) bis 1989 ein Rahmensystem CAP/NC als Softwarekomplex aus problemabhängigen und -unabhängigen Modulen (Bild 6).

Als weitere Eigenentwicklungen zur Arbeitsgangausarbeitung Drehen fließen die Komponenten NC-FMS als Fertigungsmittelspeicher (Dr. J. Sämisch), NC-TEACH als Fachspracheneditor (Dipl.-Ing. J. Michaelis), NMK für wissensbasierte Anwendungen (Dr.J.Sämisch) sowie NC-Archiv als Dateiverwaltung und TOOLBOX als Dialog- und Servicesoftware (Dr. J. Sämisch) ein ${ }^{11}$.

Die Offenheit und Erweiterbarkeit des Systems erlaubt beliebige Ausbaustufen, z. B. die Integration von Modulen zu weiteren speziellen Verfahrensanforderungen wie QUATTRO zum Drehen auf $2 \times 2-$ Achsen Bearbeitungsmaschinen ${ }^{12}$ (Dr.-Ing. U. Brümmer, Dr.-Ing. M. Hess) oder zu anderen Fertigungsverfahren wie SAFRAE zum Fräsen (Dr.-Ing. Ch. Schöne).

Neben Eigenentwicklungen sind im offenen Rahmensystem CAP/NC die kommerziell verfügbaren NC-Programmiersysteme NCM-DIALOG (ab 1990 DCAM-nc, NCSoft Wagner Schwerin) und DR 43 (FZW Karl-Marx-Stadt, ab 1990 GERFEMA Chemnitz) integriert.

\footnotetext{
${ }^{8}$ VEB Forschung, Entwicklung und Rationalisierung des Schwermaschinen- und Anlagenbaus Magdeburg (FER)

${ }^{9}$ Grafische Benutzeroberfläche für den Umgang mit PC-kompatiblen MS-DOS (1985)

${ }^{10}$ Stand 1990: FORTRAN, IBM XT/AT mit Grafik CGA, EGA, VGA und Betriebssystem MS-DOS

${ }^{11}$ in Zusammenarbeit mit POLYGRAPH Planeta Radebeul und NILES Stellantriebe Dresden

12 in Zusammenarbeit mit Forschungs- und Entwicklungszentrum Werkzeugmaschinen (FZW) KarlMarx-Stadt
} 

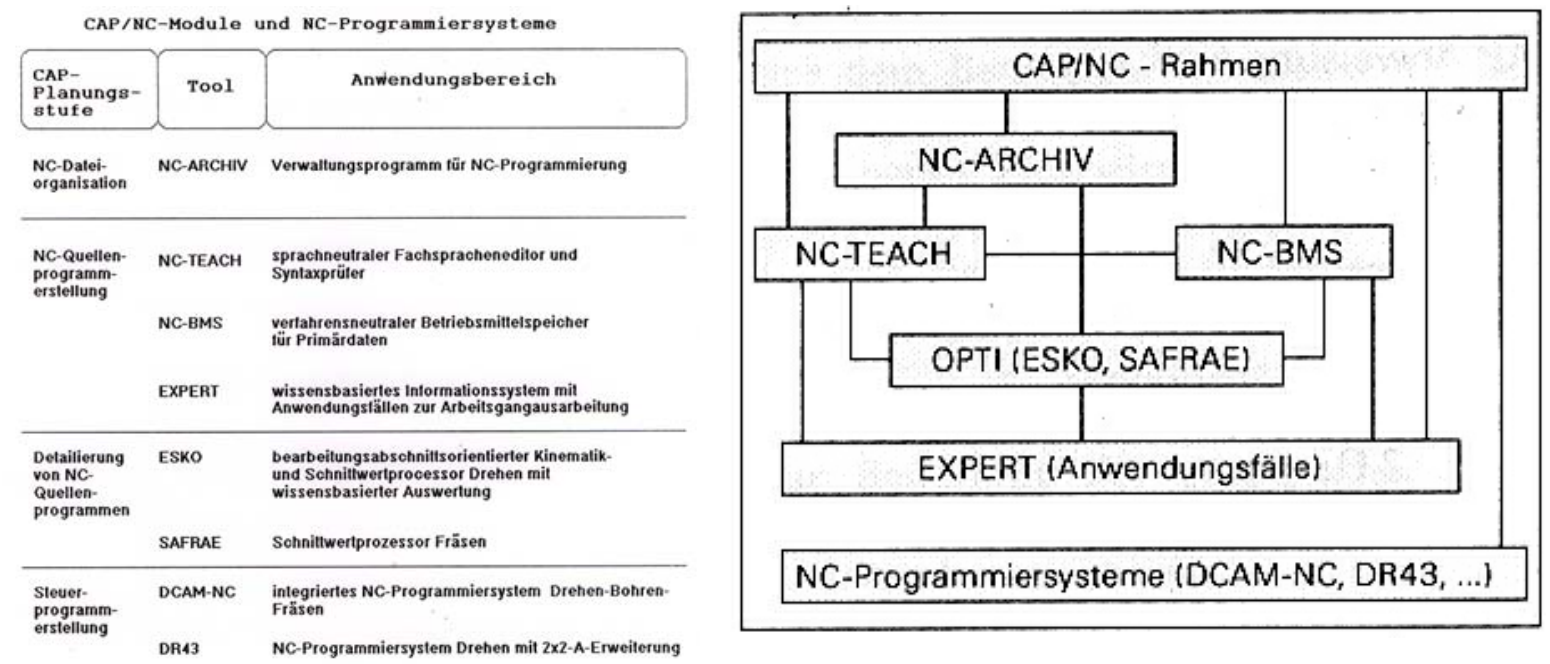

Bild 6: CAP/NC - Tools (lks.) und informationelle Verknüpfungen der Module (rts.)

Mit der Einbeziehung einer neuen wissensbasierten Systemkomponente - auf der Grundlage der Methode der Nichtmetrischen Klassifizierung (NMK) von Informationen (Dr. J. Sämisch) - in ESKO (1988) gelingt eine neue Qualität der Benutzerunterstützung zur Bewertung von Einflussgrößen auf die komplizierten technologischen Zusammenhänge der optimierten Schnittwerte (Bild 7). Für Technologische Operationen können Einflussgrößen auf das System der technischen Grenzen erstmalig im grafisch-interaktiven Dialog auf gängiger PC-Technik ergründet, bewertet und zielgerichtet verändert werden. Damit erreichen die Möglichkeiten zur Optimierung von Schnittwerten, zur Bewertung der eingesetzten Fertigungsmittel und zur Einflussnahme auf das Berechnungsergebnis ein praktikables Niveau für einen, über den Experten hinausgehenden, breiten Anwenderkreis.

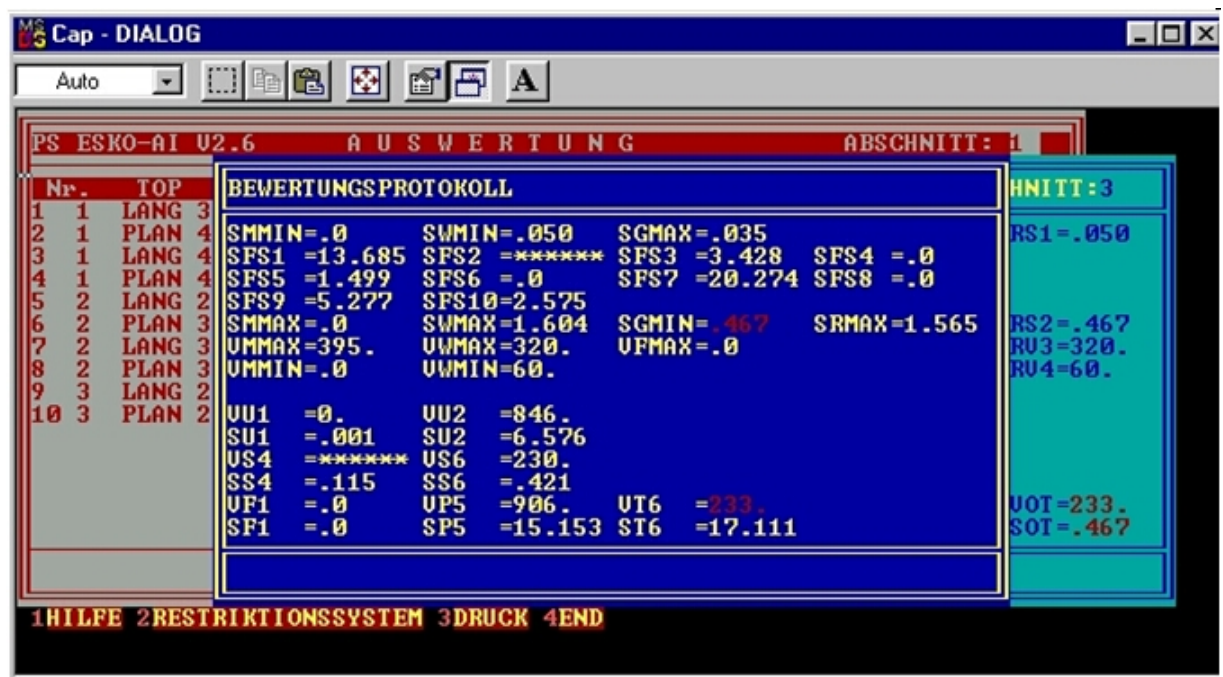

Bild 7: Modul ESKO (Drehen): Auswertung Technologischer Operationen mit Protokoll zur Bewertung des komplexen Restriktionssystems der technischen Grenzen und einer wissensbasierten Hilfe für ergebnisrelevante Einflussparameter

Die Methode zur wissensbasierten Kinematik- und Schnittwertermittlung beim Drehen wird erstmals zur 7. int. IFIP/IFAC Konferenz „Software for Manufacturing" PROLAMAT' 88 in Dresden vorgestellt und u. a. in der Fachzeitschrift ZWF-CIM 84 (1989) veröffentlicht. 
Für das - aus dem Anwendungsfall zur computerunterstützten Wissensakquisition für Restriktionssysteme entwickelte Modul EXPERT (Dr. J. Sämisch) werden bis 1991 weitere technisch-technologischen Problemstellungen aufbereitet. Damit eröffnet sich die Möglichkeit für den Arbeitsplaner und NC-Programmierer ein wissensbasiertes Umfeld sowohl mit objektiven (allgemein vorhandenes Wissen) als auch subjektiven (Wissen des Anwenders) Wissensquellen aufbauen. Zahlreiche Anwendungsfälle, wie zur Fertigungsmittelzuordnung und zum Schneidwerkstoffeinsatz, unterstützen die implementierten CAP/NC-Module und schließen bestehende Lücken im automatisierten Informationsfluss.

Die computergestützten Wissensakquisition wird 1990 zum 15. Problemseminar "Rechnerunterstützte Fertigungstechnik" in Gaußig vorgetragen und u.a. in der Fachzeitschrift ZWF-CIM 85 (1990) veröffentlicht.

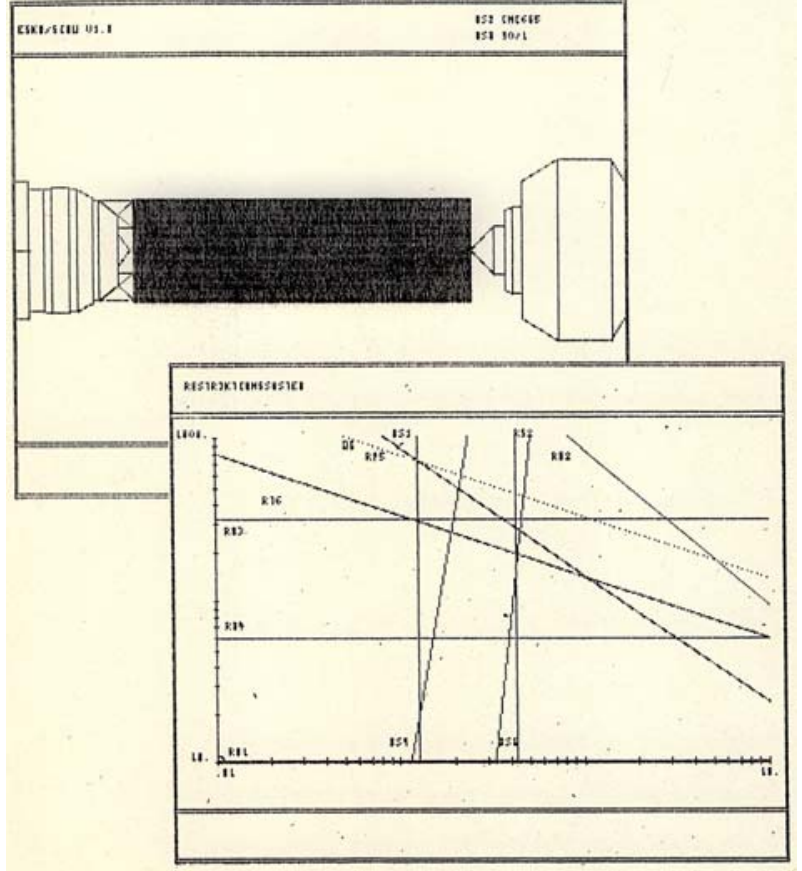

Bild 8: Modul ESKO (Drehen): Simulation
Ein paralleles Arbeitsgebiet ist die Visualisierung und Simulation technisch-technologischer Sachverhalte. Die PC-Technik gestattet für die Weiterentwicklung von ESKO zunehmend grafische Unterstützung (Grafikkarten z. B. Hercules, monochrom; später VGA, mehrfarbig). Erste realisierte Simulationen beinhalten zunächst $u$. $a$. einfache statische Darstellungen zur Werkstückgeometrie mit Einspannverhältnissen, zu Bearbeitungsabschnitten, Fertigungsmitteln und Restriktionssystemen, die wahlweise im Dialog nutzbar sind (Bild 8). Später folgen dynamische Darstellungen, die Abläufe mit eingesetzten Werkzeugen an der aktualisierten Werkstückgeometrie (Bearbeitungskinematik) simulieren.

Die aus CAP/NC resultierende Pilotlösung kommt 1989 als neue Komponente zum Komplexpraktikum „FPG II“ in der Lehre zur Anwendung. Dieses Praktikum stellt für die Studenten der Studienrichtung Produktionstechnik eine grundlegende Querschnittsveranstaltung dar. Dazu steht im Zeu-Bau der PC-Pool (CAERechenstation, Dipl.-Math. Krahmer) der Fakultät Maschinenwesen zur Verfügung. Zur Arbeitsgangausarbeitung Drehen entsteht neues übungsbegleitendes Lehrmaterial in Form eines Lehrheftes und eines Wissensspeichers (Bild 9) sowie einer Studienanleitung. Außerdem wird dementsprechend das Vorlesungsmaterial zur rechnerunterstützten NC-Programmierung grundlegend aktualisiert. 

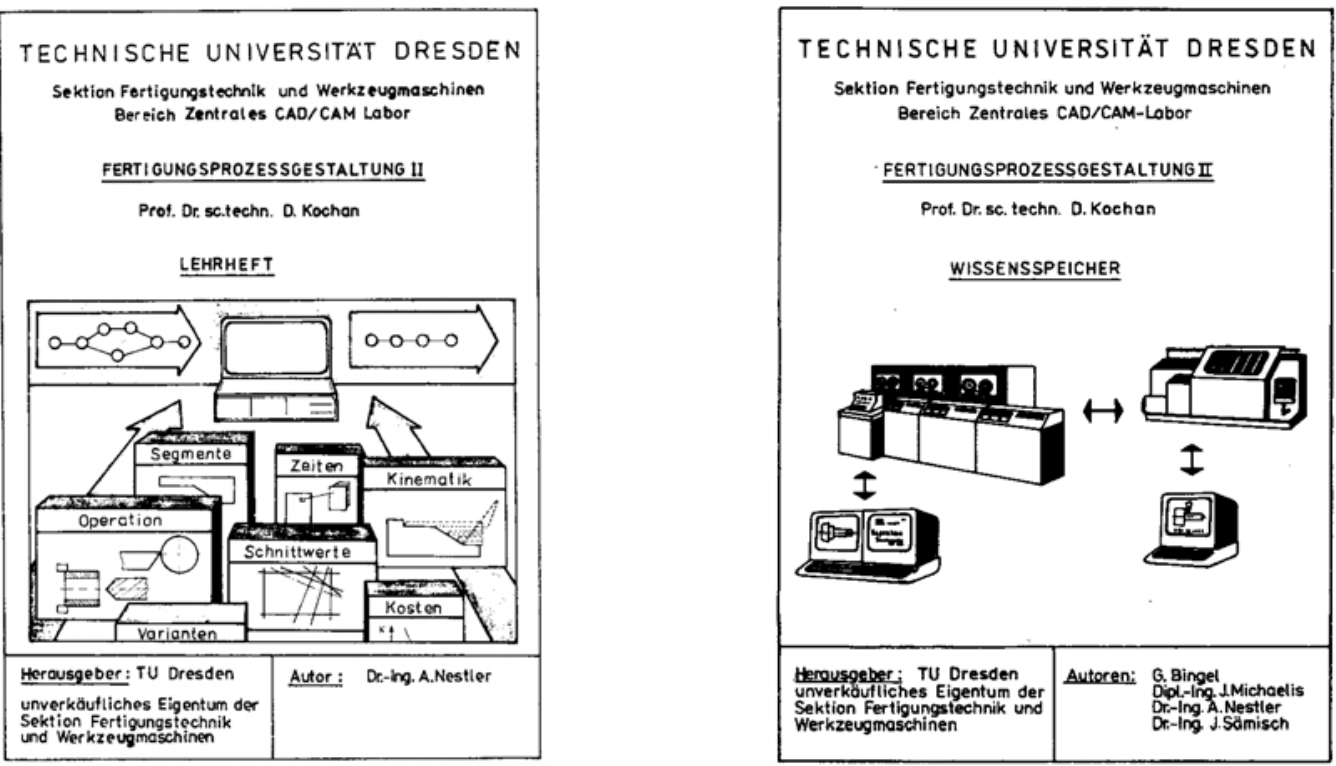

Bild 9: Lehrheft und Wissensspeicher zum Praktikum FPG II

Im Zeitraum von 1990 bis 1992 entstehen auf Initiative von D. Kochan zahlreiche neue Kontakte, u.a. zu Firmen wie Norsk Data GmbH in Mühlheim/a.d.Ruhr (Dipl.Ing. Baltersee, Dr.-Ing. Porrmann), Mähdrescherwerk Bischofswerda/Singwitz (Dipl.Ing. Schlenkrich), Traub AG in Reichenbach/Fils ( $v$. Zeppelin), MAHO AG in Pfronten (Dr.-Ing. Bähren), EXAPT Systemtechnik GmbH (Dr.-Ing. Budde) in Aachen oder auch zur Universität Twente (Dr.-Ing. van Houten). Neben Demonstrationen zu CAP/NC sind Angebotsinformationen erarbeitet, es finden Anwenderseminare statt und mögliche Projekte werden besprochen und geplant.

Auf Messen, wie der CeBIT '91 in Hannover, wird die Leistungsfähigkeit und Innovation der Forschungsergebnisse präsentiert (Bild 10). Die technischtechnologische Komplexität und Wissensunterstützung zur Kinematik- und Schnittwertermittlung für die NC-Programmierung wird derzeit von bekannten nationalen und internationalen Lösungen nicht erreicht.

Mit der Gründung des Technologie-Transfer-Zentrums für rechnerintegrierte Fertigung (CIMTT) an der TU Dresden (1991) sind die Systemkomponenten fester Bestandteil für auf die Industrie ausgerichtete Transferangebote (Bild 11). A. Nestler unterstützt zu aktuellen Fachthemen auch zahlreiche CIMTT-Seminare mit Themen wie „NC-Programmiersysteme im Vergleich“. Auf der Basis aktuell verfügbarer Rechen- und Maschinentechnik an der TU Dresden liefert er Beiträge zu Referenzlösungen für durchgängige CAD/NC-Datenflüsse.

Die Erfahrung aus diesen konstruktiven Aktivitäten zu CAP/NC subsumiert sich in der Skepsis der Partner gegenüber Modulen zur Schnittwertoptimierung bzgl. der damit verbundenen Aufwände zu den Datenbasen, insbesondere zu erforderlichen Kennwerten, die nicht trivial zu Verfügung stehen. Diese Kennwerte wie Koeffizienten und Faktoren zur Berechnung z. B. von Schnittkräften, Standzeiten u. a. oder Steifewerte von Maschinen und deren Komponenten sind zwar unter universitären Bedingungen mit Aufwänden ermittelbar bzw. bei Maschinenherstellern beschaffbar, für die breite industrielle Anwendung ist diese Datenbasis jedoch nach wie vor nicht verfügbar. 


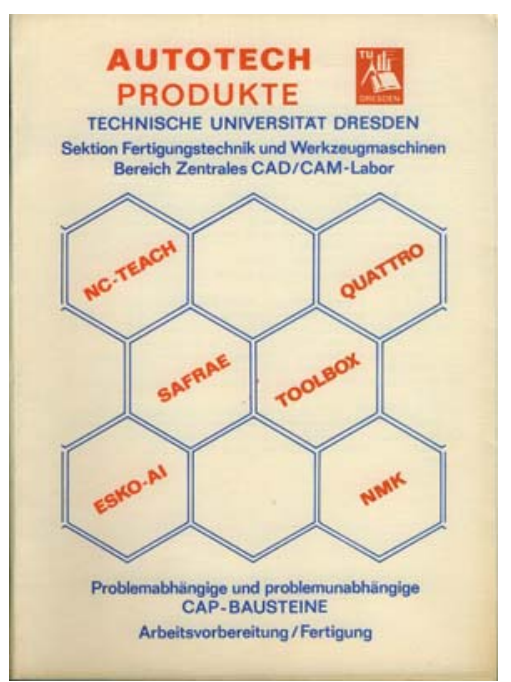

Bild 10: Messeaktivitäten mit eigenständigen Softwarelösungen, 1989 Wissenschaftsbereich ZCCL (lks.) und 1991 Institut für Fertigungsinformatik (rts.)

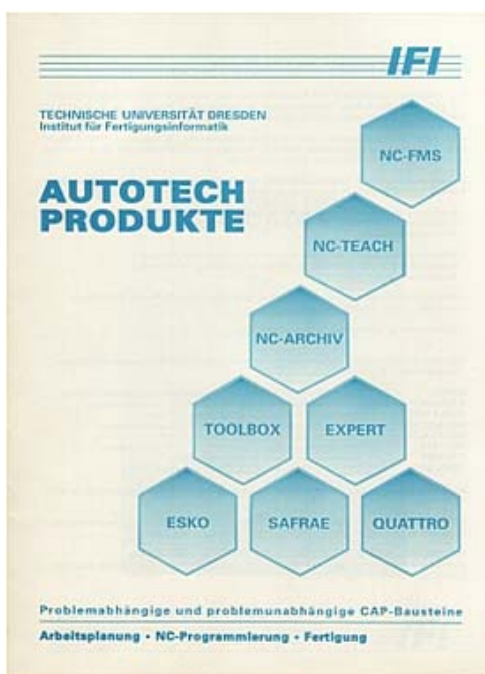

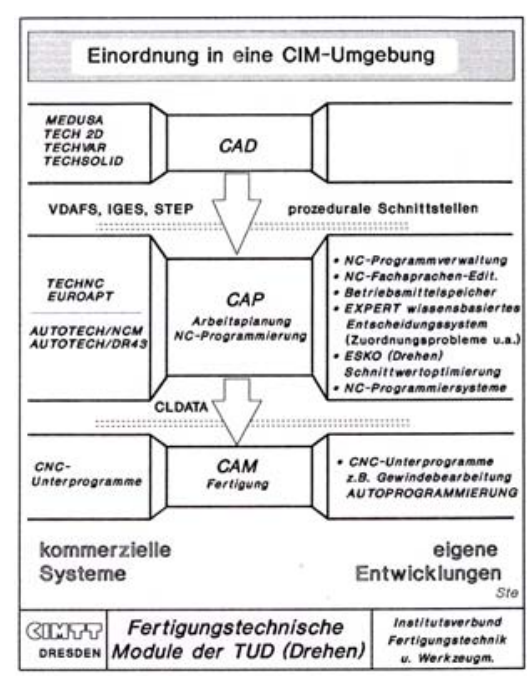

Bild 11: Transferangebot vom CIMTT der TU Dresden

\section{Mittel und Methoden von Technologiedatenbanken}

Anfang der 90er Jahre richtet sich die Forschungsarbeit stärker auf Themenkomplexe der Mittel und Methoden von Technologiedatenbanken, die bereichsübergreifend als Integrationskomponente für CAx-Systeme eine zentrale Bedeutung erlangen. Die auf ein von $D$. Kochan initiiertes und über die Forschungsvereinigung „Programmiersprachen für Fertigungseinrichtungen e.V. (FVP)“ der Arbeitsgemeinschaft industrieller Forschungsvereinigungen (AiF) gefördertes Projekt „Technologiedatenbank" bezogene Forschungsarbeit (1991-93) konzentriert sich zunächst auf das Umfeld des NC-Programmiersystems EXAPTplus der EXAPT Systemtechnik $\mathrm{GmbH}$ Aachen (Bild 12). Neben der EXAPT-Software kommen DEC-PC's und eine DEC-Workstation als Hardware zur Nutzung in Forschung und Lehre an der TU Dresden hinzu. Mit einem relativ großen Mitarbeiterkreis entstehen eine Systemplattform an der TU Dresden, Referenzlösungen für Anwender in der sächsischen Industrie ${ }^{13}$, methodische Richtlinien und Musterdatenbestände zu Bereichen der Technologiedatenorganisation sowie ein Leitfaden zur Einführungsunterstützung. Dieses erste AiF-Projekt in der Projektverantwortung von $A$. Nestler steht aber auch für eine außerordentlich wertvolle Lehrzeit zum Neuland des Drittmittel-Projektmanagements und der projektbezogenen Arbeiten nach den noch

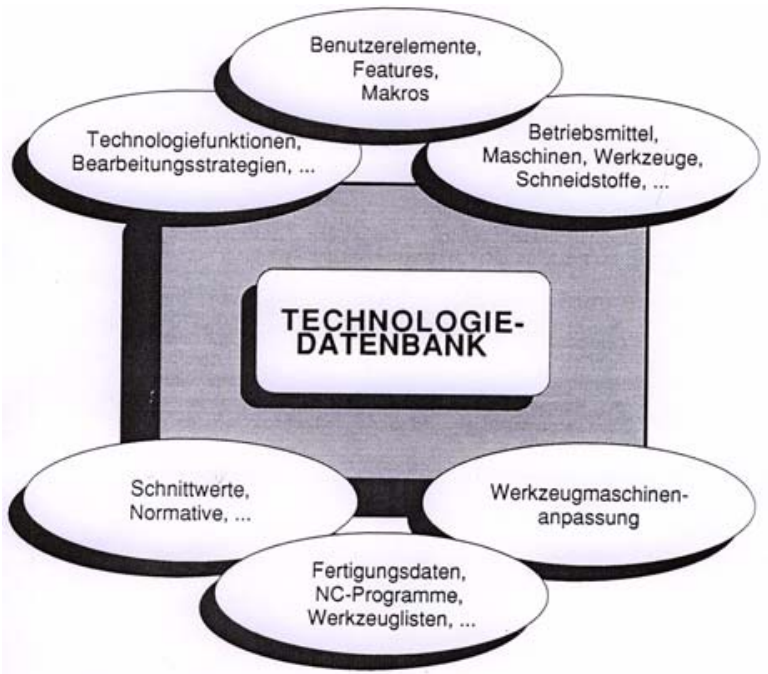
ungewohnt neuen Arbeitsweisen.

Bild 12: Gegenstand Technologiedatenbank (Bild Forschungsvereinigung Programmiersprachen e.V. Aachen)

\footnotetext{
${ }^{13}$ in Zusammenarbeit mit Union Werkzeugmaschinenfabrik Gera GmbH, Siemens AG Turbinenbau GmbH Görlitz, Wagonbau Niesky GmbH, KSB AG Halle, Berliner Bremsenwerk - Knorr-Bremsen AG
} 
Das NC-Programmiersystem EXAPTpus kommt ab 1994 in der Lehre zur Anwendung. A. Nestler entwickelt ein Praktikum, das insbesondere auf die technologische Prozessausarbeitung und die automatisierte Technologieplanung abzielt. Als Begleitmaterial für Praktikum und Vorlesung entstehen die Hefte „EXAPT-Fachsprache in Übersichten“ und „EXAPTplus Drehtechnologie und Bohr-/Frästechnologie“. Das Vorlesungsmaterial zu den Lehrveranstaltungen „Rechnerunterstützte NCProgrammierung" und "Technologiedatenbank" wird um die Programmierung mit der EXAPT-Fachsprache und zu den Technologiedatenbereichen erweitert.

Der komprimierte Stand der Ergebnisse und Erfahrungen zu NC-Programmiersystemen mit mathematischen Schnittwertoptimierungsmodellen zum Drehen und Fräsen, zur wissensbasierten Unterstützung und zum Technologiedatenmanagement ist u.a. zur IFIP Konferenz "Modelling and Simulation for Optimization of Manufacturing Systems Design and Application" 1989 in Tempe (Arizona, USA), zur IFIP TC5/WG 5.3 Working Conference „Process Planning for Complex Machining and AI-Methods" 1991 in Gaußig bei Dresden und zur 8. int. IFIP/IFAC Konferenz PROLAMAT '92 „Man in CIM“ in Tokio (Japan) aufbereitet. 1992 erhält A. Nestler die erste Gelegenheit im „kapitalistische Ausland“ an einer internationalen Konferenz teilzunehmen und wissenschaftliche Ergebnisse des Lehrstuhls Fertigungstechnik/Technologische Programmierung zu präsentieren, die er mit D. Kochan und Ch. Schöne erarbeitet hat.

Ab 1992 erhält $A$. Nestler als Oberassistent bei Prof. Dr.-Ing. habil. D. Fichtner an der neu geschaffenen Professur ${ }^{14}$ für Produktionsautomatisierung/Steuerungstechnik (PAS), ab 2002 Professur für Produktionsautomatisierung, Zerspan- und Abtragtechnik (PAZAT), des ebenfalls neu gegründeten Institutes ${ }^{15}$ für Produktionstechnik (IPT) die Gelegenheit seine Erfahrungen und Lehrveranstaltungen in den Lehrbetrieb einzubringen und die bisherige Forschungstätigkeit zu Technologiedatenbasen für die CNC-Fertigung fortzusetzen. Das betrifft insbesondere Themen zu Gestaltung von Informationsflüssen, Datenschnittstellen und Systemkomponenten sowie alternative Methoden zum Technologiedatenmanagement in Prozessketten der automatisierten Teilefertigung.

Der Stand der Forschungsergebnisse zum Thema Technologiedatenbank wird u.a. zum IFAC-Workshop „Intelligent Manufacturing Systems“" IMS'94 in Wien präsentiert.

Für die Lehre findet 1993 zur Vorlesung „Fertigungsprozessgestaltung“ eine Übungsvariante statt, bei der die manuelle und grafisch interaktive NC-Programmerstellung mit der Software des KELLER-Systems (Grundsoftware, GKE, DAM, Steuerungssimulatoren) im Rechnerraum Kutzbach-Bau E1 und zur Fertigung die Drehmaschine CTX 400 mit der Steuerung EPL 2 und die Fräsmaschine DECKEL FP4A mit der Steuerung DIALOG 11 im Versuchsfeld zum Einsatz kommen.

Zur Vorlesung „Fertigungsinformatik“ entsteht 1994 neues Praktikum. Anliegen ist das Kennenlernen ausgewählter Systeme und Schnittstellen der automatisierten Prozesskette. Fertigungsaufgabe ist ein prismatisches Werkstück. Die Informationsflüsse im Netzverbund verlaufen von der 3D-Modellierung und Zeichnungserstellung (CAD mit ICEM DDN) über die CAD/NC-Kopplung mit IGES-Schnittstelle, Technologieplanung und NC-Programmierung (CAP mit DCAM-nc) im CAE-Labor Zeu-Bau bis zur Werkstattsteuerung (CAM mit Bosch DNC/BDE-Leitsystem, Werkzeug-

\footnotetext{
${ }^{14}$ Lehrstühle werden ab 1992 durch Professuren ersetzt

15 Sektionen und Wissenschaftsbereiche werden 1991 durch Institute ersetzt
} 
voreinstellgerät Zoller) und CNC-Fertigung (Fräsmaschine MAHO 800) im Versuchsfeld Kutzbach-Bau.

Ausgehend vom Thema Technologiedatenbank besteht Notwendigkeit, sich mit neuer Forschung stärker den intelligenten Softwaretechnologien in der Anwendungsdomäne der Prozesskette der spanenden Teilefertigung widmen. Neben der Anwendung rein datenbasierter und algorithmischer Methoden beschäftigt sich A. Nestler zunehmend mit wissens- und erfahrungsbasierten Methoden.

\section{Featuretechnologie und intelligente Softwaretechnologien als neue Strategie der Produktionsautomatisierung}

Die Featuretechnologie, die ihren Ursprung in der Werkstückklassifizierung zur automatisierten Fertigung und der Makroprogrammierung zur NC-Technik hat, gewinnt mit fortschreitender Leistungsfähigkeit der Rechentechnik und den damit einhergehenden Möglichkeiten zur Anreicherung von Informationen in STEPProduktmodellen bis Ende der 90er Jahre an Bedeutung. Die sich etablierte Objektorientierung von CAD/CAM-Software ist für die weitere Forschung grundlegend.

Ein Ziel der avisierten Arbeiten ist es, der Bedeutung der Software für Innovationen im Anwendungsbereich gerecht zu werden. Anspruchsvolle Aufgaben werden mit immer höheren Anteilen softwaretechnischer Lösungen automatisiert, die zunehmend nach ihrer Intelligenz bewertet werden. Um dieser Erwartungshaltung von Anwendern entgegenzukommen, werden weltweit Anstrengungen unternommen, sog. intelligente Softwaretechnologien in Anwendungsbereichen zu etablieren. Das Interesse der eignen Forschungsarbeit zur Produktionsautomatisierung, speziell der Informationsprozesse, ist durch neue objekt- und agentenorientierte Methoden der Softwareentwicklung beeinflusst.

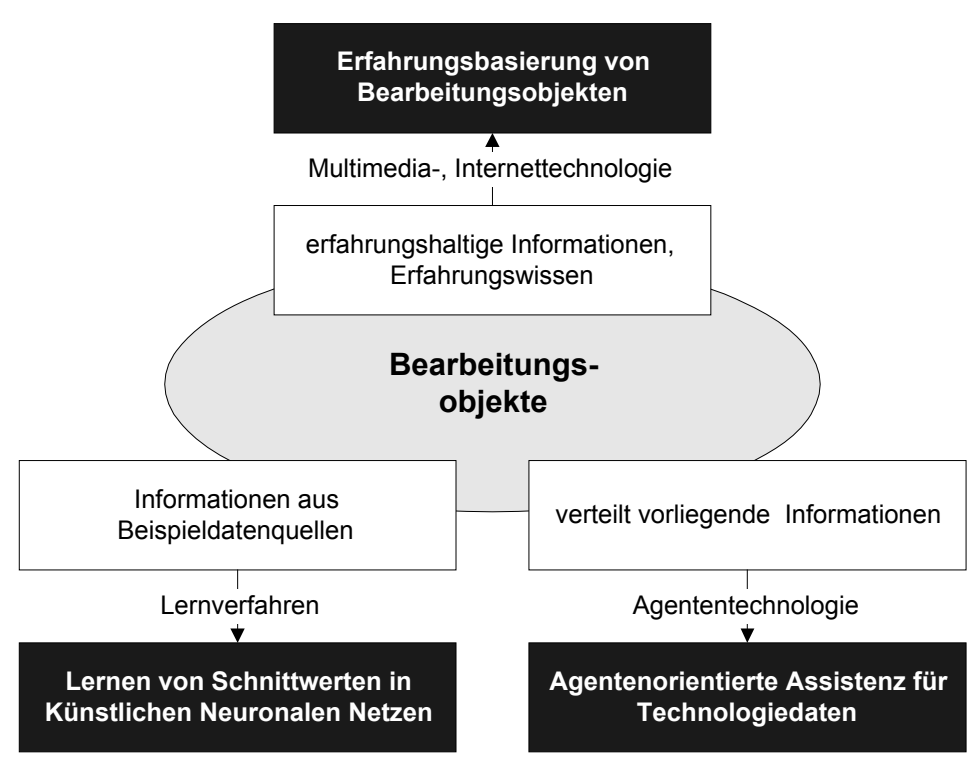

Bild 13: Bearbeitungsobjekte als Informationscontainer

Betrachtete Schwerpunkte zu Technologiedaten sind das Maschinelle Lernen, insbesondere die Anwendung Künstlicher Neuronaler Netze zur Schnittwertermittlung, der Einsatz von Multiagententechnologien, insbesondere die Assistenz und Agenten zur Beschaffung aus der Fertigung und Aufbereitung für die Planung und das Erfahrungswissen, insbesondere die Anwendung von Multimediatechnologien für komplizierte Bearbeitungsfälle. Das verbindende Objekt dieser Forschungsarbeiten ist das Bearbeitungsfeature (Bild 13), worunter ein Zerspansegment, das mit einem Werkzeug in einer Operation hergestellt wird, zu verstehen ist.

\section{Das Lernen von Schnittwerten in Künstlichen Neuronalen Netzen}

Hervorhebenswert ist die Projektverantwortung für das durch die DFG geförderte Projekt mit Grundlagenuntersuchungen zur Wissensakquisition für Schnittwerte beim 
Fräsen unter Nutzung von Künstlichen Neuronalen Netzen $(\mathrm{KNN})^{16}$. Alternativ zu den komplexen analytischen Modellen zur Berechnung von Schnittwerten können die auch auf der Grundlage von Beispieldaten gelernt und im Rahmen einer Technologiedatenbank anwendergerecht bereitgestellt werden (Bild 14).

Unter Verwendung der Entwicklungsumgebung Stuttgarter Neural Network Simulator (SNNS) entstehen im Bearbeiterkollektiv bis 1999 zahlreiche Anwendungsfälle zu vorliegenden Hersteller- bzw. Werkzeugkatalogen und Schnittwertspeichern sowie zu Berechnungsergebnissen von Optimierungssoftware ${ }^{17}$ und betriebsspezifischen Datensammlungen $^{18}$. Die praktischen Tests der Netzausgaben zu Schnittwerten durch Facharbeiter im Versuchsfeld erbringt im Durchschnitt gute Ergebnisse. Ein Leitfaden zur Anwendung von KNN verallgemeinert das erprobte Vorgehen. Zur transparenten Verwaltung der zahlreich anfallenden Dateien im Erstellungsprozess entstand ein nützliches KNN-Dateiverwaltungsmodul. Der erreichte Entwicklungstand erfordert für die erste Phase zum Maschinellen Lernen noch Expertenwissen. Die zweite Phase, die Anwendung selbst, ist bereits für jeden Nutzer ohne Kenntnisse zu KNN geeignet.

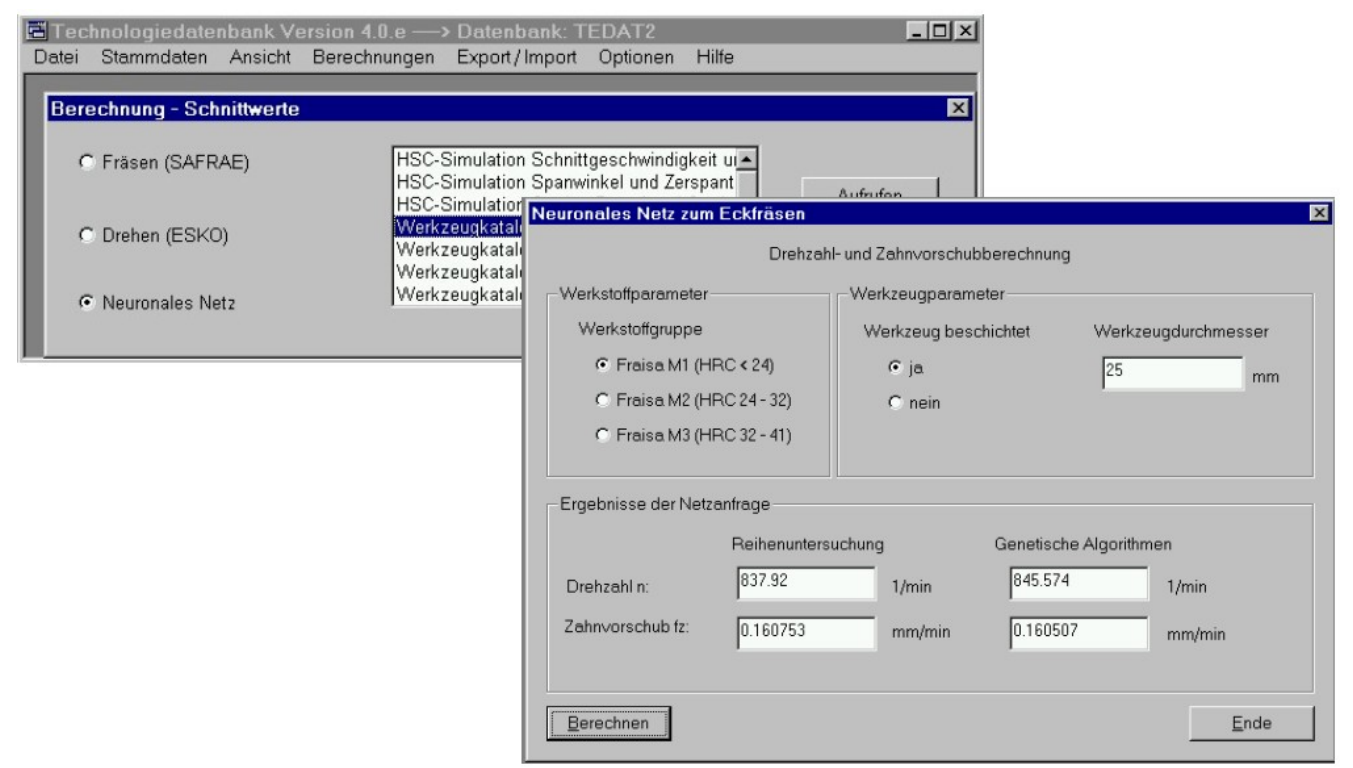

Bild 14: Technologiedatenbank mit der Funktionalität Berechnungen, hier für Schnittwerte mit mathematischen Modellen (ESKO, SAFRAE) und neuronalen Modellen (mit Ergebnissen nach zwei Methoden zur KNN-Strukturoptimierung)

Von den zahlreichen Veröffentlichungen zu diesen bemerkenswerten Ergebnissen sollen stellvertretend die aktiven Beiträge zu den Konferenzen „Computer Applications in Production Engineering “ CAPE'95 in Peking, „European Conference on Integration in Manufacturing" liM'97 in Dresden, "Flexible Automation and Intelligent Manufacturing" Faim'98 in Portland, International IFIP TC5 Prolamat'98 in Trento und "International Conference on Industrial Tools“ ICIT'99 im Maribor genannt sein.

Bedauerlich ist, dass ein vorliegendes Manuskript zur Dissertation von Dipl.-Ing. M. Schulz, der sehr kreativ und überdurchschnittlich aktiv das Projekt ausgefüllt hat, nicht zu Ende gebracht werden konnte.

\footnotetext{
${ }^{16}$ ein Teilgebiet der Künstlichen Intelligenz (KI), Neuroinformatik

${ }^{17}$ in Zusammenarbeit mit der John Moores University Liverpool

${ }^{18}$ mit Unterstützung KBA Planeta Radebeul und Siemens Turbinenbau Görlitz
} 
In der Folge entstanden erste Konzepte und Prototypen zur Ablaufautomatisierung der Datenbehandlung und Netzgenerierung für weitestgehend automatisierte Lernprozesse in Form eines KNN-Selbstlernmoduls. Das Thema kontinuierliches, adaptives Selbstlernen für aktuelle Datenbasen wird greifbar.

\section{Agentenorientiertes Assistenzsystem für verteilte Technologiedaten}

Informationen werden durch die Möglichkeiten der Vernetzung zunehmend verteilt gehalten. Die Assistenz ist dabei eine Form der Unterstützung von Informationssystemen zur Informationsbeschaffung. Für Technologiedatenbasen fehlt den Anwendern ein derartiges Organisationsinstrument, insbesondere für zeitaufwändige, immer wiederkehrende Abläufe.

Für eine proaktive Planungsunterstützung wird eine kontinuierliche Informationsversorgung aus dezentralen Datenbasen erarbeitet. Die selbstständige Beschaffung und Aufbereitung verteilt vorliegender Informationen für NC-Planungs- und Berechnungsmethoden unter Nutzung von Multiagentensystemen (MAS) ${ }^{19}$ entsteht als Forschungsbeitrag für das zunehmend kooperative Technologiedatenmanagement. Zum MAS werden Agententypen wie z. B. Maschinen-, Bearbeitungsobjekt- und Neuroagent entwickelt, die durch ihre spezifischen Aufgaben, Fähigkeiten und Kommunikation unterschiedlich agieren. Über einen sog. agentenbasierter Arbeitsplatz, realisiert als Prototyp eines Assistenzsystems AgentAP auf der Basis des Agentenprogrammiersystems Aglets (IBM) und weiterer Entwicklungswerkzeuge, werden auf Bearbeitungsobjekte bezogene Informationen von dezentralen Standorten, z.B. im Bearbeitungsprozess an CNC-Steuerungen, gesammelt (Bild 15) und durch Maschinelles Lernen zur Nutzung im Planungsprozess aufbereitet.

Damit wird zur Wiederverwendung und zum kontinuierlichen Lernen von Informationen beigetragen. Das Fazit aus diesen Arbeiten ist, dass die Agentifizierung produktionstechnischer, cyberphysikalischer Objekte in Verbindung mit dem Maschinellen Lernen intelligente, kommunizierende und zielgerichtet handelnde Objekte wie Maschine, Spannmittel, Werkzeug oder Werk-

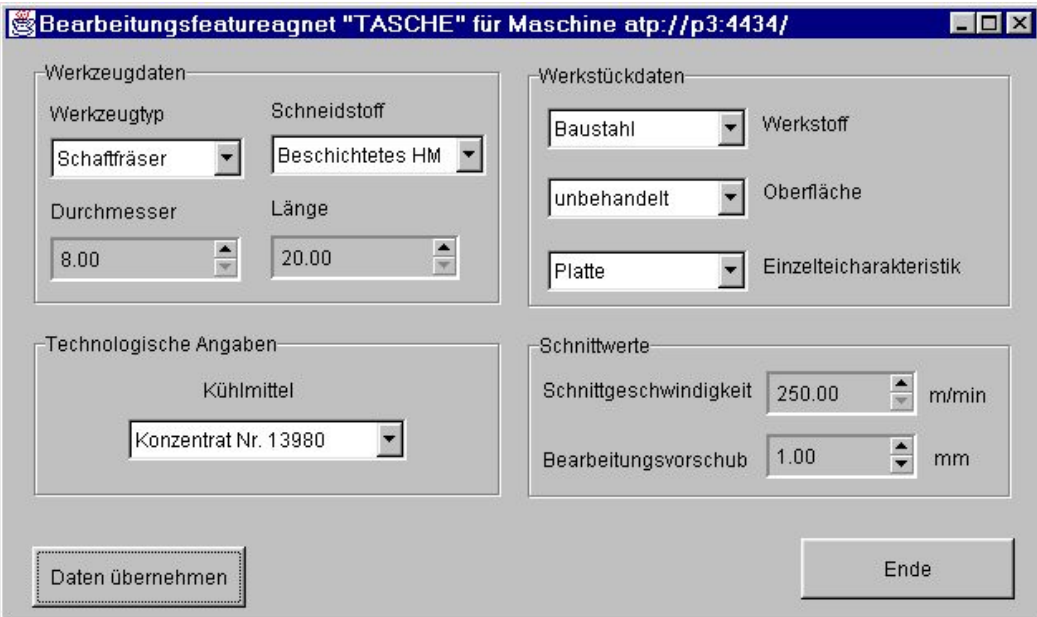

Bild 15: Ein Bearbeitungsfeatureagent repräsentiert Bearbeitungsobjekte, hier beispielhafte Informationen zur Objektklasse „Tasche“ stück ermöglicht.

Ergebnisse werden u.a. zur International "Conference on Flexible Automation \& Intelligent Manufacturing (FAIM'02) in Dresden vorgestellt und 2006 im International Journal of Computer Integrated Manufacturing veröffentlicht.

Als weiteres MAS-Anwendungsgebiet ist die agentenbasierte DNC-Software erforscht. Ein Prototyp als Programmassistent unterstützt NC-Planer bzw. NCProgrammierer und überwacht Änderungen an NC-Daten im heterogenen DNCSystem.

${ }^{19}$ ein Teilgebiet der Verteilten Künstlichen Intelligenz (VKI) 


\section{Erfahrungsbasierung von Bearbeitungsobjekten}

Das Wissen zur Vorbereitung, Planung und Durchführung einer Bearbeitungsaufgabe ist häufig personalisiert und hochgradig erfahrungsintensiv. Die Wissens- und Erfahrungsbasierung von Informationen und Informationsmodellen ist ein interdisziplinäres Forschungsanliegen ${ }^{20}$. Als Voraussetzung dazu fehlen erfahrungshaltige Informationen und technische Mittel zu deren Aufbereitung und Bereitstellung.

Zur Erfahrungsbasierung von Bearbeitungsobjekten ist eine neue Methoden entwickelt, nach der Informationen der Operationsplanung mit Erfahrungsanteilen ergänzt werden können. Als Träger erfahrungshaltiger Informationen wird das Erfahrungsobjekt mit handlungsorientierten Inhalten definiert. Zur deren Darstellung werden beliebige multimediale Informationen gebündelt. Die dazu notwendigen Daten und Dokumente verwaltet eine weiterentwickelte Technologiedatenbank.

Die Berücksichtigung von Erfahrungsgehalten in einer Datenbank-Applikation geht über die bisher übliche Abbildung von Daten in Tabellen einer relationalen Datenbank hinaus. Die realisierte Pilotlösung JTeDat ist eine in Java implementierte Anwendung. JTeDat ermöglicht für umfangreiche multimediale Datenbestände komfortable Web-Anwendungen für verteilte Arbeitsweisen mit einer größeren Anzahl von Benutzern (Bild 16).

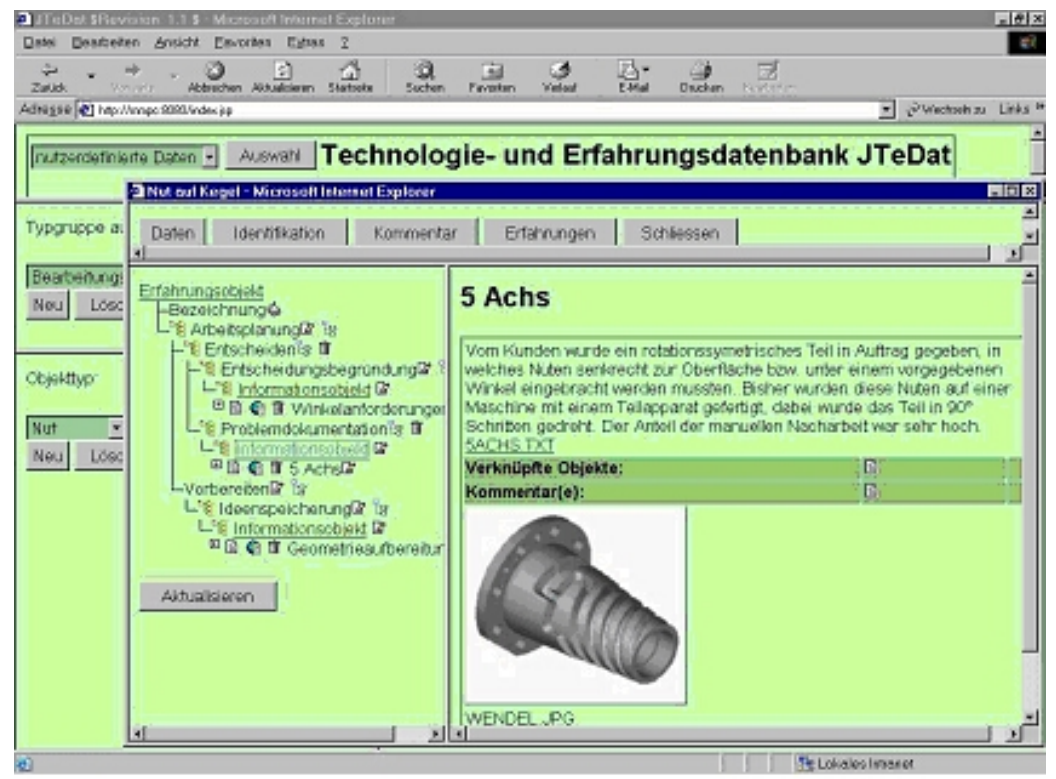

Bild 16: Web-Anwendung der Technologie- und Erfahrungsdatenbank mit Beispiel zum Erfahrungsobjekt „Nut auf Kegel“ zeigt die Erfahrungsstruktur und -inhalte

Ergebnisse sind u. a. zur IFIP Konferenz „Feature Modelling and Advanced DesignFor-The-Live-Cycle Systems“(FEATS'01) in Valenciennes und zur Konferenz „Global Project and Manufacturing Management" (MIP'04) in Siegen präsentiert.

Ein PAZAT-Fachkolloquium „Intelligente Produktionsprozesse - Softwarelösungen zur Gestaltung von Fertigungsprozessen" findet an der TU Dresden mit rund 70 Teilnehmern einschließlich Referenten von Hochschulen und aus der Industrie statt.

Diese Forschungsarbeiten führen $2005 \mathrm{im}$ Ergebnis zur durch D. Fichtner betreuten Habilitation mit dem Thema „Produktionsautomatisierung unter dem Einfluss der Informationstechnologien - Intelligente Informationsprozesse zum Technologiedatenmanagement in der Fertigung".

${ }^{20}$ beispielsweise Arbeitswissenschaft und Angewandter Informatik 


\section{Zusammenfassung zum Tätigkeitszeitraum 1977 bis 2005 als Mitarbeiter}

Im Zeitraum bis 1992 arbeitet $A$. Nestler als Mitarbeiter am Lehrstuhl Fertigungstechnik/Technologische Programmierung (D. Kochan), Wissenschaftsbereich Fertigungsprozessgestaltung der Sektion Fertigungstechnik und Werkzeugmaschinen. Später wechseln die Bezeichnungen der Wissenschaftsbereiche in Fertigungsgestaltung (1980), Zentrales CAD/CAM-Labor (1986) und Fertigungsinformatik (1990). Am daraus hervorgegangenen Institut für Fertigungsinformatik (1991) folgt nach dem Ausscheiden D. Kochans aus der Hochschule (1992) die Weiterarbeit bereits unter der Leitung von Doz. Dr.-Ing. sc. techn. Fichtner.

Mit der Gründung des Institutes für Produktionstechnik (1992) kann A. Nestler die Arbeit in seinem Fachgebiet an der neu gegründeten Professur für Produktionsautomatisierung und Steuerungstechnik (Prof. Dr.-Ing. habil. Fichtner) sowie später an der aus der Fusion mit der Professur für Zerspan- und Abtragtechnik (K. Künanz) hervorgegangen Professur für Produktionsautomatisierung, Zerspan- und Abtragtechnik (2002) fortsetzen.

Erfahrungen sammelt er bei der Projektverantwortung und -leitung (DFG, AiF, kmU), der Organisation regionaler Informationsveranstaltungen (in Zusammenarbeit mit CIMTT der TU Dresden) und überregionaler Fachtagungen (Dresdner Produktionstechnisches Kolloquium).

Er führt Lehrveranstaltungen zu CAD/CAM, NC-Programmierung und NC-Planung sowie zum Technologiedatenmanagement im Direkt- und Fernstudium sowie zur Weiterbildung durch. Damit werden an der TU Dresden ausgewählte Inhalte der „Fertigungsprozessgestaltung“ als eigene Lehrveranstaltungen „Rechnerunterstützte NC-Programmierung" und "Technologiedatenbank" weitergeführt. Zu beiden Lehrveranstaltungen entstehen im Rahmen der Reihe „Fertigungsinformatik“ Studienbriefe für das Fernstudium.

Außeruniversitär ist er sporadisch mit ausgewählten Vorlesungen an der HTW Dresden und der Berufsakademie Sachsen in Dresden tätig.

In den neunziger Jahren ist die Mitarbeit im Beirat des CNC-Zentrums Dresden eine wertvolle Ergänzung zum Lehrbetrieb an der Universität. Seit der Gründungsversammlung am 24. Juni 1993 treffen sich regelmäßig Fachvertreter der Hochschulen, Berufsausbildung und Industrie, um neue Möglichkeiten der Ausgestaltung der Facharbeit mit neuster CAD/CAM und CNC-Maschinentechnik voranzubringen.

Die Oberassistententätigkeit ist u.a. mit der Planung von Lehre und Forschung, der Öffentlichkeitsarbeit und der Zusammenstellung von Jahresstatistiken geprägt.

Mehrere Jahre unterstützt $A$. Nestler als stellvertretender Vorsitzender das Wirken der "Gesellschaft zur Förderung der angewandten Informatik" (GFal) Sachsen e.V.

Im Jahre 1999 beginnt die Mitgliedschaft im Programmkomitee der „International Conference on Industrial Tools" (ICIT).

Über 60 nationale und internationale wissenschaftliche Veröffentlichungen und aktive Teilnahme an internationalen Kongressen im Ausland (Japan, China, Österreich, Slowenien, Frankreich, Italien, USA) kennzeichnen die Aktivitäten auf dem Weg zu neuen wissenschaftlichen Erkenntnissen.

Mit dem erfolgreichen Abschluss der Habilitation im Jahr 2005 erlangt er die Befähigung, in Wissenschaft und Lehre auf dem Gebiet „Produktionsautomatisierung spanender Teileprozesse“ wirken zu können. Dem akademischen Titel Privatdozent 
(PD) geht ein Antrag auf Lehrbefugnis an der Fakultät Maschinenwesen voraus. Mit der Verleihung der Lehrbefugnis ist das Recht zur Führung der Bezeichnung "Privatdozent" verbunden.

\section{Entwicklungsweg als Leiter der Produktionsautomatisierung, Zerspan- und Abtragtechnik ab 2005}

Mit Wirkung vom 01.10.2005 wird für die bisherige Professur für Produktionsautomatisierung, Zerspan- und Abtragtechnik (PAZAT) unter Leitung von D. Fichtner die Struktureinheit Arbeitsgruppe eingerichtet. Ab dem Wintersemester 2005/06 wird A. Nestler die Leitung der selbstständigen Einheit am Institut für Formgebende Fertigungstechnik (IFF) und des Fachgebietes Produktionsautomatisierung, Zerspanund Abtragtechnik übertragen, womit alle in Lehre und Forschung auszuführenden Aktivitäten eingeschlossen sind (Bild 17).

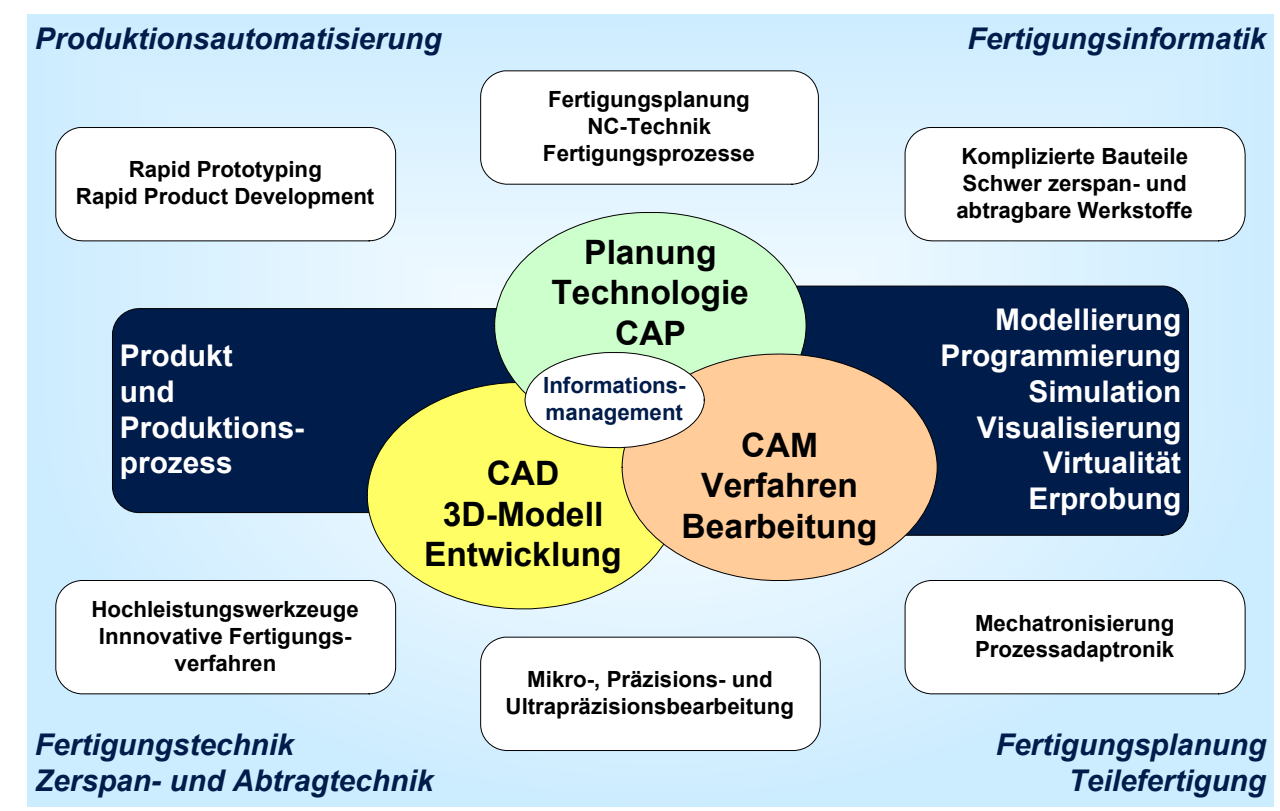

Bild 17: Lehr-, Forschungs- und Arbeitsgebiete der Arbeitsgruppe Produktionsautomatisierung, Zerspan- und Abtragtechnik (PAZAT) unter Leitung von A. Nestler

Die Arbeitsgruppe PAZAT vertritt in Lehre und Forschung als Querschnittsdisziplin die Produktionsautomatisierung und Fertigungsinformatik, weiterhin die Fertigungsplanung der Teilefertigung und die Fertigungsverfahren der Hauptgruppe Trennen mit der Zerspan- und Abtragtechnik. In diesem Zeitraum ab 2005 werden u. a. über 10 Lehrveranstaltungen im Direkt- und Fernstudium verantwortet:

- Fertigungstechnik I und II / Zerspan- und Abtragtechnik, Präzisions-, Ultrapräzisions- u. Mikrozerspanung, Abtragtechnik u. Werkzeugkonstruktion

- Fertigungsplanung I und II / Teilefertigung

- Produktionsautomatisierung, Fertigungsinformatik, Mehrachstechnologien, Rapid Product Development.

Forschungsschwerpunkte bilden die Systeme und Prozesse der automatisierten spanenden Teilefertigung. Das betrifft sowohl Verfahrensuntersuchungen an der Wirkstelle zwischen Werkzeug und Werkstück als auch Mittel und Methoden zur 
Gestaltung von Prozessketten vom Entwurf über die Planung und Simulation der Abläufe bis zur Bearbeitung auf mehrachsiger CNC-Maschinentechnik.

In konsequenter Weiterverfolgung bewährter Forschungslinien und unter Einbeziehung aktueller Erfordernisse für zukünftige Produkte und Prozesse der automatisierten spanenden Teilefertigung verfolgt $A$. Nestler schwerpunktmäßig die drei Entwicklungsrichtungen:

- Information und Software für die Produktionsautomatisierung, insbesondere Fertigungsplanung, NC-Technik und Fertigungsprozesse zur Bearbeitung komplizierter Bauteile.

Realisierte Projekte: u. a. zu den Themen virtuelle Bearbeitungsmaschine, virtuelles Werkstück, Bearbeitungsmodelle mit physikalischen Eigenschaften, Kennwerte zur physikalischen Simulation und Digitaler Zwilling.

- Technologieentwicklung zur Bearbeitung schwer zerspanbarer Werkstoffe und zur Präzisionszerspanung.

Realisierte Projekte: u. a. zu den Themen Schwerzerspanung Drehen, binderlose Schneidwerkstoffe für höchste Anforderungen, HSC-Fräsbearbeitung, Bearbeitung und Simulation zellularer metallischer Werkstoffe, Aspekte der Zerspanbarkeit bzgl. Spanform und Schleifprozesse im Trockenschliff.

- Mechatronisierung und Prozessadaptronik, insbesondere Werkzeugtechnik und Steuerungskomponenten mit Bezug zu Prozessdaten.

Realisierte Projekte: u. a. zu den Themen prozessdatenbasierte Entwicklung von Schleifwerkzeugen, Draht als Werkzeug zur Präzisionszerspanung, homogen beheizbare Werkzeugtechnik für Faserverbundbauteile und modulare aktorische Werkzeuge zur Schwerzerspanung.

Ausgewählte, als Projektleiter initiierte und betreute Forschungen, die zur Weiterentwicklung fertigungstechnisch orientierter Informatik-Anwendungen entstehen, sollen in Kurzform charakterisiert werden.

\section{Virtuelle Bearbeitungsmaschinen}

Beginnend im Jahr 2006 stellt die Technische Universität Dresden über das Media Design Center (MDC) erstmals zentrale Unterstützungsmittel für Vorhaben im Bereich e-Learning zur Verfügung.

Mit der Kenntnis zu früheren Multimedia-Projekten für die Lehre (z. B. Multimediale Lehr- und Lernumgebung Maschinenwesen) wird zunächst mit eigenen Entwicklungen das Ziel verfolgt, vorrangig zur Schulung des räumlichen Vorstellungsvermögens und für komplizierte Zusammenhänge unterstützende 3D-Simulationen vorlesungsbegleitend und zum Selbststudium anzubieten. Das geschieht anfangs nur im Rahmen der Vorlesung, in der weiteren Entwicklung werden die Inhalte über die Lernplattform OPAL ${ }^{21}$ bereitgestellt. So können ausgewählte Inhalte von Einzellehrveranstaltungen der Fertigungstechnik, Fertigungsplanung und Produktionsautomatisierung mit studentischen Projekten zur Online-Simulation von Werkzeugen (Bild 18, Bild 19), Maschinen und Planungsvorgängen umgesetzt werden.

${ }^{21}$ OPAL (Online-Plattform für Akademisches Lehren und Lernen) ist das zentrale LernmanagementSystem der Sächsischen Hochschulen. 


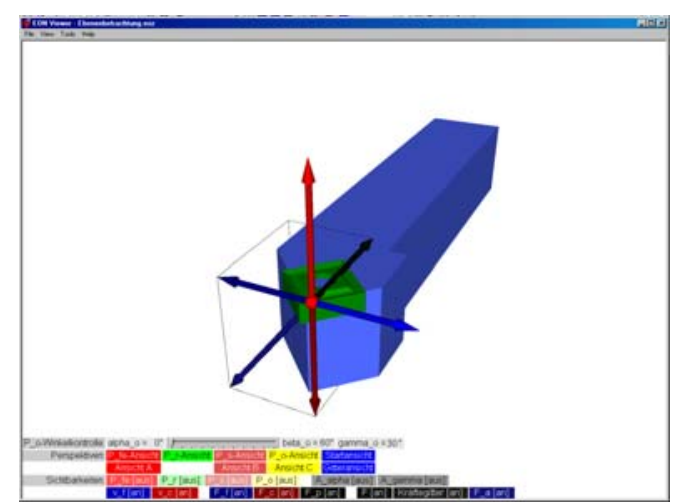

Bild 18: 3D-Modell mit interaktiv Elementen zu Kraft- und Geschwindigkeitsvektoren

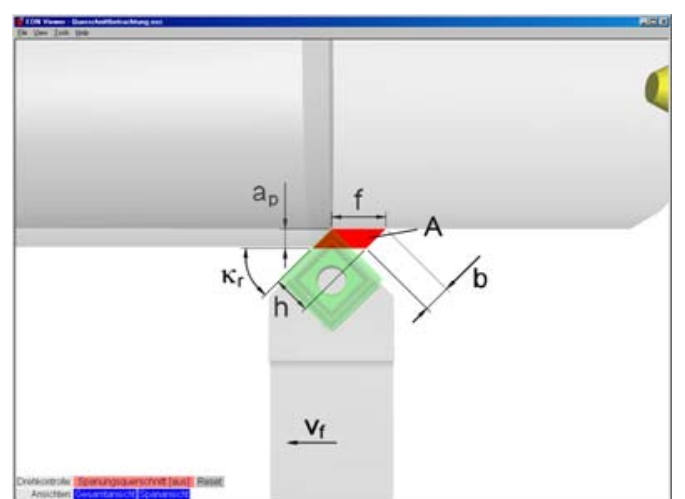

Bild 19: Dynamisch veränderliches 3DModell Spanungsquerschnitt mit relevanten Einflussparametern

Ab dem Jahr 2007 steht mit der Gründung des Zentrum Virtueller Maschinenbau (ZVM) an der TU Dresden ein Virtual-Reality-Labor mit 5-Seiten-CAVE und Powerwall zur Verfügung. A. Nestler ist mit dem Teilthema "Virtuelle Bearbeitungszentren" für Anwendungsmöglichkeiten in der Fertigungsplanung von Anfang an im Labor aktiv mit Arbeiten für Lehre und als Grundlage für die Forschung beteiligt.

Zur 3D-Visualisierung virtueller Werkzeugmaschinenmodelle steht $u$. a. die Software SolidWorks und EON-Studio zur Verfügung, die auch in den Lehrveranstaltungen „Rechentechnisches Praktikum" und „Fertigungsinformatik“ zum Einsatz kommt.

Der historische Lichtenheldt-Hörsaal im Zeuner-Bau ist in der Zeit restauriert und als moderner 3D-Präsentationsraum ausgestattet, Bis zu 400 Studenten können in den Vorlesungsstoff eingebaute interaktive 3D-Visualisierungen erleben (Bild 20).
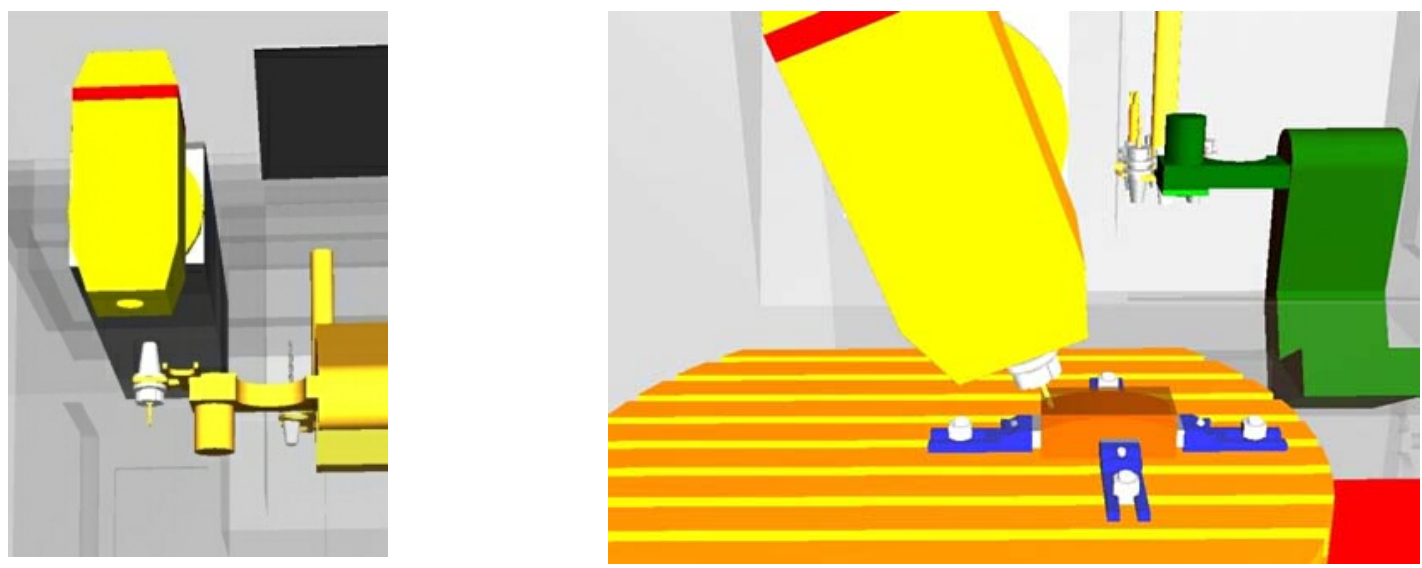

Bild 20: Virtuelle Basis-Maschine mit Teilkomponenten zu Maschine, Spannmittel, Werkstück und Werkzeug sowie steuer- und regelbaren Funktionen und NC-Programmablauf

Aus diesen anfänglichen Aktivitäten sind spätere Forschungsergebnisse zur automatisierten Konfiguration virtueller Maschinen hervorgegangen.

In Zusammenarbeit mit der Industrie ${ }^{22}$ entstehen Hardware in the Loop (HiL) Lösungen mit realer Steuerung (CNC) und virtuellen 3D-Maschinenmodellen. Die generierten Maschinenmodelle sind z. B in der Industrie begehrte Messeobjekte.

\section{Simulation Aided Manufacturing (SAM ) - virtuelles Werkstück}

${ }^{22}$ in Zusammenarbeit mit andron $\mathrm{GmbH}$, Wasserburg, später LTI Motion 
Ein von PAZAT vertretenes Fachgebiet zu Mehrachstechnologien hat mit den Freiformflächengeometrien und deren Fertigung auf mehrachsigen Fräsmaschinen eine jahrzehntelange Tradition, beginnend von den frühen Anfängen der Modellierung und Fertigung in den 1980er Jahren (D. Kochan, Dr. P. Bonitz) und fortgeführt unter veränderten Bedingungen (D. Fichtner). Die enge wissenschaftliche Verbindung von Informations- und Zerspantechnik bietet notwendige Voraussetzungen zu neuen Forschungen für Informationssysteme mit Anwendungsszenarien zur Ablaufplanung und Mehrachsbearbeitung (etwa 2003-2013).

Basis für ein potentielles Forschungsgebiet und besonders hervorhebenswert sind die Ergebnisse zur hochgenauen Bearbeitungssimulation für die simultane 5-AchsFräsbearbeitung und zur entwickelten Strategie des Simulation Aided Manufacturing (SAM) im Zusammenhang mit drei geförderten AiF-Projekten ${ }^{23}$ und der Dissertation von Dr.-Ing. Lee im Jahr 2001. Für die Materialabtragsimulation wird eine neue Methode zur Ermittlung der Hüllvolumina mit Hilfe der Gauß-Abbildung geschaffen. Die entwickelte Software „NC Simulation Kernel“ (NCSK) kann zu jedem beliebigen Zeitpunkt des Bearbeitungsablaufes die Geometrie hochgenau und mit hervorragender Performance berechnen. In Verbindung mit dem Einsatz bekannter Zerspankraftmodelle sind realitätsnahe Oberflächenstrukturen visualisierbar (Bild 21, Bild 22).

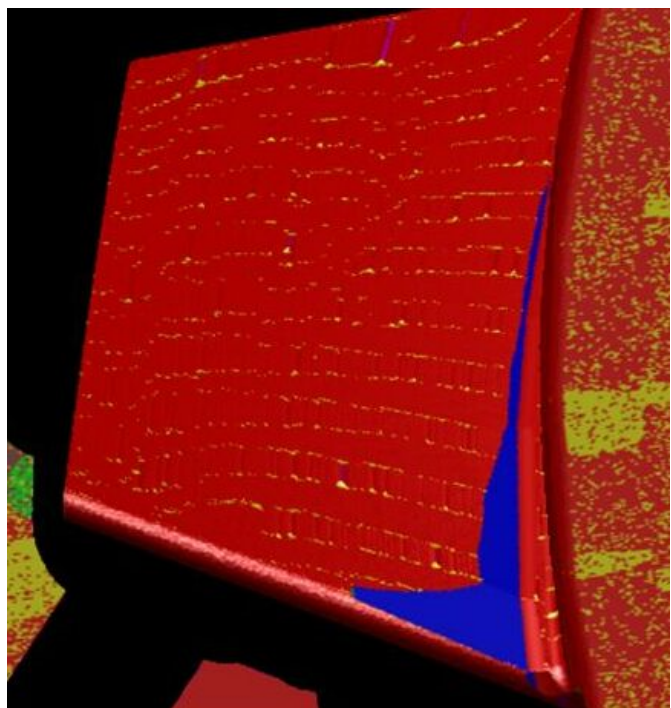

Bild 21: Virtuelles Werkstück - Makrogeometrie mit Restmaterial (blau)

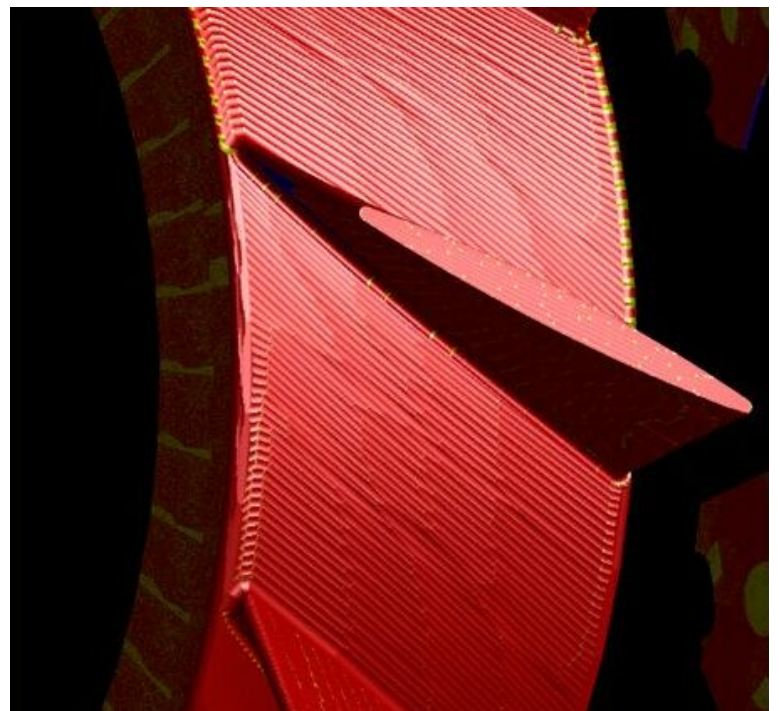

Bild 22: Virtuelles Werkstück - Mikrogeometrie mit Werkzeug-Rillenprofil

In der Weiterführung gelingt die Übertragung der Ergebnisse zum Mehrachsfräsen beliebig freigeformter Flächen auf das Gewindefräsen schraubenartiger Strukturen. Es entsteht ein Softwaremodul für durchgängig integriertes Engineering und virtuell unterstützte Prozessabsicherung von Profilwerkzeugen.

\section{Bearbeitungsmodelle mit physikalischen Eigenschaften}

3D-Visualisierung auf der Basis rein geometrischer Modelleigenschaften des Gesamtsystems Maschine-Spannmittel-Werkstück-Werkzeug in Verbindung mit der Kinematik ist 2012 Stand der Technik. Erst die Erweiterung der Forschung auf die "Physiksimulation" führt zu einer neuen Qualität von Simulationsmodellen für Bearbeitungsabläufe auf virtuellen Maschinen. Die bereits in den 1970er und 1980er

\footnotetext{
${ }^{23}$ FlexMill (2007-2009), OptiMill (2009-2011) und ViProfil (2010-2013). FlexMill ist noch vor dem Eintritt von Prof. Fichtner in den altersbedingten Ruhestand initiiert und beantragt worden.
} 
Jahren u. a. durch D. Kochan und H.-J. Jacobs publizierten Forschungsarbeiten zu technologisch leistungsfähigen mathematischen Modellen finden eine Fortführung.

Mit der Schaffung von Grundlagen zu virtuellen Planungswerkzeugen in der technologischen Arbeitsvorbereitung unter Einbezug der Prozessphysik (Simulation Computer Aided Planning) werden technisch-technologische Prozessmodelle mit physikalischen Eigenschaften des Zerspanprozesses zur Ablaufplanung des Verfahrens Fräsen auf Bearbeitungszentren erarbeitet. Der gewählte neue mechatronische Ansatz wird in Zusammenarbeit mit der Industrie auf der Basis der Simulationsplattform SimulationX prototypisch realisiert (Bild 23) ${ }^{24}$.

Die Ergebnisse zum erreichten Entwicklungsstand des Themas virtuelle Maschine für die Fertigungsplanung sind vom Bearbeiterkollektiv u. a. zu der - maßgeblich durch $D$. Kochan initiierten - internationalen IFIP TC5 Konferenz NEW PROLAMAT 2013 in Dresden veröffentlicht.

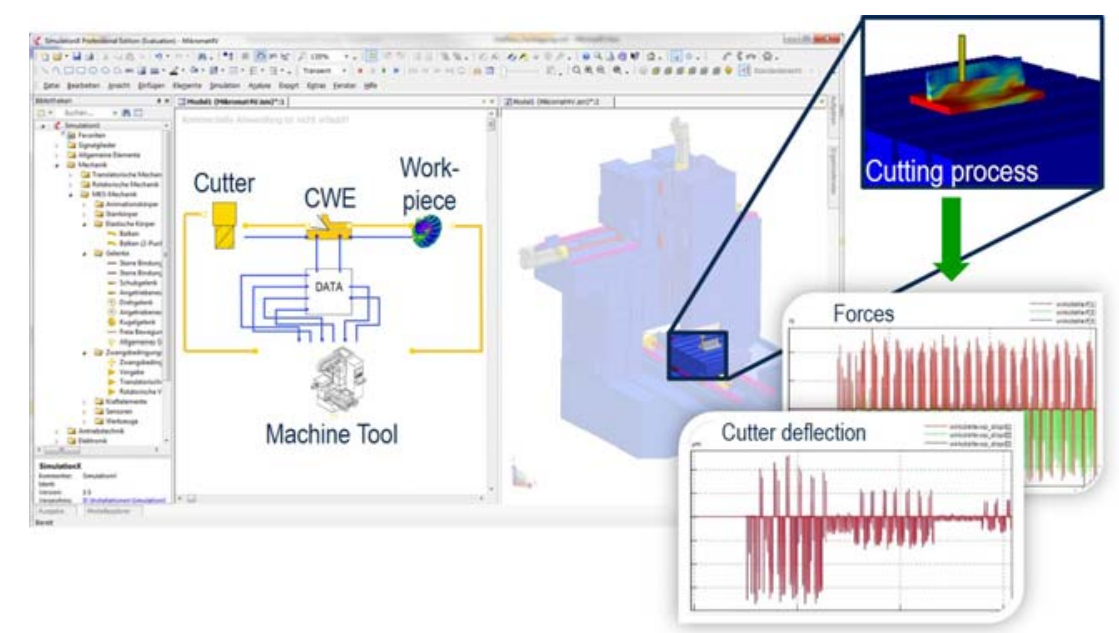

Bild 23: Systemsimulation zur Werkzeugmaschine Mikromat 4V in SimulationX

\section{Kennwerte zur physikalischen Simulation}

Eine aus den Anforderungen zur realitätsnahen Simulation abgeleitete Forderung ist die Bereitstellung von Kennwerten für technisch-technologisch fundierte mathematische Modelle. Das Beispiel der kraftmodellbasierten Auslegung von NCWerkzeugbahnen steht auch im Jahr 2015 für das ungelöste Problem der Technologiedatenbasen, in denen die als Berechnungsvoraussetzung erforderlichen Kennwerte fehlen. So sind z. B. für die Berechnung der Schnittkraftkomponente nach Kienzle Daten zur spezifischen Schnittkraft $\left(\mathrm{k}_{\mathrm{c}}-\right.$ Werte) erforderlich, die seit Jahrzehnten lediglich material-, zeit- und kostenintensiv empirisch ermittelt werden können und damit aktuell de facto nicht zur Verfügung stehen. Die bereits in den 1970er Jahren u. a. durch H.-J. Jacobs forcierten Forschungsarbeiten zur Spanungsoptimierung verwenden zur Ermittlung von Kennwerten u.a. eine reale Versuchsfeldwerkzeugmaschine als sogenannte Richtwertmaschine in Verbindung mit Auswertesoftware.

Die neue Richtwertmaschine ist der Rechner in Verbindung mit intelligenten Softwarekomponenten. Einen neuen Weg zur aufwandarmen Verfügbarkeit von $\mathrm{k}_{\mathrm{c}^{-}}$ Werten in den Datenbasen, z. B. für NC-Panungs- und Simulationssysteme, verfolgt der gewählte Ansatz auf der Grundlage moderner Technologien der Maschinen- und

\footnotetext{
${ }^{24}$ in Zusammenarbeit mit ITI GmbH Dresden
} 
Prozessdatenermittlung über CNC und MES-Systeme. Am Beispiel der Fräsbearbeitung ist eine Methode erprobt, nach der die spezifische Schnittkraft $k_{c}$ aus der laufenden Fräsearbeitung ermittelt wird.

Der Komplexität der Aufgabe entsprechend ist die automatisierte Ermittlung von Kennwerten mit Hilfe der Methode des Maschinellen Lernens durch KNN für eine sich kontinuierlich erweiternde Datenbasis angemessen. Damit gelingt es, aus neues Wissen für Technologiedatenbasen zu generieren. Ein hoffnungsvoller Schritt zur Prozesssimulation schnittkraftabhängiger Restriktionen ist gelungen. Die Entwicklung ist als Softwareprodukt umgesetzt, getestet und validiert (Bild 24 und Bild 25) ${ }^{25}$.

Die Forschungsergebnisse des Bearbeiterkollektivs sind u.a. im Jahr 2018 im Rahmen der CIRP-Konferenz "Intelligent Computation in Manufacturing Engineering“ in Neapel veröffentlicht. Für die Weiterentwicklung ist ein Folgeprojekt mit einem NC-Programmiersystemhersteller in Vorbereitung.

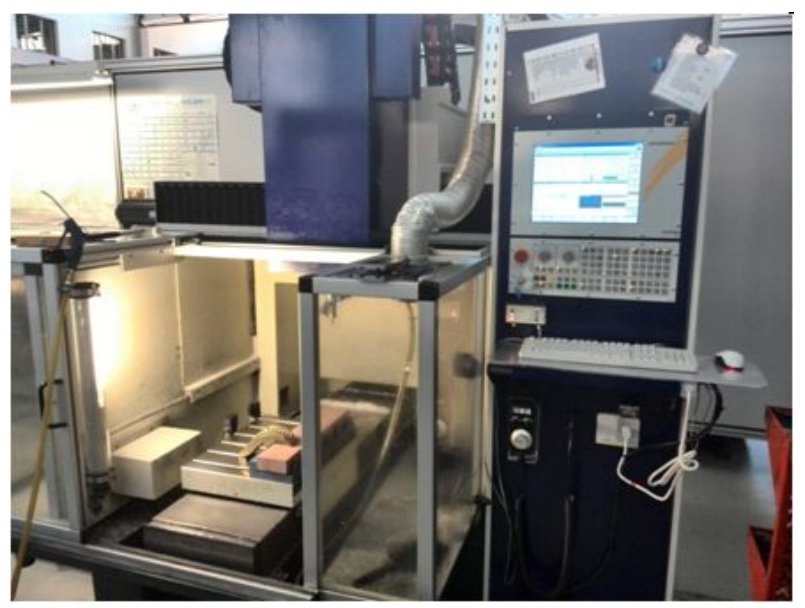

Bild 24: Fräsmaschine Mikromat 4V mit andronic 3060 zur Prozessdatengewinnung

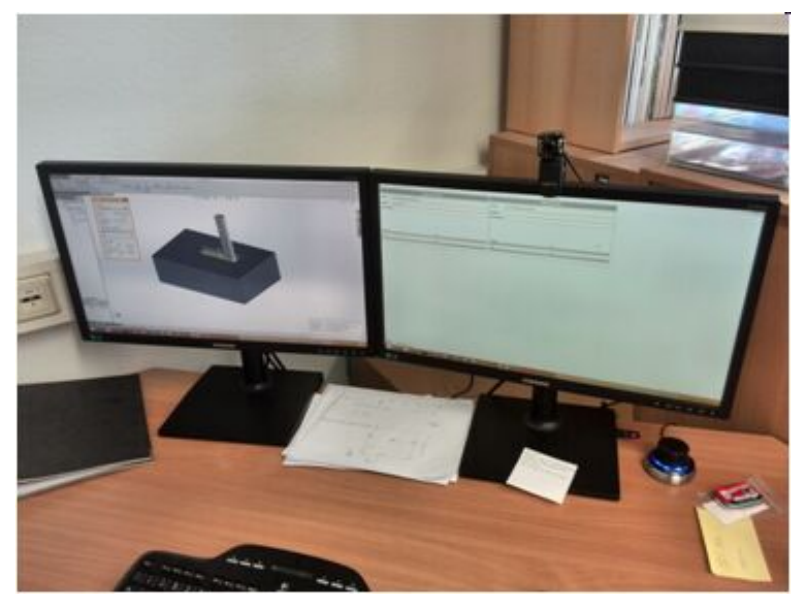

Bild 25: Leitstandsrechner mit MES-System und Software zur Prozessdatenverarbeitung

\section{Echtzeitdaten für die Fertigungsplanung - Digitaler Zwilling}

Das Thema Digitaler Zwilling greift in alle Bereiche der Produktionstechnik ein. Im Zusammenhang mit Prozessdaten und einer Technologieplattform ist die Verbindung des realen Einzelteils mit seinem digitalen Abbild ein zukunftsorientierter Ansatz.

Entwickelt wird ein intelligentes Analyse- und Optimierungssystem, dass die Vorgabe von realitätsnahen Fertigungsdaten für die Fertigungsplanung erlaubt. Kern des neuen Systems sind sogenannte digitale Prozess-Zwillinge, die für jedes real gefertigte Werkstück erzeugt werden und damit die jeweiligen bauteilindividuellen Fertigungsprozesse möglichst realistisch abbilden. Dieses digitale Abbild beruht maßgeblich auf Prozessdaten, die unmittelbar aus dem spanenden Bearbeitungsprozess resultieren und in Verbindung mit zugehörigen Planungsdaten zur Prognose für verbesserte Prozesse in der Fertigungsplanung eingesetzt werden (Bild 26).

${ }^{25}$ in Zusammenarbeit mit N\&P Informationssysteme GmbH Meerane 


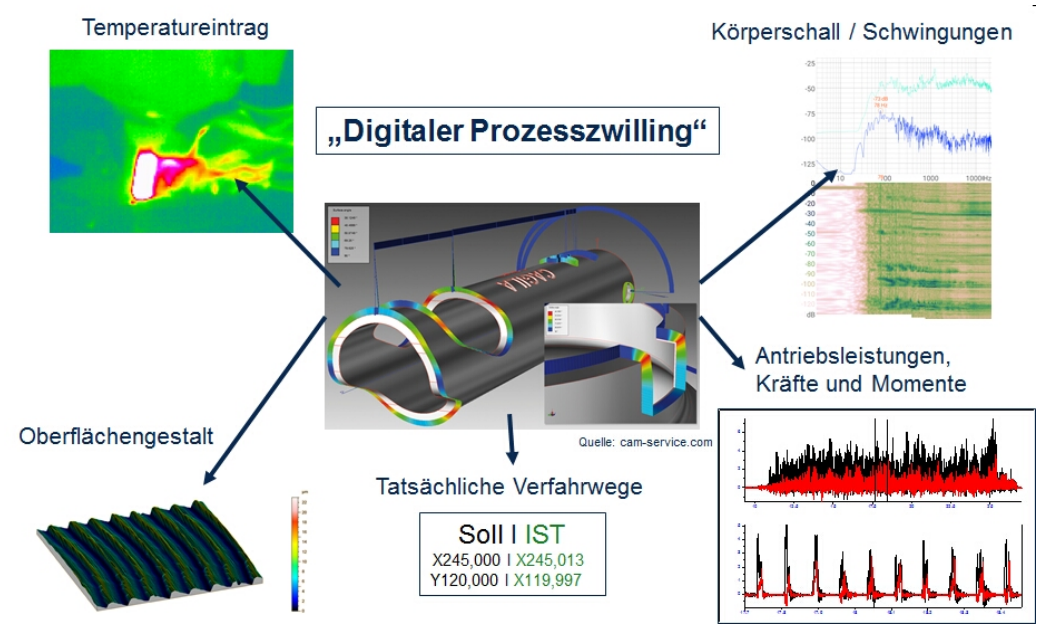

Bild 26: Potentiale zum Forschungsschwerpunkt digitaler Prozess-Zwilling

Der Prototyp ist auf der technischen Basis einer 3-Achs Fräsmaschine mit einer Steuerung HEIDENHAIN TNC620 entstanden. Alle notwendigen Daten zur Erstellung eines digitalen Prozesszwillings sind in einem neuen dazu entwickelten Informationsmodell gespeichert. Auf der Informationsbasis lassen sich nun eine Vielzahl von Prozesskenngrößen, wie Schnittkräfte, Schwingungen, dynamische Instabilitäten usw. berechnen und visualisieren.

Diese viel beachtete Forschung zum digitalen Prozess-Zwilling ist Ausdruck der konsequenten Weiterentwicklung des dargestellten Forschungsschwerpunktes. Mit diesem Entwicklungsschritt erhält z. B. das virtuelle Werkstück zusätzlich eine realitätsnahe Historie, welche zur Gewinnung neuen Wissens zur Prozessauslegung oder zur immer mehr an Bedeutung gewinnenden Prozessdokumentation reichlich Zukunftspotential für viele Anwendungsbranchen besitzt ${ }^{26}$.

\section{Lernplattform Fertigungsprozessplanung und -durchführung}

Als strategisch angelegte Weiterführung der erprobten und gelehrten Arbeitsweisen liegt eine Konzeption für eine Lernplattform als digitales Hilfsmittel zur Fertigungsprozessplanung und -durchführung für Lehrende und Lernende vor, welche im Rückblick als „Fertigungsprozessgestaltung 4.0“ eingeordnet werden kann. Mit dem sächsisch-tschechischen Kooperationsprojekt POKROK.digital, ein durch SAB / Europäischer Fonds für regionale Entwicklung (EFRE) mit rund 1,1 Mio. Euro gefördertes Gemeinschaftsprojekt ${ }^{27}$ ist das ermöglicht worden.

Für eine prototypische Realisierung einer Lernplattform mit computervermittelter Realität von Planungs- und Produktionsprozessen kommt mit der Entwicklungsumgebung Unity eine sogenannte Game-Engine mit einer aus der Spieleentwicklung stammende Technologie zum Einsatz, welche die Erstellung und interaktive Ablaufsteuerung im Zusammenhang mit unterschiedlichsten Medien wie Grafik, Video, Animation unterstützt und Entwicklungen für alle wesentlichen Plattformen gestattet (Bild 27). Damit wird für Lehr- und Lernaufgaben die Welt des interaktiven 3D mit den Anforderungen des digitalen Maschinenbaues verbunden.

\footnotetext{
${ }^{26}$ in Zusammenarbeit mit PMG Precision Mechanics Group GmbH, Wilsdruff; HSU, Institut für Automatisierungstechnik, Hamburg; QualityPark AviationCenter $\mathrm{GmbH}$, Hamburg

27 in Zusammenarbeit mit TU Liberec, Universität Usti nad Labem, Fachmittelschule Usti nad Labem und TU Bergakademie Freiberg
} 

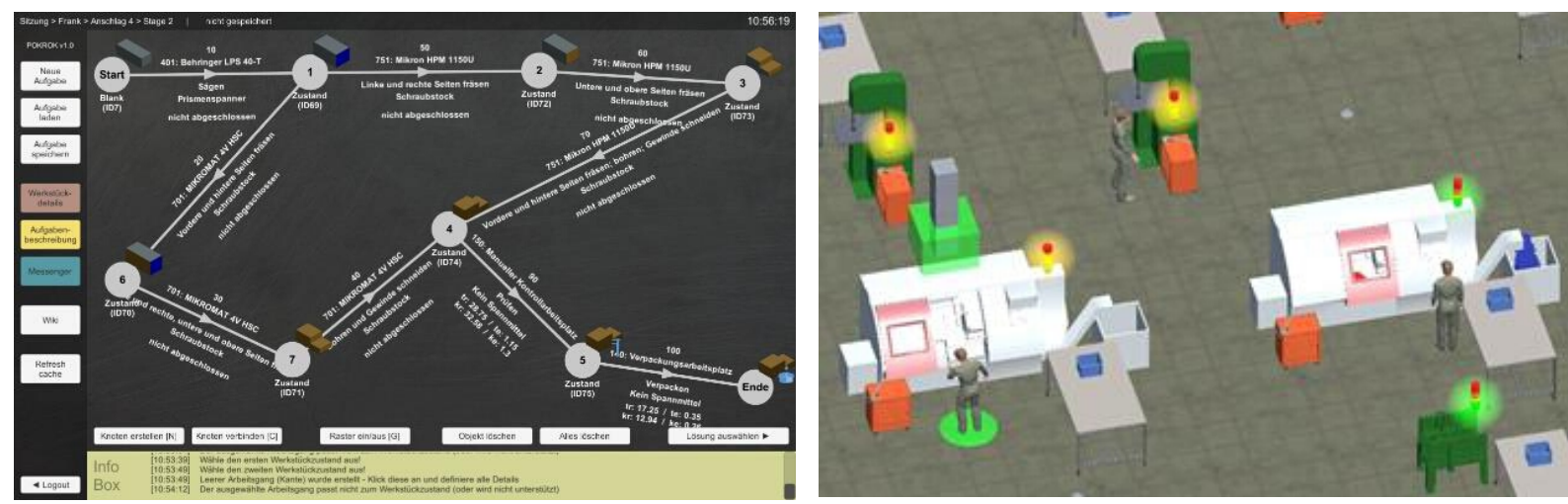

Bild 27: Game-Technologien für die Prozessplanung, Fertigungsplanung und -steuerung. Interaktiver Prozessgraph (lks.) und virtuelle Fertigung (rts.)

Die kurz dargestellten aktuellen Ergebnisse $\mathrm{zu}$ fertigungstechnisch orientierten Informatik-Anwendungen sind auch Bestandteil des 21. PAZAT-Fachkolloquiums „Digitalisierung und Vernetzung von Systemen und Prozessen der spanenden Teilefertigung" im Jahr 2018 an der TU Dresden. Mit rund 80 Teilnehmern einschließlich Referenten von Hochschulen und aus der Industrie bestätigt sich die große Resonanz und das Interesse an den gewählten Themen.

\section{Zusammenfassung zum Tätigkeitszeitraum 2005 bis 2019 als Leiter}

In den zu vertretenden Fachgebieten werden 68 Diplomanden betreut, über 60 Projekte (etwa zur Hälfte öffentlich geförderte Projekte und Industrieprojekte) geleitet und weitere rund 100 Publikationen veröffentlicht. Mit Unterstützung der Doktorväter K. Künanz und D. Fichtner finden 6 Dissertationen ihren Abschluss.

Als Beauftragter des Praktikantenamtes vertritt $A$. Nestler seit 2007 die Fakultät Maschinenwesen zum jährlichen Praktikantentag, auf dem Vorschläge für den Fakultätentag für Maschinenbau und Verfahrenstechnik (FTMV) erarbeitet werden.

In der Fakultät Maschinenwesen übernimmt er 2005 weiterhin von D. Fichtner die Verantwortung für das durch die Deutsch-Französische Hochschule (DFH) geförderte Doppeldiplomstudium der TU Dresden mit der ENSAM (Ecole National Supérieure d'Arts et Métiers) in Metz.

Ebenso wird die aktive Mitarbeit im Forschungsbeirat der GFal Berlin (Vorstandsvorsitzender Prof. A. Iwainsky) weitergeführt. Seit 2005 ist A. Nestler als Fachgutachter tätig.

Seit 2013 ist $A$. Nestler Vorsitzender des Freundeskreises der Dresdner Zerspanund Abtragtechnik (FDZAT) e. V. und organisiert in Zusammenarbeit mit der Arbeitsgruppe PAZAT jährlich sehr gut besuchte Fachkolloquien mit wechselnden Themen. Die jährlich zur PAZAT Fachtagung stattfindende Verleihung des Alfred-RichterPreises für herausragende Leistungen junger Wissenschaftler bzw. verdienstvoller Fachkollegen aus dem In- und Ausland sowie aus Industrie und Hochschule gehört zur Traditionspflege. Seit 2005 sind 22 Preisträger mit einem Preisgeld von je 1000 Euro bedacht worden.

Eine Form der respektablen Anerkennung sind Ehrenkolloquien für ausgewählte und verdienstvolle Hochschullehrer. Zu gegebenen besonderen Anlässen werden $H_{\text {.- }}$ J. Jacobs (2006, 70. Geburtstag), K. Künanz (2007, 70. Geburtstag) und D. Kochan (2010, 75. Geburtstag) gewürdigt. Die Verleihung des Alfred-Richter-Ehrenpreises ist die höchste Form der Anerkennung durch FDZAT für herausragende Hochschullehrer. Altmagnifizenz Prof. Dr.-Ing. habil. Dr.-Ing. E. h. H. Weber (TU 
Chemnitz) und Altmagnifizenz Dr.-Ing. Dr. sc. techn. Dr. sc. oec. H.-J. Jacobs (TU Dresden) erhielten den Preis 2011 in würdiger Form.

Mitarbeiter-, Absolventen- und Doktorandentreffen der Dresdner Produktionsautomatisierung, Zerspan- und Abtragtechnik sind ein weiteres Anliegen zur Kontaktpflege und Kommunikation. Die überaus große Resonanz auf derartige zusätzliche Großveranstaltungen lohnen die Mühe der Organisatoren. Anlässlich des 100. Geburtstages von Prof. $H$. Seidel findet 2008 das 1. PAZAT-Doktorandentreffen statt. Es folgt anlässlich des 100. Geburtstages von Prof. A. Richter am 2011 ein 2. PAZAT-Absolventen-, Doktoranden- und Mitarbeitertreffen. Das erste Absolventenbuch mit rund 1600 registrierten Absolventen erscheint. Ein 3. PAZAT-Absolventen-, Doktoranden- und Mitarbeitertreffen findet 2018 aus Anlass der Jubiläen „65 Jahre Lehrstuhl Produktionsautomatisierung, Zerspan- und Abtragtechnik“ und „20 Jahre FDZAT e. V." statt.

Im Jahr 2012 geschieht die Einverleibung der Arbeitsgruppe PAZAT in die neu berufene Professur für Formgebende Fertigungsverfahren (Prof. Dr.-Ing. A. Brosius), die wiederum mit in das Institut für Fertigungstechnik integriert wird.

Im Januar 2016 erfolgt durch den Rektor der TU Dresden, Herrn Prof. Hans MüllerSteinhagen, die Berufung zum außerplanmäßigen Professor.

Seit November 2016 ist PAZAT Mitglied im Kooperationsnetzwerk für Intelligente Produktionstechnologien (InPro) - Industrie 4.0. Produktionstechnologien auf der Basis intelligenter Softwaretechnologien sind schon seit Jahrzehnten im Focus der universitären Forschung der Institute für Fertigungsinformatik, insbesondere am Lehrstuhl für Technologische Programmierung (D. Kochan), und wurden mit der Professur für Produktionsautomatisierung und Steuerungstechnik (D. Fichtner) in ausgewählten Richtungen erfolgreich weiter verfolgt. Nachdem dieser Forschungsschwerpunkt $\mathrm{zu}$ den automatisierten spanenden Prozessen der Teilefertigung folgerichtig in die Gegenwart geführt ist, erfährt die angewandte Forschung mit Initiativen zu Industrie 4.0 eine umfassende öffentliche Förderung.

Mit dem altersbedingten Ausscheiden von A. Nestler am 01.11.2019 wird die Bezeichnung „Produktionsautomatisierung, Zerspan- und Abtragtechnik“ von A. Brosius in eine Abteilung "Technologien der Trennenden Fertigungsverfahren“ umbenannt. 
Dr. sc. techn. Manfred Rahmig, geb. am 10.12.1957 in Oelsnitz/Vogtland

Ein Beitrag für das TUD-Archiv anlässlich des 85. Geburtstages von Prof. Dr. sc. techn. Kochan am 4. Juli 2020

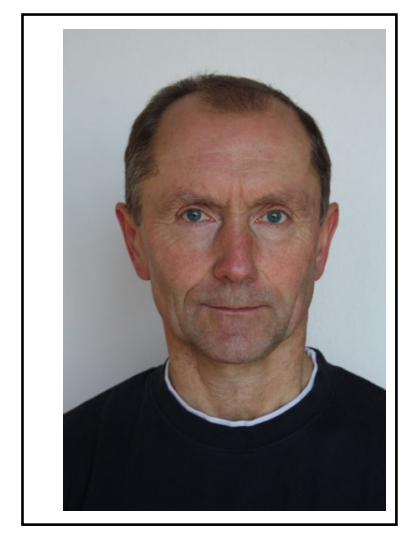

\section{Zusammenfassender Rückblick auf eine 1978 an der TU Dresden, Sektion Fertigungstechnik, begonnene fachliche Entwicklung zum „Fertigungsprozessgestalter“, die schon zeitig in Richtung CAD/CAM Automobilbau abdriftete}

Es begann mit einem studentischen freiwilligen Praktikum an der BESM6 in Leipzig, zusammen mit Dieter Haas, einem Studienkollegen, der später auch bei Prof. Kochan promoviert hat. Gerade einmal die ersten Kenntnisse in EDV (Elektronischer Datenverarbeitung) im Grundstudium erworben, sollten für das Programmpaket AUTENT (siehe Beitrag Prof. Dr. Bonitz) am Großrechner Programme getestet werden. Das war wohl damals noch die Programmiersprache Algol. Die Details sind mir leider entfallen; es kann auch sein, dass der Name AUTENT zu dem Zeitpunkt noch gar nicht geboren war.

Und so ging es dann weiter, immer gefördert von den Partnern TUD und PKW-Kombinat. Ich glaube den Hintergedanken „billige studentische Arbeitskraft“ gab es damals noch nicht. Aber das spielte auch keine Rolle. Rechentechnik und Software steckten ja noch in den Kinderschuhen, und es war schon etwas Besonderes, wenn man sich bei ROBOTRON die Nacht um die Ohren schlagen durfte oder Zutritt zur „geheimen“ TU Rechentechnik hatte.

Das alles hat dann zwei Doktorarbeiten abgeworfen und dazu geführt, dass das WTZ Automobilbau (Außenstelle Dresden) und die Gruppe um Dr. Bonitz mein erster Arbeitgeber wurde.

Vielleicht wäre es ja auch einmal mit Lehre und Forschung weitergegangen, aber es kam die Wende - und plötzlich konnte man an den tollen Programmsystemen, die man zur Genüge aus der Literatur kannte, selbst mitwirken. Der Einstieg war ICEM DDN von Control Data, damals auf der Cyber180 Mainframe bei Volkswagen im Einsatz. Wie zu erwarten war - auch hier wird nur mit Wasser gekocht. Das war beruhigend. Dann kam das Zeitalter der Workstations. Es herrschte eine Aufbruchstimmung, und bald wurde mit der ICEM Systems GmbH eine eigene Softwarefirma gegründet, die vorwiegend für den Kunden im Automobilbau da war. Zu schnelles Wachstum ist auch nicht gut, und nach drei Jahren wurde die Firma gesplittet.

Der neue Arbeitgeber war die Intergraph Deutschland GmbH. Hier musste man nicht mehr FORTRAN programmieren, sondern es gab eine Art objektorientiertes C. Das war ein Meilenstein. Die Workstations hießen Clipper, das Betriebssystem Clix, das CAD/CAM Basissystem war EMS. Speziell für den Automobilbau hatte man in Hannover ein „Center of Competence“ gegründet, das sozusagen der strategische Türöffner zur Automobilindustrie werden sollte. Aber leider wollte man auf der anderen Seite die Tür nicht öffnen. Die Luft wurde allmählich dünn, und das Center of Competence 1998 aufgelöst. 
Nach einem kurzen Zwischenspiel bei VW GEDAS ging ich im gleichen Jahr zurück zu ICEM Systems, deren Mutterfirma inzwischen Parametric Technology hieß. Der damalige Chef, Peter Salzmann, auch ein ehemaliges AUTENT Gewächs, hat mir hoch und heilig versprochen, dass ich nie mehr FORTRAN programmieren muss. Nebenbei bemerkt: Auch 2020 haben wir noch FORTRAN code im Produkt! Aber PTC wurde auch nicht glücklich mit ICEM und verkaufte die Firma an eine Investitionsgruppe aus England.

Ein neues Produkt wurde designed: ICEM Style. Man wollte mit den ICEM Surf Restriktionen brechen und entschied sich für die Entwicklung einer moderneren Datenbasis inklusive Laufzeitmodell. Da ich aus Intergraph-Zeiten hier einige Erfahrungen mitbrachte, konvertierte ich plötzlich vom „Experten für getrimmte Flächen und Datenaustausch“ zum Datenbasis Entwickler. ICEM Style war eigentlich eine tolle Sache und wurde sogar einmal verkauft - aber dann klopfte Dassault Systemes an die Tür und winkte mit einer GOLD Partnership.

Für eine Venture Capital Company die Gelegenheit: 2010 wurde ICEM Systems von Dassault schließlich komplett übernommen. Das Style Produkt wurde eingestellt, meine Datenbasis überlebte als inzwischen natives externes Format von ICEM Surf.

Wie ist der Stand heute?

Die Softwareprodukte im Bereich Automobilkonstruktion tun eigentlich das, was sie sollen. Es gibt die beiden grundsätzlichen Ansätze

- Schwerpunkt Parametrik (erfordert durchdachte Konstruktionsabläufe), z.B. CATIA

- Schwerpunkt Freie Formgestaltung (Kontrollpunkte zupfen), z.B. ICEM Surf

Aber der Konkurrenzdruck ist hoch. Gefragt sind neue Modelle in immer kürzeren Zeiträumen bei Minimierung der Kosten. Allgemein besteht die Auffassung, dass man durch Verschmelzung beider Konzepte noch einiges herausholen kann. Es gab der Integrationsprojekte schon einige, mehr oder weniger mit mäßigem Erfolg.

Im Rahmen von https://www.3ds.com/de/ueber-3ds/3dexperience-plattform/ haben wir im Moment mit IDX (ICEM Design Experience) gerade wieder einmal einem neuen Versuch in Arbeit. Dabei geht es nicht nur um Integration von bestehenden Konzepten, sondern auch um Anwendung komplett neuer Ideen.

Allerdings, die eigentlich finale Frage ist die: Will das der Anwender? Fühlt er sich nicht etwa überfordert? Ist es für einen gut, wenn der Computer immer schon im Voraus weiß, was man denn als nächstes vorhat? Oder ist es besser, selbst die Kontrolle zu behalten? Die Entwicklung geht weiter. Was technisch machbar ist, wird umgesetzt werden. Ob es zum Wohlbefinden des Automobilkonstrukteurs beiträgt, sei dahingestellt. Um mit den Worten unseres geschätzten, leider schon verstorbenen ehemaligen Kollegen und Lehrers Gerhard Franz zu schließen: „,... ich hab“ da ja so meine Zweifel“". 
In den 1970er Jahren als Frau ein Maschinenbaustudium aufzunehmen, war nicht unbedingt ungewöhnlich, wenngleich das Verhältnis männlicher zu weiblicher Studentenzahl einer Seminargruppe von 4 : 24 nicht gerade ein Ausdruck von Wunschstudium für Mädchen war. Zugegebenermaßen stellte es auch für mich zunächst nicht die erste Wahl dar, schien aber interessant genug, um in der Sektion Fertigungstechnik und Werkzeugmaschinen zu beginnen.

Nach drei Jahren Studium im Bereich Schweiß- und Fügetechnik und einer Meinungsverschiedenheit mit dem Direktorat Erziehung und Ausbildung gab es für das letzte Studienjahr einen Wechsel in den Fachbereich Technologische Programmierung. Prof. Kochan hatte für eine neue studentische Heimat gesorgt. FORTRAN war bis dahin eine Unbekannte für mich, aber sehr schnell galt es, sich in die Tiefen der Programmierung für Werkzeugmaschinen einzuarbeiten. Das dies ein erfreuliches Fach sein kann, bewies das Angebot, nach dem Studium die Promotion anzugehen.

Der Beginn der 1980er Jahre war geprägt von Forschungsarbeiten und Diskussionen um die Verknüpfung verschiedener Prozessebenen der Fertigungsvorbereitung und automatisierten Fertigung, um CNC und DNC - am Ende sollte die weitestgehend bedienerlose Fertigung stehen. Die eigene Promotion war ein Baustein dieser Vision: „Grundlagen zur funktionellen Gestaltung numerischer Steuerungssysteme für die bedienerlose Fertigung und Entwicklung eines multivalent nutzbaren Funktionsbausteins“. Damit sollte die Fragestellung gelöst werden, wie Werkzeuge bei unvorhergesehenen Störungssituationen gefahrlos wieder in die Ausgangsposition für einen Neustart geführt werden können, ohne dass ein Mensch eingreifen muss. Die neu entwickelte Steuerungsstruktur sollte - wie bisher nicht üblich - modular und somit flexibel für unterschiedlichste Anwendungszwecke produzierbar und einsetzbar sein. (Vielleicht ist es ganz lohnenswert sich ins Gedächtnis zu rufen, dass zu jener Zeit für Programme als Speichermedium noch Lochstreifen genutzt wurden und das verwendete Mikrorechnersystem K 1520 auf einer 8-bit-Mikroprozessortechnik beruhte.)

Einer praktischen Umsetzung in die Produktion nach einer sehr fruchtbaren Zusammenarbeit mit dem VEB Numerik „Karl Marx“ - größter Produzent von Werkzeugmaschinensteuerungen in der DDR und im RGW - während der Promotionsphase verhinderte die alles verändernde Wende.

Wie für viele Andere auch nach diesem vollständigen Umbruch eröffneten sich auch für mich neue Horizonte - und die mussten auch nicht ganz freiwillig neu erobert werden. Die beim VEB Mikromat angesiedelte Arbeitsgruppe, die ich leiten durfte und welche die neue Steuerungskonzeption gemeinsam mit dem VEB Numerik realisieren sollte, wurde alsbald aufgelöst.

Nach einem kurzen Intermezzo als Beteiligte und Gründerin der GIB (Gesellschaft für Industrieberatung) - auch andere Doktoranden Prof. Kochans waren beteiligt - ließ mich auch für die nächsten Jahrzehnte die Software nicht los. 
Nach einer eher zufällig abgesendeten Bewerbung bei der Firma SAP (damals ca. 1600 Mitarbeiter, heute mehr als 100.000) und einem Vorstellungsgespräch in Walldorf war ich ab September 1990, also noch zu offiziellen DDR-Zeiten - eine von 70 Neuen (davon 6 Frauen) aus mehr als 2000 Bewerbern. Fortan wechselte meine Berufsbezeichnung von ,Berater ${ }^{\star} \mathrm{zu}$,Consultant ${ }^{\star}$, später ,Principal Solution Consultant‘ usw. Der Inhalt dieser Tätigkeit blieb für die nächsten 19 Jahre mit Variationen allerdings gleich. Die betriebswirtschaftliche Software, die SAP für alle Unternehmensprozesse erstellt - Vertrieb, Einkauf, Materialwirtschaft, Produktionsplanung und -steuerung, Instandhaltung, Finanzbuchhaltung und Controlling - in Unternehmen so einzuführen, dass die jeweiligen Abläufe effizienter und transparenter werden. Die Softwarearchitektur war - um sie bestmöglich Kundenanforderungen anzupassen - modular aufgebaut. Das war - ohne zum damaligen Zeitpunkt Weiteres im Detail zu kennen - ein ausschlaggebender Punkt meiner Bewerbung, erinnerte es mich doch an die Idee der modularen Steuerungssoftware. Bei der Wahl der Softwarekomponente, auf die ich mich in der Beratungstätigkeit spezialisieren würde, gab es kein Zögern - natürlich PPS, Produktionsplanung und -steuerung. Die Beratungsschwerpunkte reichten dabei von Bereichen wie Demand Management, Long Term Planning, Material Resource Planning and Execution, Shop Floor Control and Execution, Production Order Scheduling and Rescheduling, Integration von MES (Manufacturing Execution Systems), Capacity Management und natürlich die Integration in jeweils angrenzende Unternehmensbereiche. Die fachliche Beratungstätigkeit wurden ergänzt um Risiko- und Change Management, Project Portfolio Management im Sinne der strategischen Ausrichtung großer Unternehmen, usw. Im Übrigen machte ich sehr bald die Erfahrung, dass Vollzeit-Berufstätigkeit oder gar technische Studienrichtungen und Berufsbilder in der alten BRD ein eher ungewöhnliches Betätigungsfeld für Frauen darstellten.

Die Beratungstätigkeit begann mit dem in Mainframe-Architektur strukturierten System R/2, in den 1990er Jahren erfolgte bei vielen Kunden nach und nach der Wechsel auf das in Client-ServerArchitektur basierten System R/3 noch mit den klassischen ERP-Funktionen, zum Jahrtausendwechsel dominierte der Schritt ins Internetzeitalter und später das Cloudcomputing die Entwicklung - Kunden konnten nun nicht nur unternehmensintern, sondern auch über Unternehmensgrenzen hinweg ihre Prozesse integriert abwickeln. Die Software entwickelte sich sowohl vom UI als auch hinsichtlich neuer Branchenspezifika beträchtlich, was in jedem Fall die Beratungstätigkeit beeinflusste. Häufig wurden neue Anforderungen aus Kundenprojekten in die Entwicklung getragen und wurden später zu Standardfunktionalität. Während unzähliger Beratungsprojekte, von Klein- und Mittelständlern bis zu Großkonzernen, von Sachsenring bis BASF, war einer meiner Beratungsschwerpunkte die Variantenkonfiguration, eine komplexe Softwareapplikation zur Umsetzung von kundenindividueller Einzelfertigung, welche in allen unternehmerischen Bereichen und somit Softwaremoduln - angefangen von der Konstruktion, über Vertrieb und Produktion bis hin zum Controlling eine anspruchsvolle Abbildung der spezifischen Kundenprozesse darstellte. Mehr und mehr übernahm ich auch die Leitung 
von Projekten für die Einführung der SAP-Software, teilweise mit der Verantwortung für ein Budget von mehreren Millionen Euro Projektkosten.

Nach 19 Jahren interessanter Beratungstätigkeit bot sich die Möglichkeit der Rückkehr an den Heimatort Dresden mit einem beruflichen Wechsel innerhalb von SAP an. Betrachtet man den Softwarezyklus, wechselte ich fast vom Ende - der Einführung und Produktivsetzung im jeweiligen Unternehmen - ganz an den Anfang, in den Bereich Research. In dieser Zeit leitete ich für SAP zwei jeweils dreijährige Forschungsprojekte, die sich sehr frühzeitig mit cloudbasierten Business-Softwarelösungen befassten. Thematisch waren beide angesiedelt im Bereich Mobile Computing und Internet of Things. Es ging nun darum, nicht die schon existierende Software an die vielfältigsten Unternehmenserfordernisse anzupassen, sondern langfristig vorausschauend zu erforschen und prototypisch zu entwickeln, welche Funktionalitäten in einigen Jahren bei Kunden benötigt und eingesetzt werden würden.

Auch eine große Firma wie SAP wickelt solche Forschung in der Regel nicht allein ab. Kooperationen mit Klein- und mittelständischen Firmen, Forschungsinstituten und Universitäten und anderen Konzernen mit tangierenden Interessen spielen bei solchen, meist von BMBF oder EU geförderten Projekten eine Rolle. Eines dieser Projekte befasste sich mit der Idee, eine Vielzahl relevanter Produktdaten, die während eines Produktlebenszyklus entstehen, direkt am und mit Objekten zu verknüpfen und diese für diverse Interaktionen in verschiedensten Anwendungsszenarien nutzbar zu machen - das ,Semantic Product Memory“. Auf der Basis enorm weiter entwickelter Speicher- und Sensortechnik wurden - teilweise mit Kunden - Prototypen entwickelt, die auf der verschiedenen Messen, wie die Hannover Messe, vorgestellt wurden. Auf dieser Basis konnte das nächste Forschungsprojekt begonnen werden - Ressourcenschonung durch intelligente Kommunikation, zu einem Zeitpunkt, als komplexe Cloudplattformen für unterschiedliche Anwendungsbereiche und Visionen vom Business Web entstanden. Im Konkreten ging es um die Konzeption von neuen, mobilen Kundendienstleistungen im Unternehmens- und Endkundenbereich, so dass diese remote und kollaborativ über Unternehmensgrenzen hinweg im After Sales Prozesse effektiver und transparenter abgebildet werden können. Die zunächst prototypische Umsetzung mit einer Vielzahl von Unternehmen mündete schließlich in die Überleitung vom Research-Bereich in die Entwicklung zur anwendungsreifen Marktlösung ,Asset Intelligence Network ${ }^{`}$ - ein Erfolg, der nur wenigen Forschungsprojekten bis dato beschieden war. Dieses cloudbasierte Netzwerk dient als globales Asset Register für ein erweitertes Netzwerk von autorisierten Geschäftspartnern (OEM, Manufacturer, Service Provider). Unternehmensübergreifend kann so auf Engineering Daten, kritische Alerts etc. zugegriffen und Serviceprozesse als End-to-end-Prozess vom Design bis zur Inbetriebnahme deutlich effektiver abgewickelt werden.

Die Wahl in den Aufsichtsrat der SAP und die neunjährigen Mitgliedschaft zwischen 1998 - 2007 brachte einen zusätzlichen Aufgabenschwerpunkt mit einer völlig anderen Perspektive auf das 
Unternehmen SAP. Im Mittelpunkt standen die Beratung und die Überprüfung der operativen Tätigkeit des Vorstandes, die Diskussionen und Entscheidungen zur strategischen Ausrichtung des Konzerns, zu Produktentwicklungen, zu Akquisitionen, strukturellen Änderungen, Internationalisierung verschiedener Geschäftszweige, das Einbringen von Mitarbeiterinteressen und - im Speziellen Kundenerfahrungen und -anforderungen.

Abschließend sei angemerkt, dass es - zumindest aus meiner Sicht - im Leben immer sehr viel mehr Dinge gibt, die man genauer kennlernen möchte. Daher absolvierte ich in den 2000er Jahren berufsbegleitend ein Masterstudium - diesmal in einer komplett anderen, einer geisteswissenschaftlichen Fachrichtung. Der Abschluss als Master of Arts befasste sich mit dem Thema „Nonprofit mit Aktienkapital - Chancen einer marktwirtschaftlich geprägten Organisationsform und deren Ausgestaltung im Kulturbereich“.

Darüber hinaus führen mich als langjähriges Mitglied der Deutschen Gesellschaft für Polarforschung seit Jahren immer wieder Expeditionen und Forschungsaufenthalte in die hohe Arktis. Die Forschungsergebnisse zur insbesondere russischen Polargeschichte werden auf Kongressen, in wissenschaftlichen Publikationen und auf für die Öffentlichkeit zugänglichen Events, wie Ausstellungen und Themenveranstaltungen, präsentiert. 
Prof. Dr.-Ing. habil. Peter Bonitz, geb. am 22.11.1940 in Göttingen,

Voglerstr, 30, 01277 Dresden, Tel.: 0351-3102806

E-Mail: fachbuch@bonitz-web.de

Ein Beitrag für das TUD-Archiv

anlässlich des 85. Geburtstages (am 28. Juni 2020) von

Prof. Dr. sc. techn. Kochan (Nachfeier am 4. Juli 2020)

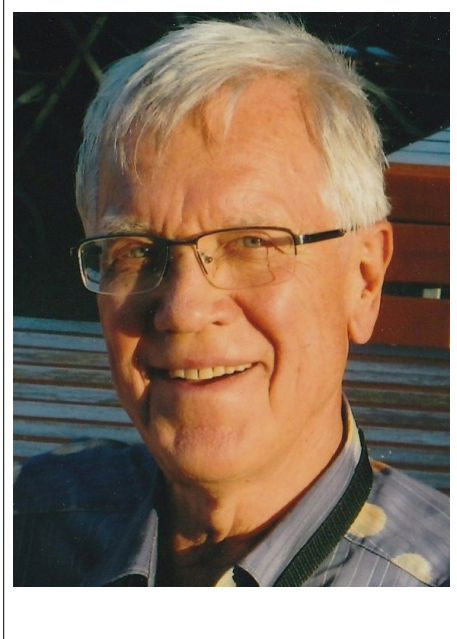

Rückblick auf eine jahrzehntelange interdisziplinäre Zusammenarbeit zwischen der TU Dresden, der Westsächsischen Hochschule Zwickau (früher Technische Hochschule Zwickau) und dem Automobilbau, speziell bezüglich des Karosseriebaues über den gesamten Bereich beginnend mit dem Design, weiter über die mathematische Beschreibung der Karosserieoberflächen bis zur NC-Fertigung der Formen in den Umformwerkzeugen für die Blechteile.

Entstamme zwar nicht der damaligen Sektion Fertigungstechnik und Werkzeugmaschinen/ Fachrichtung Fertigungsprozessgestaltung mit Spezialisierung Technologische Programmierung (fortan hier abgekürzt mit FT/TP), sondern habe meine fachlichen Wurzeln in der Geodäsie/Photogrammetrie/Mathematik (Sonderstudienplan in Diffentialgeometrie) an der TUD, denke aber gerne an die Zeit 1977 als (vom Automobilbau bezahlten) „Forschungs-Gast“" in einem Zimmer der o.g. Sektion im Zeunerbau und später in der Dürerstraße (bis etwa 1982) in der gemeinsamen Gruppe TUD/PKW-Kombinat, die dann schließlich als CAD/CAM-Forschungsgruppe Karosseriebau in einem Nebengebäude des Karosseriewerkes Dresden in der Arnoldstraße bis zur Wende 1989/90 tätig war. Das Ergebnis - das System AUTENT (in Langform: Automobilkarosserieentwurfsystem) ist auch mit den Namen der damaligen TP-Absolventen Bernd Burkhardt (bzgl. CAM- Frästechnik) und Manfred Rahmig (bzgl. CAD-Drahtmodelle, u.a.) sowie mit den Prüfungen der AUTENTSoftware durch den wissenschaftlichen Mitarbeiter der Sektion FT, Dipl.-Math. Gerhard Franz, verbunden. Ausführlich sind die vielfältigen fachlichen Hintergründe und Entwicklungsstufen an Hochschulen und im Automobilbau selbst im Vorwort meines Fachbuches beschrieben, siehe http://www.bonitz-web.de/fb_public/01_vorwort/01_preface.pdf

$\mathrm{Zu}$ meinem eigenen fachlichen Lebenslauf und auch, wie es nach der Wende in Hannover bei Control Data bzw. ICEM Technologies weitergegangen ist, siehe www.bonitz-web.de.

Prof. Kochan, als Förderer, hat dazu beigetragen, dass die mathematische Software von AUTENT national (auch branchenübergreifend, z.B. durch Nutzung im System GRAFIS der DDR-Schuhindustrie in Weißenfels) und international (z.B. durch Vermittlung meiner Vortragsmöglichkeit zur internationalen PROLAMAT-Konferenz 1979 in Ann Arbor/Michigan/USA) bekannt wurde. Als Sachverständiger auf dem Gebiet der FT/TP wurde Prof. Kochan folgerichtig auch einer der vier Gutachter bei meiner Promotion B im Jahre 1987 an der Technischen Hochschule Zwickau. Im gleichen Jahr verlieh mir der Senat der Hochschule die „FACULTAS DOCENDI“ (Lehrbefähigung) für das Fachgebiet CAD/CAM Karosseriebau. Dies war die Voraussetzung für die Umwandlung des akademischen Grades „Dr. sc. techn.“ in „Dr.-Ing. habil.“ im Jahre 1992 .

Im November 2005 ging ich planmäßig mit 65 Jahren in Rente und hatte in den Jahren 2006 bis 2009 endlich Zeit, das oben erwähnte Fachbuch zu schreiben. Es ist auch ein Beleg für die o.g. interdisziplinäre Zusammenarbeit. 


\title{
Fachlicher Lebenslauf
}

\author{
Name: Dr.-Ing. Ingolf Berndt
}

Geburtsdatum: 30. April 1957 in Dresden

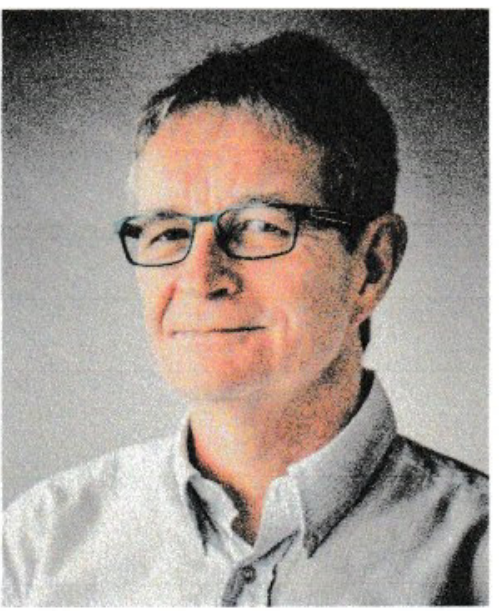

$10 / 1987$

Dissertation an der TU Dresden, Fakultät Maschinenwesen, Fachrichtung Fertigungsprozessgestaltung, Prof. Kochan

Thema ${ }_{n}$ Zur Beschreibung von technischen Objekten aus der Sicht der Anforderungen der rechnergestützten Fertigungsplanung"

\section{3/1982 - 12/1993 Wissenschaftlicher Assistent und Oberassistent an der TU Dresden, Fakultät Maschinenwesen, Fachrichtung Fertigungsprozessgestaltung}

Demonstration eines Verfahrens zur werkstückbezogenen Arbeitswerteermittlung beim Mehrachsfräsen auf dem Messestand des Technologiezentrums Dresden auf der SYSTEC 1990. Durch die Ankopplung zum Digitalisiersystem scancad der Firma BCT GmbH Dortmund konnte ein durchgängiger Informationsfluss von der Aufnahme von Modelldaten über die CAD-Modellierung, die CNC-Datengenerierung bis hin zur CNCFertigung vorgeführt werden.

Auf Grund der großen Resonanz auf der SYSTEC 1990 wurde das Institut für Fertigungsinformatik (IFI) Vollmitglied des Projektes FAME - Five Axes Manufacturing Environment - des EG-Forschungsprogramms ESPRIT. Damit fanden die langjährigen Erfahrungen des Lehrstuhls "Fertigungstechnik/Technologische Programmierung" von Prof. Kochan internationale Anerkennung. Als Teamleiter war ich für die fachlichen und organisatorischen Abstimmungen verantwortlich. Auf der ESPRIT-Konferenz 1991 in Brüssel konnte ich als einziger ostdeutscher Vertreter auf der Ausstellung eine Pilotlösung der Informationskette vom Digitalisieren über CAD-Modellierung bis hin zur NC-Fräsweggenerierung demonstrieren. Die mathematischen Inhalte in der Projektgruppe wurden maßgeblich durch Dipl.-Math. Gerhard Franz erarbeitet.

In den Jahren 1992 und 1993 erfolgte der Aufbau eines Zentrums für Rapid Product Development am IFI. Dabei war ich für die Inbetriebnahme und Nutzung der ersten Stereolithographie-Anlage in Ostdeutschland verantwortlich. In dieser Zeit entstand auch unter meiner fachlichen Leitung ein Lehrvideo zu Rapid Prototyping und deren Nutzungsmöglichkeiten im Entwicklungsprozess mit Unterstützung des Audiovisuellen Zentrums der TU Dresden. 


\section{1/1994 - 11/1997 Geschäftsführer, SFM - Gesellschaft zur Schnellen Fertigung von Modellen $\mathbf{m b H}$}

Zu meiner Geschäftsführertätigkeit gehörten neben betriebswirtschaftlichen Aufgaben vor allem der Aufbau einer kommerziellen Fertigung von Rapid-Prototyping-Modellen mittels unterschiedlicher Verfahren (STLVerfahren, LOM-Verfahren). Mit Partnern zusammen wurde auch die Nutzung der STL-Modelle als Urmodelle für den Vakuumguss aufgebaut. Somit war eine Fertigung einer Kleinserie in unterschiedlichen Polyurethan-Materialien möglich. Damit verbunden waren umfangreiche Marketingaktionen zur Verbreitung des Wissens über die Anwendungsmöglichkeiten des Rapid Product Development.

\section{2/1997 - 03/2011 Entwicklungsingenieur/Konstrukteur, pro forma - Leistungen für den Werkzeugbau GmbH}

Die Anwendung der vollparametrischen CAD-Konstruktion im Rahmen des Werkzeug- und Formenbaus bildete den Schwerpunkt meiner Tätigkeit bei der pro forma $\mathrm{GmbH}$. Technische Basis dafür war das CADSystem PRO/ENGINEER. Dafür wurde unter meiner Leitung ein Fachbereich aufgebaut und eine Vielzahl von Konstruktionsmethoden und Werkzeuge zum Projektmanagement entwickelt. Ziel war, die Nutzung von parametrischen Modellen vom Konstruktionsentwurf über die CAD-Konstruktion einschließlich Zeichnungserstellung bis hin zur Fertigung.

Ein Anwendungsbereich stellte die Konstruktion von Kunststoff-Spritzguss-Werkzeugen dar. Unter Verwendung von vereinheitlichten parametrisierter Werkzeugaufbauten konnten Verkürzungen der Konstruktionszeit von bis zu $60 \%$ erzielt werden. Außerdem war eine automatisierte Ableitung von CADDaten für die Fertigung von Erodier-Elektroden möglich.

In Zusammenarbeit mit der MECO Eckel GmbH \& Co. KG entstanden zahlreiche Anwendungen im Bereich des Metallgusses. Für große Gussteile aus dem Bereich Automotiv, wie beispielsweise Getriebegehäuse, Kurbelgehäuse, erfolgte der Aufbau voll parametrisierter CAD-Modelle. Diese ermöglichen eine schnelle Modellpflege einschließlich Zeichnungsänderung und Ableitung von Messdaten. Auftraggeber waren dabei verschiedenste Automobilkonzerne, wie WW, AUDI, BMW und DAF.

Basierend auf diesen Gussteil-Modellen wurden parametrisierte CAD-Modelle für Druckguss-Einrichtungen und Kokillen gestaltet. Diese CAD-Modelle ermöglichen die automatisierte Ableitung von Werkzeugplatten, Schiebern, Kontureinsätzen und Gießkernen unter Beachtung von unterschiedlichen Schwindungen und lokalen Konturabweichungen (gemessene Gussabweichungen aus dem laufenden Gussprozess) sowie die Ableitung der Fertigungsdokumente. In Zusammenarbeit mit BMW konnte auch ein Schnittstellensystem zur Werkzeugverwaltung mittels SAP-System realisiert werden.

Im Rahmen dieser CAD-Konstruktionsarbeiten wurden auch verschieden Möglichkeiten zur konturnahen Kühlung in die Fertigungseinrichtungen integriert. Auch wurden Möglichkeiten der Nutzung von Methoden des Rapid Prototyping zur Herstellung von Kontureinsätzen untersucht.

\section{4/2011 - Technischer Betriebsleiter, PTZ - Prototypenzentrum GmbH}

Als technischer Betriebsleiter in der PTZ GmbH bin ich wieder auf dem Gebiet des Rapid Product Development tätig. Ich trage dabei Verantwortung für die gesamte Fertigung im Unternehmen. Sie umfasst die 3D-Druck-Techniken, Stereolithographie, Selektiv-Laser-Sintern Kunststoff und Metall, Fused Deposition Modelling Kunststoff und Metall, das Vakuumgießen von Polyurethanen, Polyamiden, Silikonen und Wachs, die Mehr-Achs-CNC-Bearbeitung und den Prototypen-Spritzguss. Für unsere Auftraggeber stellen wir Einzelteile und Kleinserien aus Kunststoff und Metall her. Zunehmend werden aber auch ganze Baugruppen gefertigt, die eine Vielzahl unterschiedlich gefertigter Einzelteile umfasst. Dabei sind unsere Produkte nicht nur im Vorserienbereich und Entwicklung gefragt sondern auch als Serienteile im Einsatz. Dabei spielen zunehmend auch Qualitätssicherungsprozesse einschließlich 3D-Vermessung eine Rolle. Neben der Organisation der Fertigung betreue ich eine Reihe von Fertigungsaufträgen und berate unsere Kunden bei Fragen der Wahl geeigneter Fertigungsverfahren und Materialien. 


\author{
Dr.-Ing. habil Christine Schöne \\ TU Dresden \\ Institut für Maschinenelemente und Maschinenkonstruktion \\ Lehrstuhl Konstruktionstechnik/CAD \\ 01062 Dresden \\ Germany
}

Christine.schoene@tu-dresden.de

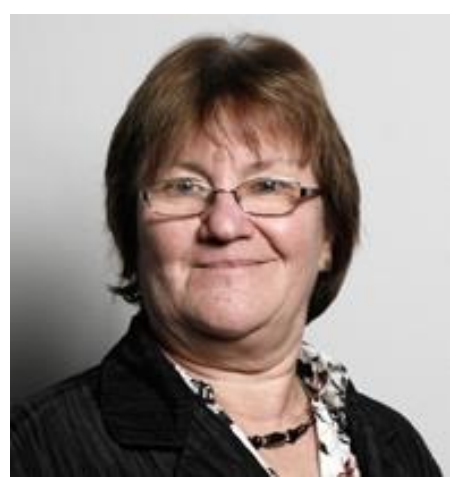

\section{Wissenschaftlicher Werdegang}

1975-1979 Studium an der TU Dresden, Sektion Fertigungstechnik und Werkzeugmaschinen, Diplomarbeit 1979 zum Thema „Algorithmen für die Schnittwertbestimmung beim Schaftfräsen“, Als Hochschullehrer war Prof. Dr.sc.techn. Kochan verantwortlich für die Diplomphase. Dr. Fichtner war zum damaligen Zeitpunkt bereits Dozent, der die Arbeit inhaltlich betreute.

1979-1992 Wissenschaftlicher Assistent an den Lehrstühlen Technologische Programmierung und ab 1990 Fertigungsinformatik. In dieser Zeit wurden die beiden Kinder geboren, wodurch sich eine Verzögerung bei der Promotion ergab.

Promotion zum Dr.-Ing. zum Thema „Arbeitsganggestaltung nach dem Optimierungsprinzip für beliebige Fräsaufgaben in CAD/CAM-Systemen 1992-2005 1992 erfolgte eine Bedarfskündigung an der TU Dresden aus unbefristeter Anstellung. Weitere Wissenschaftliche Mitarbeit im Lehrstuhl Produktionsautomatisierung/Steuerungstechnik folgte mit dem Schwerpunkt 3D-Digitalisieren, Reverse Engineering, Rapid Prototyping in Industriethemen durch Eigenfinanzierung und Themenakquisition.

seit 2005 Wissenschaftlicher Mitarbeiter, Institut für Maschinenelemente und Maschinenkonstruktion, Lehrstuhl Konstruktionstechnik/CAD, Leiterin der Arbeitsgruppe Reverse Engineering, Digitalisierdaten geeignet aufzubereiten und die Methoden weiterzuentwickeln war zwingend notwendig, wenn produktionstechnische Aufgabenstellungen Thema sind.

2009 - 2017 2009: Habilitation an der TU Dresden, Fakultät Maschinenwesen zum Thema: „Reverse Engineering für Freiformflächen in Prozessketten der Produktionstechnik“. Dieses Thema wurde bis 2017 vollumfänglich als Arbeitsgruppenleiterin mit bis zu 8 Mitarbeitern im Lehrstuhl befördert. Seit 2014 kamen Themen des Direct Manufacturing, der Herstellung von Funktionsbauteilen aus Metall und Keramik hinzu, wobei der Schwerpunkt in 
der Konstruktion, Auslegung und Arbeitsvorbereitung für diese Teile liegt.

Diese Synergie ergab sich aus der Nutzung und Behandlung diskreter Daten

in beiden Anwendungsszenarien.

\section{Mit 3D-Digitalisierung zu virtuellen und realen Prototypen - Chancen, Risiken und Grenzen}

Reverse Engineering umfasst die 3D-Digitalisierung eines Objektes, die Aufbereitung der Digitalisierdaten zu CAD-Modellen und die weitere Nutzung dieser Daten in einer CAD/CAMUmgebung zur Erstellung von physischen Objekten. Wesentliche Einsatzgebiete sind der Designbereich des Automobilbaus, der Konsumgüterbereich, der Strömungsmaschinenbau sowie der Medizin, Kunst und Kultur.

In allen Gebieten wurden zwischen 1995 bis 2017 mehr als 40 Forschungsprojekte akquiriert und bearbeitet, die durch die Industrie, AiF, DFG, SAB und BMBF finanziert wurden

Zunehmend wurden im handwerklichen Sektor Methoden des Reverse Engineering und der rechnergestützten Modellierung erfolgreich angewendet. Anhand eigener Referenzprojekte (Musikinstrumentenbau, Restaurierung Rathausmann, Präsentationsvorlage für Nationalparkmuseum, Prozesskette Zahnprothetik und Epithetik, Kieferchirurgie, Orthopädieund Rehatechnik, Optische Messtechnik zur Qualitätssicherung, Einsatzunterstützung CNCTechnik im Handwerk) wurden die Chancen der modernen Technologie sowie deren Grenzen aufgezeigt und befördert.

Seit 2014 wurden außerdem Themen im Direct Manufacturing akquiriert und befördert. Die metallbasierten Verfahren gilt es als Prozess zu betrachten, beginnend von der Konstruktion, Berechnung bis hin zur Fertigungsplanung und Fertigung. Neben Qualitätsthemen wurden Methoden der Topologieoptimierung und Konstruktionsrichtlinie befördert. Die Arbeit mit diskreten Daten im Reverse Engineering lieferte hierzu Synergieeffekte für die Prozessketten im Direct Manufacturing.

Im Januar 2018 wurde die Leitung der Arbeitsgruppe an einen Nachfolger übergeben. 